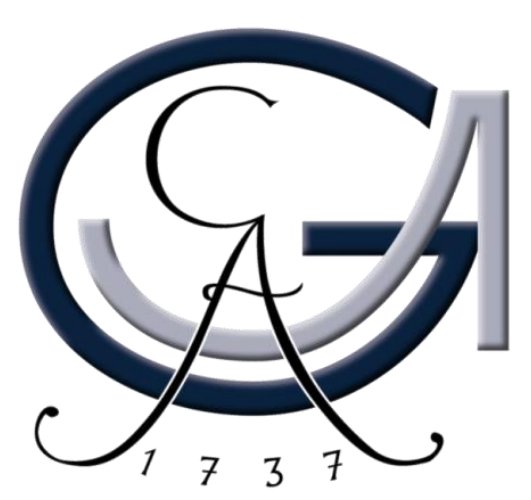

\title{
DER EINFLUSS DER UV-INITIIERTEN RAFT- POLYMERISATION AUF DIE STRUKTUREN UND EIGENSCHAFTEN VON POLYMERNETZWERKEN
}

\author{
Dissertation \\ zur Erlangung des mathematisch-naturwissenschaftlichen Doktorgrades \\ „Doctor rerum naturalium“ \\ an der Georg-August-Universität Göttingen \\ im Promotionsprogramm Chemie \\ der Georg-August University School of Science (GAUSS)
}

vorgelegt von

Rouven Christoph Henkel

aus Salzgitter

Göttingen, 2014 



\section{Betreuungsausschuss:}

Prof. Dr. P. Vana, Makromolekulare Chemie, Institut für Physikalische Chemie,

Georg-August-Universität Göttingen

Prof. Dr. M. Buback, Technische und Makromolekulare Chemie, Institut für Physikalische Chemie, Georg-August-Universität Göttingen

\section{Mitglieder der Prüfungskommission:}

Referent: Prof. Dr. P. Vana, Makromolekulare Chemie, Institut für Physikalische Chemie, Georg-August-Universität Göttingen

Korreferent: Prof. Dr. M. Buback, Technische und Makromolekulare Chemie, Institut für Physikalische Chemie, Georg-August-Universität Göttingen

\section{Weitere Mitglieder der Prüfungskommission:}

Prof. Dr. Peter Botschwina, Theoretische Chemie, Institut für Physikalische Chemie, Georg-August-Universität Göttingen

Prof. Dr. Burkhard Geil, Biophysikalische Chemie, Institut für Physikalische Chemie, Georg-August-Universität Göttingen

Jun.-Prof. Dr. Ricardo Mata, Computerchemie und Biochemie, Institut für Physikalische Chemie, Georg-August-Universität Göttingen

PD Dr. Thomas Zeuch, Physikalische Chemie II, Institut für Physikalische Chemie, Georg-August-Universität Göttingen 



\section{Danksagung}

Mein besonderer Dank gilt Prof. Dr. Philipp Vana dafür, dass er mir die Möglichkeit gegeben hat, diese sehr interessante und herausfordernde Aufgabenstellung in meiner Dissertation zu bearbeiten. Weiterhin möchte ihm für die hilfreichen und motivierenden Diskussionen sowie für seine Unterstützung danken. Auch danke ich Prof. Dr. Michael Buback für die Übernahme des Koreferats. Der Forschungsgesellschaft Druck e. V. in München danke ich dafür, dass durch ihre Initiative diese Arbeit erst möglich wurde. Weiterhin danke ich der Arbeitsgemeinschaft industrieller Forschungsvereinigungen "Otto von Guericke" e.V. (AiF) für die langjährige Finanzierung des Projekts.

Ebenfalls danke ich allen Mitgliedern der Arbeitskreise Makromolekulare Chemie sowie Technische und Makromolekulare Chemie für die gute Stimmung und vor allem die sehr gute Zusammenarbeit innerhalb und zwischen den Abteilungen, die wesentlich zum Gelingen dieser Arbeit beigetragen hat. Heike Rohmann und Sandra Lotze danke ich für viele hilfreiche Tätigkeiten, die das Arbeiten in der Abteilung erst möglich machen. Dr. Hans-Peter Vögele gebührt ebenfalls großer Dank für seine kompetente Unterstützung bei der Handhabung von diversen Messgeräten sowie für seine Bereitschaft mit großem Interesse erhaltene Messergebnisse zu diskutieren. Der Mechanik-Werkstatt des IPCs möchte ich für das große Engagement bei Planung und Herstellung der Teflonformen für die Zugversuch-Probekörper sowie des Rollkugel-Testers danken.

Vielen Dank auch an alle dauerhaften und temporären Büromitbewohner Björn Sölter, JanHendrik Schütz, Sebastian Primpke, Micheal Hendrich, Florian Ehlers, Nino Hinte, Toni Schiller, Lars Lewerdomski, Aline Selke für viele spaßige Diskussionen aller Art und die gute Stimmung im Büro. Bedanken möchte ich mich auch bei meinem Bachelorstudenten Toni Schiller und meinen Praktikanten Alana Sidoruk, Garlef Wartner und Hendrik Schwenson, die ich im Laufe meiner Promotion betreuen durfte. Sie alle haben durch ihr Interesse und ihren Einsatz zum Fortkommen meiner Forschung beigetragen. Sehr großer Dank gilt auch den Korrekturlesern dieser Arbeit Dennis Hübner, Michael Hendrich, Patrick Drawe, Ann-Christin Poeppler, Björn Sölter, Hendrik Kattner, Sebastian Seiffert für die hilfreiche Kritik und die beim Lesen geopferte Zeit. Besonders Ann-Christin möchte ich außerdem für die sehr gute Zusammenarbeit beim gemeinsamen NMR-Projekt danken.

Besonders bedanke ich mich bei meinen Eltern, meinem Bruder und meiner Freundin für die jahrelange Unterstützung und dafür, dass sie immer an mich geglaubt haben. Meiner Freundin Steffi möchte ich außerdem dafür danken, dass sie gerade in der stressigen Zeit des Schreibens dieser Arbeit immer für mich da war und so viel Verständnis aufgebracht hat. Mein ganz besonderer Dank gilt meiner Oma Adele Henkel, die mit mich mein Leben lang auf vielerlei Art und Weise unterstützt hat und bis zuletzt immer sehr großes Interesse an meiner Arbeit und meinem Lebensweg hatte. 

Meinen Eltern

und meiner Oma 



\section{Inhaltsverzeichnis}

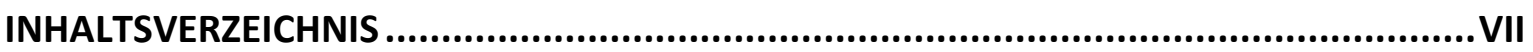

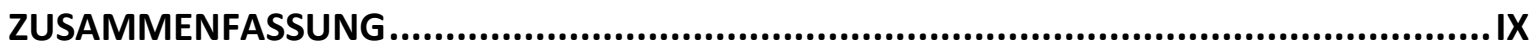

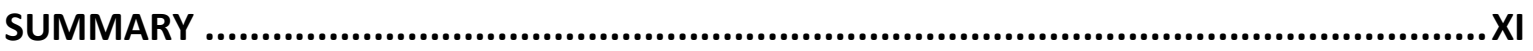

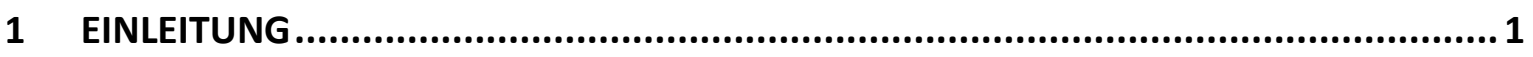

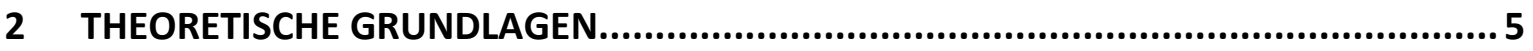

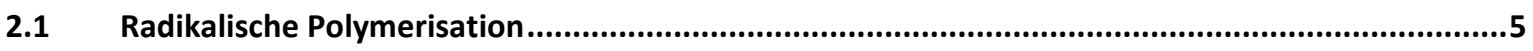

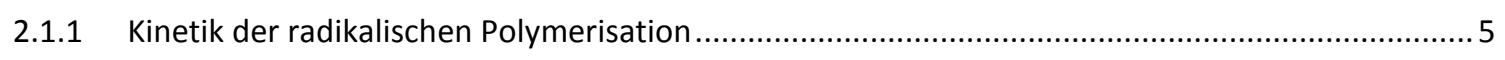

2.1.2 Reversibel-Desaktivierbare Radikalische Polymerisation (RDRP) ......................................... 10

2.1.3 Reversible Addition-Fragmentation chain Transfer (RAFT)-Polymerisation .............................. 12

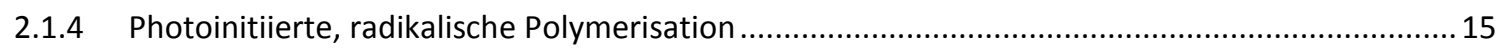

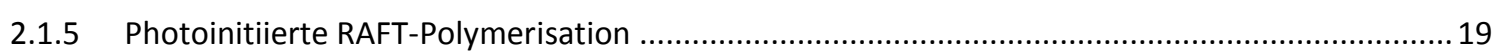

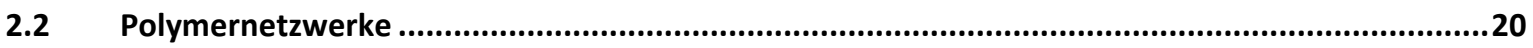

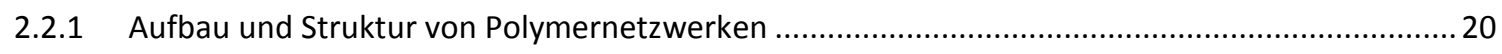

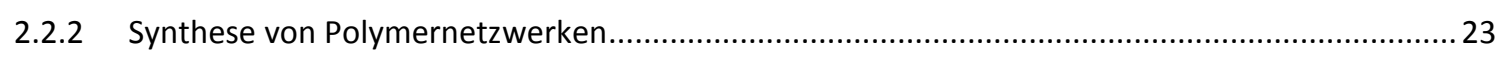

2.3 Mechanische Eigenschaften von Polymernetzwerken ......................................................27

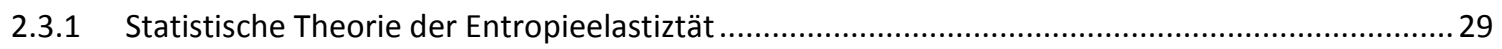

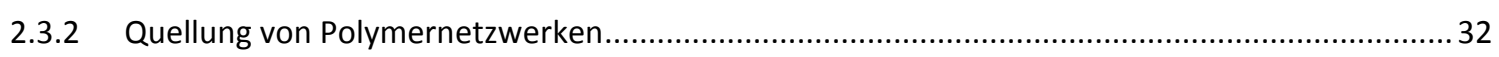

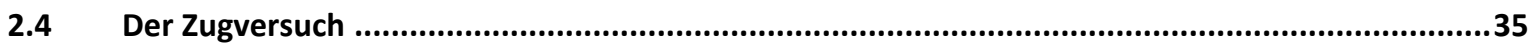

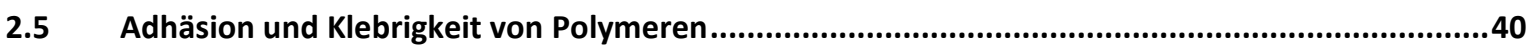

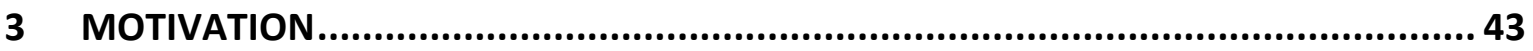

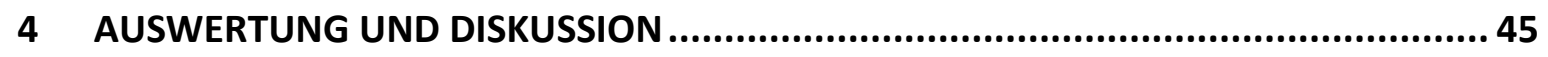

4.1 Der Einfluss der RAFT-Polymerisation auf statistische Poly(butyl-co-1,4-Butandioldiacrylat)- und Poly(ethyl-co-1,4-Butandioldiacrylat)-Netzwerke .........................................................................45

4.1.1 Einfluss der RAFT-Polymerisation auf die mechanischen Eigenschaften der Netzwerke ..............46

4.1.2 Einfluss der RAFT-Polymerisation auf die Glastemperaturen der Netzwerke ..............................71

4.1.3 Einfluss der RAFT-Polymerisation auf die Quellungseigenschaften der Netzwerke ....................74 
4.1.4 Einfluss der RAFT-Polymerisation auf die Kinetik der Reaktion .............................................93

4.1.5 Einfluss der RAFT-Polymerisation auf die Klebrigkeit der Netzwerke.......................................115

4.2 Der Einfluss der RAFT-Polymerisation auf Duroplaste ........................................................131

4.2.1 Einfluss der RAFT-Polymerisation auf Polyethlyenglycoldiacrylat-Netzwerke...........................131

4.2.2 Einfluss der RAFT-Polymerisation auf die Recyclierbarkeit von UV-härtenden Druckfarben ......154

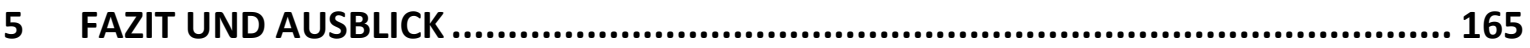

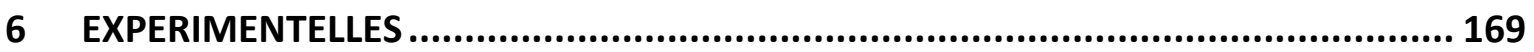

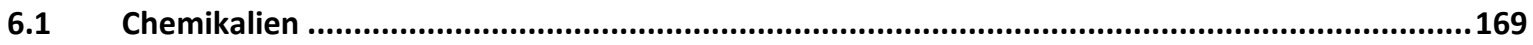

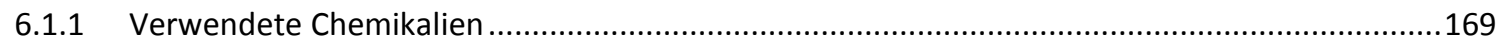

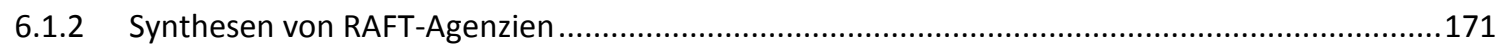

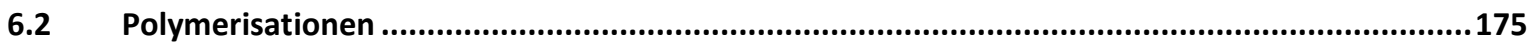

6.2.1 Synthese von Probekörpern für Zugversuche .................................................................175

6.2.2 Synthese von Probekörpern für Rasterkraftmikroskop-Messungen.........................................176

6.2.3 Synthese von Probekörpern für die NMR-Untersuchungen ................................................176

6.2.4 Zusammensetzungen der Polymerisationsmischungen ..................................................177

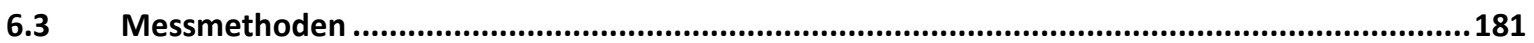

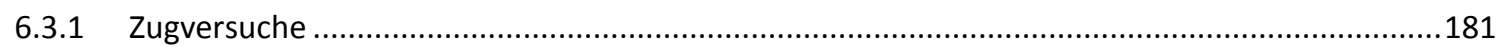

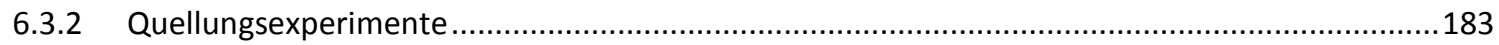

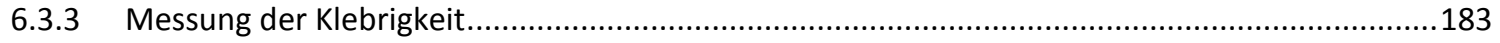

6.3.4 Fourier-Transformations-Infrarot-(FTIR)-Spektroskopie ........................................................ 186

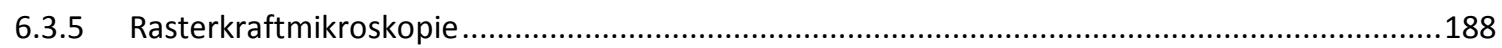

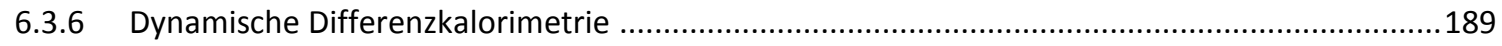

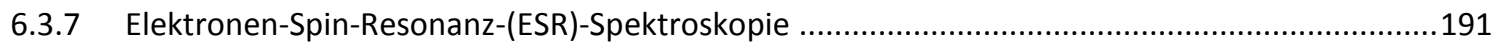

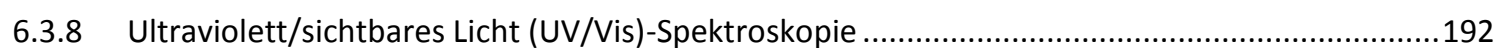

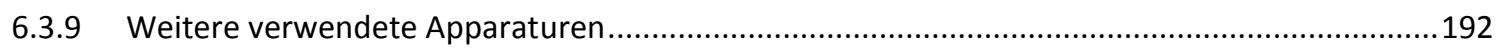

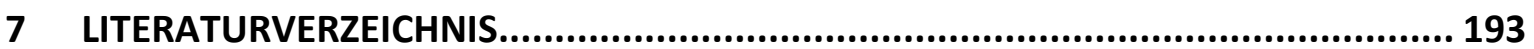

8 VERZEICHNIS DER ABKÜRZUNGEN ...................................................... 207

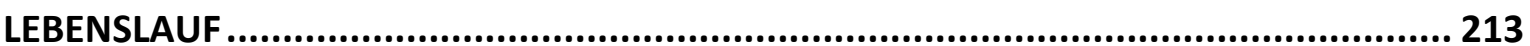




\section{Zusammenfassung}

Im Rahmen dieser Arbeit wurde der Einfluss der Reversible Addition-Fragmentation chain Transfer(RAFT)-Polymerisation auf die Mikrostrukturen und die daraus resultierenden Eigenschaften von Polymernetzwerken untersucht. Dazu wurden elastomere, statistische Poly(butylacrylat-co-1,4-Butandioldiacrylat, BA-co-BDDA)- $\quad$ und Poly(ethylacrylat-co-1,4Butandioldiacrylat, EA-co-BDDA)-Netzwerke über UV-initiierte RAFT-Polymerisation hergestellt. Es wurde der molare Anteil des Vernetzungsreagenz BDDA, sowie der des RAFTAgens $S$-Ethylpropan-2-ylonat-S'-hexyltrithiocarbonat (EPHT) variiert.

Im Vergleich zu ihren Gegenstücken aus UV-initiierter, konventioneller radikalischer Polymerisation erwiesen sie sich die RAFT-Netzwerke in Zugversuchen als weicher: Sie verfügten über kleinere Elastizitätsmoduln sowie bei geringen molaren BDDA-Anteilen von unter $1 \mathrm{~mol}-\%$ über größere Bruchdehnungen. Eine ähnliche Tendenz zeigten in Aceton durchgeführte Quellungsexperimente. Die RAFT-Netzwerke mit BDDA-Anteilen von unter 1 mol-\% zeigten höhere Volumenquellungsgrade als die entsprechenden konventionellen Netzwerke. Bei diesen Experimenten konnte gezeigt werden, dass die geringeren Netzkettendichten der RAFT-Netzwerke auf nicht in das Netzwerk eingebaute Sol-Moleküle sowie auf die Reduzierung von Nanogel-Domänen in den RAFT-Netzwerken zurückgehen. Beides ist eine Folge des durch die RAFT-Polymerisation veränderten Mechanismus der Netzwerkbildung. Große Auswirkungen hatten die veränderten Mikrostrukturen der Netzwerke auch auf deren Klebrigkeit. Eine größere Klebrigkeit der RAFT-Netzwerke konnte mittels Rollkugel- und Probe-Tack-Tests nachgewiesen werden. Durch RasterkraftmikroskopAufnahmen der Oberflächenstrukturen und deren mechanischer Eigenschaften konnte dies durch die Veränderung der Mikrostrukturen der Netzwerke erklärt werden. In ergänzend zu den Quellungsexperimenten durchgeführten, speziellen Slice-Selective Excitation(SSE)-Nuclear Magnetic Resonance (NMR)-Experimenten konnte außerdem gezeigt werden, dass die RAFTNetzwerke über eine homogenere Verteilung der Netzkettendichten verfügen als die entsprechenden konventionellen Netzwerke.

Dass sich der Mechanismus der Netzwerkbildung bei den RAFT-Netzwerken von dem der konventionellen Netzwerke unterscheidet, konnte anhand isothermer Differential Scanning Calorimetry(DSC)-Messungen gezeigt werden: Die konventionellen radikalischen Polymerisationen verliefen schneller und zeigten eine größere Selbstbeschleunigung als die RAFT-Polymerisationen. Deren Polymerisationskinetik ähnelte nicht-vernetzenden RAFTPolymerisationen. Weiterhin konnte im Zuge dieser Messungen eine Retardierung der Polymerisation gefunden werden, die durch die optische Absorption des EPHTs bei der zur UVInitiierung verwendeten Wellenlänge von $366 \mathrm{~nm}$ verursacht wurde. Diese Retardierung führte aber nicht zu den veränderten Mikrostrukturen der RAFT-Netzwerke. Dies wurde durch 
Zugversuche von Poly(BA-co-BDDA)-Netzwerken, denen das Trithiocarbonat $S$-S'-Diethyltrithiocarbonat (DET) hinzugesetzt wurde gezeigt. Die optische Absorption von DET entspricht bei $366 \mathrm{~nm}$ der des EPHTs. DET ist aufgrund seiner molekularen Struktur allerdings nicht in der Lage, als RAFT-Agens zu fungieren, sodass die mechanischen Eigenschaften der resultierenden Netzwerke denen der konventionellen Netzwerke entsprachen.

Ergänzend $\mathrm{zu}$ den Untersuchungen an den Elastomeren wurde der Einfluss des RAFTMechanismus auf die Polymerisation, sowie die Mikrostrukturen von duroplastischen Netzwerken studiert. Diese bestanden aus Polyethylenglycoldiacrylat (PEGDA)-Monomeren mit einer jeweils unterschiedlichen Anzahl von Ethylenglycol-Einheiten (Spacer) zwischen den Acrylat-Gruppen. Bei der Untersuchung der Polymerisationskinetik mittels FourierTransformations-Infrarot (FTIR)-Spektroskopie zeigte sich ebenfalls eine Retardierung der Polymerisation, die mit steigendendem Massenanteil des EPHTs in den Polymerisationsmischungen zunahm. Mittels Elektronen-Spin-Resonanz(ESR)-Spektroskopie und Rasterkraftmikroskop-Messungen konnte gezeigt werden, dass der Einfluss der RAFTPolymerisation auf den inneren Aufbau, sowie die Oberflächenstrukturen dieser Netzwerke nur sehr gering ist. Allerdings wurde die Tendenz gefunden, dass der Einfluss auf die Mikrostrukturen der Netzwerke mit einem größeren Spacer in den PEGDA-Systemen zunimmt. Zusätzlich wurden in einem Kooperationsprojekt mit der Forschungsgesellschaft Druck e. V. (FOGRA) in München erstmals die Auswirkungen der Zugabe von RAFT-Agenzien auf die Recyclierbarkeit der fertigen Druckprodukte aus industriell verwendeten UV-härtenden Druckfarben untersucht. 


\section{Summary}

In this work the influence of the Reversible Addition-Fragmentation chain Transfer (RAFT)polymerization on the microstructures and the resulting properties of polymer networks was investigated. Elastomeric, statistical poly(butylacrylat-co-1,4-butanedioldiacrylate, BA-co-BDDA)and poly(ethylacrylat-co-1,4-butanedioldiacrylate, EA-co-BDDA) networks were synthesized via UV-initiated RAFT polymerization. The amount of the crosslinker BDDA as well as the amount of the RAFT agent $S$-ethyl-propane-2-ylonate- $S$-hexyl-trithiocarbonate (EPHT) were varied.

Compared to their counterparts made from UV-initiated, conventional radical polymerization the RAFT networks were much softer as tensile tests revealed. They featured lesser elasticity moduli and with an amount of BDDA under $1 \mathrm{~mol} \%$ greater elongations at break. A similar tendency was shown in swelling experiments conducted in acetone. The RAFT networks with an amount under $1 \mathrm{~mol} \%$ BDDA featured greater volume degrees of swelling than the corresponding conventional ones. During these experiments it was found that the lower strand densities of the RAFT networks were caused by a part of the sol-fraction which is not added to the overall network as well as the reduction of nanogel domains in the RAFT networks, respectively. Both are a result of the modified mechanism of network building during the RAFT polymerization. The modified microstructures of the RAFT networks had also an enormous effect on the tackiness of the networks. The greater tackiness of the RAFT networks were investigated via rolling ball- as well as probe tack tests, respectively. Using Atomic Force Microscopy (AFM) it was found that this is a consequence of the modified surface structures as well as the mechanical properties of the surfaces. Additionally to the swelling experiments special Slice Selective Excitation (SSE)-Nuclear Magnetic Resonance (NMR)-measurements were conducted. In these experiments it was found that the RAFT networks exhibit a more homogenous distribution of the strand densities than the conventional networks.

On the basis of isothermal Differential Scanning Calorimetry (DSC)-measurements it was found that the conventional radical polymerizations proceeded much faster than the RAFT polymerizations which additionally showed a lesser self-acceleration. The kinetics of the crosslinking RAFT polymerizations were more or less similar to the kinetics of none-crosslinking RAFT polymerizations while the kinetics of crosslinking and none-crosslinking conventional radical polymerization showed a great deviation. Furthermore it was found that the RAFT polymerizations featured an increasing retardation with increasing amount of the RAFT agent. This was caused by the absorption of light at the wavelength of $366 \mathrm{~nm}$ by the RAFT agent EPHT. That this retardation was not the cause for the modified microstructures of the RAFT networks was shown in tensile tests of poly(BA-co-BDDA) networks in which the thiocarbonylthio compound $S$-S-diethyltrithiocarbonate (DET) was added. At $366 \mathrm{~nm}$ the optical 
absorption of DET is similar to EPHT but due to its molecular structure DET is not able to act as a RAFT agent. For that reason, the mechanical properties of the networks synthesized in the presence of DET were similar to networks made by conventional radical polymerization.

Additionally to the investigations which were conducted with the elastomers the influence of the RAFT mechanism on the polymerization as well as the resulting microstructures of thermosetting resins were studied. These resins were built by polyethyleneglycoldiacrylates (PEGDA) with different spacer lengths between the acrylate groups. The polymerization kinetics were investigated via Fourier-Transformation-Infrared (FTIR)-spectroscopy. Again an increasing retardation of the polymerization with increasing amount of the RAFT agent EPHT was found. Using Electron-Spin-Resonance (ESR)-spectroscopy and AFM it was found that there is just a slight influence of the RAFT mechanism on the inner and surface microstructures of these networks. But this influence increased with an increasing spacer length. Furthermore the influence of the RAFT mechanism on the recyclability of hardened UV-curing colors was investigated. This was a cooperation project with the Forschungsgesellschaft Druck e. V. in Munich. 


\section{Einleitung}

Kunststoffe sind ein essentieller Bestandteil des heutigen Lebens und finden eine vielfältige Anwendung im Alltag und in der Technik. Seit den 50er Jahren des letzten Jahrhunderts ist die Produktion weltweit von 1,7 Mio. Tonnen auf 288 Mio. Tonnen im Jahr 2012 gestiegen. ${ }^{[1]}$ Davon entfallen allein 13 Mio. Tonnen auf die Produktion von Kunststoffen in Deutschland. Größtenteils finden Kunststoffe heutzutage europa- und deutschlandweit in der Verpackungsindustrie Verwendung. Aber auch in vielen anderen Industriezweigen wie dem Baugewerbe, der Fahrzeug- und Elektroindustrie und der Konsumgüterindustrie sind sie ein wichtiger Werkstoff. Weiterhin kommen Kunststoffe im Haushalt, für Sport und Freizeit und zunehmend in der Medizin zum Einsatz. ${ }^{[1,2]}$ Aufgrund der vielfältigen Einsatzgebiete waren und sind Kunststoffe über Jahre Gegenstand intensiver Forschung und Weiterentwicklung sowohl in der Industrie als auch in der Wissenschaft. Dies gilt vor allem für die Moleküle aus denen sie aufgebaut sind, die so genannten Polymere.

Eines der Hauptziele in der Polymerchemie bestand sehr lange Zeit darin, eine Möglichkeit zu finden, die Synthese von Polymeren mit wohl definierten Architekturen wie Komposition, Topologie und Funktionalität (s. Abbildung 1-1) zu realisieren. ${ }^{[3]}$ Dieses Ziel erreichte Swarc Mitte der 50er Jahre des letzten Jahrhunderts mit der Entwicklung einer kontrollierten Polymerisationstechnik, der lebenden anionischen Polymerisation. ${ }^{[4]}$ Diese Methode stellt allerdings sehr hohe Ansprüche an die Reaktionsbedingungen. So ist sie sehr anfällig gegenüber Wasser, Sauerstoff oder anderen Verunreinigungen, was sie sehr kosten- und zeitintensiv macht. Darum konnte sie sich auch für industrielle Anwendungen nicht großflächig durchsetzen und findet darum bis heute deutlich weniger Gebrauch als z. B. die radikalische Polymerisation.

Die erste Verwendung dieser Art von Polymerisation liegt dabei schon sehr weit zurück. Im Alten Ägypten wurden während des Mumifizierungsprozesses die ersten, in diesem Fall, photoinitiierten, radikalischen Polymerisationen durchgeführt. ${ }^{[5]}$ Aber erst in den 1920er Jahren war es vor allem Staudinger, der die Grundlagen für die Erforschung der radikalischen Polymerisation legte. ${ }^{[6-8]}$ Einige Jahre später gelang es Flory, die Natur des Kettenwachstums und Abbruchs zu erkennen und als Erster die Kinetik der radikalischen Polymerisation zu beschreiben. ${ }^{[9,10]}$ Aufgrund der vielen Vorteile der radikalischen Polymerisation für industrielle Bedürfnisse entwickelte sie sich bis in die 1980er Jahre zu einer der wichtigsten industriellen Polymerisationsmethoden, sodass sie ungefähr $50 \%$ der weltweiten Kunststoffproduktion ausmachte. $^{[9]} \mathrm{Zu}$ diesen Vorteilen zählt unter anderem die Möglichkeit eine Vielzahl von verschiedensten Monomeren und die Synthese unterschiedlichster Polymerarchitekturen zu realisieren. Da eine einfache Reaktionsführung möglich ist und die Reaktion eine hohe 
Robustheit gegenüber äußeren Einflüssen zeigt, ist es möglich, eine schnelle und kostengünstige Synthese von einer Vielzahl von unterschiedlichsten Kunststoffen zu realisieren. ${ }^{[11-13]}$ Trotz der Konkurrenz von neuartigen Polymerisationsmethoden, wie z. B. der bereits genannten anionischen Polymerisation, betrug der Anteil von synthetischen Polymeren aus radikalischer Polymerisation an der weltweiten Gesamtproduktion von Kunstoffen im Jahr 2009 noch immer etwa $45 \%(\approx 100$ Millionen Tonnen) und $40 \%$ bei synthetischem Gummi $(\approx 4,6$ Millionen Tonnen). ${ }^{[9]}$ Vor allem die photoinitiierte, radikalische Polymerisation nimmt dabei in den letzten 30 Jahren wieder eine zunehmend wichtige Rolle ein. ${ }^{[14]}$

Der Nachteil der radikalischen Polymerisation ist allerdings, dass sie sehr limitiert ist in ihren Möglichkeiten, zielgenau Polymere mit genau definiertem Kompositionen, Topologien oder Funktionalitäten (s. Abbildung 1-1), woraus sich spezifische Eigenschaften ergeben, herzustellen. Da aber Polymermaterialien in vielen technischen Bereichen z. B. der Nanotechnologie ${ }^{[15,16]}$, medizinischen Bereichen z. B. der Pharmakologie ${ }^{[17]}$ und in wissenschaftlichen Bereichen zunehmend hochspezifische Aufgaben zu erfüllen haben, ist die wirtschaftliche Synthese von für den entsprechenden Verwendungszweck, zugeschnittenen Polymeren, immer häufiger von großer Bedeutung. Weiterhin ist die Kenntnis von den Struktur-Eigenschaftsbeziehungen der Polymere ein wesentlicher Faktor, um die Polymere zielgerichtet, entsprechend ihrer Aufgaben herzustellen.

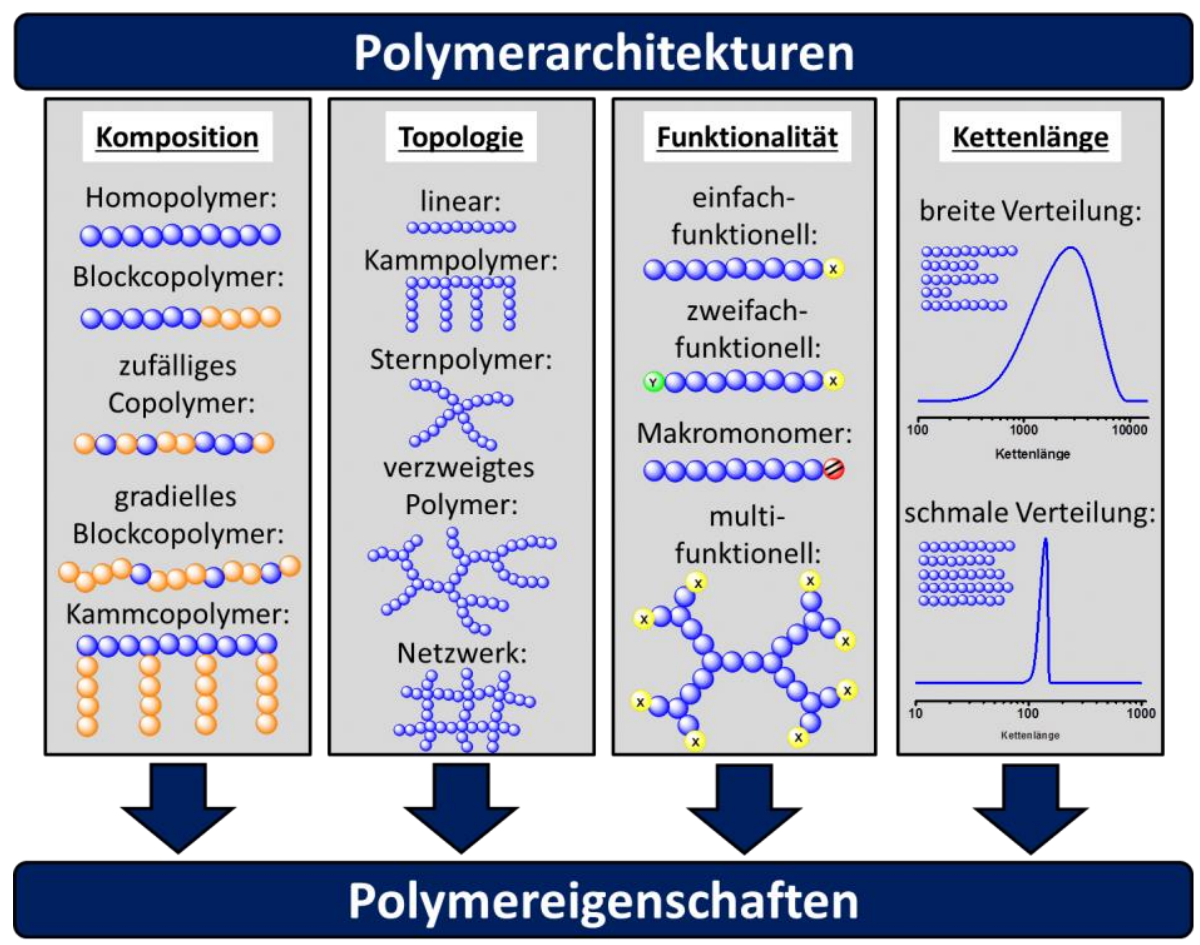

Abbildung 1-1 Möglicher Aufbau und mögliche Strukturen sowie Funktionalitäten von Makromolekülen, die aus radikalischer Polymerisation synthetisierbar sind und jeweils $\mathrm{zu}$ anderen Eigenschaften der Polymere führen. Allerdings ist für einige der Architekturen die Verwendung von speziellen Techniken, wie z. B. der anionischen Polymerisation notwendig. 
Dies gilt insbesondere für Polymernetzwerke deren Eigenschaften sehr stark von ihrem mikroskopischen Aufbau und ihrer inneren Struktur abhängen. ${ }^{[18]}$ Diese weisen bei der klassischen Synthese aus radikalischer Polymerisation häufig eine sehr große Heterogenität auf, welche es sehr aufwendig macht, die Polymernetzwerke an ihren speziellen Verwendungszweck anzupassen.

Seit der Mitte der 1980er Jahre wurden zunehmend neue Synthesemethoden entwickelt, die die Vorteile einer radikalischen Polymerisation mit den Vorteilen der kontrollierten Polymerisation vereinen. Somit ist die schnelle und kostengünstige Synthese von speziell an ihre Verwendung zugeschnittenen Polymeren und Polymernetzwerken möglich geworden. Eine dieser Methoden hat sich dabei als besonders vielfältig erwiesen: Die so genannte Reversible AdditionFragmentation chain Transfer Polymerization oder RAFT-Polymerisation. Diese ermöglicht die Synthese von einer Vielzahl unterschiedlichster Polymere mit genau definierten Nanostrukturen ${ }^{[19]}$ und findet darum auch in einigen industriellen Prozessen schon verbreitete Verwendung. ${ }^{[12,20]}$

Im Rahmen der vorliegenden Arbeit soll der Einfluss der RAFT-Polymerisation auf den Aufbau und die Strukturen von Polymernetzwerken hergestellt aus photoinitiierter, radikalischer Polymerisation untersucht werden. Darum soll im Laufe der folgenden Kapitel zunächst die theoretischen Grundlagen, der an der Polymerisation beteiligten Mechanismen erläutert werden. Außerdem wird gezeigt, wie die mikroskopischen Strukturen von Polymernetzwerken die Eigenschaften der Netzwerke beeinflussen. Anschließend werden die erhaltenen Ergebnisse vorgestellt, diskutiert und in den Rahmen der theoretischen Grundlagen eingeordnet. 



\section{Theoretische Grundlagen}

\subsection{Radikalische Polymerisation}

\subsubsection{Kinetik der radikalischen Polymerisation}

Die radikalische Polymerisation stellt eine typische Kettenwachstumsreaktion dar. Dabei wird eine Substanz, die über ein ungepaartes Elektron verfügt, die Radikalfunktion, an die Kohlenstoff-Kohlenstoff-Doppelbindung eines Monomers addiert. Dieses Monomer ist nun selber Träger der Radikalfunktion und in der Lage, immer weitere Monomere zu addieren. Der Verlauf der radikalischen Polymerisation wird in vier wesentliche Schritte unterteilt: ${ }^{[1]}$

(1) Initiierung und Kettenstart:

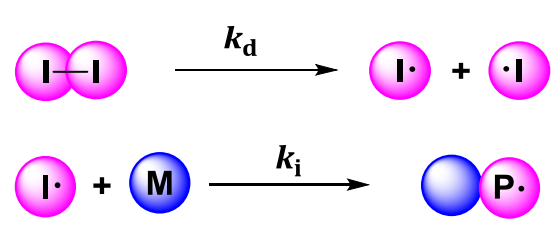

(2) Kettenwachstum:

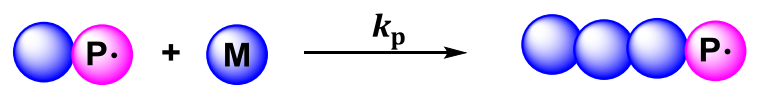

(3) Kettenabbruch:

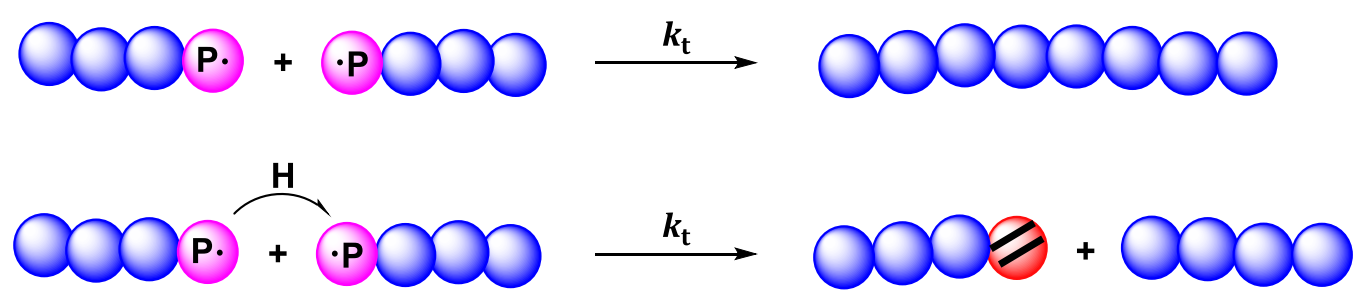

(4) Kettentransfer:

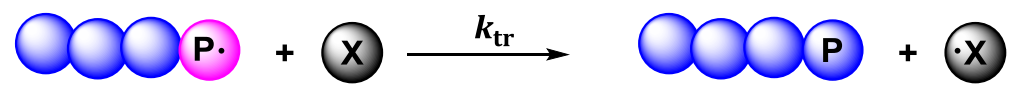

Schema 2-1 Darstellung des Reaktionsverlaufs einer radikalischen Polymerisation. 
Für eine nähere Diskussion der idealen radikalischen Polymerisationskinetik sollte von folgenden Annahmen ausgegangen werden: ${ }^{[21,22]}$

- Alle Polymerradikale besitzen die gleiche Reaktivität, unabhängig von ihrer Kettenlänge.

- Monomere werden nur durch Wachstumsschritte verbraucht.

- Nach dem Bodenstein'schen Stationaritätsprinzip ist die Radikalkonzentration während der Polymerisation konstant.

- Alle Reaktionen sind irreversibel.

- Der Kettenabbruch erfolgt ausschließlich durch Kombination oder Disproportionierung.

Der erste Schritt der radikalischen Polymerisation besteht in der Bildung von so genannten Primärradikalen aus einem Initiatormolekül. Dies kann z. B. durch eine homolytische Bindungsspaltung, sowohl thermisch, chemisch als auch photochemisch, vonstattengehen. Für den, in Schema 2-1 gezeigten Fall, dass der Initiator in zwei propagationsfähige Primärradikale zerfällt, gilt für die Reaktionsgeschwindigkeit $r_{\mathrm{d}}$ :

$$
r_{\mathrm{d}}=-\frac{1}{2} \cdot \frac{\mathrm{d} c_{\mathrm{I}-\mathrm{I}}}{\mathrm{d} t}=\frac{d c_{\mathrm{I}}}{\mathrm{d} t}=2 k_{\mathrm{d}} c_{\mathrm{I}-\mathrm{I}}
$$

mit $t$ als Reaktionszeit, $c_{\mathrm{I}-\mathrm{I}}$ als Initiatorkonzentration, $c_{\mathrm{I}}$. als Konzentration der Primärradikale und $k_{\mathrm{d}}$ als Geschwindigkoeffizient der Zerfallsreaktion. Da Radikale im Normalfall sehr reaktiv sind, kann es nach dem Zerfall des Initiators zu Nebenreaktionen kommen, sodass niemals alle gebildeten Primärradikale an ein Monomer addieren, um eine Polymerisation zu starten. Um dies zu berücksichtigen muss in Gleichung (1) ein weiterer Faktor eingeführt werden, die Initiatoreffizienz $f_{\mathrm{I}}$ :

$$
r_{\mathrm{d}}=2 k_{\mathrm{d}} \cdot f_{\mathrm{I}} \cdot c_{\mathrm{I}-\mathrm{I}}
$$

Die Initiierung verläuft deutlich langsamer als die eigentliche Kettenstartreaktion. Somit ist sie für den Beginn einer radikalischen Polymerisation geschwindigkeitsbestimmend, sodass die Anlagerung eines Primärradikals an ein Monomer für die Kinetik der Reaktion unbedeutend ist. $^{\text {[23] }}$

Der nächste Schritt einer radikalischen Polymerisation ist das Kettenwachstum (s. Schema 2-1) oder auch Propagation. Das Makroradikal wächst durch Anlagerung von weiteren Monomeren 
solange weiter bis es entweder terminiert oder die Radikalfunktion über eine Transferreaktion abgibt. Für die Reaktionsgeschwindigkeit der Propagation $r_{\mathrm{p}}$ gilt:

$$
r_{\mathrm{p}}=-\frac{\mathrm{d} c_{\mathrm{M}}}{\mathrm{d} t}=k_{\mathrm{p}} \cdot c_{\mathrm{M}} \cdot c_{\mathrm{P}}
$$

Die Propagation eines Makroradikals hängt also sowohl von der Konzentration des Monomers $c_{\mathrm{M}}$ als auch von der Konzentration der Radikalspezies $c_{\mathrm{P}}$. ab. Die Geschwindigkeit der Propagation ist also jeweils 1. Ordnung in Bezug auf die jeweilige Spezies. Der Geschwindigkeitskoeffizient der Propagation $k_{\mathrm{p}}$ ist dabei, wie oben erwähnt, idealerweise unabhängig von der Kettenlänge der Radikalspezies.

Für den Kettenabbruch oder die Terminierung existieren, wie in Schema 2-1 gezeigt, zwei Möglichkeiten: zum einen die Rekombination von zwei wachsenden Makroradikalen zu einem doppelt so langen Polymer und zum anderen die Disproportionierung. Bei letzterem wird ein Wasserstoffatom von einem Makroradikal an ein zweites übertragen, sodass sich zwei Polymere bilden. Eines dieser Polymere verfügt über eine polymerisierbare Doppelbindung und stellt somit ein Makromonomer dar. Das zweite Polymer ist genau wie das Rekombinationsprodukt ein so genanntes „totes“ Polymer. Es verfügt über keine weiteren Funktionalitäten, um an einem der Schritte einer radikalischen Polymerisation teilzunehmen. Für die Bestimmung der Reaktionsgeschwindigkeit $r_{\mathrm{t}}$ der Abbruchreaktion werden beide Möglichkeiten im Geschwindigkeitskoeffizienten $k_{\mathrm{t}}$ zusammengefasst.

$$
r_{\mathrm{t}}=-\frac{\mathrm{d} c_{\mathrm{P}}}{\mathrm{d} t}=2 k_{\mathrm{t}} \cdot c_{\mathrm{P} .}^{2}
$$

Die Geschwindigkeit der Terminierung ist also 2. Ordnung in Bezug auf die Konzentration der Radikalspezies. Es müssen immer zwei Radikale zusammentreffen um zu terminieren. Damit ist die Terminierung stark von der Fähigkeit der Radikale abhängig z. B. durch Diffusion einander so nahe zu kommen, dass eine Reaktion möglich ist. Dies kann vor allem bei z. B. netzwerkbildenden, radikalischen Polymerisationen oder auch bei sehr hohen Umsätzen dazu führen, dass es zu deutlichen Abweichungen von der idealen Kinetik kommt. Durch die steigende Viskosität der Reaktionsmischung ist es für die großen Makroradikalen zunehmend schwerer zueinander zu diffundieren. Sie können nicht mehr miteinander terminieren und die Radikalkonzentration im System steigt immer mehr an, da durch fortschreitende Initiierung weitere Radikale nachgeliefert werden. Es kommt zu einer so genannten Selbstbeschleunigung der Reaktion, bei der die Geschwindigkeit der Gesamtreaktion im Vergleich zur idealen Kinetik 
sehr stark ansteigt. Dieser Effekt wird Gel-Effekt oder auch Norrish-Trommsdorff-Effekt ${ }^{[24]}$ genannt.

Um die Geschwindigkeit der Gesamtreaktion der radikalischen Polymerisation $R_{\mathrm{P}}$ zu beschreiben, muss ein quasi-stationärer Zustand angenommen werden. In diesem Fall ist die Zahl der Radikale, die neu gebildet werden gleich der Zahl der Terminierenden. Somit können auch die Geschwindigkeitsgesetze für beide Reaktionen gleichgesetzt werden:

$$
2 k_{\mathrm{d}} \cdot f_{\mathrm{I}} \cdot c_{\mathrm{I}-\mathrm{I}}=2 k_{\mathrm{t}} \cdot c_{\mathrm{P}}{ }^{2}
$$

Wird Gleichung (5) nach der Radikalkonzentration aufgelöst und in Gleichung (4) eingesetzt, ergibt sich für die Gesamtgeschwindigkeit der radikalischen Polymerisation $R_{\mathrm{P}}$ :

$$
R_{\mathrm{P}}=-\frac{\mathrm{d} c_{\mathrm{M}}}{\mathrm{d} t}=k_{\mathrm{p}}\left(f_{\mathrm{I}} \cdot \frac{k_{\mathrm{i}}}{k_{\mathrm{t}}}\right)^{1 / 2} \cdot c_{\mathrm{M}} \cdot c_{\mathrm{I}-\mathrm{I}}^{1 / 2}
$$

Mit dem Proportionalitätsfaktor $K_{\mathrm{P}}$ :

$$
K_{\mathrm{P}}=k_{\mathrm{p}}\left(f_{\mathrm{I}} \cdot \frac{k_{\mathrm{i}}}{k_{\mathrm{t}}}\right)^{1 / 2} \cdot c_{\mathrm{I}-\mathrm{I}}^{1 / 2}
$$

folgt für die Geschwindigkeit der Gesamtreaktion:

$$
R_{\mathrm{P}}=-\frac{\mathrm{d} c_{\mathrm{M}}}{\mathrm{d} t}=K_{\mathrm{P}} \cdot c_{\mathrm{M}}
$$

Nach Integration über die Reaktionszeit von 0 bis $t$ ergibt sich:

$$
\ln \left(\frac{c_{\mathrm{M}}^{0}}{c_{\mathrm{M}}^{t}}\right)=K_{\mathrm{P}} \cdot t
$$

Für eine ideal verlaufende radikalische Polymerisation sollte die Auftragung des Logarithmus auf der linken Seite von Gleichung (9) gegen die Reaktionszeit $t$ eine Gerade ergeben. Abweichungen nach oben würden für eine Selbstbeschleunigung der Reaktion, nach unten für eine mögliche Retardierung sprechen (s. Abbildung 2-1). 


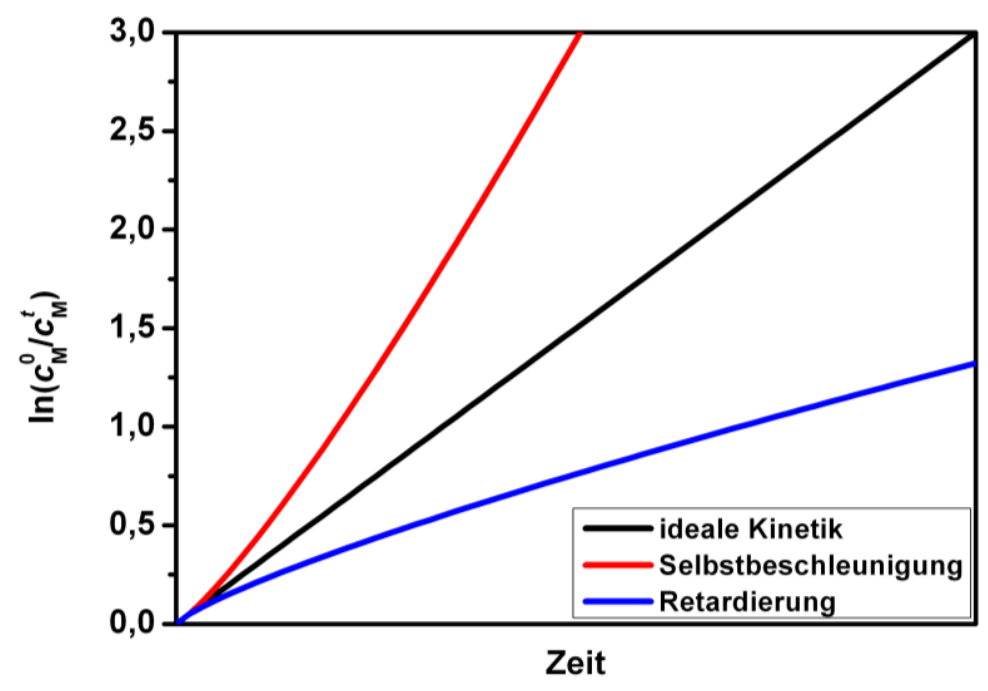

Abbildung 2-1 Auftragung der integrierten Form des Geschwindigkeitsgesetzes gegen die Reaktionszeit einer idealen (-), dem Gel-Effekt unterliegenden (-) und einer retardierten radikalischen Polymerisation (-).

Eine weitere Reaktionsmöglichkeit der Makroradikale ist, neben Wachstum und Abbruch, der Transfer der Radikalfunktion auf ein anderes Molekül X (s. Schema 2-1). Dabei kann es sich z. B. um ein Monomer, ein Polymermolekül oder auch ein spezielles Kettentransferagens (engl.: Chain Transfer Agent, CTA) handeln. Für die Geschwindigkeit des Transfers $r_{\text {tr }}$ lässt sich folgendes Geschwindigkeitsgesetz aufstellen:

$$
r_{\mathrm{tr}}=-\frac{\mathrm{d} c_{\mathrm{X}}}{\mathrm{d} t}=2 k_{\mathrm{tr}} \cdot c_{\mathrm{X}} \cdot c_{\mathrm{P}}
$$

mit $k_{\text {tr }}$ als Geschwindigkeitskoeffizient der Transferreaktion und $c_{\mathrm{X}}$ als Konzentration des Transferagens. Wenn es sich bei der Substanz X um ein speziell zugesetztes CTA handelt, so kann die Effizienz dieser Substanz anhand der Transferkonstante $K_{\mathrm{tr}}$ beschrieben werden. Hierbei handelt es sich um das Verhältnis des Geschwindigkeitskoeffizienten der Transferreaktion zu dem der Propagation:

$$
K_{\mathrm{tr}}=\frac{k_{\mathrm{tr}}}{k_{\mathrm{p}}}
$$

Der Kettentransfer hat keinen Einfluss auf die Radikalkonzentration im betrachteten System. Das bedeutet, er sollte auch keinen Einfluss auf Polymerisationsgeschwindigkeit haben. Eine Auftragung entsprechend Abbildung 2-1 sollte also für eine radikalische Polymerisation mit 
Kettentransfer den gleichen Kurvenverlauf liefern wie eine radikalische Polymerisation ohne Kettentransfer. Dies ist allerdings nur der Fall, wenn die Reinitiierung einer Polymerkette, also die Addition des CTA an ein Monomer, schnell verläuft, sodass das Radikal der Polymerisationsreaktion direkt wieder zur Verfügung steht. Die Radikalkonzentration bleibt somit konstant. Ist dies nicht der Fall, kommt es zu einer Retardierung der Reaktion. Der Transfer der Radikalfunktion hat also im idealen Fall keinen Einfluss auf die Gesamtkinetik der radikalischen Polymerisation. Allerdings wird das Wachstum einer Kette beendet. Somit hat der Transfer der Radikalfunktion hauptsächlich einen Einfluss auf die molare Masse der aus der Polymerisation resultierenden Polymere.

Wie schon ganz zu Beginn in der Einleitung erwähnt, ist der große Nachteil der radikalischen Polymerisation, dass sie aufgrund ihrer radikalischen Natur kaum Möglichkeiten bietet, Einfluss auf die Struktur und Zusammensetzung der fertigen Produkte zu nehmen. So sind die molaren Massen der resultierenden Polymere häufig sehr breit verteilt (hohe Dispersität). Weiterhin ist es kaum bzw. gar nicht möglich Einfluss auf die Mikrostrukturen der Polymere zu nehmen und somit gezielt besondere Polymerarchitekturen wie z. B. Kamm- , Sternpolymere und Netzwerke mit homogenen bzw. definierten Mikrostrukturen oder bestimmte Polymerkompositionen wie z. B. Blockcopolymere mit genau definierten Blockgrößen zu synthetisieren.

\subsubsection{Reversibel-Desaktivierbare Radikalische Polymerisation (RDRP)}

Der Begriff Reversibel-Desaktivierbare Radikalische Polymerisation (RDRP), früher auch als Kontrollierte oder Lebende Radikalische Polymerisation bezeichnet, ${ }^{[25]}$ umfasst eine große Anzahl verschiedener Methoden zur Realisierung des gewünschten Reaktionsverlaufes. Als prominenteste Vertreter der RDRP sind vor allem die Nitroxide Mediated Polymerization (NMP), ${ }^{[26]}$ die Atom Transfer Radical Polymerization (ATRP) ${ }^{[27,28]}$ und die Reversible AdditionFragmentation chain Transfer (RAFT-) Polymerisation ${ }^{[29]}$ zu nennen. Bei diesen Methoden zur Reaktionsführung einer radikalischen Polymerisation liegt das Prinzip zugrunde, die propagierenden Ketten anhand eines, zur Polymerisation hinzugegebenen Reagenz, reversibel zu desaktivieren. Während die Desaktivierung entweder durch eine Kupplungsreaktion zwischen einer stabilen Radikalspezies wie z. B. einer Nitroxid-Verbindung und der Radikalfunktion der propagierenden Kette (NMP) oder durch Übertragung eines Halogenids auf das aktive Kettenende (ATRP) erreicht wird, handelt es sich bei der RAFT-Polymerisation um einen degenerativen Kettentransfer (s. Kapitel 2.1.3). Das jeweilige, zugesetzte Reagenz reagiert mit der Radikalfunktion des wachsenden Makroradikals und bildet somit eine nicht radikale Spezies, die nicht in der Lage ist, weitere Monomereinheiten zu addieren (s. Schema 2-2). Häufig wird in diesem Zusammenhang auch von „schlafenden“ oder desaktivierten Ketten gesprochen. 


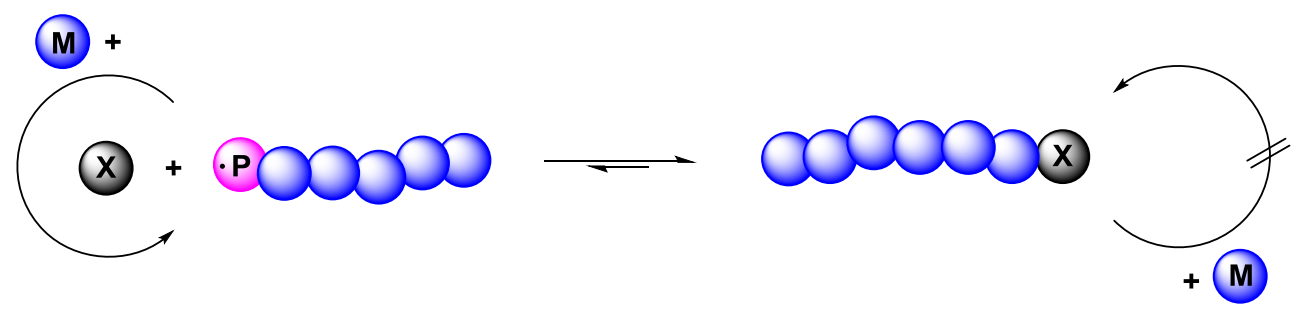

Schema 2-2 Darstellung des RDRP-Prinzips.

Es kommt zur Ausbildung eines dynamischen Gleichgewichts zwischen der propagierenden und der schlafenden Polymerkette. Bei der ATRP und der NMP liegt dieses Gleichgewicht dabei immer auf der Seite der desaktivierten Spezies, was die Anzahl von propagationsfähigen Radikalen in der Polymerisation im Allgemeinen stark reduziert. Dabei wird bei diesen beiden Methoden der so genannte Persistent Radical Effect ${ }^{[30]}$ (PRE) genutzt. Bei den zugesetzten Agenzien handelt es sich um sehr stabile Radikale (engl.: persistent radicals), die nicht in der Lage sind, an ein Monomer zu addieren und somit ein propagierendes Makroradikal zu bilden.

Daraus resultiert, dass die Wahrscheinlichkeit von Terminierungsreaktionen zwischen zwei aktiven Ketten ebenfalls deutlich reduziert ist. Damit geht häufig eine langsamere Geschwindigkeit der Polymerisation im Vergleich zur konventionellen radikalischen Polymerisation einher. Der hiervon abweichende Mechanismus der RAFT-Polymerisation wird im folgenden Kapitel näher diskutiert.

Bei einer RDRP können Polymerketten so lange weiter wachsen bis das gesamte Monomer im Reaktionsvolumen aufgebraucht ist. Die Lebensdauer der Ketten ist gegenüber einer konventionellen radikalischen Polymerisation deutlich verlängert. Zusätzlich bleibt das aktivierbare Zentrum an den Ketten auch nach einem Abbruch der Polymerisation, oder nachdem das gesamte Monomer verbraucht ist, an den Ketten erhalten. Dies ermöglicht es, weitere Polymerisationen an den Ketten durchzuführen oder bestimmte funktionelle Gruppen in die Polymere einzubringen.

Die RDRP bietet dadurch die Möglichkeit, die Nachteile der konventionellen radikalischen Polymerisation zu umgehen. Es ist mit dieser Methode möglich, Polymere mit sehr schmalen Molmassenverteilungen und eine Vielzahl von verschiedener Polymerarchitekturen (z. B. linear, sternförmig, Dendrimere, Netzwerke) oder Kompositionen (Homo-, Block, Pfropfpolymere) der Polymere zu erhalten ${ }^{[19,31,32]}$ Die RDRP ist somit in der Lage, die Vorteile einer radikalischen Polymerisation, wie z. B. die schnelle und einfache Reaktionsführung, mit den Vorteilen einer kontrollierten Polymerisation, wie z. B. Einfluss auf die Dispersität oder die Struktur der Produkte, zu verbinden.

Da im Rahmen der vorliegenden Arbeit im Wesentlichen mit der RAFT-Polymerisation gearbeitet wurde, soll diese im Folgenden näher vorgestellt und erläutert werden. 


\subsubsection{Reversible Addition-Fragmentation chain Transfer (RAFT)- Polymerisation}

Wie im vorherigen Kapitel erwähnt, stellt die RAFT-Polymerisation einen Sonderfall der RDRP dar. Bei der RAFT-Polymerisation findet an einem speziellen Transferagens, dem so genannten RAFT-Agens (s. Schema 2-3), ein reversibler, thermodynamisch neutraler (degenerativer) Kettentransfer statt. Dieser verläuft im Vergleich zur Propagation der Ketten sehr schnell. ${ }^{[33]}$ Dabei wird die Radikalfunktion über das RAFT-Agens zwischen einer aktiven, propagierenden Kette und einer inaktiven, desaktivierten Kette ausgetauscht.

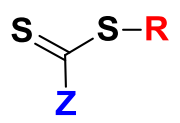

\section{R: Abgangsgruppe \\ Z: stabilisierende Gruppe}

Abbildung 2-2 Allgemeine Struktur eines RAFT-Agens.

Das RAFT-Agens verfügt immer über eine so genannte Z-Gruppe, die das während der Polymerisation auftretende Intermediatradikal stabilisieren soll und über eine R-Gruppe, die das RAFT-Agens verlassen kann und für die Initiierung einer neuen Kettenwachstumsreaktion sorgen soll. Welche Auswirkungen dies auf den Mechanismus der Reaktion hat, wird im Folgenden anhand des Reaktionsschemas einer RAFT-Polymerisation (s. Schema 2-3) erläutert.

Wie bei der konventionellen radikalischen Polymerisation findet die Initiierung über konventionelle Initiatoren statt. Die gebildeten Radikale addieren an ein Monomer und starten somit das Wachstum einer Polymerkette (1). Anschließend findet allerdings keine freie Propagation statt, sondern das Kettenradikal addiert an die Thiocarbonylfunktion des RAFTAgens. Es stellt sich ein so genanntes Vorgleichgewicht (2) ein. Die Radikalfunktion wird auf das RAFT-Agens übertragen, wobei sich ein Intermediatradikal bildet. Anschließend kommt es zur Fragmentierung des RAFT-Agens bei der die R-Gruppe abgespalten wird, wodurch sich ein Makro-RAFT-Agens und ein Radikal bilden. Dieses sollte im Folgenden in der Lage sein, eine neue Kettenwachstumsreaktion zu starten (3). Es wird bei diesem Schritt auch von Reinitiierung gesprochen. Das aus der R-Gruppe hervorgegangene Makroradikal addiert sehr schnell wieder an das im Vorgleichgewicht gebildete Makro-RAFT-Agens und es kommt zur Ausbildung des Hauptgleichgewichts der RAFT-Polymerisation (4). In diesem Schritt wird die Radikalfunktion zwischen zwei wachsenden Ketten über das RAFT-Agens reversibel ausgetauscht. Passiert dieser Austausch ausreichend schnell und ist die Konzentration des RAFT-Agens ausreichend groß, wachsen nahezu alle Ketten mit gleicher Wahrscheinlichkeit parallel nebeneinander, wodurch letztlich alle Polymere eine ähnliche Kettenlänge erreichen. 
(1) Initiierung und Kettenstart:

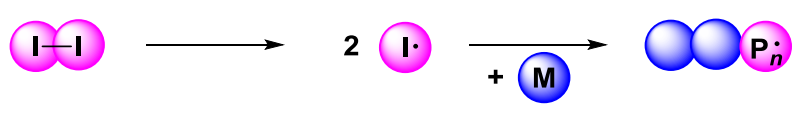

(2) Vorgleichgewicht:

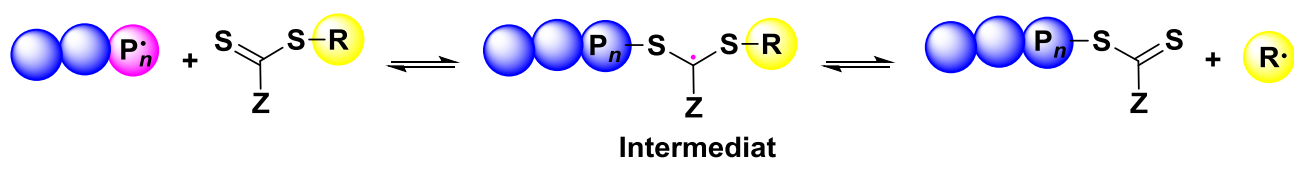

(3) Reinitiierung:

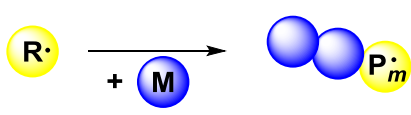

(4) Hauptgleichgewicht:

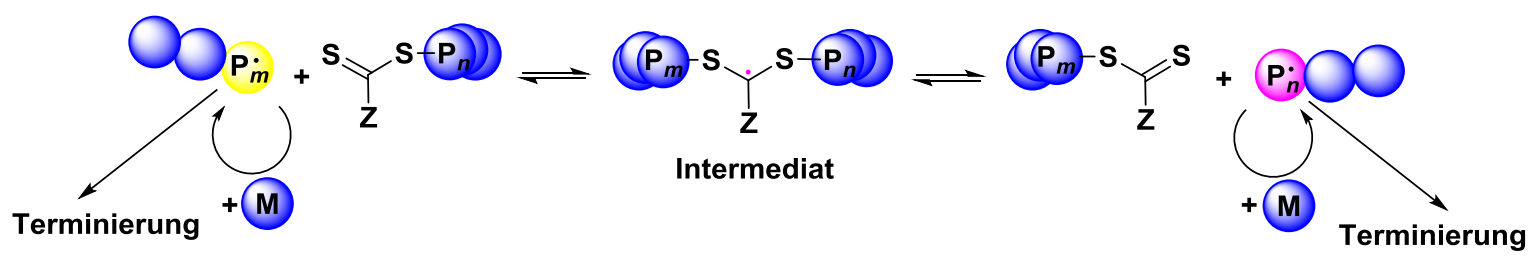

Schema 2-3 Darstellung des Reaktionsverlaufs einer RAFT-Polymerisation.

Dies führt zu einer schmalen Verteilung der molaren Massen der Polymere. Dabei besteht bei der RAFT-Polymerisation die Möglichkeit, die theoretisch möglichen Molmassen der Polymere $\bar{M}_{n}^{\text {theo }}$ über die Konzentration des RAFT-Agens $c_{\text {RAFT }}$ zu steuern. Für eine ideal verlaufende RAFT-Polymerisation gilt folgende Beziehung:

$$
\bar{M}_{n}^{\text {theo }}=\frac{c_{\mathrm{M}} \cdot M_{\mathrm{M}} \cdot C_{\mathrm{M}}}{c_{\mathrm{RAFT}}}+M_{\mathrm{RAFT}}
$$

Dabei stehen $M_{\mathrm{M}}$ und $M_{\mathrm{RAFT}}$ für die molaren Massen des Monomers bzw. des RAFT-Agens. $C_{\mathrm{M}}$ steht für den Umsatz des Monomers. Es ist erkennbar, dass die theoretischen Molmassen der Polymere mit steigender Konzentration des RAFT-Agens abnehmen.

Die Effizienz des gesamten Austauschprozesses hängt sehr stark von der chemischen Natur der Z- sowie der R-Gruppe ab. Über diese Gruppen können die RAFT-Agenzien dabei gezielt an das jeweilige Polymerisationssystem (z. B. Monomere oder Prozessführung) angepasst werden. ${ }^{[34-37]}$ 
Bei der Z-Gruppe kann es sich um unterschiedliche Gruppen handeln. Bislang wurden vor allem Phenyl- $^{[38-41]}$ (Dithioester) und Thioalkylgruppen ${ }^{[42-44]}$ (Trithiocarbonate) verwendet und beschrieben. RAFT-Agenzien mit diesen Gruppen zeichnen sich durch eine relativ einfache Synthese, ein hohes Maß an Kontrolle über die Polymerisation und eine hohe Variabilität in den verwendbaren Monomeren aus. Weiterhin sind Xanthate ${ }^{[45,46]}$ und Carbamate ${ }^{[47]}$ bekannt, die vor allem für die Polymerisation von Vinylestern geeignet sind. Je besser die Z-Gruppe in der Lage ist, das Intermediat zu stabilisieren, desto schneller erfolgt die Addition des Makroradikals (s. Abbildung 2-3). Außerdem ist die Z-Gruppe in der Lage, die C=S-Doppelbindung des RAFTAgens in Bezug auf die Addition eines Radikals zu aktivieren oder zu desaktivieren. ${ }^{[48]}$

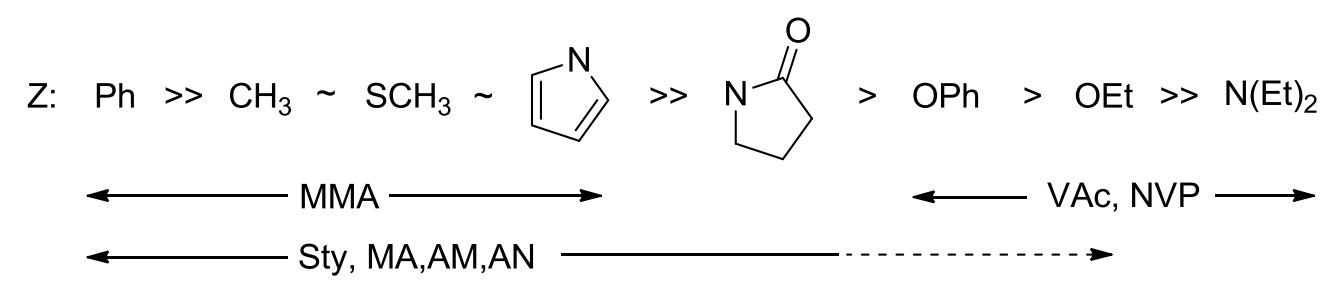

Abbildung 2-3 Beispiele für unterschiedliche Z-Gruppen. Von links nach rechts mit sinkender Rate der Addition und steigendender Rate der Fragmentierung für verschiedene Monomere (MMA: Methylmethacrylat, MA: Methacrylat, AM: Acrylamid, AN: Acrylnitril, VAc: Vinylacetat, NVP: N-Vinylpyrrolidon). Nach Matyjaszewski ${ }^{[33]}$ und Rizzardo $^{[37]}$.

Ist diese Stabilisierung allerdings zu stark ausgeprägt, kann es sein, dass es zu einer Retardierung der Reaktion kommt, da die Fragmentierung der R-Gruppe verzögert passiert. Dadurch werden Terminierungsreaktionen der Intermediat-Radikalen möglich. Dies konnte vor allem bei Dithioestern mit Phenylgruppen als Z-Gruppe beobachtet werden. ${ }^{[4]}$

Der R-Gruppe kommt im RAFT-Prozess die Aufgabe zu, eine neue radikalische Kettenreaktion zu starten. Darum ist es für den Prozess wichtig, dass die Fragmentierung der R-Gruppe schnell erfolgt. Dieser Schritt verläuft umso schneller, je stabiler das durch die Fragmentierung gebildete Radikal ist. Darum eignen sich als R-Gruppe besonders elektrophile oder über sterisch anspruchsvolle Substituenten verfügende Verbindungen wie z. B. Cyano-, oder Phenylverbindungen. ${ }^{[34,37]}$ Weiterhin ist es notwendig, dass sie schnell an das verwendete Monomer addieren und somit eine neue Kette starten. Ansonsten steigt die Wahrscheinlichkeit für das Eintreten von Terminierungsreaktionen. Darum ist darauf zu achten, dass die R-Gruppe passend zum verwendeten Monomer gewählt wird. Ein Beispiel hierfür sind die Acrylate, bei denen auch monomeranaloge Verbindungen, im Gegensatz zu der in Abbildung 2-4 gezeigten Eignung der R-Gruppen, eine sehr gute Kontrolle zeigen. ${ }^{[50]}$ 


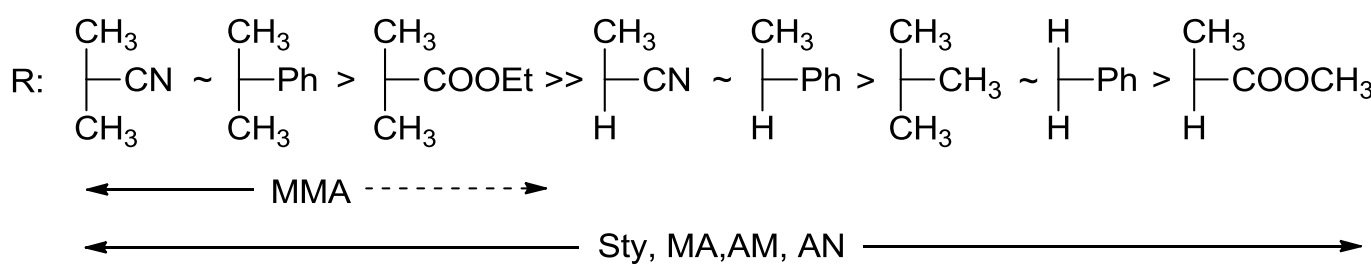

Abbildung 2-4 Beispiele für verschiedene R-Gruppen. Von links nach rechts mit sinkender Rate der Fragmentierung bei verschiedenen Monomeren. Nach Matyjaszewski ${ }^{[33]}$ und Rizzardo ${ }^{[37]}$.

In einer ideal verlaufenden RAFT-Polymerisation ist die Konzentration des RAFT-Agens deutlich größer als die Konzentration der durch den Initiator generierten Primärradikale. Darum tragen nahezu alle Polymere am Ende der Polymerisation ein RAFT-Agens als Endgruppe. Sie können in weiteren Polymerisationen als Makro-RAFT-Agenzien verwendet werden. Dies ermöglicht die Synthese von z. B. Blockcopolymere ${ }^{[51]}$ mit genau definierter Blocklänge oder die Einführung von funktionellen Gruppen wie z. B. speziellen Farbstoffen in die Polymere. ${ }^{[52]}$ Weiterhin ist die RAFT-Polymerisation in Hinblick auf die Reaktionsbedingungen sehr vielseitig. Sie ist z. B. unter hohem Druck, ${ }^{[53]}$ bei sehr hohen oder sehr niedrigen Temperaturen, ${ }^{[38,44]}$ sowie in Dispersions- ${ }^{[54]}$ oder Emulsionspolymerisation, ${ }^{[55]}$ in Substanz, ${ }^{[41]}$ in superkritischen Lösungsmitteln ${ }^{[56]}$ oder auch in wässrigen Lösungen ${ }^{[57]}$ durchführbar.

\subsubsection{Photoinitiierte, radikalische Polymerisation}

Von photoinitiierter, radikalischer Polymerisation wird gesprochen, wenn die Bildung, der zum Starten der Polymerisation benötigten Primärradikale (s. Kapitel 2.1), durch das Bestrahlen der Polymerisationsmischung mit ultravioletter (UV)-Strahlung oder sichtbarem Licht hervorgerufen wird. Die photoinitiierte, radikalische Polymerisation findet aufgrund ihrer vielen Vorteile, wie z. B. die Möglichkeit bei niedrigen Temperaturen zu arbeiten, in einer Vielzahl von technischen, medizinischen und wissenschaftlichen Prozessen Anwendung und ist darum in der Vergangenheit eingehend untersucht worden. Die Ergebnisse dieser Arbeiten wurden in einer Reihe von Übersichtsartikeln ausführlich zusammengefasst. ${ }^{[5,14,58-61]}$

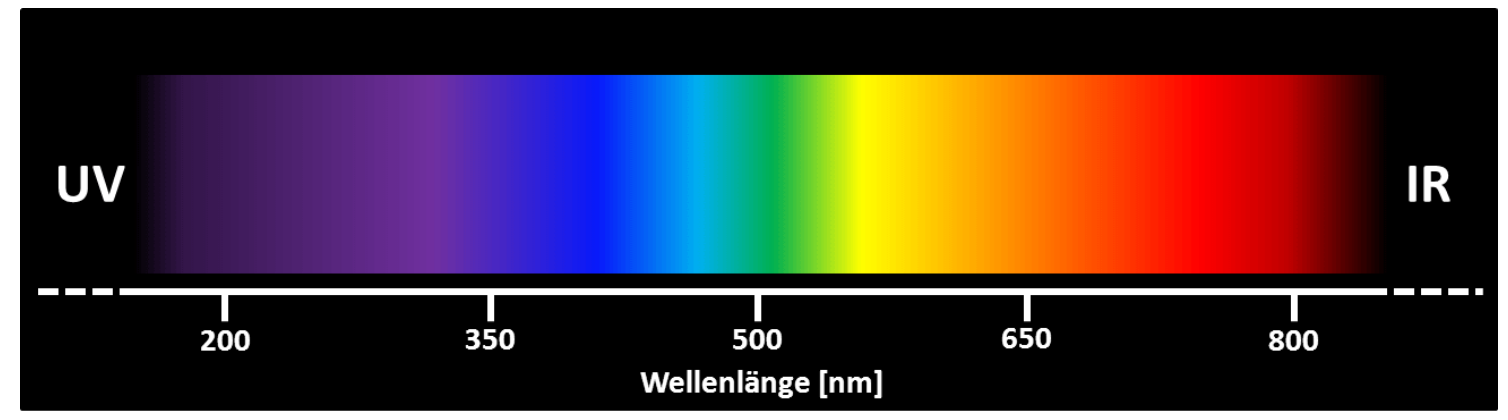

Abbildung 2-5 Schematische Darstellung des sichtbaren Lichtspektrums 
Bei UV-Strahlung und sichtbarem Licht handelt es sich um einen Teil des Spektrums der elektromagnetischen Strahlung. Dieser Teil umfasst den Wellenlängenbereich von etwa $100 \mathrm{~nm}$ bis $780 \mathrm{~nm}$ (s. Abbildung 2-5). Als sichtbares Licht wird der Teil des elektromagnetischen Spektrums von etwa $380 \mathrm{~nm}$ bis $780 \mathrm{~nm}$ bezeichnet. Zu größeren Wellenlängen schließt sich der Bereich der infraroten Strahlung an, zu niedrigeren Wellenlängen, der der ultravioletten Strahlung. Für die photoinitiierte, radikalische Polymerisation wird dabei sehr häufig UVStrahlung verwendet. Diese eignet sich sehr gut, um effizient viele Radikale zu generieren, da sie nach:

$$
E=h \cdot \frac{c}{\lambda}=h \cdot v
$$

über eine höhere Energie $E$ verfügt. Wobei $h$ für das Planck'sche Wirkungsquantum, $c$ für die Lichtgeschwindigkeit, $\lambda$ die Wellenlänge und $v$ für die Frequenz stehen. Die UV-Strahlung kann noch in weitere Bereiche unterteilt werden: ${ }^{[62]}$

- UV-A-Strahlung $(400-315 \mathrm{~nm})$,

- UV-B-Strahlung $(315-280 \mathrm{~nm})$,

- UV-C-Strahlung $(280-100 \mathrm{~nm})$.

Die Strahlung wird von den Initiatormolekülen absorbiert, wodurch diese in einen elektronisch angeregten Zustand versetzt werden. Dies geschieht vor allem bei der Arylketongruppe (s. Abbildung 2-6) sehr effizient, ${ }^{[63]}$ weshalb diese Gruppe auch in den meisten kommerziell erhältlichen Photoinitiatoren enthalten ist.

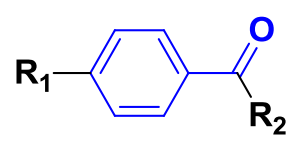

Abbildung 2-6 Die Arylketongruppe.

Die Bindungselektronen der Carbonylbindung gelangen durch die Absorption der Energie eines Photons auf ein höheres Energieniveau. Bei welcher Wellenlänge diese Anregung stattfinden kann, wird durch den Substituenten in der $\mathrm{R}_{1}$-Position beeinflusst. Vom angeregten SingulettZustand wechseln sie anschließend in den Triplett-Zustand. Das weitere Schicksal des TriplettZustands wird dabei vom Substituenten in der $\mathrm{R}_{2}$-Postion bestimmt. Handelt es sich bei diesem um eine Alkylgruppe, liegt die Bindungsenergie der C-C-Bindung zwischen der Carbonylfunktion und dem Substituenten bei etwa $65-70 \mathrm{kcal} / \mathrm{mol}$. Die durch die UV-Strahlung 
bereitgestellte Energie liegt aber bei etwa 70-80 kcal/mol, sodass es zum Bruch der C-C-Bindung kommt. Ist der Substituent eine Arylgruppe, liegt die Bindungsenergie bei 80-90 kcal/mol. Die zur Verfügung gestellte Energie reicht also nicht aus, um einen Bindungsbruch zu verursachen. Das Molekül verbleibt im angeregten Zustand bis es mit einem passenden Wasserstoffdonor reagiert und von diesem ein Wasserstoffatom abstrahiert. ${ }^{[63]}$

Bei der photoinitiierten, radikalischen Polymerisation existieren also zwei mögliche Mechanismen für die Bildung von Primärradikalen. Die erste wird als Norrish Typ I Mechanismus bezeichnet. Hierbei handelt es sich um einen unimolekularen Prozess, bei dem, wie oben beschrieben, nach der Anregung des Moleküls eine homolytische Bindungsspaltung eintritt und das Molekül somit in zwei Primärradikale zerfällt. Da die für den Bindungsbruch benötigte Energie relativ hoch ist, wird für diesen Prozess meistens UV-Strahlung benötigt.

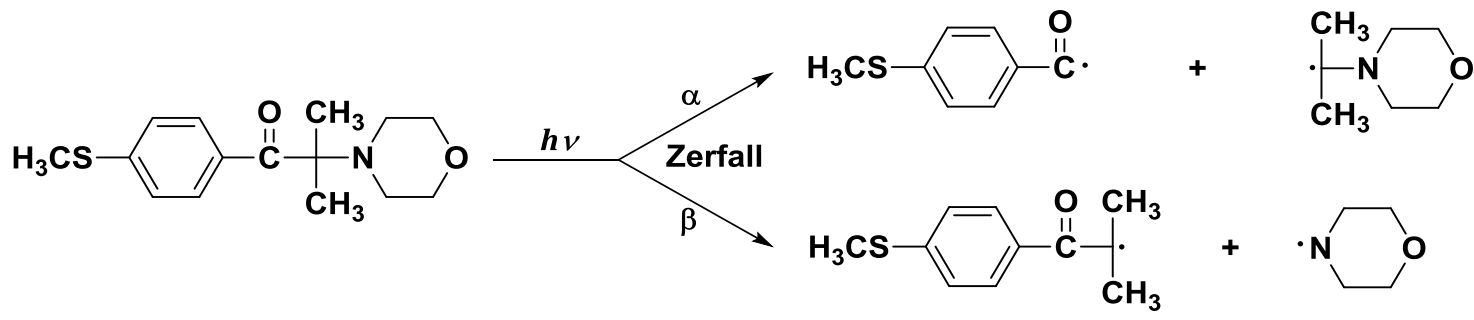

Schema 2-4 Norrish Typ 1 Mechanismus am Beispiel, des in dieser Arbeit hauptsächlich verwendeten 2-Methyl-4'(methylthio)-2-morpholinopropiophenon (MMMP). Nach Green ${ }^{[64]}$.

Im Fall des in Schema 2-4 dargestellten MMMP existieren zwei Möglichkeiten für den Zerfall, wobei der $\alpha$-Zerfall gegenüber dem $\beta$-Zerfall deutlich dominiert. Die aus dem $\alpha$-Zerfall gebildeten Primärradikale sind in der Lage, ein Kettenwachstum zu starten, sodass aus einem Initiatormolekül zwei propagierende Ketten gebildet werden können.

Die zweite Möglichkeit wird als Norrish Typ II Mechanismus bezeichnet. Hierbei handelt es sich um einen bimolekularen Prozess. Die Radikalbildung erfolgt nicht am eigentlichen Photoinitiator, sondern an einem zweiten Molekül, durch die Abstraktion eines Wasserstoffs. Bei dem zweiten Molekül kann es sich dabei z. B. um ein Lösungsmittelmolekül oder Monomer handeln. Meistens werden aber spezielle Koinitiatoren wie z. B. tertiäre Amine, Ether, Sulfide oder Thiole verwendet. Sie fungieren als Wasserstoffdonatoren und bilden nach der Abstraktion des Wasserstoffs hochreaktive Primärradikale. Durch die Verwendung dieser Koinitiatoren wird die Effizienz des Prozesses deutlich erhöht. ${ }^{[61,65]}$ 


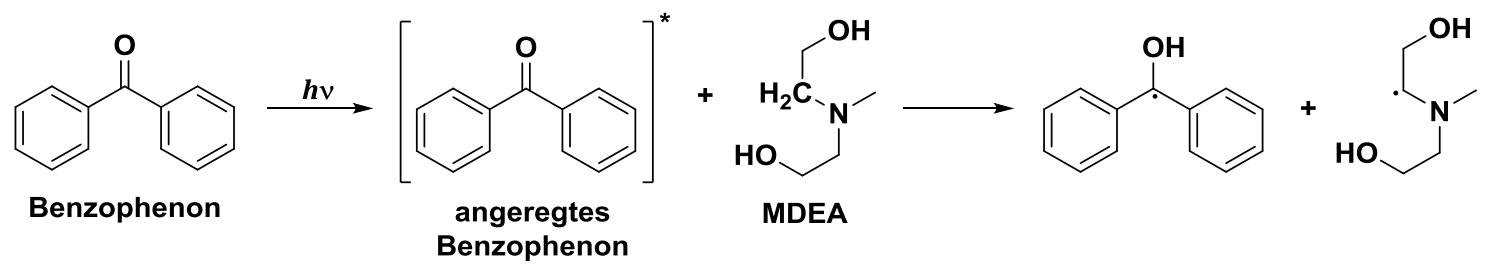

Schema 2-5 Norrish Typ II Mechanismus am Beispiel von Benzophenon und Methyldiethanolamin (MDEA) als Koinitiator. Nach Green ${ }^{[64]}$.

Bei dem in Schema 2-5 gezeigten Beispiel wird das Benzophenon durch Bestrahlung mit Licht in einen angeregten Zustand versetzt. Das angeregte Benzophenon ist nicht in der Lage, an eine Doppelbindung eines Monomers zu addieren. Erst durch die Abstraktion eines $\alpha$-Wasserstoffs an einer der Ethanolgruppen des MDEAs bildet sich ein Primärradikal, welches eine radikalische Polymerisation initiieren kann. Als häufige Nebenreaktion tritt hierbei die Rekombination von zwei Ketylradikalen zu einem Pinacolmolekül auf. Zusätzlich ist es möglich, dass die relativ stabilen Ketylradikale zur Terminierung einer schon wachsenden Kette führen können. ${ }^{[58]}$

Die Effizienz eines Initiators wird bei der UV-initiierten, radikalischen Polymerisation häufig über die Quantenausbeute angegeben. Diese steht für die Zahl der polymerisierten Monomere pro absorbiertem Photon. ${ }^{[6]}$ Für die Anregung eines Initiatormoleküls ist theoretisch ein Photon ausreichend. Darum ist der Norrish-I-Mechanismus häufig effizienter als der Norrish-IIMechanismus, da aus ersterem zwei Primärradikale hervorgehen. Der große Vorteil der Norrish-II-Systeme ist allerdings, dass eine Vielzahl von möglichen Verbindungen zur Verfügung steht, die mit sichtbarem Licht angeregt werden können. Dies reduziert die für den Prozess benötigte Energiezufuhr, was vor allem in industriellen Prozessen von Vorteil sein kann, da somit die Kosten des Prozesses gering gehalten werden können.

Aufgrund der vielen unterschiedlichen Einsatzmöglichkeiten der photoinitiierten, radikalischen Polymerisation und der Möglichkeit über den Initiator die Polymerisation zu steuern, ist es besonders wichtig, dass die Eigenschaften des Initiators genau an die gewünschten Prozessbedingungen angepasst sind. Darum sollten die Initiatoren allgemein über folgende Eigenschaften verfügen: ${ }^{[65]}$

- hohe Absorption in im Bereich der Aktivierung (Dies hängt von der Anwendung und der verwendeten Lichtquelle ab.),

- hohe Quantenausbeute, um effizient Radikale zu erzeugen,

- gute Löslichkeit im Polymerisationsmedium,

- gute Lagerfähigkeit,

- möglichst geruchlos und nicht färbend,

- nicht giftig, kostengünstig und einfach zu handhaben. 
Zur Beschreibung der Kinetik der photoinitiierten, radikalischen Polymerisation muss beachtet werden, dass der Zerfall des Initiators nur erfolgt, wenn dieser mit Licht oder UV-Strahlung bestrahlt wird. Also haben auch die Intensität $I_{0}$ der Strahlung, die Quantenausbeute $\phi_{\mathrm{Q}}$ und der molare dekadische Extinktionskoeffizient $\varepsilon_{\mathrm{E}}$ des Initiators einen Einfluss auf die Initiierungsreaktion. Darum muss Gleichung (2) entsprechend modifiziert werden, für die Geschwindigkeit einer Photoinitiierung gilt dann: ${ }^{[5]}$

$$
r_{\mathrm{d}}=2 I_{0} \cdot \phi_{\mathrm{Q}} \cdot \varepsilon_{\mathrm{E}} \cdot f_{\mathrm{I}} \cdot c_{\mathrm{I}-\mathrm{I}}
$$

Damit ergibt sich für die Polymerisationsgeschwindigkeit der Gesamtreaktion einer photoinitiierten, radikalischen Polymerisation:

$$
R_{\mathrm{P}}=-\frac{\mathrm{d} c_{\mathrm{M}}}{\mathrm{d} t}=\frac{k_{\mathrm{p}}}{k_{t}^{1 / 2}} \cdot\left(f_{\mathrm{I}} \cdot I_{0} \cdot \phi_{\mathrm{Q}} \cdot \varepsilon_{\mathrm{E}} \cdot c_{\mathrm{I}-\mathrm{I}}\right)^{1 / 2} \cdot c_{\mathrm{M}}
$$

\subsubsection{Photoinitiierte RAFT-Polymerisation}

Sowohl aus akademischer als auch industrieller Sicht ist es sinnvoll, die Vorteile der photoinitiierten, radikalischen Polymerisation (s. Kapitel 2.1.4) auch für die RAFT-Polymerisation nutzbar zu machen. ${ }^{[67]}$ In frühen Untersuchungen konnten Quinn et al. ${ }^{[68]}$ anhand der Polymerisation von Methylmethacrylat (MMA) und Styrol bei einer Bestrahlung mit UV-AStrahlung in Anwesenheit von Dithioestern als CTA zeigen, dass photoinitiierte RAFTPolymerisationen, in Abhängigkeit von der Z-Gruppe des CTAs, prinzipiell möglich ist. Die gleichzeitige Verwendung der CTAs als Photoinitiator führt zu einem deutlichen Verlust der Kontrolle über die Reaktion bei Umsätzen von höher als 30\%, der sich auf die, mit der Reaktionszeit zunehmende, Zersetzung der CTAs zurückführen ließ. Lu et al. ${ }^{[67,69]}$ untersuchten die Abhängigkeit der Zersetzung des CTAs von der chemischen Natur des CTAs. Die Anwesenheit eines klassischen Photoinitiators ermöglichte außerdem die Variation der Wellenlänge der verwendeten UV-Strahlung. Dabei zeigt sich, dass die Zersetzung sowohl von Dithioestern (DTE) als auch von Trithiocarbonaten (TTC) sehr stark von der Wellenlänge der UV-Strahlung abhängt. Unter der Bestrahlung mit dem kompletten Spektrum einer Quecksilberdampflampe zersetzten sich sowohl der Dithioester als auch das Trithiocarbonat. Wobei die Zersetzungsgeschwindigkeit des TTCs deutlich geringer war. Weiterhin konnte durch die Verwendung eines UV-Filters, der nur UV-A-Strahlung passieren lässt, diese geringe Zersetzung noch weiter reduziert werden. 
Dass eine schnelle, effiziente und gut kontrollierte photoinitiierte RAFT-Polymerisation durch die Verwendung eines geeigneten, klassischen Photoinitiators, nicht zu energiereicher (UV)Strahlung und der Verwendung eines Trithiocarbonats als CTA möglich ist, wird in vielen weiteren Untersuchungen auch von anderen Arbeitsgruppen bestätigt. ${ }^{[70-76]}$ In den letzten Jahren wurde auch der Einfluss photoinitiierten RAFT-Polymerisation auf die Kinetik sowie die Strukturen und Eigenschaften von Polymernetzwerken untersucht. ${ }^{[77-79]}$ Diese und andere Ergebnisse zu Polymernetzwerken werden im Laufe der nächsten Kapitel näher erläutert.

\subsection{Polymernetzwerke}

\subsubsection{Aufbau und Struktur von Polymernetzwerken}

Polymernetzwerke bilden einen makroskopischen Festkörper aus dreidimensional miteinander verknüpften Polymerketten. Dabei wird zwischen physikalischen oder chemischen Verknüpfungen unterschieden. Netzwerke, die über physikalische Verknüpfungen gebildet werden, heißen physikalische Netzwerke. Physikalische Verknüpfungen bilden sich durch intermolekulare Wechselwirkungen, wie z. B. ionische Wechselwirkungen und Kristallitbildung, Blockcopolymermicellen infolge von Mikrophasenseperation, Wasserstoffbrückenbindungen oder durch Verschlaufungen bzw. Verhakungen der Polymerketten. ${ }^{[80,81]}$ (s. Abbildung 2-7)

Dabei wird zwischen starken (z. B. Kristallite) und schwachen Wechselwirkungen (z. B. Wasserstoffbrückenbindungen) unterschieden. Vor allem starke physikalische Netzwerke können dem mechanischen Verhalten von chemischen Netzwerken entsprechen und sich dauerhaft wie ein Festkörper verhalten. Schwache physikalische Netzwerke sind allerdings keine dauerhaften Festkörper. Auf bestimmten Zeitskalen, die meist kürzer sind als die Relaxationszeit der Ketten oder bei mechanischen Belastungen, die geringer sind als die Streckspannung (s. Kapitel 2.4), können sie sich aber durchaus wie ein solcher verhalten. Beiden Arten physikalischer Verknüpfungen ist gemein, dass sie durch Änderungen bei den Umgebungsbedingungen, wie z. B. die Zugabe eines geeigneten Lösungsmittels oder durch Aufschmelzen, wieder aufgebrochen werden. ${ }^{[81]}$ 

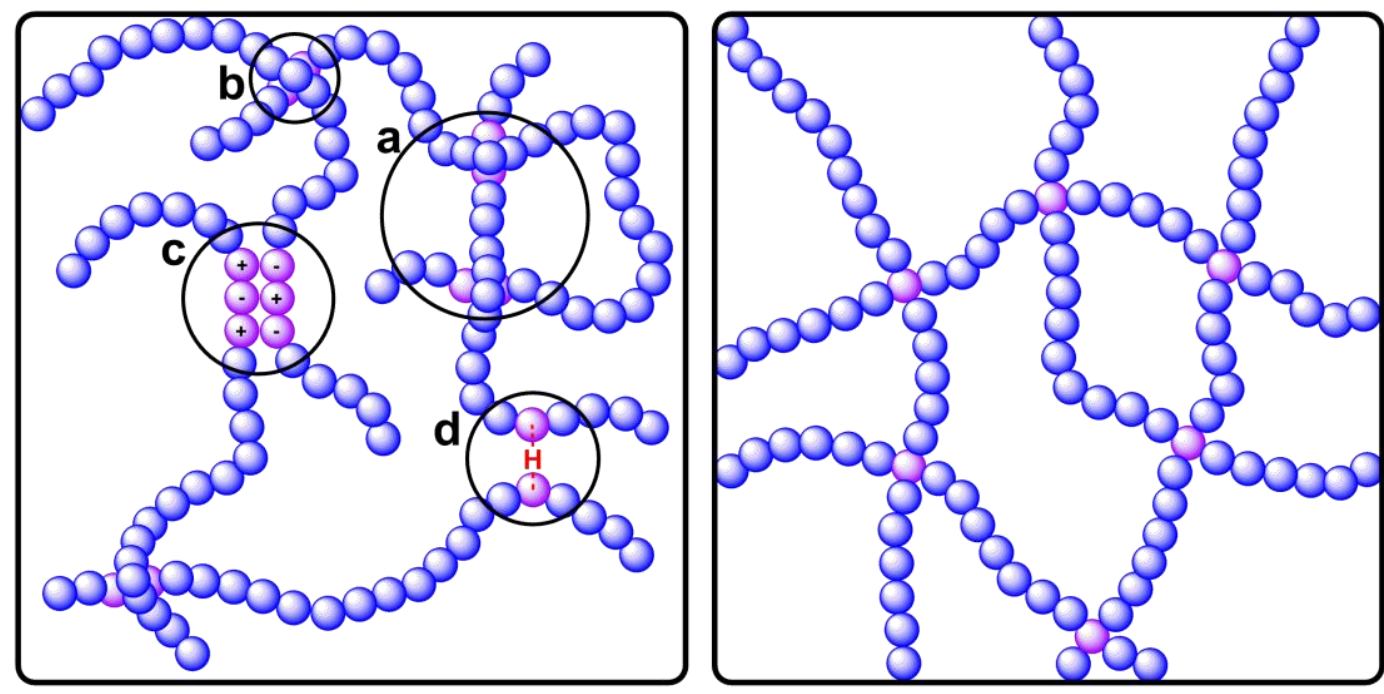

Abbildung 2-7 Links: Physikalisches Netzwerk mit verschiedenen Beispielen einer physikalischen Verknüpfung (a: Verschlaufung; b: Verhakung; c: kristalliner Bereich durch ionische WW d: Wasserstoffbrückenbindung). Rechts: chemisches Netzwerk mit kovalenten Verknüpfungen zwischen den Netzwerkketten.

Bestehen die Verknüpfungen zwischen den Polymerketten aus kovalenten Bindungen, wird von einem chemischen Netzwerk gesprochen. Im Gegensatz zu physikalischen Netzwerken sind chemische Netzwerke dauerhaft formstabil und lassen sich weder aufschmelzen noch gehen sie in Lösung. Stattdessen sind sie in der Lage, in einem geeigneten Lösungsmittel um ein Vielfaches ihres Volumens aufzuquellen, wobei ihre äußere Form beibehalten wird. In diesem gequollenen Zustand werden Polymernetzwerke als Gele bezeichnet. Bei Gelen handelt es sich um „leicht deformierbare, aber formbeständige disperse Systeme, die aus einem festen, aber beweglichen dreidimensionalen Netzwerk bestehen, das die Matrix des Dispergiermittels durchzieht“.[82] Kürzer ausgedrückt ist ein Gel eine feste Phase, die von einer fluiden Phase, z. B. einem Lösungsmittel, vollständig durchdrungen ist. ${ }^{[83]}$

Chemische Netzwerke sind Festkörper, die idealerweise aus einem einzigen, „unendlich“ großen Molekül bestehen. „Unendlich“ bedeutet in diesem Fall: sehr viel größer als ein übliches Molekül oder gar ein Polymer. ${ }^{[80]}$ Bei der Einwirkung äußerer Kräfte zeigen chemische Netzwerke ein elastisches Verhalten, welches im Gegensatz zu physikalischen Netzwerken nicht von der Dauer der Einwirkung abhängig ist. Für dieses elastische Verhalten sind die mikroskopische Struktur und der Aufbau der Netzwerke von großer Bedeutung. Auf die thermodynamische und strukturelle Herkunft dieser elastischen Eigenschaften wird in Kapitel 2.3.1 näher eingegangen.

Ein bedeutender Strukturparameter von Polymernetzwerken ist die Netzkettendichte. Sie beschreibt die Zahl der pro Volumeneinheit vorliegenden Netzketten. In einem idealen Netzwerk kann davon ausgegangen werden, dass alle Vernetzungspunkte homogen über das Netzwerk verteilt sind. Weiterhin verfügen alle Netzketten über die gleiche zahlenmittlere Molmasse $\bar{M}_{\mathrm{s}}$. Die gesamte Stoffmenge des Vernetzers $n_{\mathrm{x}}$ wurde entsprechend seiner Funktionalität $f$ 
(s. Kapitel 2.2.2) vollständig in das Netzwerk eingebaut und es liegen keine weiteren Netzwerkdefekte vor. Somit nehmen alle Netzketten an einer elastischen Verformung des gesamten Netzwerkvolumens $V$ teil. Die theoretische Netzkettendichte $v_{\text {th }}$ lässt sich beschreiben als:

$$
v_{\mathrm{th}}=\frac{n_{\mathrm{x}} f}{2 V}
$$

In der Realität bilden sich während der Vernetzung allerdings immer Defekte aus. Dies führt zu einer inhomogenen Netzwerkstruktur, die auf die folgenden Gründe zurückgeht: ${ }^{[84]}$

- nichtreagierte funktionelle Gruppen,

- Netzwerkfehler durch lose Kettenenden, Verschlaufungen und intramolekulare Ringschlüsse,

- Vorordnungen der Moleküle im unvernetzten Zustand,

- Verdünnungseffekte (inter- und intramolekulare Verknüpfung),

- unterschiedliche Reaktivität von Monomeren und Vernetzer (ungleichmäßige Verteilung der Vernetzungsstellen),

- Entmischungseffekte infolge sterischer Hinderung,

- Phasentrennung (Makro- und Mikrosynärese).

Diese Defekte haben einen erheblichen Einfluss auf die mechanischen Eigenschaften von Polymernetzwerken (s. Kapitel 2.3). So sind z. B. lose Kettenenden (s. Abbildung 2-8, b) oder intramolekulare Ringe (s. Abbildung 2-8, c) nicht elastisch aktiv, was die messbare Netzkettendichte verringert. Verschlaufungen (s. Abbildung 2-8, a) dagegen fügen zusätzliche Knotenpunkte in das Netzwerk ein. Diese sind in einem chemischen Netzwerk aufgrund der festen Verknüpfungen nicht durch Abgleiten der Ketten voneinander lösbar. Somit erhöht sich die Netzkettendichte im betrachteten Volumenelement des Netzwerks. 


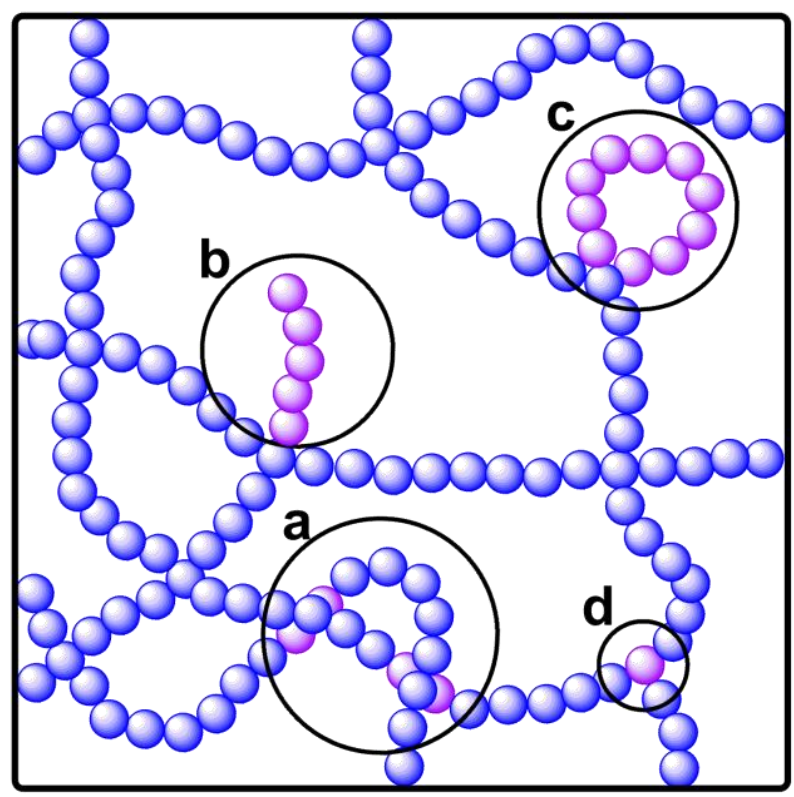

Abbildung 2-8 Beispiele für mögliche Defekte eines realen Netzwerkes (a: Verschlaufung; b: loses Kettenende; c: intramolekularer Ringschluss; d: abweichende Funktionalität eines Netzknotens).

Darum ist es sinnvoll, für die Betrachtung von realen Netzwerken eine effektive Netzkettendichte $v_{\text {eff }}$ zu formulieren (s. Kapitel 2.3.1). Diese bezieht sich ausschließlich auf die, bei einer Deformation, elastisch aktiven Netzketten. Aus dem Verhältnis dieser beiden Größen kann auf die Effizienz der Vernetzungsreaktion $\Omega$ geschlossen werden. Es gilt:

$$
\Omega=\frac{v_{\text {eff }}}{v_{\text {th }}}
$$

\subsubsection{Synthese von Polymernetzwerken}

Für die Synthese von chemisch verknüpften Polymernetzwerken existieren drei unterschiedliche Prozesse: ${ }^{[81]}$ die Kondensationspolymerisation, die nachträgliche Vernetzung von linearen Polymeren und die Kettenadditionspolymerisation.

Die Kondensationspolymerisation ist eine Stufenwachstumsreaktion bei der Monomere direkt miteinander reagieren (z. B. Säure- und Alkoholfunktionen). Es kann zu einer Vernetzung kommen, wenn eines dieser Monomere mindestens über drei funktionelle Gruppen verfügt. Andernfalls werden nur lineare Polymere gebildet. An dieser Stelle wird zunächst zwischen der chemischen Funktionalität der Monomere und der physikalischen Funktionalität der Knotenpunkte im fertigen Netzwerk unterschieden. Während es sich bei der chemischen Funktionalität um die Anzahl von funktionellen Gruppen (z. B. Doppelbindungen) im Molekül handelt, ist die physikalische Funktionalität der Netzknotenpunkte die Anzahl der von diesem 
Kontenpunkt ausgehenden Netzketten. ${ }^{[85]}$ Bei der Kondensationspolymerisation ist es so, dass im idealen Fall, welcher gegeben ist, wenn alle funktionellen Gruppen des dreifach funktionellen Monomers reagieren, drei Netzketten von einem Kontenpunkt ausgehen, was letztlich zu einer Vernetzung führt.

Die zweite Möglichkeit, eine Vernetzung herbeizuführen, ist die nachträgliche Vernetzung. Der bekannteste Vertreter dieser Art der Vernetzung ist die von Charles Goodyear entwickelte Vulkanisation. ${ }^{[86]}$ Hierbei werden die Polymerketten des natürlichen Kautschuks (cis-1,4Polyisopren) mit Hilfe von Schwefel an den in den Polymerketten enthaltenen Doppelbindungen verknüpft: Es wird Gummi erhalten. Diese Verknüpfung ist dabei zufällig und ungeordnet, sodass eine breite Verteilung der Netzkettenlängen auftritt. Weitere Möglichkeiten der nachträglichen Verknüpfung von linearen Polymeren bieten die Photovernetzung über spezielle, im Polymer enthaltene, photoreaktive Monomerbausteine ${ }^{[87]}$ oder die Verknüpfung über reaktive Endgruppen. Letzteres bietet die Möglichkeit die Dispersität der Netzkettenlängen eng zu gestalten, sofern die Polymere z. B. via RAFT-Polymerisation hergestellt wurden. Damit lassen sich relativ homogene Netzwerke herstellen. ${ }^{[88-90]}$

Die dritte Möglichkeit zur Synthese von Polymernetzwerken ist die Kettenadditionspolymerisation. Ein Beispiel hierfür ist die radikalische Polymerisation, die auch im Rahmen der vorliegenden Arbeit verwendet wurde. Hierbei existieren unterschiedliche Möglichkeiten um Netzwerke herzustellen. Zum einen die Copolymerisation von einfach(z. B. Acrylate) und zweifachfunktionellen Monomeren (z. B. Diacrylate). Dabei werden die zweifachfunktionellen Monomere (Vernetzer) statistisch in die Polymerketten eingebaut, so dass die zweite Vinylgruppe des Vernetzers seitlich an der Polymerkette verbleibt. Sie steht für den Einbau in weitere, parallel wachsende Polymerketten bereit. Ist der Vernetzer bifunktionell, bilden sich im fertigen Netzwerk Verknüpfungen mit einer Funktionalität von vier. Über die Vernetzerkonzentration kann dabei Einfluss auf die Eigenschaften der Netzwerke genommen werden: Je größer diese ist, desto mehr Kontenpunkte entstehen und das fertige Netzwerk verfügt über eine größere Netzkettendichte, wird steifer und weniger elastisch. Eine weitere Möglichkeit, um Netzwerke durch radikalische Polymerisation zu erhalten, ist die direkte Verwendung von mehrfach funktionellen Monomeren, wie z. B. Di- oder Triacrylate. Die daraus resultierenden Netzwerke verfügen über eine sehr hohe Netzkettendichte, da praktisch jeder Vernetzungspunkt auch eine Netzkette darstellt. Die besonderen Eigenschaften der resultierenden Netzwerke, wie z. B. die hohe Resistenz gegenüber äußeren Einflüssen (die Netzwerke quellen bei Kontakt mit Lösungsmitteln nahezu nicht auf) oder eine sehr schnelle Polymerisation, ${ }^{[91,92]}$ machen diese Form von chemischen Netzwerken für viele industrielle Anwendungen wie Druckfarben ${ }^{[93,94]}$ oder Beschichtungen ${ }^{[95]}$ besonders interessant. Auch im medizinischen Bereich, z. B. im Bereich von Zahnfüllungen, findet diese Form der Vernetzung verbreitete Verwendung. ${ }^{[96]}$ 
Aufgrund der Relevanz für industrielle Prozesse wurde vor allem die photoinitiierte, radikalische Polymerisation von mehrfach funktionellen Monomeren hinlänglich ihrer Kinetik seit den 90er Jahren des letzten Jahrhunderts intensiv untersucht. ${ }^{[1,92,97-107]}$ Dabei wurde unter anderem der Einfluss der Monomerfunktionalität, ${ }^{[100]}$ der Lichtintensität, ${ }^{[101]}$ der Initiatorkonzentration ${ }^{[102]}$ und der Gegenwart von Sauerstoff ${ }^{[105-107]}$ auf die Polymerisation erforscht. Des Weiteren existiert eine Reihe von Untersuchungen $\mathrm{zu}$ den mechanischen Eigenschaften der resultierenden Netzwerke. ${ }^{[93,108-112]}$

Ein großes Problem der Netzwerke aus radikalischen Polymerisationen, sowohl aus der direkten als auch aus der Copolymerisation, stellt das Fehlen einer Möglichkeit zur Kontrolle der Mikrostruktur, wie der Länge der Netzketten, der Netzwerke dar. ${ }^{[113,14]}$ Die Netzwerke sind häufig heterogen und verfügen über viele Netzwerkdefekte. Der Grund dafür ist die radikalische Natur der Reaktion. Es ist bekannt, dass in der radikalischen Polymerisation schon in einer frühen Phase der Polymerisation ein hohes Maß an Ringbildung und intramolekularer Vernetzung stattfinden, was zu stark vernetzten, kompakten Polymernanodomänen führt, den so genannten Nanogelen. ${ }^{[115]}$ Dies geht auf die schnelle Propagation der Ketten zurück, die im Bereich der Nanogel-Domänen durch einen lokalen Geleffekt noch zusätzlich beschleunigt werden können. Erst im späteren Verlauf der Reaktion wachsen diese Nanogel-Domänen über die verbleibenden, im äußeren Bereich der Nanogele liegenden seitlichen Doppelbindungen zusammen und bilden somit das Gesamtnetzwerk. ${ }^{[113,116-118]}$ Dies führt letztlich bei den resultierenden Netzwerken zu einer sehr inhomogenen Mikrostruktur, die mit großen Dichteunterschieden verbunden ist. Dies wird begleitet durch viele Netzwerkdefekte wie Verschlaufungen, Verhakungen oder intramolekularen Ringbildungen (s. Abbildung 2-9). Der Übergang von den einzelnen Nanogel-Domänen zu einem makroskopischen Netzwerk wird auch als Gelierung bezeichnet. Diese verläuft über den Gelpunkt, der den Punkt darstellt, an dem zum ersten Mal ein „unendliches“ Molekül gebildet wird.

Ein weiteres häufig auftretendes Phänomen ist die Volumenschrumpfung des fertigen Netzwerks. Nach dem Vernetzen sind sich die Monomermoleküle einander näher, da die van-der-WaalsWechselwirkungen zwischen ihnen durch kovalente Bindungen ersetzt wurden. Gerade bei der radikalischen Polymerisation kann die Schrumpfung, die bei der frühen Bildung von NanogelDomänen entsteht, nicht mehr durch eine makroskopische Schrumpfung kompensiert werden. Dadurch entstehen innere Spannungen im Netzwerk, die zu Unebenheiten, Mikrorissen oder letztlich zum Bruch des Netzwerks führen können. ${ }^{[77]}$ 


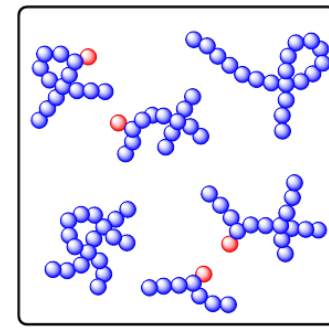

gelöstes Polymer

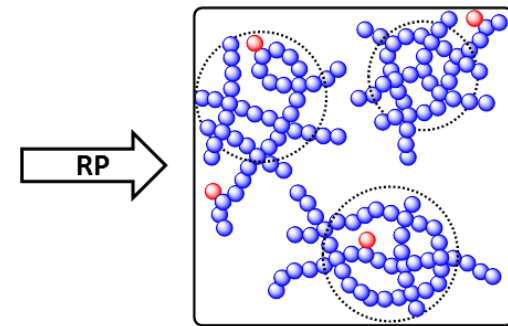

Nanogele

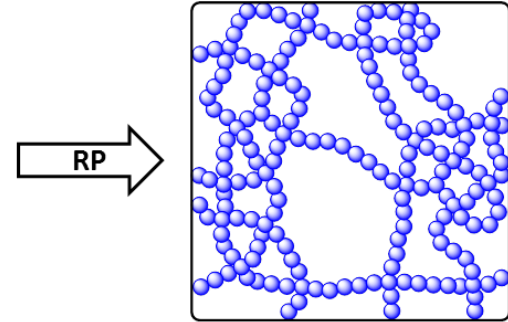

Netzwerk fortschreitende Polymerisation

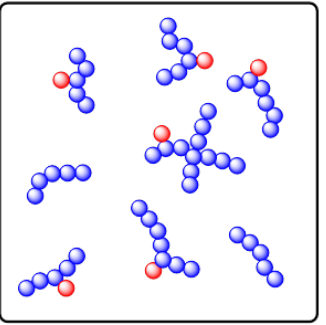

gelöstes Polymer

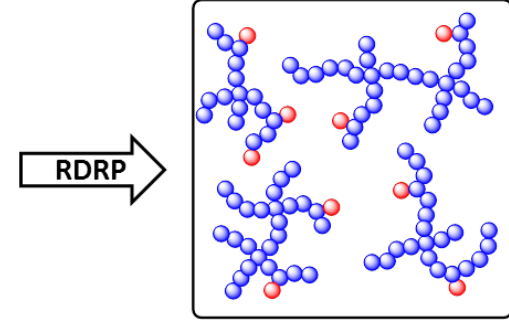

Sol

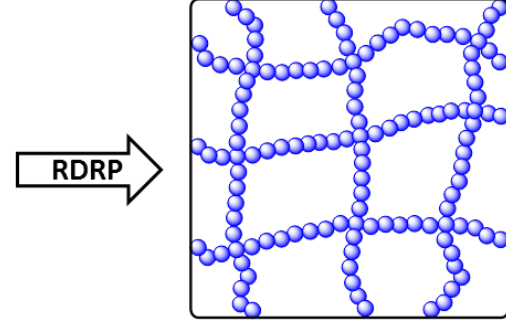

Netzwerk

Abbildung 2-9 Schematische Darstellung des Vernetzungsprozesses einer konventionellen radikalischen Polymerisation (oben) und einer RDRP (unten). Rote Kugeln symbolisieren nicht reagierte, seitliche Vinylgruppen.

Die Netzwerkbildung in einer RDRP verläuft dagegen anders. Aufgrund des immer wiederkehrenden, reversiblen Desaktivierens der Radikalfunktion nach wenigen Propagationsschritten, haben die Polymerketten die Möglichkeit zu relaxieren. Dies vermindert die Bildung von intramolekularen Ringschlüssen oder Verschlaufungen. Weiterhin wird die Wachstumswahrscheinlichkeit einer Kette besser über das gesamte System verteilt, sodass die Radikalfunktionen letztlich weiter voneinander entfernt sind. Dies führt dazu, dass sich anstatt von Nanogel-Domänen stark verzweigte Polymere mit vielen seitlichen Vinylgruppen bilden, die allerdings weiterhin in Lösung sind (s. Abbildung 2-9). Hierbei handelt es sich um die so genannte Sol-Fraktion. Im weiteren Verlauf der Polymerisation können die verzweigten Makromoleküle dieser Sol-Fraktion über die verbliebenen seitlichen Vinylgruppen zum Gesamtnetzwerk zusammenwachsen, was letztlich zu einer deutlich homogeneren Mikrostruktur der Netzwerke führt.

Dass diese Annahme tatsächlich zutreffend ist, konnte von Ide et al. ${ }^{[119]}$ für Polystyrol-Netzwerke aus NMP nachgewiesen werden. Ähnliches fanden Yu et al. ${ }^{[120]}$ bei Netzwerken aus Dimethacrylaten, die mittels ATRP synthetisiert wurden. Norisuye et al. ${ }^{[121]}$ konnten mittels Lichtstreuexperimenten eine erhöhte Homogenität von Polystyrol-Netzwerken aus RAFTPolymerisation ermitteln. Die Arbeitsgruppe um Zhu zeigte anhand von Computersimulationen und mittels mechanischer Spektroskopie, dass dies auch für Netzwerke aus photoinitiierter RAFT-Polymerisation von Oligoethylenglycoldiacrylaten zutreffend ist. ${ }^{[79,122]}$ Zhuo et al. ${ }^{[78]}$ konnten die Ergebnisse der mechanischen Analyse allerdings nicht bestätigen. Bei beiden 
Arbeiten zeigte sich allerdings, dass die Zugabe eines RAFT-Agens zur Reaktionsmischung zu einer deutlichen langsameren Polymerisation führt. Liu et al. ${ }^{[123]}$ fanden heraus, dass Polyisopropylacrylamid (pNIPAm)-Hydrogele aus RAFT-Polymerisation über eine ausgeprägtere Quellfähigkeit verfügen und überdies eine beschleunigte Entquellung zeigen. Ebenfalls für pNIPAm-Netzwerke entdeckten Bünsow et al. ${ }^{[124]}$ durch Rasterkraftmikroskopie, dass die Oberfläche von Netzwerken aus RAFT-Polymerisation deutlich homogener ist als die von Netzwerken aus konventioneller radikalischer Polymerisation. Leung und Bowman erreichten, dass Dimethacrylat-Netzwerke durch die Zugabe eines RAFT-Agens eine verringerte Schrumpfung zeigten. ${ }^{[77]}$ Huang et al. ${ }^{[125]}$ fanden durch die Zugabe eines RAFT-Agens eine erhöhte Netzkettendichte bei thermisch synthetisierten Polybutylacrylat-Netzwerken einhergehend mit erhöhter Homogenität des Netzwerks. Weiterhin gelang es Nicolay et al. ${ }^{[126]}$ Polymethylmethacrylat-Netzwerke mittels eines Trithiocarbonat-Vernetzers zu synthetisieren. Dies ermöglicht eine Reorganisation der Netzwerke, was zu deutlich höheren Quellungsgraden führt. Außerdem war es möglich, diese Netzwerke nach dem Zerschneiden wieder zusammenwachsen zu lassen.

Alle diese Untersuchungen zeigen, dass ein Einfluss der RDRP und speziell der RAFTPolymerisation auf die Mikrostruktur der Netzwerke existiert und dass sich die Eigenschaften der Netzwerke dadurch verändern können. Die Herkunft dieser Eigenschaften soll in den folgenden Kapiteln näher betrachtet werden.

\subsection{Mechanische Eigenschaften von Polymernetzwerken}

Das makroskopische, mechanische Verhalten von Polymernetzwerken beruht im Wesentlichen auf dem mikroskopischen Aufbau und der Struktur der Netzwerke. Bei einer hohen Netzkettendichte existieren entsprechend viele Netzketten in einem gewählten Volumenelement des Netzwerks. Diese Ketten sind dementsprechend kurz und verfügen über eine geringe zahlenmittlere Molmasse $\bar{M}_{\mathrm{s}}$. Da die Molmasse der Netzketten und somit die Netzkettendichte einen direkten Einfluss auf die mechanischen Eigenschaften hat, ${ }^{[109]}$ wird bei der Beschreibung von Polymernetzwerken zwischen zwei verschiedenen Arten unterschieden.

Bei dem oben beschriebenen Fall eines engmaschigen Netzwerks handelt es sich um ein duroplastisches Netzwerk. Duroplasten zeigen häufig ein mechanisches Verhalten, das einem energieelastischen Festkörper im Hook'schen Sinne ähnelt. ${ }^{[127]}$ Auf die speziellen mechanischen Eigenschaften dieser Polymerklasse wird in Kapitel 2.4 genauer eingegangen.

Netzwerke mit einer geringen Vernetzungsdichte zeigen dagegen häufig ein ganz besonderes Verhalten bei mechanischer Belastung. Dies gilt vor allem für Netzwerke, deren Schmelz- und Glastemperatur sich unter Raumtemperatur befindet. Diese als Elastomere oder auch Gummis 
bezeichneten Netzwerke ${ }^{[81]}$ lassen sich bei Anlegen einer äußeren Kraft häufig auf ein Vielfaches ihrer ursprünglichen Größe ausdehnen. Weiterhin sind sie in der Lage, nach Beendigung der Belastung die ursprüngliche Form spontan wieder einzunehmen.

Dieses Verhalten ist im Gegensatz zu energieelastischen Stoffen entropischen Ursprungs. Thermodynamisch lässt sich dies damit erklären, dass die Änderung der inneren Energie $\Delta U$ während der Deformation eines idealen Netzwerks gleich Null ist bzw. als Null angenommen wird. ${ }^{[128]}$ Dies zeigt sich bei vielen theoretischen Herleitungen ${ }^{[129-131]}$ und konnte von Meyer und Ferri für vernetzten Kautschuk schon in frühen Experimenten nachgewiesen werden. ${ }^{[132]}$

Zur Beschreibung des Gleichgewichtszustandes eines Systems kann seine freie Energie herangezogen werden. Da die Deformation von Elastomeren im Regelfall unter konstantem Volumen abläuft (s. Kapitel 2.3.1), kann in diesem Fall die Definition der Änderung der freien Energie $\Delta A_{\text {def }}$ nach Helmholtz gewählt werden. Diese ist gegeben als: ${ }^{[131]}$

$$
\Delta A_{\mathrm{def}}=\Delta U-T \Delta S
$$

Hieraus lässt sich erkennen, dass die Änderung der freien Energie des Systems nur von der Änderung der Entropie $\Delta S$ abhängt bzw. von dieser dominiert wird. Phänomenologisch lässt sich diese Tatsache dadurch erklären, dass durch die Dehnung des Netzwerks die Wahrscheinlichkeitsverteilung des Endabstandsquadrates der Netzketten einen geringeren Erwartungswert annimmt. Dies entspricht nach der Boltzmann-Gleichung einer Verringerung der Entropie im System. Dieses hat aber das Bestreben den Zustand größerer Entropie wieder einzunehmen. Das heißt im Fall eines elastomeren Polymernetzwerks, dass die Ketten das Bestreben haben, sich wieder zu verknäulen. Dieses Verhalten wird als Entropie- oder Gummielastizität bezeichnet.

Das erste statistische Modell zur Beschreibung dieses Verhaltens geht auf Kuhn ${ }^{[133,134]}$ zurück. Anhand der theoretischen Betrachtung der Entropielelastizität von Naturkautschuk konnte Kuhn zeigen, dass sich für elastomere Stoffe Gesetzmäßigkeiten ergeben, die unabhängig von der chemischen Natur der Polymere sind und sich allein auf die Zahl der Netzketten zurückführen lassen. $^{[135]}$ 


\subsubsection{Statistische Theorie der Entropieelastiztät}

\subsubsection{Das affine Netzwerkmodell ${ }^{[136,137]}$}

Kuhn ging davon aus, dass bei einer äußeren mechanischen Belastung eines Polymernetzwerkes, bei dem die Quadrate der End-zu-End-Abstandsvektoren der Netzketten $\vec{R}$ idealerweise einer Gaußverteilung folgen, jedes einzelne Volumenelement eine Deformation relativ zur makroskopischen Deformation des Netzwerks erfährt. Ein solches Volumenelement kann z. B. eine einzelne Netzkette bilden (s. Abbildung 2-10). Nach Kuhn ist also jede Netzkette anteilig an der Deformation des Gesamtnetzwerks beteiligt. Die Änderung der End-zu-End Abstandsvektoren der Netzketten während der Deformation ist dabei linear (affin).

Die Entropieänderung des Gesamtnetzwerks ergibt sich als Summe der Entropieänderungen aller Netzketten. Für die Entropieänderung eines um den Faktor $l_{(\mathrm{x}, \mathrm{y}, \mathrm{z})}$ deformierten Netzwerks in $\mathrm{x}^{-}, \mathrm{y}-$ und $\mathrm{z}-$ Richtung folgt somit:

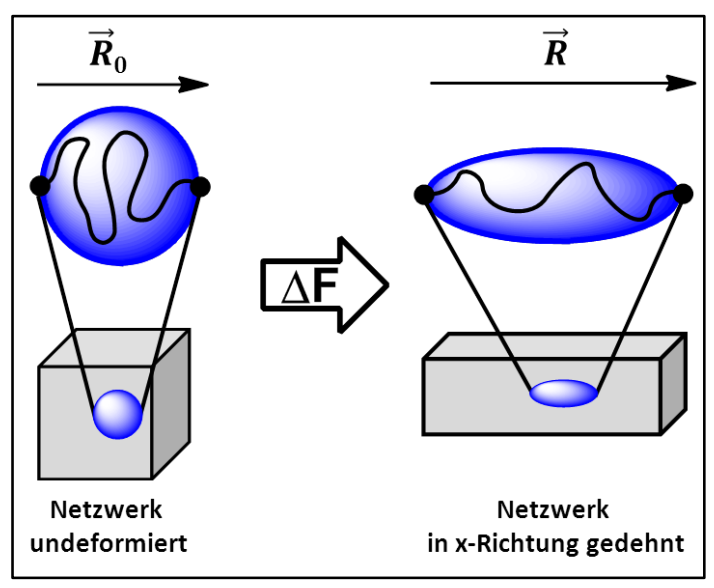

Abbildung 2-10 Affine Dehnung einer Netzkette relativ zur makroskopischen Deformation des Gesamtnetzwerks bei Anlegen einer äußeren Kraft $F$. Nach Rubinstein ${ }^{[136]}$.

$$
\Delta S=-\frac{n k_{\mathrm{B}}}{2}\left(l_{\mathrm{x}}^{2}+l_{\mathrm{y}}^{2}+l_{z}^{2}-3\right)
$$

Wobei $n$ die Zahl der Netzketten und $k_{\mathrm{B}}$ die Boltzmann-Konstante bezeichnen. Trockene, unsolvatisierte Netzwerke sind normalerweise inkompressibel; ihr Volumen ist daher während einer mechanischen Verformung konstant. Für eine uniaxiale Deformation in x-Richtung folgt somit, dass die Längenänderung in $\mathrm{x}$-Richtung mit einer isochoren Verkleinerung der $\mathrm{y}$ - und zDimension einhergeht, somit folgt für Längenänderung:

$$
l_{\mathrm{x}}=l ; \quad l_{\mathrm{y}}=l_{\mathrm{z}}=\frac{1}{\sqrt{l}}
$$

Für die Änderung der freien Energie einer uniaxialen Dehnung in x-Richtung ergibt sich somit: 


$$
\Delta A_{\mathrm{def}}=-T \Delta S=\frac{n k_{\mathrm{B}} T}{2}\left(l^{2}+\frac{2}{l}-3\right)
$$

Die Kraft $F_{\mathrm{X}}$, die dafür notwendig ist, das Netzwerk entlang seiner x-Achse um den Faktor $l$ zu deformieren, ergibt sich, wenn die Änderung der freien Energie während der Dehnung nach der Länge des Netzwerkes entlang der x-Achse nach dem Dehnen $L_{\mathrm{x}}$ abgeleitet wird. Es folgt:

$$
F_{\mathrm{x}}=\frac{\partial \Delta A_{\mathrm{def}}}{\partial L_{\mathrm{x}}}=\frac{n k_{\mathrm{B}} T}{L_{\mathrm{x} 0}} \cdot\left(l-\frac{1}{l^{2}}\right)
$$

mit $L_{\mathrm{x} 0}$ als undeformierter Länge des Netzwerks entlang der $\mathrm{x}$-Achse. Die auf die Querschnittsfläche des Netzwerks wirkende Kraft wird auch als Spannung $\sigma$ bezeichnet. Die makroskopische Deformation des Netzwerks $\gamma$ ist der Spannung proportional, die entsprechende Proportionalitätskonstante ist der Schermodul $G$. Dieser ergibt sich im affinen Netzwerkmodell $\mathrm{zu}:$

$$
G=\frac{\sigma}{\gamma}=\frac{n k_{\mathrm{B}} T}{V}=v_{\mathrm{eff}} \cdot k_{\mathrm{B}} T=\frac{\rho R T}{\bar{M}_{\mathrm{x}}}
$$

mit $\rho$ der makroskopischen Dichte des Netzwerks und $R$ der idealen Gaskonstanten. Die Zahl der Netzketten pro Volumeneinheit $(n / V)$ bildet die schon zuvor erwähnte effektive Netzkettendichte $v_{\text {eff }}$ ab (s. Kapitel 2.2.1). Hierbei handelt es sich um die Zahl der elastisch aktiven Netzketten im Netzwerk. $\bar{M}_{\mathrm{x}}$ ist die zahlenmittlere, scheinbare Molmasse dieser Netzketten.

Der Schermodul ist eine einfach, z. B. durch Zugversuche (s. Kapitel 2.4), messbare Größe, die das makroskopische, mechanische Verhalten einer Probe beschreibt. $G$ kann also über die Netzkettendichte einen idealisierten Eindruck vom Aufbau und der Struktur des Netzwerks vermitteln.

\subsubsection{Das Phantom-Netzwerkmodell ${ }^{[135-137]}$}

Der Begriff des Phantom-Netzwerks stammt ursprünglich von Flory ${ }^{[138]}$. Es handelt sich hierbei um eine Weiterentwicklung des affinen Netzwerkmodells. Während beim affinen Netzwerkmodell die Vereinfachung gemacht wird, dass die Netzknotenpunkte fest im Raum fixiert sind, also nur eine affine Positionsänderung entsprechend einer möglichen makroskopischen Deformation des Netzwerks existiert, lässt das Phantom-Netzwerkmodell eine 
Fluktuation der Netzknoten um ihre mittlere Position zu. Damit wird der Tatsache Rechnung getragen, dass in realen Netzwerken die Netzknoten je nach Funktionalität mit mehreren Netzketten verbunden sind. Hierdurch wird eine kumulative Netzwerkdeformation vermindert und die Änderung der Entropie während der Deformation verringert. Für die Änderung der freien Energie des Systems gilt dann:

$$
\Delta A_{\mathrm{def}}=n R T\left(\frac{A}{2} \eta\left(l_{\mathrm{x}}^{2}+l_{\mathrm{y}}^{2}+l_{\mathrm{z}}^{2}-3\right)-B \cdot \ln \left(\eta^{3 / 2} \cdot l_{\mathrm{x}} l_{\mathrm{y}} l_{\mathrm{z}}\right)\right)
$$

Die Konstanten $A$ und $B$ stellen hier den Struktur- und den Volumenfaktor dar. Der Dilatationsfaktor $\eta$ ergibt sich aus dem Verhältnis der mittleren quadratischen End-zu-EndAbstände von den Netzwerkketten eines isotropen, nicht deformierten Netzwerks und freien, unvernetzten Ketten in gleicher molekularer Umgebung. Da diese Werte als nahezu gleich angenommen werden können, ${ }^{[139,140]}$ nimmt $\eta$ folglich näherungsweise den Wert 1 an.

Der Volumenfaktor $B$ ist nach Flor ${ }^{[138]}$ als $2 / f$ von der Funktionalität des Vernetzers abhängig. Allerdings gilt bei der Annahme eines konstanten Volumens während der Deformation des Netzwerks, dass der zweite Term von Gleichung (24) gleich Null ist und der Wert von $B$ somit keinerlei Bedeutung hat.

Somit hängt die Verringerung der Änderung der freien Energie allein vom Strukturfaktor ab. Er stellt ein Maß für die Fluktuation der Netzknotenpunkte dar. Der Wert dieses Faktors war lange Zeit Gegenstand kontroverser Diskussionen. Während Kuhn ${ }^{[133,134]}$, Flory und Rehner ${ }^{[141,142]}$, Wall $^{[139,143,144]}$ und Hermans ${ }^{[140]}$ diesen bei $A=1$ sahen, womit letztlich wieder das affine Netzwerkmodell gelten würde, haben vor allem fames und Guth ${ }^{[145,146]}$ eine mögliche Fluktuation der Netzknoten in ihren Modellen berücksichtigt und erhielten somit einen Wert von $A=0,5$. In den folgenden Jahren verallgemeinerten Eichinger ${ }^{[147]}$ und Greassley ${ }^{[148,149]}$ diese Sichtweisen, indem sie den Wert von $A$ mit der Funktionalität des Vernetzer in Verbindung setzten und folgende Beziehung formulierten:

$$
A=\left(1-\frac{2}{f}\right)
$$

Hieraus ist zu erkennen, dass für einen tetrafunktionellen Vernetzer der Strukturfaktor den von fames und Guth vorgeschlagenen Wert von 0,5 annehmen würde. Schließlich kam Flory ${ }^{[138]}$ in späteren Arbeiten ebenfalls zu diesem Ergebnis, wobei er darauf verwies, dass es sich bei den beiden Modellen (affin, Phantom) jeweils um Extremfälle handeln muss. Unsolvatisierte Netzwerke bei geringen Deformationen würden demnach eher dem affinen Modell entsprechen, 
da Fluktuationen der Netzknoten durch die sterischen Ansprüche der sie umgebenden Polymerketten weitgehend unterdrückt sind. Dagegen sollten bei gequollenen oder stark deformierten Netzwerken die Polymerketten für eine deutlich geringere Beschränkung der Fluktuation der Netzknoten sorgen, sodass das Phantom-Netzwerkmodell seine Gültigkeit hat. Bei realen Netzwerken kommt es allerdings immer wieder zu der Ausbildung von Defekten (s. Kapitel 2.2.1). Diese können sowohl zu einer Erhöhung- (z. B. Verschlaufungen) oder Erniedrigung (z. B. freie Kettenenden) der Anzahl elastisch aktiver Vernetzungspunkte führen. Dies vergrößert oder verringert ebenfalls die Zahl der elastisch wirksamen Netzketten. Weiterhin könnten sie nicht in das Netzwerk eingebaute Moleküle enthalten, was zu einer geringen Quellung bzw. geringeren Deformation der Ketten führen kann. Darum sollte für reale Netzwerke folgende Beziehung gelten:

$$
\left(1-\frac{2}{f}\right) \leq A \leq 1
$$

Die Einführung des Strukturfaktors im Phantom-Netzwerkmodell hat ebenfalls Auswirkungen auf den Schermodul des Netzwerks. Die mögliche Fluktuation der Netzknoten würde zu einem weicheren Netzwerk führen und den Modul somit verringern. Er ergibt sich zu:

$$
G=v_{\mathrm{eff}} k_{\mathrm{B}} T \cdot \frac{f-2}{f}=\frac{\rho R T}{\bar{M}_{\mathrm{x}}} \cdot\left(1-\frac{2}{f}\right)=\frac{\rho R T \cdot A}{\bar{M}_{\mathrm{x}}}
$$

\subsubsection{Quellung von Polymernetzwerken}

Wie schon in Kapitel 2.2.1 erwähnt, gehen Polymernetzwerke bei Zugabe eines geeigneten Lösungsmittels nicht in Lösung, sondern quellen um das Vielfache ihrer ursprünglichen Größe auf. Dies geschieht durch Aufnahme von Lösungsmittelmolekülen in das Netzwerk. Es kommt also zu einer Mischung von Polymer und Lösungsmittel. Thermodynamisch gesehen ist der Mischungsvorgang zweier Komponenten freiwillig, wenn die Änderung der molaren freien Mischungsenthalpie $\Delta \bar{A}_{\text {mix }}$ während des Mischungsvorganges negativ ist.

Da bei der Quellung das Volumen des Netzwerks nicht konstant ist, wird für die thermodynamische Betrachtung der Quellung die Gibbs'sche freie Enthalpie verwendet. Diese ist gegeben durch:

\footnotetext{
i Obwohl das Formelzeichen $A$ in der Literatur eigentlich nur für die freie Energie nach Helmholtz verwendet wird, wird es in dieser Arbeit auch für die Gibbs'sche freie Enthalpie verwendet, um mögliche Verwechslungen mit dem Schermodul $G$ zu vermeiden.
} 


$$
\Delta \bar{A}_{\text {mix }}=\Delta \bar{H}_{\text {mix }}-T \Delta \bar{S}_{\text {mix }}
$$

mit $\Delta \bar{H}_{\text {mix }}$ als Mischungsenthalpie und $\Delta \bar{S}_{\text {mix }}$ als Mischungsentropie. Eine negative freie Enthalpie ist gegeben, wenn die Mischungsentropie positiv ist. Dass dies für PolymerLösungsmittel-Systeme der Fall ist, wurde unabhängig voneinander von Flory ${ }^{[150]}$ und Huggins ${ }^{[151]}$ untersucht und verifiziert. Die molare Mischungsenthalpie hängt dabei von der Güte des Lösungsmittels ab. ${ }^{[152-154]}$ So wird sie z. B. bei Verwendung eines athermischen Lösungsmittels gleich Null, was den Einfluss des entropischen Teils von Gleichung (28) deutlich macht. Mit Hilfe der Flory-Huggins-Theorie ${ }^{[81,155]}$ besteht die Möglichkeit, die molare freie Enthalpie für verschiedene Lösungsmittel-Polymer-Systeme zu bestimmen.

Für die Änderung der freien Enthalpie der Quellung darf allerdings nicht nur die freie Mischungsenthalpie betrachtet werden. Durch die Aufnahme von Lösungsmittelmolekülen in das Netzwerk kommt es zur Streckung der Netzwerkketten. Es muss also auch die freie Energie der elastischen Ausdehnung der Netzwerkketten $A_{\text {def }}$ in Betracht gezogen werden. Dieser Zusammenhang wurde von Flory und Rehner ${ }^{[141]}$ in Bezugnahme auf die Arbeit Frenkels ${ }^{[156]}$ so ausgedrückt, dass sich die Änderung der freien Enthalpie der Quellung $\Delta A_{\mathrm{Q}}$ aus der freien Entropie der Mischung und der freien elastischen Enthalpie zusammensetzt:

$$
\Delta A_{\mathrm{Q}}=\Delta A_{\mathrm{mix}}+\Delta A_{\mathrm{def}}
$$

Die freie elastische Energie geht dabei aus der Theorie der Entropieelastizität (s. Kapitel 2.3) hervor. Sie steigt für eine Kette bei affiner Deformation mit wachsendem Kettenendabstand $R_{0}$ nach:

$$
A_{\mathrm{def}} \approx k_{\mathrm{B}} T \frac{\left(l \cdot R_{0}\right)^{2}}{R_{\mathrm{ref}}^{2}}
$$

Dabei bezeichnet $R_{\text {ref }}^{2}$ die mittlere Fluktuation der Kettenendabstände. Mit zunehmender Aufnahme von Lösungsmittel in das Netzwerk und somit zunehmender Dehnung der Netzketten erreicht das System irgendwann den Punkt an dem die Beträge der freien Mischungsenthalpie und der freien elastischen Enthalpie gleich sind, so dass $\Delta A_{\mathrm{Q}}=0$ wird. Das System erreicht ein Quellungsgleichgewicht, an dem sich die Netzketten nicht weiter entknäueln. Nach den statistischen Betrachtungen der Quellung von Flory und Rehner ${ }^{[141,142]}$ gilt im Quellungsgleichgewicht folgender Zusammenhang: ${ }^{[112]}$ 


$$
\ln \left(1-\Phi_{\mathrm{Pol}}\right)+\Phi_{\mathrm{Pol}}+\chi \Phi_{\mathrm{Pol}}^{2}+v_{\mathrm{eff}} \cdot V_{\mathrm{mol}(\mathrm{LM})} \cdot\left(\Phi_{\mathrm{Pol}}^{\frac{1}{3}}-\frac{\Phi_{\mathrm{Pol}}}{2}\right)=0
$$

$\Phi_{\text {Pol }}$ ist der Volumenbruch des Polymers, $V_{\text {mol(s) }}$ stellt das Molvolumen des Lösungsmittels dar und $\chi$ ist der so genannte Flory-Huggins-Wechselwirkungsparameter. Dieser beschreibt die Abweichung eines Lösungsmittels vom athermischen Verhalten. Darum gilt, je kleiner der Flory-Huggins-Wechselwirkungsparameter ist, desto idealer verhält sich das Lösungsmittel. Die ersten drei Terme der Flory-Rehner-Gleichung (31) lassen sich dabei auf die Änderung der freien Mischungsenthalpie und die letzten beiden Therme auf die Änderung der freien elastischen Energie während der Quellung zurückführen. ${ }^{[18]}$

Die Streckung der Ketten während der Quellung zeigt, dass diese letztlich auch als eine Art mechanische Eigenschaft des Netzwerks angesehen werden kann, was wiederum eine makroskopische Betrachtung dieses Prozesses ermöglicht. Bei der makroskopischen Ausdehnung des Netzwerks nimmt es auch an Volumen zu. Diese Ausdehnung ist dabei in alle Raumrichtungen gleichgroß. Die lineare Deformation $L_{\mathrm{D}}$ ergibt sich zu

$$
L_{\mathrm{D}}=\left(\frac{V_{\mathrm{eq}}}{V_{0}}\right)^{1 / 3}
$$

mit $V_{\text {eq }}$ dem Gesamtvolumen des Gels im Quellungsgleichgewicht und $V_{0}$ dem Volumen des reinen, ungequollenen Netzwerks. Dabei setzt sich das Gleichgewichtsvolumen aus dem Volumen des Polymeren $V_{\text {Pol }}$ und dem Volumen des Lösungsmittels im Netzwerk $V_{\text {LM }}$ zusammen:

$$
V_{\mathrm{eq}}=V_{\mathrm{Pol}}+V_{\mathrm{LM}}
$$

Im Quellungsgleichgewicht kann der Volumenquellungsgrad $Q_{V}$ mit:

$$
Q_{V}=\frac{V_{\mathrm{eq}}}{V_{\mathrm{Pol}}}
$$


definiert werden. Der Kehrwert des Quellungsgrades stellt dabei den Volumenbruch des Polymeren im gequollenen Zustand dar: ${ }^{[157]}$

$$
\Phi_{\mathrm{Pol}}=\frac{1}{Q_{V}}
$$

Eine weitere in der allgemeinen Laborpraxis häufig besser zugängliche Möglichkeit, den Quellungsgrad zu ermitteln, ist die Massenänderung des Netzwerks während des Quellens. Diese ist durch einfache gravimetrische Messungen eines Probekörpers zugänglich. Die hieraus zu erhaltende Größe ist der Massenquellungsgrad $Q_{m}$. Unter der Annahme, dass das Molvolumen der einzelnen Polymersegmente (Monomereinheiten) gleich dem Molvolumen des Lösungsmittels ist, entspricht der Volumenbruch des Polymers dem Grundmolenbruchs $x_{\text {Pol }}^{*}$ des Polymers. ${ }^{[85]}$ Entsprechend Gleichung (35) ist $Q_{m}$ der Kehrwert des Grundmolenbruchs im gequollenen Zustand. Somit kann der Massenquellungsgrad nach Rehage ${ }^{[153]}$ angegeben werden als:

$$
Q_{m}=\frac{1}{x_{\text {Pol }}^{*}}=\frac{m_{L M}+m_{\mathrm{Pol}}}{m_{\mathrm{Pol}}}
$$

Sind die Dichten des Polymers $\rho_{\text {Pol }}$ und des verwendeten Lösungsmittels $\rho_{\mathrm{LM}}$ sehr ähnlich, so kann in guter Näherung davon ausgegangen werden, dass der Massenquellungsgrad dem Volumenquellungsgrad entspricht. Weichen die Dichten zu stark voneinander ab, kann diese Näherung nicht gemacht werden. Der Volumenquellungsgrad ergibt sich somit über den Massenquellungsgrad nach:

$$
Q_{V}=\frac{\left(Q_{m}-1\right) \cdot \rho_{\mathrm{Pol}}}{\rho_{\mathrm{LM}}}+1
$$

\subsection{Der Zugversuch}

Eine sehr verbreitete Methode, die mechanischen Eigenschaften von Werkstoffen und somit auch Polymerproben zu messen, ist der Zugversuch. Hierbei werden normierte Proben bekannter Abmessung in die Klemmbacken einer Zugmaschine eingespannt. Eine der Klemmbacken wird 
anschließend mit konstanter Geschwindigkeit von der anderen entlang einer festen Achse wegbewegt. Dadurch wirkt eine Kraft auf die Probe, die in dieser nach:

$$
\sigma=\frac{1}{L_{\mathrm{y} 0} L_{\mathrm{z} 0}} \cdot F
$$

eine Spannung im Material erzeugt, die der entlang der Achse anliegenden Kraft $F$ proportional ist. Dabei steht das Produkt $L_{\mathrm{y} 0} L_{\mathrm{z} 0}$ für die Querschnittfläche der Probe zu Beginn der Messung. Abbildung 2-11 zeigt einen solchen Probekörper, die Schultern rechts und links dienen als Einspannflächen in die Klemmbacken. Der schmalere Steg zwischen den Schultern dient als Sollbruchstelle, außerdem ist das Material hier unbeeinflusst vom Druck der Klemmen. ${ }^{[158]}$

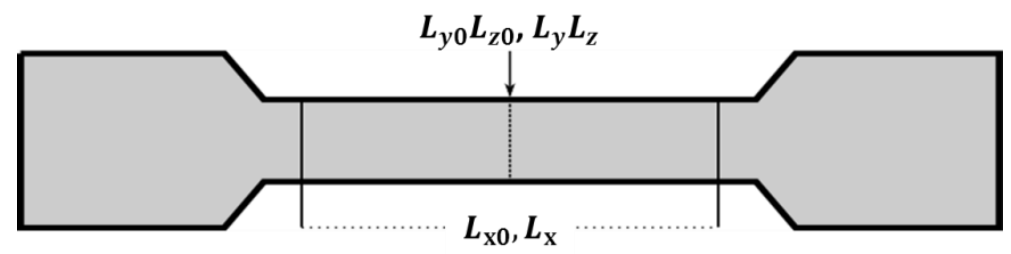

Abbildung 2-11 Schematische Darstellung eines typischen Probekörper bei einem Zugversuch. $L_{\mathrm{y} 0} L_{\mathrm{z} 0}$ ist die Querschnittsfläche, $L_{\mathrm{x} 0}$ die Länge der Probe zu Beginn der Messung. $L_{\mathrm{y}} L_{\mathrm{z}}$ und $L_{\mathrm{x}}$ sind die Querschnittsfläche und die Länge der Probe nach begonnener Messung.

Durch die anliegende Spannung wird die Probe, vor allem entlang des schmalen Stegs, gedehnt. Nach Gleichung (38) ist die Spannung hier am höchsten. Diese Dehnung $\gamma$ lässt sich berechnen nach:

$$
\gamma=\frac{L_{\mathrm{x}}-L_{\mathrm{x} 0}}{L_{\mathrm{x} 0}}=\frac{\Delta L}{L_{\mathrm{x} 0}}
$$

Im Bereich kleiner Dehnungen ist diese vollständig reversibel (elastischer Bereich). Hier ergibt sich eine Proportionalität der durch die Spannung herbeigeführten Dehnung. Nach dem Hook'schen Gesetz gilt:

$$
\sigma=E \cdot \gamma
$$

Die Proportionalitätskonstante $E$ wird als Elastizitäts- oder kurz E-Modul bezeichnet. Vor allem im englischen Sprachraum ist die Bezeichnung Young-Modul (nach Thomas Young) ${ }^{[159,160]}$ 
gebräuchlich. Im elastischen Bereich wird der E-Modul auch als Gleichgewichtsmodul (engl.: equilibrium modulus) bezeichnet. ${ }^{[161-164]}$

Es gilt: Je größer der E-Modul ist, desto steifer ist auch das Probenmaterial. Dies lässt sich sehr anschaulich am Messverlauf eines Zugversuches zeigen, dem so genannten SpannungsDehnungs-Diagramm (s. Abbildung 2-12). Aus der Steigung der Spannungs-Dehnungs-Kurve (SDK) bei geringen Dehnungen im elastischen Bereich, kann der Elastizitätsmodul erhalten werden.

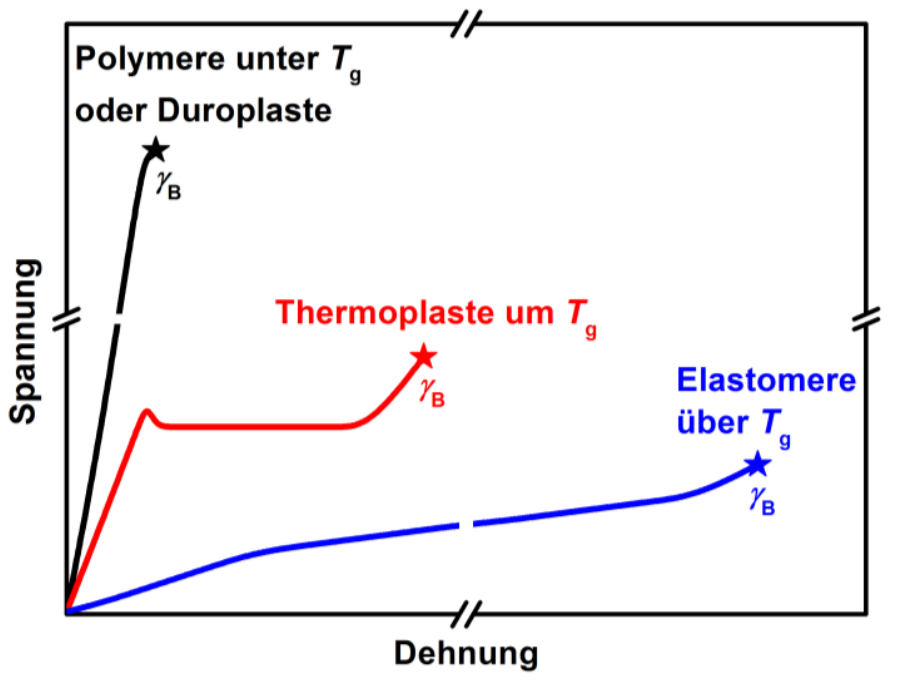

Abbildung 2-12 Beispielhafte Verläufe von Spannungs-Dehnungs-Kurven verschiedener Polymerarten bei unterschiedlichen Temperaturen relativ zu ihrem Glaspunkt bis zum Versagen des Materials, der Bruchdehnung $\gamma_{B}$. Nach Tieke ${ }^{[165]}$.

Einen sehr starken Einfluss auf den Betrag des Elastizitätsmoduls hat neben der chemischphysikalischen Struktur der Polymere auch die Temperatur, bei der die Messungen durchgeführt werden. Vor allem der Wert der Glastemperatur der Probe relativ zur Messtemperatur (i. d. R. Raumtemperatur) ist dabei entscheidend für die mechanischen Eigenschaften der Probe und somit für den Verlauf der Messung. Als Glastemperatur $T_{\mathrm{g}}$ wird die Temperatur bezeichnet, bei der die amorphen Bereiche eines Polymers aufweichen, wenn es aufgeheizt wird, bzw. erstarren wenn das Polymer abgekühlt wird.

Polymere, die sich bei Raumtemperatur unter ihrer Glastemperatur befinden, wie z. B. Polystyrol, sind sehr steif und verhalten sich nahezu wie energieelastische Materialien. Sie erreichen nur geringe Dehnungen bei sehr hohen Spannungen bevor sie brechen. Dies gilt ebenfalls für duroplastische Materialien, wie z. B. Netzwerke aus multifunktionellen (Meth-)Acrylaten. Bei diesen ist der Einfluss der Temperatur auf die mechanischen Eigenschaften allerdings sehr gering. Wenn sie überhaupt über eine Glastemperatur verfügen, befindet sich diese häufig erst bei relativ hohen Temperaturen (s. Abbildung 2-13). Erst danach ändern sie ihr mechanisches 
Verhalten und werden zunehmend lederartig, bis sie sich bei sehr hohen Temperaturen schließlich thermisch zersetzten.

Thermoplasten, also teilkristalline, lineare Polymere, wie z. B. Polyethylen hoher Dichte (HDPE), zeigen im Bereich ihrer Glastemperatur einen deutlich anderen Verlauf der SpannungsDehnungs-Kurve. Zunächst steigt die Spannung im elastischen Bereich mit der Dehnung an. Sie durchläuft ein Maximum: die Streckgrenze. Hier kommt es zur Einschnürung der Probe. Dabei nimmt ihr Querschnitt ab, was die auf sie wirkende Spannung zunächst sinken lässt. Anschließend bleibt die Spannung konstant. In dieser Phase kommt es zu einer parallelen Ausrichtung der Polymere in der Probe. Die Probe wird verstreckt. Nach vollständiger Verstreckung steigt die Spannung wieder an, bis die Probe von Kettenriss oder Abgleiten benachbarter Ketten schließlich versagt und bricht. Dieser Vorgang wird auch als Kaltverstreckung bezeichnet. ${ }^{[158,165]}$

Elastomere, wie z. B. Gummi oder vernetztes Polybutylacrylat (pBA), befinden sich bei Raumtemperatur weit über ihrer Glastemperatur. Ihre E-Moduln sind deutlich geringer als die der vorherigen Beispiele. Auch ist bei Elastomeren der elastische Bereich deutlich länger als bei Duro- oder Thermoplasten. Das heißt, sie lassen sich häufig um sehr große Beträge, nahezu vollständig reversibel dehnen, bis es bei sehr großen Dehnungen von bis zu mehreren $100 \%$ zum Bruch kommt. Die dabei anliegenden Spannungen sind allerdings sehr klein.

Einen Überblick über das unterschiedliche mechanische Verhalten der verschiedenen Polymerarten bei unterschiedlichen Temperaturen liefert Abbildung 2-13.

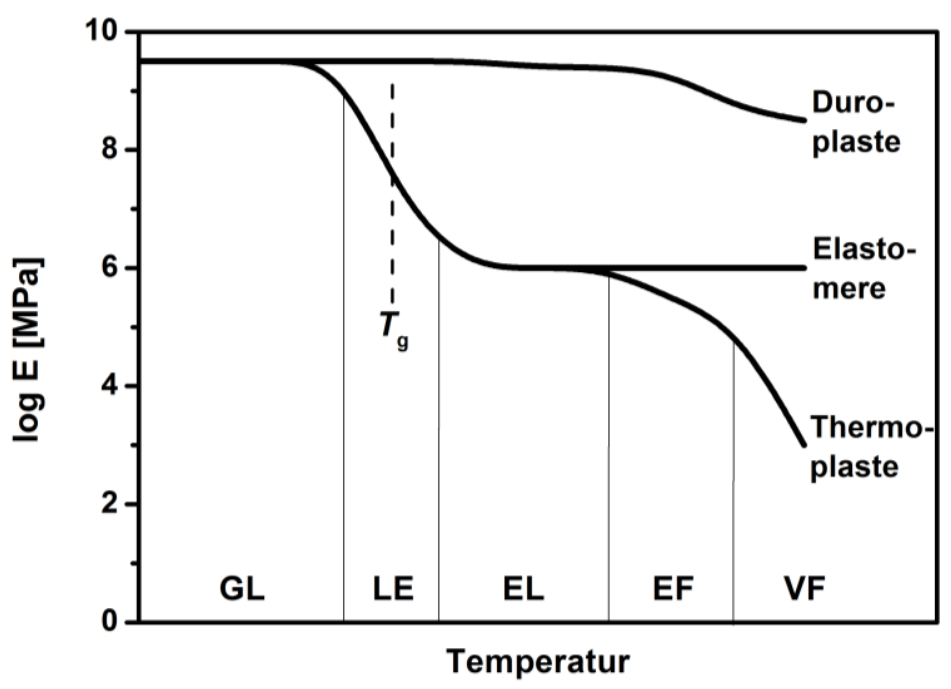

Abbildung 2-13 Typischer Verlauf des Elastizitätsmoduls mit steigender Temperatur. Während der E-Modul eines Duroplasten nahezu keine Abhängigkeit von der Temperatur zeigt, lassen sich für Elastomere und Thermoplaste unterschiedliche Bereiche definieren. GL: Glaszustand, LE: lederartiges Verhalten, EL: elastomeres Verhalten (gummielastisches Plateau). Bei Thermoplasten zusätzlich: EF: elastomeres Fließen, VF: viskoses Fließen. $T_{\mathrm{g}}$ : Glastemperatur. Nach Tieke ${ }^{[131]}$ und Elias ${ }^{[159]}$. 
Eine wichtige Größe für die Untersuchung von Polymeren, vor allem von Elastomeren, ist der Schermodul $G$, da sich mit diesem z. B. die Netzkettendichte oder die Molmasse der Netzketten berechnen lassen (s. Kapitel 2.3.1). Der Schermodul lässt sich aber aus dem E-Modul nach:

$$
G=\frac{1}{3} E
$$

berechnen. Somit bieten Zugversuche eine sehr gute Möglichkeit mit einem relativ einfachen, aber standardisierten experimentellen Aufbau, Daten zu erhalten, aus denen Rückschlüsse auf die mikroskopische Struktur von Elastomeren gezogen werden können.

Zusätzlich können dem Spannungs-Dehnungs-Diagramm noch weitere Informationen entnommen werden. So entspricht z. B. die Fläche unter der Spannungs-Dehnungs-Kurve bei Thermoplasten der Energie, die benötigt wird, um das Material zu zerreißen und stellt somit ein Maß für die Sprödigkeit des Materials bei mechanischer Belastung dar. ${ }^{[166]}$ Die Zähigkeit ist dabei der Widerstand des Materials gegen einen Bruch. ${ }^{[167]}$ Sie ergibt sich im Allgemeinen durch die Fähigkeit des Materials, sich bei einer mechanischen Belastung elastisch oder plastisch zu verformen. Bei Elastomeren entspricht die Fläche unter der SDK der Deformationsarbeit pro Einheitsvolumen $w$. Bei einer uniaxialen Deformation der Probe (dies ist beim Zugversuch gegeben) ist sie definiert als: ${ }^{[131]}$

$$
w=\frac{1}{2} G\left[l^{2}+\frac{2}{l}-3\right]
$$

Dabei steht $l$ für das Dehnverhältnis. Es ist definiert als:

$$
l=\frac{L_{\mathrm{x}}}{L_{\mathrm{x} 0}}
$$

Der Vergleich von Gleichung (42) mit Gleichung (21) zeigt, dass die Deformationsarbeit der Änderung der freien Energie im System entspricht. Weiterhin ist auch die Bruchdehnung $\gamma_{\mathrm{B}}$ für viele technische Anwendungen eine sehr wichtige Kenngröße und somit häufig von großem Interesse, da sie zeigt, bei welchen Belastungen das Material versagt. Außerdem ermöglicht sie, zusammen mit dem Modul, die Einteilung der Kunststoffe in unterschiedliche Klassen. ${ }^{[159]}$ 


\subsection{Adhäsion und Klebrigkeit von Polymeren}

Einige Polymere zeigen die besondere Fähigkeit, andere Materialien an ihren Oberflächen dauerhaft miteinander zu verbinden bzw. zu verkleben. Diese Polymere werden auch als Adhäsive oder auch engl. Pressure Sensitve Adhesives (PSA) bezeichnet. Sie werden in vielen, verschiedenen industriellen Bereichen angewendet, wie z. B. der Verpackungsindustrie, der Elektroindustrie oder der Medizin. ${ }^{[168,169]}$ Weiterhin sind sie im Alltag sehr weit verbreitet. So werden sie z. B. für Klebebänder oder selbstklebende Aufkleber verwendet. ${ }^{[170]}$ Typische PSAs sind Polymere wie Kautschuk, Silikone, Polyester, Polyurethane, Polyether oder Polyacrylate. ${ }^{[171]}$ Die am häufigsten verwendete Gruppe von Polymeren sind dabei die Polyacrylate. Deren Anteil am weltweiten PSA-Markt beträgt etwa $40 \% .{ }^{[172]}$ Polyacrylate werden in unterschiedlichsten Formen als PSAs verwendet: z. B. als Dispersionspolymerpartikel, ${ }^{[173-175]}$ Elastomere $^{[176]}$ oder auch als Schmelzen, die nachträglich, z. B. durch UV-Bestrahlung, teilvernetzt werden. ${ }^{[172,177]}$ Polyacrylate zeichnen sich durch eine hohe Stabilität gegenüber äußeren Einflüssen aus. Sie sind z. B. unempfindlich gegen eine mögliche Oxidation oder UV-Strahlung. Zudem besitzen sie gute thermische Eigenschaften, eine geringe Giftigkeit und sind kostengünstig. ${ }^{[178]}$ Weiterhin verfügen sie über ausgezeichnete adhäsive und kohäsive Eigenschaften, welche entscheidend sind für die Fähigkeit, andere Materialien (Adhärens) dauerhaft zu verbinden. ${ }^{[171,179]}$

Die Adhäsion bezeichnet dabei die Wechselwirkungen des Polymers mit den Oberflächen der anderen Materialien und kann in zwei Anteile unterteilt werden: zum einen die sofortige Adhäsion bzw. Klebrigkeit und zum andern die dauerhafte Haftung des anderen Materials am PSA, allgemein nur als Adhäsion bezeichnet. Hierbei handelt es sich um die Energie, die aufgebracht werden muss, um das PSA wieder von der Oberfläche des Adhärens vollständig zu lösen. Die Kohäsion dagegen bezeichnet die Wechselwirkungen zwischen den einzelnen Polymermolekülen. Sie stellt sozusagen den inneren Zusammenhalt der Polymerphase dar und entscheidet über die Fähigkeit des PSAs beim Anlegen einer Scherkraft an seiner Position zu verbleiben. ${ }^{[170,180]}$

Von besonderer Bedeutung für das makroskopische Verkleben von PSA und Adhärens ist aber vor allem die Klebrigkeit des PSAs. Von Klebrigkeit oder engl. Tack wird dann gesprochen, wenn das PSA in der Lage ist, schon bei geringem Druck und kurzer Kontaktzeit starke Wechselwirkungen mit dem Adhärens zu entwickeln. ${ }^{[175,181]}$ Hierfür sind die viskoelastischen Eigenschaften des Polymers von sehr großer Bedeutung. ${ }^{[168,175,181-185]}$ Diese hängen wiederum sehr stark von der Glastemperatur des verwendeten Polymers ab (s. Kapitel 2.4). Vor allem Polymere mit niedriger Glastemperatur, wie z. B. die schon genannten Polyacrylate, zeigen bei Raumtemperatur eine sehr ausgeprägte Klebrigkeit, da sie aufgrund der damit verbundenen guten Fließfähigkeit der Ketten zu einer sehr ausgeprägten Benetzung des Adhärens in der Lage sind. Eine gute Benetzung des Adhärens ist notwendig, um den Kontakt auf molekularer Ebene, z. B. durch Säure-Base-Wechselwirkungen, Wasserstoffbrücken oder Van-der-Waals- 
Wechselwirkungen, zu ermöglichen. Außerdem wird die Kontaktfläche zwischen PSA und Adhärens vergrößert, was wiederum die zwischen den Materialien wirkende Kraft erhöht. Weiterhin kann eine gute Benetzung dafür sorgen, dass mögliche Unebenheiten des Adhärens ausgeglichen werden und die Kontaktfläche somit noch mehr zunimmt. Diese Faktoren führen letztlich zu einer starken makroskopischen Verbindung des Adhärens mit dem PSA. ${ }^{[170,185]}$ Die Zahl und Größe der direkten Kontaktpunkte zwischen PSA und Adhärens innerhalb der makroskopischen Kontaktfläche steigt außerdem mit der Kontaktzeit und dem Druck, mit dem Adhärens und PSA zusammengebracht werden. ${ }^{[182]}$

Während die Klebrigkeit ein sehr kurzzeitiges Phänomen ist, sind für den dauerhaften Halt des PSAs auf dem Adhärens die kohäsiven und adhäsiven Prozesse verantwortlich. Um beide wieder zu trennen, müssen entweder die adhäsiven Kräfte zwischen PSA und Adhärens, die während des Kontakts entstanden sind, oder der kohäsive Zusammenhalt im PSA überwunden werden. Wie gut dies möglich ist, hängt vor allem von der Zusammensetzung des PSAs ab. Um den kohäsiven Zusammenhalt des PSAs zu verstärken, werden in der Regel teilvernetzte Polymere verwendet, die entweder während der Polymerisation, ${ }^{[171,178,186]}$ oder nachträglich, z. B. durch Bestrahlung mit UV-Strahlung, ${ }^{[172,177]}$ zu einem geringen Grad miteinander verknüpft werden. Die Vernetzung hat eine Verringerung der Klebrigkeit zur Folge, beeinflusst aber zusätzlich zu den kohäsiven Eigenschaften ebenfalls die dauerhaften adhäsiven Eigenschaften positiv. Die Vernetzung ermöglicht die Bildung von Fibrillen während des Ablösens des Adhärens vom PSA. Bei Fibrillen handelt es sich um mikroskopisch kleine Fäden, die die für die Trennung von PSA und Adhärens, benötigte Energie beträchtlich erhöhen und somit die Verbindung der beiden Stoffe verstärkt. ${ }^{[185,187]}$ Im Wesentlichen wird die Fibrillenbildung dadurch hervorgerufen, dass Polymerketten in der Lage sind, miteinander zu verhaken, was bei teilvernetzten Polymeren deutlich häufiger vorkommt. Zusätzlich sind diese Verhakungen dauerhafter als bei rein linearen Polymeren. Tobing konnte außerdem zeigen, dass mit der Zunahme der mittleren Kettenlänge zwischen zwei Verhakungen $\bar{M}_{\mathrm{e}}$ die Klebkraft ebenfalls zunimmt. ${ }^{[178]}$

Eine Alternative zu teilvernetzten Polymeren können aber auch Copolymere bieten, bei denen eine, oder mehrere Polymerkomponenten über eine höhere Glastemperatur verfügen. Diese können sich ebenfalls wie ein klassisches PSA verhalten. ${ }^{[174,175]}$ Dies zeigt, dass für den Prozess des Verklebens von PSA und Adhärens neben den thermischen Eigenschaften auch der strukturelle Aufbau des PSAs von großer Bedeutung ist, da dieser auch einen starken Einfluss auf die viskoelastischen Eigenschaften hat.

Trotz großer Schwierigkeiten bei der korrekten, fehlerfreien Messung der Klebrigkeit, ${ }^{[181,184]}$ existieren einige experimentelle Möglichkeiten, diese zu ermitteln, wie z. B. der so genannte Probe Tack Test. ${ }^{[188,189]}$ Hier wird mit einem Stempel auf das PSA gedrückt und dieser anschließend mit einer konstanten Geschwindigkeit wieder abgezogen. Die dafür notwendige Kraft wird dabei z. B. über einen Kraftaufnehmer gemessen. Anhand dieser Methode konnten für unterschiedliche Adhäsive, die in Abbildung 2-14 gezeigten Haftkurven erhalten werden. Dabei 
sollte beachtet werden, dass die Kurven $\mathbf{A}$ und $\mathbf{C}$ ebenfalls als Grenzfälle für ein teilvernetztes PSA, bei $\mathbf{A}$ sehr langsamen Abziehen des Stempels oder $\mathbf{C}$ sehr schnellem Abziehen auftreten können. ${ }^{[181]}$ Der Verlauf von Kurve B ist eine Folge der Ausbildung von Fibrillen zwischen PSA und Adhärens.

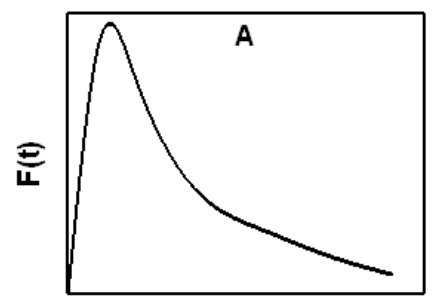

$\mathbf{t}$

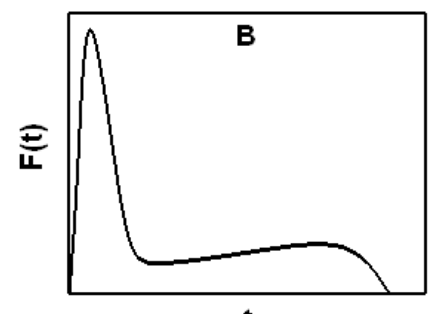

$\mathbf{t}$

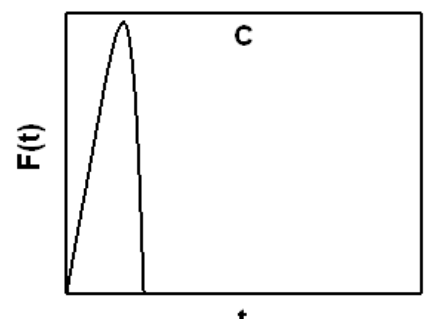

$\mathbf{t}$

Abbildung 2-14 Beispiele für Haftkurven von unterschiedlichen Adhäsiven, erhalten durch einen Probe Tack Test. A: viskoses Adhäsiv (z. B. niedermolekulare Flüssigkeit), B: viskoelastisches Adhäsiv (z. B. teilvernetztes PSA), C: elastisches Adhäsiv (z. B. elastomeres PSA). Nach Verdier ${ }^{[181]}$.

Eine weitere, apparativ weniger aufwendige Möglichkeit bietet der so genannte Rollkugeltest oder engl. Rolling Ball Tack Test. ${ }^{[173]}$ Dabei wird eine Metallkugel bekannten Gewichts und Durchmessers eine Rampe mit genormten Abmessungen ${ }^{[190]}$ herabgerollt. Am Fuß der Rampe befindet sich eine dünne Schicht des zu messenden PSAs. Dabei gilt, je weiter die Kugel über die Probe rollt, desto weniger klebrig ist das PSA. Die hieraus ermittelten Werte entsprechen zwar wissenschaftlich gesehen nicht direkt der Klebrigkeit oder der Adhäsion; hierbei würde es sich um eine Kraft bzw. Energie handeln. Sie eignen sich aber um vergleichende Messungen an unterschiedlichen PSAs durchzuführen. 


\section{Motivation}

Die RAFT-Polymerisation verbindet die Vorteile einer radikalischen Polymerisation mit den Vorteilen einer kontrollierten Polymerisation. Sie erlaubt die Synthese von speziell für die entsprechende Verwendung zugeschnittenen Polymeren und Polymermaterialien mit einem Minimum an apparativem Aufwand.

Aus diesem Grund ist die RAFT-Polymerisation von hohem Wert für Wissenschaft und Technik, denn viele moderne Anwendungen stellen häufig genau definierte Ansprüche an die verwendeten Polymermaterialien. Um die Polymere zielgenau herstellen und einsetzen zu können, ist die Kenntnis von ihren Struktur-Eigenschaftsbeziehungen von wesentlicher Bedeutung. Dies gilt in besonderem Maße für Polymernetzwerke.

Die mechanischen Eigenschaften dieser besonderen Klasse von synthetischen Materialien hängen sehr stark von ihrer Mikrostruktur ab. Diese weist bei der klassischen Synthese durch radikalische Polymerisation eine sehr große Heterogenität auf, die sowohl die elastischen als auch die Quellungseigenschaften der Netzwerke sehr stark beeinflusst. Die aus der RAFTPolymerisation erhaltenen Netzwerke sollten eine weitaus homogenere Struktur aufweisen. Somit wären sie den aus der radikalischen Polymerisation erhaltenen Gelen in vielen Anwendungsbereichen überlegen. Weiterhin sollte die RAFT-Polymerisation neben ihrem Einfluss auf den inneren Aufbau der Netzwerke ebenfalls deren Oberflächenstrukturen beeinflussen und somit einen Einfluss auf die Eigenschaften zu haben, die von dieser abhängen, wie z. B. die Klebrigkeit.

Trotz Untersuchungen zum Einfluss der RAFT-Polymerisation auf die Mikrostrukturen und die Eigenschaften von Polymernetzwerken, sind noch viele Fragen offen. Zum einen wurden widersprüchliche Ergebnisse erhalten und zum anderen ist die Zahl an untersuchten Monomersystemen noch relativ gering. Weiterhin fehlt es an Studien, die sich eingehend mit dem Einfluss der RAFT-Polymerisation auf unterschiedliche Eigenschaften der Netzwerke befassen und diese in ein Gesamtverhältnis zueinander stellen.

Das Ziel der vorliegenden Arbeit ist es darum, das Spektrum an untersuchten Monomeren zu erweitern und den Einfluss des RAFT-Mechanismus auf die Polymerisationskinetik und unterschiedliche Eigenschaften der Netzwerke, von ihrem Verhalten bei mechanischer Belastung bis hin zu ihrer Klebrigkeit, zu bestimmen. Dabei soll speziell der Einfluss der RAFTPolymerisation auf Polymernetzwerke aus UV-initiierter, radikalischer Polymerisation untersucht werden, da diese eine industriell sehr verbreitete Methode ist, um Polymernetzwerke herzustellen. 



\section{Auswertung und Diskussion}

\subsection{Der Einfluss der RAFT-Polymerisation auf statistische Poly(butyl-co-1,4-Butandioldiacrylat)- und Poly(ethyl- co-1,4-Butandioldiacrylat)-Netzwerke}

Zunächst wurde der Einfluss der RAFT-Polymerisation auf elastomere Polymernetzwerke untersucht. Dazu wurden systematisch Serien von statistischen Netzwerken auf Butylarcrylat(BA)- und Ethylacrylat(EA)-Basis synthetisiert. Die Synthese erfolgte als photoinitiierte, radikalische Polymerisation. Als Photoinitiator diente 2-Methyl-4'-(methylthio)2-morpholinopropiophenon (MMMP) (s. Schema 2-4). Als Vernetzungsreagenz, im Folgenden als Vernetzer abgekürzt, diente 1,4-Butandioldiacrylat (BDDA). Dieser Vernetzer wurde gewählt, da er den verwendeten Monomeren chemisch sehr ähnlich ist, sodass angenommen werden kann, dass er mit gleicher Wahrscheinlichkeit wie das<smiles>C=CC(=O)OCCCC</smiles>

BA<smiles>C=CC(=O)OCC</smiles>

EA<smiles>C=CC(=O)OCCCCOC(=O)C=C</smiles>

BDDA sichergestellt. Die Monomere wurden gewählt, da deren Polymerisation auch bei Raumtemperatur sehr schnell ist (s. Tabelle 4-1), was es ermöglichte, in einem angemessenen Zeitrahmen viele Proben für die

Abbildung 4-1 Strukturen der verwendeten Monomere $n$-Butylacrylat (BA) und Ethylacrylat (EA) sowie des Vernetzungsreagenz 1,4-Butandioldiacrylat (BDDA).

unterschiedlichen Untersuchungsmethoden herzustellen. Außerdem verfügen die resultierenden Polymere über eine sehr niedrige Glastemperatur (s. Tabelle 4-1), wodurch die gebildeten Netzwerke sich bei Raumtemperatur wie Elastomere verhalten können.

Tabelle 4-1 Glastemperaturen und Propagationskoeffizienten für die verwendeten Polyacrylate (Literaturwerte).

\begin{tabular}{ccc}
\hline Polymer & $\boldsymbol{k}_{\mathbf{p}}\left[\mathbf{L m o l}^{-\mathbf{1}} \mathbf{s}^{-1}\right]$ & Glastemperatur $\left[{ }^{\circ} \mathbf{C}\right]$ \\
\hline Poly-BA & $16100^{\mathrm{a}}$ & $-41,5^{\mathrm{b}}$ \\
Poly-EA & $14900^{\mathrm{c}}$ & $-24,0^{\mathrm{d}}$ \\
\hline
\end{tabular}

\footnotetext{
${ }^{\mathrm{a}}$ berechnet aus Werten nach Buback et al. ${ }^{[236]}$

${ }^{\mathrm{b}}$ nach Fuente ${ }^{[237]}$

${ }^{c}$ abgeschätzt aus Werten für BA und MA nach Beuermann et al. ${ }^{[238]}$

d nach Hughes et al. ${ }^{[239]}$
} 
Sehr intensiv wurde das System Poly(Butylacrylat-co-1,4-Butandioldiacrylat) (Poly(BA-coBDDA)) untersucht. Das System Poly(Ethylacrylat-co-1,4-Butandioldiacrylat) (Poly(EA-co-BDDA) diente als Kontrollsystem, um mögliche beim Poly(BA-co-BDDA) gefundene Effekte und Einflüsse der RAFT-Polymerisation auf die Eigenschaften und die Synthese der Netzwerke zu bestätigen.

\subsubsection{Einfluss der RAFT-Polymerisation auf die mechanischen Eigenschaften der Netzwerke}

\subsubsection{Variation des molaren BDDA-Anteils}

Zunächst wurde untersucht, wie sich die mechanischen Eigenschaften von Poly(BA-co-BDDA)- und Poly-(EA-coBDDA)-Netzwerken mit dem molaren Anteil des Vernetzers BDDA verändern. Dazu wurden bei einem konstanten molaren Anteil von 2 mol-\%o des RAFTAgens $S$-Ethylpropan-2-ylonat- $S^{\prime}$-hexyltrithiocarbonat (EPHT) (bezogen auf die Stoffmenge des Monomers) eine

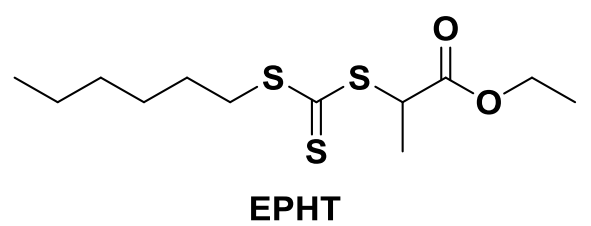

Abbildung 4-2 Struktur des verwendeten RAFT Agens S-Ethylpropan-2-ylonat- $S^{\prime}-$ hexyltrithiocarbonat (EPHT). Reihe von Netzwerken mit steigendem Gehalt an BDDA hergestellt und die entsprechenden E-Moduln per Zugversuch gemessen. Um den Einfluss der RAFT-Polymerisation auf die mechanischen Eigenschaften zu spezifizieren, wurden analog dazu, Netzwerke ohne die Zugabe von EPHT hergestellt und ebenfalls per Zugversuch vermessen. In allen Polymerisationsmischungen waren 2 Gew.-\% (bezogen auf die Masse des Monomers) MMMP als Photoinitiator enthalten. Genaue Angaben zu den Konzentrationen der beteiligten Substanzen und den Zusammensetzungen der Polymerisationsmischungen, die für die Synthese der Netzwerke verwendet wurden, sowie die Synthese- und Messbedingungen sind in Kapitel 6.2.4 sowie Kapitel 6.2.1 und Kapitel 6.3.1 zu finden.

Ein Eindruck von der Wirkung der RAFT-Polymerisation auf die Strukturen der Netzwerke, konnte schon direkt nach dem Aushärten der Probekörper für die Zugversuche erhalten werden.

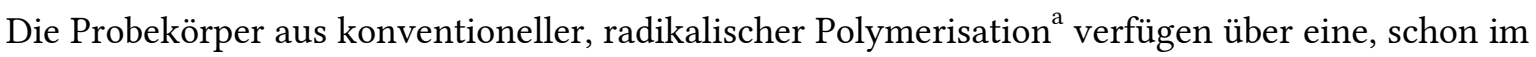
makroskopischen Bereich erkennbar, heterogenere Oberfläche. Anhand von LichtmikroskopAufnahmen ist dies an den weißen Flächen auf den Probekörpern zu erkennen. Hier wird das Licht der weißen Leuchtdioden, mit denen der Probeteller des Mikroskops beleuchtet wird, an den Unebenheiten der Oberflächen reflektiert, wodurch diese weiß erscheinen (s. Abbildung 4-3 und Abbildung 4-4; jeweils obere Bildreihe).

\footnotetext{
${ }^{a}$ Zur besseren Unterscheidung der radikalischen Polymerisation und der RAFT-Polymerisation, wird die radikalische Polymerisation mit dem Zusatz konventionell versehen. Die daraus resultierenden Netzwerke werden im Folgenden als konventionelle Netzwerke, die aus RAFT-Polymerisation dementsprechend als RAFT-Netzwerke bezeichnet.
} 


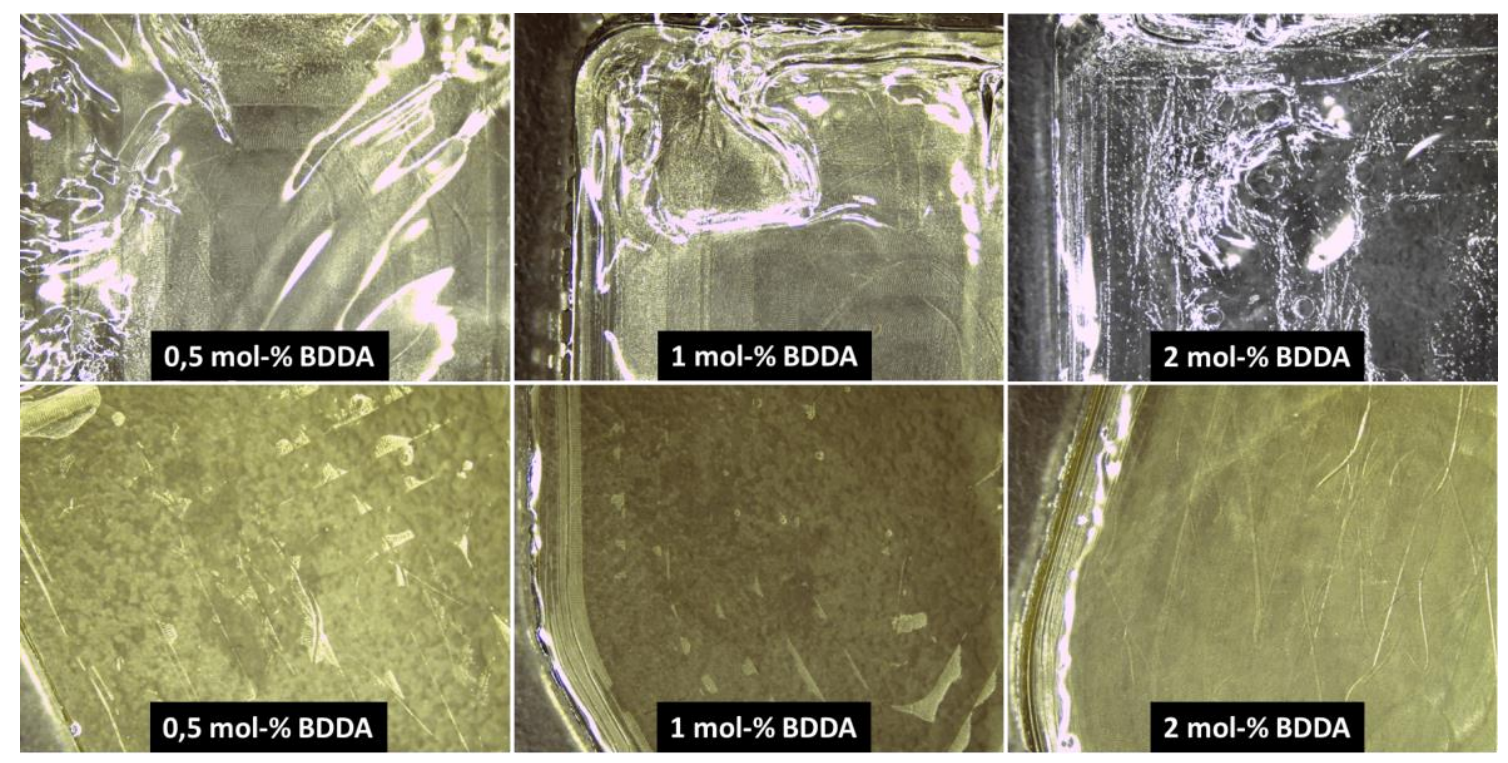

Abbildung 4-3 Lichtmikroskop-Aufnahmen von Poly(BA-co-BDDA)-Netzwerken mit 0,5 mol-\%, 1 mol-\% und 2 mol-\% des Vernetzers BDDA. Es handelt sich hierbei um Bildausschnitte der Zugversuch-Probekörper. Oben: Netzwerke aus UV-initiierter, konventioneller radikalischer Polymerisation. Unten: Netzwerke aus UV-initiierter RAFTPolymerisation mit jeweils 2 mol-\%o EPHT als RAFT-Agens. Als Photoinitiator wurde bei allen Netzwerken 2 Gew.-\% MMMP verwendet. Die Lichtintensität betrug $9 \mathrm{~mW} / \mathrm{cm}^{2}$ bei $366 \mathrm{~nm}$. Die Polymerisationszeit betrug $30 \mathrm{~min}$. Alle Polymerisationen wurden bei Raumtemperatur durchgeführt.

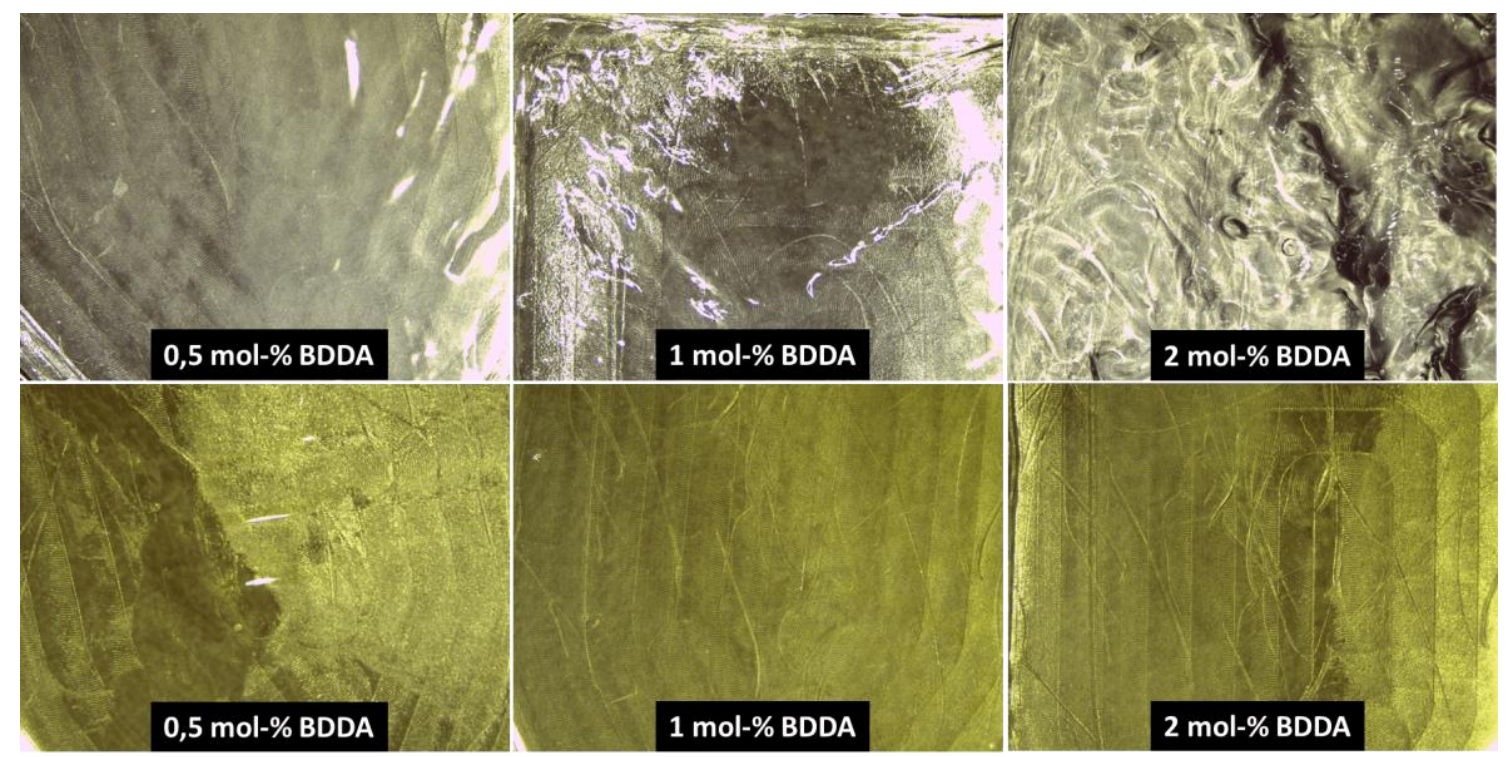

Abbildung 4-4 Lichtmikroskop-Aufnahmen von Poly(EA-co-BDDA)-Netzwerken mit 0,5 mol-\%, 1 mol-\% und 2 mol-\% des Vernetzers BDDA. Es handelt sich hierbei um Bildausschnitte der Zugversuch-Probekörper. Oben: Netzwerke aus UV-initiierter, konventioneller radikalischer Polymerisation. Unten: Netzwerke aus UV-initiierter RAFTPolymerisation mit jeweils 2 mol-\%o EPHT als RAFT-Agens. Als Photoinitiator wurde bei allen Netzwerken 2 Gew.-\% MMMP verwendet. Die Lichtintensität betrug $9 \mathrm{~mW} / \mathrm{cm}^{2}$ bei $366 \mathrm{~nm}$. Die Polymerisationszeit betrug $30 \mathrm{~min}$. Alle Polymerisationen wurden bei Raumtemperatur durchgeführt. 
Bei den Poly(EA-co-BDDA)-Netzwerken zeigt sich sogar eine klare Zunahme der Unebenheiten der Oberfläche mit zunehmenden, molaren Anteilen des Vernetzers BDDA. Bei den Probekörpern aus RAFT-Polymerisation dagegen ist die Oberfläche makroskopisch gesehen sehr glatt. Das Licht wird kaum von ihren Oberflächen reflektiert, sodass auch kaum weiße Flächen zu finden sind. Dafür sind die Rückseiten der Probekörper mit dem Fräßmuster der Teflonformen, in denen die Probekörper ausgehärtet wurden, zu erkennen (s. Abbildung 4-3 und Abbildung 4-4; untere Bildreihe). Eine Erklärung für diese Beobachtung könnte sein, dass bei den konventionellen Netzwerken hohe innere Spannungen existieren, die $\mathrm{zu}$ den Oberflächenunebenheiten führen (s. Kapitel 2.2.2). Diese scheinen bei den RAFT-Netzwerken entsprechend weniger oder gar nicht aufzutreten, was zu homogeneren Oberflächen führt.

Anschließend an die Lichtmikroskop-Aufnahmen wurden die Zugversuche durchgeführt. Bei diesen wurden die Spannungs-Dehnungs-Kurven (SDK) der Probekörper aufgezeichnet. Die Messbedingungen und Parameter sind in Kapitel 6.3.1 zu finden. Abbildung 4-5 zeigt beispielhaft für alle gemessenen Poly(BA-co-BDDA)-Netzwerke jeweils eine SDK eines konventionellen und eines RAFT-Netzwerks mit einem jeweiligen molaren Anteil von 0,5, 1 und 2 mol- $\%$ des Vernetzers BDDA. Die Spannungs-Dehnungs-Kurven stehen repräsentativ für alle, für das jeweilige Netzwerk, durchgeführten Messungen. Der Übersichtlichkeit halber wird auf die Darstellung weiterer Spannungs-Dehnungs-Kurven verzichtet.
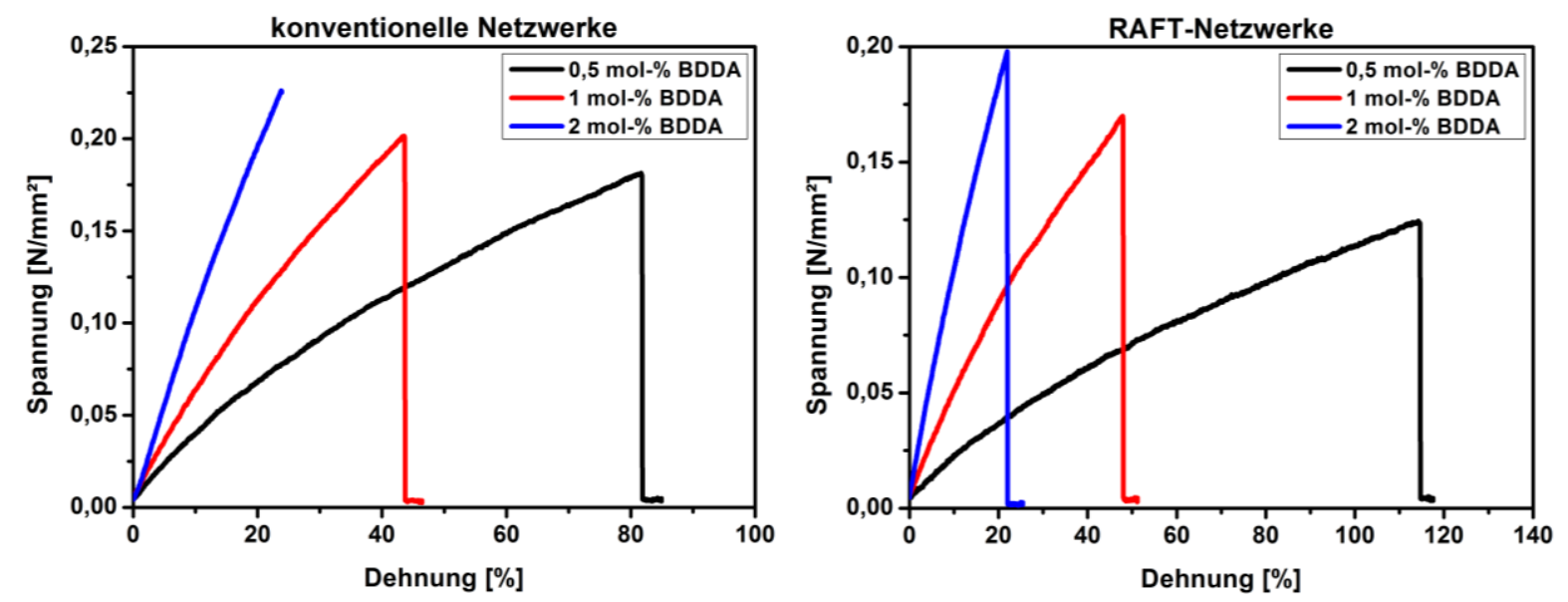

Abbildung 4-5 Mittels Zugversuch gemessene Spannungs-Dehnungs-Kurven von Poly(BA-co-BDDA)-Netzwerken mit 0,5 mol-\%, 1 mol-\% und 2 mol-\% des Vernetzers BDDA. Links: Aus UV-initiierter, konventioneller radikalischer Polymerisation. Rechts: Aus UV-initiierter RAFT-Polymerisation mit einem molaren Anteil von 2 mol-\%o vom RAFTAgens EPHT. Die Zugversuche wurden mit einer Zuggeschwindigkeit von $25 \mathrm{~mm} / \mathrm{min}$ bei einer Temperatur von $21^{\circ} \mathrm{C}$ durchgeführt. Die gezeigten Kurven stehen beispielhaft für alle durchgeführten Messungen. 
Abbildung 4-6 zeigt analog zur obigen Abbildung die Spannungs-Dehnungs-Kurven von drei Poly(EA-co-BDDA)-Netzwerken mit unterschiedlichen molaren Anteilen vom Vernetzer BDDA. Auch hier gilt, dass aus Gründen der besseren Übersichtlichkeit auf die SDKs von weiteren Netzwerken verzichtet wurde.
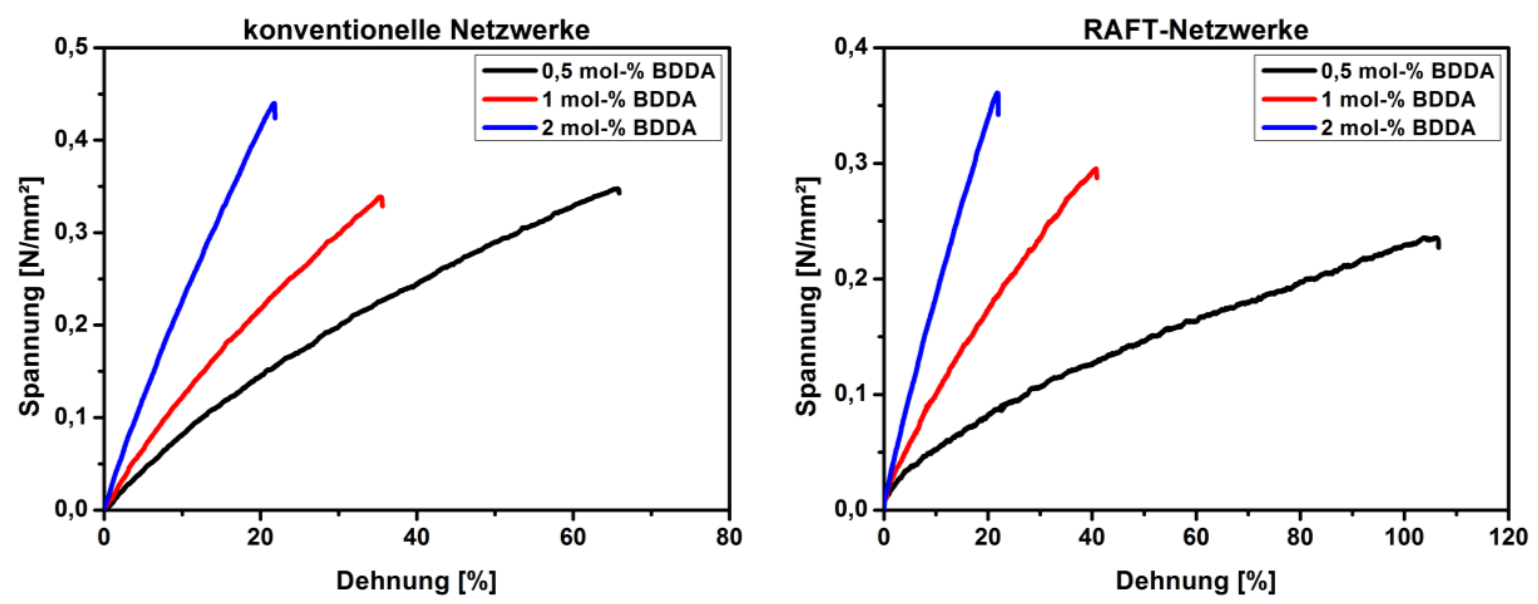

Abbildung 4-6 Mittels Zugversuch gemessene Spannungs-Dehnungs-Kurven von Poly(EA-co-BDDA)-Netzwerken mit 0,5 mol-\%, $1 \mathrm{~mol}-\%$ und $2 \mathrm{~mol}-\%$ des Vernetzers BDDA. Links: Aus UV-initiierter, konventioneller radikalischer Polymerisation. Rechts: Aus UV-initiierter RAFT-Polymerisation mit einem molaren Anteil von 2 mol-\%o vom RAFTAgens EPHT. Die Zugversuche wurden mit einer Zuggeschwindigkeit von $25 \mathrm{~mm} / \mathrm{min}$ bei einer Temperatur von $21^{\circ} \mathrm{C}$ durchgeführt. Die gezeigten Kurven stehen beispielhaft für alle durchgeführten Messungen.

Die Kurvenverläufe lassen erkennen, dass es sich bei den Netzwerken eindeutig um Elastomere handelt (s. Abbildung 2-12). Besonders deutlich ist dies bei den RAFT-Netzwerken mit einem molaren Anteil von 0,5 mol-\% BDDA zu erkennen. Sie erreichen sehr große Dehnungen von über $100 \%$ bei relativ geringen Spannungen. Aber auch die konventionellen Netzwerke mit diesem molaren Anteil des Vernetzers BDDA ähneln in ihren Verläufen sehr stark dem Verlauf eines typischen Elastomers (s. Abbildung 2-12, blaue Kurve). Mit zunehmendem molaren Anteil von BDDA in den Netzwerken steigen die erreichten Spannungen, während die Bruchdehnungen abnehmen. Auch wenn die Verläufe der Spannungs-Dehnungs-Kurven auf den ersten Blick denen eines Duroplasten ähneln (s. Abbildung 2-12, schwarze Kurve), so sind die erreichten Spannungen viel zu niedrig für einen Duroplasten und die Bruchdehnungen entsprechend zu hoch. Typische Werte für die erreichten Spannungen bei Duroplasten liegen deutlich höher. ${ }^{[159]}$ Aufgrund der Verläufe der SDKs kann ausgeschlossen werden, dass es sich bei den Probekörpern um Thermoplaste handelte. Es ist weder eine Streckgrenze noch ein Einschnürungsbereich zu erkennen. Diese Messungen sind ein erster guter Hinweis darauf, dass tatsächlich eine Vernetzung der Polymere vorliegt. 
Aus allen gemessenen Spannungs-Dehnungs-Kurven wurden anschließend der E-Modul, die Bruchdehnung $\gamma_{\mathrm{B}}$ und die Deformationsarbeit $w$ der jeweiligen Netzwerke ermittelt. Die entsprechenden Methoden werden in Kapitel 6.3.1 näher erläutert.

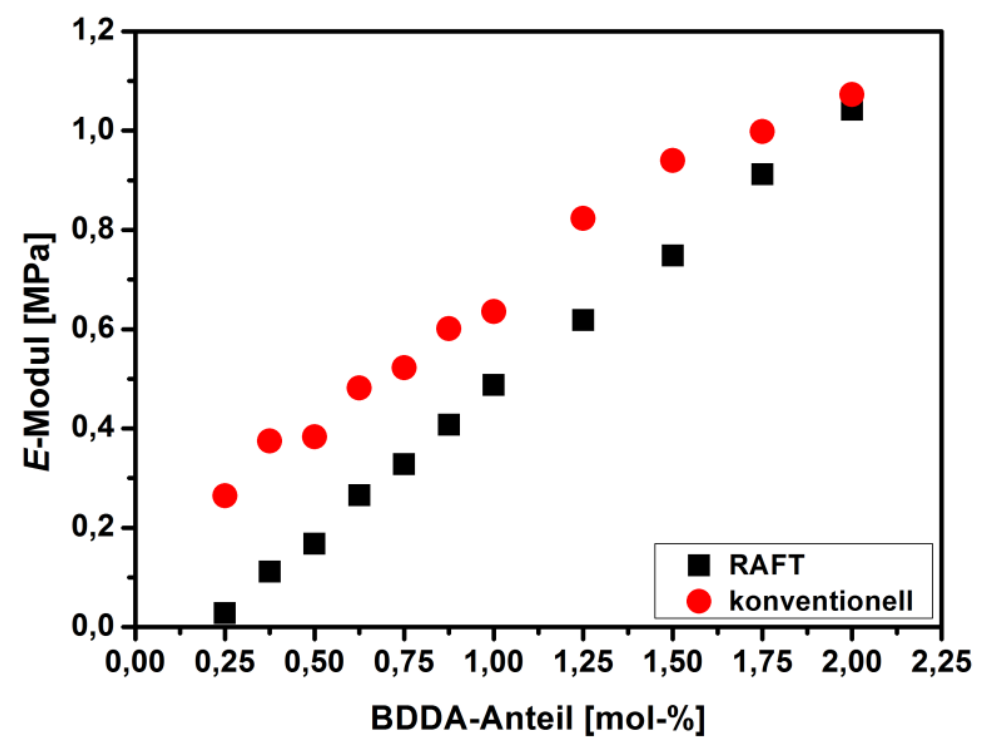

Abbildung 4-7 Mittels Zugversuch ermittelte E-Moduln der hergestellten Poly(BA-co-BDDA)-Netzwerke, aufgetragen gegen den prozentualen molaren Anteil von BDDA für die Netzwerke aus UV-initiierter RAFT-Polymerisation mit 2 mol-\% EPHT (•) sowie aus UV-initiierter, konventioneller radikalischer Polymerisation (•). Die Zugversuche wurden mit einer Zuggeschwindigkeit von $25 \mathrm{~mm} / \mathrm{min}$ bei einer Temperatur von $21{ }^{\circ} \mathrm{C}$ durchgeführt.

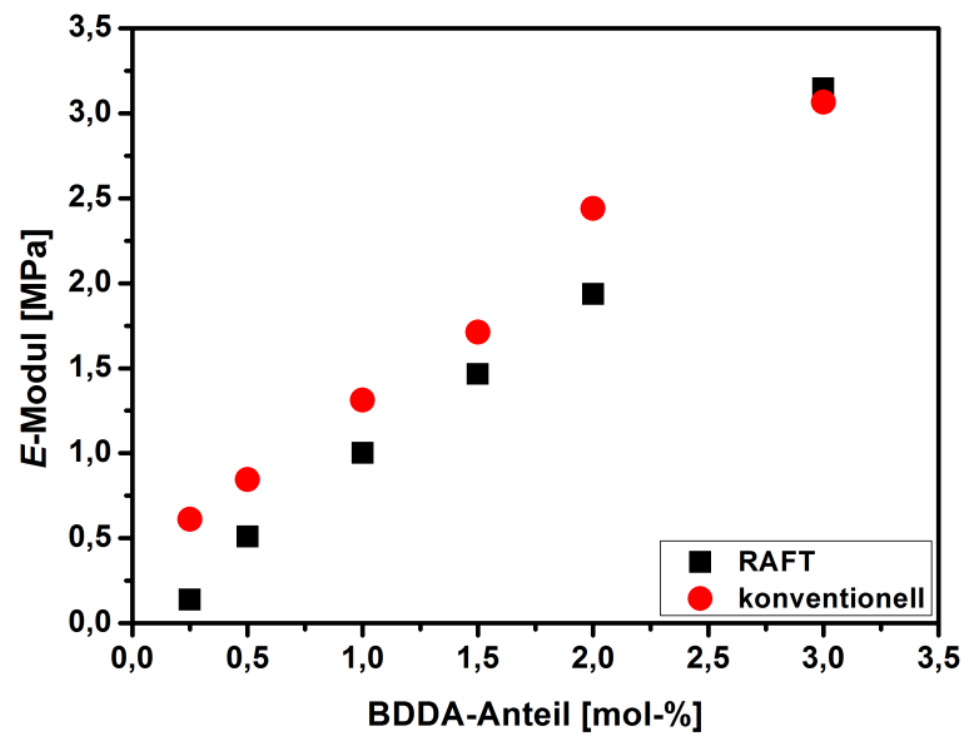

Abbildung 4-8 Mittels Zugversuch ermittelte E-Moduln der hergestellten Poly(EA-co-BDDA)-Netzwerke, aufgetragen gegen den prozentualen molaren Anteil von BDDA für die Netzwerke aus UV-initiierter RAFT-Polymerisation mit 2 mol-\% EPHT (•) sowie aus UV-initiierter, konventioneller radikalischer Polymerisation (•). Die Zugversuche wurden mit einer Zuggeschwindigkeit von $25 \mathrm{~mm} / \mathrm{min}$ bei einer Temperatur von $21^{\circ} \mathrm{C}$ durchgeführt. 
Abbildung 4-7 zeigt den Verlauf der ermittelten E-Moduln von Poly(BA-co-BDDA)-Netzwerken im Bereich eines molaren BDDA-Anteils von 0,25 bis 2 mol-\%. Abbildung 4-8 zeigt den Verlauf der ermittelten E-Moduln von Poly(EA-co-BDDA)-Netzwerken im Bereich von 0,25 bis 3 mol-\% BDDA. Es ist wie erwartet erkennbar, dass die E-Moduln der Netzwerke, sowohl aus konventioneller radikalischer-, als auch aus RAFT-Polymerisation mit steigendem molaren BDDA-Anteil ebenfalls ansteigen. Dies stimmt mit Gleichung (16) überein, wonach in der Theorie die Netzkettendichte mit steigendem molaren Anteil eines Vernetzers zunimmt. Eine größere Netzkettendichte wiederum führt nach Gleichung (23) bzw. Gleichung (27) zu einem größeren Schermodul und somit auch zu einem größeren E-Modul (s. Gleichung (41)).

Deutlich zu erkennen ist auch ein Effekt durch die Zugabe des RAFT-Agens zu den Polymerisationsmischungen der Netzwerke. Die E-Moduln der Netzwerke aus konventioneller radikalischer Polymerisation liegen durchgehend über denen aus RAFT-Polymerisation, wobei dieser Unterschied bei den Poly(EA-co-BDDA)-Netzwerken weniger stark ausgeprägt ist (s. Abbildung 4-8). Erst bei hohen molaren Anteilen von 2 mol-\% BDDA bei den Poly(BA-coBDDA)- und 3 mol-\% BDDA bei den Poly(EA-co-BDDA)-Netzwerken nähern sich die E-Moduln der konventionellen und der RAFT-Netzwerke an. Weiterhin ist erkennbar, dass die Werte der konventionellen Netzwerke viel stärker um eine gedachte Gerade streuen als die der RAFTNetzwerke (s. Abbildung 4-7). Besonders deutlich wird dies für die Poly(BA-co-BDDA)-Netzwerke. Der Grund hierfür liegt wahrscheinlich in der größeren Brüchigkeit der konventionellen Netzwerke im Vergleich zu den RAFTNetzwerken. Aufgrund dessen war es deutlich problematischer, die Probekörper aus der Teflonform, in der sie synthetisiert wurden, zu entnehmen, ohne dass es zu kleinen Defekten an den Probekörpern kam. Weiterhin waren die Oberflächen makroskopisch uneinheitlicher, was ebenfalls zu abweichenden E-Moduln geführt haben kann. Diese Unebenheiten waren ebenfalls auf den für die Zugversuche entscheidenden Stegen (s. Kapitel 2.4) der Probekörper $\mathrm{zu}$ erkennen, wie in Abbildung 4-9 deutlich wird.

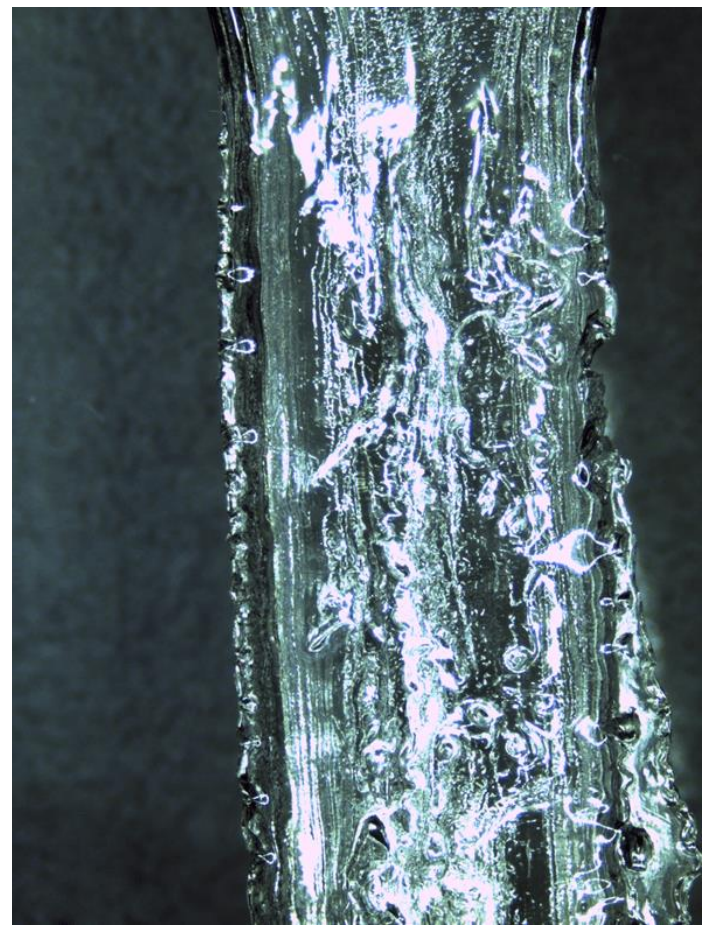

Abbildung 4-9 Lichtmikroskop-Aufnahme von einem Steg eines Zugversuch-Probekörpers eines konventionellen Poly(BA-co-BDDA)-Netzwerks mit einem molaren Anteil von 2 mol-\% BDDA. 
Die größtenteils niedrigeren E-Moduln der RAFT-Netzwerke zeigen eindeutig, dass diese weicher sind als die konventionellen Gegenstücke. Da die Konzentration des Vernetzers jeweils immer identisch ist, kann es sich bei diesem Effekt nur um eine Folge des RAFT-Mechanismus auf die innere Struktur der Netzwerke handeln. Erst bei hohen molaren Anteilen des Vernetzers BDDA scheint der Effekt der RAFT-Polymerisation geringer zu werden und der Vernetzer die Ausbildung der Netzwerkstruktur zu dominieren. Im Allgemeinen zeigt sich, dass die Poly(EAco-BDDA)-Netzwerken durchgehend etwa doppelt so große E-Moduln besitzen und somit deutlich steifer sind als die entsprechenden Poly(BA-co-BDDA)-Netzwerke.

Zusätzlich zu den E-Moduln wurden die Bruchdehnungen der RAFT- und konventionellen Netzwerke ermittelt. Diese wurden gegen den molaren BDDA-Anteil aufgetragen (s. Abbildung 4-10 und Abbildung 4-11).

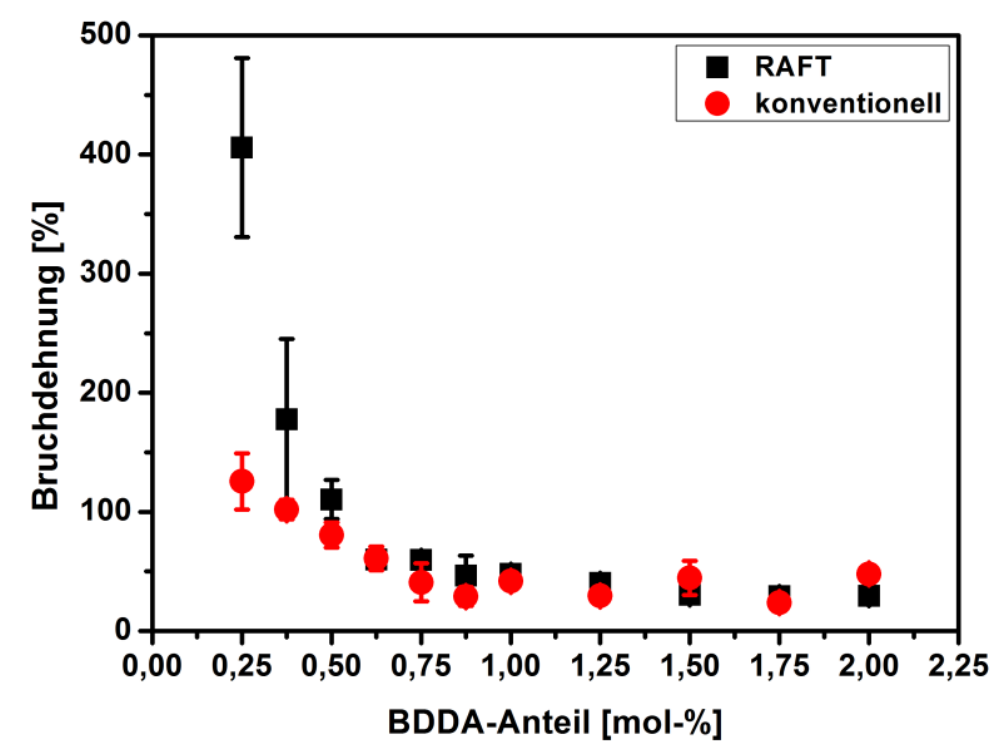

Abbildung 4-10 Mittels Zugversuch ermittelte Bruchdehnungen der Poly(BA-co-BDDA)-Netzwerke, aufgetragen gegen den prozentualen molaren Anteil von BDDA für die Netzwerke aus UV-initiierter RAFT-Polymerisation mit 2 mol-\% EPHT (•) sowie aus UV-initiierter, konventioneller radikalischer Polymerisation (•). Die Fehlerbalken repräsentieren die Standardabweichungen der Bruchdehnungen. Die Zugversuche wurden mit einer Zuggeschwindigkeit von $25 \mathrm{~mm} / \mathrm{min}$ bei einer Temperatur von $21^{\circ} \mathrm{C}$ durchgeführt. 


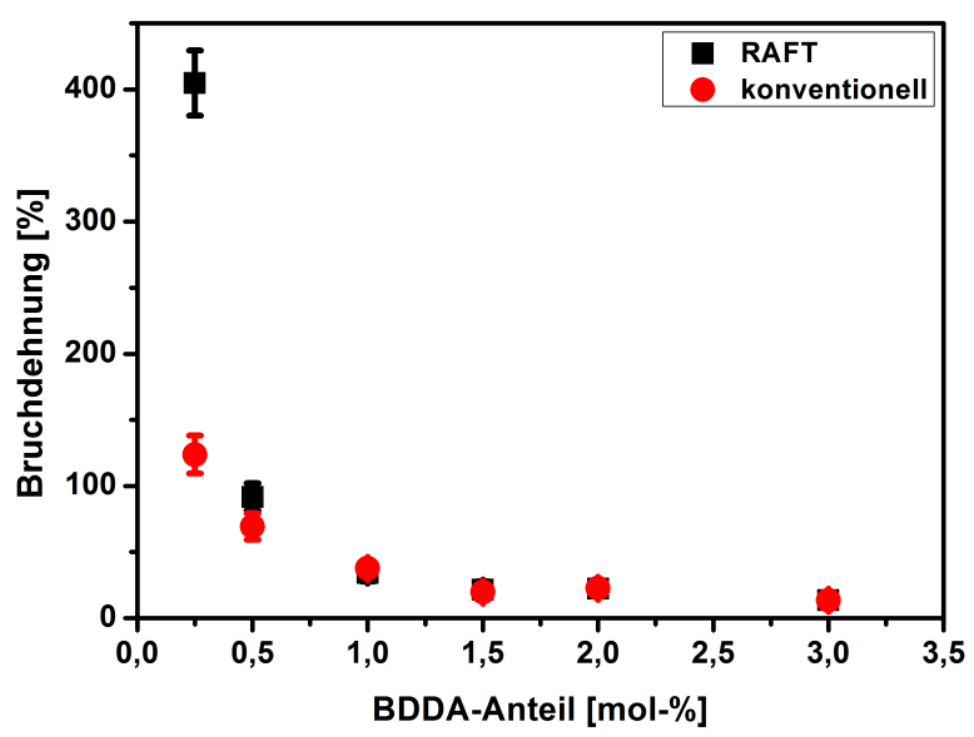

Abbildung 4-11 Mittels Zugversuch ermittelte Bruchdehnungen der Poly(EA-co-BDDA)-Netzwerke, aufgetragen gegen den prozentualen molaren Anteil von BDDA für die Netzwerke aus UV-initiierter RAFT-Polymerisation mit 2 mol-\% EPHT (•) sowie aus UV-initiierter, konventioneller radikalischer Polymerisation (•). Die Fehlerbalken repräsentieren die Standardabweichungen der Bruchdehnungen. Die Zugversuche wurden mit einer Zuggeschwindigkeit von $25 \mathrm{~mm} / \mathrm{min}$ bei einer Temperatur von $21^{\circ} \mathrm{C}$ durchgeführt.

Sowohl bei den Poly(BA-co-BDDA)- als auch bei den Poly(EA-co-BDDA)-Netzwerken steigen die Bruchdehnungen deutlich an, sobald die jeweiligen Netzwerke unter 1 mol-\% BDDA enthalten. Folglich können sie also zunehmend weiter gedehnt werden ohne zu reißen. Das bedeutet, dass die Netzketten zunehmend weiter gestreckt (entknäuelt) werden können. Dass dieser Anstieg für die Netzwerke aus RAFT-Polymerisation unter 0,5 mol-\% BDDA trotz einer deutlich größeren Standardabweichung viel größer ist, beweist, dass die Netzketten dieser Netzwerke noch besser bzw. weiter entknäuelt werden können als die der konventionellen Netzwerke. Dies ist ein Hinweis darauf, dass die RAFT-Netzwerke über weniger Netzknotenpunkte verfügen als die konventionellen Netzwerke. Ab einem molaren BDDA-Anteil von über 1 mol-\% liegen die ermittelten Bruchdehnungen der RAFT- und der konventionellen Netzwerke zwischen $30 \%$ und $40 \%$. Das bedeutet, dass die RAFT-Polymerisation hier nur einen Einfluss auf die E-Moduln der Netzwerke hat, nicht aber auf die Bruchdehnungen. Weiterhin fällt auf, dass sowohl die RAFTals auch die konventionellen Netzwerke, unabhängig von ihrer chemischen Natur, jeweils nahezu gleiche Bruchdehnungen erreichen (s. Abbildung 4-10 und Abbildung 4-11).

Die mit abnehmendem molaren BDDA-Anteil zunehmende Bruchdehnung legt den Schluss nahe, dass auch zunehmend mehr Energie benötigt wird, um die Netzwerke zu zerreißen. Es muss mehr Deformationsarbeit aufgebracht werden, da aufgrund einer größeren Bruchdehnung auch die Fläche unter der Spannungs-Dehnungs-Kurve ansteigen würde. Tabelle 4-2 und Tabelle 4-3, zeigen die entsprechenden Daten für einige beispielhaft ausgewählte Netzwerke. 
Tabelle 4-2 Mittels Zugversuch ermittelte Deformationsarbeit pro Volumenelement von Poly(BA-co-BDDA)Netzwerken aus UV-initiierter RAFT- sowie UV-initiierter, konventioneller radikalischer Polymerisation. Die Zugversuche wurden mit einer Zuggeschwindigkeit von $25 \mathrm{~mm} / \mathrm{min}$ bei einer Temperatur von $21^{\circ} \mathrm{C}$ durchgeführt.

\begin{tabular}{|c|c|c|}
\hline \multirow{2}{*}{ BDDA-Anteil [mol-\%] } & \multicolumn{2}{|c|}{ Deformationsarbeit $\left[\mathrm{J} / \mathbf{m m}^{3}\right]$} \\
\hline & RAFT & konventionell \\
\hline 2 & $3,6 \pm 1,0$ & $3,9 \pm 1,4$ \\
\hline 1 & $4,5 \pm 0,7$ & $4,6 \pm 1,0$ \\
\hline 0,5 & $9,2 \pm 2,5$ & $8,4 \pm 2,0$ \\
\hline 0,25 & $11,0 \pm 3,1$ & $11,6 \pm 3,5$ \\
\hline
\end{tabular}

Tabelle 4-3 Mittels Zugversuch ermittelte Deformationsarbeit pro Volumenelement von Poly(EA-co-BDDA)Netzwerken aus UV-initiierter RAFT- sowie UV-initiierter, konventioneller radikalischer Polymerisation. Die Zugversuche wurden mit einer Zuggeschwindigkeit von $25 \mathrm{~mm} / \mathrm{min}$ bei einer Temperatur von $21^{\circ} \mathrm{C}$ durchgeführt.

\begin{tabular}{|c|c|c|}
\hline \multirow{2}{*}{ BDDA-Anteil [mol-\%] } & \multicolumn{2}{|c|}{ Deformationsarbeit $\left[\mathrm{J} / \mathrm{mm}^{3}\right]$} \\
\hline & RAFT & konventionell \\
\hline 2 & $4,6 \pm 0,3$ & $5,6 \pm 1,0$ \\
\hline 1 & $5,2 \pm 2,1$ & $7,5 \pm 2,0$ \\
\hline 0,5 & $11,9 \pm 2,5$ & $14,1 \pm 3,8$ \\
\hline 0,25 & $32,5 \pm 8,1$ & $24,6 \pm 4,3$ \\
\hline
\end{tabular}

In Bezug auf einen zunehmenden molaren Anteil von BDDA erweist sich diese Annahme als korrekt. Werden allerdings die Deformationsarbeiten von RAFT- und konventionellen Netzwerken bei gleichem Anteil von BDDA betrachtet, zeigt sich, dass diese im Rahmen der Standardabweichung, sowohl für Poly(BA-co-BDDA)- als auch für Poly(EA-co-BDDA)-Netzwerke nahezu gleich sind, wobei dieses für Erstere weitaus deutlicher wird. Das bedeutet, dass bei der Betrachtung der Deformationsarbeiten, die an den Netzwerken geleistet wurden, der Einfluss des BDDAs über den des RAFT-Agens dominiert. Weiterhin wird deutlich, dass die Poly(EA-coBDDA)-Netzwerke tendenziell eine größere Deformationsarbeit pro Volumenelement benötigen, um zu reißen. Dies lässt sich auf ihren höheren E-Modul zurückführen, da die Bruchdehnungen nahezu denen der Poly(BA-co-BDDA)-Netzwerke entsprechen.

Dass trotz signifikant größerer Bruchdehnungen der RAFT-Netzwerke bei einem molaren Anteil von unter 1 mol-\% BDDA nahezu gleiche Deformationsarbeiten benötigt werden, um die Probekörper zu zerreißen, erscheint zunächst unerwartet. Allerdings werden die Netzwerke mit der Zugabe von EPHT weicher. Somit nimmt die Kraft, die benötigt wird, um die Probekörper bis zum Bruch zu dehnen, deutlich ab. Die Werte der Integrale der Spannungs-Dehnungs-Kurven von konventionellen und RAFT-Netzwerken unterscheiden sich folglich kaum voneinander. Diese Tatsache wird beispielhaft in Abbildung 4-12 für ein zwei Poly(BA-co-BDDA)-Netzwerke 
aus RAFT- bzw. konventioneller radikalischer Polymerisation aber mit gleichem BDDA-Anteil von 0,25 mol-\% verdeutlicht. Trotz großer Unterschiede bei der maximal erreichten Spannung und der Bruchdehnung sind die Werte der Integrale nahezu gleich. Bei fünf vermessenen Probekörpern ergibt sich im Mittel also für alle Netzwerke eine ähnliche notwendige Deformationsarbeit, um die Proben bis zum Reißen zu dehnen.

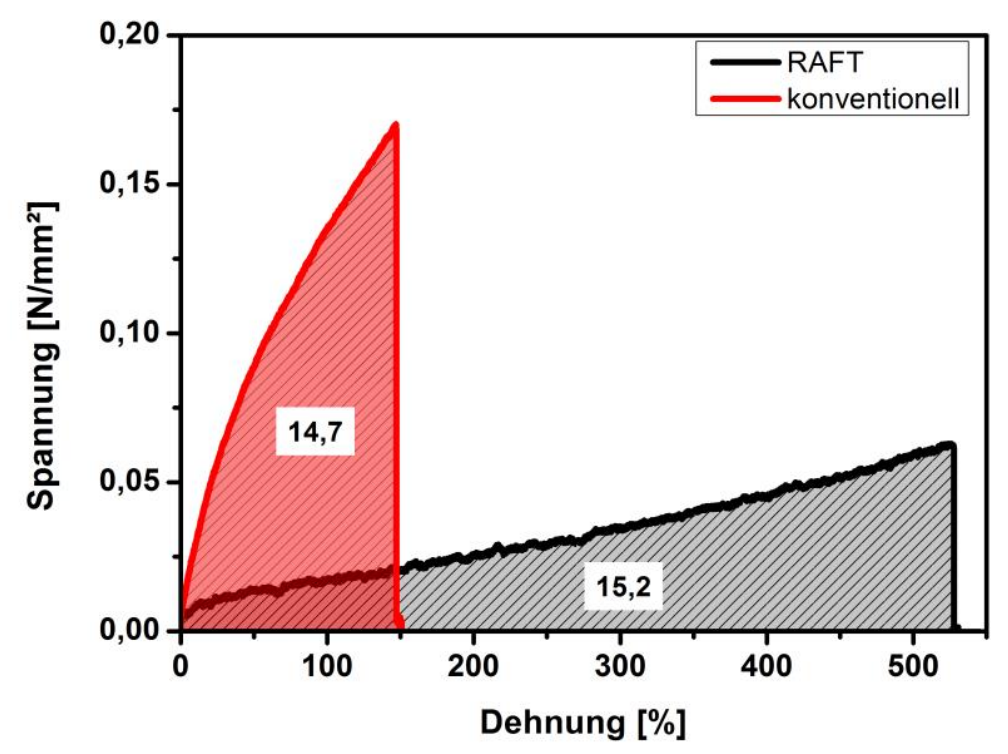

Abbildung 4-12 Beispielhafte Verläufe von mittels Zugversuch gemessene Spannungs-Dehnungs-Kurven zweier Poly(BA-co-BDDA)-Probekörper mit 0,25 mol-\% BDDA als Vernetzer. Schwarz: aus UV-initiierter RAFTPolymerisation mit 2 mol-\%o EPHT; Rot: aus UV-initiierter, konventioneller radikalischer Polymerisation. Die Werte der Integrale sind auf den jeweiligen Flächen abzulesen. Die Zugversuche wurden mit einer Zuggeschwindigkeit von $25 \mathrm{~mm} / \mathrm{min}$ bei einer Temperatur von $21^{\circ} \mathrm{C}$ durchgeführt.

Anschließend an die Zugversuche wurden, mittels der aus den Spannungs-Dehnungs-Kurven hervorgehenden Daten, die effektiven Netzkettendichten $v_{\text {eff }}$ der Netzwerke berechnet. Dazu wurden die gemessenen E-Moduln über Gleichung (41) in die entsprechenden Schermoduln umgerechnet. Anschließend ist es möglich, durch Umstellen von Gleichung (27) nach:

$$
v_{\mathrm{eff}}=\frac{G}{R T \cdot A}
$$

die $v_{\text {eff }}$ zu berechnen. Die einzige Variable in Gleichung (44) ist dabei der Wert für den Strukturfaktor $A$. Je nachdem welches Modell zur Beschreibung der Entropieelastizität (s. Kapitel 2.3.1) verwendet wird, kann $A$ den Wert 1 oder 0,5 annehmen. Darum wurden die Netzkettendichten für beide Modelle berechnet und mit den theoretisch möglichen Werten, 
berechnet aus Gleichung (16), verglichen. Abbildung 4-13 zeigt diesen Vergleich zunächst nur für die Poly(BA-co-BDDA)-Netzwerke.
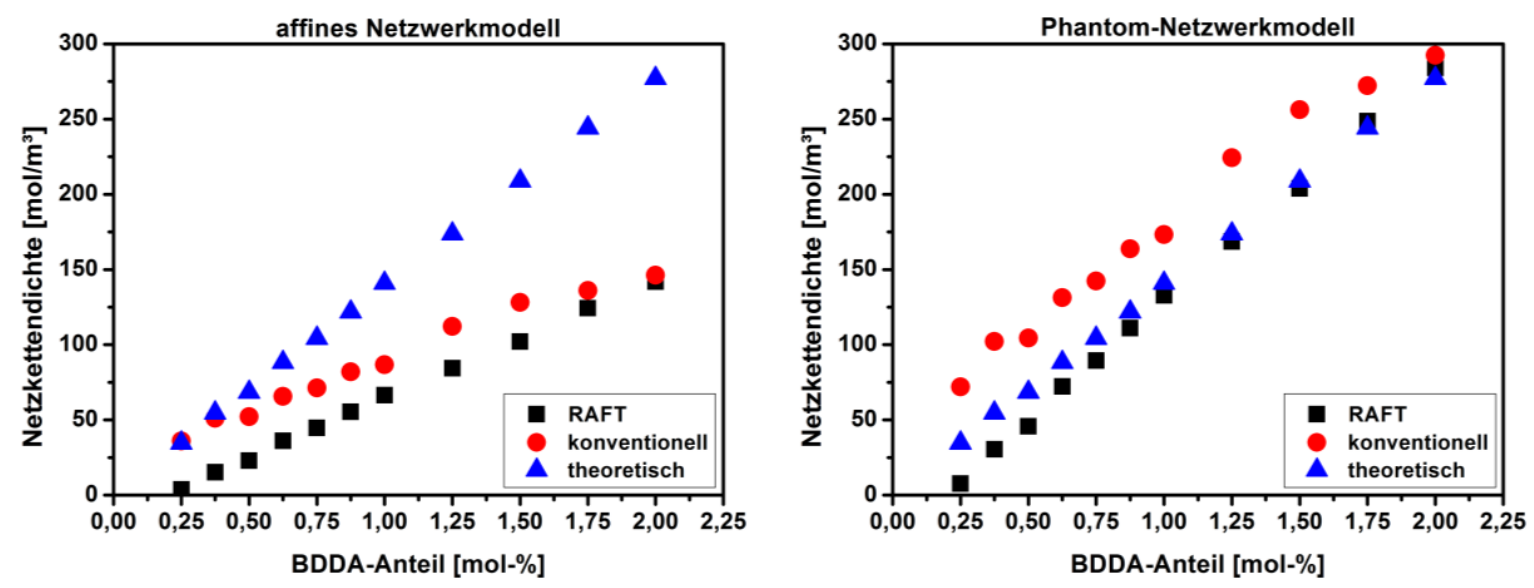

Abbildung 4-13 Aus den experimentell ermittelten Schermodulen errechnete Netzkettendichten der Poly(BA-coBDDA)-Netzwerke aus UV-initiierter RAFT-Polymerisation mit 2 mol-\%。 EPHT als RAFT-Agens (-) sowie aus UVinitiierter, konventioneller radikalischer Polymerisation (๑) im Vergleich zu der theoretischen Netzkettendichte eines idealen Netzwerks (४). Links: nach dem affinen Netzwerkmodell errechnet; Rechts: nach dem PhantomNetzwerkmodell errechnet.

Die Netzkettendichten weichen unter der Annahme, dass ein affines Netzwerk vorliegt sowohl für die RAFT- als auch für die konventionellen Poly(BA-co-BDDA)-Netzwerke sehr stark von den theoretischen Werten ab, obwohl die effektiven Netzkettendichten der konventionellen Netzwerke bei einem geringen molaren Anteil von unter 0,5 mol-\% mit den theoretischen Werten übereinstimmen (s. Abbildung 4-13, rechts). Wird angenommen, dass es sich bei den Poly(BA-coBDDA)-Netzwerken um ein Phantomnetzwerk handelt, ist die Übereinstimmung der effektiven Netzkettendichten mit den theoretischen Werten insgesamt deutlich besser. Ab einem molaren Anteil von 1 mol-\% BDDA ist diese für die RAFT-Netzwerke sogar nahezu vollständig (s. Abbildung 4-13, links). Diese Netzwerke verhalten sich also nahezu wie ein ideales Phantomnetzwerk. Dies lässt den Schluss zu, dass in den RAFT-Netzwerken bei höheren molaren Anteilen von über 1 mol-\% BDDA alle Netzketten an der mechanischen Verformung des Netzwerks beteiligt waren und nur eine geringe Zahl an Netzwerkdefekten aufgetreten ist. Dass die Netzkettendichte darunter unter den theoretischen Werten liegt, zeigt, dass nicht alle Netzketten an der mechanischen Verformung teilgenommen haben. Möglicherweise war die Vernetzungsreaktion in diesem Bereich nicht vollständig und es kam zur Bildung von freien Kettenenden oder nicht vollständig funktionellen Netzknotenpunkten und somit einem nicht vollständigen Einbau von Sol-Polymeren. Da bekannt ist, dass RAFT-Netzwerke über eine größere Zahl von freien Kettenenden verfügen als konventionelle Netzwerke ${ }^{[123]}$, sind somit die 
aus den Zugversuchen erhaltenen Ergebnisse bestätigt. ${ }^{a}$ Die insgesamt höheren Werte der konventionellen Netzwerke bedeuten, dass mehr Netzketten an der Deformation beteiligt waren als theoretisch möglich.

Die höheren Netzkettendichten der konventionellen Netzwerke lassen sich demnach durch das Auftreten von Verhakungen und Verschlaufungen erklären. Jeder dieser Netzwerkdefekte erhöht die Zahl an Netzknotenpunkten im Netzwerk und somit auch die effektive Netzkettendichte. Die Folge ist, dass die Netzwerke deutlich steifer erscheinen als sie entsprechend der Theorie sein sollten. Dies entspricht den erhaltenen Ergebnissen und kann durch das vermehrte Auftreten von Nanogel-Domänen in konventionellen Netzwerken erklärt werden. Dass die konventionellen Netzwerke über Verhakungen und Verschlaufungen in großer Zahl verfügen müssen, zeigt auch die Vernetzungseffizienz, bei der es sich entsprechend Gleichung (17) um das Verhältnis der effektiven Netzkettendichte zu der theoretischen handelt. Im idealen Fall, wenn der Vernetzer vollständig in das Netzwerk eingebaut wurde und ansonsten keine Netzwerkdefekte vorliegen, sollte dieses 1 betragen.

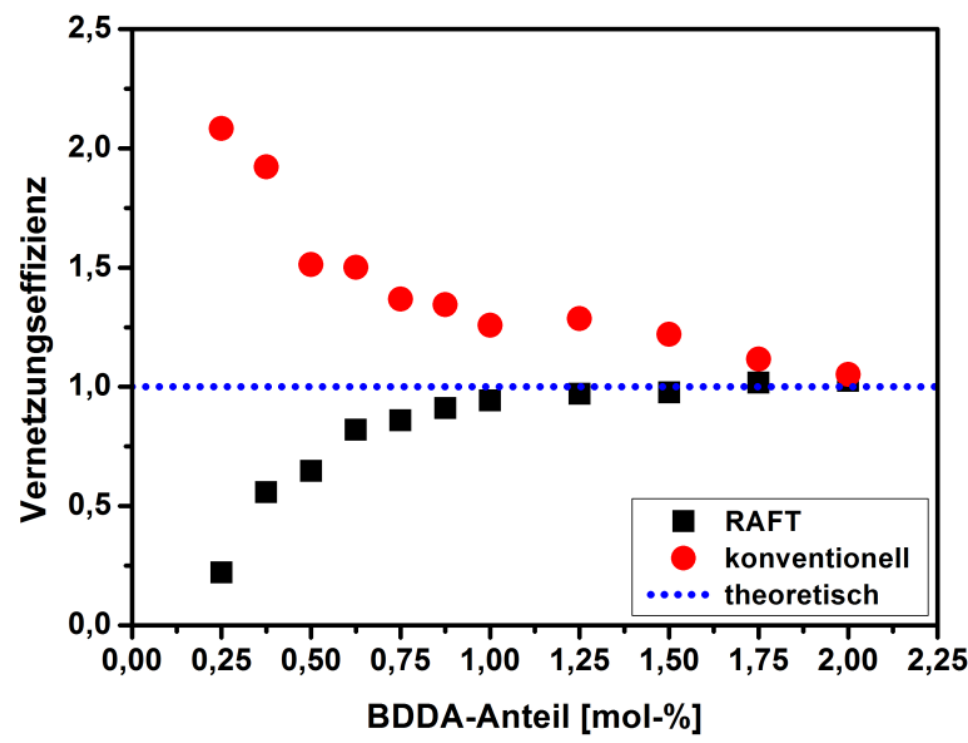

Abbildung 4-14 Effizienz der Vernetzungsreaktion für Poly(BA-co-BBDA)-Netzwerke aus UV-initiierter RAFTPolymerisation mit 2 mol-\%o EPHT (ロ) und aus UV-initiierter, konventioneller radikalischer Polymerisation (•) im Vergleich zur theoretischen Vernetzungseffizienz eines idealen Netzwerks $(\cdots)$.

In Abbildung 4-14 ist zu erkennen, dass die Vernetzungseffizienz der konventionellen Poly(BAco-BDDA)-Netzwerke bei geringen molaren Anteilen des Vernetzers BDDA deutlich über dem idealen Wert liegt und mit zunehmendem molaren BDDA-Anteil abnimmt: Sie verbleibt allerdings immer über dem idealen Wert.

\footnotetext{
${ }^{a}$ Dieser Punkt wird im Kapitel 4.1.3 noch ausführlicher diskutiert.
} 
Dagegen ist die Vernetzungseffizienz der RAFT-Netzwerke bei geringen molaren Anteilen von unter 1 mol-\% BDDA zunächst deutlich unter dem idealen Wert von 1, was die Vermutung einer nicht vollständig verlaufenen Vernetzungsreaktion unterstützt. Bei den RAFT-Netzwerken nähert sich die Vernetzungseffizienz ebenfalls mit steigendem molaren Anteil von BDDA dem idealen Wert an. Ab einem molaren Anteil von 1 mol-\% BDDA korrespondieren die effektiven Vernetzungsdichten im beobachteten Bereich gut mit den theoretischen.

Eine mögliche Erklärung des Verlaufs der Vernetzungseffizienz $\Omega$ für die konventionellen Netzwerke kann durch den Schermodul geliefert werden. Dieser setzt sich entsprechend Gleichung (45) bei realen Netzwerken aus einer Summe von zwei Anteilen zusammen. Zum einen, ein durch chemische Vernetzungen hervorgerufener Anteil $G_{\mathrm{x}}$ und zum anderen der durch physikalische Verhakungen der Netzketten hervorgerufene Anteil $G_{\mathrm{e}}$, sodass sich der Schermodul aus folgender Gleichung ergibt: ${ }^{[191]}$

$$
G \cong G_{\mathrm{x}}+G_{\mathrm{e}} \approx \rho R T\left(\frac{1}{\bar{M}_{\mathrm{x}}}+\frac{1}{\bar{M}_{\mathrm{e}}}\right)
$$

Dabei ist $\bar{M}_{\mathrm{x}}$ die zahlenmittlere, scheinbare Netzkettenlänge zwischen zwei chemischen Verknüpfungen und $\bar{M}_{\mathrm{e}}$ die zahlenmittlere, scheinbare Kettenlänge zwischen zwei physikalischen Verhakungen. Der Schermodul wird also bei Netzwerken mit kurzen Netzketten von der molaren Masse zwischen zwei chemischen Vernetzungen dominiert $\left(\bar{M}_{\mathrm{x}}<\bar{M}_{\mathrm{e}}\right)$. Bei Netzwerken mit langen Netzketten wird der Schermodul allerdings von der molaren Masse zwischen zwei Verhakungen $\left(\bar{M}_{\mathrm{x}}>\bar{M}_{\mathrm{e}}\right)$ dominiert. ${ }^{[191]}$ Das bedeutet für die Poly(BA-co-BDDA)Netzwerke, dass mit abnehmendem molaren Anteil des Vernetzers BDDA der Schermodul zunehmend von physikalischen Verhakungen bestimmt wird. Dies führt zu längeren Netzketten. Da Verhakungen und Verschlaufungen im Wesentlichen bei den konventionellen Netzwerken auftreten, werden diese deutlich stärker von ihnen beeinflusst.

Obwohl ungequollene Netzwerke eher mit dem affinen Modell beschrieben werden sollten (s. Kapitel 2.3.1), zeigt die gute Übereinstimmung der theoretischen mit den gemessenen Werten, dass das Phantom-Netzwerkmodell für die RAFT-Netzwerke durchaus geeigneter ist als das affine Netzwerk-Modell. Eine mögliche Erklärung hierfür ist, dass sich die Poly(BA-co-BDDA)Netzwerke bei Raumtemperatur weit über ihrer Glastemperatur befinden und somit sehr weich sind, was eine Beweglichkeit der Netzknotenpunkte erlauben würde. Außerdem kann die Übereinstimmung der effektiven Netzkettendichten mit den theoretischen nur bei den RAFTNetzwerken beobachtet werden. Diese sind weicher als die konventionellen Netzwerke und entsprechen somit eher dem Phantom-Netzwerkmodell, das weichere Netzwerke voraussagt (s. Abschnitt 2.3.1.2). 
Für die Poly(EA-co-BDDA)-Netzwerke wurde ebenfalls der Vergleich der errechneten Netzkettendichten, jeweils aus dem affinen und dem Phantom-Netzwerkmodell, mit den theoretischen Werten durchgeführt (s. Abbildung 4-15).
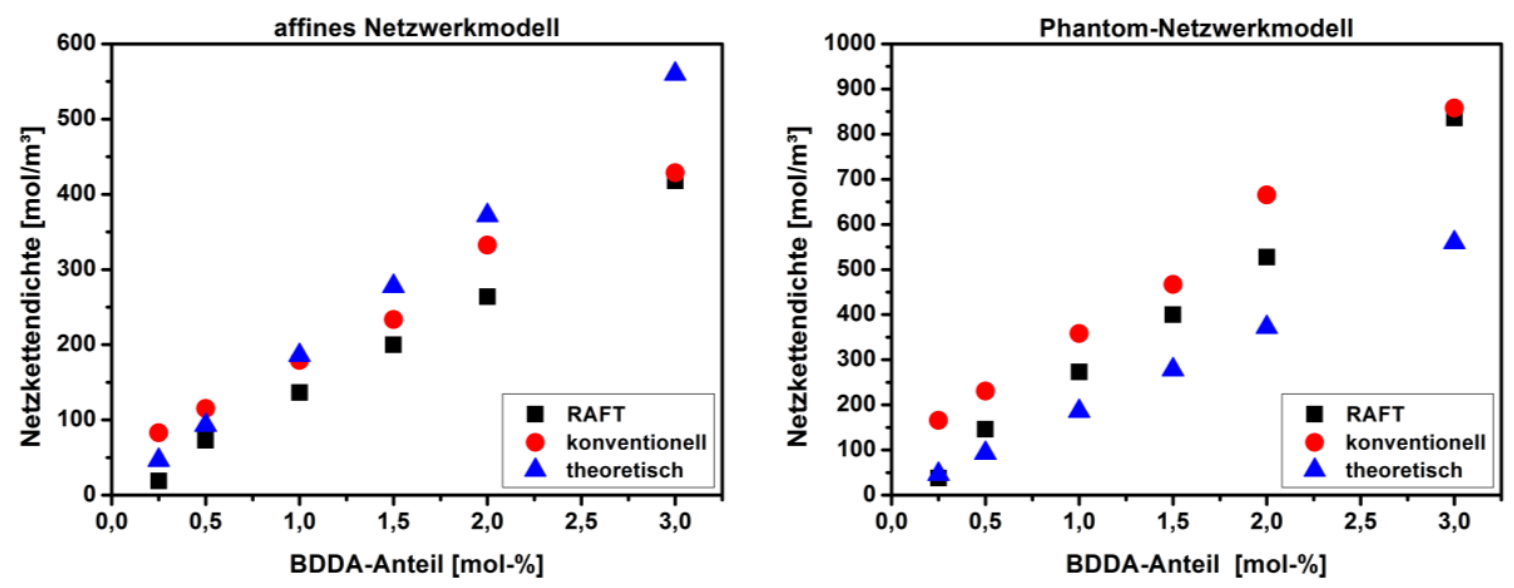

Abbildung 4-15 Aus den experimentell ermittelten Schermodulen errechnete Netzkettendichten der Poly(EA-coBDDA)-Netzwerke aus UV-initiierter RAFT-Polymerisation mit 2 mol-\%o EPHT als RAFT-Agens (๘) sowie aus UVinitiierter, konventioneller radikalischer Polymerisation (๑) im Vergleich zu der theoretischen Netzkettendichte eines idealen Netzwerks ( $\mathbf{\Delta})$. Links: nach dem affinen Netzwerkmodell errechnet; Rechts: nach dem PhantomNetzwerkmodell errechnet.

Hier zeigt sich, im Gegensatz zu den Poly(BA-co-BDDA)-Netzwerken, ein anderes Bild: Entsprechend den Erwartungen (s. Kapitel 2.3.1) stimmen die aus dem affinen Netzwerkmodell ermittelten effektiven Netzkettendichten der Poly(EA-co-BDDA)-Netzwerke eher mit den theoretischen Werten überein. Dies trifft für dieses System bei einem molaren Anteil von unter 1 mol-\% BDDA sowohl für die RAFT- als auch für die konventionellen Netzwerke zu. Bei höheren molaren Anteilen von BDDA stimmen die effektiven Netzkettendichten der konventionellen Netzwerke eher mit den theoretischen Werten überein. Wird das PhantomNetzwerkmodell den Berechnungen der effektiven Netzkettendichten zugrunde gelegt, wird deutlich, dass RAFT-Netzwerke insgesamt den theoretischen Werten näher sind. Dies ist vor allem bei geringen molaren Anteilen von unter 1 mol-\% BDDA der Fall. Bei höheren molaren BDDA-Anteilen weichen sie ebenfalls sehr stark von den theoretischen Werten ab, obwohl sie diesen trotzdem noch näher sind als die effektiven Netzkettendichten der konventionellen Netzwerke. Insgesamt ist das affine Netzwerkmodell zur Beschreibung der Poly(EA-co-BDDA)Netzwerke geeigneter.

Allerdings ergeben sich mit steigendem molarem BDDA-Anteil bei beiden Modellen deutliche Abweichungen der effektiven Netzkettendichten von den theoretischen Werten. Eine mögliche Erklärung für dieses Verhalten könnte sein, dass nach Treloar die Theorie der Entropieelastizität nur Geltung hat, wenn der E-Modul der Netzwerke unter $1 \mathrm{MPa}$ beträgt. ${ }^{[108]}$ Dies ist für die 
Poly(BA-co-BDDA)-Netzwerke immer der Fall (s. Abbildung 4-7). Darum ist bei diesen diese Bedingung erfüllt. Die Netzwerke zeigen eine relativ gute Übereinstimmung mit der Theorie über den gesamten beobachteten Bereich. Bei den Poly(EA-co-BDDA)-Netzwerken ist diese Bedingung nur bis zu einem Anteil von 1 mol-\% BDDA erfüllt (s. Abbildung 4-8), sodass sich für diese Netzwerke nur in diesen Bereichen sinnvolle Vergleiche mit der Theorie anstellen lassen. Sofern dies berücksichtigt wird, kann anhand der vorliegenden Messwerte konstatiert werden, dass sich konventionelle Netzwerke entsprechend der Theorie eher durch das affine Netzwerkmodell beschreiben lassen, während die weicheren RAFT-Netzwerke deutlich besser durch das PhantomNetzwerkmodell beschrieben werden.

Um die Netzwerke noch weiter zu charakterisieren, wurden die scheinbaren Molmassen $\bar{M}_{\mathrm{x}}$ der Netzketten aus der effektiven Netzkettendichte berechnet. Es gilt:

$$
\bar{M}_{\mathrm{x}}=\frac{\rho}{v_{\mathrm{eff}}}
$$

Die makroskopische Dichte der Netzwerke $\rho$ wurde sowohl für Poly(BA-co-BDDA)- als auch für Poly(EA-co-BDDA)-Netzwerke mit unterschiedlichen molaren BDDA- und EPHT-Anteilen mittels Messungen mit einem Pyknometer bestimmt. Auf die Verwendung von Literaturwerten wurde verzichtet, da sich diese auf unvernetzte Polymere beziehen. In den betrachteten Variationsbereichen des molaren EPHT- und des molaren BDDA-Anteils konnten bei beiden Netzwerken keine signifikanten Unterschiede in den Dichten festgestellt werden. Darum wurden die Mittelwerte aus allen Messungen berechnet. Die somit erhaltenen Dichten sind in Tabelle 4-4 zu finden.

Tabelle 4-4 Mittels Pyknometer gemessene Dichten der untersuchten Poly(BA-co-BDDA)- und Poly(EA-co-BDDA)Netzwerke bei $21^{\circ} \mathrm{C}$.

\begin{tabular}{cc}
\hline Netzwerk & Dichte $\left[\mathbf{g} / \mathbf{c m}^{\mathbf{3}}\right]$ \\
\hline Poly(BA-co-BDDA) & 1,02 \\
Poly(EA-co-BDDA) & 1,13 \\
\hline
\end{tabular}

Für die anschließende Berechnung der scheinbaren Molmassen wurden für Poly(BA-co-BDDA)Netzwerke die effektiven Netzkettendichten aus dem Phantom-Netzwerkmodell und für die Poly(EA-co-BDDA)-Netzwerke die entsprechenden Werte aus dem affinen Netzwerkmodell zugrunde gelegt. 
Unter der Annahme, dass der Vernetzer ideal statistisch in das Netzwerk eingebaut wird und keine weiteren Netzwerkdefekte vorliegen, kann auch die theoretische Molmasse der Netzketten eines idealen Netzwerks $\bar{M}_{\mathrm{s}}$ bestimmt werden. Dazu werden die Gleichungen (16) und (49) kombiniert, sodass folgt:

$$
\bar{M}_{\mathrm{s}}=\frac{\rho}{v_{\mathrm{th}}}=\frac{2 \rho V}{n_{\mathrm{x}} \cdot f}
$$

Die somit erhaltenen scheinbaren Molmassen der Netzketten wurden aufgrund der besseren Übersichtlichkeit doppelt-logarithmisch gegen den molaren Anteil von BDDA in den Netzwerken aufgetragen (s. Abbildung 4-16 und Abbildung 4-17). Zum Vergleich wurden die theoretischen Molmassen der Netzketten hinzugefügt.

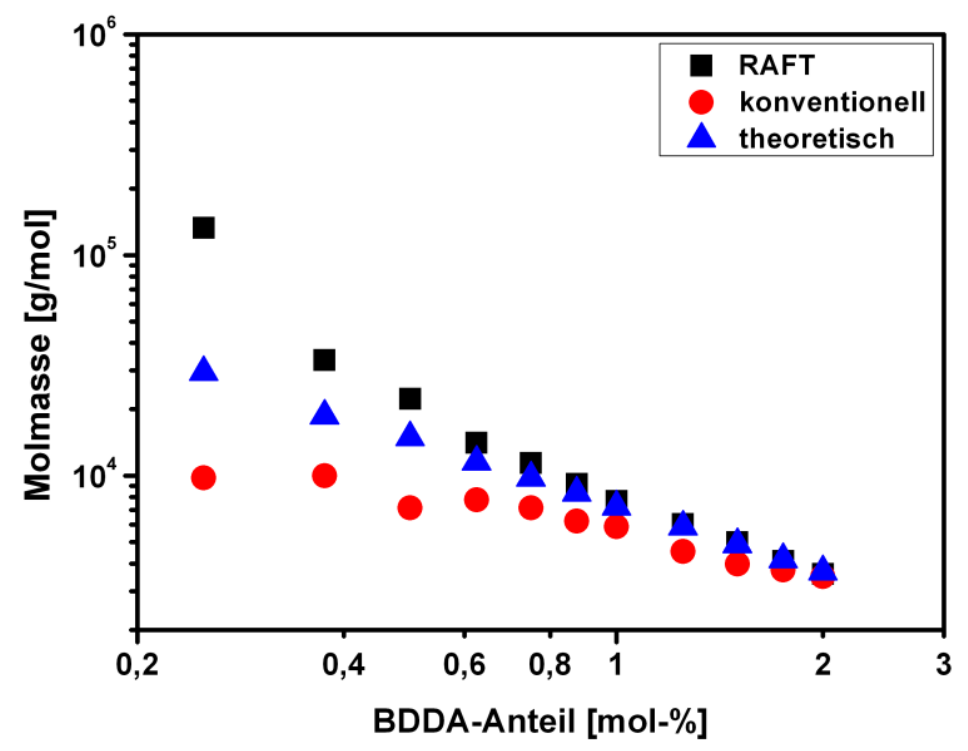

Abbildung 4-16 Doppelt-logarithmische Auftragung der scheinbaren Molmassen der Netzketten, berechnet aus Gleichung (46), von Poly(BA-co-BDDA)-Netzwerken aus UV-initiierter RAFT-Polymerisation mit 2 mol-\%o EPHT als RAFT-Agens (•) und UV-initiierter, konventioneller radikalischer Polymerisation(•) im Vergleich zu den theoretischen Molmassen ( $\mathbf{\wedge})$, berechnet aus Gleichung (47). 


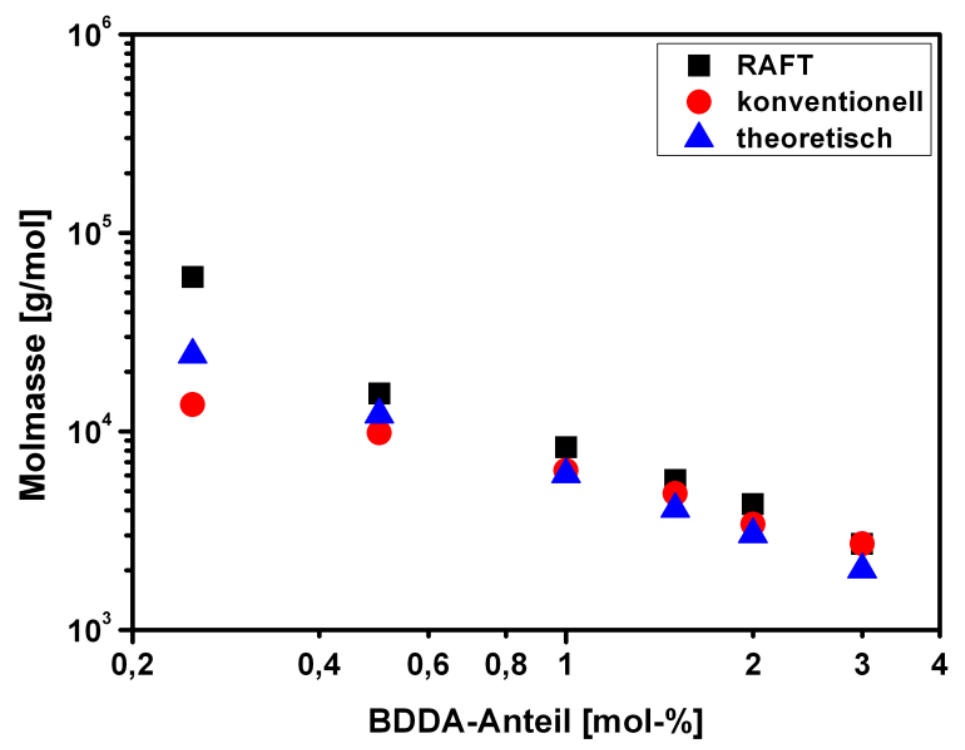

Abbildung 4-17 Doppelt-logarithmische Auftragung der scheinbaren Molmassen der Netzketten, berechnet aus Gleichung (46), von Poly(EA-co-BDDA)-Netzwerken aus UV-initiierter RAFT-Polymerisation mit 2 mol-\%o EPHT als RAFT-Agens (•) und UV-initiierter, konventioneller radikalischer Polymerisation(•) im Vergleich zu den theoretischen Molmassen (₫), berechnet aus Gleichung (47).

Wie erwartet steigen die Molmassen mit abnehmenden prozentualen molaren BDDA-Anteil in den Netzwerken sowohl für die Poly(BA-co-BDDA)- als auch für die Poly(EA-co-BDDA)Netzwerke an. Dabei fällt auf, dass dieser Anstieg für die Poly(BA-co-BDDA)-RAFT-Netzwerke sehr deutlich ausfällt und bis ca. 0,75 mol-\% BDDA nahezu exakt mit dem theoretischen Werten korrespondiert. Bei geringeren molaren BDDA-Anteilen weichen die Molmassen der RAFTNetzwerke deutlich von den theoretischen Werten nach oben ab. Die konventionellen Netzwerke dagegen zeigen ab ca. 0,75 mol-\% BDDA nur noch einen sehr geringen Anstieg mit abnehmendem BDDA-Anteil und weichen dementsprechend bei geringeren molaren Anteilen des Vernetzers nach unten von den theoretischen Werten ab.

Bei den Poly(EA-co-BDDA)-RAFT-Netzwerken sind die scheinbaren Molmassen durchweg größer als die theoretischen und steigen parallel zu diesen mit abnehmendem molaren BDDAAnteil an. Ebenso steigen auch die Werte der konventionellen Netzwerke. Erst bei einem molaren BDDA-Anteil von 0,25 mol-\% kommt zu einer deutlicheren Abweichung nach oben für das RAFT-Netzwerk und nach unten für das konventionelle Netzwerk.

Wie bei den vorherigen Betrachtungen tritt die Abweichung der Werte der RAFT- und der konventionellen Netzwerke ab einem molaren Anteil von unter 1 mol-\% auf. Dies bestätigt wieder, dass die effektive Netzkettendichte der RAFT-Netzwerke in diesem Bereich deutlich geringer ist als die der konventionellen Netzwerke, obwohl in beiden Fällen der molare BDDAAnteil identisch war. Wieder bestätigt sich, dass physikalische Netzknotenpunkte in Form von 
Verhakungen oder Verschlaufungen in den konventionellen Netzwerken deutlich häufiger auftreten müssen als in den RAFT-Netzwerken, die sich nahezu ideal entsprechend der theoretischen Netzwerke verhalten.

\subsubsection{Einfluss der Reaktionszeit auf die mechanischen Eigenschaften der RAFT- Netzwerke}

Um zu überprüfen, ob die Bestrahlungsdauer bzw. die Reaktionszeit einen Einfluss auf die mechanischen Eigenschaften der Netzwerke hat, wurden für eine Kombination von BDDA (0,5 mol-\%) und EPHT (2 mol-\%) Zugversuch-Probekörper unterschiedlich lang ausgehärtet und anschließend mittels Zugversuch hinsichtlich ihrer E-Moduln und Bruchdehnungen untersucht. Diese Kombination des molaren Anteils des Vernetzer und des RAFT-Agens wurde gewählt, da hier ein deutlicher Einfluss des RAFT-Agens auf die mechanischen Eigenschaften des Netzwerks zu erkennen war (s. Abschnitt 4.1.1.1). Dieser war bei niedrigen Anteilen von BDDA zwar noch deutlicher, allerdings waren diese Probekörper sehr weich und darum sehr anspruchsvoll in ihrer Handhabung. Die erhaltenen Ergebnisse der Zugversuche sind in Tabelle 4-5 zu finden.

Tabelle 4-5 Elastizitätsmoduln eines RAFT-Netzwerkes (0,5 mol-\% BDDA, 2 mol-\% EPHT). Die Synthese erfolgte mit unterschiedlichen Reaktionszeiten bei Raumtemperatur. Als Photoinitiator wurden 2 Gew.-\% MMMP verwendet. Die Lichtintensität betrug $9 \mathrm{~mW} / \mathrm{cm}^{2}$ bei $366 \mathrm{~nm}$. Die Zugversuche wurden mit einer Zuggeschwindigkeit von $25 \mathrm{~mm} / \mathrm{min}$ bei einer Temperatur von $21^{\circ} \mathrm{C}$ durchgeführt.

\begin{tabular}{ccc}
\hline Reaktionszeit [min] & E-Modul [MPa] & Bruchdehnung [\%] \\
\hline 10 & $0,149 \pm 0,014$ & $130 \pm 10$ \\
20 & $0,188 \pm 0,012$ & $117 \pm 25$ \\
30 & $0,168 \pm 0,011$ & $122 \pm 13$ \\
40 & $0,189 \pm 0,009$ & $113 \pm 7$ \\
50 & $0,174 \pm 0,016$ & $104 \pm 2$ \\
60 & $0,181 \pm 0,010$ & $113 \pm 6$ \\
120 & $0,174 \pm 0,012$ & $127 \pm 18$ \\
\hline
\end{tabular}

Wie zu erkennen ist, hat die weitere Erhöhung der Reaktionszeit über 20 Minuten keinen signifikanten Einfluss auf die mechanischen Eigenschaften der Netzwerke. Die Werte des E-Moduls streuen um einen Wert von ca. 0,180 MPa. Weiterhin zeigen diese Ergebnisse auch, dass die Reproduzierbarkeit der E-Modul-Messungen per Zugversuch sehr gut ist, was die Standardabweichung der E-Moduln sehr gering werden lässt. Die Bruchdehnungen der Netzwerke nehmen zunächst mit der Reaktionszeit ab, was bei stärker vernetzten Netzwerken auch zu erwarten ist. Allerdings steigen sie auch bei langen Reaktionszeiten wieder deutlich an, 
was letztlich zeigt, dass diese ebenfalls keine signifikante Abhängigkeit von der Reaktionszeit haben. Dies liegt in der Kinetik der Reaktion begründet und wird in Kapitel 4.1.4 diskutiert.

\subsubsection{Variation des molaren EPHT-Anteils}

Zusätzlich zu den Untersuchungen über den Einfluss des molaren Anteils des Vernetzers BDDA auf die mechanischen Eigenschaften der Poly(BA-co-BDDA) und Poly(EA-co-BDDA)-Netzwerke, wurde der Einfluss des molaren Anteils des RAFT-Agens EPHT untersucht. Dazu wurde, wie schon bei den Untersuchungen im vorhergehenden Abschnitt 4.1.1.2, ein fester molarer Anteil von 0,5 mol-\% BDDA (bezogen auf die Stoffmenge des Monomers) gewählt. Der molare Anteil des RAFT-Agens EPHT wurde von 0 bis 4 mol-\%o variiert. In allen Polymerisationsmischungen waren 2 Gew.-\% (bezogen auf die Masse des Monomers) MMMP als Photoinitiator enthalten.

Zunächst wurden wie schon zuvor (s. Abschnitt 4.1.1.1), sowohl für die Poly(BA-co-BDDA)- als auch die Poly(EA-co-BDDA)-Netzwerke, Ausschnitte der Zugversuch-Probekörper mittels Lichtmikroskop aufgenommen, um einen ersten Eindruck vom Einfluss des RAFT-Agens auf die Netzwerke zu bekommen (s. Abbildung 4-18 und Abbildung 4-19).

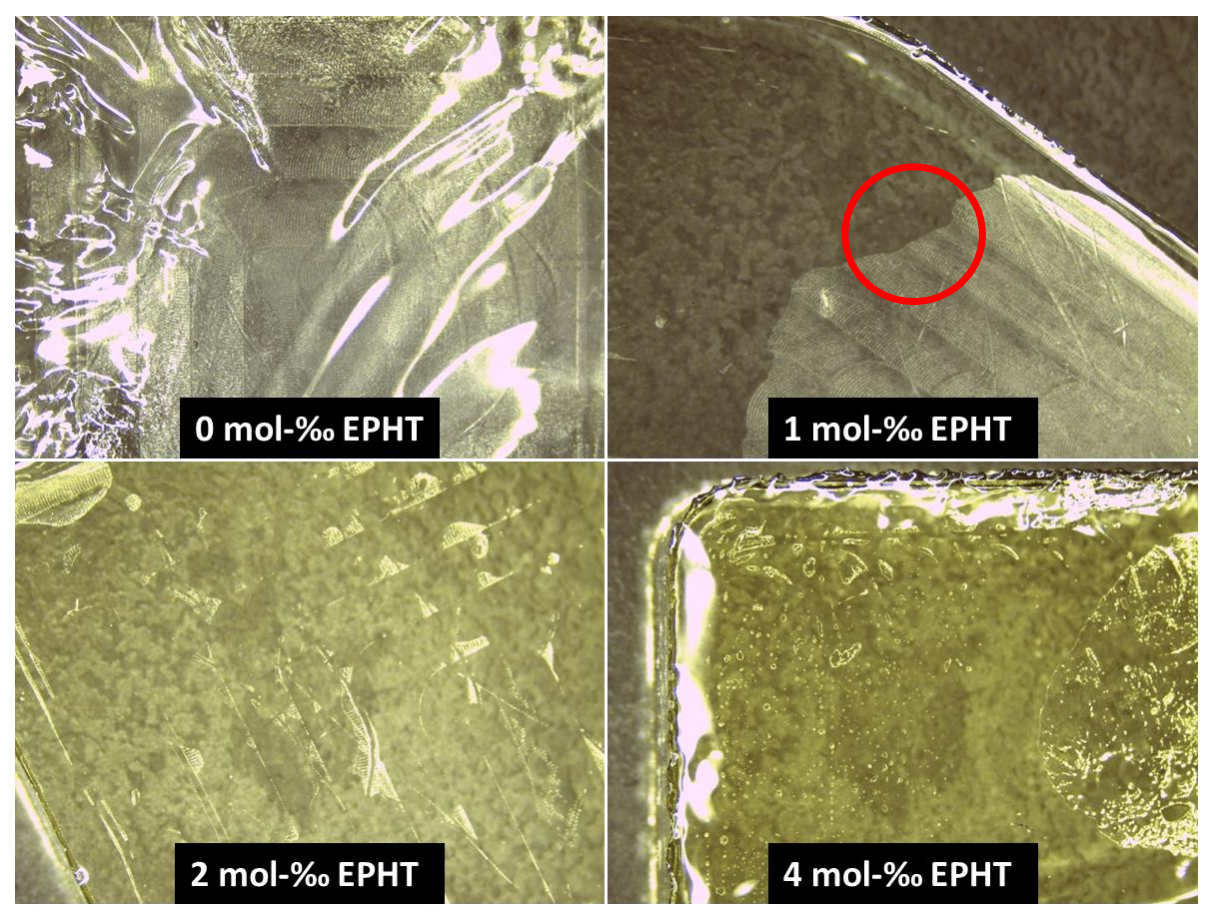

Abbildung 4-18 Lichtmikroskop-Aufnahmen von Poly(BA-co-BDDA)-Netzwerken mit 0,5 mol-\% BDDA als Vernetzer aus UV-initiierter konventioneller radikalischer Polymerisation, sowie aus UV-initiierter RAFT-Polymerisation mit 1 mol-\%o, 2 mol-\%o sowie 4 mol-\%o des RAFT-Agens EPHT. Als Photoinitiator wurde bei allen Netzwerken 2 Gew.-\% MMMP verwendet. Die Lichtintensität betrug $9 \mathrm{~mW} / \mathrm{cm}^{2}$ bei $366 \mathrm{~nm}$. Die Polymerisationszeit betrug für das konventionelle und das RAFT-Netzwerk mit 1 mol-\%o EPHT $30 \mathrm{~min}, 45 \mathrm{~min}$ für das RAFT-Netzwerk mit 2 mol-\%o und 80 min für das RAFT-Netzwerk mit 4 mol-\%. Alle Polymerisationen wurden bei Raumtemperatur durchgeführt. 


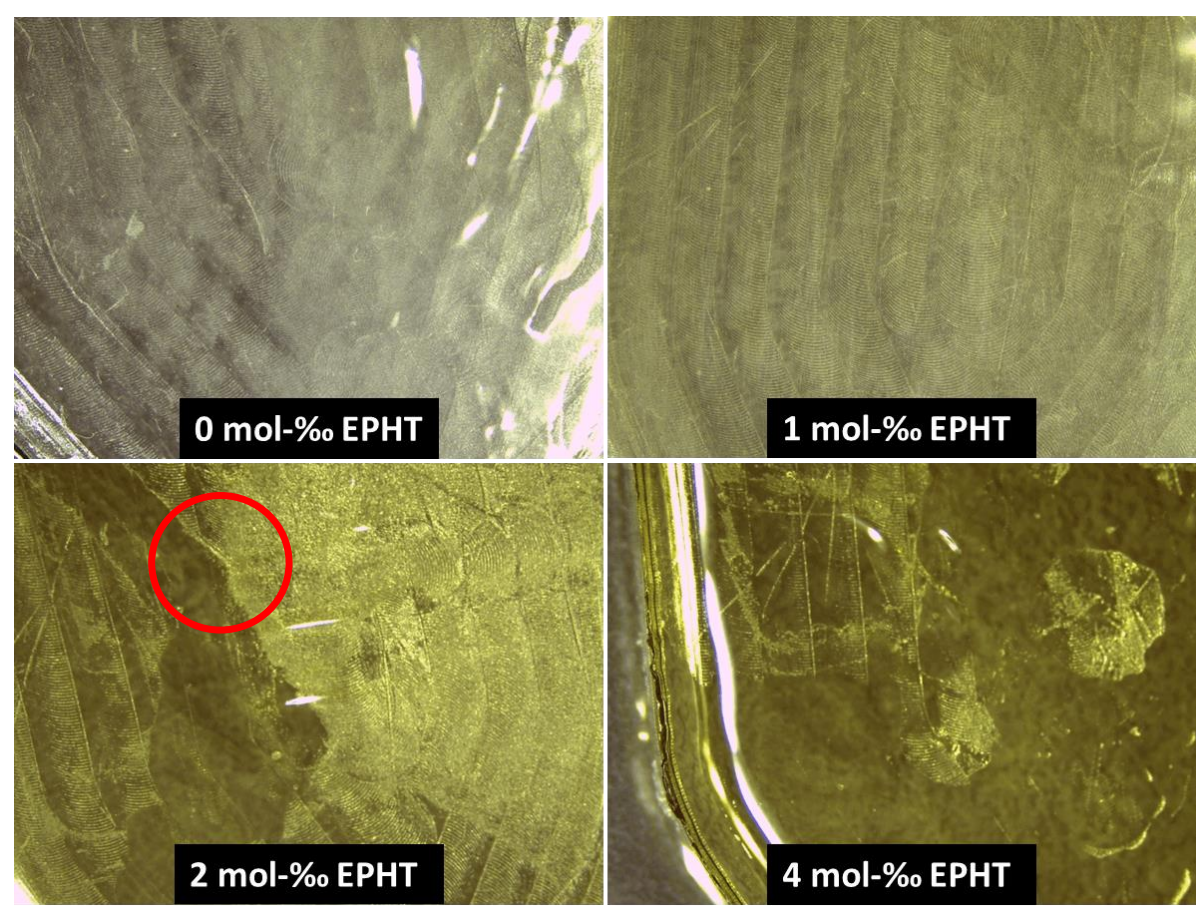

Abbildung 4-19 Lichtmikroskop-Aufnahmen von Poly(EA-co-BDDA)-Netzwerken mit 0,5 mol-\% BDDA als Vernetzer aus UV-initiierter konventioneller radikalischer Polymerisation, sowie aus UV-initiierter RAFT-Polymerisation mit 1 mol-\%o, 2 mol-\%o sowie 4 mol-\%o des RAFT-Agens EPHT. Als Photoinitiator wurde bei allen Netzwerken 2 Gew.-\% MMMP verwendet. Die Lichtintensität betrug $9 \mathrm{~mW} / \mathrm{cm}^{2}$ bei $366 \mathrm{~nm}$. Die Polymerisationszeit betrug für das konventionelle und das RAFT-Netzwerk mit 1 mol-\% EPHT $30 \mathrm{~min}, 45 \mathrm{~min}$ für das RAFT-Netzwerk mit 2 mol-\%o und 80 min für das RAFT-Netzwerk mit 4 mol-\%o. Alle Polymerisationen wurden bei Raumtemperatur durchgeführt.

Es ist erkennbar, dass schon die Zugabe einer sehr geringen Menge von 1 mol-\%o EPHT deutliche Auswirkungen auf die makroskopische Oberfläche der Netzwerke hat. Diese wurde gleichmäßiger und ebener, was ein Hinweis darauf ist, dass sich auch die inneren Strukturen des Netzwerks im Vergleich zum konventionellen Netzwerk verändert haben. Eine weitere Zugabe von EPHT hatte nur einen Einfluss auf die Farbe der Probekörper. Weiterhin fiel auf, dass die Probekörper mit steigendem EPHT-Anteil zunehmend klebriger wurden. In Abbildung 4-18 und Abbildung 4-19 ist zu erkennen: Die Probekörper mit größerem EPHT-Anteil treten intensiver mit der Glasoberfläche in Kontakt, auf der sie sich für die Mikroskop-Aufnahmen befanden. Die roten Kreise in den Abbildungen markieren Bereiche, in denen eine klare Grenzlinie zwischen dunklen und hellen Bereichen zu erkennen ist. Die dunklen Bereiche gehen auf einen sehr engen Kontakt auf molekularer Ebene zwischen Glas und Probekörper zurück. In den hellen Bereichen liegt der Probekörper nur locker auf der Glasoberfläche auf. Dieser Effekt wird in Kapitel 4.1.5 ausführlich diskutiert.

Anschließend an die Lichtmikroskop-Aufnahmen wurden erneut Zugversuche durchgeführt. Abbildung 4-20 zeigt die erhaltenen Spannungs-Dehnungs-Kurven der Poly(BA-co-BDDA)- 
Netzwerke. Abbildung 4-21 zeigt die SDKs der Poly(EA-co-BDDA)-Netzwerke. Wieder wurden aufgrund der besseren Übersichtlichkeit jeweils nur die SDKs eines Probekörpers ausgewählt und aufgetragen.

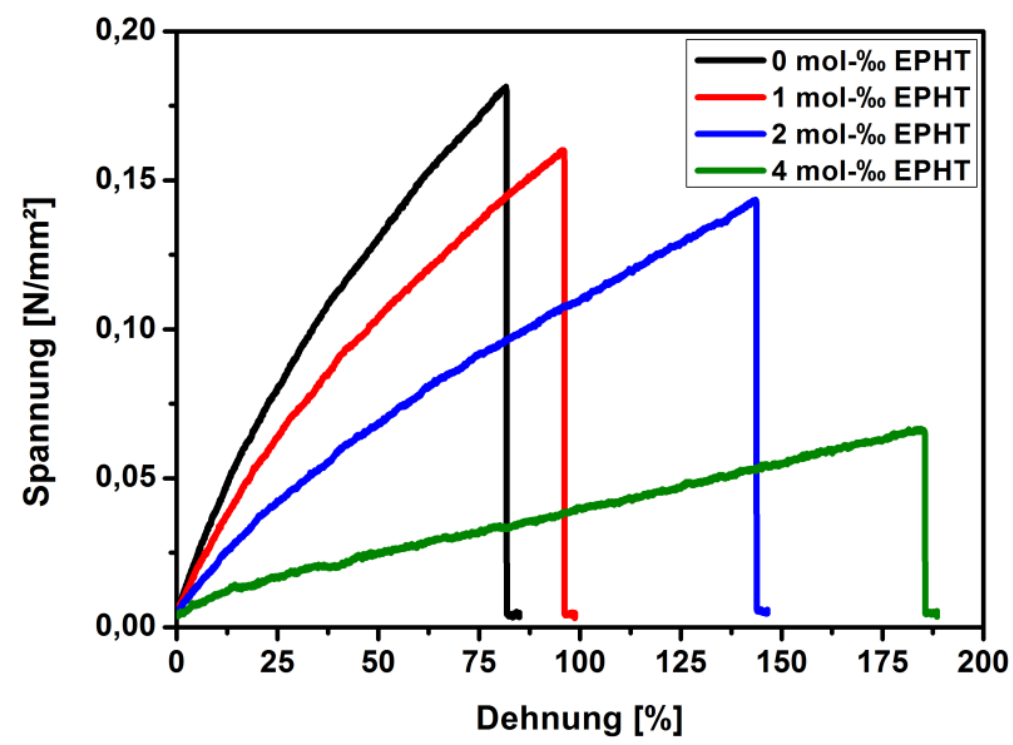

Abbildung 4-20 Mittels Zugversuch gemessene Spannungs-Dehnungs-Kurven von Poly-(BA-co-BDDA)-Netzwerken mit einem molaren Anteil von 0,5 mol-\% vom Vernetzer BDDA aus UV-initiierter, konventioneller radikalischer Polymerisation, sowie aus UV-initiierter RAFT-Polymerisation mit 1 mol-\%o, 2 mol-\%o sowie 4 mol-\%o des RAFT-Agens EPHT. Die Zugversuche wurden mit einer Zuggeschwindigkeit von $25 \mathrm{~mm} / \mathrm{min}$ bei einer Temperatur von $21^{\circ} \mathrm{C}$ durchgeführt. Die gezeigten Kurven stehen beispielhaft für alle durchgeführten Messungen.

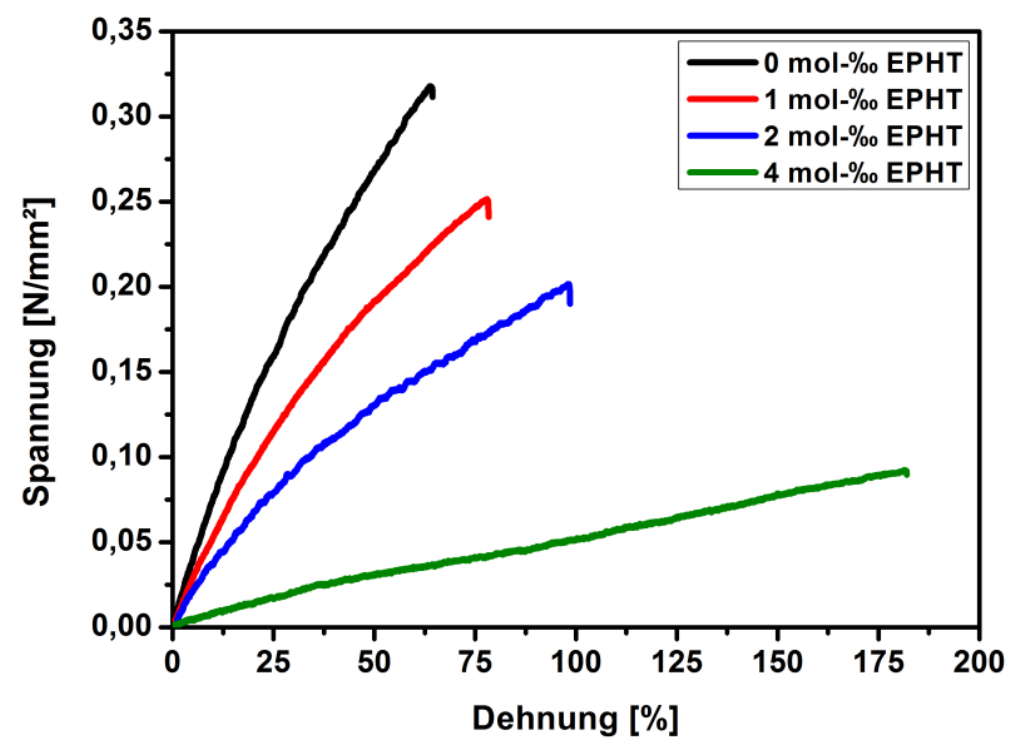

Abbildung 4-21 Mittels Zugversuch gemessene Spannungs-Dehnungs-Kurven von Poly-(EA-co-BDDA)-Netzwerken mit einem molaren Anteil von 0,5 mol-\% vom Vernetzer BDDA aus UV-initiierter, konventioneller radikalischer Polymerisation, sowie aus UV-initiierter RAFT-Polymerisation mit 1 mol-\%, 2 mol-\%o sowie 4 mol-\%o des RAFT-Agens 
EPHT. Die Zugversuche wurden mit einer Zuggeschwindigkeit von $25 \mathrm{~mm} / \mathrm{min}$ bei einer Temperatur von $21^{\circ} \mathrm{C}$ durchgeführt. Die gezeigten Kurven stehen beispielhaft für alle durchgeführten Messungen.

Trotz des immer gleichen molaren Anteils des Vernetzers BDDA in den Netzwerken unterscheiden sich die Verläufe der SDKs sowohl der Poly(BA-co-BDDA)- als auch der Poly(EAco-BDDA)-Netzwerke mit ansteigendem molarem Anteil vom RAFT-Agens EPHT erheblich voneinander. Je mehr EPHT in den Netzwerken enthalten ist, desto geringere Spannungen werden benötigt, um die Probekörper bis zum Bruch zu dehnen. Die Beträge der Bruchdehnungen werden dabei immer größer. Eine Auftragung der E-Moduln und der Bruchdehnungen gegen den molaren Anteil des RAFT-Agens EPHT in den jeweiligen Netzwerken ergibt folgende Verläufe (s. Abbildung 4-22 und Abbildung 4-23).

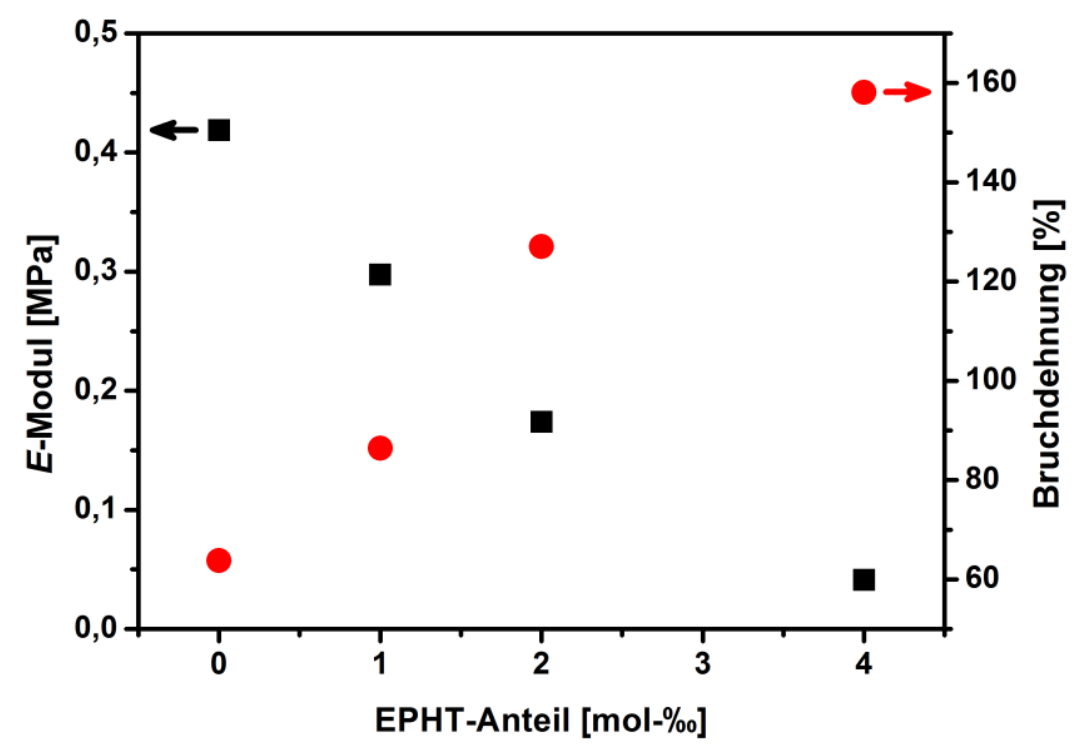

Abbildung 4-22 Mittels Zugversuch ermittelte E-Moduln und Bruchdehnungen von Poly(BA-co-BDDA)-Netzwerken mit 0,5 mol-\% BDDA als Vernetzer aus UV-initiierter, konventioneller radikalischer Polymerisation, sowie aus UVinitiierter RAFT-Polymerisation mit 1 mol-\%o, 2 mol-\%o sowie 4 mol-\%o des RAFT-Agens EPHT. Die Zugversuche wurden mit einer Zuggeschwindigkeit von $25 \mathrm{~mm} / \mathrm{min}$ bei einer Temperatur von $21^{\circ} \mathrm{C}$ durchgeführt. Die gezeigten Kurven stehen beispielhaft für alle durchgeführten Messungen. 


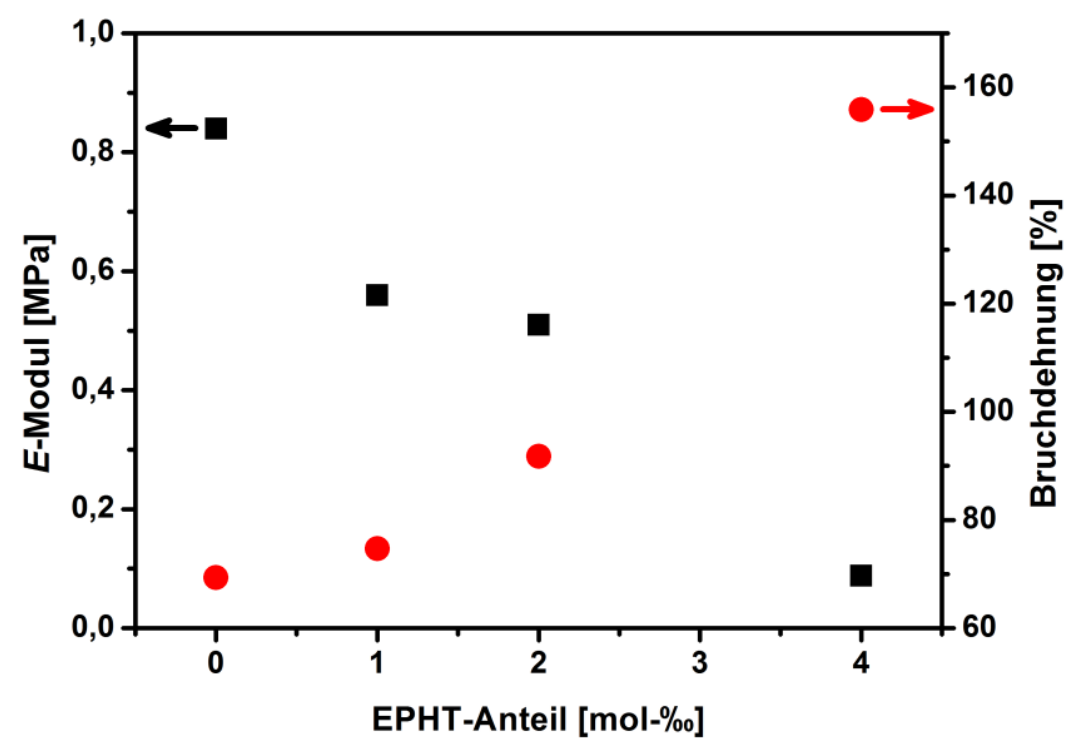

Abbildung 4-23 Mittels Zugversuch ermittelte E-Moduln und Bruchdehnungen von Poly(EA-co-BDDA)-Netzwerken mit 0,5 mol-\% BDDA als Vernetzer aus UV-initiierter, konventioneller radikalischer Polymerisation, sowie aus UVinitiierter RAFT-Polymerisation mit 1 mol-\%o, 2 mol-\%o sowie 4 mol-\%o des RAFT-Agens EPHT. Die Zugversuche wurden mit einer Zuggeschwindigkeit von $25 \mathrm{~mm} / \mathrm{min}$ bei einer Temperatur von $21{ }^{\circ} \mathrm{C}$ durchgeführt. Die gezeigten Kurven stehen beispielhaft für alle durchgeführten Messungen.

Es ist erkennbar, dass die E-Moduln sowohl bei den Poly(BA-co-BDDA)- als auch bei den Poly(EA-co-BDDA)-Netzwerken von 0 bis 4 mol-\% EPHT nahezu linear um ca. eine Zehnerpotenz abnehmen. Bei den Poly(BA-co-BDDA)-Netzwerken sinken sie von 0,42 MPa beim konventionellen Netzwerk auf 0,041 MPa bei dem RAFT-Netzwerk mit 4 mol-\%o EPHT. Bei den Poly(EA-co-BDDA)-Netzwerken erfolgt eine Abnahme von 0,84 $\mathrm{MPa}$ auf 0,088 $\mathrm{MPa}$. Die Bruchdehnungen dagegen steigen bei beiden Netzwerken um nahezu $100 \%$ an: bei den Poly(BAco-BDDA)-Netzwerken von $64 \%$ beim konventionellen Netzwerk bis auf $158 \%$ bei dem RAFTNetzwerk mit 4 mol-\%o EPHT und bei den Poly(EA-co-BDDA)-Netzwerken von $69 \%$ beim konventionellen Netzwerken bis auf $158 \%$ bei dem RAFT-Netzwerk mit 4 mol-\%o EPHT. Der Einfluss, den das RAFT-Agens EPHT auf die mechanischen Eigenschaften ausübt, ist bei den Poly(BA-co-BDDA)- und Poly(EA-co-BDDA)-Netzwerken jeweils vollkommen gleich. In beiden Fällen führt die Zugabe einer gleichen Menge des RAFT-Agens EPHT zu einer prozentual gleichen Erweichung des Netzwerks. Somit kann festgestellt werden, dass der schon zuvor gefundene Einfluss des RAFT-Agens auf die mechanischen Eigenschaften der Netzwerke (s. Abschnitt 4.1.1.1) von der zugegebenen Menge des RAFT-Agens abhängt.

Anschließend wurde aus den E-Moduln der Netzwerke nach Gleichung (41) die Schermoduln berechnet, womit schließlich über die effektiven Netzkettendichten die scheinbaren molaren Massen der Netzketten ermittelt werden konnten. Weiterhin konnte aus den Integralen der 
Spannungs-Dehnungs-Kurven die Deformationsarbeit pro Einheitsvolumen bestimmt werden, die benötigt wird, um die Netzwerke bis zum Bruch zu dehnen. Die Werte der scheinbaren Molmassen und der Deformationsarbeiten sind für die Poly(BA-co-BDDA)-Netzwerke in Tabelle 4-6 und für die Poly(EA-co-BDDA)-Netzwerke in Tabelle 4-7 zusammengefasst. Auf die Angabe der effektiven Netzkettendichten wird an dieser Stelle verzichtet, da diese nach Gleichung (46) umgekehrt proportional zu den scheinbaren molaren Massen ist.

Tabelle 4-6 Scheinbare Molmassen der Netzketten im Vergleich zu den theoretischen Werten und die Deformationsarbeiten pro Einheitsvolumen für Poly(BA-co-BDDA)-Netzwerke mit einem molaren Anteil von 0,5 mol-\% des Vernetzers BDDA aus UV-initiierter, konventioneller radikalischer Polymerisation, sowie aus UV-initiierter RAFT-Polymerisation mit 1 mol-\%, 2 mol-\%o sowie 4 mol-\%o des RAFT-Agens EPHT.

\begin{tabular}{cccc}
\hline EPHT-Anteil[mol-\%o $]$ & $\overline{\boldsymbol{M}}_{\mathbf{x}}[\mathbf{g} / \mathbf{m o l}]$ & $\overline{\boldsymbol{M}}_{\mathbf{x}}^{\text {th }}[\mathbf{g} / \mathbf{m o l}]$ & Deformationsarbeit $\left[\mathbf{J} / \mathbf{m m}^{3}\right]$ \\
\hline 0 & 8760 & 25634 & $8,4 \pm 2,0$ \\
1 & 12340 & 25634 & $6,8 \pm 0,2$ \\
2 & 21110 & 25634 & $9,2 \pm 2,5$ \\
4 & 89100 & 25634 & $5,7 \pm 0,7$ \\
\hline
\end{tabular}

Tabelle 4-7 Scheinbare Molmassen der Netzketten im Vergleich zu den theoretischen Werten und die Deformationsarbeiten pro Einheitsvolumen für Poly(EA-co-BDDA)-Netzwerke mit einem molaren Anteil von 0,5 mol-\% vom Vernetzer BDDA aus UV-initiierter, konventioneller radikalischer Polymerisation, sowie aus UV-initiierter RAFT-Polymerisation mit 1 mol-\%o, 2 mol-\%o sowie 4 mol-\%o des RAFT-Agens EPHT.

\begin{tabular}{cccc}
\hline EPHT-Anteil [mol-\%] $]$ & $\overline{\boldsymbol{M}}_{\mathbf{\mathbf { x }}}[\mathbf{g} / \mathbf{m o l}]$ & $\overline{\boldsymbol{M}}_{\mathbf{X}}^{\text {th }}[\mathbf{g} / \mathbf{m o l}]$ & Deformationsarbeit $\left[\mathbf{J} / \mathbf{m m}^{\mathbf{3}}\right]$ \\
\hline 0 & 8720 & 20024 & $14,2 \pm 3,1$ \\
1 & 13170 & 20024 & $10,5 \pm 2,2$ \\
2 & 13710 & 20024 & $11,9 \pm 2,5$ \\
4 & 83960 & 20024 & $7,0 \pm 2,5$ \\
\hline
\end{tabular}

Die scheinbaren Molmassen weichen zum Teil deutlich von den theoretischen Werten ab. Während die konventionellen Netzwerke als Folge ihrer hohen Moduln und somit ebenfalls hohen Netzkettendichten niedrigere Werte aufweisen, sind die scheinbaren Molmassen der Netzwerke mit einem molaren Anteil von 4 mol-\%o EPHT deutlich größer als die theoretisch möglichen Molmassen der Netzketten. Dies kann entsprechend auch über den niedrigen Wert des E-Moduls und der damit einhergehenden niedrigen Netzkettendichte erklärt werden. Der Grund hierfür liegt, wie schon im vorherigen Abschnitt diskutiert, an der Reduzierung von Verhakungen und Verschlaufungen der Netzketten durch die Zugabe des RAFT-Agens. Die hier erhaltenen Daten legen nahe, dass diese Netzwerkdefekte bei einem molaren Anteil von 1 mol-\%o EPHT noch nicht vollständig reduziert wurden. Die scheinbaren Molmassen sind kleiner als die 
theoretischen. Bei einem molaren Anteil von 2 mol-\%o EPHT sind die scheinbaren Molmassen der Netzketten des Poly(BA-co-BDDA)-Netzwerks nahezu deckungsgleich mit den theoretischen. Die scheinbaren Molmassen der Netzketten des Poly(EA-co-BDDA)-Netzwerks sind eindeutig kleiner als der theoretische Wert, was sich allerdings sehr wahrscheinlich auf den relativ hohen E-Modul zurückführen lässt. Abbildung 4-23 zeigt, dass der E-Modul deutlich zu groß ist, wenn eine ansonsten lineare Abnahme des E-Moduls mit zunehmendem molarem Anteil des RAFT-Agens EPHT angenommen wird. Es kann also davon ausgegangen werden, dass die scheinbaren Molmassen der Netzketten hier wahrscheinlich gut mit den theoretischen übereinstimmen würden. Bei den Netzwerken mit einem molaren Anteil von 4 mol-\%o EPHT sind die scheinbaren Molmassen der Netzketten viel größer als sie theoretisch möglich sein sollten. Das bedeutet, dass in diesen Netzwerken nahezu keine Verhakung und Verschlaufungen mehr vorhanden sind, sondern eher andere Netzwerkdefekte vorliegen, wie z. B. freie Kettenenden, welche die effektiven Netzkettendichten reduzieren und somit die Molmassen der Netzketten größer erscheinen lassen. Weiterhin fällt auf, dass die scheinbaren Molmassen der Netzketten beider Netzwerkarten die gleichen Werte annehmen. Dies ist eine Folge der unterschiedlichen Berechnungsgrundlagen für diese Werte. Während für die Poly(BA-co-BDDA)-Netzwerke entsprechend der vorhergehenden Ergebnisse das Phantomnetzwerk-Modell zugrunde gelegt wurde, wurde für die Poly(EA-co-BDDA)-Netzwerke das affine Netzwerkmodell verwendet. Nach Gleichung (45) ergibt sich bei einem Wert von $A=0,5$ für das Phantomnetzwerk im Vergleich zum affinen Netzwerk, bei $\operatorname{dem} A=1$ ist, eine doppelt so große Netzkettendichte. Da die $E$ Moduln und damit auch die G-Moduln, der Poly(BA-co-BDDA)-Netzwerke genau halb so groß sind wie die der Poly(EA-co-BDDA)-Netzwerke, ergeben sich für beide Netzwerkarten identische effektive Netzkettendichten und somit auch identische scheinbare Molmassen der Netzketten.

Die Deformationsarbeiten zeigen bei beiden Netzwerk-Arten in dem Bereich von 0 bis 2 mol-\%o im Rahmen der Standardabweichung keinerlei Abhängigkeit vom molaren Anteil des RAFTAgens in den Netzwerken. Dies war zu erwarten, da der molare Anteil des Vernetzers BDDA konstant war. Allerdings zeigt sich bei beiden Netzwerkarten, dass die Deformationsarbeiten der Netzwerke mit einem molaren Anteil von 4 mol-\%o deutlich geringer sind als die der anderen Netzwerke. Dies unterstützt die Annahme, dass in diesen Netzwerken tatsächlich weniger elastisch effektive Netzketten existieren. Eine geringere Anzahl von Netzketten würde dementsprechend auch weniger Energie benötigen, um zerrissen zu werden. Ein anschaulicher Vergleich ist an dieser Stelle ein dünnes Seil, das eher reißen würde als ein dickes, sofern beide aus dem gleichen Material gefertigt sind. 


\subsubsection{Abschließende Bemerkungen}

In diesem Kapitel konnte gezeigt werden, dass die Zugabe eines RAFT-Agens in die Polymerisationsmischungen von statistischen Poly(BA-co-BDDA)- und Poly(EA-co-BDDA)Netzwerken dazu führt, dass die resultierenden Netzwerke deutlich weicher sind als die entsprechenden konventionellen Netzwerke. Diese Erweichung ist dabei abhängig vom molaren Anteil des RAFT-Agens in der Polymerisationsmischung: Mit zunehmendem molaren Anteil des RAFT-Agens werden die Netzwerke zunehmend weicher. Dies geht auf die Verringerung von Verhakungen und Verschlaufungen der Netzketten zurück, wobei bei sehr hohen molaren Anteilen des RAFT-Agens wahrscheinlich vermehrt freie Kettenenden auftreten, die das Netzwerk noch weicher werden lassen. Diese Veränderung der inneren mikroskopischen Strukturen der Netzwerke geht dabei mit einer Veränderung der makroskopischen Oberflächen der Netzwerke einher, die mit Zugabe des RAFT-Agens deutlich ebenmäßiger werden. Weiterhin konnte gezeigt werden, dass sich die Poly(BA-co-BDDA)-Netzwerke aus RAFT-Polymerisation entgegen bisheriger Annahmen (s. Kapitel 2.3.1) treffend mit dem Phantom-Netzwerkmodell beschrieben werden können und somit einem idealen Modellnetzwerk sehr nah sind.

\subsubsection{Einfluss der RAFT-Polymerisation auf die Glastemperaturen der Netzwerke}

Eine Größe, welche die mechanischen Eigenschaften der Polymere wesentlich beeinflusst, ist ihre Glastemperatur. Wie in Kapitel 2.4 schon erwähnt, erstarrt ein Polymer bei seiner Glastemperatur, wenn es abgekühlt wird. Im Gegenzug erweicht es beim Erwärmen über die Glastemperatur. Dieser Prozess wird auch Glasumwandlung genannt.

Der Grund für diese Veränderungen ist in der Beweglichkeit der Polymerkettensegmente zu finden. Oberhalb der Glastemperatur sind Rotationsbewegungen der Segmente möglich. Dabei handelt es sich vermutlich um trans-gauche Umwandlungen der Bindungswinkel von Monomeren, die über größere Bereiche verlaufen und eine Art Kurbelwellenbewegung verursachen (s. Abbildung 4-24). ${ }^{[193,194]}$ Unterhalb der Glastemperatur frieren diese Bewegungen ein, sodass das Polymer auch makroskopisch erstarrt. Neben thermischen Faktoren wie z. B. der Heizrate mit der das Polymer abgekühlt oder aufgeheizt wird, haben vor allem konstitutionelle Faktoren einen Einfluss auf die Glasumwandlung. ${ }^{[195]}$ So führt z. B. eine Vernetzung dazu, dass die Rotation der Segmente behindert wird, da diese die Ketten näher aneinander rücken lässt und somit die Bewegungsmöglichkeiten der Kettensegmente einschränkt. Dies hat zur Folge, dass die Glastemperatur steigt.

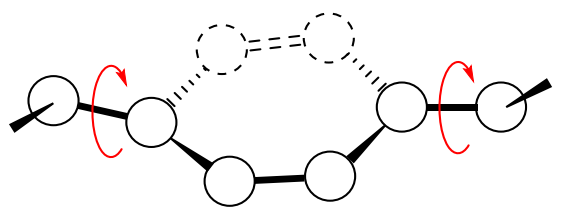

Abbildung 4-24 Kurbelwellenbewegung eines Kettensegments. Nach Cowie ${ }^{[192]}$. 
Die Glastemperaturen der Poly(BA-co-BDDA)- und Poly(EA-co-BDDA)-Netzwerke wurden per dynamischer Differenzkalorimetrie (gebräuchlicher ist die englische Bezeichnung: Differential Scanning Calorimetry, kurz: DSC) gemessen. Informationen $\mathrm{zu}$ den Messungen und zur Auswertung der Thermogramme sind in Kapitel 6.3.6 zu finden.

Zunächst wurde untersucht, welchen Einfluss eine Erhöhung des molaren BDDA-Anteils in den Netzwerken auf deren Glastemperatur hatte. Dazu wurden jeweils eine unvernetzte Probe Poly-BA bzw. Poly-EA sowie jeweils drei Netzwerke, die alle mittels RAFT-Polymerisation hergestellt worden sind, ausgewählt und die Glastemperaturen dieser Proben gemessen. Die Ergebnisse der Messungen sind in Tabelle 4-8 zu finden.

Tabelle 4-8 Glastemperaturen von Poly-BA und Poly-EA sowie Poly(BA-co-BDDA)- und Poly(EA-co-BDDA)Netzwerken aus UV-initiierter RAFT-Polymerisation mit 0,5 mol-\%, 1 mol-\% und 2 mol-\% des Vernetzers BDDA. Der molare Anteil des RAFT-Agens EPHT beträgt immer 2 mol-\%.

\begin{tabular}{ccc}
\hline BDDA-Anteil [mol-\%] & $\boldsymbol{T}_{\mathrm{g}}^{\mathbf{p B A}}\left[{ }^{\circ} \mathbf{C}\right]$ & $\boldsymbol{T}_{\mathrm{g}}^{\mathbf{p E A}}\left[{ }^{\circ} \mathbf{C}\right]$ \\
\hline 0 & $-45,5$ & $-14,8$ \\
0,5 & $-42,8$ & $-11,8$ \\
1 & $-41,8$ & $-11,8$ \\
2 & $-39,8$ & $-8,2$ \\
\hline
\end{tabular}

Da eine höhere Vernetzer-Konzentration eine höhere Netzkettendichte zur Folge hat (s. Abschnitt 4.1.1.1), führt die Erhöhung des molaren BDDA-Anteils entsprechend der Erwartungen auch zu einer Erhöhung der Glastemperaturen der Netzwerke. Zusätzlich wurde der Einfluss des molaren Anteils des RAFT-Agens EPHT auf die Glastemperaturen der Poly(BAco-BDDA)- und Poly(EA-co-BDDA)-Netzwerke untersucht. Hierzu wurde, wie schon zuvor (s. Abschnitt 4.1.1.3), bei konstantem molarem BDDA-Anteil von 0,5 mol-\%, die Konzentration des RAFT-Agens EPHT erhöht. Die Ergebnisse der Messungen sind in Tabelle 4-9 zu finden

Tabelle 4-9 Glastemperaturen von Poly(BA-co-BDDA)- und Poly(EA-co-BDDA)-Netzwerken aus UV-initiierter konventioneller radikalischer Polymerisation, sowie aus UV-initiierter RAFT-Polymerisation mit 1 mol-\%o, 2 mol-\%o und 4 mol-\% des RAFT-Agens EPHT. des RAFT-Agens EPHT. Der molare Anteil des Vernetzers BDDA beträgt immer $0,5 \mathrm{~mol}-\%$.

\begin{tabular}{ccc}
\hline EPHT-Anteil [mol-\%o] & $\boldsymbol{T}_{\mathrm{g}}^{\mathbf{p B A}}\left[{ }^{\circ} \mathbf{C}\right]$ & $\boldsymbol{T}_{\mathrm{g}}^{\mathbf{p E A}}\left[{ }^{\circ} \mathbf{C}\right]$ \\
\hline 0 & $-40,9$ & $-10,7$ \\
1 & $-42,7$ & $-10,5$ \\
2 & $-42,8$ & $-11,8$ \\
4 & $-42,9$ & $-12,9$ \\
\hline
\end{tabular}


Durch die Zugabe des RAFT-Agens EPHT kommt es bei den Poly(BA-co-BDDA)-Netzwerken zu einer Abnahme der Glastemperatur. Auch wenn die weitere Abnahme der Glastemperatur nur sehr gering ist, ist die Tendenz zu insgesamt niedrigeren Glastemperaturen mit steigendender EPHT-Konzentration doch klar zu erkennen. Gleiches gilt für die Poly(EA-co-BDDA)-Netzwerke. Obwohl die Glastemperatur zunächst sogar ansteigt, sinkt sie anschließend klar ab. Da eine Erhöhung des molaren EPHT-Anteils, wie im vorherigen Kapitel gezeigt, zu einer Verringerung der Netzkettendichte der Netzwerke führt, ist auch hier die Abnahme der Glastemperaturen nachvollziehbar.

Alle Zugversuche wurden bei Raumtemperatur $\left(21^{\circ} \mathrm{C}\right)$ durchgeführt. Aufgrund der insgesamt geringen Änderungen der Glastemperaturen, im Vergleich zu den im Mittel viel höheren Raumtemperaturen (diese Unterschiede betrugen ungefähr $60{ }^{\circ} \mathrm{C}$ bei den Poly(BA-co-BDDA)- und ungefähr $30^{\circ} \mathrm{C}$ bei den Poly(BA-co-BDDA)-Netzwerken), sollten diese Änderungen keinen Einfluss auf die gemessenen E-Moduln und Bruchdehnungen der Netzwerke gehabt haben. 


\subsubsection{Einfluss der RAFT-Polymerisation auf die Quellungseigenschaften der Netzwerke}

\subsubsection{Variation des molaren BDDA-Anteils}

Eine weitere wesentliche Eigenschaft von elastomeren Polymernetzwerken ist, neben ihren besonderen mechanischen Eigenschaften, die Fähigkeit in einem geeigneten Lösungsmittel aufzuquellen (s. Kapitel 2.3.2).

Darum wurden im Anschluss an die Zugversuche Quellungsexperimente mit den hergestellten Netzwerken durchgeführt. Dazu wurden Proben der Netzwerke mehrere Tage bei Raumtemperatur in einem Überschuss Aceton aufbewahrt und die Änderung der Masse der Probekörper mittels Gravimetrie verfolgt. Der genaue Ablauf der Experimente ist in Kapitel 6.3.2 zu finden. Mittels der Gleichungen (36) und (37) wurde anschließend der Volumenquellungsgrad der Netzwerke berechnet. Die erhaltenen Werte wurden aus Gründen der Übersichtlichkeit in einer doppelt logarithmischen Auftragung gegen den molaren Anteil des Vernetzers BDDA aufgetragen (s. Abbildung 4-25 und Abbildung 4-26). Weiterhin macht diese Art der Auftragung Parallelen in den Verläufen der Volumenquellungsgrade bei steigendem molaren BDDA-Anteil zwischen den Poly(BA-co-BDDA)- und Poly(EA-co-BDDA)-Netzwerken deutlich.

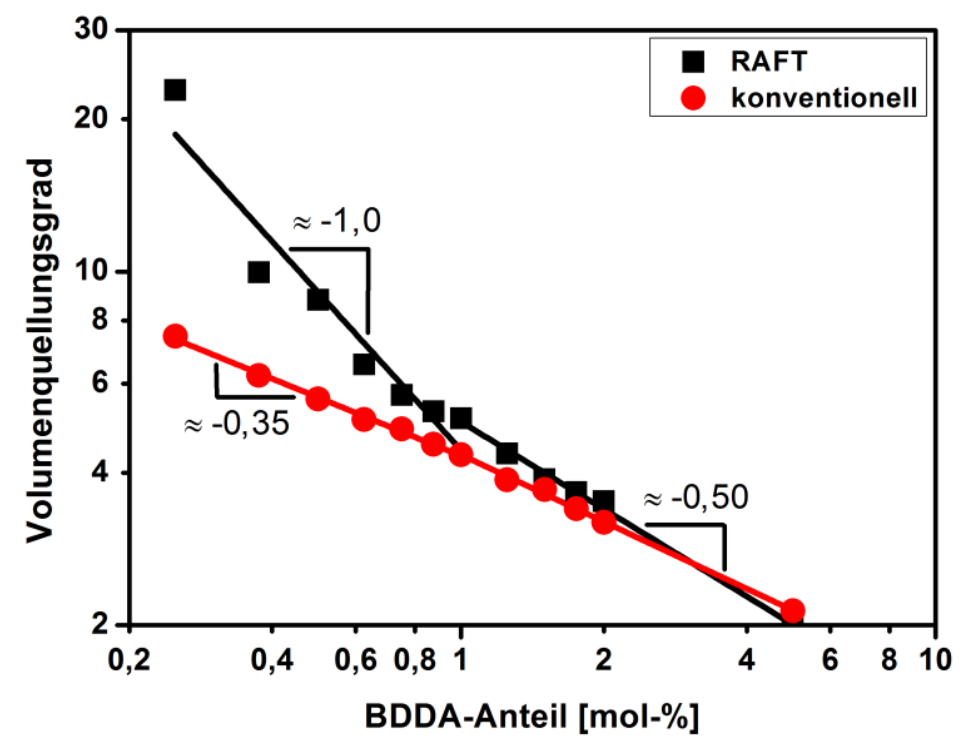

Abbildung 4-25 Doppelt-logarithmische Auftragung des Volumenquellungsgrades von Poly(BA-co-BDDA)Netzwerken in Aceton gegen den molaren Anteil des Vernetzers BDDA in den Netzwerken. (๘): für Netzwerke aus UV-initiierter RAFT-Polymerisation mit 2 mol-\%o des RAFT-Agens EPHT. (•): für Netzwerke aus UV-initiierter, konventioneller radikalischer Polymerisation. Die Quellungsexperimente fanden bei Raumtemperatur statt. Die eingezeichneten Steigungsdreiecke beziehen sich jeweils auf einen Bereich ober- bzw. unterhalb von 1 mol-\% BDDA. 


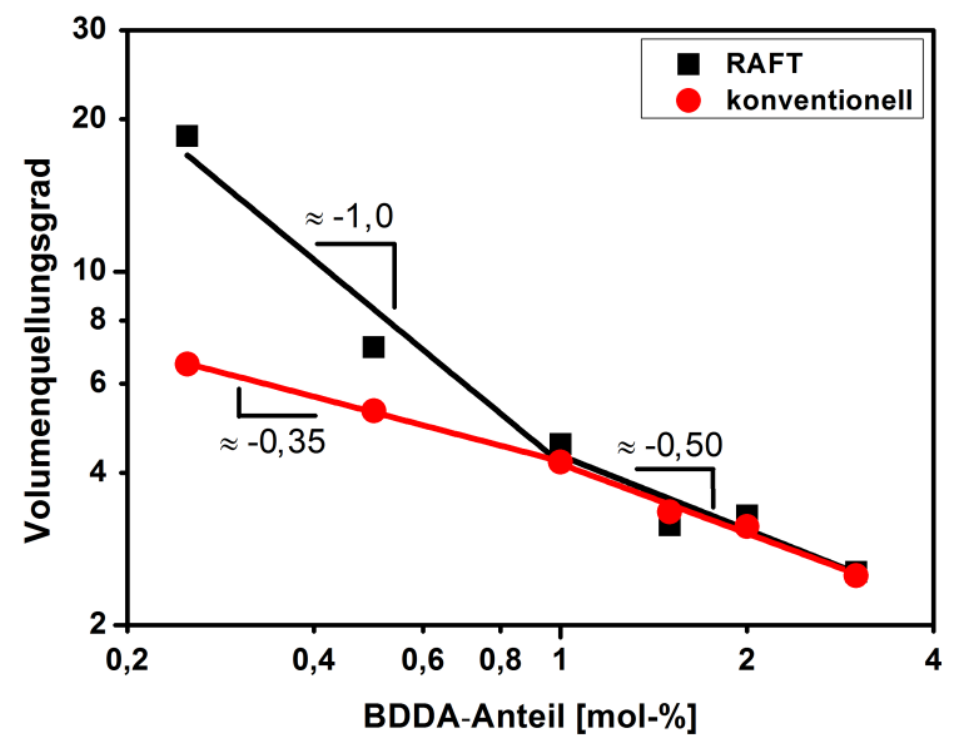

Abbildung 4-26 Doppelt-logarithmische Auftragung des Volumenquellungsgrades von Poly(EA-co-BDDA)Netzwerken in Aceton gegen den molaren Anteil des Vernetzers BDDA in den Netzwerken. (ロ): für Netzwerke aus UV-initiierter RAFT-Polymerisation mit 2 mol-\%o des RAFT-Agens EPHT. (•): für Netzwerke aus UV-initiierter, konventioneller radikalischer Polymerisation. Die Quellungsexperimente fanden bei Raumtemperatur statt. Die eingezeichneten Steigungsdreiecke beziehen sich jeweils auf einen Bereich ober- bzw. unterhalb von 1 mol-\% BDDA.

Es ist erkennbar, dass die Volumenquellungsgrade mit steigendem molarem Anteil BDDA in den Netzwerken abnehmen. Es zeigt sich ebenfalls, dass diese Abnahme für die RAFT-Netzwerke im Vergleich zu den konventionellen Netzwerken im Bereich von unter 1 mol-\% BDDA unterschiedlich stark ist. Während die RAFT-Netzwerke entlang einer in Abbildung 4-25 und Abbildung 4-26 eingefügten Ausgleichsgeraden mit der Steigung -1 verlaufen, ist die Abnahme der Volumenquellungsgrade der konventionellen Netzwerke geringer. Sie verlaufen entlang einer Ausgleichsgeraden mit der Steigung $-0,35$.

$\mathrm{Ab}$ einem molaren BDDA -Anteil von 1 mol-\% ist die Steigung für beide Netzwerke nahezu gleich. Dies wird vor allem bei den Poly(EA-co-BDDA)-Netzwerken deutlich (s. Abbildung 4-26). Wie schon bei den scheinbaren Molmassen der Netzketten oder den Bruchdehnungen der Netzwerke (s. Kapitel 4.1.1.1) wird erkennbar, dass ab einem molaren Anteil von 1 mol-\% BDDA die jeweiligen Netzwerke aufgrund ihrer Volumenquellungsgrade kaum noch unterscheidbar sind. Die Steigungen der Ausgleichsgeraden der Volumenquellungsgrade sind in Tabelle 4-10 zusammengefasst. 
Tabelle 4-10 Steigungen der an die Volumenquellungsgrade von Poly(BA-co-BDDA)- und Poly(EA-co-BDDA)Netzwerken aus UV-initiierter RAFT-Polymerisation mit 2 mol-\%o des RAFT-Agens EPHT und UV-initiierter, konventioneller radikalischer Polymerisation angepassten Geraden (s. Abbildung 4-25 und Abbildung 4-26). Die Geraden wurden jeweils an einen Bereich ober- bzw. unterhalb von 1 mol-\% BDDA angepasst.

\begin{tabular}{cccc}
\hline Netzwerk & & oberhalb 1 mol-\% BDDA & unterhalb 1 mol-\% BDDA \\
\hline \multirow{2}{*}{ Poly-(BA-co-BDDA) } & RAFT & $-1,04$ & $-0,57$ \\
& konv. & $-0,38$ & $-0,44$ \\
\multirow{2}{*}{ Poly-(EA-co-BDDA) } & RAFT & $-1,01$ & $-0,48$ \\
& konv. & $-0,32$ & $-0,46$ \\
\hline
\end{tabular}

Eine Erhöhung des Quellungsgrades durch die Zugabe eines RAFT-Agens in die Polymerisationsmischung konnte schon von Liu et al. bei Hydrogelen beobachtet werden. ${ }^{[123]}$ Dies wurde auf eine höhere Anzahl von freien Kettenenden in den RAFT-Netzwerken zurückgeführt. Die freien Kettenenden erhöhen die Beweglichkeit im Netzwerk und ermöglichen somit die Aufnahme einer größeren Menge des Lösungsmittels während der Quellung.

Bezogen auf die hier vorliegenden Daten, bedeutet dies, dass ab einem molaren BDDA-Anteil von unter 1 mol-\% die Anzahl dieser freien Kettenenden in den RAFT-Netzwerken signifikant höher ist als in den konventionellen, was zu einem deutlich höheren Volumenquellungsgrad der RAFT-Netzwerke führt. Bei molaren BDDA-Anteilen von über 1 mol-\% dominiert der Einfluss des Vernetzers über den des RAFT-Agens und führt dazu, dass sich die Zahl der freien Kettenenden sich deutlich reduziert und die Volumenquellungsgrade der RAFT- und der konventionellen Netzwerke nicht mehr unterscheidbar sind. Auch dieses Verhalten ist mit den Beobachtungen von Liu et al. konform. Eine Erhöhung der Anzahl der freien Kettenenden durch die RAFT-Polymerisation sollte allerdings nicht mehr auftreten, wenn ein bifunktionelles RAFTAgens verwendet wird. In diesem Fall sollte es nicht zu einer Erhöhung des Quellungsgrades kommen.

Um dies zu überprüfen wurde das bifunktionelle RAFT-Agens Bis(S-Ethylpropan-2-ylonat)- $S$-1,4butylbistrithiocarbonat (BEPBT) (s. Abbildung 4-27) hergestellt. 2 mol-\%o BEPBT wurden bei der Synthese eines Poly(BA-co-BDDA)-Netzwerks als RAFT-Agens verwendet. Eine Probe des Netzwerks wurde in Aceton gequollen und dessen Volumenquellungsgrad bestimmt. Anschließend konnte das Ergebnis mit dem Volumenquellungsgrad eines konventionellen und eines Netzwerks mit EPHT als RAFT-Agens verglichen werden (s. Tabelle 4-11).

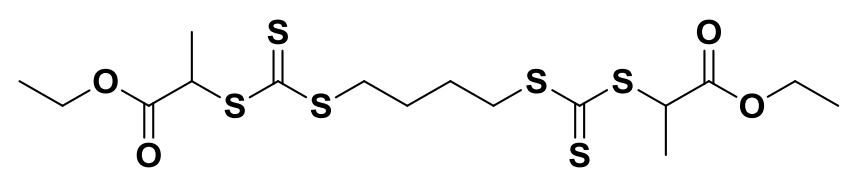

Abbildung 4-27 Strukturformel des bifunktionellen RAFT-Agens $\operatorname{Bis(S-Ethylpropan-2-ylonat)-S'-1,4-butylbistrithiocarbonat~(BEPBT).~}$ 
Tabelle 4-11 Volumenquellungsgrade in Aceton von Poly(BA-co-BDDA)-Netzwerken mit 0,5 mol-\% BDDA als Vernetzer aus UV-initiierter RAFT-Polymerisation. Eines der Netzwerke wurde in Anwesenheit von 2 mol-\%o des RAFT-Agens EPHT, das andere in Anwesenheit des RAFT-Agens BEPBT synthetisiert. Zum Vergleich wurde der Volumenquellungsgrad eines konventionellen Poly(BA-co-BDDA)-Netzwerken mit 0,5 mol-\% BDDA als Vernetzer hinzugefügt.

\begin{tabular}{cc}
\hline RAFT-Agens & Volumenquellungsgrad \\
\hline konventionell & 5,2 \\
EPHT & 8,8 \\
BEPBT & 8,3 \\
\hline
\end{tabular}

Anhand dieser Werte zeigt sich, dass die Anzahl der freien Kettenenden in den Netzwerken nicht der alleinige Grund für die höheren Volumenquellungsgrade der RAFT-Netzwerke sein kann. Sollte dies der Fall sein, so müsste der Volumenquellungsgrad des BEPBT-Netzwerks, dem des konventionellen Netzwerks entsprechen. Wie in Tabelle 4-11 aber zu erkennen ist, entspricht der Volumenquellungsgrad dieses Netzwerks im Rahmen eines möglichen Fehlers aber dem des EPHT-Netzwerks und nicht dem des konventionellen Netzwerks. Darum muss noch ein weiterer Grund für die höheren Volumenquellungsgrade der RAFT-Netzwerke existieren.

In diesen Zusammenhang passt eine weitere Beobachtung, die während der Quellungsversuche gemacht werden konnte. Die Probekörper hatten zum Teil deutlich an Gewicht verloren, nachdem sie nach den beendeten Messungen wieder getrocknet wurden. Dies war umso stärker ausgeprägt, je geringer der molare BDDA-Anteil in den Netzwerken war. Diese zum Teil erheblichen Gewichtsverluste lassen sich auf die Existenz von so genannten Extrahierbaren zurückführen. Hierbei handelt es sich um Polymere der Sol-Fraktion, die nicht in das Netzwerk integriert wurden und daher mit Hilfe eines Lösungsmittels aus dem Netzwerk extrahiert werden können. In Tabelle 4-12 und Tabelle 4-13 sind für vier Poly(BA-co-BDDA)- und für vier Poly(EAco-BDDA)-Netzwerke die prozentualen Anteile dieser Extrahierbaren am Polymergewicht vor der Quellung zusammengefasst.

Tabelle 4-12 Prozentualer Gewichtsanteil der extrahierbaren Sol-Fraktion am Gewicht von Poly(BA-co-BDDA)Netzwerken mit 2 mol-\%, 1 mol-\%, 0,5 mol-\% und 0,25 mol-\% des Vernetzers BDDA aus UV-initiierter RAFTPolymerisation mit 2 mol-\%o des RAFT-Agens EPHT und UV-initiierter, konventioneller radikalischer Polymerisation.

\begin{tabular}{ccc}
\hline \multirow{2}{*}{ BDDA-Anteil [mol-\%] } & \multicolumn{2}{c}{ Anteil Extrahierbare [\%] } \\
& RAFT & konventionell \\
\hline 2 & 3,12 & 2,79 \\
1 & 4,48 & 3,08 \\
0,5 & 7,64 & 3,51 \\
0,25 & 40,02 & 6,85 \\
\hline
\end{tabular}


Tabelle 4-13 Prozentualer Gewichtsanteil der extrahierbaren Sol-Fraktion am Gewicht von Poly(EA-co-BDDA)Netzwerken mit 2 mol-\%, 1 mol-\%, 0,5 mol-\% und 0,25 mol-\% des Vernetzers BDDA aus UV-initiierter RAFTPolymerisation mit 2 mol-\%o des RAFT-Agens EPHT und UV-initiierter, konventioneller radikalischer Polymerisation.

\begin{tabular}{ccc}
\hline \multirow{2}{*}{ BDDA-Anteil [mol-\%] } & \multicolumn{2}{c}{ Anteil Extrahierbare [\%] } \\
RAFT & konventionell \\
\hline 2 & 7,44 & 6,05 \\
1 & 7,02 & 5,59 \\
0,5 & 11,37 & 5,29 \\
0,25 & 34,04 & 8,40 \\
\hline
\end{tabular}

Hierbei zeigt sich im Fall der RAFT-Netzwerke eine deutliche Zunahme der Extrahierbaren mit abnehmendem molaren BDDA-Anteil von 2 auf 0,25 mol-\%. Bei den Poly(BA-co-BDDA)Netzwerken steigt der Gewichtsanteil der Extrahierbaren am ungequollenen Netzwerk von rund $3 \%$ auf rund $40 \%$ an, bei den Poly(EA-co-BDDA)-Netzwerken von rund 7,5\% auf $34 \%$. Bei den konventionellen Netzwerken fällt diese Zunahme dagegen nur sehr gering aus. Sowohl bei den Poly(BA-co-BDDA)- als auch bei den Poly(EA-co-BDDA)-Netzwerken zeigt sich, dass sich die Differenz zwischen den Gewichtsanteilen der Extrahierbaren der RAFT-Netzwerke zu denen der konventionellen Netzwerke ab einem molaren BDDA-Anteil von unter 1 mol-\% im Netzwerk zu vergrößern beginnt. Dies ist völlig analog zu den Verläufen der Volumenquellungsgrade (s. Abbildung 4-25 und Abbildung 4-26). Der Grund hierfür sind Netzwerkdefekte wie Verhakungen und Verschlaufungen, sowie intramolekulare Ringe, die zu Nanogel-Domänen in den Netzwerken führen. Diese treten bei konventionellen Netzwerken, sowie bei den RAFTNetzwerken mit einem BDDA-Anteil von über 1 mol-\% wahrscheinlich deutlich häufiger auf (s. Kapitel 2.2.1 und Kapitel 4.1.1). Die sehr dichten Netzwerkstrukturen in den NanogelDomänen können die Auswaschung von der Extrahierbaren während der Quellung verhindern, sodass die weiterhin zum Quellungsgleichgewicht beitragen und die Volumenquellungsgrade somit geringer bleiben.

Anhand der Extrahierbaren können somit die unterschiedlichen Verläufe der Volumenquellungsgrade der RAFT- und der konventionellen Netzwerke erklärt werden. Bei einem Anstieg des Gewichtsanteils der Extrahierbaren steigt auch der Volumenquellungsgrad des entsprechenden Netzwerks überdurchschnittlich an. Dies ist eine Folge der geringeren effektiven Netzkettendichten, die sich aus der Existenz der Extrahierbaren ergeben.

Die effektive Netzkettendichte der Netzwerke ist nach der Flory-Rehner-Gleichung mit dem Quellungsgleichgewicht verbunden. Wird Gleichung (31) umgeformt ergibt sich die effektive Netzkettendichte nach: 


$$
v_{\mathrm{eff}}=\frac{-\ln \left(1-\Phi_{\mathrm{Pol}}\right)-\Phi_{\mathrm{Pol}}-\chi \Phi_{\mathrm{Pol}}^{2}}{V_{\mathrm{mol}(\mathrm{LM})} \cdot\left(\Phi_{\mathrm{Pol}^{3}} \frac{1}{3}-\frac{\Phi_{\mathrm{Pol}}}{2}\right)}
$$

Die effektive Netzkettendichte sinkt also mit einem abnehmenden Volumenbruch des Polymers. Da dieser wiederum nach Gleichung (35) der Kehrwert des Volumenquellungsgrades ist, bedeutet dies, dass ein hoher Volumenquellungsgrad eine niedrige effektive Netzkettendichte zur Folge hat. Allerdings darf in diesem Zusammenhang nur der Teil des Polymers betrachtet werden, der auch tatsächlich mit dem Netzwerk verbunden ist, da nur dieser an der Verformung des Netzwerks während der Quellung teilnimmt und somit einen Einfluss auf das Gleichgewicht hat, das den Volumenquellungsgrad festlegt. Darum ist es nicht überraschend, dass Netzwerke mit einem hohen Anteil an Extrahierbaren über einen hohen Volumenquellungsgrad und somit über eine niedrige Netzkettendichte verfügen, obwohl diese nach Gleichung (16) theoretisch nur von der Stoffmenge des Vernetzers abhängen sollte. Es ist so, dass wenige lange, effektiv an der Verformung beteiligte Netzketten leichter zu verformen sind als viele kurze, wodurch das Quellungsgleichgewicht zu höheren Quellungsgraden verschoben wird. Aufgrund größerer Maschenweiten kann mehr Lösungsmittel in das Netzwerk eindringen, weiterhin können die Ketten, da sie weniger Restriktionen anderer Ketten unterliegen, entsprechend während der Quellung weiter gedehnt werden.

Anhand der Volumenquellungsgrade konnte im Anschluss an die Quellungsexperimente über die Gleichungen (48) und (46) die scheinbaren Kettenlängen der Netzwerkketten berechnet werden. Um dies zu realisieren, muss allerdings der Flory-Huggins-Koeffizient bekannt sein, oder mit folgender Gleichung abgeschätzt werden: ${ }^{[178]}$

$$
\chi=0,34+\frac{V_{\mathrm{mol}(\mathrm{LM})}}{R T}\left(\delta_{\mathrm{Pol}}-\delta_{\mathrm{LM}}\right)
$$

Dabei ist $\delta_{\text {Pol }}$ der Löslichkeitsparameter des Polymers und $\delta_{\mathrm{LM}}$ der Löslichkeitsparameter des Lösungsmittels, bei dem es sich bei den durchgeführten Versuchen um Aceton handelte. Die Konstante 0,34 stellt einen "fudge factor" dar, der die Unterschiede des freien Volumens zwischen Polymer und Lösungsmittel berücksichtigt. ${ }^{[178]}$ Die Löslichkeitsparameter können aus, in der Literatur tabellierten, Werten direkt erhalten oder berechnet werden. Für die beteiligten Stoffe Poly-BA, -EA und Aceton sind sie zusammen mit dem Flory-Huggins Parameter des jeweiligen Polymernetzwerkes in Tabelle 4-14 zusammengefasst. 
Tabelle 4-14 Löslichkeitsparameter $\delta$ von Poly-BA, Poly-EA und Aceton, sowie die mittels Gleichung (49) errechneten Flory-Huggins-Parameter $\chi$ dieser Substanzen.

\begin{tabular}{ccc}
\hline Substanz & $\boldsymbol{\delta}\left[(\mathbf{M P a})^{\mathbf{1} / 2}\right]$ & $\chi$ \\
\hline Poly-BA & $18,21^{\mathrm{a}}$ & 0,29 \\
Poly-EA & $19,00^{\mathrm{b}}$ & 0,31 \\
Aceton & $19,94^{\mathrm{c}}$ & \\
\hline
\end{tabular}

Die somit für die Poly(BA-co-BDDA)- und Poly(EA-co-BDDA)-Netzwerke erhaltenen scheinbaren Molmassen der Netzketten $\bar{M}_{\mathrm{x}}$ sind in Abbildung 4-28 und Abbildung 4-29 doppeltlogarithmisch gegen den molaren Anteil des Vernetzers BDDA in den Netzwerken aufgetragen. Zum Vergleich wurden die theoretischen Molmassen der Netzketten $\bar{M}_{\mathrm{s}}$, die nach Gleichung (47) berechnet wurden, hinzugefügt.

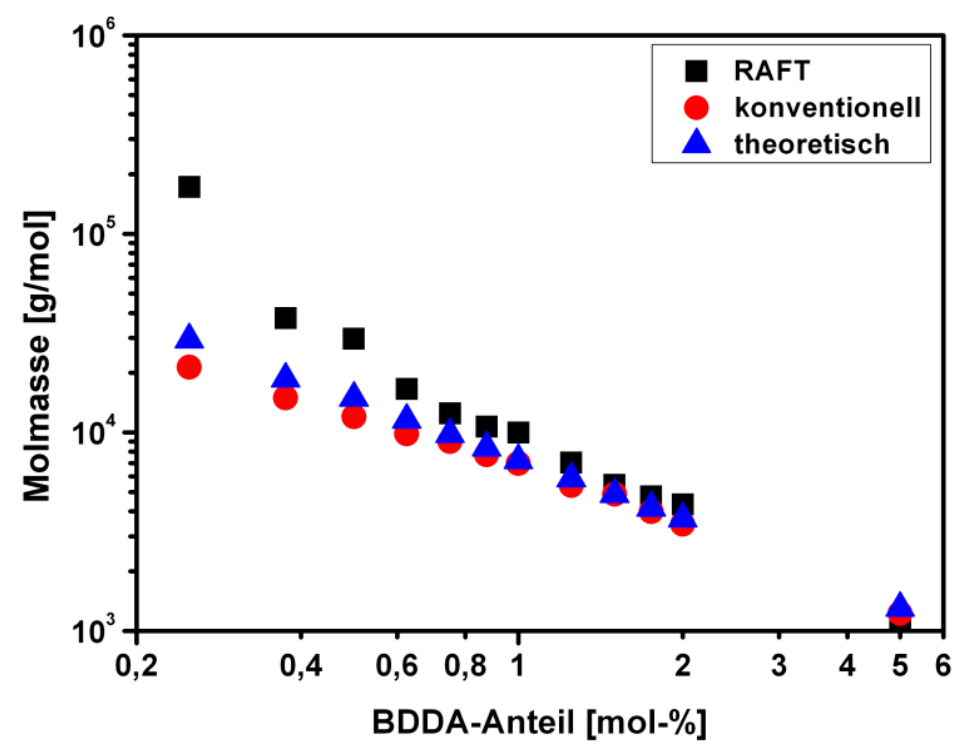

Abbildung 4-28 Doppelt-logarithmische Auftragung der scheinbaren Molmassen der Netzketten, berechnet aus den Gleichungen (48) und (46) von Poly(BA-co-BDDA)-Netzwerken aus UV-initiierter RAFT-Polymerisation mit 2 mol-\%o EPHT als RAFT-Agens (•) und UV-initiierter, konventioneller radikalischer Polymerisation (•) im Vergleich zu den theoretischen Molmassen ( $\mathbf{\wedge})$, berechnet aus Gleichung (47).

\footnotetext{
ach Tobing et al. ${ }^{[178]}$

${ }^{b}$ nach Barton ${ }^{[240]}$

${ }^{\mathrm{c}}$ berechnet nach Meichsner ${ }^{[241]}$
} 


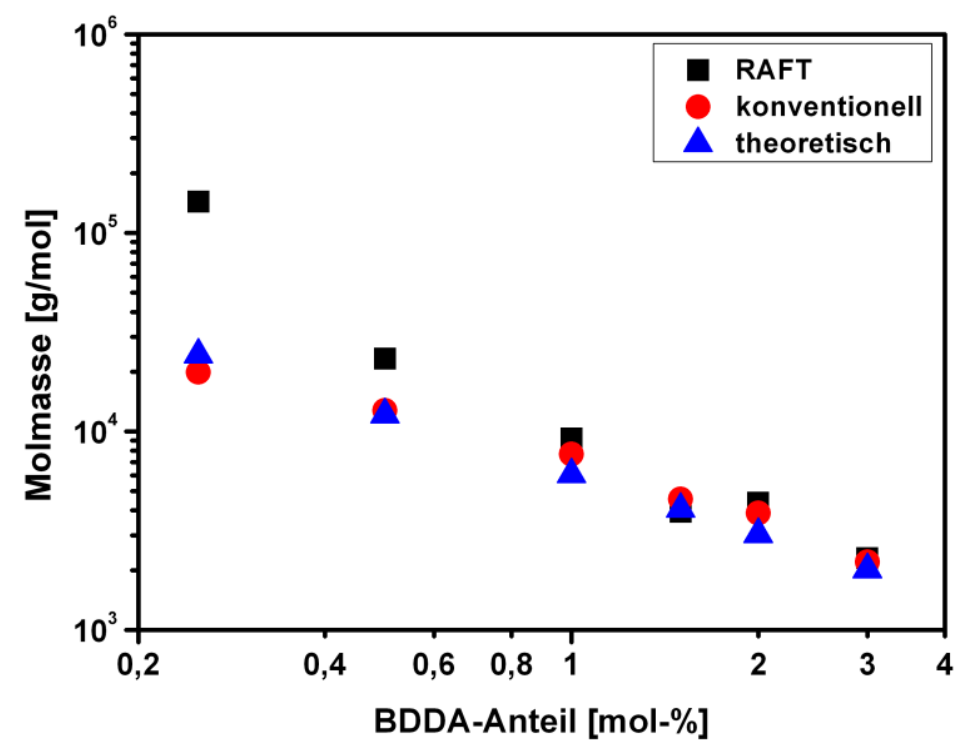

Abbildung 4-29 Doppelt-logarithmische Auftragung der scheinbaren Molmassen der Netzketten, berechnet aus den Gleichungen (48) und (46) von Poly(EA-co-BDDA)-Netzwerken aus UV-initiierter RAFT-Polymerisation mit 2 mol-\%o EPHT als RAFT-Agens (•) und UV-initiierter, konventioneller radikalischer Polymerisation (•) im Vergleich zu den theoretischen Molmassen (৯), berechnet aus Gleichung (47).

Es zeigt sich, dass die scheinbaren Molmassen, die nach dieser Methode bestimmt wurden, insgesamt höher liegen als die aus den Zugversuchen ermittelten Werte (s. Abbildung 4-16 und Abbildung 4-17), was dazu führt, dass in diesem Fall die Werte der konventionellen Poly(BA-coBDDA)- und Poly(EA-co-BDDA)-Netzwerke besser mit den theoretischen Molmassen übereinstimmen. Ein möglicher Grund hierfür könnte sein, dass sich die Extrahierbaren deutlich stärker auf den Volumenquellungsgrad auswirken als auf den E-Modul. Das bedeutet: Sind mehr Extrahierbare vorhanden, erscheinen die Molmassen der Netzketten aus den Quellungsexperimenten größer als aus den Zugversuchen. Dies geht möglicherweise darauf zurück, dass die Extrahierbaren bei den Zugversuchen noch in den Netzwerken enthalten sind. Somit können die durch physikalische Verhakungen zum gemessenen E-Modul beitragen, während sie bei der Quellung aus dem System entfernt werden und somit keinen Effekt auf die gemessenen Quellungsgrade haben können. Insgesamt lässt sich aber festhalten, dass durch Abbildung 4-28 und Abbildung 4-29, trotz leichter Unterschiede in den berechneten Molmassen aus Quellungsund Zugversuchen, die bekannten Tendenzen der scheinbaren Molmassen (s. Abbildung 4-16 und Abbildung 4-17) bestätigt werden. 


\title{
4.1.3.2 Variation des molaren EPHT-Anteils
}

Zusätzlich zu den vorangegangenen Untersuchungen des Quellungsverhaltens der Poly(BA-coBDDA)- und Poly(EA-co-BDDA)-Netzwerke in Abhängigkeit vom molaren Anteil des Vernetzers (s. Abschnitt 4.1.3.1) wurde die Abhängigkeit des Quellungsverhaltens vom molaren Anteil des RAFT-Agens EPHT in den Netzwerken untersucht.

Analog zu den Untersuchungen der mechanischen Eigenschaften der Netzwerke (s. Abschnitt 4.1.1.3) wurden für die hier durchgeführten Messungen wieder die Netzwerke mit einem molaren BDDA-Anteil von 0,5 mol-\% ausgewählt. Es wurden die Volumenquellungsgrade und der prozentuale Anteil der Extrahierbaren an der Masse des Polymers vor der Quellung ermittelt. Die erhaltenen Werte wurden für die Poly(BA-co-BDDA)-Netzwerke (s. Abbildung 4-30) und für die Poly(EA-co-BDDA)-Netzwerke (s. Abbildung 4-31) gegen den molaren Anteil des RAFT-Agens EPHT in den Netzwerken aufgetragen. Auf eine doppeltlogarithmische Auftragung wurde an dieser Stelle verzichtet, da diese die Werte der konventionellen Netzwerke nicht erfassen würde.

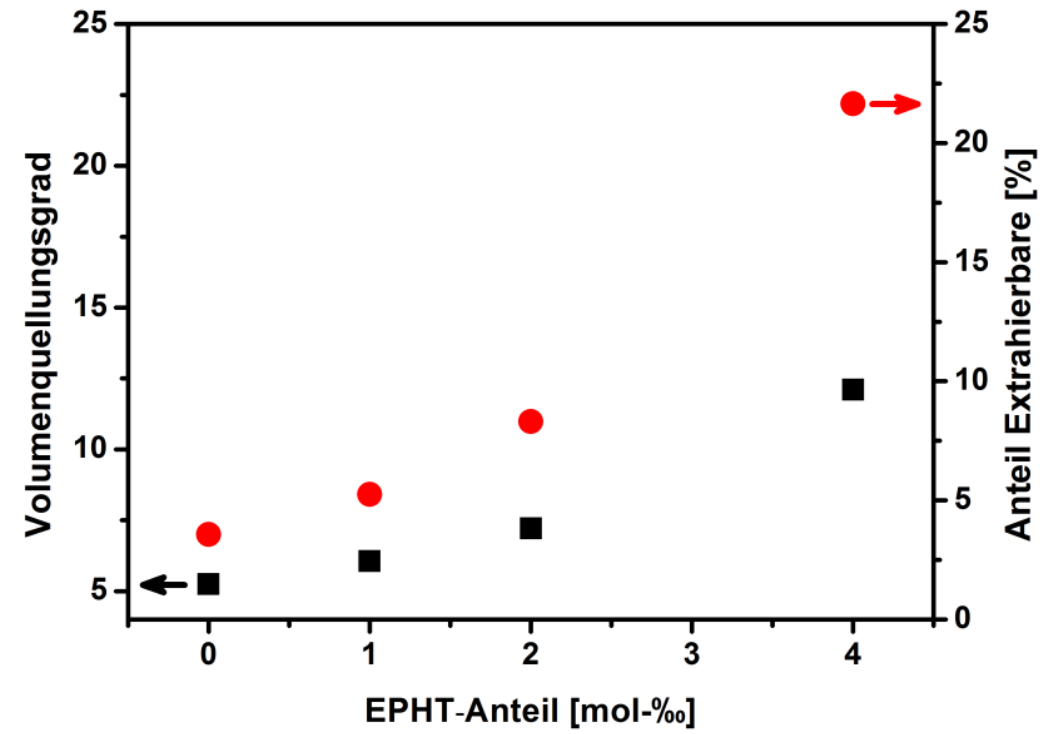

\begin{abstract}
Abbildung 4-30 Volumenquellungsgrad eines Poly(BA-co-BDDA)-Netzwerks mit 0,5 mol-\% des Vernetzers BDDA aus UV-initiierter, konventioneller radikalischer Polymerisation sowie von Poly(BA-co-BDDA)-Netzwerken mit 0,5 mol-\% des Vernetzers BDDA aus RAFT-Polymerisation mit 1 mol-\%o, 2 mol-\%o und 4 mol-\%o des RAFT-Agens EPHT (घ). Ergänzend: der korrespondierende Gewichtsanteil der Extrahierbaren im Netzwerk vor der Quellung (•). Die Quellungsexperimente wurden mit Aceton als Quellungsmittel bei Raumtemperatur durchgeführt.
\end{abstract}

Der Volumenquellungsgrad der Poly(BA-co-BDDA)-Netzwerke steigt von 5,6 beim konventionellen Netzwerk bis auf 12,1 beim RAFT-Netzwerk mit einem molaren Anteil von 4 mol-\% EPHT. Trotz des gleichen molaren BDDA-Anteils verdoppelt sich der 
Volumenquellungsgrad nur durch die Zugabe des RAFT-Agens. Der Gewichtsanteil der Extrahierbaren nimmt analog dazu von 3,5\% beim konventionellen auf bis $21,7 \%$ beim RAFTNetzwerk mit einem molaren Anteil von 4 mol-\%o EPHT zu. Der Gewichtsanteil der Extrahierbaren in den Netzwerken steigt also fast um das Siebenfache an, was im Verhältnis deutlich stärker ist, als der Anstieg des Volumenquellungsgrads.

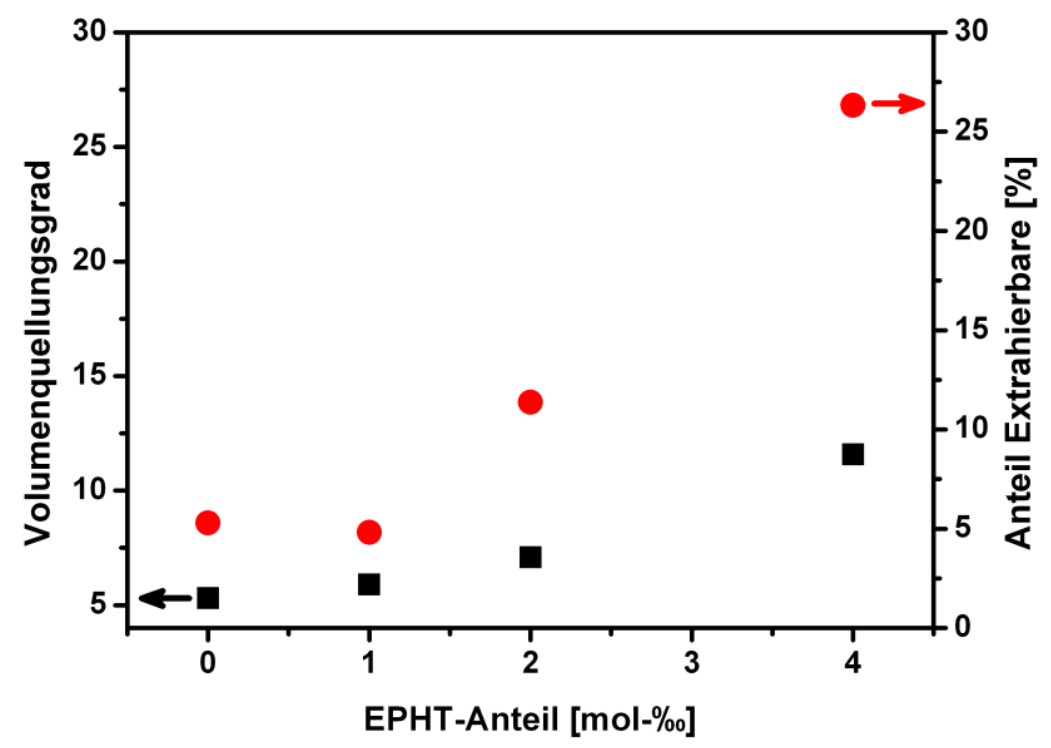

\begin{abstract}
Abbildung 4-31 Volumenquellungsgrad eines Poly(BA-co-BDDA)-Netzwerks mit 0,5 mol-\% des Vernetzers BDDA aus UV-initiierter, konventioneller radikalischer Polymerisation sowie von Poly(EA-co-BDDA)-Netzwerken mit 0,5 mol-\% des Vernetzers BDDA aus RAFT-Polymerisation mit 1 mol-\%, 2 mol-\%o und 4 mol-\%o des RAFT-Agens EPHT (๘). Ergänzend: der korrespondierende Gewichtsanteil der Extrahierbaren im Netzwerk vor der Quellung (•). Die Quellungsexperimente wurden mit Aceton als Quellungsmittel bei Raumtemperatur durchgeführt.
\end{abstract}

Bei den Poly(EA-co-BDDA)-Netzwerken steigt der Volumenquellungsgrad von 5,3 beim konventionellen bis 11,6 beim RAFT-Netzwerk mit einem molaren Anteil von 4 mol-\%o EPHT, zwar nicht ganz so deutlich, aber doch nahezu um das Doppelte an. Die Extrahierbaren steigen von $5,3 \%$ bis zu 26,3\% um etwa das Fünffache, was im Rahmen möglicher Wägefehler dem Anstieg der Poly(BA-co-BDDA)-Netzwerke entspricht. Es finden sich, bei beiden Netzwerkarten, die schon im vorherigen Abschnitt gefundenen, Abhängigkeiten des Volumenquellungsgrades und des Massenanteils der Extrahierbaren vom molaren Anteil des RAFT-Agens EPHT.

Wieder können die steigenden Volumenquellungsgrade der Netzwerke auf den zunehmenden Gewichtsanteil der Extrahierbaren und die damit einhergehende, größere Anzahl von freien Kettenenden in den Netzwerken zurückgeführt werden. Die Gründe für den zunehmenden Gewichtsanteil der Extrahierbaren lassen sich anhand dieser Daten sehr gut erklären. Nach Gleichung (12) sinkt die Molmasse eines Polymers aus RAFT-Polymerisation mit steigender 
Konzentration des RAFT-Agens. Bezogen auf eine statistische Vernetzungsreaktion bedeutet dies, dass die Moleküle der Sol-Fraktion zunehmend kleiner werden müssen. Kleinere SolMoleküle haben dementsprechend weniger Vernetzer-Moleküle eingebaut und verfügen somit über weniger seitliche Doppelbindungen. Dies reduziert die Wahrscheinlichkeit, dass das SolMolekül mit in das Netzwerk eingebaut wird. Außerdem besteht auch die Möglichkeit, dass die seitlichen Doppelbindungen so weit voneinander entfernt sind, dass sie sich nicht mehr miteinander verknüpfen können. Dies kann sogar soweit führen, dass die Sol-Moleküle zu klein sind und eine Vernetzung gar nicht mehr stattfinden kann. Diese Beobachtungen konnten z. B. bei der ATRP schon mehrfach gemacht werden. ${ }^{[196,197]}$ Da es sich bei dieser, wie auch bei der RAFT-Polymerisation, um eine RDRP handelt und die Netzwerkbildung somit analog verläuft, können diese Ergebnisse auf die RAFT-Polymerisation übertragen werden.

Mittels Gleichung (46) wurden wieder die mittleren, scheinbaren Molmassen der Netzketten bestimmt. Die dazu benötigten effektiven Netzkettendichten wurden über die Flory-RehnerGleichung berechnet. Die somit erhaltenen Werte sind in Tabelle 4-15 zusammengefasst. Zum Vergleich wurden wieder die theoretischen Molmassen nach Gleichung (47) hinzugefügt.

Tabelle 4-15 Scheinbare Molmassen $\bar{M}_{\mathrm{x}}$, berechnet aus den Gleichungen (48) und (46) und theoretische $\bar{M}_{\mathrm{S}}$ Molmassen der Netzketten, berechnet aus Gleichung (47), von Poly(BA-co-BDDA)- und Poly(EA-co-BDDA)-Netzwerken mit 0,5 mol-\% des Vernetzers BDDA aus UV-initiierter, konventioneller radikalischer Polymerisation sowie aus UV-initiierter RAFT-Polymerisation mit 1 mol-\%o, 2 mol-\%o und 4 mol-\%o des RAFT-Agens EPHT.

\begin{tabular}{ccccc}
\hline EPHT-Anteil & \multicolumn{2}{c}{ Poly(BA-co-BDDA) } & \multicolumn{2}{c}{ Poly(EA-co-BDDA) } \\
[mol-\%o $]$ & $\overline{\boldsymbol{M}}_{\mathbf{x}}$ [g/mol] & $\overline{\boldsymbol{M}}_{\mathbf{S}}$ [g/mol] & $\overline{\boldsymbol{M}}_{\mathbf{x}}$ [g/mol] & $\overline{\boldsymbol{M}}_{\mathbf{s}}$ [g/mol] \\
\hline 0 & 10450 & 25634 & 12750 & 20024 \\
1 & 14130 & 25634 & 14280 & 20024 \\
2 & 20040 & 25634 & 23230 & 20024 \\
4 & 54300 & 25634 & 32590 & 20024 \\
\hline
\end{tabular}

Die scheinbaren Molmassen steigen mit dem molaren Anteil des RAFT-Agens EPHT in den Netzwerken deutlich an. Sowohl bei den Poly(BA-co-BDDA)- als auch bei den Poly(EA-coBDDA)-Netzwerken befinden sich die scheinbaren Molmassen der konventionellen Netzwerke deutlich unter den theoretischen Werten. Die RAFT-Netzwerke mit einem molaren EPHT-Anteil von 4 mol-\%o dagegen besitzen scheinbare Molmassen, die deutlich über den theoretischen Werten liegen, was den Ergebnissen aus den Zugversuchen (s. Abschnitt 4.1.1.3) entspricht. Dies ist eine Folge des steigenden Anteils von Extrahierbaren in den Netzwerken, da diese, wie oben erwähnt, zu freien Kettenenden in den Netzwerken führen, die nicht effektiv zum Quellungsgleichgewicht beitragen und somit bei der Berechnung der effektiven Netzkettendichte 
über die Flory-Rehner-Gleichung nicht erfasst werden. Die deutlich unter der theoretischen Molmasse liegenden Werte der Netzwerke mit einem molaren Anteil von 1 mol-\%o EPHT zeigen, dass die Konzentration des RAFT-Agens noch zu gering war, um für eine ausreichende Verringerung der seitlichen Doppelbindungen in den Sol-Molekülen zu sorgen. Dies lässt sich daran belegen, dass der Gewichtsanteil der Extrahierbaren bei beiden Netzwerkarten $5 \%$ beträgt. Außerdem besteht die Möglichkeit, dass die geringe Konzentration des RAFT-Agens die Polymerisation nicht ausreichend kontrollieren konnte und trotz seiner Anwesenheit in der Polymerisationsmischung immer noch eine große Menge Nanogel-Domänen gebildet wurden. Dadurch existiert wieder eine Vielzahl von Verhakungen und Verschlaufungen im System. Diese würden, die durch das RAFT-Agens hervorgerufenen, freien Kettenenden kompensieren, was die effektive Netzkettendichte erhöhen würde. Es fällt auf, dass die in Tabelle 4-15 erkennbaren Verläufe der scheinbaren Molmassen sehr stark an die schon aus Abschnitt 4.1.1.3 bekannten Verläufe der scheinbaren Molmassen aus den Zugversuchen erinnern (s. Tabelle 4-6 und Tabelle 4-7). Da sowohl das Quellungsverhalten als auch die mechanischen Eigenschaften der Netzwerke sehr stark von deren mikroskopischer Struktur und Aufbau und vor allem ihrer effektiven Netzkettendichte abhängen (s. Kapitel 2.2.1), konnte dieses Ergebnis so erwartet werden. 


\subsubsection{Untersuchung der Netzwerkstrukturen mittels Kernresonanz-Spektroskopie}

Ergänzend zu den gravimetrischen Quellungsuntersuchungen existiert die Möglichkeit, die Quellung von Polymernetzwerken mittels Kernresonanz- (gebräuchlicher ist die englische Bezeichnung: Nuclear Magnetic Resonance, kurz: NMR)-Spektroskopie zu verfolgen. ${ }^{[198]}$ Umfangreiche Studien dazu wurden von Ann-Christin Poeppler an thermisch synthetisierten, konventionellen Polystyrol-co-1,6-Divinylbenzol(Poly(ST-co-DVB))-Netzwerken am Institut für Anorganische Chemie der Georg-August-Universität Göttingen in der Arbeitsgruppe von Prof. Stalke durchgeführt. ${ }^{[199]}$

In einem gemeinsamen Kooperationsprojekt wurden die in der vorliegenden Arbeit studierten Poly(BA-co-BDDA)- und Poly(EA-co-BDDA)-Netzwerke von Ann-Christin Poeppler ebenfalls mittels NMR-Spektroskopie untersucht. Dabei bieten vor allem die Poly(BA-co-BDDA)Netzwerke ein großes Potential für die NMR-Analytik in anisotroper Umgebung. ${ }^{[200]}$ Sie quellen hervorragend in vielen verschiedenen Lösungsmitteln und sind darum sehr variabel einsetzbar. Weiterhin erreichen sie sehr schnell ihr Quellungsgleichgewicht und sind aufgrund ihrer sehr niedrigen Glastemperatur auch für Tieftemperaturmessungen bis $-90{ }^{\circ} \mathrm{C}$ zu verwenden. Auf der anderen Seite bietet die Untersuchung der Netzwerke mittels NMR für die Polymerforschung die Möglichkeit, die Homogenität von Netzwerken mit geringer Fehleranfälligkeit zu bestimmen. Außerdem besteht bei der NMR-Spektroskopie das Potential, die Messungen mit bildgebenden Verfahren ${ }^{[201]} \mathrm{zu}$ kombinieren und somit den Einblick in die Netzwerkstruktur noch weiter zu optimieren.

Im Gegensatz zu der in der Polymeranalytik üblichen ${ }^{1} \mathrm{H}$-NMR-Spektroskopie wurde bei diesen Messungen auf das ${ }^{2} \mathrm{H}$-Signal eines deuterierten Lösungsmittels zurückgegriffen. Dazu wurden speziell synthetisierte Netzwerk-Zylinder (s. Kapitel 6.2.3) in einem NMR-Röhrchen (ID: $4 \mathrm{~mm}$ ) mit deuteriertem Aceton unter- und überschichtet (s. Abbildung 4-34), sodass die Netzwerke im Wesentlichen nur entlang der Längsachse des NMR-Röhrchens aufquellen konnten. Aufgrund dieser einachsigen Quellung findet die Streckung der Netzketten nur entlang dieser Achse statt, wodurch die Räume zwischen den Netzketten (Kavitäten) ebenfalls nur entlang dieser Achse gestreckt werden (s. Abbildung 4-32). In den gestreckten Kavitäten befinden sich die Aceton- $d_{6}$ Moleküle in einer anisotropen Umgebung. Dies hat einen deutlichen Effekt auf das Messsignal, welcher im Folgenden kurz erläutert wird. 


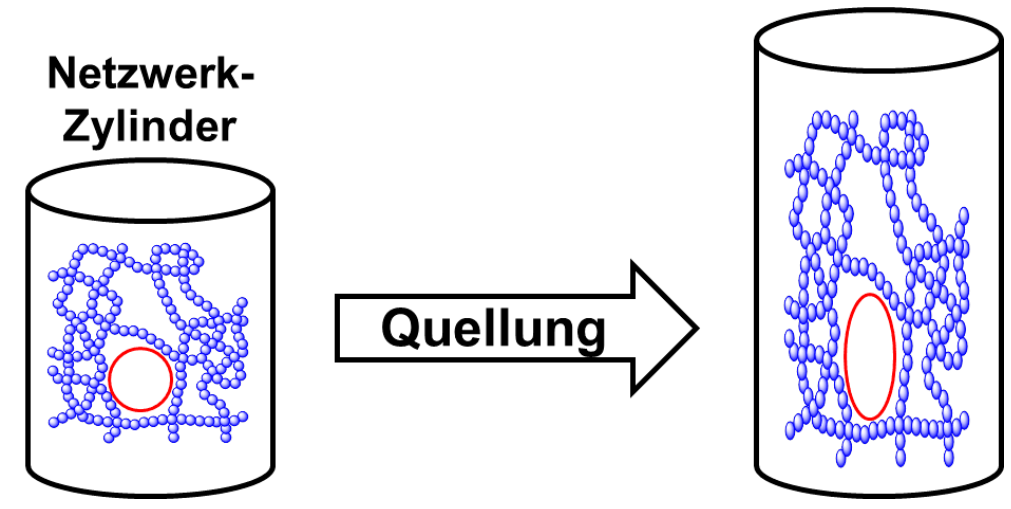

Abbildung 4-32 Schematische Darstellung einer einachsigen Quellung eines Polymernetzwerk-Zylinders.

Die Moleküle des verwendeten Lösungsmittels Aceton- $d_{6}$ zeigen in einer isotropen Umgebung ein Singulett im ${ }^{2} \mathrm{H}-\mathrm{NMR}$ Spektrum, da sie sich frei im Raum bewegen können. In einer anisotropen Umgebung dagegen, ist ihre freie Beweglichkeit eingeschränkt und es kommt $\mathrm{zu}$ einer leichten Vorzugsausrichtung. Wechselwirkungen, die in isotroper Lösung zu null gemittelt werden, können dann als sogenannte Restkopplungen beobachtet werden. Es kommt z. B. für Deuterium (Kernspin: $I=1) \quad \mathrm{zu}$ einer quadrupolaren Restaufspaltung des NMR-Signals in ein Dublett (s. Abbildung 4-33). Eine solche anisotrope Umgebung stellt z. B. ein Polymernetzwerk dar. Die Moleküle befinden sich dabei in den Kavitäten, die durch die Netzwerkketten aufgespannt werden. Deren Größe wird von den Molmassen der Netzketten bestimmt. Je nachdem wie groß die Kavitäten des Netzwerks sind, ist diese Aufspaltung größer (hohe Netzkettendichte) oder kleiner (niedrige Netzkettendichte). Es

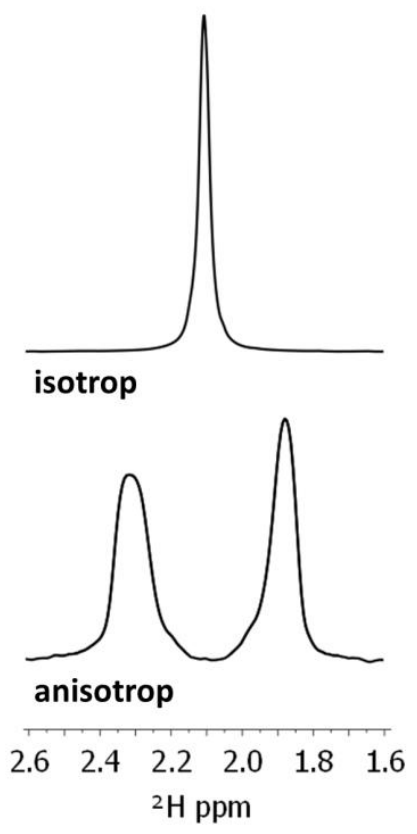

Abbildung 4-33 Aufspaltung des ${ }^{2} \mathrm{H}-$ NMR-Signals von Aceton- $d_{6}$ in einer anisotropen Umgebung. ist also möglich, mittels der NMR-Spektroskopie zu erkennen, ob Polymernetzwerke über eine hohe oder niedrige Netzkettendichte verfügen. Zusätzlich bietet sie sogar die Möglichkeit, die Verteilung dieser Netzkettendichten über das Netzwerk zu ermitteln. Um dies sichtbar zu machen, muss eine besondere Messmethode verwendet werden: Die so genannte schicht-selektive Anregungs- (oder engl.: slice-selective exitation, kurz: SSE)Technik. ${ }^{[202,203]}$ Bei dieser Technik wird die Polymerprobe in insgesamt 13 Schichten aufgeteilt, die sich in einem Abstand von $2 \mathrm{~mm}$ befinden. Die Dicke dieser Schichten beträgt 0,55 mm. Innerhalb dieser Schichten wird das ${ }^{2}$ H-NMR-Signal des Lösungsmittels aufgenommen. Es wird für jede Schicht ein Spektrum erhalten, die anschließend übereinander gelegt werden (s. Abbildung 4-34), wodurch ein Überblick über die gesamte Probe erhalten wird. 


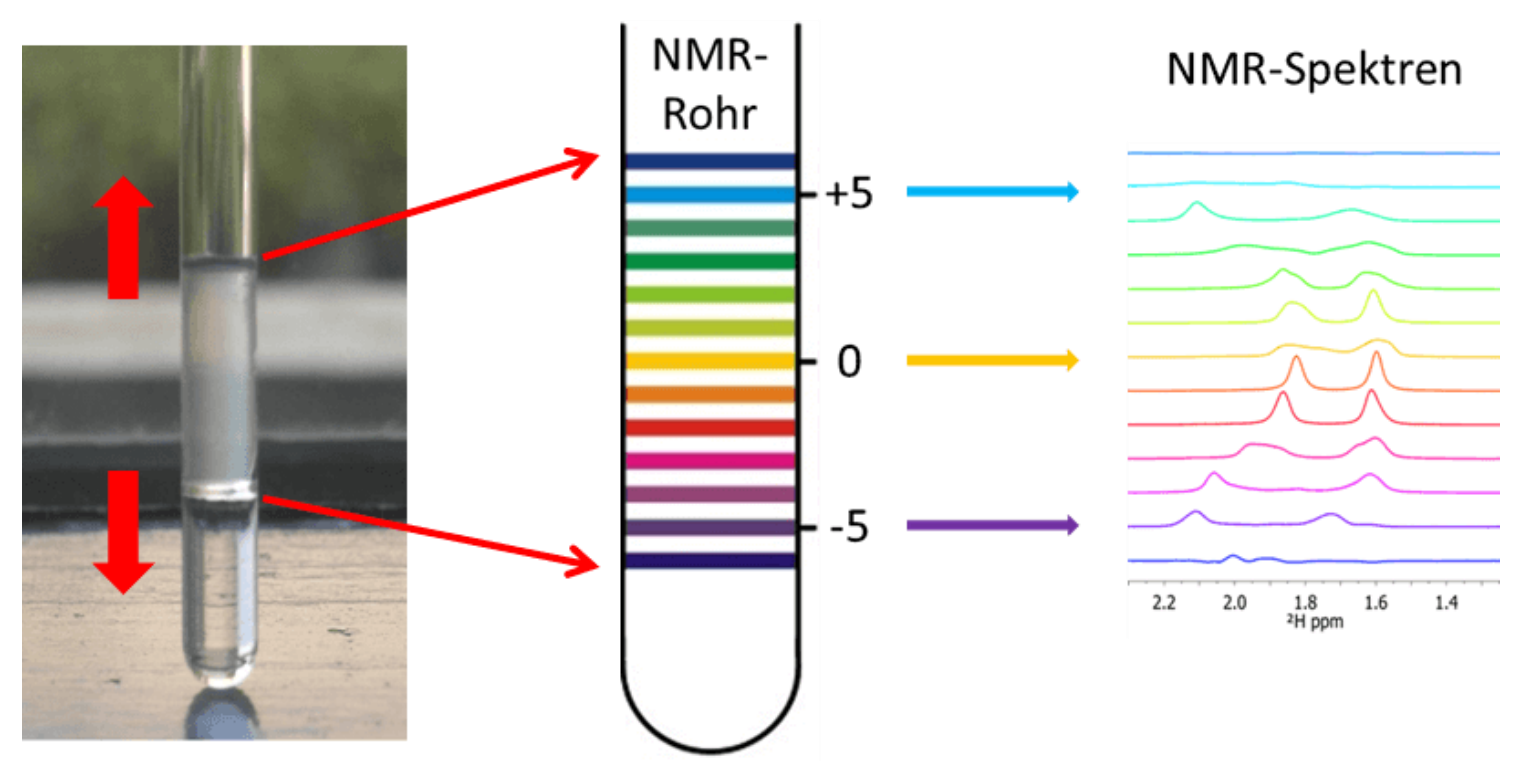

Abbildung 4-34 Foto eines Poly(ST-co-DVB)-Netzwerk-Zylinders in einem NMR-Röhrchen unter- und überschichtet mit einem Lösungsmittel und eine schematische Darstellung der Schichten, in die der Polymer-Zylinder bei der SSETechnik unterteilt wird sowie beispielhafte NMR-Spektren für die einzelnen Schichten. Nach Poeppler ${ }^{[199]}$.

Es wurden Poly(BA-co-BDDA)- und Poly(EA-co-BDDA)-Netzwerke mit jeweils unterschiedlichen molaren Anteilen des Vernetzers BDDA und des RAFT-Agens EPHT untersucht. Diese sind in Tabelle 4-16 zu finden. Die Synthesemethode zur Herstellung der Netzwerk-Probekörper wird in Kapitel 6.2.3 ausführlich erläutert.

Tabelle 4-16 Molare Anteile des Vernetzers BDDA und des RAFT-Agens EPHT an den per SSE-NMR-Technik untersuchten Poly(BA-co-BDDA)- und Poly(EA-co-BDDA)-Netzwerken. Die genauen Zusammensetzungen der Netzwerke sind in Kapitel 6.2.4 zu finden.

\begin{tabular}{cccc}
\hline \multicolumn{2}{c}{ Poly(BA-co-BDDA) } & \multicolumn{2}{c}{ Poly(EA-co-BDDA) } \\
BDDA [mol-\%] & EPHT [mol-\%o] & BDDA [mol-\%] & EPHT [mol-\%] \\
\hline 0,5 & 0 & 0,5 & 0 \\
0,5 & 1 & 0,5 & 1 \\
0,5 & 2 & & \\
1 & 0 & & \\
1 & 2 & & \\
\hline
\end{tabular}

Abbildung 4-35 zeigt die ${ }^{2}$ H-NMR-Spektren von Poly(BA-co-BDDA)-Netzwerken mit einem konstanten, molaren BDDA-Anteil von 0,5 mol-\%. Variiert wurde bei diesen Netzwerken der molare EPHT-Anteil in den Netzwerken von 0 bis 2 mol-\%. Netzwerke mit höherem molarem EPHT-Anteil konnten nicht vermessen werden, da sie zu weich und zu klebrig waren, sodass es unmöglich war, sie in den NMR-Röhrchen zu platzieren. 


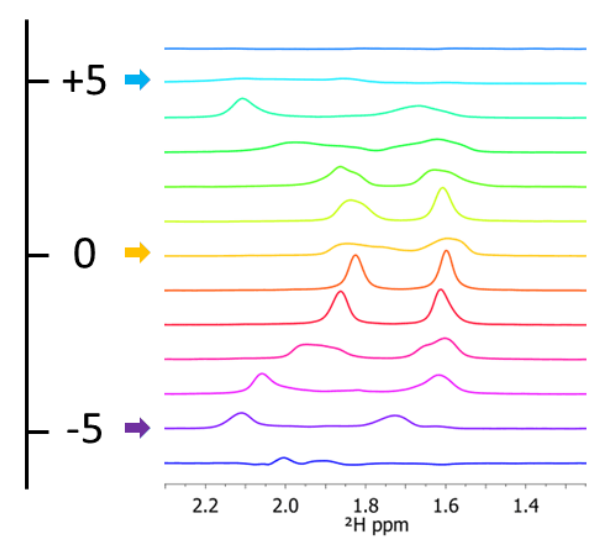

Schicht

$$
0 \text { mol-\%o EPHT }
$$

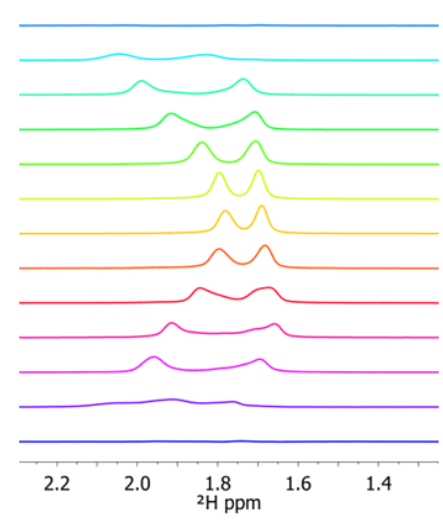

$1 \mathrm{~mol}-\%$ o EPHT

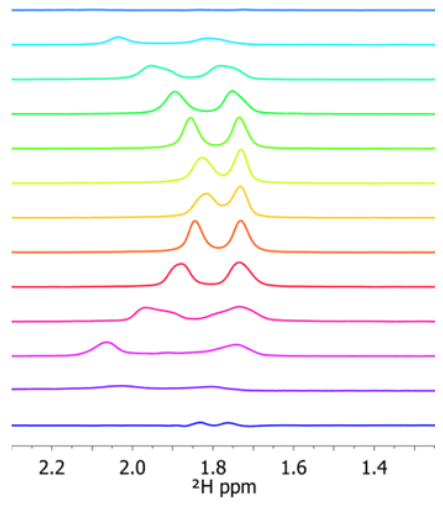

2 mol-\%o EPHT

Abbildung 4-35 Schicht-selektive ${ }^{2} \mathrm{H}-\mathrm{NMR}-$ Spektren von Aceton- $d_{6}$ in Poly(BA-co-BDDA)-Netzwerken mit einem molaren Anteil von 0,5 mol-\% des Vernetzers BDDA aus UV-initiierter, konventioneller radikalischer Polymerisation sowie aus RAFT-Polymerisation mit 1 mol-\%o und 2 mol-\%o des RAFT-Agens EPHT. Die Messung erfolgte 24 Stunden nachdem die Proben angesetzt wurden. Nach Poeppler ${ }^{[199]}$.

Mit der Zugabe des RAFT-Agens EPHT zu den Polymerisationsmischungen der Netzwerke zeigt sich eine geringere Aufspaltung des Aceton- $d_{6}{ }^{2} \mathrm{H}-\mathrm{NMR}$-Signals. Dies geht auf die schon zuvor beschriebene geringere Netzkettendichte der RAFT-Netzwerke zurück, die zu einer geringeren Restriktion der Lösungsmittelmoleküle führt. Weiterhin ist die Abweichung der Aufspaltungen untereinander in den verschiedenen Schichten bei den RAFT-Netzwerken geringer als beim konventionellen Netzwerk. Das bedeutet, die Kavitäten der RAFT-Netzwerke sind homogener über das Netzwerk verteilt. Allerdings muss auch festgestellt werden, dass die weitere Erhöhung des molaren EPHT-Anteils zu keiner weiteren signifikanten Veränderung der Signalaufspaltung oder der Abweichung führt. Weiterhin wurden bei Messungen von Poly(BA-co-BDDA)Netzwerken aus RAFT-Polymerisation (0,5 mol-\% BDDA; 2 mol-\%o bei denen die Extrahierbaren vor dem Platzieren der Polymer-Zylinder in den NMR-Röhrchen entfernt wurden, festgestellt, dass diese keinen Einfluss auf die Messwerte haben.

In weiteren Experimenten wurden anschließend die gleichen Messungen wie zuvor mit Poly(BAco-BDDA)-Netzwerken mit einem molaren Anteil von 1 mol-\% BDDA durchgeführt. Dabei wurden wieder ein konventionelles und ein RAFT-Netzwerk mit einem molaren EPHT-Anteil vom 2 mol-\%o synthetisiert und per NMR vermessen. Die resultierenden Spektren sind in Abbildung 4-36 zu finden. 


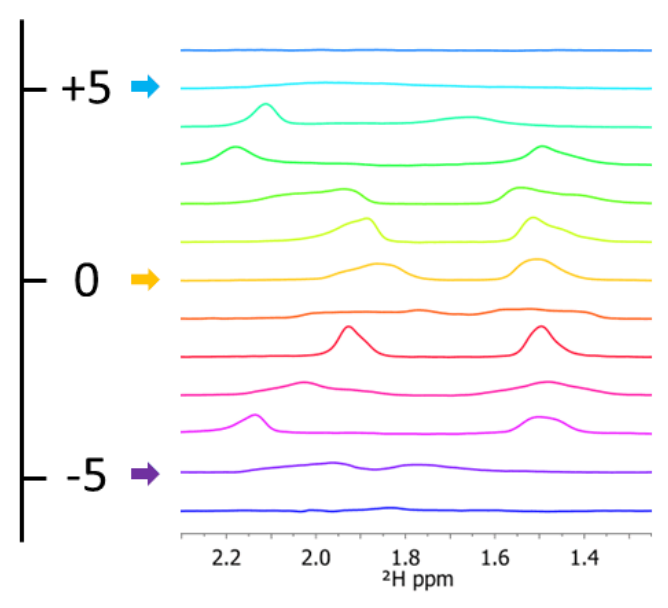

Schicht

\section{0 mol-\%o EPHT}

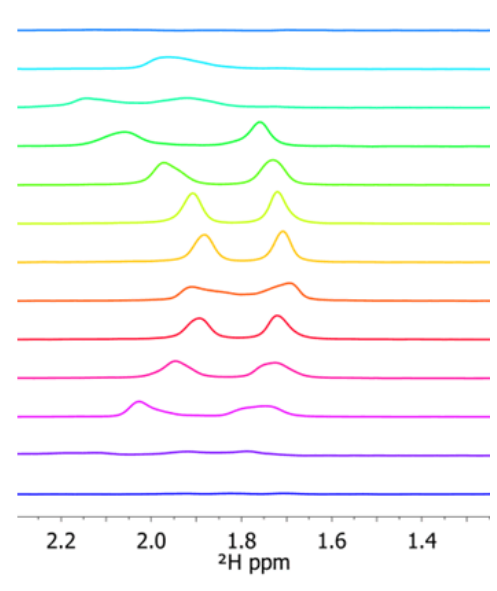

2 mol-\%o EPHT

\begin{abstract}
Abbildung 4-36 Schicht-selektive ${ }^{2}$ H-NMR-Spektren von Aceton- $d_{6}$ in Poly(BA-co-BDDA)-Netzwerken mit einem molaren Anteil von 1 mol-\% des Vernetzers BDDA aus UV-initiierter, konventioneller radikalischer Polymerisation sowie aus RAFT-Polymerisation 2 mol-\%o des RAFT-Agens EPHT. Die Messung erfolgte 24 Stunden nachdem die Proben angesetzt wurden. Nach Poeppler ${ }^{[199]}$.
\end{abstract}

Wieder ist eine deutlich geringere Aufspaltung und eine geringere Abweichung der Signale der unterschiedlichen Schichten zu beobachten. An diesen Messungen ist dieses Verhalten sogar noch deutlicher zu erkennen als bei den vorherigen. Auch lässt sich hier wieder der Einfluss des Vernetzers auf die Netzkettendichte und somit auf die Größe der Kavitäten erkennen. Die Aufspaltung dieser Netzwerke ist in beiden Fällen klar größer als bei den entsprechenden Netzwerken mit einem molaren BDDA-Anteil von 0,5 mol-\%.

Eine detaillierte Auswertung der vorliegenden Daten führte zu den folgenden Ergebnissen: ${ }^{[199]}$

- Die Konzentration des Lösungsmittels in den Netzwerken ist unabhängig davon, ob ein RAFT- oder konventionelles Netzwerk vorlag.

- Die RAFT-Netzwerke erreichen das Quellungsgleichgewicht etwas schneller.

- Der Betrag der Signalaufspaltung ist bei den RAFT-Netzwerken ungefähr halb so groß wie bei den konventionellen und über den gesamten Messbereich gleichmäßiger verteilt.

- Die Linienbreiten der Signale bei den RAFT-Netzwerken sind einheitlicher als bei den konventionellen.

Vor allem die letzten zwei Punkte lassen sich auf eine homogenere Netzwerkstruktur der RAFTNetzwerke zurückführen. Der zweite und der dritte Punkt belegen die geringeren Restriktionen der Netzketten in den RAFT-Netzwerken (schnellere Quellung) und die niedrigeren 
Netzkettendichten (geringere Aufspaltung). Somit bestätigen die NMR-Messungen alle in den vorherigen Kapiteln gemachten Ergebnisse und Vermutungen.

Die zusätzlich zu den Poly(BA-co-BDDA)-Netzwerken vermessenen Poly(EA-co-BDDA)Netzwerke zeigen den gleichen Einfluss des RAFT-Agens auf die Aufspaltung und die Linienbreiten der NMR-Signale, was wiederum bedeutet, dass die RAFT-Netzwerke über eine homogenere aber nicht so dichte Struktur verfügen wie die konventionellen Netzwerke. Außerdem zeigten die Messungen, dass bei gleichem molaren BDDA-Anteil in den Netzwerken die Poly(EA-co-BDDA)-Netzwerke über eine höhere Netzkettendichte als die Poly(BA-co-BDDA)Netzwerke verfügen. Erkennen lässt sich dies an der größeren Aufspaltung des Messsignals (s. Abbildung 4-37). Somit belegen diese Messungen, dass die in den vorherigen Kapiteln ermittelten höheren E-Moduln und niedrigeren Quellungsgrade dieser Netzwerke ganz eindeutig auf eine dichtere Struktur (höhere Netzkettendichte) zurückzuführen sind.

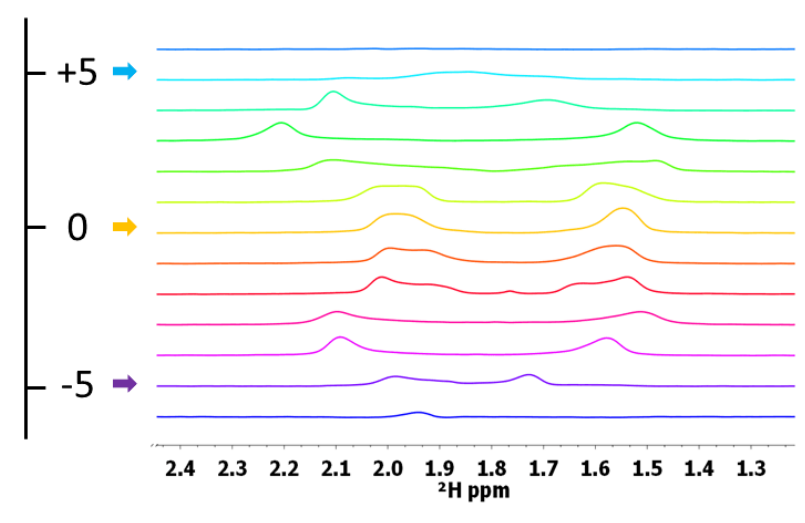

Schicht
0 mol-\%o EPHT

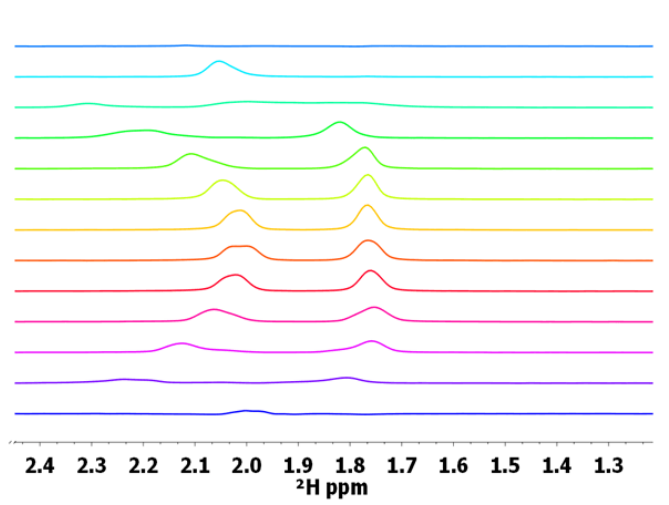

$1 \mathrm{~mol}$ \%

Abbildung 4-37 Schicht-selektive ${ }^{2}$ H-NMR-Spektren von Aceton- $d_{6}$ in Poly(BA-co-BDDA)-Netzwerken mit einem molaren Anteil von 0,5 mol-\% des Vernetzers BDDA aus UV-initiierter, konventioneller radikalischer Polymerisation sowie aus RAFT-Polymerisation mit 1 mol-\%o des RAFT-Agens EPHT. Die Messung erfolgte 24 Stunden nachdem die Proben angesetzt wurden.

\subsubsection{Abschließende Bemerkungen}

In diesem Kapitel konnte gezeigt werden, dass die Zugabe eines RAFT-Agens in die Polymerisationsmischungen von statistischen Poly(BA-co-BDDA)- und Poly(EA-co-BDDA)Netzwerken dazu führt, dass die resultierenden Netzwerke über einen höheren Volumenquellungsgrad verfügen als konventionelle Netzwerke mit dem gleichen molaren Anteil eines Vernetzers. Weiterhin ist der Gewichtsanteil einer extrahierbaren Sol-Fraktion unter einem molaren BDDA-Anteil von 1 mol-\% in den RAFT-Netzwerken viel größer als in konventionellen Netzwerken, was wahrscheinlich der Grund für die unterschiedlichen Quellungseigenschaften 
ist. Die gleichen Effekte (Zunahme des Volumenquellungsgrades und der Extrahierbaren) zeigen sich bei einer Erhöhung des molaren Anteils des RAFT-Agens EPHT.

Ergänzend wurde mittels NMR-Messungen unter Verwendung der SSE-Technik gezeigt, dass die RAFT-Netzwerke über eine eindeutig homogenere Struktur verfügen als die konventionellen Netzwerke. Außerdem konnten die zuvor in Zug- und Quellungsversuchen ermittelten geringeren Netzkettendichten der RAFT-Netzwerke mit diesen Messungen bestätigt werden. 


\subsubsection{Einfluss der RAFT-Polymerisation auf die Kinetik der Reaktion}

\subsubsection{Variation des molaren BDDA-Anteils}

Die kinetischen Messungen erfolgten für die Poly(BA-co-BDDA)-Netzwerke mittels isothermer DSC. Dabei wird die Temperatur in der Messkammer konstant gehalten und Reaktionswärme der Polymerisation aufgezeichnet. Diese Messungen sind in der Lage, einen wesentlichen Beitrag zur Aufklärung der, mit steigendem molaren Anteil des Vernetzers BDDA, ähnlicher werdenden mechanischen Eigenschaften der konventionellen und der RAFT-Netzwerke zu leisten. Gemessen wurden die Reaktionsverläufe von Polymerisationsmischungen für RAFT-Netzwerke mit einem molaren EPHT-Anteil von 2 mol-\%o (bezogen auf die Stoffmenge des Monomers). Die molaren Anteile des Vernetzers BDDA wurden von 0,5 bis 5 mol-\% (bezogen auf die Stoffmenge des Monomers) variiert. In allen Polymerisationsmischungen waren 2 Gew.-\% (bezogen auf die Masse des Monomers) MMMP als Photoinitiator enthalten.

Abbildung 4-38 zeigt die während der Polymerisation aufgetretenen Wärmeströme von drei beispielhaft ausgewählten RAFT-Polymerisationsmischungen mit einem BDDA-Anteil von 0,5 mol-\%, 2 mol-\% und 5 mol-\%. Vergleichend wurde der Wärmestrom einer konventionellen Polymerisation mit einem molaren BDDA-Anteil von 0,5 mol-\% hinzugefügt. Die genauen Zusammensetzungen der Polymerisationsmischungen sind in Abschnitt 6.2.4.1 zu finden.

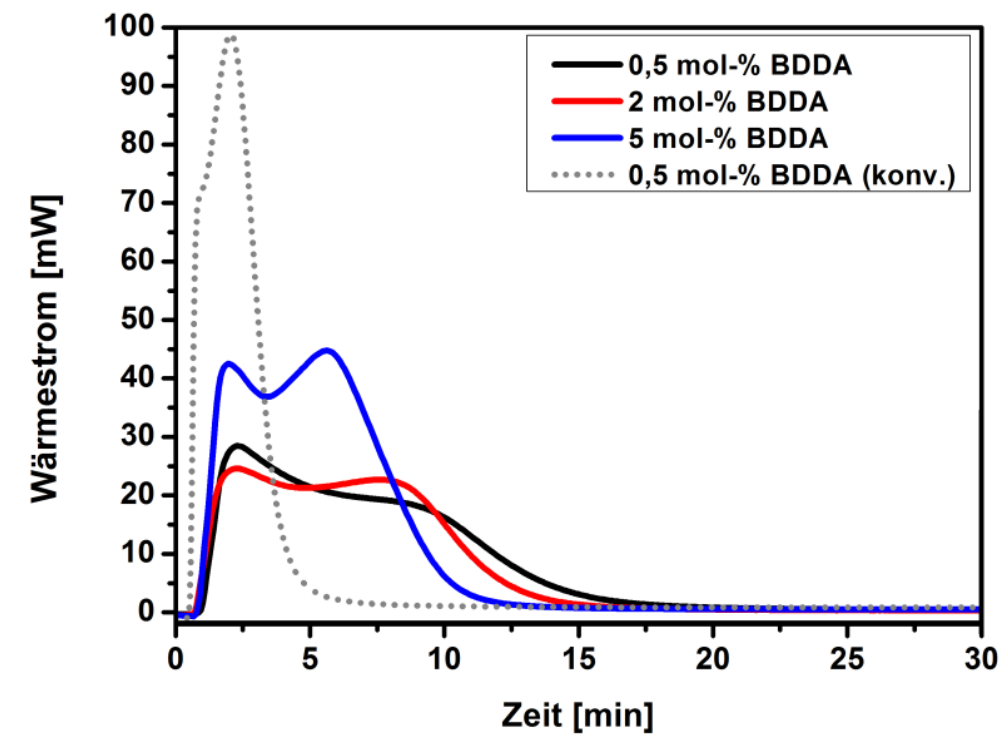

Abbildung 4-38 Mittels isothermer DSC $\left(21^{\circ} \mathrm{C}\right)$ aufgezeichnete Wärmeströme von UV-initiierten, radikalischen Polymerisationen ausgewählter Poly(BA-co-BDDA)-Netzwerke aus RAFT-Polymerisation mit einem molaren Anteil von 2 mol-\% EPHT als RAFT-Agens und 0,5 mol-\% (-), 2 mol-\% (-) und 5 mol-\% (-) vom Vernetzer BDDA. Zum Vergleich: Der Wärmestrom einer konventionellen Polymerisation mit 0,5 mol-\% BDDA ( ․). Als Photoinitiator wurden bei allen Polymerisationen 2 Gew.-\% MMMP verwendet. Die Lichtintensität betrug $9 \mathrm{~mW} / \mathrm{cm}^{2}$ bei $366 \mathrm{~nm}$. 
Die in Abbildung 4-38 zu erkennende Totzeit resultiert daher, dass bei jeder Messung zunächst $30 \mathrm{~s}$ gewartet wurde, bevor die UV-Lampe auf dem Kalorimeter platziert wurde und die Polymerisation starten konnte. Dies diente der Ermittlung einer Basislinie bei der Auswertung der Kurven.

Um aus den gemessenen Reaktionswärmen den Umsatz $C$ und die Reaktionsgeschwindigkeit $R_{\mathrm{P}}$ der Polymerisationen zu ermitteln, mussten die erhaltenen Wärmeströme entsprechend der in der Literatur beschriebenen Methoden ${ }^{[78,204]}$ umgerechnet werden. Dazu wurde von der Annahme ausgegangen, dass die Wärmetönung der Reaktion allein auf die während der Polymerisation umgesetzten Doppelbindungen zurückgeht und andere Reaktionen, wie z. B. der Zerfall des Initiators, keine Rolle spielen. Es folgt für die Berechnung des Monomerumsatzes $C_{\mathrm{M}}$ der Reaktion:

$$
C_{\mathrm{M}}=\frac{\int_{0}^{t} \mathrm{~d} H / \mathrm{d} t}{\Delta H_{0}^{\mathrm{th}}}=\frac{\Delta H_{t}}{\Delta H_{0}^{\mathrm{th}}}
$$

$(\mathrm{d} H / \mathrm{d} t)$ stellt den gemessenen Wärmestrom dar. $\Delta H_{t}$ ist das Integral des Wärmestroms und entspricht somit der gesamten Reaktionswärme, die während der Polymerisation frei wird. $\Delta H_{0}^{\text {th }}$ bezeichnet die theoretische Wärme für den vollständigen Umsatz aller in der Polymerisationsmischung vorhandenen Doppelbindungen und beträgt für die AcrylatDoppelbindung: $86,25 \mathrm{~kJ} / \mathrm{mol}^{[91]}$

Da die üblichen Probenmengen deutlich kleiner waren als ein Mol, mussten die theoretischen Wärmen für jede Probe neu berechnet werden. Eine Erläuterung dazu findet sich in Abschnitt 6.3.6.2. Bei bekannter Probenmasse war es somit möglich, die theoretische Wärme für jede Probe einzeln zu berechnen und die gemessenen Wärmeströme während der Berechnung des Monomerumsatzes über das Probengewicht zu normieren. Bei der Integration der Wärmeströme wurde die vordere Integrationsgrenze so gewählt, dass der Zeitpunkt $t=0$ dem Beginn der Wärmeströme entspricht, wodurch die Totzeit aus den Wärmestrom-Kurven (s. Abbildung 4-38) in der Auswertung keine Rolle mehr spielt. Abbildung 4-39 zeigt die nach der obigen Methode errechneten Umsatz/Zeit-Kurven der drei ausgewählten RAFT-NetzwerkPolymerisationsmischungen. Vergleichend wurde wieder die Umsatz/Zeit-Kurve der konventionellen Polymerisation hinzugefügt. 


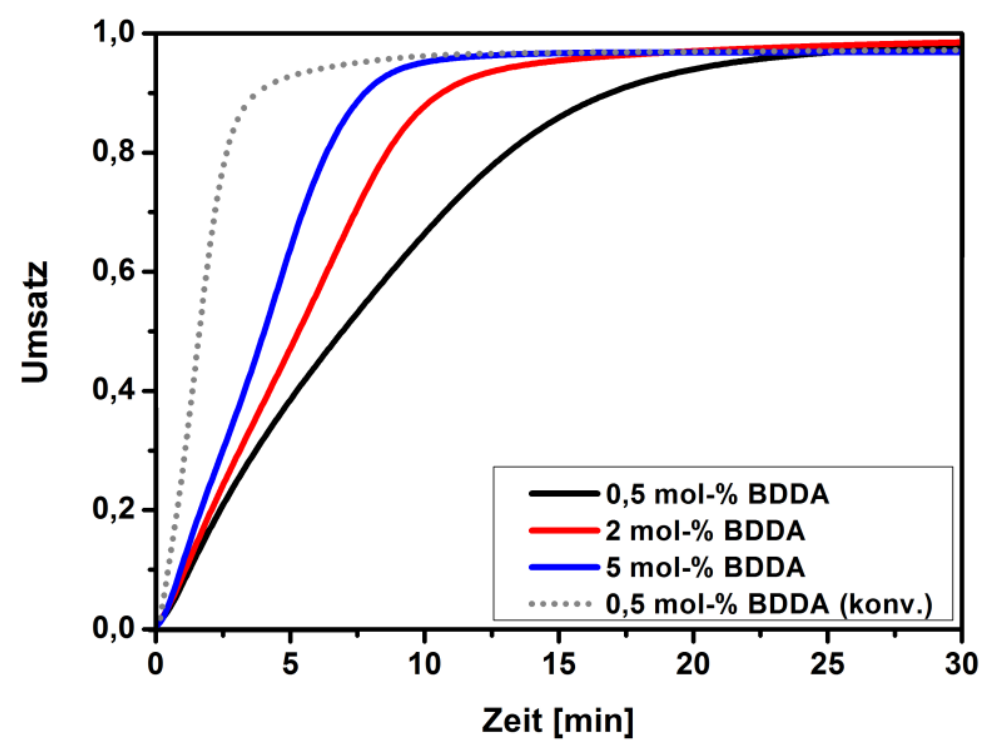

Abbildung 4-39 Aus isothermer DSC $\left(21^{\circ} \mathrm{C}\right)$ erhaltene Umsatz/Zeit-Kurven von UV-initiierten, radikalischen Polymerisationen ausgewählter Poly(BA-co-BDDA)-Netzwerke aus RAFT-Polymerisation mit einem molaren Anteil von 2 mol-\%o EPHT als RAFT-Agens und 0,5 mol-\% (-), 2 mol-\% (-) und 5 mol-\% (-) vom Vernetzer BDDA. Zum Vergleich: Die Umsatz/Zeit-Kurve einer konventionellen Polymerisation mit 0,5 mol-\% BDDA ( $\cdots)$. Als Photoinitiator wurden bei allen Polymerisationen 2 Gew.-\% MMMP verwendet. Die Lichtintensität betrug $9 \mathrm{~mW} / \mathrm{cm}^{2}$ bei $366 \mathrm{~nm}$.

Es ist erkennbar, dass alle Polymerisationsmischungen nach 30 Minuten sehr hohe Monomerumsätze von über $97 \%$ erreichen. Die maximalen Monomerumsätze $C_{\mathrm{M}}^{\max }$ der Polymerisationen sind in Tabelle 4-17 zusammengefasst. Da die Auswertung der DSCMesskurven, und somit die Berechnung des Monomerumsatzes, bei der Integration der Messwerte sehr anfällig für Verschiebungen der Basislinie ist, kann davon ausgegangen werden, dass der gefundene Monomerumsatz für alle drei Netzwerke im Rahmen eines möglichen Fehlers gleich ist und seinen maximal erreichbaren Wert bei allen durchgeführten Polymerisationen erreicht hat. Bei den Umsatz/Zeit-Kurven der RAFT-Polymerisationen in Abbildung 4-39 ist zu Beginn eine kurze Inhibierungsphase zu erkennen. Ein möglicher Grund dafür könnte in der Polymerisationsmischung vorhandener Sauerstoff sein, der als Biradikal in der Lage ist, mit der wachsenden Polymerkette zu terminieren. Allerdings fällt auf, dass die konventionelle Polymerisation keine erkennbare Inhibierungsphase zeigt. Da alle Polymerisationsmischungen auf die gleiche Weise für die Polymerisation vorbereitet wurden (s. Abschnitt 6.3.6.1), sollte die Inhibierung durch Sauerstoff bei allen Polymerisationsmischungen ebenfalls gleich gewesen sein. Da dies nicht der Fall ist, ist es möglich, dass noch mindestens ein weiterer Grund für die Inhibierung in den RAFT-Systemen vorhanden ist oder dass der Sauerstoff in diesen nur langsamer abgebaut wurde. Dies wird in Abschnitt 4.1.4.3 ausführlich diskutiert. 
Weiterhin ist in Abbildung 4-39 zu erkennen, dass die maximalen Umsätze der RAFTPolymerisationen bei einem höheren molaren BDDA-Anteil deutlich früher erreicht werden. Da sowohl die Initiatorkonzentration als auch die Lichtintensität in allen Experimenten gleich war, kann dieses Verhalten nur auf einen mit der Vernetzermenge zunehmenden Gel-Effekt zurückgeführt werden. Um diesen zu verdeutlichen, wurde mittels Gleichung (51) aus den Monomerumsätzen der Polymerisationen die Polymerisationsgeschwindigkeit berechnet: ${ }^{[78,204]}$

$$
R_{\mathrm{P}}=\frac{\mathrm{d} C_{\mathrm{M}}}{\mathrm{d} t}=\frac{\mathrm{d} H / \mathrm{d} t}{\Delta H_{0}^{\mathrm{th}}}
$$

und gegen den Monomerumsatz der Polymerisationen aufgetragen (s. Abbildung 4-40).

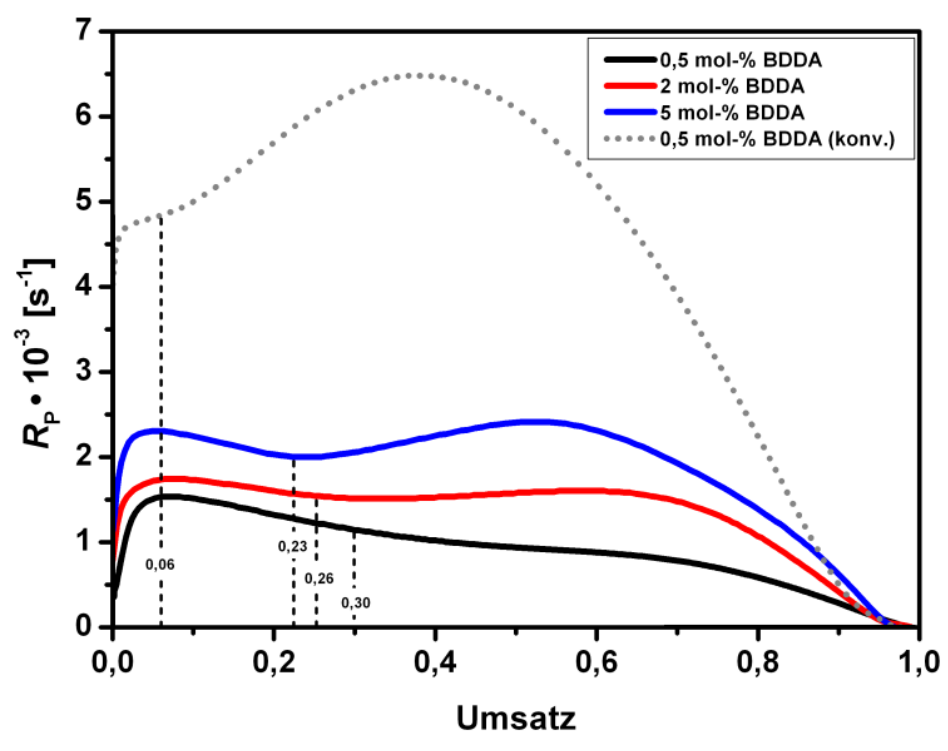

Abbildung 4-40 Polymerisationsgeschwindigkeit $R_{\mathrm{P}}$ aufgetragen gegen den Monomerumsatz $C_{\mathrm{M}}$ von UV-initiierten, radikalischen Polymerisationen ausgewählter Poly(BA-co-BDDA)-Netzwerke aus RAFT-Polymerisation mit einem molaren Anteil von 2 mol-\%o EPHT als RAFT-Agens und 0,5 mol-\% (-), 2 mol-\% (-) und 5 mol-\% (-) vom Vernetzer BDDA. Zum Vergleich: Die $R_{\mathrm{P}} / C_{\mathrm{M}}$-Auftragung einer konventionellen Polymerisation mit 0,5 mol-\% BDDA ( $\left.\cdots\right)$. Alle Polymerisationen fanden bei $21^{\circ} \mathrm{C}$ statt, mit 2 Gew.-\% MMMP als Photoinitiator und einer Lichtintensität von $9 \mathrm{~mW} / \mathrm{cm}^{2}$ bei $366 \mathrm{~nm}$.

Die Verläufe der $R_{\mathrm{P}} / C_{\mathrm{M}}$-Auftragungen lassen erkennen, dass sich die Reaktionsverläufe der einzelnen Polymerisationsmischungen deutlich voneinander unterscheiden. Aufgrund der zuvor beschriebenen Inhibierung kommt es bei den RAFT-Polymerisationen zu Beginn zu einem Anstieg der Polymerisationsgeschwindigkeiten. In dieser Auftragung zeigt sich allerdings, dass bei der konventionellen Polymerisation doch eine geringe Inhibierung stattgefunden hat. Diese ist aber deutlich geringer als bei den RAFT-Polymerisationen, bei denen sie für alle 
Polymerisationen nahezu gleich ist. Weiterhin geht aus Abbildung 4-40 hervor, dass die Polymerisationsgeschwindigkeiten der RAFT-Polymerisationen bei ungefähr $6 \%$ Monomerumsatz ein Maximum durchlaufen, während die Polymerisationsgeschwindigkeit der konventionellen Polymerisation immer stärker zunimmt. Diese erreicht ihr Maximum erst bei einem Monomerumsatz von etwa $50 \%$.

Dieser Anstieg hat seinen Ursprung in der Selbstbeschleunigung der Polymerisation infolge des Gel-Effekts (s. Kapitel 2.1.1). Die RAFT-Polymerisationen zeigen ebenfalls einen Gel-Effekt, wobei dieser mit abnehmendem molaren BDDA-Anteil deutlich schwächer wird. Während bei der Polymerisationsmischung mit 5 mol-\% BDDA ein erneuter Anstieg der Polymerisationsgeschwindigkeit dazu führt, dass das erste Maximum bei einem Monomerumsatz von etwa $55 \%$ sogar noch übertroffen wird, zeigt die Polymerisationsgeschwindigkeit der RAFT-Polymerisation mit 2 mol-\% BDDA nur einen moderaten Anstieg mit einem zweiten Maximum bei $65 \%$ Monomerumsatz. Die Polymerisationsgeschwindigkeit der RAFT-Polymerisation mit 0,5 mol-\% BDDA steigt nicht ein zweites Mal an, sondern bildet zu höheren Monomerumsätzen über $50 \%$ eine Schulter aus. Dies zeigt die Tendenz, dass ein höherer molarer Anteil des Vernetzers BDDA zu einem stärkeren Gel-Effekt führt, was eine immer schneller werdende Polymerisation zur Folge hat. Dabei fällt auf, dass die RAFT-Polymerisationen trotz höherer molarer BDDA-Anteile ( 2 mol-\% und 5 mol-\%) immer noch deutlich langsamer sind als die konventionelle radikalische Polymerisation mit nur 0,5 mol-\% BDDA. Weiterhin ist bei ihnen die Selbstbeschleunigung deutlich weniger ausgeprägt. Dass die konventionelle Polymerisation trotz des geringen BDDAAnteils viel schneller verläuft als die RAFT-Polymerisation und der Gel-Effekt schon bei sehr geringen Umsätzen auftritt, lässt sich auf die schon sehr früh in der Polymerisation gebildeten Nanogel-Domänen zurückführen, die schon bei geringen Monomerumsätzen zu einer lokalen Selbstbeschleunigung der Polymerisation führen können (s. Kapitel 2.2.2). Die genauen Werte der Polymerisationsgeschwindigkeit $R_{\mathrm{P}}$ der durchgeführten Polymerisation, die ohne einen GelEffekt zu Beginn der Polymerisation am höchsten sein sollte, wurden nach der anfänglichen Inhibierung bei $6 \%$ Monomerumsatz in Abbildung 4-40 abgelesen und sind in Tabelle 4-17 zusammengefasst.

Bei den RAFT-Polymerisationen verhindert der RAFT-Mechanismus die Bildung von NanogelDomänen. Stattdessen verläuft sie über eine Sol-Fraktion, die zwar aus mehrfach verzweigten Makromolekülen besteht, welche allerdings zunächst noch kein Netzwerk bilden. Dadurch setzt der Gel-Effekt bei den beobachteten RAFT-Polymerisationen, ähnlich einer nicht-vernetzenden Polymerisation, erst bei höheren Monomerumsätzen mit der steigenden Viskosität der Polymerisationsmischung ein. Darum ist er im Vergleich zu einer konventionellen vernetzenden radikalischen Polymerisation verzögert (s. Abbildung 2-9). Weiterhin sollte dies auch den Gelpunkt, also die Bildung des Gesamtnetzwerks, zu höheren Monomerumsätzen verschieben. Dabei sollte beachtet werden, dass der Gelpunkt nicht zwangsläufig mit dem Eintreten des GelEffekts gleichzusetzten ist. Eine Selbstbeschleunigung kann aufgrund von zunehmender 
Viskosität der Polymerisationslösung auch bei den RAFT-Polymerisationen schon eintreten, wenn nur Sol-Moleküle vorhanden sind und das Gesamtnetzwerk noch nicht gebildet wurde. Die genauen Werte der Monomerumsätze bei denen der Gel-Effekt eintritt $C_{\mathrm{M}}^{\mathrm{Gel}}$, wurden in Abbildung 4-40 an dem Punkt an dem die $R_{\mathrm{P}} / C_{\mathrm{M}}$-Kurve keine lineare Abnahme mehr zeigt, abgelesen und sind in Tabelle 4-17 zusammengefasst.

Tabelle 4-17 Maximale Monomerumsätze $C_{\mathrm{M}}^{\max }$, Reaktionsgeschwindigkeiten $R_{\mathrm{P}}$ und Monomerumsätze, bei denen der Gel-Effekt einsetzt $C_{\mathrm{M}}^{\mathrm{Gel}}$ von RAFT-Polymerisationen Poly(BA-co-BDDA)-Netzwerken mit einem molaren Anteil von 2 mol-\%o EPHT als RAFT-Agens, aber unterschiedlichen molaren Anteilen vom Vernetzer BDDA und eine konventionelle Polymerisation mit 0,5 mol-\% BDDA. Die Werte wurden aus Abbildung 4-39 und Abbildung 4-40 entnommen.

\begin{tabular}{ccccc}
\hline BDDA-Anteil [mol-\%] & EPHT-Anteil [mol-\%] & $\boldsymbol{C}_{\mathrm{M}}^{\max }[\%]$ & $\boldsymbol{R}_{\mathbf{P}}\left[\mathbf{s}^{-1}\right]$ & $\boldsymbol{C}_{\mathrm{M}}^{\mathrm{Gel}}[\%]$ \\
\hline 0,5 & 2 & 98 & $1,5 \cdot 10^{-3}$ & 30 \\
2 & 2 & 99 & $1,7 \cdot 10^{-3}$ & 26 \\
5 & 2 & 97 & $2,3 \cdot 10^{-3}$ & 23 \\
0,5 & 0 & 98 & $4,8 \cdot 10^{-3}$ & 6 \\
\hline
\end{tabular}

Da die Erhöhung der BDDA-Konzentration in den RAFT-Netzwerken schon bei geringen Monomerumsätzen wieder zu einer verstärkten Bildung von Nanogel-Domänen führen kann, kommt es schon im frühen Stadium der Polymerisation zu einer Erhöhung der Polymerisationsgeschwindigkeit. Dadurch wird der Reaktionsverlauf der RAFT-Polymerisationen dem der konventionellen radikalischen Polymerisation immer ähnlicher. Allerdings wird diese Ähnlichkeit erst bei der RAFT-Polymerisation mit 5 mol-\% BDDA deutlicher erkennbar. Dies bestätigt sich auch durch eine Auftragung der integrierten Form des Geschwindigkeitsgesetztes (s. Gleichung (9)) gegen die Zeit, der so genannten Pseudo-Erste-Ordnung-Auftragung (s. Abbildung 4-41). 


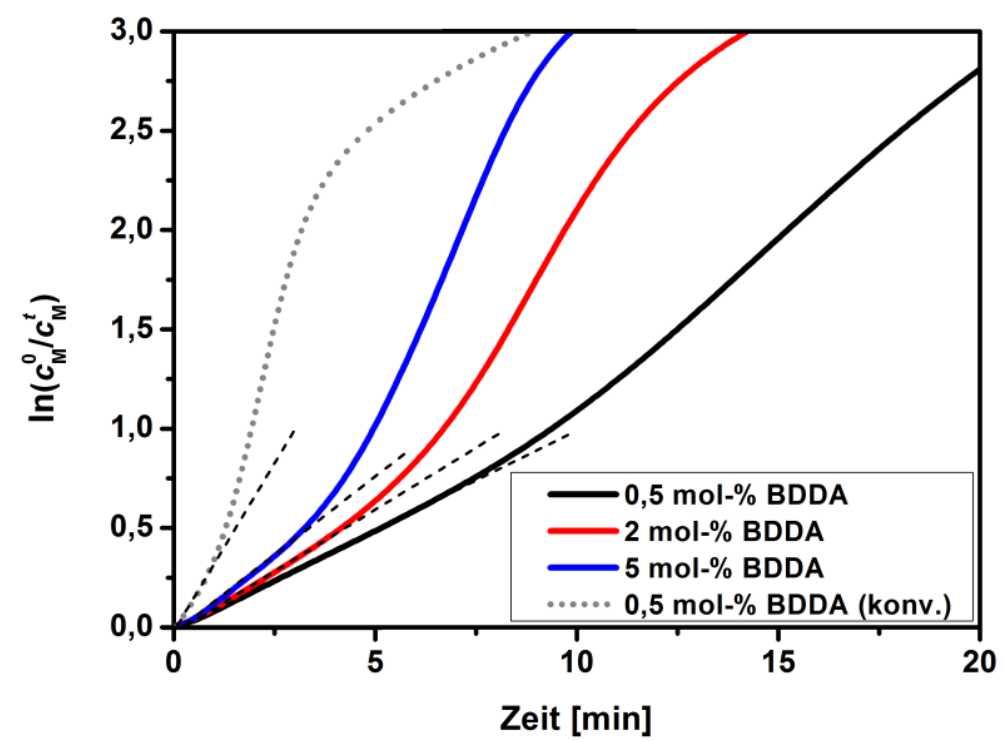

Abbildung 4-41 Pseudo-Erste-Ordnung-Auftragung von UV-initiierten, radikalischen Polymerisationen ausgewählter Poly(BA-co-BDDA)-Netzwerke aus RAFT-Polymerisation mit einem molaren Anteil von 2 mol-\%o EPHT als RAFTAgens und 0,5 mol-\% (-), 2 mol-\% (-) und 5 mol-\% (-) vom Vernetzer BDDA. Zum Vergleich: Die Auftragung einer konventionellen Polymerisation mit 0,5 mol-\% BDDA ( ․). Die gestrichelten Linien stellen eine lineare Anpassung der anfänglichen nach idealer Polymerisationskinetik verlaufenden Bereiche der Kurven dar. Alle Polymerisationen fanden bei $21^{\circ} \mathrm{C}$ statt, mit 2 Gew.-\% MMMP als Photoinitiator und einer Lichtintensität von $9 \mathrm{~mW} / \mathrm{cm}^{2}$ bei $366 \mathrm{~nm}$.

Nach der kurzen Inhibierungsphase zeigen alle Kurven in Abbildung 4-41 einen linearen Anstieg entsprechend der idealen Kinetik einer radikalischen Polymerisation mit konstanter Radikalkonzentration (s. Abbildung 2-1). Dieser ist bei der konventionellen Polymerisation am stärksten und steigt bei den RAFT-Polymerisationen mit größer werdenden BDDA-Anteil an (s. Abbildung 4-41). Bei der konventionellen Polymerisation setzt der Gel-Effekt nahezu direkt nach dem Beginn der Polymerisation ein, wodurch die Radikalkonzentration im System ansteigt und diese den linearen Bereich verlässt. Bei den RAFT-Polymerisationen wird dieser Punkt mit sinkendem BDDA-Anteil immer später erreicht. Die Steigung der linearen Anpassungen an die Messkurven in Abbildung 4-41 entspricht dem Proportionalitätsfaktor $K_{\mathrm{P}}$. Dieser ist über Gleichung (7) mit den Geschwindigkeitskoeffizienten $k_{\mathrm{p}}, k_{\mathrm{i}}$ und $k_{\mathrm{t}}$ verknüpft, die in die Geschwindigkeitsgesetze der einzelnen Teilreaktion der radikalischen Polymerisation eingehen (s. Kapitel 2.1.1). Unter der Annahme, dass die Propagationsgeschwindigkeit und die Geschwindigkeit des Initiatorzerfalls innerhalb der RAFT-Systeme gleich sind, kann der zunehmende Wert von $K_{\mathrm{P}}$ nur aus einer, mit steigendendem BDDA-Anteil, kleiner werdenden Terminierungsgeschwindigkeit resultieren. Dieser Effekt beruht wahrscheinlich auf einer höheren Mikroviskosität der stärker verzweigten Sol-Molekülem, die bei höherem BDDA-Anteil in der Polymerisationsmischung vorhanden sind. Schon bei geringen Monomerumsätzen führt dies zu einer schnelleren Polymerisation. Dies erklärt auch die lokale Selbstbeschleunigung in 
den Nanogel-Domänen des konventionellen Netzwerks. Die Werte von $K_{\mathrm{P}}$ sind in Tabelle 4-18 gemeinsam mit dem Zeitpunkt, an dem der Gel-Effekt einsetzt $t_{\mathrm{Gel}}$ zusammengefasst.

Tabelle 4-18 Proportionalitätsfaktor $K_{\mathrm{P}}$ und die Zeitpunkte, an denen der Gel-Effekt einsetzt $t_{\mathrm{Gel}}$ von RAFTPolymerisationen Poly(BA-co-BDDA)-Netzwerken mit einem molaren Anteil von 2 mol-\%o EPHT als RAFT-Agens, aber unterschiedlichen molaren Anteilen vom Vernetzer BDDA und eine konventionelle Polymerisation mit 0,5 mol-\% BDDA. Die Werte wurden aus Abbildung 4-41 entnommen.

\begin{tabular}{cccc}
\hline BDDA-Anteil [mol-\%] & EPHT-Anteil [mol-\%] & $\boldsymbol{K}_{\mathbf{P}}[\mathbf{1} / \mathbf{m i n}]$ & $\boldsymbol{t}_{\mathbf{G e l}}[\mathbf{m i n}]$ \\
\hline 0,5 & 2 & 0,10 & 8 \\
2 & 2 & 0,13 & 5 \\
5 & 2 & 0,16 & 3 \\
0,5 & 0 & 0,32 & 1 \\
\hline
\end{tabular}

Anhand des Wertes von $t_{\text {Gel }}$ für das RAFT-Netzwerk mit 0,5 mol-\% BDDA und der zugehörigen Umsatz/Zeit-Kurve (s. Abbildung 4-39) kann auch die Unabhängigkeit der mechanischen Eigenschaften des Netzwerks von der Reaktionszeit erklärt werden (s. Abschnitt 4.1.1.2).

Wenn nach einer Polymerisationszeit von etwa acht Minuten die Viskosität der Polymerisationsmischung so groß wird, dass der Gel-Effekt einsetzt, haben die Sol-Moleküle eine Größe erreicht, dass sie kurz darauf in der Lage sind, zum Gesamtnetzwerk zusammenzuwachsen und somit den Gelpunkt zu erreichen (s. Kapitel 2.2.2). Dies zeigt sich daran, dass bei der Probe mit einer Polymerisationszeit von zehn Minuten schon ein Netzwerk gebildet war. Dieses vernetzte anschließend durch weitere Polymerisation nur noch weiter, bis nach ca. 20 Minuten der vollständige Monomerumsatz nahezu erreicht war (s. Abbildung 4-39). Darum zeigen sich in Abschnitt 4.1.1.2 bei den Probekörpern mit einer Polymerisationszeit ab 20 Minuten keine Unterschiede mehr in den mechanischen Eigenschaften.

Die Steigung der Anfangsbereiche der Pseudo-Erste-Ordnung-Auftragungen $K_{\mathrm{P}}$ ermöglicht es weiterhin, die Koeffizienten $k_{\mathrm{p}}, k_{\mathrm{i}}$ und $k_{\mathrm{t}}$ zu bestimmen. Da der Fokus dieser Arbeit jedoch auf den Struktur/Eigenschaftsbeziehungen der Netzwerke liegt, soll auf tiefergehende kinetische Berechnungen an dieser Stelle verzichtet werden. Nichtsdestotrotz belegt der mit dem BDDAAnteil zunehmende Gel-Effekt, dass Netzwerke mit einer höheren Netzkettendichte gebildet werden. Die in diesem Abschnitt bestimmten Daten bestätigen somit die bei den Zugversuchen (s. Abschnitt 4.1.1.1) und den Quellungsexperimenten (s. Abschnitt 4.1.3.1) gemachten Beobachtungen. 


\subsubsection{Variation des molaren EPHT-Anteils}

Ergänzend zu den vorherigen Messungen wurde auch der Einfluss des molaren EPHT-Anteils auf die Polymerisationskinetik der Poly(BA-co-BDDA)-Netzwerke untersucht. Dazu wurden die analog zu den in den Abschnitten 4.1.1.3 und 4.1.3.2 untersuchten Polymerisationsmischungen der Netzwerke mit einem molaren BDDA-Anteil von 0,5 mol-\% ausgewählt und der molare EPHT-Anteil von 0 bis 4 mol-\%o (bezogen auf die Stoffmenge des Monomers) variiert. Die genauen Zusammensetzungen der Polymerisationsmischungen sind in Abschnitt 6.2.4.1 zu finden. Die Reaktionswärmen der Polymerisationen wurden wieder mittels isothermer DSCMessungen bestimmt. Anhand der Gleichungen (50) und (51) wurde der Monomerumsatz und die Polymerisationsgeschwindigkeit der Reaktionen berechnet.

Schon bei einer Auftragung des Monomerumsatzes gegen die Reaktionszeit zeigt sich ein deutlicher Einfluss der RAFT-Agens-Menge auf die Umsatz/Zeit-Kurven der Polymerisationen (s. Abbildung 4-42).

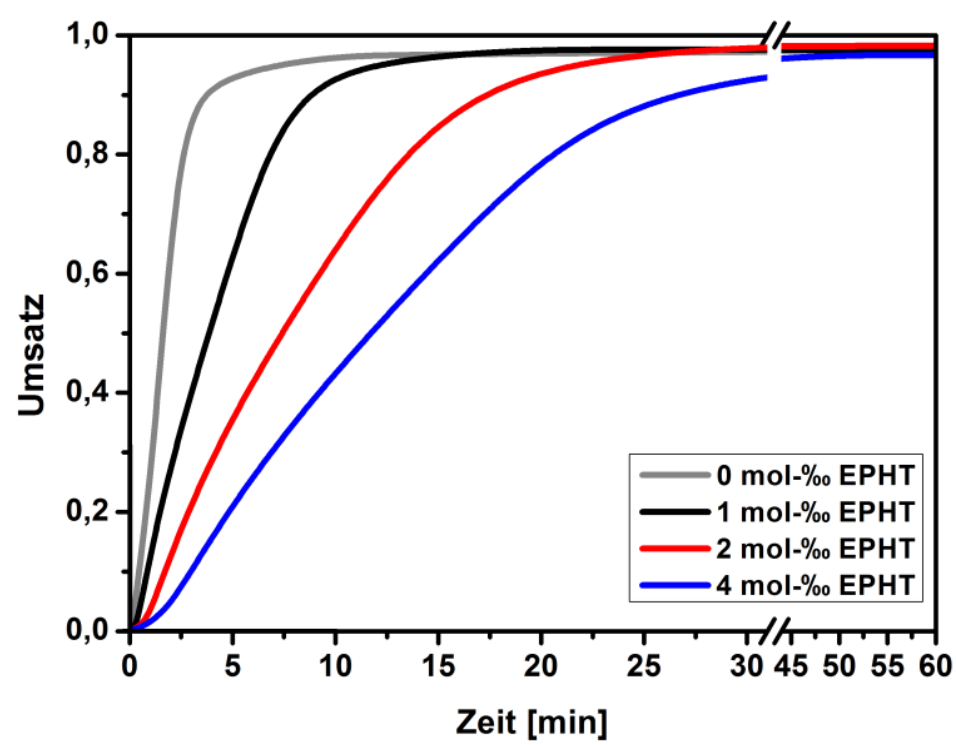

Abbildung 4-42 Aus isothermer DSC erhaltene Umsatz/Zeit-Kurven von UV-initiierten, radikalischen Polymerisationen ausgewählter Poly(BA-co-BDDA)-Netzwerke mit einem molaren Anteil von 0,5 mol-\% BDDA als Vernetzer ohne das RAFT-Agens EPHT (-) sowie mit 1 mol-\%o (-), 2 mol-\%o (-) und 4 mol-\%o (-) EPHT. Alle Polymerisationen fanden bei $21^{\circ} \mathrm{C}$ statt, mit 2 Gew.-\% MMMP als Photoinitiator und einer Lichtintensität von $9 \mathrm{~mW} / \mathrm{cm}^{2}$ bei $366 \mathrm{~nm}$.

In Abbildung 4-42 ist erkennbar, dass die Inhibitionsperiode am Beginn der Polymerisationen, ausgelöst durch in den Polymerisationsmischungen vorhandenen Restsauerstoff mit steigendem EPHT-Anteil, immer länger andauert. Weiterhin verlaufen die Polymerisationen insgesamt immer langsamer (s. Abbildung 4-43), was dazu führt, dass die vollständigen Monomerumsätze 
zunehmend später erreicht werden (s. Abbildung 4-42). Es ist also möglich, dass das EPHT zu einer Retardierung der Polymerisation beiträgt bzw. führt. Die möglichen Gründe für diese Retardierung der RAFT-Polymerisationen werden in Abschnitt 4.1.4.3 ausführlich diskutiert. Die maximalen Monomerumsätze der Polymerisationen sind zusammen mit den Polymerisationsgeschwindigkeiten bei $6 \%$ Umsatz in Tabelle 4-19 zusammengefasst.

Zunächst soll noch auf einen anderen Effekt der RAFT-Polymerisation auf die Bildung von statistischen Poly(BA-co-BDDA)-Netzwerken eingegangen werden, der in Abbildung 4-43 sehr anschaulich wird.

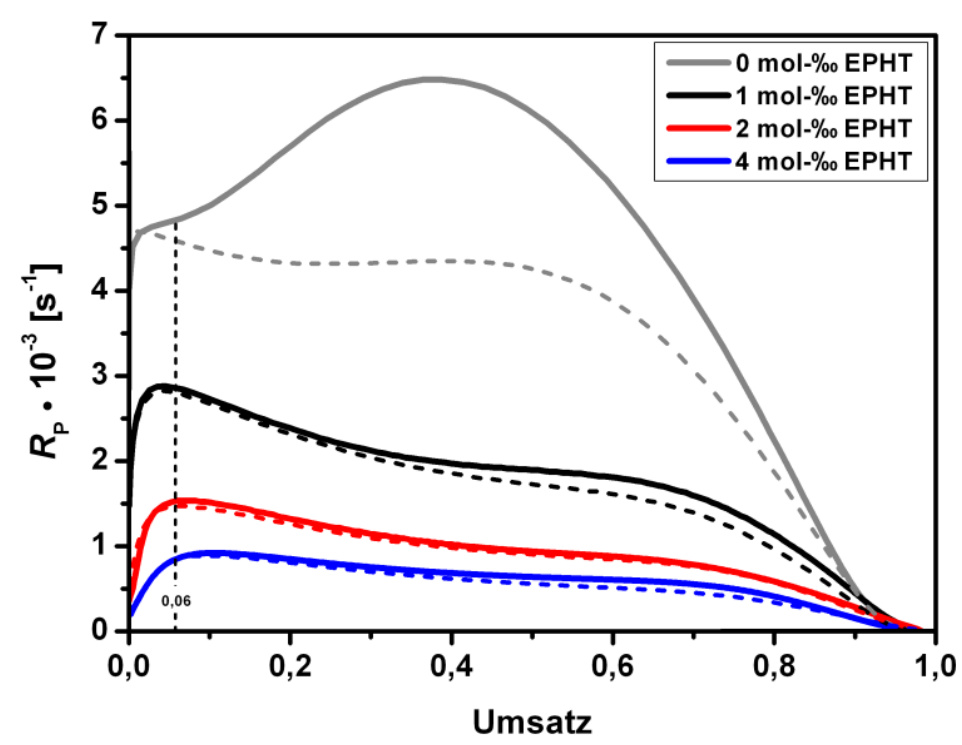

Abbildung 4-43 Polymerisationsgeschwindigkeit $R_{\mathrm{P}}$ aufgetragen gegen den Monomerumsatz $C_{\mathrm{M}}$ von UV-initiierten, radikalischen Polymerisationen ausgewählter Poly(BA-co-BDDA)-Netzwerke mit einem molaren Anteil von 0,5 mol-\% BDDA als Vernetzer ohne das RAFT-Agens EPHT (-) sowie mit 1 mol-\%o (-), 2 mol-\%o (-) und 4 mol-\%o (-) EPHT. Zum Vergleich: Die $R_{\mathrm{P}} / C_{\mathrm{M}}$-Auftragungen UV-initiierter, radikalischer BA-Homopolymerisationen ohne (--) sowie mit 1 mol-\%o (--), 2 mol-\%o (--) und 4 mol-\%o (--) des RAFT-Agens EPHT. Alle Polymerisationen fanden bei $21{ }^{\circ} \mathrm{C}$ statt, mit 2 Gew.-\% MMMP als Photoinitiator und einer Lichtintensität von $9 \mathrm{~mW} / \mathrm{cm}^{2}$ bei $366 \mathrm{~nm}$.

In dieser Abbildung wurde zusätzlich zu den Verläufen der Polymerisationsgeschwindigkeiten der Poly(BA-co-BDDA)-Netzwerke die Verläufe der entsprechenden nicht vernetzten Polymerisationen hinzugefügt und als gestrichelte Linien dargestellt. Bei den konventionellen Polymerisationen ist untereinander ein deutlicher Unterschied im Reaktionsverlauf zu erkennen. Während das vernetzende System schon bei sehr geringem Umsatz, $(<10 \%)$ aufgrund der Bildung von Nanogel-Domänen eine sehr ausgeprägte Selbstbeschleunigung zeigt, tritt diese im nichtvernetzenden System erst bei einem höheren Umsatz (ca. 20\%) ein. Diese ist auch deutlich weniger ausgeprägt. 
Die Verläufe der Polymerisationsgeschwindigkeiten der RAFT-Systeme sind dagegen jeweils nahezu identisch, unabhängig davon, ob das System vernetzt oder nicht. Das bedeutet, dass die Kinetik einer vernetzenden RAFT-Polymerisation der einer linearen bzw. einer nicht vernetzenden Verzweigungspolymerisation folgt. Somit bestätigten diese Messungen der Polymerisationskinetik die in Abbildung 2-9 gemachte Annahme, dass in einer RDRP, zu der die RAFT-Polymerisation zählt, die Bildung von Nanogel-Domänen verhindert wird und die Netzwerkbildung tatsächlich über eine Sol-Fraktion verlaufen muss. Weiterhin zeigt der Vergleich der vernetzenden mit den nicht-vernetzenden RAFT-Polymerisationen, dass eine mögliche verzögerte Vernetzung bzw. ein verzögerter Gelpunkt nicht der Grund für die Retardierung der Polymerisationen sein kann, denn diese wird ebenfalls von den nichtvernetzenden Polymerisationen gezeigt.

Zur Diskussion eines möglichen verzögerten Gelpunkts aufgrund des RAFT-Agens EPHT bietet es sich an, die integrierte Form des Geschwindigkeitsgesetztes gegen die Reaktionszeit aufzutragen. Die somit erhaltene Pseudo-Erste-Ordnung-Auftragung ist in Abbildung 4-43 zu finden.

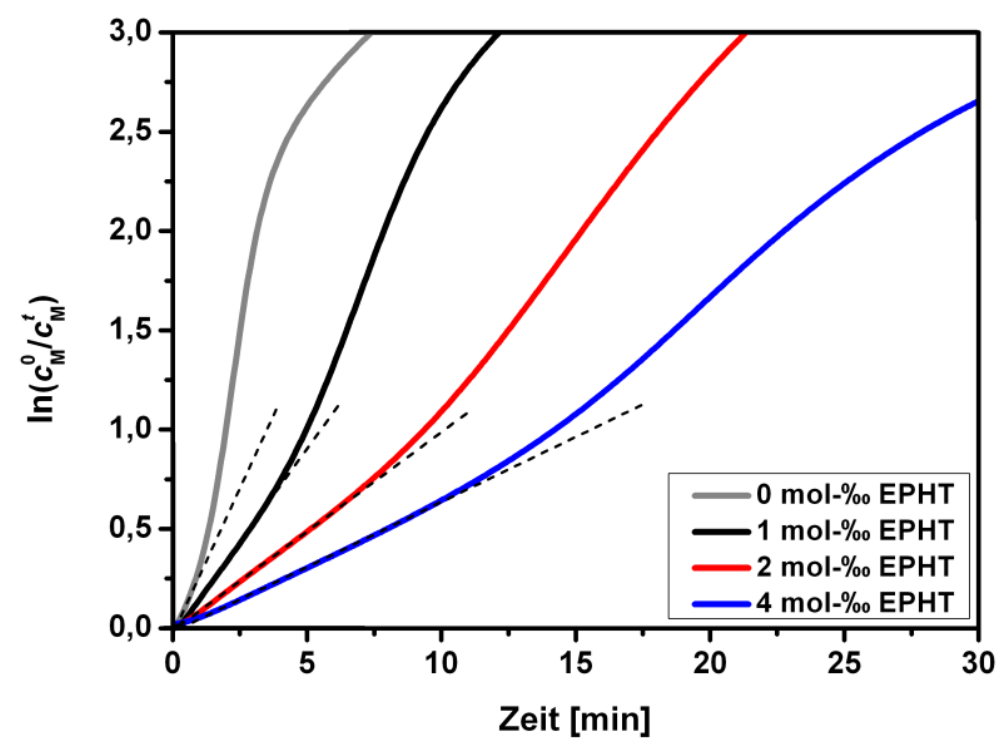

Abbildung 4-44 Pseudo-Erste-Ordnung-Auftragung von UV-initiierten, radikalischen Polymerisationen ausgewählter Poly(BA-co-BDDA)-Netzwerke mit einem molaren Anteil von 0,5 mol-\% BDDA als Vernetzer ohne (-) sowie mit 1 mol-\%o (-), 2 mol-\%o (-) und 4 mol-\%o (-) des RAFT-Agens EPHT. Die gestrichelten Linien stellen eine lineare Anpassung der anfänglichen nach idealer Polymerisationskinetik verlaufenden Bereiche der Kurven dar. Alle Polymerisationen fanden bei $21^{\circ} \mathrm{C}$ statt, mit 2 Gew.-\% MMMP als Photoinitiator und einer Lichtintensität von $9 \mathrm{~mW} / \mathrm{cm}^{2}$ bei $366 \mathrm{~nm}$.

Abbildung 4-44 zeigt, dass nach einer kurzen Inhibierungsphase alle Kurven einen linearen Anstieg entsprechend der idealen Kinetik einer radikalischen Polymerisation mit konstanter 
Radikalkonzentration zeigen. Weiterhin nehmen die Steigungen der Kurven mit zunehmendem EPHT-Anteil in den Polymerisationsmischungen ab. Auch der Gel-Effekt tritt später ein. Letzteres kann dadurch erklärt werden, dass mit steigender RAFT-Konzentration im System die Ketten der Sol-Fraktion entsprechend Gleichung (12) immer kürzer werden. Dies führt zu einem geringeren Anstieg der Viskosität des Systems während der Polymerisation. Die Makroradikale bleiben länger im System beweglich und die Terminierung kann länger mit gleichbleibender Geschwindigkeit stattfinden, bevor sie sich durch den Gel-Effekt deutlich verlangsamt. Weiterhin verfügen die gebildeten verzweigten Makromoleküle bei geringeren Molmassen über weniger seitliche Doppelbindungen, die für die für die Verzweigung der Sol-Moleküle aber unerlässlich sind. Folglich sind die Sol-Moleküle bei zunehmendem EPHT-Anteil weniger verzweigt und verfügen somit auch über geringere Mikroviskositäten (s. Abschnitt 4.1.4.1). Dies zeigt sich in den geringeren Steigungen der Kurven aus Abbildung 4-44. Weiterhin führt dies dazu, dass in diesen Systemen der Gelpunkt auch deutlich später bzw. erst bei größeren Umsätzen eintritt, da für diese eine bestimmte Anzahl von seitlichen Doppelbindungen in den Sol-Molekülen vorhanden sein muss. Die Bildung des Netzwerks würde die Beweglichkeit der Makroradikale im System endgültig verringern und somit zwangsläufig zu einem Gel-Effekt führen.

Tabelle 4-19 Maximale Monomerumsätze $C_{\mathrm{M}}^{\max }$, Polymerisationsgeschwindigkeiten $R_{\mathrm{P}}$, Proportionalitätsfaktor $K_{\mathrm{P}}$ und die Zeitpunkte, an denen der Gel-Effekt einsetzt $t_{\text {Gel }}$ von Polymerisationsmischungen für Poly(BA-co-BDDA)Netzwerken mit einem molaren Anteil von 0,5 mol-\% BDDA als Vernetzer, aber unterschiedlichen molaren Anteilen vom RAFT-Agens EPHT. Die Werte wurden aus Abbildung 4-42, Abbildung 4-43 sowie Abbildung 4-44 entnommen.

\begin{tabular}{cccccc}
\hline $\begin{array}{c}\text { EPHT-Anteil } \\
{[\mathbf{m o l - \%}]}\end{array}$ & $\begin{array}{c}\text { BDDA-Anteil } \\
{[\mathbf{m o l - \%}]}\end{array}$ & $\begin{array}{c}\boldsymbol{C}_{\mathbf{M}}^{\max } \\
{[\%]}\end{array}$ & $\begin{array}{c}\boldsymbol{R}_{\mathbf{P}} \\
{\left[\mathbf{s}^{-\mathbf{1}}\right]}\end{array}$ & $\begin{array}{c}\boldsymbol{K}_{\mathbf{P}} \\
{[\mathbf{1} / \mathbf{m i n}]}\end{array}$ & $\begin{array}{c}\boldsymbol{t}_{\text {Gel }} \\
{[\mathbf{m i n}]}\end{array}$ \\
\hline 0 & 0,5 & 97 & $4,8 \cdot 10^{-3}$ & 0,32 & 1 \\
1 & 0,5 & 97 & $2,8 \cdot 10^{-3}$ & 0,19 & 4 \\
2 & 0,5 & 98 & $1,5 \cdot 10^{-3}$ & 0,10 & 8 \\
4 & 0,5 & 97 & $0,8 \cdot 10^{-3}$ & 0,06 & 9 \\
\hline
\end{tabular}

Die geringer werdenden Molmassen der Sol-Fraktion können aber weder die abnehmenden Polymerisationsgeschwindigkeiten erklären noch, dass die anfängliche Inhibierungsphase in den RAFT-Polymerisationen mit steigendem EPHT-Anteil immer länger andauert (s. Abbildung 4-42). Darum sollen im folgenden Abschnitt mögliche Gründe für diese Effekte ausführlich diskutiert werden. 


\subsubsection{Mögliche Gründe für die Retardierung der RAFT-Polymerisationen}

\section{Backbiting}

Zunächst soll auf einen Aspekt eingegangen werden, der die Kinetik der Polymerisation von $n$-Butylacrylat wesentlich beeinflusst. Hierbei handelt es sich um den intramolekularen Transfer des Kettenendradikals eines propagierenden Makromoleküls auf die Polymerkette, das so genannte Backbiting. Diese Reaktion und deren Auswirkung auf die Polymerisationskinetik ist von verschiedenen Arbeitsgruppen ausführlich untersucht worden. ${ }^{\text {[205-210] }}$

Das Backbiting geschieht über einen sechsgliedrigen Übergangszustand unter Wasserstoffabspaltung am Makromolekül (s. Schema 4-1). Dabei entsteht aus einem sekundären Kettenendradikal (engl.: secondary chain-end radical, SPR) ein tertiäres Radikal am Rückgrat des Makromoleküls (engl.: midchain radical, MCR).
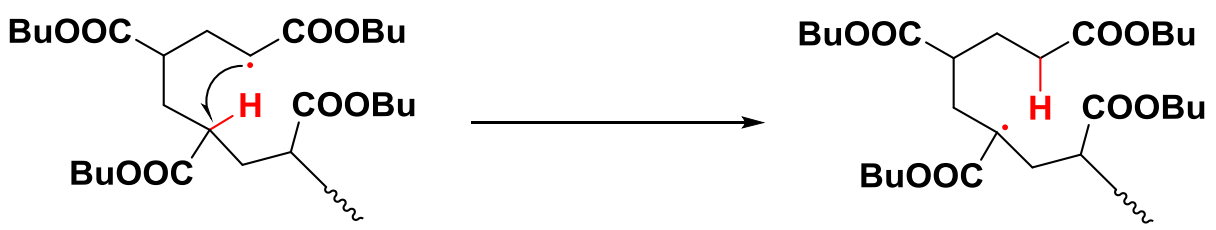

Schema 4-1 Prinzipieller Verlauf der Bildung eines tertiären Radikals (MCR) am Rückgrat eines Makromoleküls durch intramolekularen Transfer eines sekundären Kettenendradikals (SPR) an einem Poly-BA-Makromolekül während einer radikalischen Polymerisation (Backbiting). Nach Buback et al. ${ }^{[11]}$.

Der Propagationsschritt eines MCRs mit einem Monomer ist im Vergleich zum Propagationsschritt eines SPRs langsamer. Darum kann das vermehrte Auftreten von MCRs zu einer Verringerung der Netto-Propagationsgeschwindigkeit führen. Vor allem bei höheren Temperaturen ist dies bei der Betrachtung der Polymerisationskinetik von BA nicht mehr zu vernachlässigen. ${ }^{[210]}$ Aber auch bei Raumtemperatur kann diese Reaktion und die daraus resultierende Bildung der MCRs einen Einfluss auf die Polymerisationskinetik haben. ${ }^{[205]}$ Bei den in dieser Arbeit betrachteten Systemen kann allerdings davon ausgegangen werden, dass die Vernetzung und die damit einhergehende Verlangsamung der Terminierungsreaktion und die folgende Selbstbeschleunigung die Kinetik der Polymerisationen dominiert.

Weiterhin wurde herausgefunden, dass die Anzahl der aus Backbiting resultierenden Kurzkettenverzweigungen in einer RDRP deutlich verringert ist, was bedeutet, dass in einer RDRP weniger Backbiting stattfindet. Dies wurde auf die in einer RDRP geringere Konzentration von hochreaktiven Kurzkettenradikalen zurückgeführt. ${ }^{[212]}$ Weiterhin wurde auch die unterschiedliche Verteilung der Radikale in konventionellen und RDRP-Systemen als möglicher Grund ausgemacht. ${ }^{[213]}$ Anhand von Monte Carlo-Simulationen von konventionellen radikalischen Polymerisationen und RDRPs konnte schließlich gezeigt werden, dass die mittlere 
Lebensdauer eines SPRs in einer RDRP zu gering ist, um zu Backbiting zu führen, da der Transfer zum CTA viel schneller verläuft als eine mögliche Backbiting-Reaktion. ${ }^{[214]}$ Somit kann davon ausgegangen werden, dass das Backbiting als Grund für die gefundene Retardierung der RAFTPolymerisationen nicht in Frage kommt.

\section{Der RAFT-Mechanismus und Kreuzterminierung}

Eine Retardierung der Polymerisation ist in RAFT-Polymerisationen schon öfters beobachtet worden. Allerdings handelt es sich hierbei um einen Effekt, der bei der Verwendung von Dithiobenzoaten auftritt. ${ }^{[215]}$ Die Radikalfunktion ist dem während der RAFT-Polymerisation auftretenden Intermediat-Radikal (s. Schema 2-3) über den Phenylring delokalisiert (s. Schema 4-2).

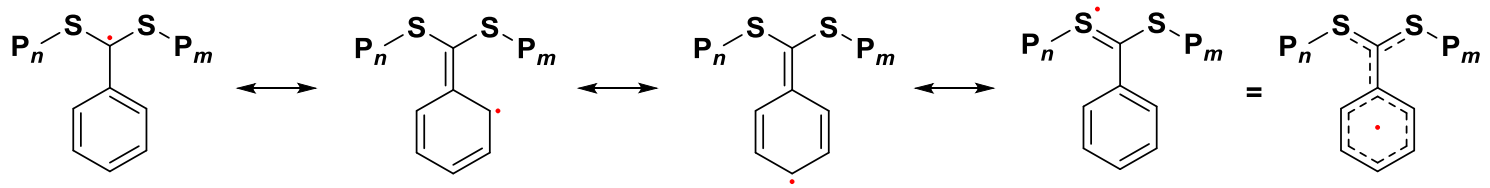

Schema 4-2 Resonanzstrukturen des Dithiobenzoat-Intermediats in einer RAFT-Polymerisation.

Die damit einhergehende höhere Stabilität des Dithiobenzoat-Intermediatradikals führt dazu, dass sich die Lebensdauer dieses Radikals deutlich erhöht, wodurch eine Terminierungsreaktion am Intermediat möglich wird, bevor die Fragmentierung eintritt. Dies wird auch als Kreuzterminierung bezeichnet.

Die Kreuzterminierung kann in bestimmten Fällen sogar zu einer Inhibierung der Polymerisation führen (s. Schema 4-3). Die im Rahmen dieser Arbeit durchgeführten Polymerisationen

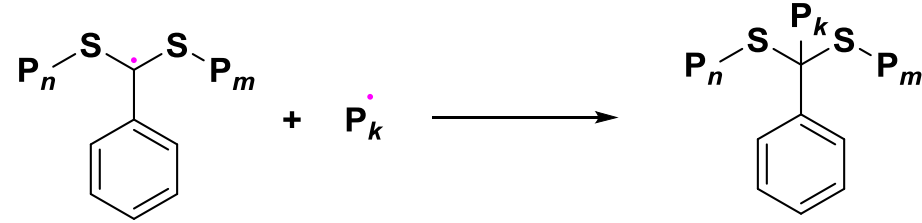

Schema 4-3 Beispiel für eine mögliche Kreuzterminierung eines Dithioester-Intermediat-Radikals wurden allerdings in Gegenwart des Trithiocarbonats EPHT durchgeführt. Da TrithiocarbonatIntermediate aber nicht stabil genug sind und sehr schnell fragmentieren, sind sie nicht in der Lage, eine signifikante Retardierung zu bewirken. ${ }^{[216]}$ Somit kann davon ausgegangen werden, dass dies ebenfalls nicht Grund für die in den isothermen DCS-Messungen gefundene Retardierung der RAFT-Polymerisationen ist.

Die bisher diskutierten möglichen Gründe für die gefundene Retardierung der RAFTPolymerisationen sind allerdings Beobachtungen, die vor allem bei thermisch-initiierten Polymerisationen gemacht wurden. Da es sich bei den in dieser Arbeit untersuchten 
Polymerisationen aber um eine UV-initiierte, radikalische Polymerisationen handelte, muss noch eine weitere Möglichkeit, wie es zu einer Retardierung der Polymerisation durch das RAFTAgens gekommen sein kann, in Betracht gezogen werden.

\section{Absorption der UV-Strahlung}

Dabei handelt es sich um eine mögliche Absorption des RAFT-Agens bei der von der UV-Lampe eingestrahlten Wellenlänge von $366 \mathrm{~nm}$. Nach Gleichung (14) ist die Zerfallsgeschwindigkeit des Photoinitiators bei der UV-initiierten, radikalischen Polymerisation abhängig von der Intensität der in die Polymerisationslösung einfallenden Strahlung. Diese hat somit nach Gleichung (15) auch einen Einfluss auf die Polymerisationsgeschwindigkeit. Kommt es zu einer signifikanten Absorption von Strahlung durch das RAFT-Agens, hat dies einen langsameren Initiatorzerfall zur Folge, der zu einer Retardierung der Polymerisation führt.

Wird die Verringerung der Lichtintensität allein durch Absorption der Strahlung hervorgerufen, so wird diese auch als Absorbanz bezeichnet, die mittels UV/Vis-Spektroskopie gemessen werden kann. Dazu wurde eine Lösung des RAFT-Agens EPHT sowie eine Lösung des Photoinitiators MMMP mit jeweils bekannter Konzentration im Monomer $n$-Butylacrylat hergestellt. Die bei den gegeben Konzentrationen der Substanzen erhaltenen Absorbanzen bei einer Wellenlänge von $366 \mathrm{~nm}$ und einer Schichtdicke von $1 \mathrm{~cm}$ sind in Tabelle 4-20 zu finden.

Tabelle 4-20 Mittels UV/Vis-Spektroskopie gemessene Absorbanzen des RAFT-Agens EPHT und des Photoinitiators MMMP bei $22{ }^{\circ} \mathrm{C}$ mit $n$-Butylacrylat als Lösungsmittel und einer Wellenlänge von $366 \mathrm{~nm}$. Die Schichtdicke betrug $1 \mathrm{~cm}$.

\begin{tabular}{ccc}
\hline Substanz & Konzentration $[\mathbf{m o l} / \mathbf{m L}]$ & Absorbanz bei $\mathbf{3 6 6} \mathbf{~ n m}$ \\
\hline EPHT & $1,7 \cdot 10^{-7}$ & 0,05 \\
MMMP & $1,0 \cdot 10^{-7}$ & 0,10 \\
\hline
\end{tabular}

Obwohl die Konzentrationen der beiden Substanzen nicht vollständig gleich sind und auch nicht den Konzentrationen dieser Substanzen in den Polymerisationsmischungen entsprechen, ist es möglich, die Absorbanzen für alle Konzentrationen zu berechnen. Nach dem Lambert-Beer'schen Gesetz (s. Gleichung (52)) hängt die Absorbanz bei einer bestimmten Wellenlänge $A_{\lambda}$ linear von der Konzentration der absorbierenden Spezies ab. Es gilt:

$$
A_{\lambda}=\varepsilon_{\lambda} \cdot c_{\mathrm{EPHT}} \cdot d
$$


Dabei steht $\varepsilon_{\lambda}$ für den molaren dekadischen Absorptionskoeffizienten und $d$ für die Schichtdicke der Probe. Ist die Konzentration der absorbierenden Spezies bekannt, kann mit der gemessenen Absorbanz mit:

$$
\varepsilon_{\lambda}=\frac{A_{\lambda}}{c_{\mathrm{EPHT}} \cdot d}
$$

der molare dekadische Absorptionskoeffizient für die entsprechende Wellenlänge (366 nm) berechnet werden. Für EPHT beträgt dieser $\varepsilon_{\lambda}=3,0 \cdot 10^{5} \mathrm{~mL} \cdot(\mathrm{mol} \cdot \mathrm{cm})^{-1}$ für $\mathrm{MMMP}$ $\varepsilon_{\lambda}=9,9 \cdot 10^{5} \mathrm{~mL} \cdot(\mathrm{mol} \cdot \mathrm{cm})^{-1}$. Mit Gleichung (52) können nun wiederum die Absorbanzen für alle verwendeten EPHT- und MMMP-Konzentrationen berechnet werden. Diese sind in Tabelle 4-21 zusammengefasst.

Tabelle 4-21 Molare Anteile des RAFT-Agens EPHT und Massenanteile des Photoinitiators MMMP, die entsprechenden Konzentrationen sowie die daraus resultierenden Absorbanzen in $n$-Butylacrylat bei einer Wellenlänge von $366 \mathrm{~nm}$ und einer Schichtdicke von $1 \mathrm{~cm}$.

\begin{tabular}{cccccc}
\hline $\begin{array}{c}\text { EPHT-Anteil } \\
\text { [mol-\%o }]\end{array}$ & $\boldsymbol{c}_{\text {EPHT }}[\mathbf{m o l} / \mathbf{m L}]$ & $\boldsymbol{A}_{\boldsymbol{\lambda}}$ & $\begin{array}{c}\text { MMMP-Anteil } \\
\text { [Gew.-\%] }\end{array}$ & $\boldsymbol{c}_{\text {MMMP }}[\mathbf{m o l} / \mathbf{m L}]$ & $\boldsymbol{A}_{\boldsymbol{\lambda}}$ \\
\hline 0 & 0 & 0 & 2 & $7,95 \cdot 10^{-5}$ & 79 \\
1 & $0,87 \cdot 10^{-5}$ & 2,6 & 2 & $7,95 \cdot 10^{-5}$ & 79 \\
2 & $1,74 \cdot 10^{-5}$ & 5,2 & 2 & $7,95 \cdot 10^{-5}$ & 79 \\
4 & $3,47 \cdot 10^{-5}$ & 10,4 & 2 & $7,95 \cdot 10^{-5}$ & 79 \\
\hline
\end{tabular}

Angesichts dieser sehr hohen Absorbanzen in Tabelle 4-21 sollte beachtet werden, dass die Schichtdicken der Polymerisationsmischungen bei den kinetischen DSC-Messungen kleiner waren als $1 \mathrm{~mm}$ (Tiefe des DSC-Probenpfännchens), was zu kleineren Absorbanzen geführt hat. ${ }^{\text {a }}$ Auch wenn die Absorbanzen des EPHTs bei den verwendeten Konzentrationen immer deutlich kleiner waren als die des MMMP, zeigen diese Ergebnisse doch, dass eine nicht zu vernachlässigende Absorption durch das EPHT vorliegt. Somit ist die Absorption der UVStrahlung durch das EPHT der wahrscheinlichste Grund für die Retardierung der RAFTPolymerisationen. Weiterhin kann sie auch die mit steigendem EPHT-Anteil zunehmenden Inhibitionsphasen der RAFT-Polymerisationen erklären (s. Abschnitt 4.1.4.2). Durch die Absorption des RAFT-Agens und dem daraus resultierenden langsameren Initiatorzerfall stehen

\footnotetext{
a Die genauen Schichtdicken $\mathrm{zu}$ bestimmen ist nicht trivial. Zum einen verändern diese sich durch Volumenschrumpfung während der Polymerisation und zum andern ist es nicht möglich die Netzwerke aus den Pfännchen zu entfernen ohne sie dabei stark zu beschädigen, da sie sehr klebrig sind.
} 
zu Beginn der Reaktion weniger Primärradikale zur Verfügung, die den in den Polymerisationsmischungen enthalten Sauerstoff „unschädlich“ machen.

Um sicherzustellen, dass die Retardierung der Polymerisationen nicht der Grund für die veränderten mechanischen und Quellungseigenschaften der Netzwerke war (s. Kapitel 4.1.1 und Kapitel 4.1.3), wurde das Trithiocarbonat $S$-S -Diethyltrithiocarbonat (DET) synthetisiert und statt des EPHTs zu den Polymerisationsmischungen zugegeben. Deren genaue Zusammensetzungen sind in Kapitel 6.2.4 zu finden.

DET sollte aufgrund seines chemischen Aufbaus nicht in der Lage sein, einen RAFTMechanismus zu etablieren (s. Schema 4-4). Es verfügt aber trotzdem über die farbgebende und somit die Absorption hervorrufende Trithiocarbonat-Gruppe, womit es über eine dem EPHT ähnliche Absorbanz von 0,05 bei einer Konzentration von $1,1 \cdot 10^{-7} \mathrm{~mol} / \mathrm{mL}$ in $n$-Butylacrylat verfügt. Dies sollte wieder eine geringere Strahlungsintensität in den Polymerisationsmischungen und somit einen langsameren Initiatorzerfall zur Folge haben, was zu einer Retardierung der Polymerisation führen sollte.
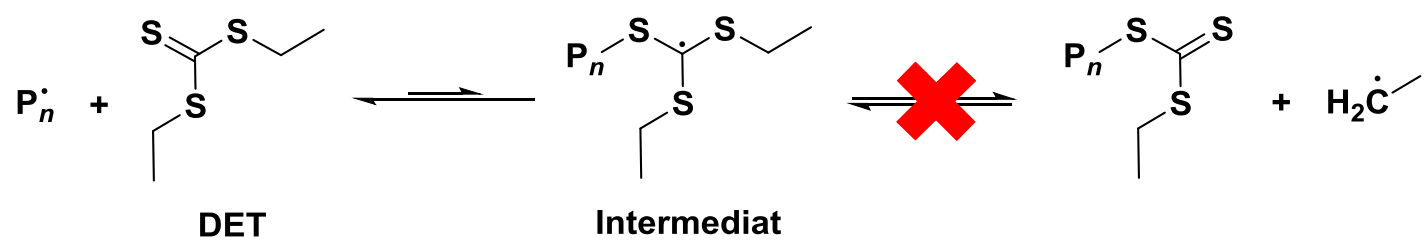

Schema 4-4 Addition eines propagierenden Radikals an ein DET-Molekül.

Um dies zu überprüfen, wurden entsprechend der vorhergehenden Untersuchungen in Abschnitt 4.1.4.2 die Reaktionswärme der Polymerisationen mittels isothermer DSC aufgenommen und anhand der Gleichungen (50) und (51) der Monomerumsatz sowie die Polymerisationsgeschwindigkeit berechnet. Eine Auftragung der beiden Größen gegeneinander zeigt, dass die erwartete Retardierung in den DET-Systemen auftritt (s. Abbildung 4-45). Dies wird durch die, bei steigendem DET-Anteil in den Polymerisationsmischungen, sinkenden Polymerisationsgeschwindigkeiten deutlich. Weiterhin ist anhand der Verläufe der Kurven aus Abbildung 4-45 zusätzlich erkennbar, dass diese Polymerisationen anders verlaufen sind als die mit EPHT als CTA (s. Abbildung 4-43). Im Gegensatz zu diesen nehmen die Polymerisationsgeschwindigkeiten der DET-Systeme mit steigendem Monomerumsatz zu. Sie zeigen also eine stärkere Selbstbeschleunigung als die EPHT-Systeme. Die größere Ähnlichkeit mit der konventionellen Polymerisation ist der erste Hinweis, dass keine RAFT-Polymerisation stattgefunden haben kann. Somit kann der RAFT-Mechanismus als Grund für die Retardierung der Polymerisationen ausgeschlossen werden. 


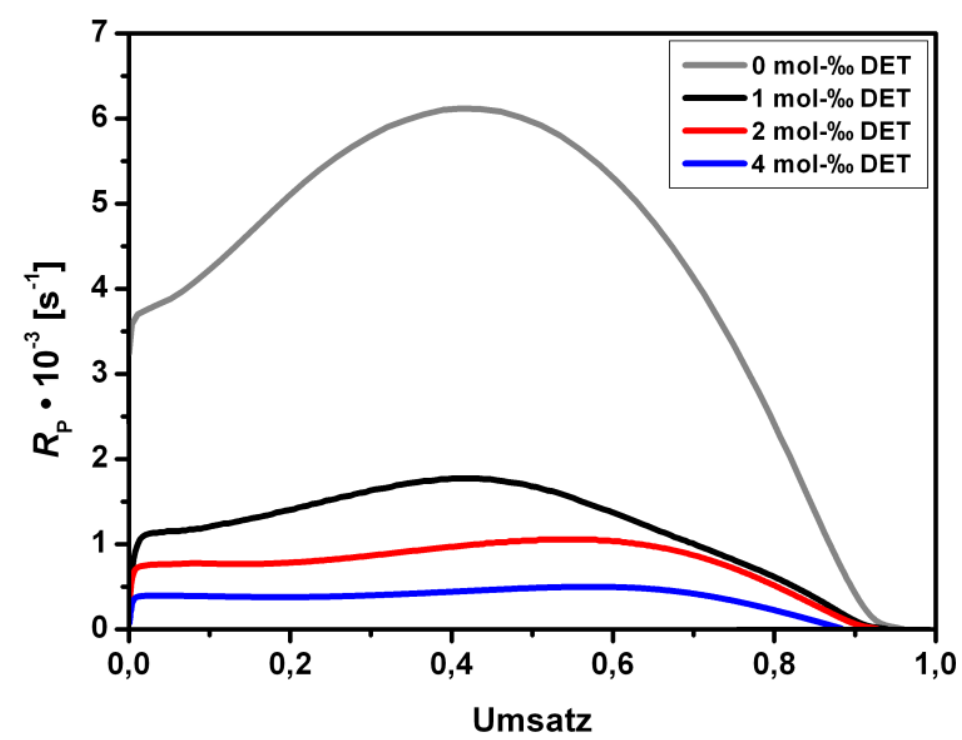

Abbildung 4-45 Polymerisationsgeschwindigkeit $R_{\mathrm{P}}$ aufgetragen gegen den Monomerumsatz $C_{\mathrm{M}}$ von UV-initiierten, radikalischen Polymerisationen ausgewählter Poly(BA-co-BDDA)-Netzwerke mit einem molaren Anteil von 0,5 mol-\% BDDA als Vernetzer ohne das Trithiocarbonat DET (-), sowie mit 1 mol-\%o (-), 2 mol-\%o (-) und 4 mol-\%o (-) DET. Alle Polymerisationen fanden bei $21^{\circ} \mathrm{C}$ statt, mit 2 Gew.-\% MMMP als Photoinitiator und einer Lichtintensität von $9 \mathrm{~mW} / \mathrm{cm}^{2}$ bei $366 \mathrm{~nm}$.

Anschließend wurden von dem Netzwerk, in dem ein molarer Anteil von 2 mol-\% DET enthalten war, Probekörper für Zugversuche entsprechend der in Kapitel 6.2.1 beschriebenen Vorschrift hergestellt. Die Zugversuche dienten wieder der Ermittlung der E-Moduln und der Bruchdehnungen der Netzwerke. Zusätzlich wurden wieder der Volumenquellungsgrad und die Fraktion der Extrahierbaren in Aceton bestimmt. Diese Werte wurden mit denen eines konventionellen und eines RAFT-Netzwerkes mit 2 mol-\%o EPHT als RAFT-Agens verglichen (s. Tabelle 4-22).

Tabelle 4-22 Ergebnisse der Zug- und Quellungsversuche eines Poly(BA-co-BDDA)-Netzwerks mit 0,5 mol-\% BDDA als Vernetzer und 2 mol-\%o vom Trithiocarbonat DET im Vergleich mit den Ergebnissen eines konventionellen sowie eines RAFT-Netzwerkes mit ansonsten analogen Zusammensetzungen und Versuchsbedingungen (s. Kapitel 6.2).

\begin{tabular}{lccc}
\hline Netzwerkeigenschaft & DET & konventionell & EPHT \\
\hline E-Modul [MPa] & 0,37 & 0,38 & 0,17 \\
Bruchdehnung [\%] & 80,4 & 80,6 & 110,4 \\
Volumenquellungsgrad & 5,4 & 5,6 & 8,8 \\
Extrahierbare [\%] & 6,5 & 3,5 & 7,6 \\
\hline
\end{tabular}


Die Daten aus Tabelle 4-22 lassen klar erkennen, dass die Poly(BA-co-BDDA)-Netzwerke mit dem Trithiocarbonat DET anstatt des RAFT-Agens EPHT in ihren mechanischen und Quellungseigenschaften eher einem konventionellen Netzwerk entsprechen. Das bedeutet, dass auch ihr innerer Aufbau und ihre Mikrostruktur, denen eines konventionellen Netzwerks ähneln müssen. Trotz der auch in den DET-Systemen vorhandenen Retardierung der Polymerisation müssen die resultierenden Netzwerke über eine Vielzahl von Netzwerkdefekten in Form von Verhakungen und Verschlaufungen verfügen, die zu einer höheren Netzkettendichte führen als theoretisch durch die enthaltene Stoffmenge des Vernetzers BDDA möglich ist. Dies wiederum hat einen höheren E-Modul und eine geringere Bruchdehnung zur Folge.

Auch zeigen diese Ergebnisse, dass die Menge an Extrahierbaren nur einen geringen Einfluss haben kann. Denn diese ist bei den DET-Netzwerken ähnlich hoch wie bei den EPHTNetzwerken. Trotzdem sind die Unterschiede der mechanischen und Quellungseigenschaften zwischen den beiden Netzwerken deutlich zu erkennen. Die Menge an Extrahierbaren ist mit knapp 7 \% möglicherweise immer noch zu klein, und kann im Vergleich zum Einfluss, der in den Netzwerken vorhandenen Verhakungen und Verschlaufungen, vernachlässigt werden. Dies ist ein weiterer Beleg dafür, dass die Retardierung nicht auf den RAFT-Mechanismus zurückgeführt werden kann, sondern vermutlich durch die Absorption der Trithiocarbonat-Gruppe bei der eingestrahlten Wellenlänge und einer daraus resultierenden Absenkung der Radikalkonzentration im System hervorgerufen wird.

\subsubsection{Variation des molaren EPHT-Anteils bei Poly(EA-co-BDDA)-Netzwerken}

Um die Ergebnisse aus den DSC-Messungen der Poly(BA-co-BDDA)-Netzwerke in einem anderen Monomersystem zu bestätigen, wurde der Einfluss des molaren EPHT-Anteils in den Polymerisationsmischungen auf die Polymerisationskinetik auch für Poly(EA-co-BDDA)Netzwerke untersucht. Dazu wurde der molare Anteil vom Vernetzer BDDA mit 0,5 mol-\% (bezogen auf die Stoffmenge des Monomers) wieder konstant gehalten und der molare Anteil vom RAFT-Agens EPHT von 0 bis 4 mol-\%o variiert (bezogen auf die Stoffmenge des Monomers). Die genauen Zusammensetzungen der Polymerisationsmischungen sind in Tabelle 6-5 zu finden.

Aufgrund des im Vergleich mit BA zehnfach höheren Dampfdrucks des Ethylacrylats, war es allerdings nicht möglich, diese Messungen mittels isothermer DSC durchzuführen. Durch den Stickstoffstrom in der Messzelle verdampfte das EA in großen Mengen, bevor es polymerisieren konnte. Darum wurden die Umsätze der Polymerisationen mittels Fourier-TransformationsInfrarot-(FTIR)-Spektroskopie bestimmt. Informationen zur Durchführung der Messungen sind in Kapitel 6.3.4 zu finden. Die erhaltenen Umsatz/Zeit-Kurven sind in Abbildung 4-46 dargestellt. 


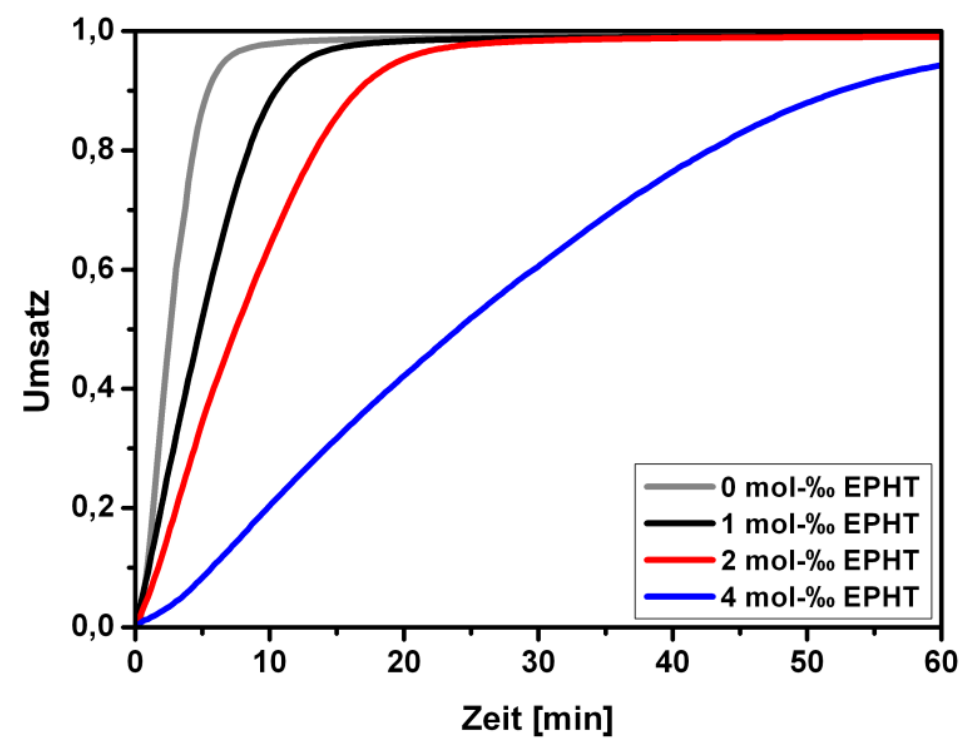

Abbildung 4-46 Aus FTIR-Spektroskopie erhaltene Umsatz/Zeit-Kurven von UV-initiierten, radikalischen Polymerisationen ausgewählter Poly(EA-co-BDDA)-Netzwerke mit einem molaren Anteil von 0,5 mol-\% BDDA als Vernetzer ohne das RAFT-Agens EPHT (-) sowie mit 1 mol-\%o (-), 2 mol-\%o (-) und 4 mol-\%o (-) EPHT. Alle Polymerisationen fanden bei $21^{\circ} \mathrm{C}$ statt, mit 2 Gew.-\% MMMP als Photoinitiator und einer Lichtintensität von $9 \mathrm{~mW} / \mathrm{cm}^{2}$ bei $366 \mathrm{~nm}$.

An den Verläufen des Monomerumsatzes zeigt sich ein ähnliches Bild wie bei den Poly(BA-coBDDA)-Netzwerken. Mit steigendem molaren EPHT-Anteil beginnt die Reaktion zunehmend verzögert, der Anstieg des Umsatzes ist langsamer und der maximale Umsatz wird deutlich später erreicht. Die maximalen Umsätze sind in Tabelle 4-23 zusammengefasst. Um die Retardierung der Reaktion noch $\mathrm{zu}$ verdeutlichen, wurde die Reaktionskinetik wieder mittels Gleichung (9) berechnet und gegen die Reaktionszeit aufgetragen (s. Abbildung 4-47). ${ }^{\mathrm{a}}$

\footnotetext{
${ }^{a}$ Auf die Bestimmung der Polymerisationsgeschwindigkeiten wurde verzichtet, da aufgrund der im Vergleich zu den DSC-Messungen geringeren Messwertdichte der FTIR-Spektroskopie diese nur unter erheblicher Glättung der Kurven möglich gewesen wäre. Dies hätte die erhaltenen Ergebnisse massiv verfälscht.
} 


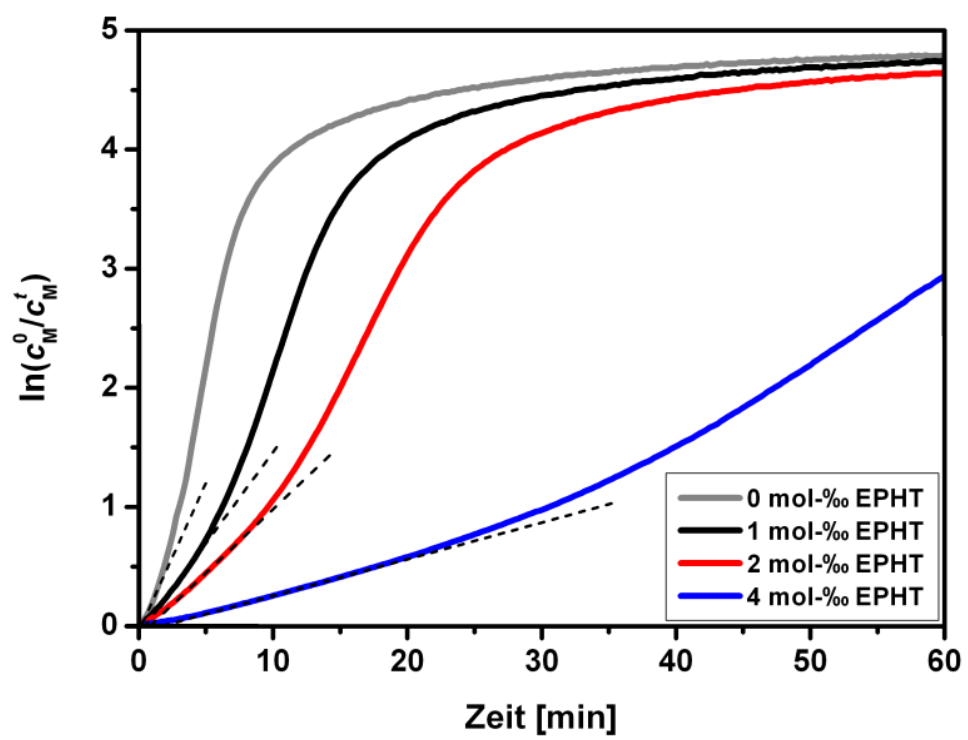

Abbildung 4-47 Pseudo-Erste-Ordnung-Auftragung von UV-initiierten, radikalischen Polymerisationen ausgewählter Poly(EA-co-BDDA)-Netzwerke mit einem molaren Anteil von 0,5 mol-\% BDDA als Vernetzer ohne das RAFT-Agens EPHT (-) sowie mit 1 mol-\%o (-), 2 mol-\%o (-) und 4 mol-\%o (-) EPHT. Die gestrichelten Linien stellen eine lineare Anpassung der anfänglichen nach idealer Polymerisationskinetik verlaufenden Bereiche der Kurven dar. Alle Polymerisationen fanden bei $21{ }^{\circ} \mathrm{C}$ statt. Als Photoinitiator wurden bei allen Polymerisationen 2 Gew.-\% MMMP verwendet. Die Lichtintensität betrug $9 \mathrm{~mW} / \mathrm{cm}^{2}$ bei $366 \mathrm{~nm}$.

An der abnehmenden Steigung der Kurven wird die zunehmende Retardierung der Polymerisation mit steigendem molaren EPHT-Anteil deutlich. Zusätzlich zeigt sich auch bei dieser Auftragung, dass der Gel-Effekt hier zunehmend später eintritt. Die aus dieser Auftragung entnommenen Steigungen und Zeitpunkte sind in Tabelle 4-23 zusammengefasst.

Tabelle 4-23 Maximale Monomerumsätze $C_{\mathrm{M}}^{\max }$, Proportionalitätsfaktor $K_{\mathrm{P}}$ sowie die Zeitpunkte, an denen der GelEffekt einsetzt $t_{\mathrm{Gel}}$ von Polymerisationsmischungen für Poly(EA-co-BDDA)-Netzwerken mit einem molaren Anteil von 0,5 mol-\% BDDA als Vernetzer, aber unterschiedlichen molaren Anteilen vom RAFT-Agens EPHT. Die Werte wurden aus Abbildung 4-46 und Abbildung 4-47 entnommen.

\begin{tabular}{cccc}
\hline EPHT-Anteil [mol-\%] & $\boldsymbol{C}_{\mathbf{M}}^{\text {max }}[\%]$ & $\boldsymbol{K}_{\mathbf{P}}$ & $\boldsymbol{t}_{\mathrm{Gel}}[\mathbf{m i n}]$ \\
\hline 0 & 99 & 0,32 & 2 \\
1 & 99 & 0,16 & 5 \\
2 & 99 & 0,11 & 7 \\
4 & 98 & 0,03 & 20 \\
\hline
\end{tabular}

Trotz einer anderen Messmethode als bei der Untersuchung der Polymerisationskinetik der Poly(BA-co-BDDA)-Netzwerke (s. Abschnitte 4.1.4.1 und 4.1.4.2) und unterschiedlichen 
Monomeren, werden hier die gleichen Tendenzen in den Polymerisationsverläufen erkennbar. Somit können diese Messungen als Bestätigung dafür angesehen werden, dass die Effekte des RAFT-Agens EPHT auf die Kinetik einer UV-initiierten, statistisch-vernetzenden Polymerisation bei verschiedenen Acrylat-Monomeren auftreten. Da sich außerdem wieder eine deutliche Retardierung der RAFT-Polymerisationen zeigt, kann davon ausgegangen werden, dass dafür die Absorption der UV-Strahlung durch die Trithiocarbonat-Gruppe verantwortlich ist.

\subsubsection{Abschließende Bemerkungen}

Mittels isothermer DSC konnte gezeigt werden, dass bei der Polymerisation von Poly(BA-coBDDA)-Netzwerken nach dem RAFT-Mechanismus die Reaktionen mit einem höheren molaren BDDA-Anteil früher den maximalen Umsatz erreichen und dass diese über eine früher einsetzende und stärkere Selbstbeschleunigung verfügen. Diese lässt sich auf einen stärkeren Gel-Effekt zurückführen, der bei Netzwerken mit einer größeren Netzkettendichte ausgeprägter ist. Darum werden die RAFT-Polymerisationen in ihrem Verlauf den konventionellen, radikalischen Polymerisationen immer ähnlicher.

Weiterhin konnte gezeigt werden, dass eine Erhöhung des molaren EPHT-Anteils in den Polymerisationsmischungen zu einer deutlichen Retardierung der Polymerisationen führt. Als wahrscheinlichste Ursache für dieses Phänomen konnte nach Ausschluss weiterer möglicher Gründe die Absorption von UV-Strahlung durch das RAFT-Agens ermittelt werden. Anhand eines Systems, das ein Trithiocarbonat enthielt, welches allerdings nicht in der Lage ist, einen RAFT-Mechanismus zu etablieren, konnte gezeigt werden, dass die Veränderung der mechanischen- und der Quellungseigenschaften von Poly(BA-co-BDDA)-Netzwerken nicht durch die Retardierung der Polymerisation hervorgerufen werden, sondern aus dem durch den RAFTMechanismus hervorgerufenen veränderten Mechanismus der Netzwerkbildung resultieren. 


\subsubsection{Einfluss der RAFT-Polymerisation auf die Klebrigkeit der Netzwerke}

Während der Untersuchung der mechanischen Eigenschaften und des Quellungsverhaltens der Poly(BA-co-BDDA)- und Poly(EA-co-BDDA)-Netzwerke (s. Abschnitte 4.1.1.3 und 4.1.3.2) zeigte sich, dass diese, vor allem mit zunehmenden molaren Anteil des RAFT-Agens EPHT in der Polymerisationsmischung, signifikant klebriger wurden. Um diese Eigenschaft nachzuweisen, wurden zunächst Rollkugel-Tests (s. Kapitel 2.5) mit diesen Netzwerken durchgeführt.

Die Messungen (s. Abschnitt 6.3.3.1) wurden direkt nach der Synthese, 30 Minuten nach der Synthese und 24 Stunden nach der Synthese bei Raumtemperatur durchgeführt. Die erhaltenen Ergebnisse wurden für die Poly(BA-co-BDDA)-Netzwerke in Tabelle 4-24 und für die Poly(EA-coBDDA)-Netzwerke in Tabelle 4-25 zusammengefasst. Während der Rollkugel-Tests zeigte sich, dass die Poly(BA-co-BDDA)-Netzwerke so klebrig waren, dass eine Hartmetallkugel für die Messungen verwendet werden musste. Diese hat eine Dichte von $14,3 \mathrm{~g} / \mathrm{cm}^{3}$. Das bedeutet, dass sie schwerer ist als eine für diese Tests standardmäßig verwendete Edelstahlkugel. Dies stellte sicher, dass die Kugel eine kleine Strecke über das Netzwerk rollen konnte, ohne sofort festzukleben, da ihre kinetische Energie am Ende der Rampe des Rollkugeltesters höher war als die einer Edelstahlkugel.

Tabelle 4-24 Bei Rollkugel-Tests ermittelte Längen der Laufstrecke einer Hartmetallkugel über ein Poly-(BA-coBDDA)-Netzwerk aus UV-initiierter, konventioneller radikalischer Polymerisation sowie Netzwerken aus UVinitiierter RAFT-Polymerisation mit 1 mol-\%o, 2 mol-\%o und 4 mol-\%o des RAFT-Agens EPHT. Alle Netzwerke enthielten 0,5 mol-\% des Vernetzers BDDA. Alle Messungen fanden bei Raumtemperatur direkt nach der Synthese des jeweiligen Netzwerks, 30 Minuten nach der Synthese und 24 Stunden nach der Synthese statt. Die gezeigten Werte entsprechen einem Mittelwert aus jeweils fünf Durchläufen, die Fehler entsprechen den Standardabweichungen. Weiterhin wurden die Mittelwerte aus den Messungen bei den drei verschiedenen Zeitpunkten ermittelt.

\begin{tabular}{ccccc}
\hline Anteil EPHT [mol-\%o] & \multicolumn{4}{c}{ Laufstecke der Kugel [cm] } \\
& $\mathbf{2}$ in & 30 min & 24 h & Mittelwerte \\
\hline 0 & $5,7 \pm 0,9$ & $4,4 \pm 0,2$ & $5,3 \pm 0,5$ & $5,2 \pm 0,6$ \\
1 & $2,8 \pm 0,4$ & $3,1 \pm 0,1$ & $2,6 \pm 0,2$ & $2,8 \pm 0,2$ \\
2 & $2,4 \pm 0,4$ & $2,4 \pm 0,2$ & $2,0 \pm 0,4$ & $2,3 \pm 0,2$ \\
4 & $1,7 \pm 0,4$ & $1,6 \pm 0,1$ & $1,3 \pm 0,4$ & $1,5 \pm 0,2$ \\
\hline
\end{tabular}

Eine Veränderung der Klebrigkeit mit der Zeit kann anhand der vorliegenden Daten nicht gefunden werden. Im Rahmen der Standardabweichungen zeigt sich kein signifikanter Unterschied zwischen der Laufstrecke der Kugel direkt nach der Synthese, sowie 30 Minuten und 24 Stunden später (s. Tabelle 4-24). Darum wurden die Mittelwerte aus allen Messungen berechnet. An diesen Mittelwerten ist erkennbar, dass schon eine Zugabe von 1 mol-\%o EPHT eine deutliche Zunahme der Klebrigkeit zur Folge hatte. Die Strecke, die von der Kugel auf dem 
Netzwerk zurückgelegt wurde, nahm nahezu um die Hälfte ab. Beim weiteren Anstieg des EPHTAnteils zeigte sich nur noch eine geringe Verkürzung der Strecke. Die Tendenz, dass mit steigendem EPHT-Anteil in den Poly(BA-co-BDDA)-Netzwerken deren Klebrigkeit zunimmt, ist hier aber eindeutig (s. Tabelle 4-24). Ein ähnlicher Einfluss des EPHTs auf die Eigenschaften der Poly(BA-co-BDDA)-Netzwerke konnte schon bei den Quellungsuntersuchungen mittels SSENMR gefunden werden (s. Abschnitt 4.1.3.3). Für die Rollkugel-Tests der Poly(EA-co-BDDA)Netzwerke wurde anschließend eine Edelstahlkugel verwendet. Wie in Tabelle 4-25 zu erkennen ist, reichte die Klebrigkeit des konventionellen Netzwerks nicht aus, um diese leichtere Kugel entlang einer Strecke von $10 \mathrm{~cm}$ zum Stillstand zu bringen.

Tabelle 4-25 Bei Rollkugel-Tests ermittelte Längen der Laufstrecke einer Edelstahlkugel über ein Poly-(EA-co-BDDA)Netzwerk aus UV-initiierter, konventioneller radikalischer Polymerisation sowie Netzwerken aus UV-initiierter RAFTPolymerisation mit 1 mol-\%o, 2 mol-\%o und 4 mol-\%o des RAFT-Agens EPHT. Alle Netzwerke enthielten 0,5 mol-\% des Vernetzers BDDA. Alle Messungen fanden bei Raumtemperatur direkt nach der Synthese des jeweiligen Netzwerks, 30 Minuten nach der Synthese und 24 Stunden nach der Synthese statt. Die gezeigten Werte entsprechen einem Mittelwert aus jeweils fünf Durchläufen, die Fehler entsprechen den Standardabweichungen. Weiterhin wurden die Mittelwerte aus den Messungen bei den drei verschiedenen Zeitpunkten ermittelt.

\begin{tabular}{ccccc}
\hline EPHT-Anteil [mol-\%o] & \multicolumn{4}{c}{ Laufstecke der Kugel [cm] } \\
& $\mathbf{2} \mathbf{~} \mathbf{m i n}$ & $\mathbf{3 0} \mathbf{~ m i n}$ & $\mathbf{2 4} \mathbf{~ h}$ & Mittelwerte \\
\hline 0 & & & & \\
1 & $6,3 \pm 0,9$ & $6,1 \pm 0,5$ & $8,4 \pm 1,5$ & $6,9 \pm 1,3$ \\
2 & $4,3 \pm 0,7$ & $3,9 \pm 0,5$ & $5,5 \pm 0,9$ & $4,6 \pm 0,8$ \\
4 & $2,0 \pm 0,9$ & $1,5 \pm 0,3$ & $2,6 \pm 0,5$ & $2,0 \pm 0,5$ \\
\hline
\end{tabular}

Aber auch bei diesen Netzwerken war keine eindeutige Abhängigkeit der Messwerte von dem Zeitpunkt der Messung erkennbar. Darum wurden auch hier wieder die Mittelwerte aus allen Messungen bestimmt (s. Tabelle 4-25). Es zeigt sich, dass schon bei der Zugabe von 1 mol-\%o EPHT zu der Polymerisationsmischung die Klebrigkeit des resultierenden Netzwerks deutlich zunahm. Eine weitere Zugabe von EPHT führte auch für die Poly(EA-co-BDDA)-Netzwerke zu einer zunehmenden Klebrigkeit und somit zu einer kürzeren Laufstrecke der Kugel über das Netzwerk. Es fällt auf, dass der Einfluss des RAFT-Agens auf die Klebrigkeit der Netzwerke ausgeprägter ist als bei den Poly(BA-co-BDDA)-Netzwerken. Weiterhin wurde sowohl bei den Poly(BA-co-BDDA)- als auch bei den Poly(EA-co-BDDA)-Netzwerken beobachtet, dass die Kugel eine Spur auf den Oberflächen der RAFT-Netzwerke hinterließ, diese sich aber nach 30 Minuten wieder deutlich verringerte und innerhalb von $24 \mathrm{~h}$ nahezu vollständig verschwand. Dies spricht dafür, dass sich auf den Oberflächen der Netzwerke eine fluide Phase befinden muss. Diese Tatsache wird im Verlauf dieses Kapitels noch näher untersucht.

Um einen Eindruck von der Haftkraft der Netzwerke zu gewinnen, wurden auch Probe-TackVersuche durchgeführt (s. Kapitel 2.5). Allerdings erwies sich die Durchführung dieser Versuche 
als nicht trivial. Der Kraftaufnehmer der Zugmaschine, an der die Zugversuche durchgeführt wurden, war nicht empfindlich genug, um die Haftkräfte der Netzwerke hinreichend genau zu messen. Darum wurden die Haftkräfte der Netzwerke mittels eines empfindlicheren Kraftaufnehmers ermittelt. Bei dem verfügbaren Aufbau war es jedoch nicht möglich, den Stempel mit konstanter Geschwindigkeit von der Probe $\mathrm{zu}$ entfernen, da dies nur per Handschraube möglich war. Außerdem waren die Messintervalle mit rund drei Sekunden zwischen zwei Messwerten relativ groß. Eine genaue Beschreibung der Probe-Tack-Versuche kann in Abschnitt 6.3.3.2 gefunden werden. Die erhaltenen Verläufe der aufgenommen Haftkraftkurven zeigen allerdings, dass es doch möglich war, reproduzierbare Werte zu erhalten und auch die Unterschiede zwischen einem konventionellen und einem RAFT-Netzwerk deutlich zu zeigen (s. Abbildung 4-48 und Abbildung 4-49). Trotzdem soll darauf hingewiesen werden, dass diese Werte nur als relative Vergleichswerte zwischen den konventionellen und RAFTNetzwerken dienen können und keine absoluten Haftkräfte darstellen. In Abbildung 4-48 und Abbildung 4-49 sind beispielhaft für jedes Monomer zwei Messreihen zu sehen: Eine für ein konventionelles Netzwerk und die Zweite für ein RAFT-Netzwerk mit 2 mol-\%o EPHT. Der molare Anteil des Vernetzers BDDA betrug beiden Fällen 0,5 mol-\%. Da sich zwei Messungen in einem Diagramm befinden, wurde die Zeitachse nicht absolut gewählt, sondern stellt nur einen relativen Verlauf dar. Wie schon erwähnt, soll nur der relative Unterschied der Haftkräfte zwischen den konventionellen und RAFT-Netzwerken verdeutlicht werden. Darum wird im Weiteren nicht auf die Beträge der Messwerte eingegangen und nur ihre relativen Verhältnisse zueinander diskutiert.

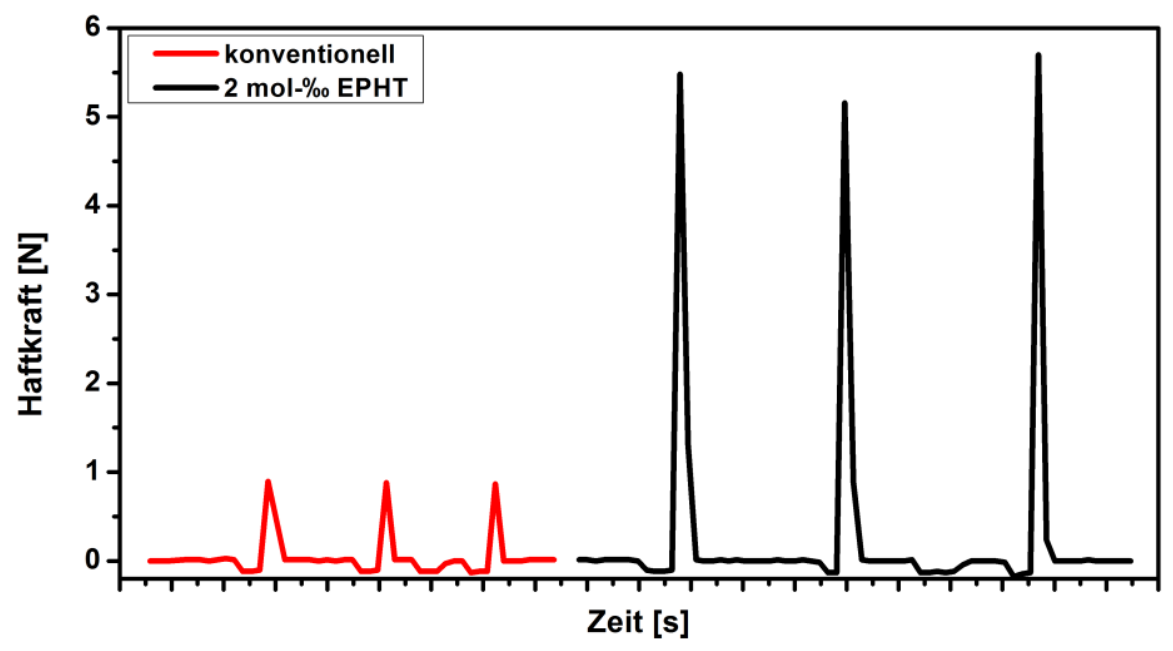

Abbildung 4-48 24 Stunden nach der Synthese bei Raumtemperatur durchgeführte Probe-Tack-Messungen eines Poly(BA-co-BDDA)-Netzwerks aus UV-initiierter konventioneller radikalischer Polymerisation (-) sowie eines Poly(BA-co-BDDA)-Netzwerks aus UV-initiierter RAFT-Polymerisation mit 2 mol-\% EPHT als RAFT-Agens (-). Beide Netzwerke enthielten 0,5 mol-\% BDDA als Vernetzer. 


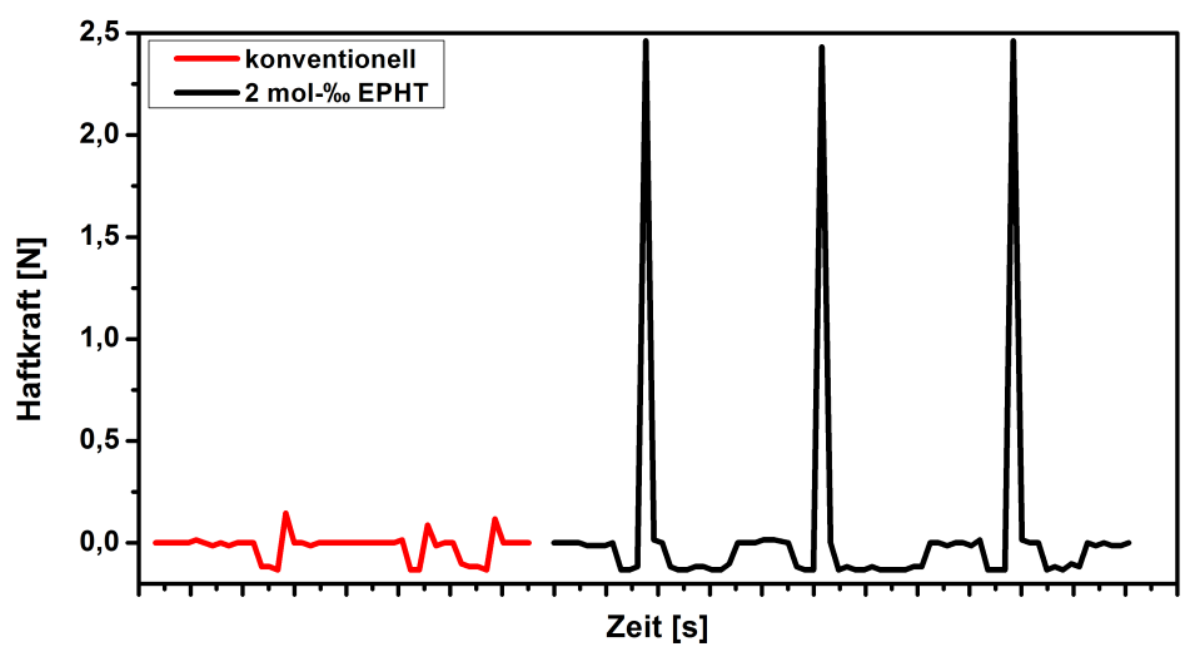

Abbildung 4-49 24 Stunden nach der Synthese bei Raumtemperatur durchgeführte Probe-Tack-Messungen eines Poly(EA-co-BDDA)-Netzwerks aus UV-initiierter konventioneller radikalischer Polymerisation (-) sowie eines Poly(EA-co-BDDA)-Netzwerks aus UV-initiierter RAFT-Polymerisation mit 2 mol-\% EPHT als RAFT-Agens (-). Beide Netzwerke enthielten 0,5 mol-\% BDDA als Vernetzer.

Die Haftkraftkurven zeigen, dass sich der erwartete Trend, der sich aus den Rollkugel-Tests ergab, bestätigt wird. Die RAFT-Netzwerke verfügen über signifikant höhere Haftkräfte. Das Maximum der Kurven bezeichnet den Punkt an dem der Stempel sich von der Probe ablöst. Negative Werte für die Kraft treten immer dann auf, wenn sich der Stempel auf den Proben befindet. Weiterhin sind die Haftkräfte der Poly(BA-co-BDDA)-Netzwerke höher als die der Poly(EA-co-BDDA)-Netzwerke.

Die Form der Messkurven entspricht der zu erwarteten Form einer elastomeren Haftkraftkurve (s. Abbildung 2-14). Trotz der klar elastomeren Haftkraftkurven, konnte vor allem bei den Proben mit relativ viel EPHT (2 und 4 mol-\%o) die Bildung von Fibrillen beobachtet werden. Diese waren aber sehr dünn und konnten darum wahrscheinlich nicht von dem Kraftaufnehmer registriert werden, da dieser dafür zu unempfindlich war.

Da Klebrigkeit und Haftung vor allem Fähigkeiten sind, die die Oberflächen von Stoffen betreffen, wurden mittels Rasterkraftmikroskopie (gebräuchlicher ist der englische Begriff: Atomic Force Microscopy, AFM) versucht, eine mögliche Erklärung für die bisher gemachten Beobachtungen zu finden. Die AFM ist in der Lage, mikroskopische Oberflächenstrukturen sichtbar zu machen. ${ }^{[217]}$ Zusätzlich bietet sie die Möglichkeit, die mechanischen Eigenschaften wie z. B. die Adhäsion einer Oberfläche direkt zu bestimmen. ${ }^{[18]}$ Die bei den durchgeführten AFM-Messungen erhaltenen Aufnahmen aus dem Messkanal der Adhäsion wurden mit dem Programm NanoScope Analysis (Version 1.4) ausgewertet (s. Abschnitt 6.3.5.2). Dabei wurden die 
mittleren Adhäsionen ${ }^{\mathrm{a}}$ der Netzwerke bestimmt. Die mittleren Adhäsionen waren, im Rahmen möglicher Messfehler und der Streuung der Messwerte sowie aufgrund kleiner Unterschiede bei der Kalibrierung, bei allen Netzwerken nahezu gleich, egal ob und wie viel EPHT in den Netzwerken enthalten war (s. Tabelle 4-26).

Tabelle 4-26 Mittels AFM im ScanAsyst-Modus bestimmte mittlere Adhäsionen von Poly(BA-co-BDDA)- und Poly(EA-co-BDDA)-Netzwerken mit 0,5 mol-\% des Vernetzers BDDA aus UV-initiierter, konventioneller radikalischer Polymerisation sowie aus UV-initiierter RAFT-Polymerisation mit 1 mol-\%, 2 mol-\%o und 4 mol-\%o des RAFT-Agens EPHT.

\begin{tabular}{ccc}
\hline EPHT-Anteil [mol-\%) & $\begin{array}{c}\text { Adhäsion [nN] } \\
\text { Poly(BA-co-BDDA) }\end{array}$ & $\begin{array}{c}\text { Adhäsion [nN] } \\
\text { Poly(EA-co-BDDA) }\end{array}$ \\
\hline 0 & 6,4 & 5,8 \\
1 & 5,7 & 4,6 \\
2 & 6,8 & 4,8 \\
4 & $13,7^{\mathrm{b}}$ & 6,2 \\
\hline
\end{tabular}

Der Grund für dieses Verhalten ist, dass mittels AFM nur die Mikroadhäsion zwischen einzelnen Molekülen und der wenige Nanometer dünnen Messspitze gemessen werden kann. Da es sich sowohl bei den konventionellen als auch bei den RAFT-Netzwerken chemisch gesehen jeweils um die gleiche Substanz handelt (entweder Poly-BA oder Poly-EA), ist es nachvollziehbar, dass auch die Adhäsion der einzelnen Moleküle gleich ist. Somit ergibt sich über die betrachtete Messfläche eine nahezu gleiche mittlere Adhäsion für die jeweiligen Netzwerke. Um die Zunahme der Klebrigkeiten der Netzwerke mit steigendem molaren Anteil des RAFT-Agens EPHT zu verstehen, müssen die Oberflächenstrukturen der Netzwerke betrachtet werden (s. Abbildung 4-50). Auf der Oberfläche des konventionellen Netzwerks lassen sich im Höhenprofil (s. Abbildung 4-50, links oben) viele runde Erhöhungen erkennen. Diese Erhöhungen zeigen jeweils nur eine geringe Adhäsion (s. Abbildung 4-50, rechts oben), dafür aber einen hohen Modul (s. Abbildung 4-50, links unten) und eine niedrige Deformation (s. Abbildung 4-50, rechts unten). $\mathrm{Zu}$ dem aus den AFM-Messungen erhaltenen Modul muss erwähnt werden, dass es sich hierbei um den Dejarguin-Muller-Toporov (DMT)-Modul ${ }^{[219,220]}$ handelt. Dieser kann in den E-Modul umgerechnet werden, ${ }^{[221]}$ worauf aber verzichtet wurde, da der DMT-Modul durchaus geeignet ist, um einen relativen Unterschied in der Steifigkeit der Probenoberfläche aufzuzeigen.

\footnotetext{
a Der Begriff mittlere Adhäsion bezeichnet die Adhäsion, welche die höchste Verteilung über die gesamte aufgenommene Fläche aufweist.

${ }^{b}$ Dieser deutlich abweichende Wert geht wahrscheinlich eher auf Unterschiede in der Kalibrierung des Mikroskops zurück, als auf eine reale höhere mittlere Adhäsion dieses Netzwerks (s. Kapitel 6.3.5).
} 


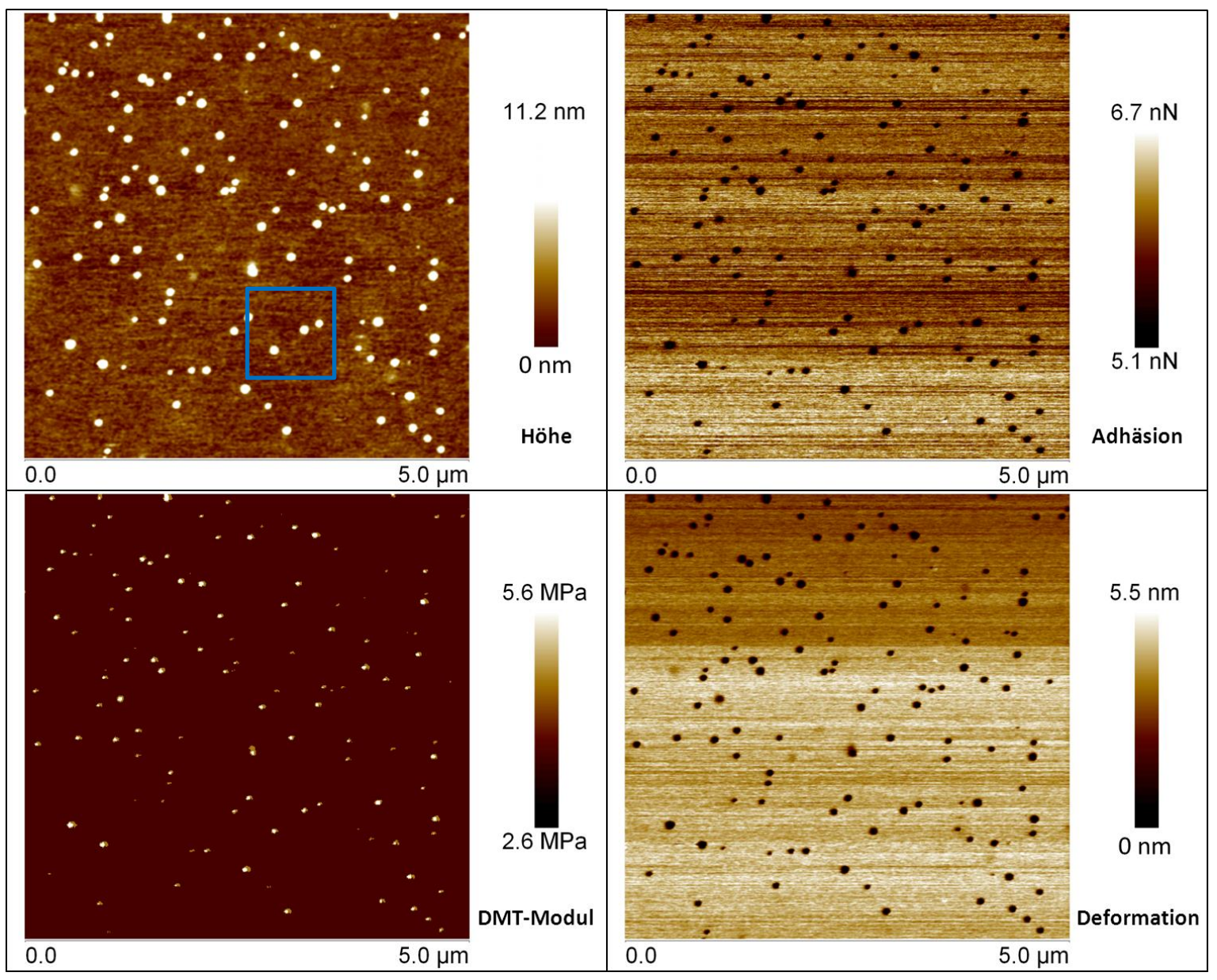

Abbildung 4-50 Rasterkraftmikroskop-Aufnahme eines konventionellen Poly(BA-co-BDDA)-Netzwerks mit 0,5 mol-\% BDDA als Vernetzer. Die einzelnen Bilder stellen unterschiedliche Kanäle der gleichen Messung dar. Links oben: Höhenprofil der Netzwerkoberfläche. Rechts oben: Adhäsion der Netzwerkoberfläche. Links unten: DMT-Modul der Netzwerkoberfläche. Rechts unten: Deformation der Netzwerkoberfläche beim Kontakt mit der Messspitze. Die Messung wurde im ScanAsyst-Modus durchgeführt. Die Kantenlänge beträgt $5 \times 5 \mu \mathrm{m}$. Die Scan-Frequenz betrug 0,5 Hz. Es wurden 512 Linien pro Bild aufgenommen.

Es kann festgehalten werden, dass diese Erhöhungen deutlich steifer sind und weniger Interaktion mit der Messspitze des Mikroskops zeigen als die sie umgebende Matrix des Netzwerks. Bei diesen Erhöhungen kann es sich weder um Verunreinigungen, wie z. B. Staub noch um Strukturen, die einer anderen Oberfläche (z. B. dem Probenträger) entstammen, handeln da die Netzwerkproben bis zu 2 mm dick waren. Für Staubpartikel sind die Erhöhungen zu klein und vor allem zu gleichmäßig verteilt und geformt. Außerdem wurden die Proben direkt nach der Synthese so gelagert, dass sie vor Staub geschützt waren (s. Kapitel 6.2.2). Somit bleibt als einzige Erklärung, dass es sich hierbei um Nanogel-Domänen handelt, die während der Polymerisation entstanden sind und anschließend beim vollständigen Aushärten des Netzwerkes an der Oberfläche festgehalten wurden. Solche Nanogel-Domänen sollten aufgrund ihrer sehr hohen Netzkettendichte, die mit sehr kurzen Netzketten einhergeht, deutlich steifer sein als ihre 
Umgebung. Zusätzlich sollte dies auch die Wechselwirkungen mit der Messspitze verringern, da die Netzketten in ihrer Beweglichkeit deutlich eingeschränkt sind, wodurch auch ein Eindringen der Messspitze in das Polymer und dessen Deformation verringert werden sollte.

Über diese Eigenschaften verfügen die Erhöhungen tatsächlich (s. Abbildung 4-50). Auch die im Vergleich zu der Umgebung niedrigere Adhäsion lässt sich durch die höhere Vernetzung der Nanogel-Domänen erklären. Kurze Netzketten sind in ihrer Beweglichkeit sehr eingeschränkt und somit weniger in der Lage, mit anderen Stoffen in einen engen Kontakt zu treten, sodass adhäsive Wechselwirkungen entstehen können.

Ein Höhenquerschnitt durch eine solche Erhöhung zeigt, dass sie nicht nur in x-y-Dimensionen gleichmäßig rund ist, sondern dass sie ebenfalls in z-Richtung eine runde Form aufweist, jedoch trotzdem fließend in das umgebende Netzwerk übergeht (s. Abbildung 4-51).
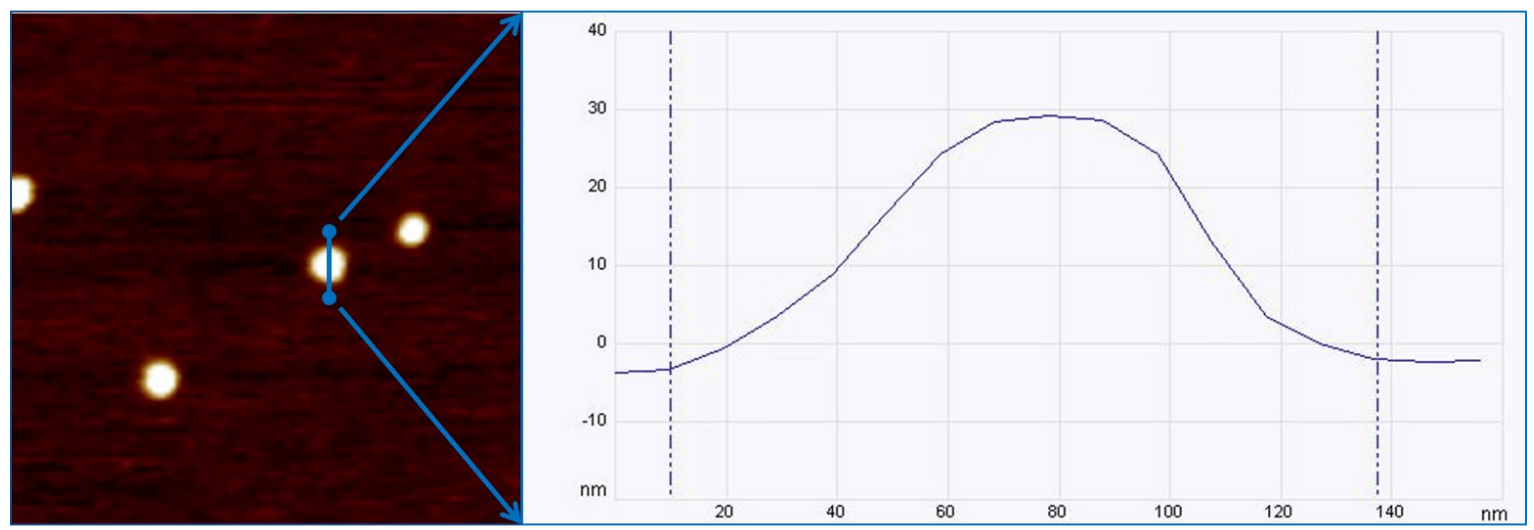

Abbildung 4-51 $1 \times 1 \quad \mu \mathrm{m}$ Bildausschnitt (vergrößerte Ansicht) aus der Aufnahme des Höhenprofils des konventionellen Poly(BA-co-BDDA)-Netzwerks (s. Abbildung 4-50, blaues Quadrat) sowie ein mit dem Programm NanoScope Analysis (Version 1.4) mittels der Funktion Section erstellter Höhenquerschnitt einer Erhöhung. Der hier gezeigte Bildausschnitt entstammt der nicht nachbearbeiteten Aufnahme des Höhenprofils der Oberfläche des Netzwerks (s. Abschnitt 6.3.5.2).

Abbildung 4-52 zeigt die Höhenstruktur-Aufnahmen der Oberflächen von Poly(BA-co-BDDA)Netzwerk aus RAFT-Polymerisation und als Referenz die Höhenstruktur-Aufnahmen einer Probe aus linearem Poly-BA, welches ebenfalls per RAFT-Polymerisation synthetisiert wurde. Auf die Darstellung der anderen Messkanäle des Rasterkraftmikroskops wurde im Folgenden verzichtet, da die beim konventionellen Netzwerk gemachten Beobachtungen immer wieder auftraten: Erhöhungen auf der Oberfläche zeigten geringere Adhäsionen, waren steifer und weniger deformierbar als das sie umgebende Netzwerk. 


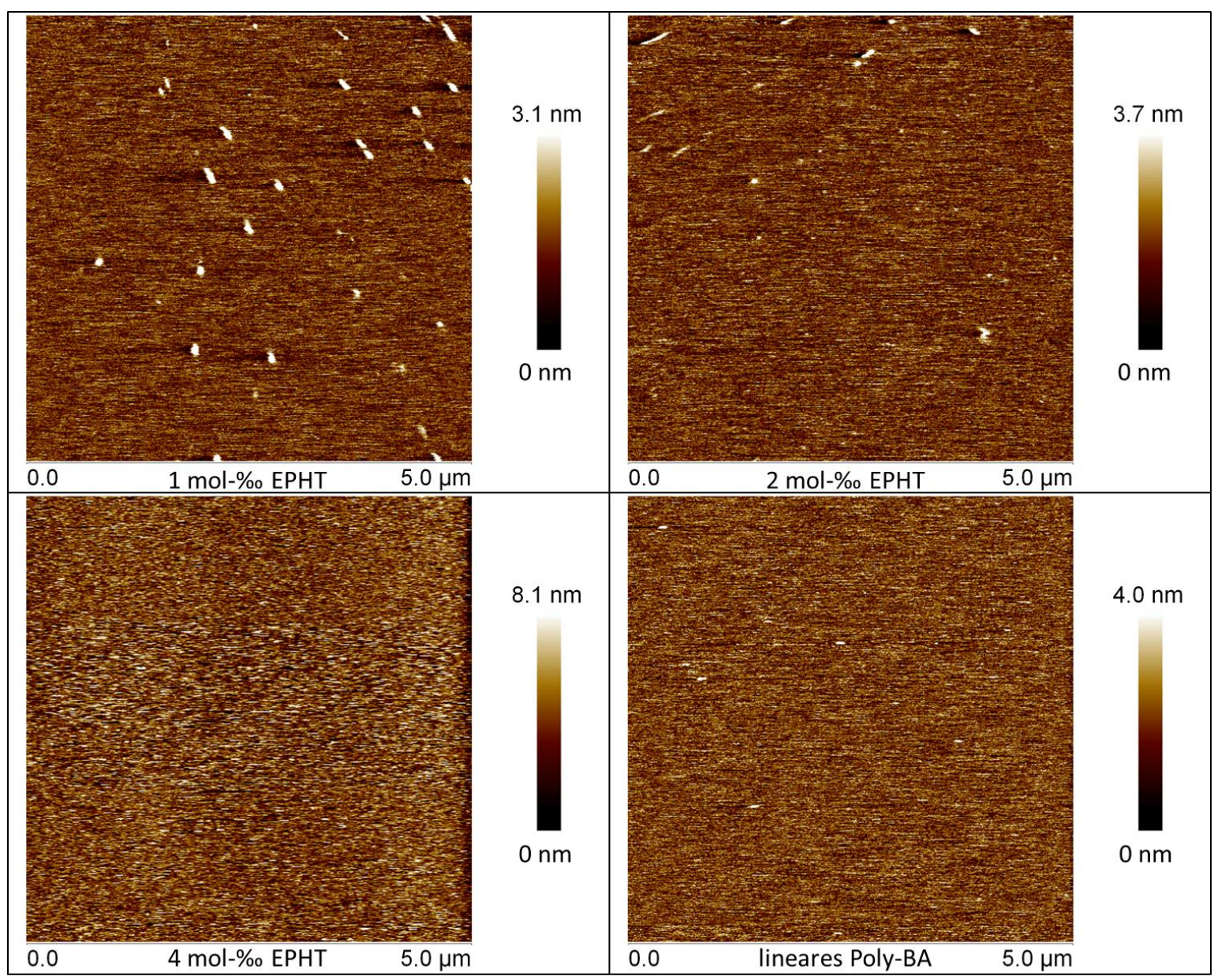

Abbildung 4-52 Rasterkraftmikroskop-Aufnahmen von Poly(BA-co-BDDA)-Netzwerken mit 0,5 mol-\% BDDA als Vernetzer aus UV-initiierter RAFT-Polymerisation mit 1 mol-\%o, 2 mol-\%o und 4 mol-\%o des RAFT-Agens EPHT, sowie von linearem Poly-BA aus RAFT-Polymerisation mit 2 mol-\% EPHT. Alle Bilder zeigen die Oberflächenstrukturen der Proben. Die Messungen wurden im ScanAsyst-Modus durchgeführt. Die Kantenlänge beträgt $5 \times 5 \mu$ m. Die ScanFrequenz betrug 0,5 Hz. Es wurden 512 Linien pro Bild aufgenommen.

Schon bei der Zugabe von 1 mol-\%o des RAFT-Agens EPHT nimmt die Zahl der Erhöhungen deutlich ab (s. Abbildung 4-52, links oben). Außerdem sind die zu erkennenden Erhöhungen nicht mehr rund geformt und deutlich kleiner als die der konventionellen Netzwerke. Bei weiterer Erhöhung des molaren EPHT-Anteils nimmt die Anzahl der Erhöhungen weiter ab, bis sie bei 4 mol-\% EPHT nicht mehr zu finden sind und die Oberfläche so eben ist, dass nur noch Rauschen aufgezeichnet wurde. Sie ähnelt deutlich der Aufnahme des linearen Poly-BAs (s. Abbildung 4-52, rechts unten).

Die Reduzierung der Erhöhungen mit der Zunahme des molaren Anteils von EPHT in den Polymerisationsmischungen ist eine weitere Bestätigung dafür, dass es sich bei diesen um Nanogel-Domänen handelt. Entsprechend der Vorhersagen aus der Literatur (s. Kapitel 2.2.2) und den bisher im Rahmen dieser Arbeit durchgeführten Untersuchungen sollten die RAFTNetzwerke über keine oder nur sehr wenige Nanogel-Domänen verfügen. Somit stellen die 
vorliegenden AFM-Aufnahmen eine visuelle Bestätigung dar, dass die bisher nur durch makroskopische Beobachtungen angestellte Vermutung korrekt ist und in RAFT-Netzwerken weniger oder keine Nanogel-Domänen vorhanden sind. Die mittleren Durchmesser der NanogelDomänen und deren Anzahl wurden mit dem Programm NanoScope Analysis (Version 1.4) ermittelt und in Tabelle 4-27 zusammengefasst.

Tabelle 4-27 Anzahl und mittlerer Durchmesser der Nanogel-Domänen von Poly(BA-co-BDDA)-Netzwerken mit 0,5 mol-\% BDDA aus UV-initiierter, konventioneller radikalischer Polymerisation sowie aus UV-initiierter RAFTPolymerisation mit 1 mol-\%o, 2 mol-\%o und 4 mol-\%o des RAFT-Agens EPHT. Die Daten wurden mit der Partikelanalysefunktion des Programms NanoScope Analysis (Version 1.4) den Höhenprofilen der Netzwerkoberflächen entnommen (s. Abbildung 4-50 und Abbildung 4-52).

\begin{tabular}{ccccc}
\hline Netzwerke & konv. & 1 mol-\%o EPHT & 2 mol-\%o EPHT & 4 mol-\%o EPHT \\
\hline Anzahl der Nanogele $/ \mu \mathrm{m}^{2}$ & 5,2 & 2,0 & 0,5 & - \\
Mittlerer Durchmesser & $77 \mathrm{~nm}$ & $71 \mathrm{~nm}$ & $82 \mathrm{~nm}$ & - \\
\hline
\end{tabular}

An Tabelle 4-27 ist die geringer werdende Anzahl der Erhöhungen mit steigendem molaren EPHT-Anteil deutlich nachzuvollziehen. Dass die mittleren Durchmesser kaum beeinflusst werden, könnte daran liegen, dass sich die Form der Erhöhungen vom konventionellen zum RAFT-Netzwerk mit 4 mol-\%o EPHT stark verändern. Sie erscheinen nicht mehr rund sondern eher länglich und für die Auswertungssoftware größer.

Anschließend zu AFM-Messungen der Poly(EA-co-BDDA)-Netzwerke wurden zusätzlich Poly(EA-co-BDDA)-Netzwerke mit 0,5 mol-\% BDDA als Vernetzer hinsichtlich der Höhenprofile und der mechanischen Eigenschaften ihrer Oberflächen untersucht. Wieder wurde der EPHTAnteil der Netzwerke von 0 bis 4 mol-\%o variiert. Die Messungen erfolgten analog zu den vorherigen (s. Abschnitt 6.3.5.2). Abbildung 4-53 zeigt die erhaltenen Höhenprofile der Oberflächen dieser Netzwerke. 


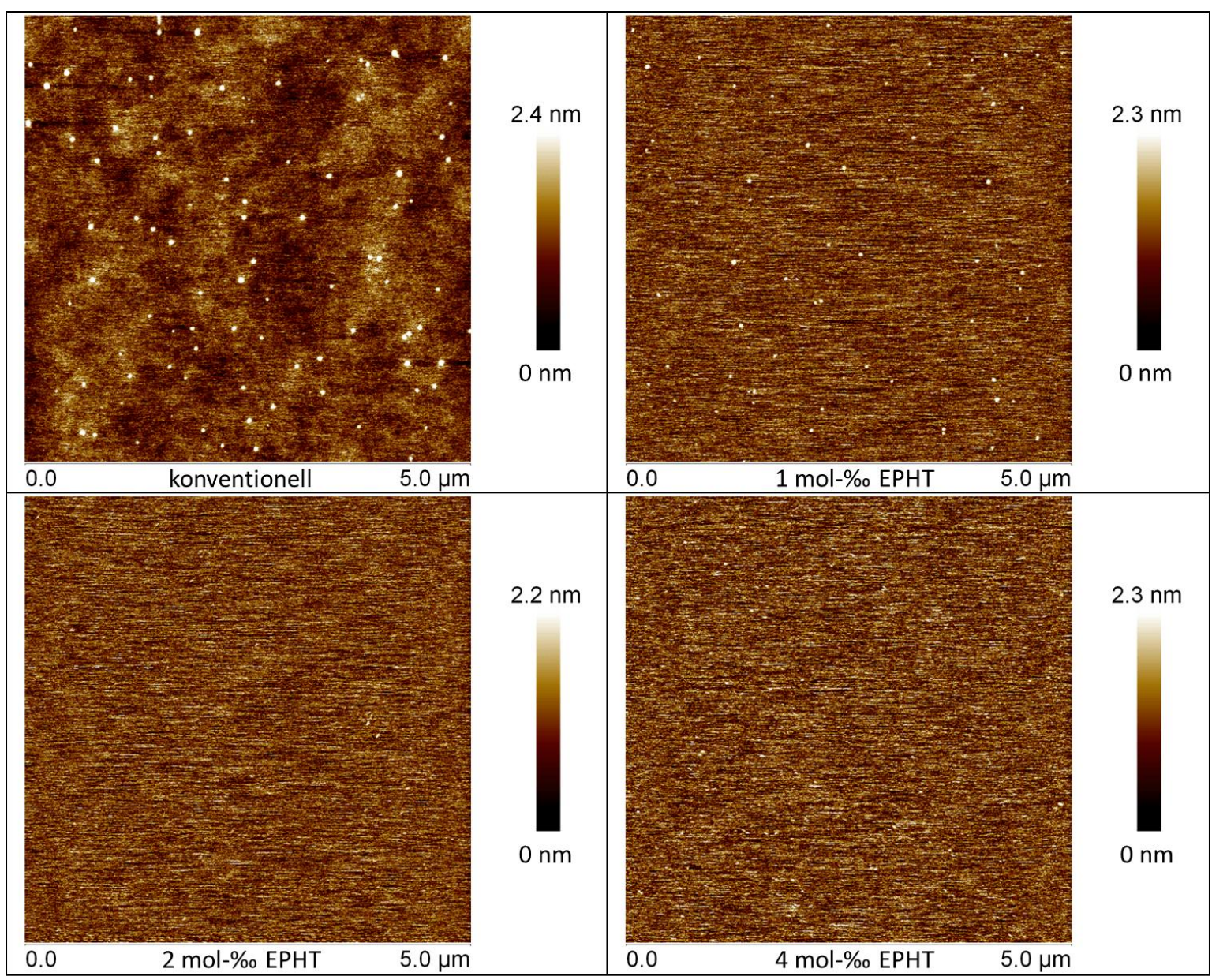

Abbildung 4-53 Rasterkraftmikroskop-Aufnahmen von Poly(EA-co-BDDA)-Netzwerken mit 0,5 mol-\% BDDA aus UV-initiierter, konventioneller radikalischer Polymerisation sowie aus UV-initiierter RAFT-Polymerisation mit 1 mol\%, 2 mol-\%o und 4 mol-\%o des RAFT-Agens EPHT. Alle Bilder zeigen die Oberflächenstrukturen der Proben. Die Aufnahmen wurden im ScanAsyst-Modus durchgeführt. Die Kantenlänge betrug $5 \times 5 \mu \mathrm{m}$. Die Scan-Frequenz beträgt 0,5 Hz. Es wurden 512 Linien pro Bild aufgenommen.

Es ist ersichtlich, dass die Nanogel-Domänen der Poly(EA-co-BDDA)-Netzwerke über einen deutlich kleineren Durchmesser und eine geringere Höhe verfügen als die der Poly(BA-coBDDA)-Netzwerke (s. Abbildung 4-50 und Abbildung 4-52). Dadurch können die Erhöhungen schon bei einem molaren Anteil von 2 mol-\% EPHT auf den Höhenprofil-Aufnahmen nicht mehr vom Rauschen unterschieden werden (s. Abbildung 4-53). Bei der Betrachtung der Aufnahmen aus dem Messkanal des DMT-Moduls zeigt sich allerdings, dass auf der Oberfläche des genannten Netzwerks durchaus noch klar abgegrenzte steifere Bereiche befinden. Dies lässt die Vermutung zu, dass doch noch Nanogel-Domänen vorhanden sind. Darum wurde zur Ermittlung der Anzahl und des mittleren Durchmessers der Nanogel-Domänen auf die Aufnahmen dieses Messkanals zurückgegriffen. Dabei muss beachtet werden, dass diese Aufnahmen nicht nur Eigenschaften der Oberfläche zeigen, sondern auch Strukturen, die direkt unter dieser liegen sichtbar werden können, sofern diese die mechanischen Eigenschaften der Oberfläche beeinflussen. Somit können 
auch Strukturen gefunden werden, die im Höhenprofil der Oberflächen nicht erkennbar sind. Die erhaltenen Werte sind in Tabelle 4-28 zusammengefasst.

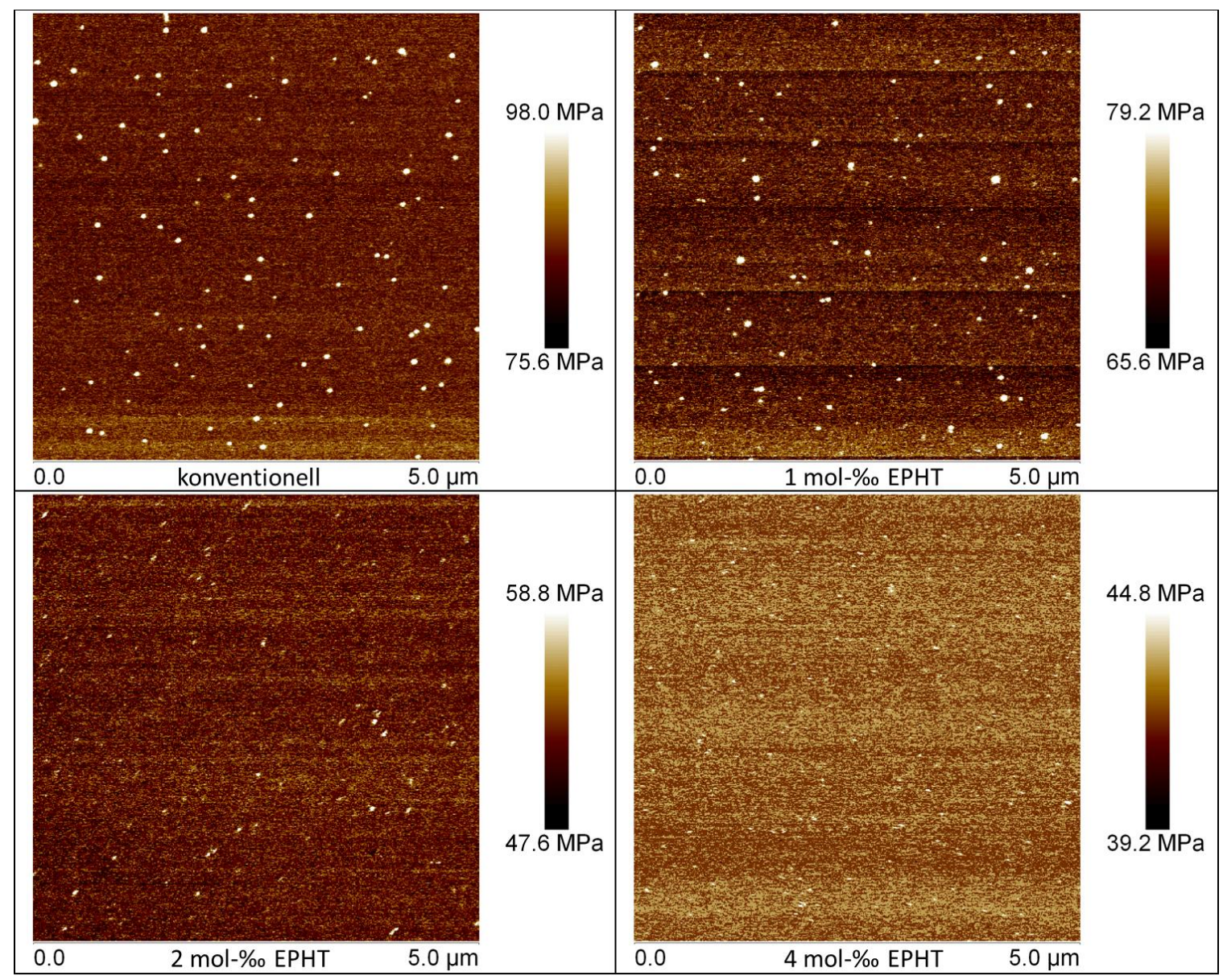

Abbildung 4-54 Rasterkraftmikroskop-Aufnahmen von Poly(EA-co-BDDA)-Netzwerken mit 0,5 mol-\% BDDA aus UV-initiierter, konventioneller radikalischer Polymerisation sowie aus UV-initiierter RAFT-Polymerisation mit 1 mol\%, 2 mol-\%o und 4 mol-\%o des RAFT-Agens EPHT. Alle Bilder zeigen den DMT-Modul der Proben. Die Aufnahmen wurden im ScanAsyst-Modus durchgeführt. Die Kantenlänge beträgt $5 \times 5 \mu \mathrm{m}$. Die Scan-Frequenz betrug $0,5 \mathrm{~Hz}$. Es wurden 512 Linien pro Bild aufgenommen.

Tabelle 4-28 Anzahl und mittlerer Durchmesser der Nanogel-Domänen von Poly(EA-co-BDDA)-Netzwerken mit 0,5 mol-\% BDDA aus UV-initiierter, konventioneller radikalischer Polymerisation sowie aus UV-initiierter RAFTPolymerisation mit 1 mol-\%o, 2 mol-\%o und 4 mol-\%o des RAFT-Agens EPHT. Die Daten wurden mit der Partikelanalysefunktion des Programms NanoScope Analysis (Version 1.4) aus den Aufnahmen des DMT-Moduls entnommen (s. Abbildung 4-53).

\begin{tabular}{ccccc}
\hline Netzwerke & konv. & 1 mol-\%o EPHT & 2 mol-\%o EPHT & 4 mol-\%o EPHT \\
\hline Anzahl der Nanogele $/ \mu \mathrm{m}^{2}$ & 5,0 & 4,0 & 3,0 & 2,9 \\
Mittlerer Durchmesser & $40 \mathrm{~nm}$ & $34 \mathrm{~nm}$ & $17 \mathrm{~nm}$ & $14 \mathrm{~nm}$ \\
\hline
\end{tabular}


Es ist zu erkennen, dass die Anzahl der Nanogel-Domänen abnimmt, genauso wie deren mittlerer Durchmesser, wo diese Abnahme sogar noch deutlicher ausfällt (s. Tabelle 4-28). Insgesamt zeigt sich also wieder eine ähnliche Tendenz wie bei den Poly(BA-co-BDDA)-Netzwerken, dass es mit steigendem EPHT-Anteil in den Netzwerken zu einer Reduzierung der Nanogel-Domänen kommt. Dies korreliert mit der Zunahme der Klebrigkeit der Netzwerke (s. Tabelle 4-24 und Tabelle 4-24). Über die Reduzierung der Anzahl bzw. der Fläche der Nanogel-Domänen mit steigendem EPHT-Anteil in den Netzwerken kann deshalb auch die zunehmende Klebrigkeit der Netzwerke erklärt werden. Die Nanogele stehen über die eigentliche Oberfläche des Netzwerks hinaus (s. Abbildung 4-55). Da sie steifer sind als die Oberfläche des umgebenden Netzwerks, verhindern sie somit einen ausreichend dichten Kontakt des Netzwerks mit einem Adhärens. Zusätzlich wird durch sie die Kontaktfläche deutlich reduziert. Die Nanogele beanspruchen nur einen sehr kleinen Teil der Gesamtfläche des Netzwerks. Da sie auch weniger adhäsiv sind als das umgebende Netzwerk, ist es nachvollziehbar, dass Netzwerke, die über viele dieser Nanogele auf der Oberfläche verfügen, auch makroskopisch weniger Klebrigkeit und Haftkraft zeigen.

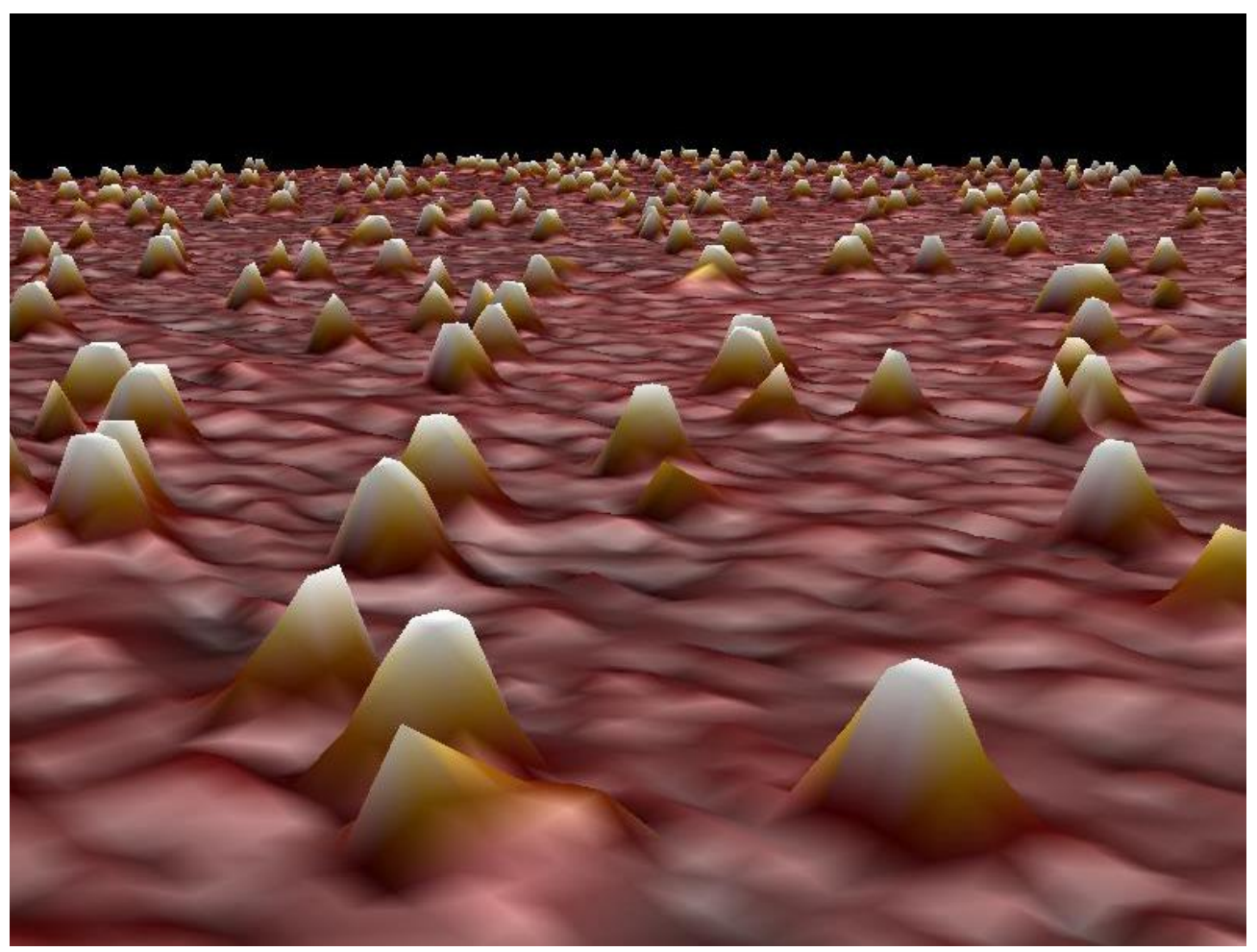

Abbildung 4-55 Ausschnitt aus einer perspektivischen, dreidimensionalen Rasterkraftmikroskop-Aufnahme des Höhenprofils von der Oberfläche eines konventionellen Poly(BA-co-BDDA)-Netzwerks mit 0,5 mol-\% BDDA als Vernetzer mit einem Zoomfaktor von 10 und einem Z-Achsen-Aspektverhältnis 0,01. Der Ausschnitt wurde einer AFM-Aufnahme mit $15 \mu \mathrm{m}$ Kantenlänge entnommen.

Ein weiterer Aspekt, der bei der Betrachtung der makroskopischen Klebrigkeit und Haftung beachtet werden muss, ist die Struktur der Oberfläche, die die Nanogele umgibt. Während sie bei 
den konventionellen Netzwerken jeweils noch über eine Mikrostruktur verfügt (zu erkennen im Höhenprofil des Netzwerks an den größeren, eher gelben bzw. braunen Bereichen in Abbildung 4-50), bilden sich mit der Zugabe des RAFT-Agens sehr glatte Oberflächen, die keinerlei Struktur mehr zeigen. Diese Beobachtung ähnelt der Änderung der makroskopischen Oberflächenstrukturen, die ebenfalls mit der Zugabe von EPHT sehr glatt wurden (s. Abbildung 4-3 und Abbildung 4-4).

Bei der Betrachtung der Rasterkraftmikoskopaufnahmen fällt weiterhin auf, dass die Aufnahme des Poly(BA-co-BDDA)-Netzwerks mit 4 mol-\%o EPHT und, abgesehen von den Nanogelen, auch die des Poly(BA-co-BDDA)-Netzwerks mit 2 mol-\%o EPHT, der des linearen Poly-BAs sehr stark ähnelt (s. Abbildung 4-52). Alle Oberflächen sind so glatt, dass die Aufnahmen nur noch Rauschen zeigen. Gleiches ergibt sich für die Poly(EA-co-BDDA)-Netzwerke, wobei hier der Unterschied zur Oberfläche des konventionellen Netzwerks noch deutlicher wird, da diese noch ausgeprägtere Strukturen aufweist als das konventionelle Poly(BA-co-BDDA)-Netzwerk.

Das lineare Poly-BA verfügt über eine sehr glatte Oberfläche, weil es bei Raumtemperatur fließen kann und einer sehr viskosen Flüssigkeit ähnelt. Es treten also keine Unebenheiten auf der Oberfläche auf, da diese durch beweglichen Polymere ausglichen werden. Ähnliches könnte bei den RAFT-Netzwerken auch geschehen. Wie schon in Kapitel 4.1.3 diskutiert, steigt bei diesen der Anteil der nicht ins Netzwerk eingebauten Sol-Fraktion. Es besteht die Möglichkeit, dass SolMoleküle an der Oberfläche der Netzwerke angereichert werden. Bei der Polymerisation von bifunktionellen Acrylaten konnte beobachtet werden, dass die Monomere, bei denen es sich um Präpolymere handelt, durch die Volumenkontraktion des Netzwerks aus diesem ausgeschlossen werden. ${ }^{[22]}$ Ähnliches könnte hier auch der Fall sein. Die Netzwerkketten ziehen sich zusammen, schließen die Extrahierbaren aus dem Netzwerk aus und drängen diese somit an die Oberfläche, sodass sie sich mit zunehmenden molaren EPHT-Anteil immer mehr wie eine Oberfläche eines nicht-vernetzten Polymers verhält. Da die konventionellen Netzwerke über nahezu keine Extrahierbaren verfügen, können diese auch nicht auf der Oberfläche angereichert werden und die Oberfläche entspricht den molekularen Verhältnissen im Netzwerkinneren. Die Ansammlung der Sol-Fraktion auf der Oberfläche könnte Beobachtungen wie die zufließenden Spuren bei den Rollkugel-Tests und die Fibrillenbildung bei den Probe-Tack-Tests erklären. Dies wäre nur beweglichen, nicht mit dem Netzwerk kovalent verbunden, Polymeren möglich. Zusätzlich zu der Reduzierung der Mikrogele aufgrund des RAFT-Mechanismus wird das zuvor Genannte ein wesentlicher Grund für die Erhöhung der Klebrigkeit sein, denn durch die Sol-Fraktion auf der Oberfläche sind die Netzwerke in der Lage, die Oberflächen eines Adhärens besser zu benetzen.

Bei Erhöhung des molaren Anteils von BDDA, sollten die Nanogel-Domänen größer bzw. zahlreicher werden, da mehr Vernetzer die Wahrscheinlichkeit erhöht, dass es z. B. zu intramolekularen Ringschlüssen kommt, die Verhakungen zur Folge haben. Um diese Annahme zu überprüfen, wurden für die Poly(BA-co-BDDA)- und die Poly(EA-co-BDDA)-Netzwerke noch 
zusätzlich Rasterkraftmikroskop-Aufnahmen von Netzwerken mit 1 mol-\% und 2 mol-\% BDDA aus konventioneller sowie RAFT-Polymerisation mit 2 mol-\%o EPHT durchgeführt. Wie in Abbildung 4-56 zu erkennen ist, treffen die zuvor angestellten Annahmen voll und ganz zu.

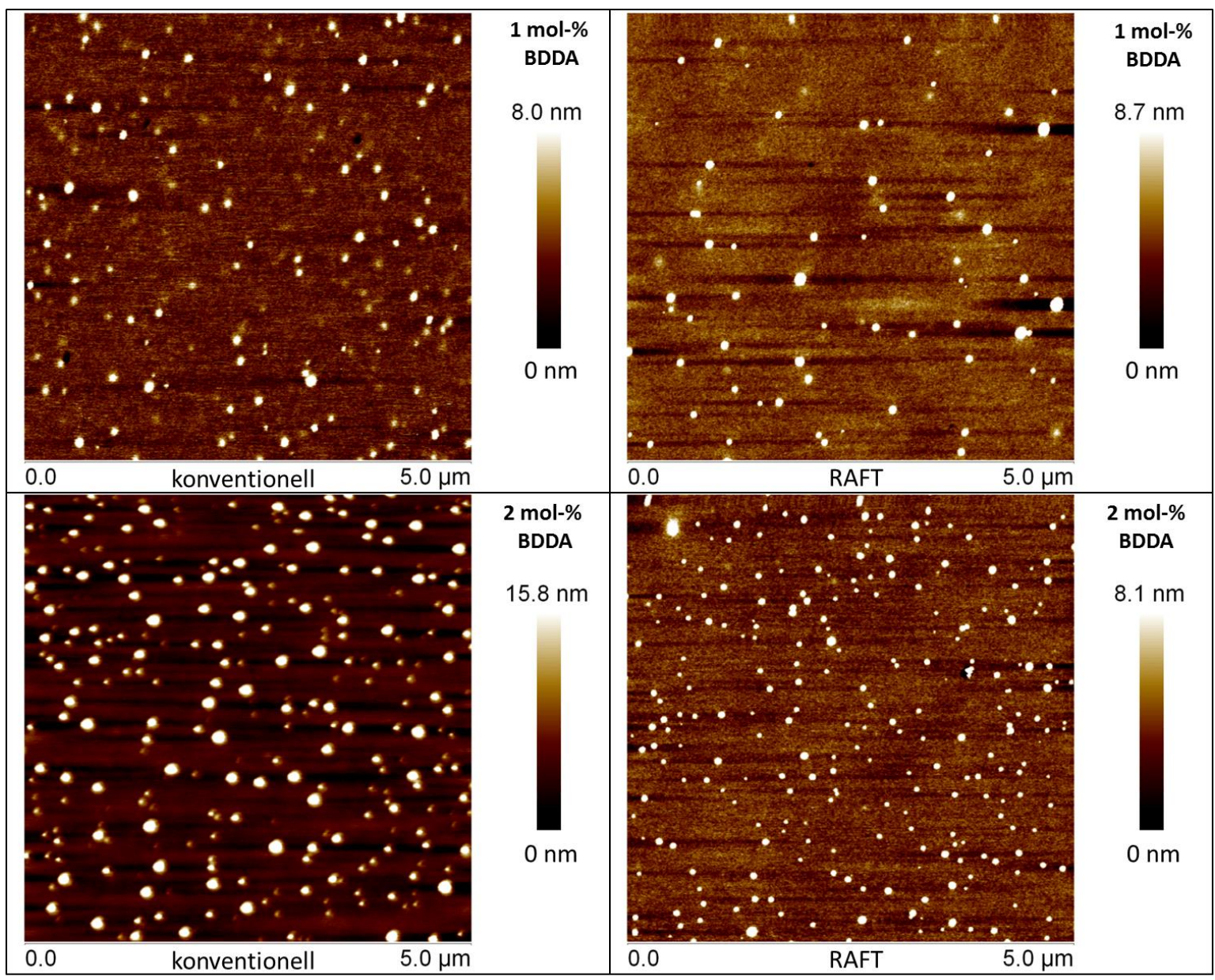

Abbildung 4-56 Rasterkraftmikroskop-Aufnahmen von konventionellen Poly(BA-co-BDDA)-Netzwerken (linke Spalte) mit 1 mol-\% BDDA (oben) und 2 mol-\% BDDA (unten), sowie Poly(BA-co-BDDA)-Netzwerken aus RAFTPolymerisation (rechte Spalte) mit 2 mol-\% EPHT als RAFT-Agens mit 1 mol-\% BDDA (oben) und 2 mol-\% BDDA (unten). Die Bilder zeigen Oberflächenstrukturen der Proben. Die Aufnahmen wurden im ScanAsyst-Modus durchgeführt. Die Kantenlänge beträgt $5 \times 5 \mu \mathrm{m}$. Die Scan-Frequenz beträgt $0,5 \mathrm{~Hz}$. Es wurden 512 Linien pro Bild aufgenommen.

Es ist deutlich zu erkennen, dass bei der Erhöhung des molaren BDDA-Anteils die Anzahl bzw. die Größe der Nanogele, sowohl bei den konventionellen als auch bei den RAFT-Netzwerken, signifikant zunimmt. Weiterhin wird deutlich, dass es bei Anwesenheit des RAFT-Agens EPHT zu einer Reduzierung der Anzahl bzw. der Größe der Nanogel-Domänen kommt. Eine genauere Auswertung der Anzahl und Größe der Nanogele ist in Tabelle 4-29 zu finden. 
Tabelle 4-29 Anzahl und mittlere Durchmesser der Nanogel-Domänen von Poly(BA-co-BDDA)-Netzwerken mit 1 mol-\% sowie 2 mol-\% BDDA aus UV-initiierter, konventioneller radikalsicher Polymerisation sowie aus UV-initiierter RAFT-Polymerisation mit 2 mol-\% EPHT als RAFT-Agens. Die Daten wurden mit der Partikelanalysefunktion des Programms NanoScope Analysis (Version 1.4) den Höhenprofilen der Netzwerkoberflächen entnommen (s. Abbildung 4-56).

\begin{tabular}{ccccc}
\hline \multirow{2}{*}{ Netzwerke } & \multicolumn{2}{c}{ 1 mol-\% BDDA } & \multicolumn{2}{c}{ 2 mol-\% BDDA } \\
& konv. & RAFT & konv. & RAFT \\
\hline Anzahl der Nanogele/ $\mu^{2}$ & 5,2 & 3,1 & 10,0 & 9,9 \\
Mittlerer Durchmesser & $46 \mathrm{~nm}$ & $66 \mathrm{~nm}$ & $89 \mathrm{~nm}$ & $55 \mathrm{~nm}$ \\
\hline
\end{tabular}

Die genaue Betrachtung zeigt, dass das RAFT-Agens für die Netzwerke je nach Vernetzermenge einen unterschiedlichen Effekt hat. Bei den Netzwerken mit 1 mol-\% BDDA nimmt die Anzahl der Nanogele deutlich ab, während ihr mittlerer Durchmesser sogar steigt. Bei den Netzwerken mit 2 mol-\% BDDA bleibt die Anzahl gleich, aber die mittleren Durchmesser der NanogelDomänen nehmen stark ab. Dass bei beiden RAFT-Netzwerken im Gegensatz zu dem Netzwerk mit 0,5 mol-\% BDDA überhaupt solche Nanogel-Domänen erkennbar sind, ist ein weiteres Indiz dafür, dass die Vernetzermenge über das RAFT-Agens dominiert. Schon bei den Quellungsversuchen wurde gezeigt, dass der Anteil der Extrahierbaren, also die Sol-Fraktion, bei Netzwerken mit molaren Anteilen von BDDA von über 1 mol-\% nicht mehr signifikant von denen der konventionellen Netzwerke abwich. Dies ist weiterhin auch ein Indiz für die Rolle der Sol-Fraktion für die Oberflächenstrukturen. Anhand dieser Aufnahmen ist ebenfalls zu erkennen, dass neben den Nanogel-Domänen auf und unter der Oberfläche Nanogel-Domänen existieren. Vor allem beim konventionellen Netzwerk mit 1 mol-\% BDDA (s. Abbildung 4-56, links oben) scheint es, als ob sich die kleineren der auf der Aufnahme zu erkennenden Nanogele zu großen Teilen unter der Oberfläche befinden.

Vergleichend wurden wieder analoge AFM-Aufnahmen von Poly(EA-co-BDDA)-Netzwerken mit 1 mol-\% sowie 2 mol-\% BDDA durchgeführt. Die Ergebnisse der Auswertung der AFMAufnahmen der Poly(EA-co-BDDA)-Netzwerke wurden in Tabelle 4-30 zusammengefasst.

Tabelle 4-30 Anzahl und mittlere Durchmesser der Nanogel-Domänen von Poly(EA-co-BDDA)-Netzwerken mit 1 mol-\% sowie 2 mol-\% BDDA aus konventioneller und RAFT-Polymerisation mit 2 mol-\%o EPHT als RAFT-Agens. Die Daten wurden mit der Partikelanalysefunktion des Programms NanoScope Analysis (Version 1.4) den Höhenprofilen der Netzwerkoberflächen entnommen.

\begin{tabular}{ccccc}
\hline \multirow{2}{*}{ Netzwerke } & \multicolumn{2}{c}{ 1 mol-\% BDDA } & \multicolumn{2}{c}{ 2 mol-\% BDDA } \\
& konv. & RAFT & konv. & RAFT \\
\hline Anzahl der Nanogele/ $\mu^{2}$ & 14,4 & 4,2 & - & 5,0 \\
Mittlerer Durchmesser: & $24 \mathrm{~nm}$ & $23 \mathrm{~nm}$ & - & $27 \mathrm{~nm}$ \\
\hline
\end{tabular}


Die Anzahl der Nanogele nimmt auch hier bei der Zugabe des RAFT-Agens ab. Bei Erhöhung des molaren BDDA-Anteils nimmt sie hingegen wieder zu. Auch dies konnte schon bei den Poly(BAco-BDDA)-Netzwerken beobachtet werden. Die mittleren Durchmesser der Nanogele bleiben hier allerdings konstant.

\subsubsection{Abschließende Bemerkungen}

Es konnte gezeigt werden, dass die Klebrigkeit von statistischen Poly(BA-co-BDDA)- und Poly(EA-co-BDDA)-Netzwerken mit der Zugabe des RAFT-Agens EPHT zu den jeweiligen Polymerisationsmischungen deutlich zunimmt. Dies hat mehrere Gründe: Zum einen reduziert der RAFT-Mechanismus die Zahl und Größe der Nanogel-Domänen auf den Oberflächen der Netzwerke, wie die durchgeführten AFM-Aufnahmen belegen. Weiterhin reichern sich die Extrahierbaren an der Oberfläche der Netzwerke an, was die Bildung von Fibrillen ermöglicht, welche die Klebrigkeit der Netzwerke erhöhen (s. Kapitel 2.5). Als letzter Grund für die zunehmende Klebrigkeit sei genannt, dass die Netzwerke mit steigendem molaren EPHT-Anteil weicher werden, wodurch die Energiedissipation beim Kontakt des Netzwerks mit einem Adhärens verbessert wird und eine größere Oberflächenenergie beim Trennen der beiden Materialien aufgebracht werden muss. 


\subsection{Der Einfluss der RAFT-Polymerisation auf Duroplaste}

Da es sich bei Duroplasten um einen z. B. in industriellen Anwendungen sehr häufig verwendeten Netzwerktyp handelt (s. Kapitel 2.2.2), sollte der Einfluss des RAFT-Mechanismus auf diese Netzwerke ebenfalls untersucht werden. Dazu wurden zunächst bifunktionelle Polyethylenglycoldiacrylate hinsichtlich ihrer Polymerisationskinetik und der Mikrostrukturen der aus den unterschiedlichen Monomeren resultierenden Netzwerke untersucht. Anschließend wurden, als industriell verwendete Systeme, UV-härtende Druckfarben untersucht.

\subsubsection{Einfluss der RAFT-Polymerisation auf Polyethlyenglycoldiacrylat- Netzwerke}

Es wurden drei Polyethylenglycoldiacrylate (PEGDA) mit unterschiedlicher Anzahl von Ethylenglycol-Einheiten zwischen den Acrylat-Gruppen untersucht. Abbildung 4-57 zeigt die chemische Grundstruktur der drei verwendeten Polyethlyenglycoldiacrylate. Variiert wurde die Anzahl der Ethylenglycol-Einheiten zwischen den Acrylatgruppen von $n=3$ über $n=10$ bis $n=13$. Dies führt zu mittleren Molmassen der PEG-Diacylate von $250 \mathrm{~g} / \mathrm{mol}, 575 \mathrm{~g} / \mathrm{mol}$ und $700 \mathrm{~g} / \mathrm{mol}$. Im Folgenden werden die entsprechenden Monomere darum mit

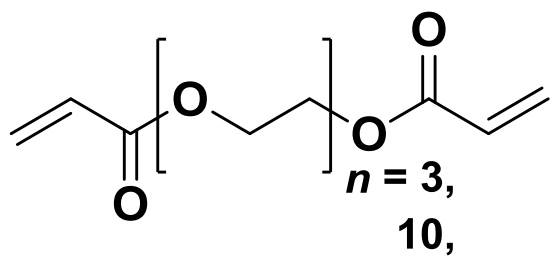

13.

Abbildung 4-57 Grundstruktur der verwendeten Polyethylendiacrylate mit $n=3$, 10 und 13 Ethylenglycol-Einheiten zwischen den Acrylat-Gruppen. PEGDA-250, PEGDA-575 und PEGDA-700 bezeichnet.

Es sollte herausgefunden werden, ob der, durch die unterschiedliche Anzahl von EthylenglycolEinheiten, unterschiedliche Abstand der Acrylat-Funktionen (häufig nach engl.: Spacer genannt) einen Einfluss auf die Effekte des RAFT-Mechanismus auf die resultierenden Netzwerke hatte. Bei Polydimethacrylaten mit unterschiedlicher Spacer-Länge, die in der Literatur sehr ausgiebig untersucht worden sind, hatten sich große Unterschiede in der Reaktionskinetik sowie in den Eigenschaften und Strukturen der resultierenden Netzwerke gezeigt. Dies wurde sowohl für konventionelle ${ }^{[91,92]}$ als auch für ATRP- ${ }^{[204]}$ und RAFT-Polymerisationen ${ }^{[78,79]}$ gefunden.

Zur Untersuchung des Einflusses der RAFT-Polymerisation auf die Eigenschaften und Strukturen der aus den PEG-Diacrylaten gebildeten Netzwerke wurden zunächst die Umsatz/Zeit-Kurven mittels FTIR-Spektroskopie aufgenommen und anschließend die Oberflächen der Netzwerke mittels Rasterkraftmikroskopie vermessen. Unterstützend wurde die Entwicklung der während 
der Polymerisation auftretenden Radikale mittels Elektronen Spin Resonanz (ESR)-Spektroskopie verfolgt.

Eine Untersuchung der mechanischen Eigenschaften mittels Zugversuch war nicht möglich. Die Probekörper zerbrachen, vermutlich aufgrund von ausgeprägter Volumenschrumpfung (s. Kapitel 2.2.2) schon während der Aushärtung (PEGDA-250) oder sie brachen spätestens beim Herauslösen aus der Polymerisationsform (PEGDA-575). Ein weiteres Problem war auch die hohe Viskosität der Polymerisationslösungen (vor allem PEGDA-700), wodurch es nicht möglich war, die Polymerisationsform ausreichend schnell $\mathrm{zu}$ füllen und $\mathrm{zu}$ verhindern, dass die Polymerisation bereits durch Umgebungslicht startete. Dies führte zu inneren Störungen wie z. B. fest einpolymerisierten Luftblasen in den Probekörpern.

\subsubsection{Einfluss des RAFT-Mechanismus auf die Polymerisationskinetik}

Zunächst wurde die Reaktionskinetik der Polymerisationen mittels FTIR-Spektroskopie verfolgt. Bevor der Einfluss der RAFT-Polymerisation auf die Reaktionskinetik der Polymerisationen untersucht wurde, wurden zunächst die Umsatz/Zeit-Kurven der konventionellen, UV-initiierten radikalischen Polymerisation der PEG-Diacrylate aufgenommen (s. Abbildung 4-58). ${ }^{\mathrm{a}}$

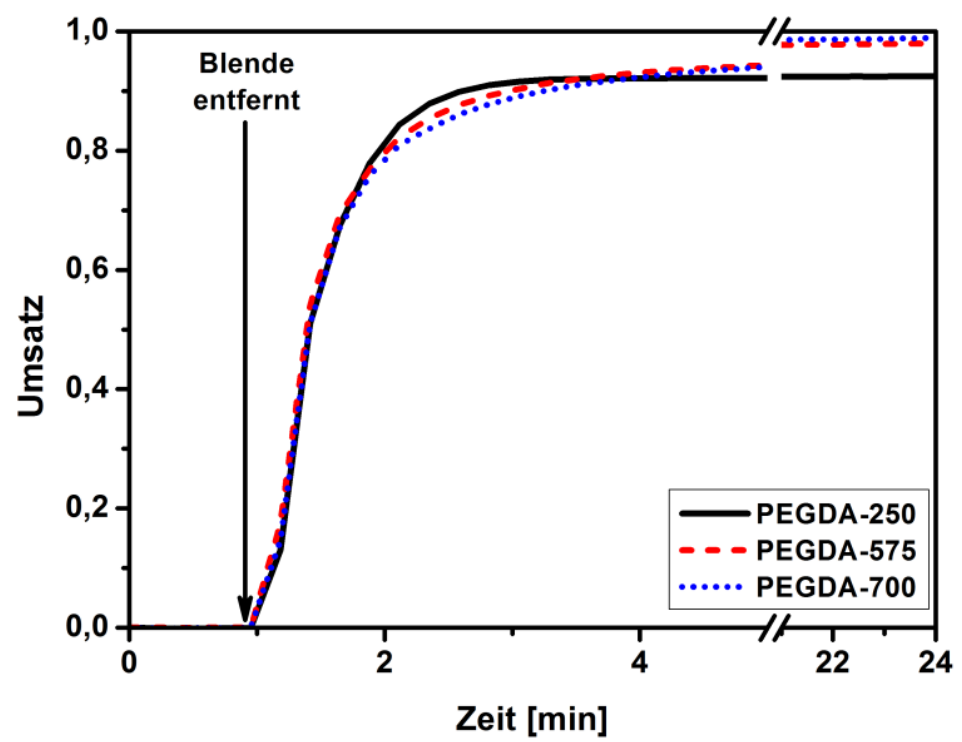

Abbildung 4-58 Umsatz/Zeit-Kurven von konventionellen, UV-initiierten radikalischen Polymerisationen von drei PEG-Diacrylaten mit Spacer-Längen von $n=3,10$ und 13 Ethylenglycol-Einheiten, bei einer Umgebungstemperatur von $21^{\circ} \mathrm{C}$ und einer Strahlungsintensität von $9 \mathrm{~mW} / \mathrm{cm}^{2}$ bei einer Wellenlänge von $366 \mathrm{~nm}$. Als Photoinitiator wurden 2 Gew.-\% MMMP verwendet. Der Zeitpunkt, bei dem die Blende zwischen UV-Lampe und Messzelle entfernt wurde, wurde mit einem Pfeil markiert.

\footnotetext{
${ }^{a}$ Die Totzeit zu Beginn der Kurven geht darauf zurück, dass zunächst fünf Messungen ohne UV-Bestrahlung vorgenommen wurden, um eine Basislinie zu erhalten. Nach diesen fünf Messungen wurde eine Blende, die sich vor der Lampe befand, entfernt. Da diese Totzeit die Übersichtlichkeit der folgenden Abbildungen verbessert und die weitere Auswertung nicht beeinflusste, wurde sie beibehalten.
} 
Nach dem Beginn der Bestrahlung starten alle Polymerisationen direkt. Der Umsatz steigt zunächst für alle drei PEG-Diacrylate in gleicher Form an. Schon nach 45 Sekunden Reaktionszeit erreichen alle Polymerisationen einen Umsatz von über $70 \%$. Während die Polymerisation von PEGDA-250 eine Minute später seinen maximalen Umsatz von $92 \%$ erreicht und stoppt, laufen die Polymerisationen von PEGDA-575 und PEGDA-700 weiter, bis sie nach 20 Minuten Reaktionszeit ihren maximalen Umsatz erreichen. Die maximalen Umsätze sind zusammen mit den maximalen Reaktionsgeschwindigkeiten in Tabelle 4-31 zusammengefasst.

Dass die PEG-Diacrylate mit den größeren Molmassen einen höheren Umsatz als das PEGDA-250 erreichen, liegt in den größeren Abständen der Acrylat-Gruppen begründet. Dies führt dazu, dass die Netzwerke, die aus diesen Monomeren gebildet werden, weniger dicht sind und über eine größere molekulare Flexibilität verfügen. ${ }^{[79]}$ Darum bleibt eine gewisse Mobilität von kleineren Spezies wie Monomer und Initiatormoleküle in diesen Systemen länger erhalten, was auch nach der Bildung eines Netzwerks weitere Propagationsreaktionen ermöglicht. Somit werden bei PEGDA-575 und 700 höhere Monomerumsätze erreicht, als bei PEGDA-250.

Tabelle 4-31 Maximale Umsätze $C_{\mathrm{M}}^{\max }$ und maximale Reaktionsgeschwindigkeiten $R_{\mathrm{P}}^{\max }$ von konventionellen, UVinitiierten radikalischen Polymerisationen von drei PEG-Diacrylaten mit Spacer-Längen von $n=3,10$ und 13 Ethylenglycol-Einheiten bei einer Umgebungstemperatur von $21{ }^{\circ} \mathrm{C}$ und einer Strahlungsintensität von $9 \mathrm{~mW} / \mathrm{cm}^{2}$ bei einer Wellenlänge von $366 \mathrm{~nm}$. Als Photoinitiator wurden 2 Gew.-\% MMMP verwendet.

\begin{tabular}{ccc}
\hline PEGDA & $\boldsymbol{C}_{\mathbf{M}}^{\max }[\%]$ & $\boldsymbol{R}_{\mathbf{P}}^{\max }\left[\mathbf{s}^{-\mathbf{1}}\right]$ \\
\hline 250 & 92 & 1,17 \\
575 & 98 & 1,16 \\
700 & 99 & 1,17 \\
\hline
\end{tabular}

Anschließend wurden die Umsatz/Zeit-Kurven von RAFT-Polymerisationen der PEG-Diacrylate gemessen. Aufgrund der unterschiedlichen Molmassen der Monomere wurde bei den folgenden Untersuchungen der Massenanteil des RAFT-Agens EPHT (bezogen auf die Masse des Monomers) in den Polymerisationsmischungen variiert und nicht wie zuvor der molare Anteil (s. Kapitel 4.1). Somit ist sichergestellt, dass der Anteil des RAFT-Agens bei den verschiedenen Monomeren vergleichbar groß ist. Außerdem ist der Massenanteil für industrielle Anwendungen, in denen multifunktionelle Monomere häufig verwendet werden, eine übliche Größe. Abbildung 4-59 zeigt die Umsatz/Zeit-Kurven von RAFT-Polymerisation von PEGDA-250 mit einem Massenanteil des RAFT-Agens EPHT von 1 bis $5 \%$. Zusätzlich wurde als Vergleich die Umsatz/Zeit-Kurve des konventionellen Systems hinzugefügt. Die genauen Zusammensetzungen der Polymerisationsmischungen sowie die gewählten Reaktionsbedingungen und eine Beschreibung der Auswertung der aufgenommenen FTIR-Spektren sind in Abschnitt 6.2.4.3 und Kapitel 6.3.4 zu finden. 


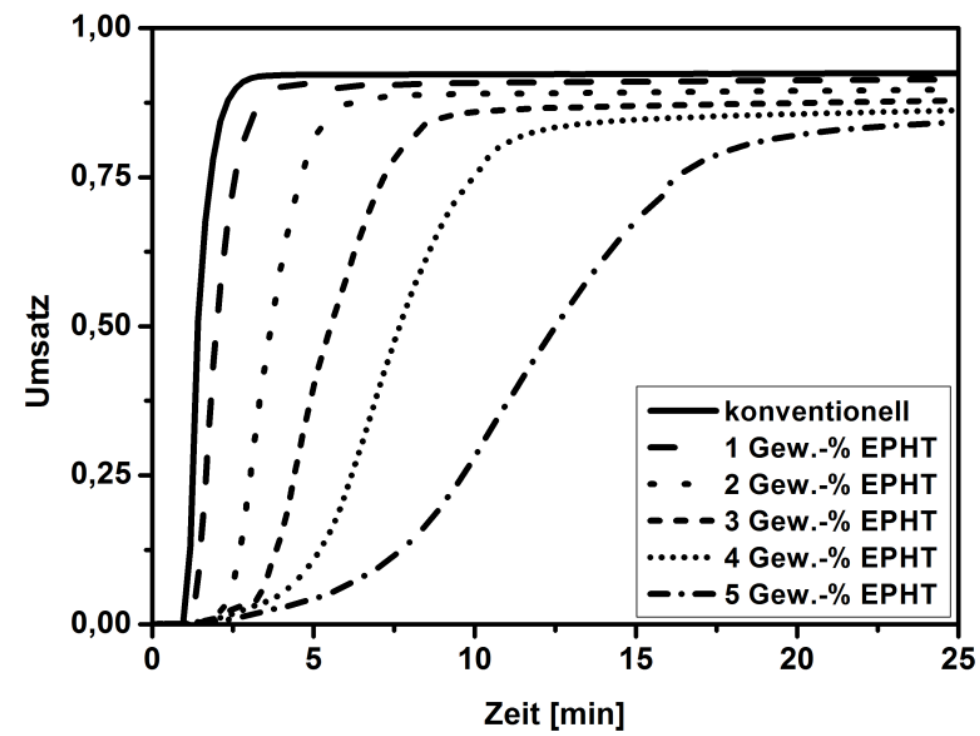

Abbildung 4-59 Umsatz/Zeit-Kurven von RAFT-Polymerisationen von PEGDA-250 mit unterschiedlichen Massenanteilen des RAFT-Agens EPHT bei $21^{\circ} \mathrm{C}$. Die Strahlungsintensität betrug $9 \mathrm{~mW} / \mathrm{cm}^{2}$ bei einer Wellenlänge von $366 \mathrm{~nm}$. Als Photoinitiator wurden 2 Gew.-\% MMMP verwendet. Vergleichend wurde die Umsatz/Zeit-Kurve einer konventionellen PEGDA-250-Polymerisation hinzugefügt.

Es zeigt sich, dass ähnlich wie bei den statistischen Netzwerken (s. Kapitel 4.1.4) der maximale Umsatz mit zunehmendem Massenanteil des RAFT-Agens in der Polymerisationsmischung immer später erreicht wird, und dass die Reaktion verzögert startet. Weiterhin nimmt der maximale Umsatz mit steigendem EPHT-Anteil ab. Ersteres kann wieder auf die retardierende Wirkung des EPHTs zurückgeführt werden, die auf die Absorption der UV-Strahlung durch das RAFT-Agens zurückgeht. Würde dies auch die abnehmenden maximalen Monomerumsätze der Reaktion vollständig erklären, so müsste sich aufgrund der gleichen Massenanteile des RAFTAgens bei den anderen Monomeren der gleiche Effekt zeigen, da dieser Effekt ausschließlich von der Konzentration von EPHT in den Polymerisationsmischungen abhängt (s. Abschnitt 4.1.4.2). Wie an Abbildung 4-60 und Abbildung 4-61 zu erkennen ist, ist dies aber nicht der Fall. 


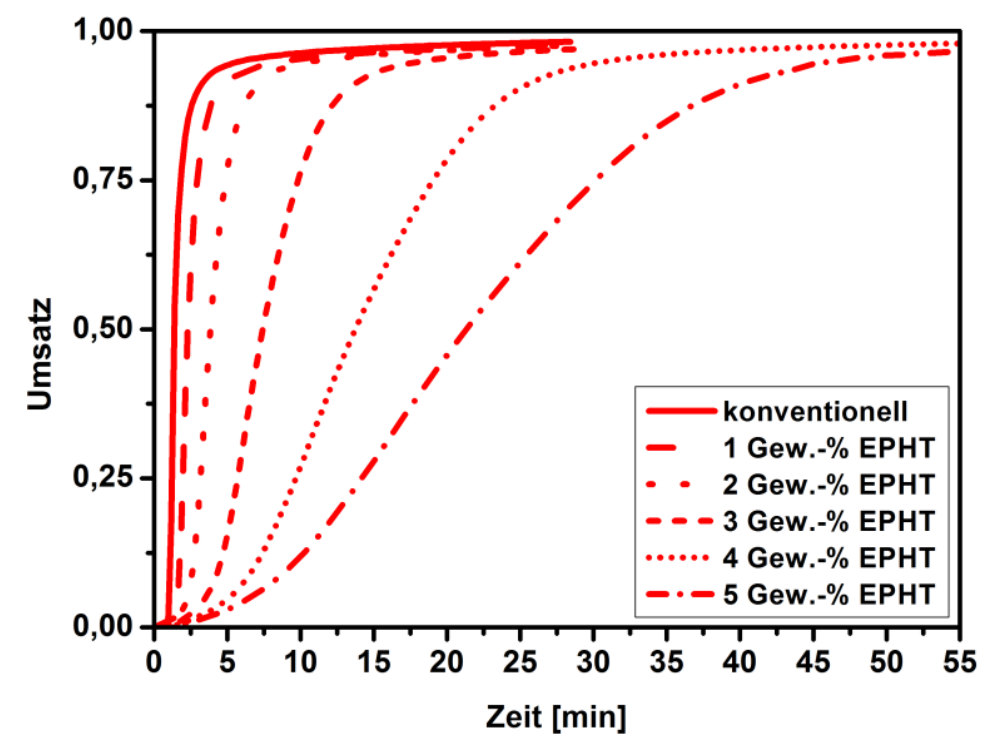

Abbildung 4-60 Umsatz/Zeit-Kurven von RAFT-Polymerisationen von PEGDA-575 mit unterschiedlichen Massenanteilen des RAFT-Agens EPHT bei $21^{\circ} \mathrm{C}$. Die Strahlungsintensität betrug $9 \mathrm{~mW} / \mathrm{cm}^{2}$ bei einer Wellenlänge von $366 \mathrm{~nm}$. Als Photoinitiator wurden 2 Gew.-\% MMMP verwendet. Vergleichend wurde der Umsatz/Zeit-Kurve einer konventionellen PEGDA-575-Polymerisation hinzugefügt.

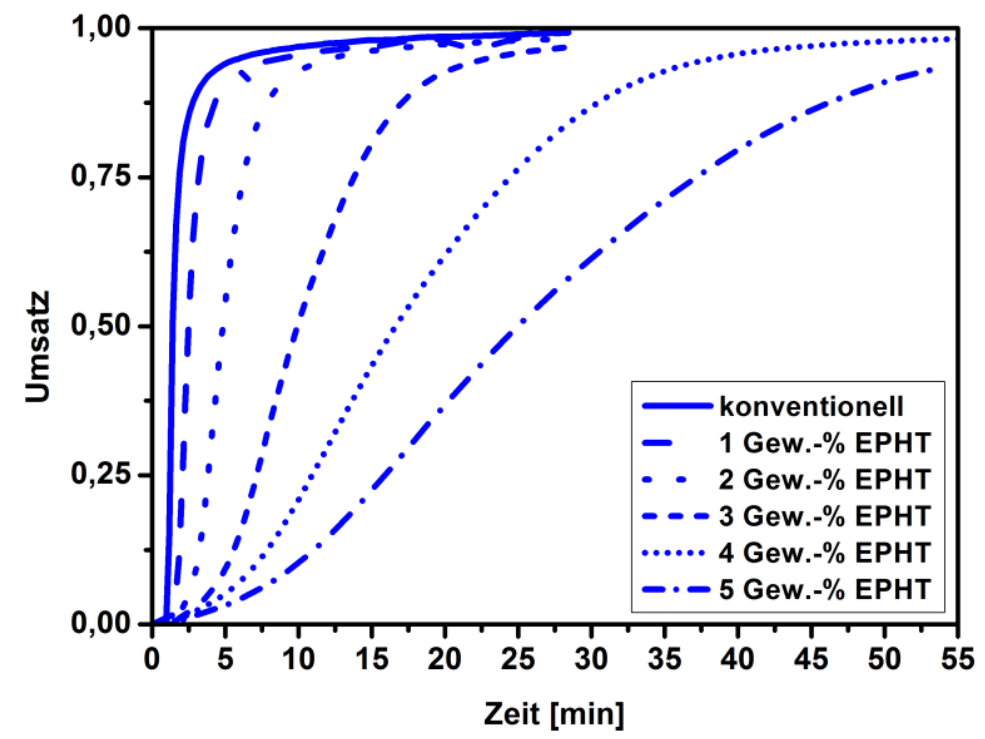

Abbildung 4-61 Umsatz/Zeit-Kurven von RAFT-Polymerisationen von PEGDA-700 mit unterschiedlichen Massenanteilen des RAFT-Agens EPHT bei $21{ }^{\circ} \mathrm{C}$. Die Strahlungsintensität betrug $9 \mathrm{~mW} / \mathrm{cm}^{2}$ bei einer Wellenlänge von $366 \mathrm{~nm}$. Als Photoinitiator wurden 2 Gew.-\% MMMP verwendet. Vergleichend wurde der Umsatz/Zeit-Kurve einer konventionellen PEGDA-700-Polymerisation hinzugefügt. 
Die Monomerumsätze der RAFT-Polymerisationen von PEGDA-575 und PEGDA-700 erreichen sogar mit bis zu 5 Gew.-\% EPHT in den Polymerisationsmischungen Werte von nahezu $100 \%$, wobei diese maximalen Umsätze bei steigender RAFT-Menge später erreicht werden. Wenn die Zeitpunkte verglichen werden, bei denen das System seinen maximalen Umsatz erreicht, zeigt sich folgender Trend. Bei gleicher RAFT-Menge nimmt die Verzögerung mit steigender Molmasse des Monomers zu. Diese Verzögerung geht wahrscheinlich nicht auf die Absorption des EPHTs zurück. Es besteht die Möglichkeit, dass die Umsatz/Zeit-Kurven von PEGDA-575 und PEGDA-700 im Rahmen des experimentellen Fehlers gleich sind. Allerdings sie kann ihre Herkunft auch im Einfluss des RAFT-Mechanismus auf die Bildung der Netzwerke und somit auf die Reaktionskinetik haben. Mittels ESR-Spektroskopie konnte außerdem nachgewiesen werden, dass die Polymerisationen der PEGDA-250-Monomere nicht durch einen vollständigen Verbrauch des Photoinitiators beendet worden ist (s. Abschnitt 4.2.1.2).

Eine weitere mögliche Erklärung für den abnehmenden Umsatz der PEGDA-250 Polymerisationen liegt in der ebenfalls abnehmenden Polymerisationsgeschwindigkeit (s. Tabelle 4-33) begründet. Aus den Polymerisationen multifunktioneller (Meth-)Acrylate ist bekannt, dass eine schnellere Polymerisation zu höheren Umsätzen führen kann. Dies wird auf eine Verzögerung in der Volumenschrumpfung zurückgeführt. Aufgrund dieser ist das freie Volumen im System größer, was wiederum den beteiligten Spezies länger eine größere Mobilität und somit höhere Umsätze ermöglicht. ${ }^{[1,92]}$ Zusätzlich ist es möglich, dass die größere Wärmeentwicklung einer schnelleren Polymerisation eine mögliche Verglasung des Systems länger verhindern kann, da das System länger über seiner Glastemperatur verbleibt. Dies würde ebenfalls die Mobilität von kleinen Spezies in der Polymerisationsmischung länger erhalten und den Umsatz der Reaktion vergrößern.

Für die PEGDA-575- und PEGDA-700-Systeme haben diese Faktoren kaum einen Einfluss, da sie sowieso eine größere Mobilität der niedermolekularen Spezies erlauben, wie die Umsatz/ZeitKurven der konventionellen Polymerisationen zeigen. (s. Abbildung 4-58). Hinzu kommt, dass der Einfluss auf die Strukturen der Netzwerke bei den Monomeren größerer Molmasse größer sein sollte. Im PEGDA-250-System ist der Abstand zwischen dem propagierenden Radikal und einer seitlichen Doppelbindung viel kürzer. Somit kann es in diesen Systemen eher zu intramolekularen Ringbildungen und anderen Netzwerkdefekten kommen. Dadurch kommt es zu einer ausgeprägten Bildung von Nanogel-Domänen, die die Homogenität des Netzwerks reduzieren. Dies konnte bei RAFT-Polymerisationen von bifunktionellen Polymethacrylaten nachgewiesen werden. ${ }^{[78,79]}$ Hinzu kommt, dass das Konzentrationsverhältnis von Monomer zu RAFT-Agens mit steigender Molmasse der Monomere immer kleiner wird (s. Tabelle 4-32). Das bedeutet, dass auf jedes Monomermolekül mehr RAFT-Moleküle kommen, was die Wahrscheinlichkeit einer Reaktion eines propagierenden Radikals mit einem EPHT-Molekül deutlich erhöht. Dies wiederum reduziert die Bildung von Nanogel-Domänen und erhält länger 
eine gewisse Beweglichkeit der propagierenden Makromoleküle im System. Dies verzögert die Selbstbeschleunigung und lässt die Polymerisation langsamer werden.

Tabelle 4-32 Monomer- und RAFT-Konzentrationen sowie die Konzentrationsverhältnisse bezogen auf eine Monomermasse von $1 \mathrm{~g}$ und einen RAFT-Anteil von $1 \mathrm{Gew} . \%$ (0,01 g).

\begin{tabular}{cccc}
\hline PEGDA & $\boldsymbol{c}_{\mathbf{M}}[\mathbf{m o l} / \mathbf{m L}]$ & $\boldsymbol{c}_{\mathrm{EPHT}}[\mathbf{m o l} / \mathbf{m L}]$ & $\boldsymbol{c}_{\mathbf{M}} / \boldsymbol{c}_{\mathrm{EPHT}}$ \\
\hline 250 & $4,4 \cdot 10^{-3}$ & $3,8 \cdot 10^{-5}$ & 117 \\
575 & $2,0 \cdot 10^{-3}$ & $3,8 \cdot 10^{-5}$ & 51 \\
700 & $1,6 \cdot 10^{-3}$ & $3,8 \cdot 10^{-5}$ & 42 \\
\hline
\end{tabular}

Dies wird auch in den Polymerisationsgeschwindigkeiten deutlich, die mit höherer EPHT-Menge abnehmen (s. Tabelle 4-33). Mit zunehmender Molmasse der Monomere ist diese Abnahme bei steigendem EPHT-Anteil in den Polymerisationsmischungen immer deutlicher. Der Einfluss des RAFT-Agens auf die Reaktionskinetik von PEG-Diacrylaten steigt also mit steigender Molmasse der Monomere.

Tabelle 4-33 Maximale Umsätze $C_{\mathrm{M}}^{\max }$ und maximale Reaktionsgeschwindigkeiten $R_{\mathrm{P}}^{\max }$ von UV-initiierten RAFTPolymerisationen von drei PEG-Diacrylaten mit Spacer-Längen von $n=3,10$ und 13 Ethylenglycol-Einheiten bei einer Umgebungstemperatur von $21^{\circ} \mathrm{C}$ und einer Strahlungsintensität von $9 \mathrm{~mW} / \mathrm{cm}^{2}$ bei einer Wellenlänge von $366 \mathrm{~nm}$. Als Photoinitiator wurden 2 Gew.-\% MMMP verwendet.

\begin{tabular}{cccc}
\hline PEGDA & EPHT-Anteil [Gew.-\%] & $\boldsymbol{C}_{\mathbf{M}}^{\max }[\%]$ & $\boldsymbol{R}_{\mathbf{P}}^{\max }\left[\mathbf{s}^{-\mathbf{1}}\right]$ \\
\hline 250 & 1 & 91 & 0,77 \\
250 & 2 & 90 & 0,43 \\
250 & 3 & 88 & 0,27 \\
250 & 4 & 87 & 0,18 \\
250 & 5 & 85 & 0,09 \\
\hline 575 & 1 & 98 & 0,77 \\
575 & 2 & 97 & 0,38 \\
575 & 3 & 97 & 0,16 \\
575 & 4 & 98 & 0,07 \\
575 & 5 & 97 & 0,04 \\
\hline 700 & 1 & 100 & 0,63 \\
700 & 2 & 98 & 0,26 \\
700 & 3 & 97 & 0,09 \\
700 & 4 & 98 & 0,05 \\
700 & 5 & 95 & 0,03 \\
\hline
\end{tabular}




\subsubsection{Untersuchungen der PEGDA-Systeme mittels ESR-Spektroskopie}

Die Elektronen-Spin-Resonanz-(ESR)-Spektroskopie ermöglicht die Detektion von paramagnetischen Spezies, wie z. B. die bei einer radikalischen Polymerisation auftretenden Radikale. Diese können anhand ihres ESR-Spektrums identifiziert werden. Weiterhin ist es mittels zeitaufgelöster ESR-Spektroskopie möglich, die Konzentration der Radikale im Verlauf der Polymerisation zu ermitteln. Diese ist proportional zum Doppelintegral des ESR-Spektrums der Radikale. $^{[44,96,97,207,223-225]}$

Die Untersuchung der PEGDA-Systeme erfolgte jeweils für eine konventionelle Polymerisation und für eine RAFT-Polymerisation mit einem Massenanteil von 1 Gew.-\% EPHT (bezogen auf die Masse des Monomers) als RAFT-Agens. Die Zusammensetzungen der Polymerisationsmischungen entsprachen denen aus dem vorigen Abschnitt und sind in Abschnitt 6.2.4.3 zu finden. Im Unterschied $\mathrm{zu}$ den vorangegangenen kinetischen Untersuchungen (s. Abschnitt 4.2.1.1) wurde bei den ESR-Experimenten, aufgrund der Geometrie des Spektrometers, eine andere Lichtquelle verwendet. Eine genaue Beschreibung des Versuchsaufbaus und des Messablaufs ist in Kapitel 6.2.4.3 zu finden. Während jeder Polymerisation wurde eine Serie von 60 ESR-Spektren aufgenommen. Nach einigen Spektren (fünf bei den konventionellen Polymerisationen, vier bei den RAFT-Polymerisationen) wurde die UV-Strahlung in die Messkammer des Spektrometers eingekoppelt und die Polymerisation gestartet. Anschließend wurden 45 Spektren während der Polymerisation aufgenommen. Bei einer Messdauer von 5,24 Sekunden pro Spektrum ergab sich somit eine Polymerisationszeit von ungefähr fünf Minuten. Dies entspricht der Zeit, die die konventionellen PEGDA-Systeme benötigten, um ihren vollständigen Umsatz zu erreichen wie aus Abbildung 4-59, Abbildung 4-60 und Abbildung 4-61 hervorgeht. Anschließend wurden bei allen Systemen noch 10 Spektren ohne weitere UV-Bestrahlung aufgenommen, um einen möglichen Abbau der Radikalkonzentration im System zu verfolgen.

Abbildung 4-62 zeigt drei ausgewählte ESR-Spektren des PEGDA-250-Systems. Nach dem 5. Spektrum (schwarz) wurde die Polymerisation gestartet und mit der UV-Bestrahlung der Probe in der Messkammer des Spektrometers begonnen. Nach dem 50. Spektrum (rot) wurde die UVBestrahlung beendet. Das 58. Spektrum (blau) ist ca. 50 Sekunden nach dem Ende der Bestrahlung aufgenommen worden. Alle Spektren wurden normiert, indem sie durch die höchste gemessene Signalintensität der jeweiligen Messreihe geteilt wurden. 


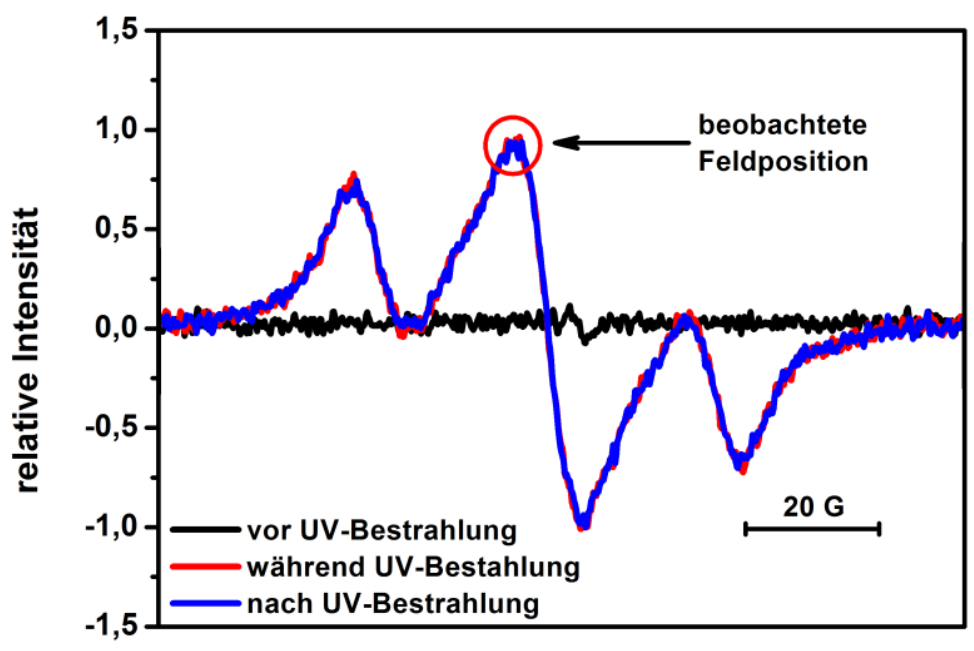

Abbildung 4-62 Normierte ESR-Spektren von PEGDA-250 vor (-), während (-) und nach (-) der UV-initiierten, konventionellen radikalischen Polymerisation bei $21^{\circ} \mathrm{C}$. Die Intensität der UV-Strahlung betrug $100 \mathrm{~mW} / \mathrm{cm}^{2}$ bei $365 \mathrm{~nm}$. Als Photoinitiator wurden 2 Gew.-\% MMMP verwendet. Die Durchlaufzeit einer Messung betrug 5,24 s, als Modulationsamplitude wurden $3 \mathrm{G}$ gewählt, die Modulationsfrequenz betrug $100 \mathrm{kHz}$, die Verstärkung des Empfängers war $60 \mathrm{~dB}$. Die Mikrowellenbrücke wurde mit einer Leistung von 0,63 mW bei einer Dämpfung von $25 \mathrm{~dB}$ betrieben. Die markierte Feldposition wurde für die weitere Auswertung verwendet.

Erwartungsgemäß ist vor dem Einkoppeln des Lichts in die Messzelle des Spektrometers kein ESR-Signal zu erkennen. Das letzte Spektrum vor dem Auskoppeln der UV-Strahlung (50. Spektrum) zeigt dagegen ein sehr deutliches Signal, das dem SPR des Acrylat-Makroradikals ähnelt. ${ }^{[225]}$ Es fällt auf, dass die Signalstärke auch 50 Sekunden nach dem Ende der UV-Bestrahlung noch nicht signifikant abgenommen hat, was bedeutet, dass sich noch immer Radikale im System befinden. Um die absolute Konzentration der Radikale zu bestimmen, ist eine vorherige Kalibrierung mit Lösungen verschiedener Konzentration eines stabilen (persistenten) Radikals notwendig. ${ }^{[225]}$ Diese Lösungen müssen exakt den untersuchten Systemen entsprechen. Da bei den durchgeführten Messungen bis zu vollständigem Monomerumsatz polymerisiert wurde, ist ein Ansetzten einer solchen Kalibierlösung in diesem Fall nicht möglich.

Allerdings kann die Radikalkonzentration qualitativ über die Signalintensität, die proportional zum Doppelintegral des Spektrums ist, trotzdem abgebildet werden. Dazu wurde für jedes aufgenommene Spektrum das Maximum des ESR-Signals ermittelt (s. Abbildung 4-62, rote Markierung) und gegen die Polymerisationszeit aufgetragen. Als Ergebnis wird die Entwicklung der maximalen Signalintensität über die Zeit erhalten (s. Abbildung 4-63). 


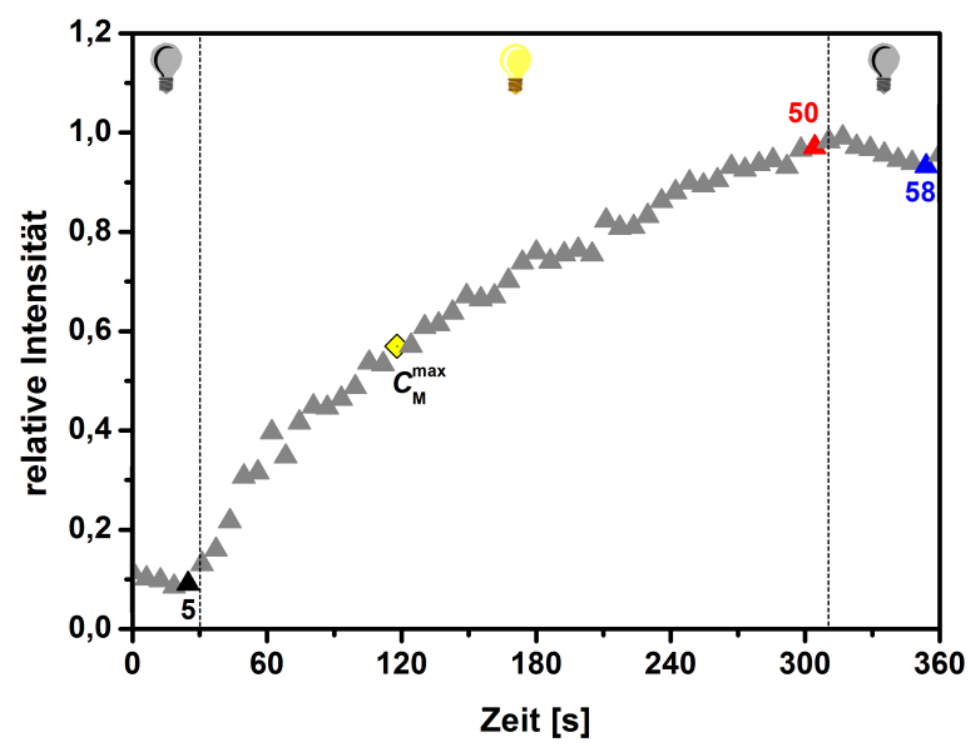

Abbildung 4-63 Entwicklung der maximalen relativen Intensität des ESR-Spektrums von PEGDA-250 während einer UV-initiierten, konventionellen radikalischen Polymerisation bei $21^{\circ} \mathrm{C}$. Die Intensität der UV-Strahlung betrug $100 \mathrm{~mW} / \mathrm{cm}^{2}$ bei $365 \mathrm{~nm}$. Als Photoinitiator wurden 2 Gew.-\% MMMP verwendet. Die Durchlaufzeit einer Messung betrug 5,24 s, als Modulationsamplitude wurden $3 \mathrm{G}$ gewählt, die Modulationsfrequenz betrug $100 \mathrm{kHz}$, die Verstärkung des Empfängers war $60 \mathrm{~dB}$. Die Mikrowellenbrücke wurde mit einer Leistung von 0,63 mW bei einer Dämpfung von $25 \mathrm{~dB}$ betrieben. Markiert wurde der Zeitpunkt bei dem in FTIR-Experimenten der maximale Umsatz erreicht war (s. Abbildung 4-58). Die zu den weiteren markierten Datenpunkten gehörenden ESR-Spektren sind in Abbildung 4-62 dargestellt.

Abbildung 4-63 zeigt, dass die Signalintensität nach dem Einkoppeln der UV-Strahlung zunächst deutlich ansteigt. Nachdem die Polymerisation ihren maximalen Umsatz nahezu erreicht (s. Abbildung 4-58) hat, ${ }^{\text {a }}$ wird der Anstieg der Signalintensität etwas geringer, sie nimmt aber weiterhin im Verlauf der Polymerisation zu. Nach dem Ende der UV-Bestrahlung bleibt die Signalintensität nahezu konstant. Das bedeutet, dass trotz fehlender Initiierung die Radikalkonzentration nahezu konstant bleibt, also die im System vorhandenen Radikale nicht z. B. durch Terminierung abgebaut werden. Der Grund hierfür ist das sehr dichte Netzwerk, das aus den PEGDA-250-Monomeren gebildet wird. Die Radikale sind innerhalb des Netzwerks gefangen und können einander nicht mehr erreichen, um zu terminieren. Darum kommt es auch schon nach Erreichen des maximalen Umsatzes zu einer weiteren Anreicherung von Radikalen im System. Schon im frühen Stadium der Polymerisation können Radikale in den gebildeten Nanogel-Domänen gefangen sein, sodass es ihnen nicht mehr möglich ist, mit einem anderen Radikal zu terminieren. ${ }^{[97]}$ Durch fortwährende Photoinitiierung werden bei andauernder UV-

\footnotetext{
${ }^{a}$ Da die Lichtintensität bei den ESR-Experimenten größer war, könnte der maximale Monomerumsatz eher erreicht worden sein, als bei den FTIR-Messungen (s. Abschnitt 4.2.1.1).
} 
Bestrahlung immer neue Radikale gebildet, sodass die Signalintensität immer weiter ansteigt, bis die Bestrahlung beendet wird.
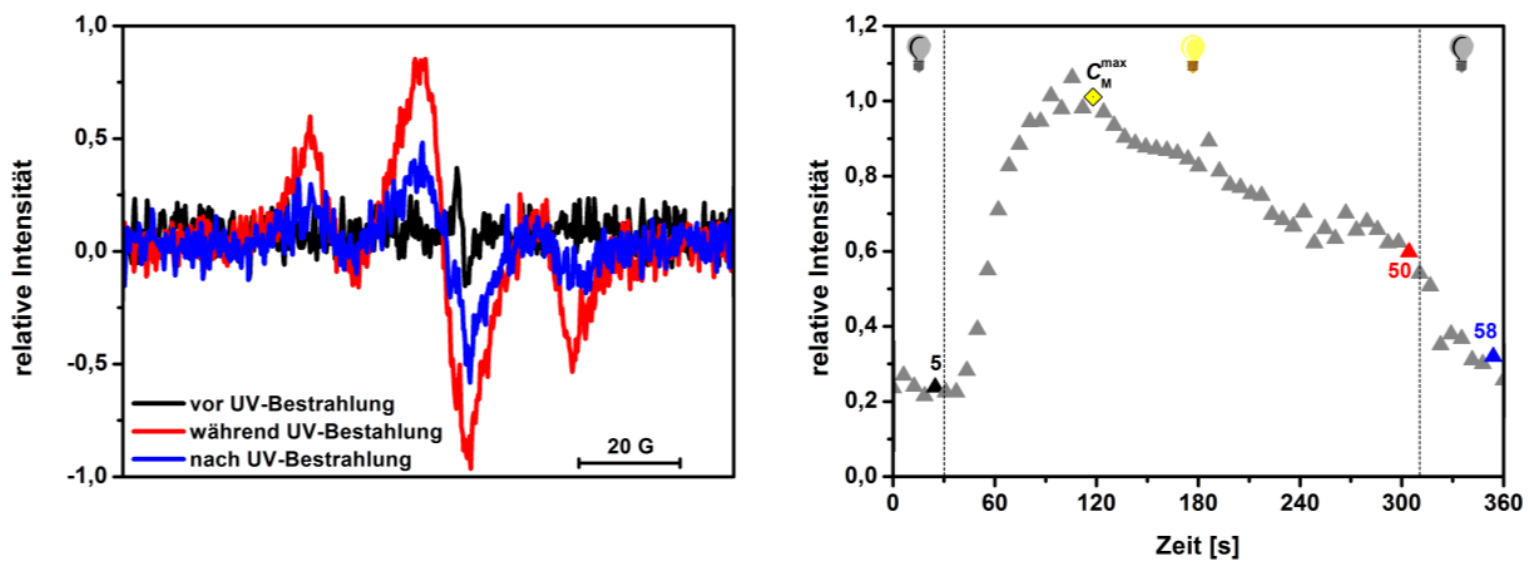

Abbildung 4-64 Links: ESR-Spektren von PEGDA-575 vor (-), während (-) und nach (-) der UV-initiierten, konventionellen radikalischen Polymerisation bei $21{ }^{\circ} \mathrm{C}$. Rechts: Die Entwicklung der maximalen relativen Intensität des ESR-Spektrums von PEGDA-575 während einer UV-initiierten, konventionellen radikalischen Polymerisation. Die Intensität der UV-Strahlung betrug $100 \mathrm{~mW} / \mathrm{cm}^{2}$ bei $365 \mathrm{~nm}$. Als Photoinitiator wurden 2 Gew.-\% MMMP verwendet. Die Durchlaufzeit einer Messung betrug 5,24 s, als Modulationsamplitude wurden $3 \mathrm{G}$ gewählt, die Modulationsfrequenz betrug $100 \mathrm{kHz}$, die Verstärkung des Empfängers war $60 \mathrm{~dB}$. Die Mikrowellenbrücke wurde mit einer Leistung von 0,63 $\mathrm{mW}$ bei einer Dämpfung von $25 \mathrm{~dB}$ betrieben. Markiert wurde der Zeitpunkt bei dem in FTIRExperimenten der maximale Umsatz erreicht war (s. Abbildung 4-58).
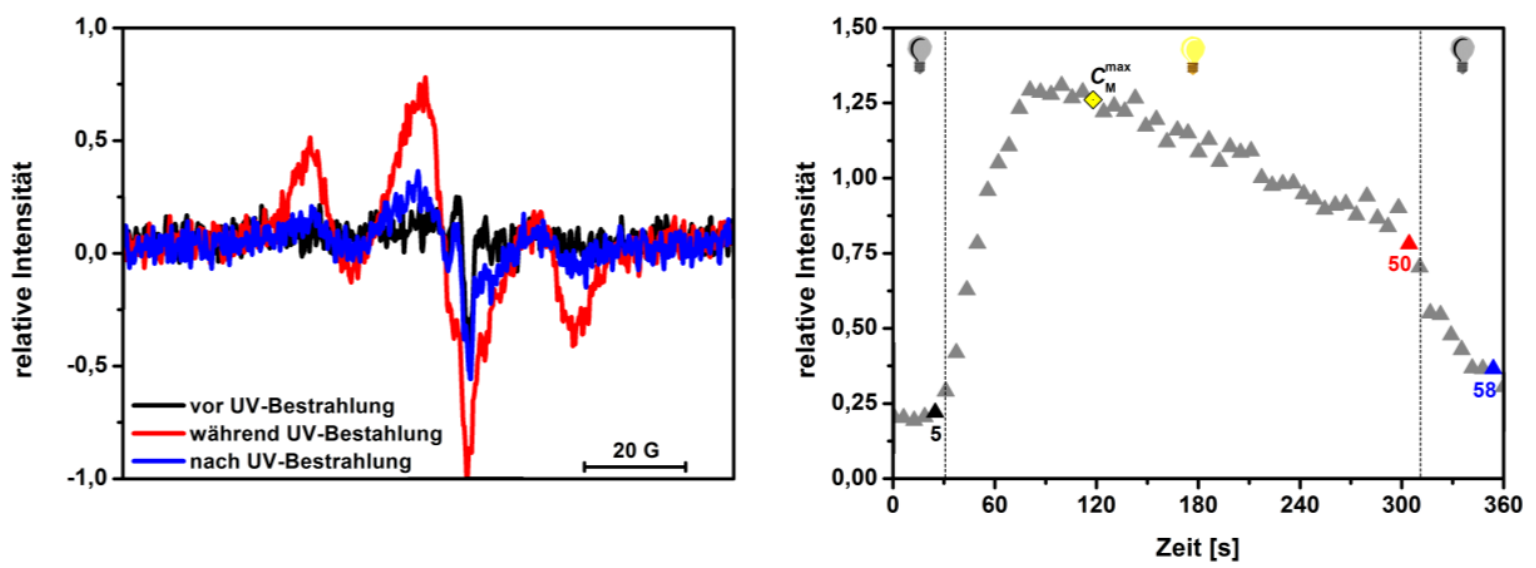

Abbildung 4-65 Links: ESR-Spektren von PEGDA-700 vor (-), während (-) und nach (-) der UV-initiierten, konventionellen radikalischen Polymerisation bei $21{ }^{\circ} \mathrm{C}$. Rechts: Die Entwicklung der maximalen relativen Intensität des ESR-Spektrums von PEGDA-700 während einer UV-initiierten, konventionellen radikalischen Polymerisation. Die Intensität der UV-Strahlung betrug $100 \mathrm{~mW} / \mathrm{cm}^{2}$ bei $365 \mathrm{~nm}$. Als Photoinitiator wurden 2 Gew.-\% MMMP verwendet. Die Durchlaufzeit einer Messung betrug 5,24 s, als Modulationsamplitude wurden $3 \mathrm{G}$ gewählt, die Modulationsfrequenz betrug $100 \mathrm{kHz}$, die Verstärkung des Empfängers war $60 \mathrm{~dB}$. Die Mikrowellenbrücke wurde mit einer Leistung von 0,63 $\mathrm{mW}$ bei einer Dämpfung von $25 \mathrm{~dB}$ betrieben. Markiert wurde der Zeitpunkt bei dem in FTIRExperimenten der maximale Umsatz erreicht war (s. Abbildung 4-58). 
Es wird deutlich, dass das PEGDA-575- (s. Abbildung 4-64) und das PEGDA-700-System (s. Abbildung 4-65) andere Verläufe der ESR-Signalintensitäten zeigen als das PEGDA-250System (s. Abbildung 4-63). Der maximale Umsatz ist auch bei diesen Polymerisationen nach maximal 90 Sekunden nahezu erreicht (s. Abbildung 4-58). Bis dahin steigt die Signalintensität stark an. Anschließend beginnt sie zu fallen. In den weniger dichten PEGDA-575- und PEGDA700-Netzwerken ist es den Radikalen besser möglich, mit anderen Radikalen zu terminieren als im PEGDA-250-Netzwerk. Da aber auch durch die andauernde UV-Bestrahlung neue Radikale nachgeliefert werden, erfolgt dieser Radikalabbau relativ langsam. Nachdem die UV-Bestrahlung beendet wurde, sinkt die Signalintensität bei beiden Systemen innerhalb des beobachteten Zeitraums sehr schnell nahezu auf den Anfangswert vor der Polymerisation ab, da die noch vorhandenen Radikale nach und nach terminieren.

Bei der Betrachtung der ESR-Signale der RAFT-Polymerisation von PEGDA-250 (s. Abbildung 4-66) fällt im Vergleich zu dem Signal der konventionellen radikalischen Polymerisation (s. Abbildung 4-62) auf, dass das ESR-Signal eine andere Form hat. Dies geht auf die Überlagerung des Signals der propagierenden Acrylat-Radikale und des Signals des RAFTIntermediat-Radikals zurück. ${ }^{[225]}$
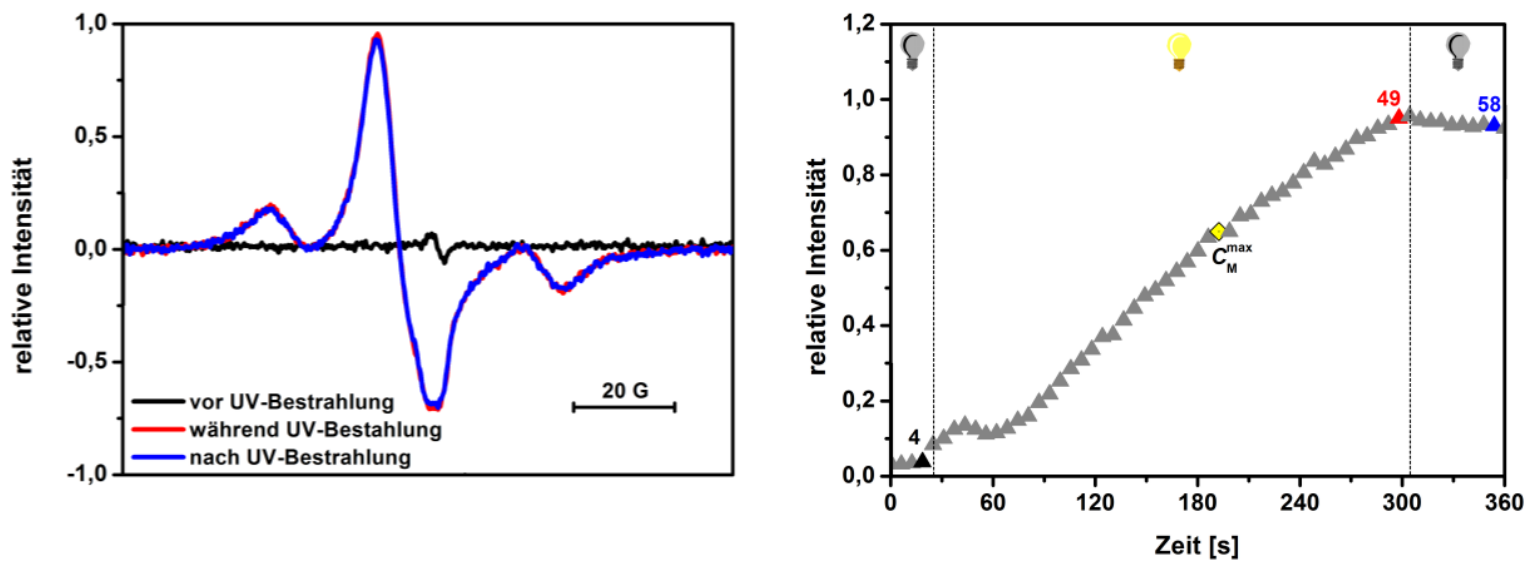

Abbildung 4-66 Links: ESR-Spektren von PEGDA-250 vor (-), während (-) und nach (-) der UV-initiierten RAFTPolymerisation. Rechts: Die Entwicklung der maximalen relativen Intensität des ESR-Spektrums von PEGDA-250 während einer UV-initiierten RAFT-Polymerisation. Die Intensität der UV-Strahlung betrug $100 \mathrm{~mW} / \mathrm{cm}^{2}$ bei $365 \mathrm{~nm}$. Als Photoinitiator wurden 2 Gew.-\% MMMP verwendet. Die Durchlaufzeit einer Messung betrug 5,24 s, als Modulationsamplitude wurden $3 \mathrm{G}$ gewählt, die Modulationsfrequenz betrug $100 \mathrm{kHz}$, die Verstärkung des Empfängers war $60 \mathrm{~dB}$. Die Mikrowellenbrücke wurde mit einer Leistung von 0,63 $\mathrm{mW}$ bei einer Dämpfung von $25 \mathrm{~dB}$ betrieben. Markiert wurde der Zeitpunkt bei dem in FTIR-Experimenten der maximale Umsatz erreicht war (s. Abbildung 4-59).

Im Allgemeinen ähnelt der Verlauf der maximalen Signalintensitäten in Bezug auf die Polymerisationszeit dem des konventionellen PEGDA-250-Systems (s. Abbildung 4-62). Nach dem Beginn der UV-Bestrahlung nach vier aufgenommenen Spektren steigt sie an und erreicht 
ihren maximalen Wert am Ende der Belichtungszeit. Anschließend bleibt sie im beobachteten Zeitraum wieder nahezu konstant und sinkt nur ganz unwesentlich ab. Dies kann darauf schließen lassen, dass trotz der Zugabe des RAFT-Agens das PEGDA-250-Netzwerk eine so dichte Struktur aufweist, dass eine Terminierung der während der Polymerisation entstehenden Radikale auch danach kaum möglich ist.
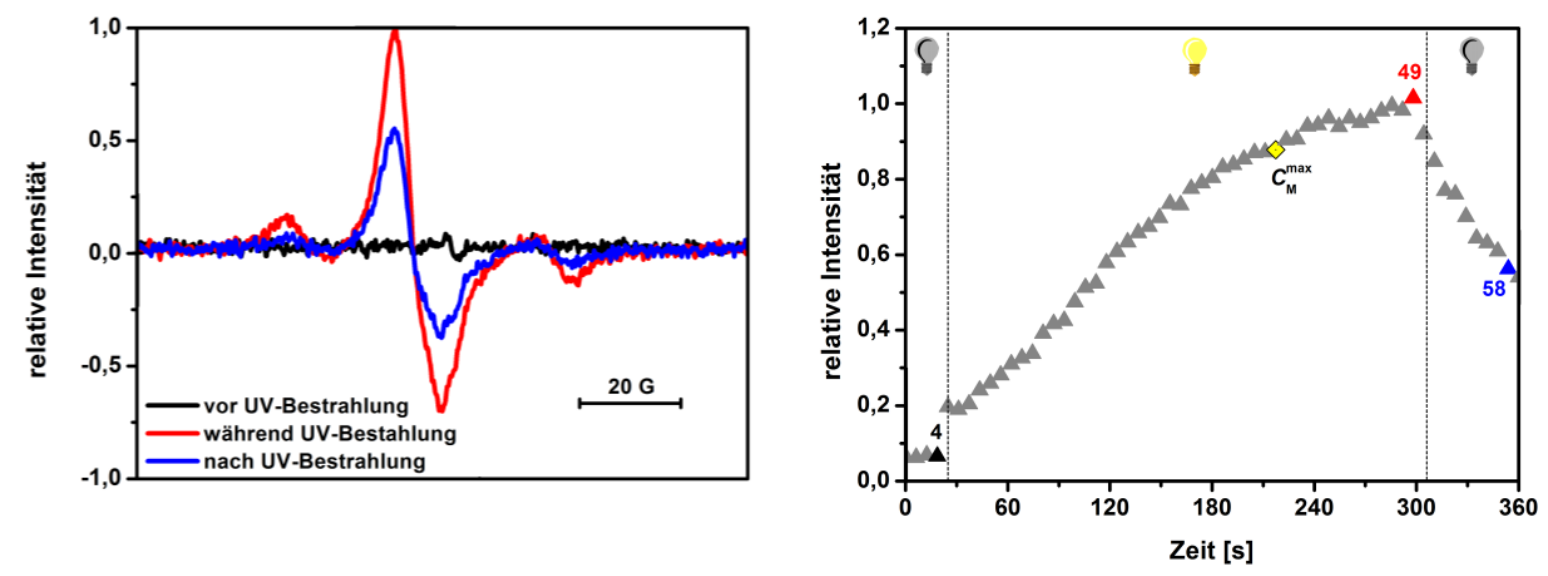

Abbildung 4-67 Links: ESR-Spektren von PEGDA-575 vor (-), während (-) und nach (-) der UV-initiierten RAFTPolymerisation. Rechts: Die Entwicklung der maximalen relativen Intensität des ESR-Spektrums von PEGDA-575 während einer UV-initiierten RAFT-Polymerisation. Die Intensität der UV-Strahlung betrug $100 \mathrm{~mW} / \mathrm{cm}^{2}$ bei $365 \mathrm{~nm}$. Als Photoinitiator wurden 2 Gew.-\% MMMP verwendet. Die Durchlaufzeit einer Messung betrug 5,24 s, als Modulationsamplitude wurden 3 G gewählt, die Modulationsfrequenz betrug $100 \mathrm{kHz}$, die Verstärkung des Empfängers war $60 \mathrm{~dB}$. Die Mikrowellenbrücke wurde mit einer Leistung von 0,63 $\mathrm{mW}$ bei einer Dämpfung von $25 \mathrm{~dB}$ betrieben. Markiert wurde der Zeitpunkt bei dem in FTIR-Experimenten der maximale Umsatz erreicht war (s. Abbildung 4-60).

Die Verläufe der maximalen Signalintensität gegen die Polymerisationszeit bei den RAFTPolymerisationen der PEGDA-575- (s. Abbildung 4-66) und PEGDA-700-Systeme (s. Abbildung 4-67) unterscheiden sich deutlicher von denen der konventionellen radikalischen Polymerisationen (vgl. Abbildung 4-64 und Abbildung 4-65).

Nach Beginn der UV-Bestrahlung steigt die maximale Signalintensität solange an bis die Bestrahlung beendet wird. Dies liegt zum einen an der durch die Zugabe des RAFT-Agens leicht verzögerten Polymerisation (es steht länger Monomer zur Verfügung) und zum anderen an der auch nach dem Erreichen des maximalen Umsatzes weiter stattfindenden Bildung von RAFTIntermediat-Radikalen. Diese verfügen im Vergleich zu propagierenden Radikalen über eine längere Lebensdauer. Außerdem können sie durch den während der UV-Bestrahlung immer fortwährenden Initiatorzerfall immer wieder neu aus den Primärradikalen gebildet werden, sodass immer mehr Intermediat-Radikale im System vorhanden sind. Erst nach dem Abschalten der UV-Bestrahlung werden keine neuen RAFT-Intermediat-Radikale mehr gebildet, sodass diese mit der Zeit terminieren und die ESR-Signalintensität sowohl im PEGDA-575- als auch im PEGDA-700-System abnimmt. 
Im beobachteten Zeitraum sinkt die Signalintensität nach dem Ende der Bestrahlung allerdings nicht wie bei den konventionellen PEGDA-575- und PEGDA-700-Systemen auf den Anfangswert ab. Dies kann auf die größere Stabilität der RAFT-Intermediate zurückgehen. Somit lassen diese Messungen keine Rückschlüsse auf die Strukturen der Netzwerke zu. Für einen Vergleich der Netzwerkstrukturen zwischen einem konventionellen Netzwerk und einem RAFT-Netzwerk eignen sich die im folgenden Abschnitt diskutierten Ergebnisse der RasterkraftmikroskopMessungen besser.
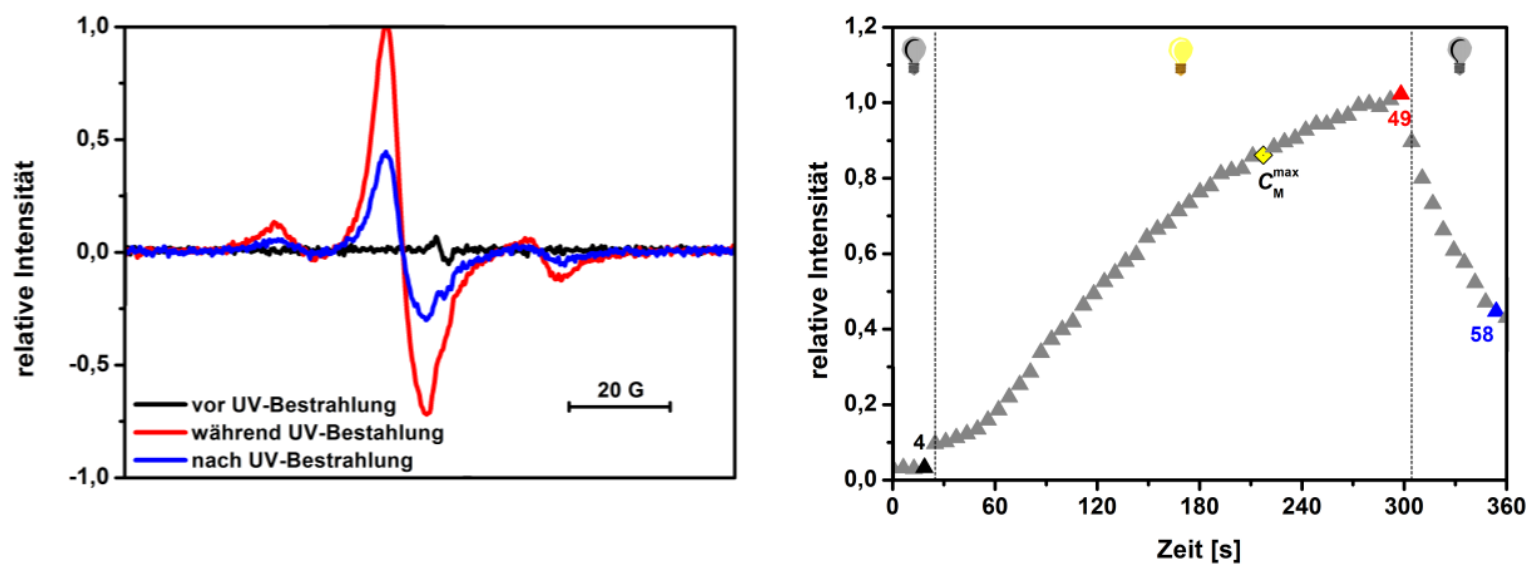

Abbildung 4-68 Links: ESR-Spektren von PEGDA-700 vor (-), während (-) und nach (-) der UV-initiierten RAFTPolymerisation. Rechts: Die Entwicklung der maximalen relativen Intensität des ESR-Spektrums von PEGDA-700 während einer UV-initiierten RAFT-Polymerisation. Die Intensität der UV-Strahlung betrug $100 \mathrm{~mW} / \mathrm{cm}^{2} \mathrm{bei} 365 \mathrm{~nm}$. Als Photoinitiator wurden 2 Gew.-\% MMMP verwendet. Die Durchlaufzeit einer Messung betrug 5,24 s, als Modulationsamplitude wurden $3 \mathrm{G}$ gewählt, die Modulationsfrequenz betrug $100 \mathrm{kHz}$, die Verstärkung des Empfängers war 60 dB. Die Mikrowellenbrücke wurde mit einer Leistung von 0,63 $\mathrm{mW}$ bei einer Dämpfung von $25 \mathrm{~dB}$ betrieben. Markiert wurde der Datenpunkt bei dem in FTIR-Experimenten der maximale Umsatz erreicht war (s. Abbildung 4-61).

\subsubsection{Einfluss der RAFT-Polymerisation auf die Mikrostrukturen der Netzwerke}

Zur Aufklärung der Mikrostrukturen der PEGDA-Netzwerke wurden RasterkraftmikroskopAufnahmen von Probekörpern aus konventioneller radikalischer Polymerisation und RAFTPolymerisation mit 2 Gew.-\% EPHT (bezogen auf die Masse des Monomers) in den Polymerisationsmischungen durchgeführt. Die Höhenprofile der Proben lassen auf die Gesamtstruktur des Netzwerks schließen und ermöglichen einen direkten Vergleich der aus den unterschiedlichen PEG-Diacrylaten resultierenden Netzwerke. Weiterhin bestand wie auch schon in Kapitel 4.1.5 die Möglichkeit, mittels weiterer zur Verfügung stehenden Messkanäle des Rasterkraftmikroskops, Informationen über die mechanischen Eigenschaften der Netzwerkoberflächen zu erhalten. Dadurch war es möglich, das Wissen über die PEGDANetzwerke noch $\mathrm{zu}$ vertiefen und einen besseren Eindruck vom Einfluss der RAFTPolymerisation auf die Strukturen der Netzwerke zu erhalten. Abbildung 4-69 zeigt die 
Höhenprofile der AFM-Aufnahmen von PEGDA-250-Netzwerken aus konventioneller und RAFT-Polymerisation.

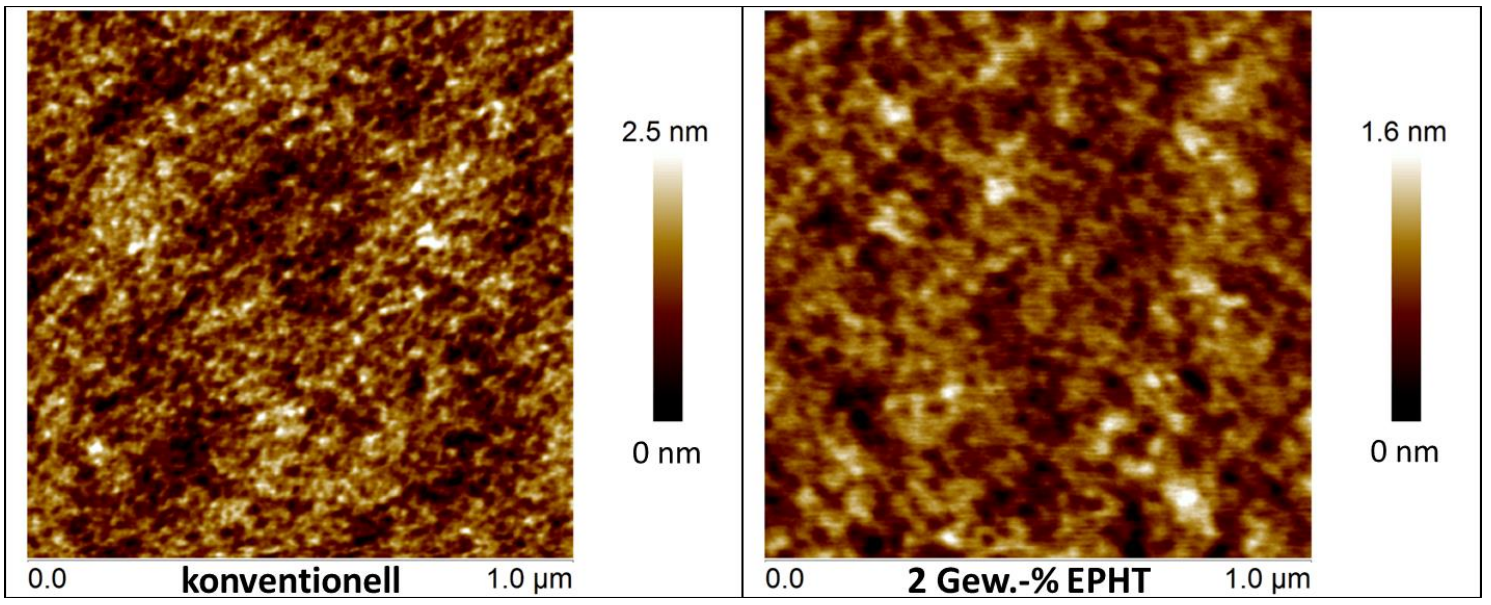

Abbildung 4-69 Rasterkraftmikroskop-Aufnahmen der Höhenprofile der Oberflächen von PEGDA-250-Netzwerken. Links: aus konventioneller, radikalischer Polymerisation. Rechts: aus RAFT-Polymerisation mit 2 Gew.-\% EPHT. Die Aufnahmen wurden im ScanAsyst-Modus durchgeführt. Die Kantenlänge beträgt $1 \times 1 \mu \mathrm{m}$. Die Scan-Frequenz beträgt 0,5 Hz. Es wurden 512 Linien pro Bild aufgenommen.

Auf den ersten Blick sind die Unterschiede zwischen dem Höhenprofil des konventionellen PEGDA-250-Netzwerks und dem entsprechenden RAFT-Netzwerk gering. In beiden Aufnahmen lassen sich höhere Bereiche erkennen, in denen sich in etwa kreisrunde tiefere Bereiche befinden. Dass die Erhöhungen beim konventionellen Netzwerk ca. $1 \mathrm{~nm}$ höher sind als beim RAFTNetzwerk, ist nicht signifikant genug, um davon auszugehen, dass die RAFT-Polymerisation zu einer ebeneren Oberfläche führt. Allerdings ist zu erkennen, dass sich vor allem die tieferen Bereiche vom konventionellen zum RAFT-Netzwerk vergrößern, wie eine Auswertung ${ }^{\mathrm{a}}$ der Aufnahmen mit dem Programm Nanoscope Analysis 1.4 zeigt (s. Tabelle 4-34).

Tabelle 4-34 Mittlere Anzahl, Fläche und Durchmesser der tieferen Bereiche von PEGDA-250-Netzwerken aus konventioneller, radikalischer und RAFT-Polymerisation mit 2 Gew.-\% EPHT als RAFT-Agens. Die Daten wurden mit der Partikelanalysefunktion des Programms NanoScope Analysis (Version 1.4) den Höhenprofilen der Netzwerkoberflächen entnommen (s. Abbildung 4-69).

\begin{tabular}{cccc}
\hline Netzwerk & Anzahl & Fläche $\left[\mathbf{n m}^{2}\right]$ & Durchmesser $[\mathbf{n m}]$ \\
\hline konventionell & 285 & 1700 & 19 \\
RAFT & 152 & 3300 & 25 \\
\hline
\end{tabular}

\footnotetext{
${ }^{a}$ Die Auswertung erfolgte mit einer absoluten Höhenskala. Erfasst wurden jeweils Bereiche, die tiefer lagen als die Hälfte der maximalen Erhöhungen.
} 
Mit der Vergrößerung der tieferen Bereiche geht erwartungsgemäß eine Verringerung der Anzahl einher. Der RAFT-Mechanismus hat also durchaus einen Einfluss auf die Struktur der PEGDA-250-Netzwerke.

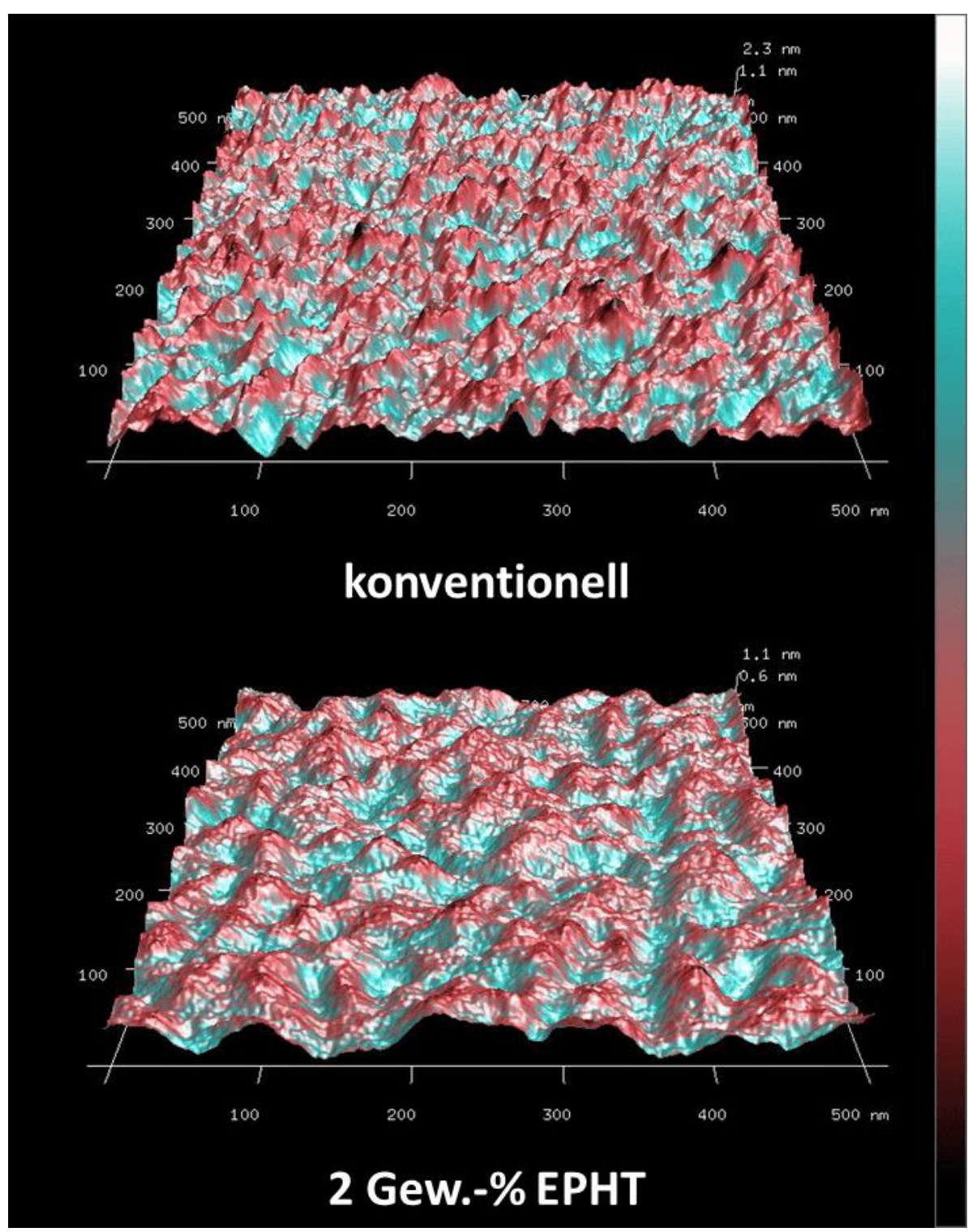

Abbildung 4-70 Dreidimensionale Darstellung von AFM-Aufnahmen des Höhenprofils von PEGDA-250-Netzwerken. Oben: aus konventioneller radikalischer Polymerisation. Unten: aus RAFT-Polymerisation mit 2 Gew.-\% EPHT. Über das Höhenprofil wurde in einer anderen Farbskala der Messkanal für die Adhäsion der Oberflächen gelegt (schwarz: niedrige Adhäsion, Weiß: hohe Adhäsion). Die Aufnahmen wurden im ScanAsyst-Modus durchgeführt. Die Kantenlänge beträgt $500 \times 500 \mathrm{~nm}$. Die Scan-Frequenz betrug 0,5 Hz. Es wurden 512 Linien pro Bild aufgenommen.

Ähnliche Oberflächenstrukturen wurden schon bei vernetzten Dimethacrylaten beobachtet. Der Grund für das Auftreten dieser höheren und tieferen Bereiche auf den Oberflächen der Netzwerke liegt in der während des Aushärtens auftretenden Phasenseparation von Netzwerk und Monomeren. Bei höheren Umsätzen sinkt die Mischbarkeit von Monomeren und schon 
vernetzten Bereichen deutlich. Darum schließen die vernetzten Bereiche bei zunehmender Vernetzungsdichte das Monomer aus und kollabieren zu einem ungequollenen, sehr dichten Netzwerk. Die Monomere können nun durch weiter fortlaufende Initiierung weitere vernetzte Bereiche ausbilden und wieder Monomer ausschließen, sodass die monomerreichen Bereiche zunehmend kleiner werden. Dies kann so lange fortgeführt werden, bis zunehmende Verglasung den Reaktionsprozess stoppt und hochvernetzte, harte Bereiche neben weniger bzw. gering vernetzten, weichen Bereichen vorliegen. ${ }^{[22]}$ Diese Tatsache wird in Abbildung 4-70 noch deutlicher. $\mathrm{Zu}$ sehen sind dreidimensionale Abbildungen der Oberflächen des konventionellen und des RAFT-PEGDA-250-Netzwerks. Als zusätzliche Informationen wurden die Daten des Messkanals der Adhäsion über die Höhenprofile gelegt, sodass die zu erkennende Farbskala für die adhäsiven Eigenschaften der Oberflächen steht. Es wird deutlich, dass die tiefer liegenden Bereiche der Netzwerkoberflächen über eine höhere Adhäsion verfügen als die höher liegenden. Dies unterstützt die zuvor aufgestellte These, dass die tieferen Bereiche nicht so stark vernetzt sind wie die höheren. Wie in Kapitel 4.1.5 gezeigt wurde, verfügen geringer vernetze Bereiche über eine höhere Adhäsion als sehr stark vernetzte Bereiche. Die mittlere Adhäsion der beiden Netzwerke kann mit 9,7 nN für das konventionelle Netzwerk und 8,1 $\mathrm{nN}$ für das RAFT-Netzwerk im Rahmen der Messgenauigkeit als identisch angesehen werden. Dies konnte so auch erwartet werden, denn beide Netzwerke bestehen aus PEGDA-250-Monomeren und somit aus den gleichen Grundbausteinen.

Dass im Gegensatz zu den elastomeren Netzwerken (s. Kapitel 4.1.5) die Nanogel-Domänen in diesen Netzwerken nicht zu erkennen sind, liegt in der geringen Molmasse des PEGDA-250 begründet. Die während der Polymerisation entstehenden Domänen sind so klein, dass sie in das Gesamtnetzwerk integriert werden, ohne sich von diesem stark zu unterscheiden. Außerdem ist die Wahrscheinlichkeit sehr groß, dass das Gesamtnetzwerk über eine sehr ähnliche Netzkettendichte verfügt wie die Nanogel-Domänen.

An Abbildung 4-70 wird ebenfalls deutlich, dass sich neben den tieferen Bereichen auch die höheren Bereiche in ihrer Ausdehnung verbreitern. Eine mögliche Erklärung für die größeren monomerreichen Bereiche auf der Oberfläche der Netzwerke kann im geringeren Monomerumsatz der RAFT-Polymerisation verglichen mit der konventionellen Polymerisation (s. Abbildung 4-59) gefunden werden. Außerdem besteht durchaus die Möglichkeit, dass die RAFT-Polymerisation trotz der bifunktionellen Monomere zu einer Verringerung der NanogelDomänen geführt hat. Dies wiederum würde bedeuten, dass das Netzwerk weniger dicht ist und zum Teil noch von Monomer aufgequollen sein kann, wodurch die Ausdehnung der vernetzten Bereiche größer wird als beim konventionellen Netzwerk. Außerdem unterliegen weniger vernetze Bereiche einer geringeren Volumenschrumpfung (s. Kapitel 2.2.2).

Weiterhin gilt auch für die PEGDA-Netzwerke, dass die Bereiche mit einer höheren Adhäsion über einen geringeren DMT-Modul verfügen. Dies ist in Abbildung 4-71 am Beispiel eines PEGDA-575-Netzwerks aus RAFT-Polymerisation deutlich zu erkennen. In dieser Abbildung 
wurde zusätzlich zur Farbskala der Adhäsion die des DMT-Moduls über das dreidimensional dargestellte Höhenprofil des Netzwerks gelegt.

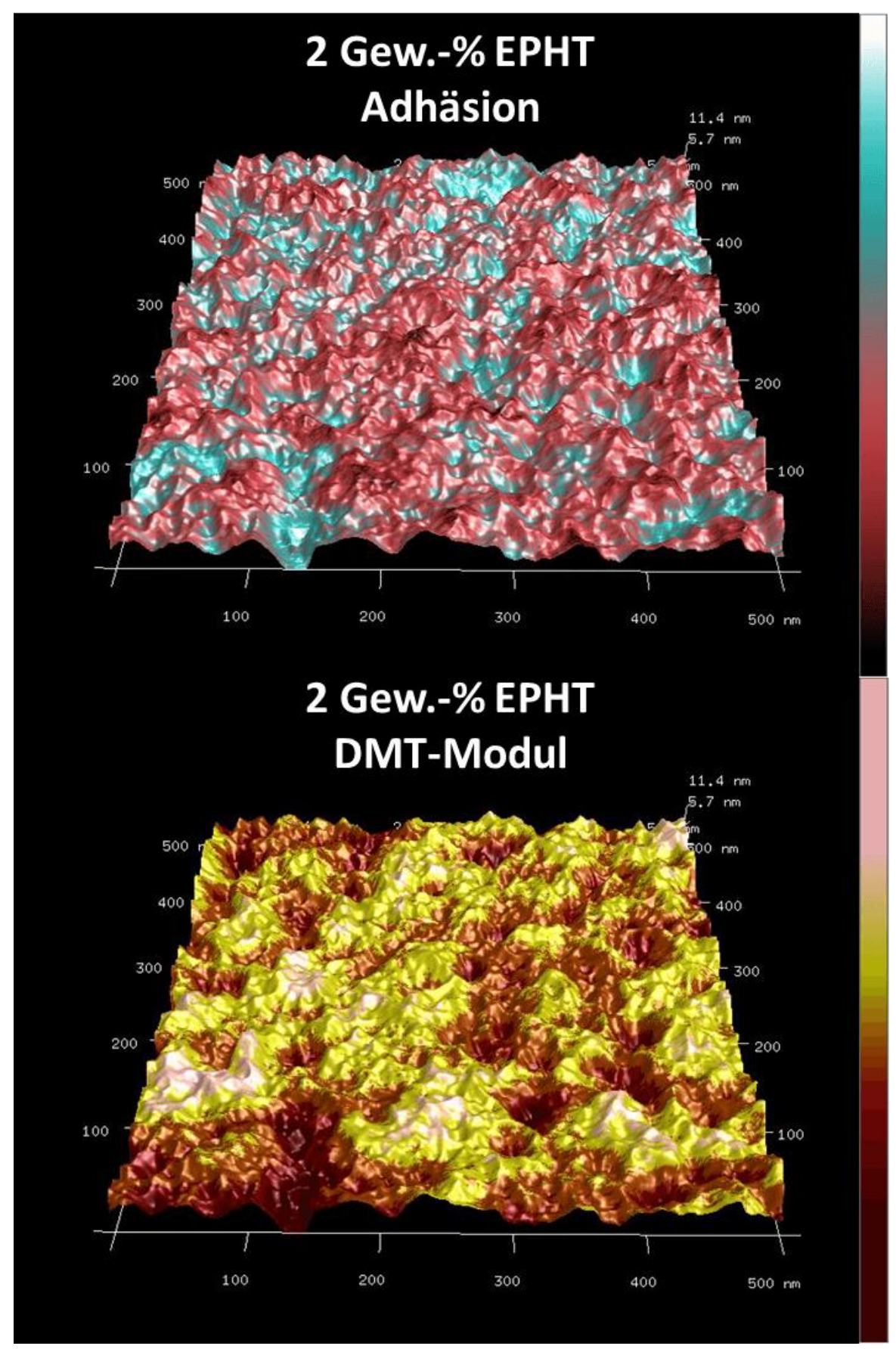

Abbildung 4-71 Dreidimensionale Darstellung von AFM-Aufnahmen des Höhenprofils eines PEGDA-575-Netzwerks aus RAFT-Polymerisation mit 2 Gew.-\% EPHT als RAFT-Agens. Im oberen Bild wurde über das Höhenprofil in einer anderen Farbskala der Messkanal für die Adhäsion der Oberflächen gelegt (schwarz: niedrige Adhäsion, weiß: hohe Adhäsion). Im unteren Bild wurde über das Höhenprofil in einer anderen Farbskala der Messkanal für den DMT-Modul der Oberflächen gelegt (braun: niedriger Modul, rosa: hoher Modul). Die Aufnahmen wurden im ScanAsyst-Modus durchgeführt. Die Kantenlänge beträgt $500 \times 500 \mathrm{~nm}$. Die Scan-Frequenz betrug 0,5 Hz. Es wurden 512 Linien pro Bild aufgenommen. 
Bei den PEGDA-575-Netzwerken wurde wieder ein direkter Vergleich der Oberflächenstrukturen eines konventionellen und eines RAFT-Netzwerks durchgeführt. (s. Abbildung 4-72).

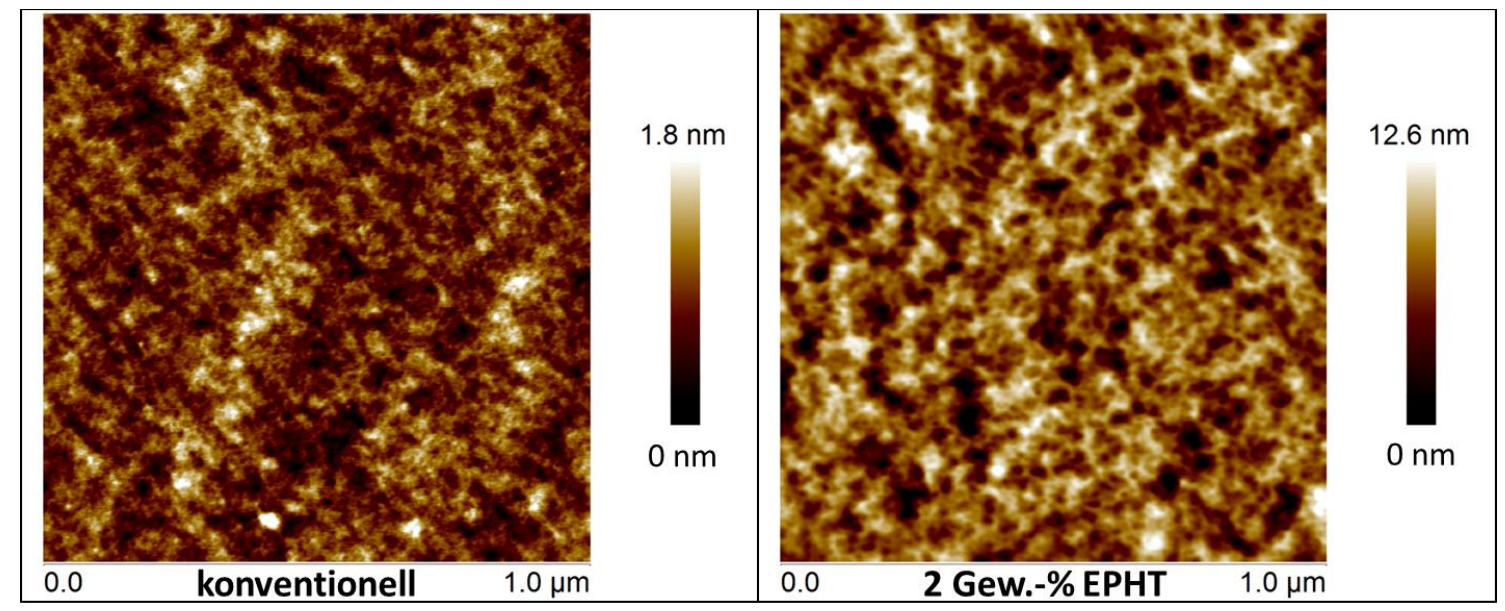

Abbildung 4-72 Rasterkraftmikroskop-Aufnahmen der Höhenprofile der Oberflächen von PEGDA-575-Netzwerken. Links: aus konventioneller radikalischer Polymerisation. Rechts: aus RAFT-Polymerisation mit 2 Gew.-\% EPHT. Die Aufnahmen wurden im ScanAsyst-Modus durchgeführt. Die Kantenlänge beträgt $1 \times 1 \mu \mathrm{m}$. Die Scan-Frequenz betrug 0,5 Hz. Es wurden 512 Linien pro Bild aufgenommen.

Es scheint, als ob die Phasenseparation im RAFT-Netzwerk noch deutlich ausgeprägter wäre als im konventionellen und dem PEGDA-250-Netzwerk. Die Höhenunterschiede sind beim RAFTNetzwerk viel ausgeprägter. Außerdem scheint es als ob das RAFT-Netzwerk insgesamt aus filigraneren Strukturen aufgebaut ist, wodurch es weniger kompakt wirkt. Dies bestätigt sich durch die Auswertung der Aufnahmen hinsichtlich Anzahl und Größe der tieferen Bereiche (s. Tabelle 4-35). Die Anzahl verringert sich deutlich, während die mittlere Fläche und der Durchmesser dieser Bereiche zunehmen.

Tabelle 4-35 Mittlere Anzahl, Fläche und Durchmesser der tieferen Bereiche von PEGDA-575-Netzwerken aus konventioneller radikalischer und RAFT-Polymerisation mit 2 Gew.-\% EPHT als RAFT-Agens. Die Daten wurden mit der Partikelanalysefunktion des Programms NanoScope Analysis (Version 1.4) den Höhenprofilen der Netzwerkoberflächen entnommen (s. Abbildung 4-72).

\begin{tabular}{cccc}
\hline Netzwerk & Anzahl & Fläche $\left[\mathbf{n m}^{2}\right]$ & Durchmesser [nm] \\
\hline konventionell & 524 & 1360 & 6 \\
RAFT & 153 & 3100 & 32 \\
\hline
\end{tabular}

Dass der Einfluss hier größer ist als bei den PEGDA-250-Netzwerken, geht wahrscheinlich auf die größeren Molmassen der Monomere zurück. Der Abstand der Doppelbindungen voneinander ist deutlich größer, wodurch die PEGDA-575-Netzwerke weniger dicht sind als die PEGDA-250Netzwerke. Dies ermöglicht bei diesen Netzwerken einen höheren Umsatz und führt zu einer 
Verlangsamung der Polymerisation, wie im vorherigen Kapitel gezeigt wurde. Abbildung 4-72 belegt die Vermutung, dass diese Effekte auf die weniger dichten Netzwerkstrukturen der RAFTNetzwerke zurückgeführt werden können. Da die Polymerisation von PEGDA-575 mit steigendem EPHT-Anteil langsamer wird, ist viel mehr Zeit vorhanden, sodass es zu einer ausgeprägteren Phasenseparation kommen kann. Weiterhin ist es nachvollziehbar, dass die weiter voneinander entfernten Doppelbindungen durchaus dazu führen können, dass die weniger stark vernetzten Bereiche zunehmen. Sollte dies tatsächlich einen Einfluss haben, müsste die Phasenseparation bei den PEGDA-700-Netzwerken noch ausgeprägter sein. Wie in Abbildung 4-73 zu erkennen ist, ist dies der Fall.

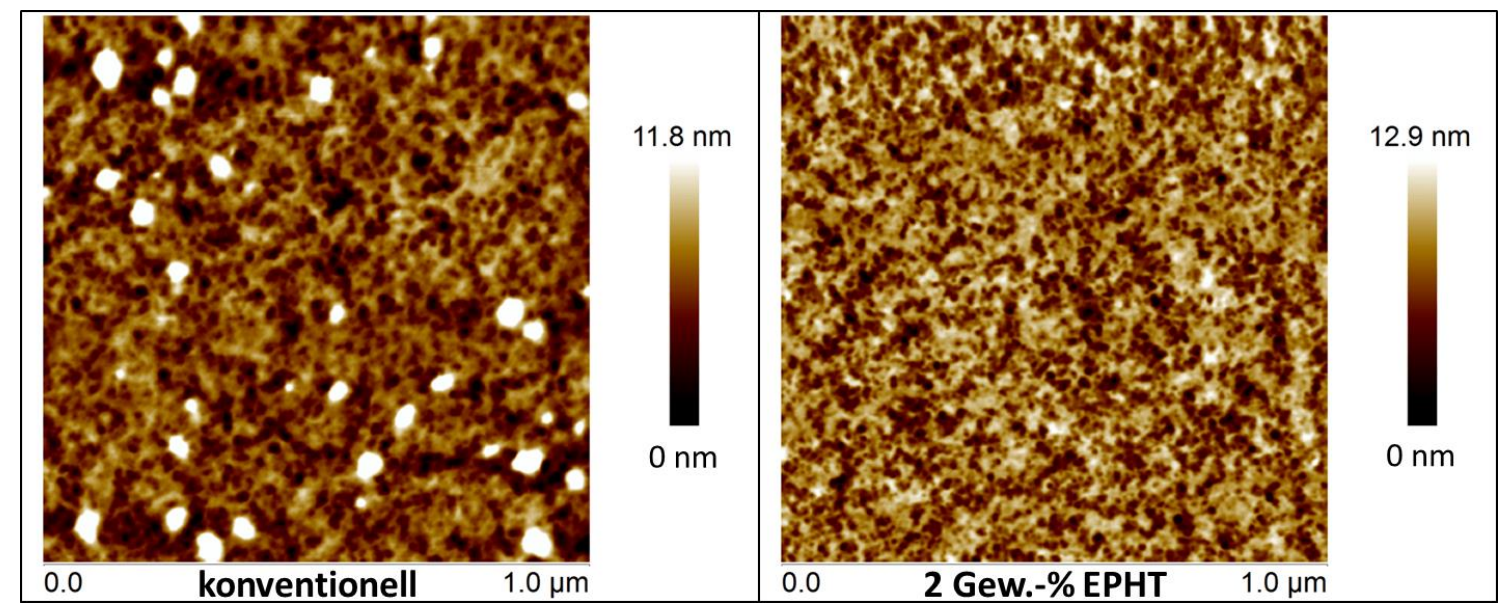

Abbildung 4-73 Rasterkraftmikroskop-Aufnahmen der Höhenprofile der Oberflächen von PEGDA-700-Netzwerken. Links: aus konventioneller radikalischer Polymerisation. Rechts: aus RAFT-Polymerisation mit 2 Gew.-\% EPHT. Die Aufnahmen wurden im ScanAsyst-Modus durchgeführt. Die Kantenlänge beträgt $1 \times 1 \mu \mathrm{m}$. Die Scan-Frequenz betrug 0,5 Hz. Es wurden 512 Linien pro Bild aufgenommen.

Schon das konventionelle PEGDA-700-Netzwerk zeigt eine sehr ausgeprägte und definierte Separation der stark vernetzten und monomerreichen bzw. wenig vernetzten Bereiche. In ihren strukturellen Aufbauten ähneln sich das konventionelle und das PEGDA-700-Netzwerk aus RAFT-Polymerisation sehr, wie anhand von Tabelle 4-36 deutlich wird. Der Aufbau der Netzwerke erinnert sehr stark an gyroide Strukturen, wie sie bei Blockcopolymeren vorkommen (s. Abbildung 4-74). Die rote Matrix wird in diesem Fall von dem vollständig ausgehärteten Netzwerk aufgebaut, während die Gyroide aus den weniger vernetzten, monomerreichen Bereichen gebildet werden.

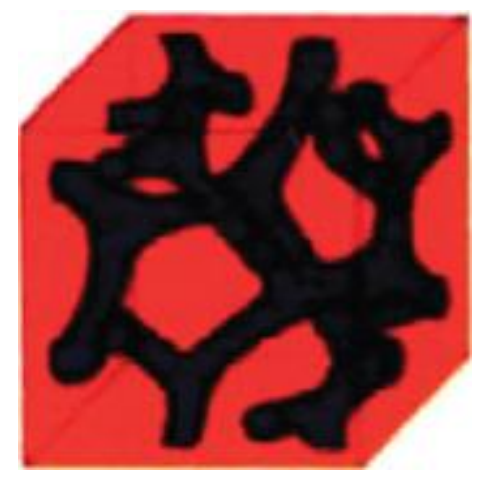

Abbildung 4-74 Gyroide Mikrophasenseperation eines Blockcopolymers. Aus Mai et al. ${ }^{[226]}$.

\footnotetext{
${ }^{a}$ Die Verwendung dieser Abbildung erfolgt mit der freundlichen Erlaubnis der Royal Society of Chemistry.
} 
Tabelle 4-36 Mittlere Anzahl, Flächen und Durchmesser der tieferen Bereiche von PEGDA-700-Netzwerken aus konventioneller radikalischer und RAFT-Polymerisation mit 2 Gew.-\% EPHT als RAFT-Agens. Die Daten wurden mit der Partikelanalysefunktion des Programms NanoScope Analysis (Version 1.4) den Höhenprofilen der Netzwerkoberflächen entnommen (s. Abbildung 4-73).

\begin{tabular}{cccc}
\hline Netzwerk & Anzahl & Fläche $\left[\mathbf{n m}^{2}\right]$ & Durchmesser [nm] \\
\hline konventionell & 159 & 3360 & 24 \\
RAFT & 165 & 3800 & 17 \\
\hline
\end{tabular}

In Anzahl, Fläche und Durchmesser unterscheiden sich die tieferen Bereiche der Netzwerke kaum voneinander. Der viel deutlichere Unterschied zwischen den Oberflächenstrukturen der beiden PEGDA-700-Netzwerke ist, dass die beim konventionellen Netzwerk vorhandenen Erhöhungen beim RAFT-Netzwerk nicht mehr existieren. Eine Partikelsuche mit dem Programm Nanoscope Analysis 1.4, die alle Erhöhungen über $6 \mathrm{~nm}$ erfasste, zeigte, dass sich diese sehr deutlich in Anzahl und Ausdehnung verringern (s. Tabelle 4-37).

Tabelle 4-37 Mittlere Anzahl, Flächen und Durchmesser der höheren Bereiche von PEGDA-700-Netzwerken aus konventioneller radikalischer und RAFT-Polymerisation mit 2 Gew.-\% EPHT als RAFT-Agens. Die Daten wurden mit der Partikelanalysefunktion des Programms NanoScope Analysis (Version 1.4) den Höhenprofilen der Netzwerkoberflächen entnommen (s. Abbildung 4-73).

\begin{tabular}{cccc}
\hline Netzwerk & Anzahl & Fläche $\left[\mathbf{n m}^{2}\right]$ & Durchmesser [nm] \\
\hline konventionell & 41 & 780 & 28 \\
RAFT & 15 & 50 & 7 \\
\hline
\end{tabular}

Da in der Polymerisationsmischung für die konventionellen Netzwerke außer PEGDA-700 und dem Photoinitiator keine weiteren Substanzen vorhanden waren und alle Synthesen unter den gleichen Bedingungen durchgeführt wurden (s. Kapitel 6.2.2), kann es sich bei den Erhöhungen nur um Nanogel-Domänen handeln, wie sie schon bei den elastomeren Netzwerken nachgewiesen werden konnten (s. Kapitel 4.1.5). Genau wie die Erhöhungen bei diesen, verfügen die beim konventionellen PEGDA-700-Netzwerk auftretenden Erhöhungen mit maximal $46 \mathrm{MPa}$ über einen deutlich höheren DMT-Modul als das umgebende Netzwerk. Dieses hat im Mittel einen DMT-Modul von 15 MPa. Diese Unterschiede werden auch in Abbildung 4-75 deutlich, wo der entsprechende Messkanal über die dreidimensionale Darstellung des Höhenprofils der PEGDA-700-Netzwerke gelegt wurde. Die Erhöhungen stechen deutlich sowohl topographisch als auch in ihrer Färbung hervor. Bei diesen Netzwerken zeigt sich also zum ersten Mal bei den PEG-Diacrylat-Netzwerken eine Verringerung der Heterogenität des Netzwerks durch die Zugabe eines RAFT-Agens zu der Polymerisationsmischung, da abgesehen von den Erhöhungen die Verteilung der harten und weichen Netzwerkbereiche über die gesamte Oberfläche sehr 
gleichmäßig ist. Sind keine Nanogel-Domänen mehr vorhanden, bleibt nur noch die Netzwerkmatrix.

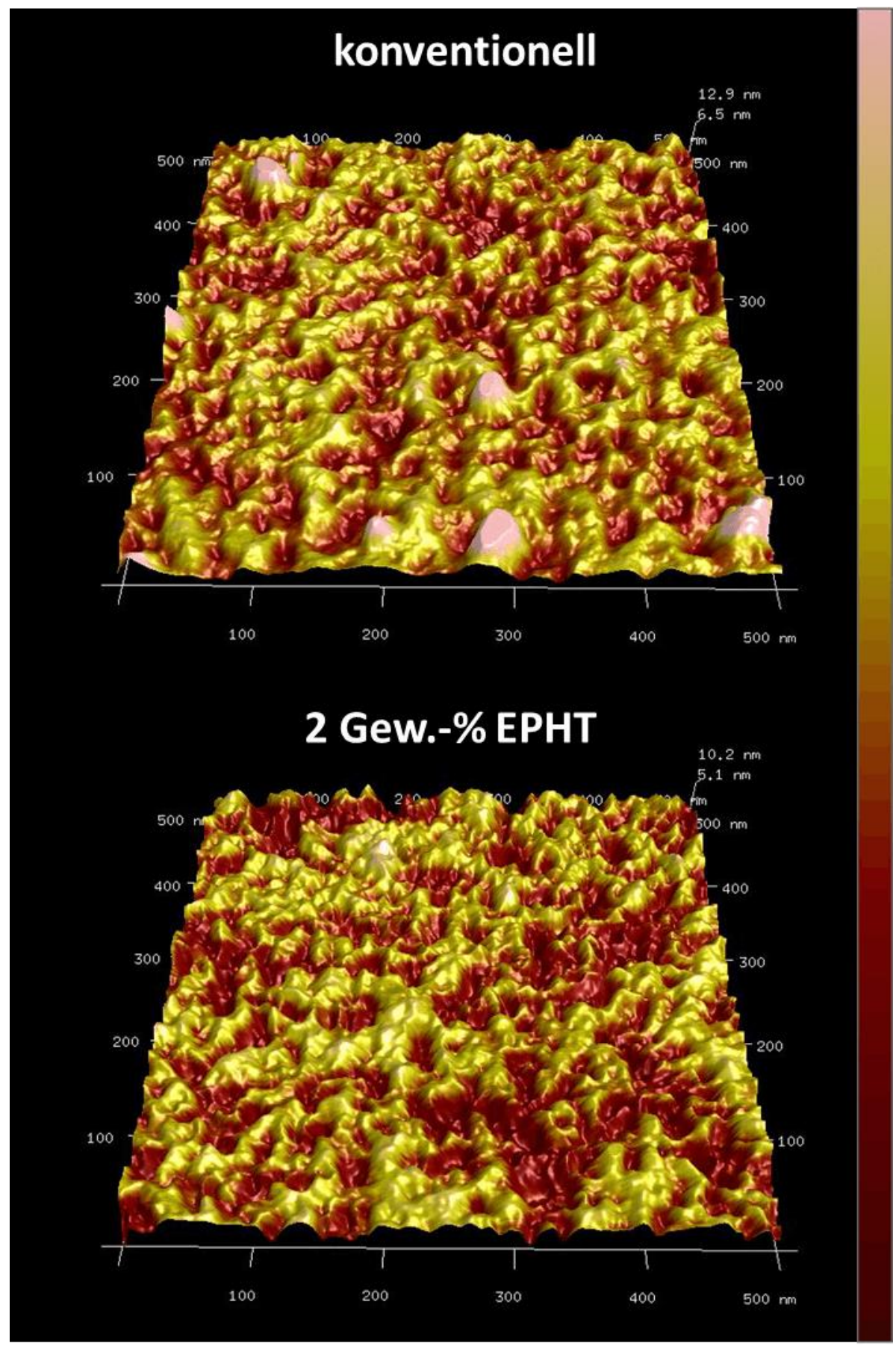

Abbildung 4-75 Dreidimensionale Darstellung von AFM-Aufnahmen des Höhenprofils von PEGDA-700-Netzwerken. Oben: aus konventioneller radikalischer Polymerisation. Unten: aus RAFT-Polymerisation mit 2 Gew.-\% EPHT. Über das Höhenprofil wurde in einer anderen Farbskala der Messkanal für den DMT-Modul der Oberflächen gelegt (braun: niedriger Modul, rosa: hoher Modul). Die Aufnahmen wurden im ScanAsyst-Modus durchgeführt. Die Kantenlänge beträgt $500 \times 500 \mathrm{~nm}$. Die Scan-Frequenz betrug 0,5 Hz. Es wurden 512 Linien pro Bild aufgenommen. 
Dass sich bei dem konventionellen PEGDA-700-Netzwerk überhaupt wieder größere, sich von der umgebenden Netzwerkmatrix abhebende Nanogel-Domänen bilden, wird mit der SpacerLänge der PEGDA-700-Moleküle zusammenhängen. Durch den relativ großen Abstand der Doppelbindungen in den PEGDA-700-Molekülen ist es möglich, dass es wieder zu Verhakungen und Verschlaufungen von Netzketten kommt und sich somit Nanogel-Domänen von größeren Ausmaßen bilden können. Außerdem ist das Eigenvolumen der Monomere in diesem System auch deutlich größer als in den anderen PEGDA-Systemen.

\subsubsection{Abschließende Bemerkungen}

Es konnte gezeigt werden, dass die Zugabe des RAFT-Agens EPHT zu bifunktionellen PEGDiacrylaten sowohl einen Einfluss auf die Kinetik der Polymerisation dieser Monomere als auch einen Einfluss auf die Oberflächenstrukturen der daraus resultierenden Netzwerke haben kann. Das RAFT-Agens führte bei allen Polymerisationen zu einer Verlangsamung der Polymerisation. Bei den PEGDA-250-Monomeren kam zusätzlich hinzu, dass nicht mehr der vollständige Umsatz erreicht wurde, während dies bei den beiden anderen PEG-Diacrylaten mit einer deutlichen Verzögerung immer noch möglich war. Bei den PEGDA-700-Monomeren war die Verlangsamung der Reaktion am stärksten.

Durch die Rasterkraftmikroskop-Aufnahmen konnte nachgewiesen werden, dass die RAFTPolymerisation bei den PEGDA-250-Netzwerken zu einer geringen Vergrößerung von dicht vernetzten und weniger dicht vernetzten Bereichen führte. Bei den PEGDA-575-Netzwerken kam es durch die RAFT-Polymerisation zu einer ausgeprägteren Phasenseparation. Bei den PEGDA700-Netzwerken führte die RAFT-Polymerisation zu einer Verringerung von Nanogel-Domänen und somit zu einer deutlich homogeneren Netzwerkstruktur. Somit kann die am Anfang des Kapitels aufgestellte Vermutung, dass der RAFT-Mechanismus einen unterschiedlichen Einfluss auf PEG-Diacrylate mit unterschiedlichen Spacer-Längen hat, als bestätigt angesehen werden. 


\subsubsection{Einfluss der RAFT-Polymerisation auf die Recyclierbarkeit von UV- härtenden Druckfarben}

In einem Kooperationsprojekt mit der Forschungsgesellschaft Druck e. V. (FOGRA) in München sollte der Einfluss der RAFT-Polymerisation auf die Recyclierbarkeit von UV-härtenden Druckfarben untersucht werden. Bei UV-härtenden Druckfarben oder Lacken handelt es sich, genau wie bei den PEG-Acrylaten, um mehrfachfunktionelle Acrylate. Meistens sind diese Oligomere mit Molmassen von einigen 100 bis $10.000 \mathrm{~g} / \mathrm{mol}^{[227]}$ Sie werden auch als Präpolymere bezeichnet. Allerdings sind ihre genauen chemischen Strukturen häufig nicht bekannt, da es sich bei diesen Präpolymeren um kommerziell vertriebene Produkte handelt, deren genaue Zusammensetzung und chemische Struktur von den Herstellern nicht bekannt gegeben wird. Sie bilden nach der Polymerisation genau wie die PEG-Acrylate ein duroplastisches Netzwerk, das je nach der Zusammensetzung der Druckfarbe eine unterschiedliche Struktur haben kann. Dies kann beim Recycling der Drucksachen zu erheblichen Problemen führen.

Beim Recyclingprozess geht es darum, die Farben vom bedruckten Papier zu trennen und dieses möglichst frei von Farbrückständen wieder zurück zu gewinnen. Dieser Prozess wird auch Deinking genannt. Die bedruckten Produkte werden dabei in einem mechanischen Prozess zerkleinert. Über einen Flotationsprozess sollen die Farbpartikel von den Papierfasern getrennt werden (s. Abbildung 4-76). Die Bruchstücke der Druckfarben sind allerdings sehr heterogen in Größe und Struktur, was den Flotationsprozess technisch sehr aufwendig macht. Außerdem ist die Trennwirkung zwischen verschiedenen Deinking-Ansätzen häufig sehr

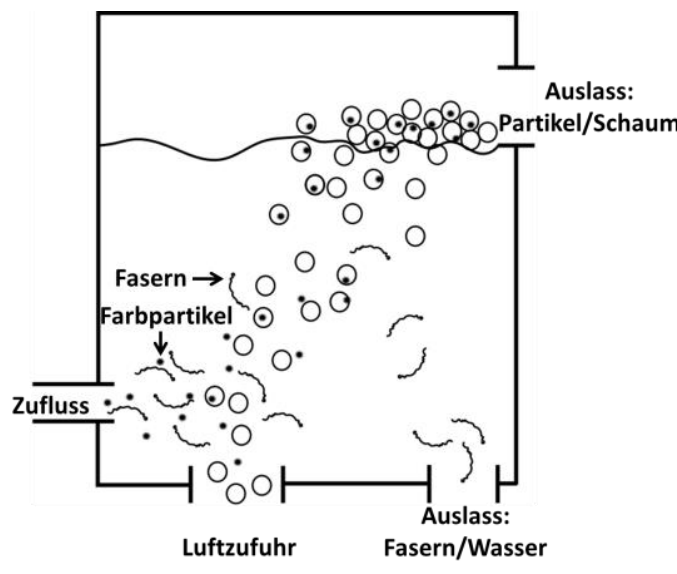

Abbildung 4-76 Schematische Darstellung des Flotationsprozesses zum Deinking von Druckprodukten. unterschiedlich. Die weite Größenverteilung der Farbpartikel geht auf die heterogenen Mikrostrukturen des Netzwerks zurück. Wie schon gezeigt werden konnte, ist die RAFTPolymerisation in der Lage, die Mikrostrukturen von Polymernetzwerken zu verändern, sodass diese homogener werden. Dies eröffnet die Möglichkeit, im Flotationsprozess eine engere Größenverteilung der Farbpartikel zu erhalten und diesen somit zu effizienter zu gestalten.

Im Rahmen dieser Arbeit soll ein Teil der Ergebnisse aus den gemeinsam mit der FOGRA durchgeführten Studien vorgestellt werden, um einen Überblick über diese Thema zu liefern. Es handelt sich hierbei um eine Möglichkeit, die RAFT-Polymerisation für kommerziell verwendete 
Systeme nutzbar zu machen. Es sei darauf hingewiesen, dass weitere Informationen aus dem von der FOGRA veröffentlichen Projekt-Bericht entnommen werden können. ${ }^{\text {[228] }}$

\subsubsection{Zusammensetzungen der untersuchten Lack-Systeme}

Untersucht wurden in diesem Projekt unterschiedliche Polymerisationsmischungen, die in diesem Zusammenhang auch Formulierungen genannt werden. Bei dem primär verwendeten Präpolymer handelte es sich um das multifunktionelle Präpolymer Laromer $9013^{\circledR}$, das von der BASF kommerziell vertrieben wird und dessen genaue chemische Struktur unbekannt ist, außer dass es ein dreifach-funktionelles, Polyetheracrylat darstellt. ${ }^{[229]}$ Dieses wurde mit zwei unterschiedlichen Photoinitiator-Kombinationen versetzt. Eine der Musterformulierungen wurde mit einem Norrish-I-Photointiatorsystem aus drei verschiedenen Photoinitiatoren versehen (s. Tabelle 4-38.) Die zweite Musterformulierung wurde mit einem Norrish-IIPhotoinitiatorsystem versehen (s. Tabelle 4-39).

Tabelle 4-38 Massenanteile der für die Grundformulierungen der Norrish-I-Systeme verwendeten Substanzen.

\begin{tabular}{cc}
\hline Substanz & Anteil in der Formulierung [Gew.-\%] \\
\hline Laromer 9013 & 88,4 \\
Luricin TPO-L & 4,6 \\
Irgacure 379 & 4,6 \\
Irgacure 907 (MMMP) & 2,4 \\
\hline
\end{tabular}

Tabelle 4-39 Massenanteile der für die Grundformulierungen der Norrish-I-Systeme verwendeten Substanzen.

\begin{tabular}{cc}
\hline Substanz & Anteil in der Formulierung [Gew.-\%] \\
\hline Laromer 9013 & 89 \\
Benzophenon & 5 \\
MDEA & 6 \\
\hline
\end{tabular}

Diese sehr hohen Photoinitiator-Konzentrationen werden verwendet, um die inhibierende Wirkung des beim Drucken vorhandenen Sauerstoffs zu kompensieren und somit eine sehr schnelle Aushärtung der Drucksachen sicherzustellen.

Zur Untersuchung des Einflusses der RAFT-Polymerisation auf die gehärteten Farben wurden diese Musterformulierungen für die meisten durchgeführten Experimente mit 0,1 Gew.-\%, 0,5 Gew.-\% oder 1 Gew-\% des RAFT-Agens EPHT versetzt. 
Diese Musterformulierungen wurden in den Laboren der FOGRA entwickelt und zusammengestellt. Weiterhin wurden auch Formulierungen mit 2,5 Gew.-\% und 5 Gew.-\% EPHT hergestellt (bezogen auf die Masse des Monomers).

Zusätzlich wurde in diesem Projekt die Auswirkung der multifunktionellen RAFT-Agenzien 1,4-Butandiolbis(2-(hexyltrithiocarbonat)propanoat) und Dipentaerythriol-hexakis(3-(S-methoxopropionattrithiocarbonat))propanoat (s. Abbildung 4-77) auf die Härtung der Formulierungen und deren Eigenschaften erprobt. $^{\text {[228] }}$

Abbildung 4-78 zeigt eine Reihe von Musterformulierungen, die nach Beendigung des Projektes im Sonnenlicht gehärtet wurden. Diese Musterformulierungen bilden

a)

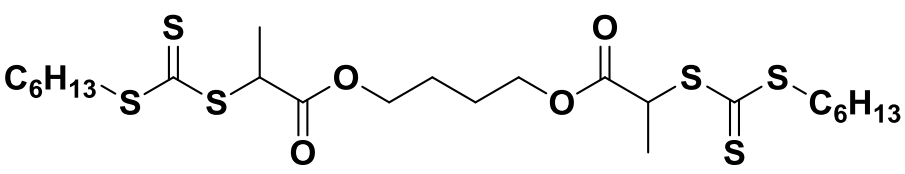

b)<smiles>COC(=O)C(C)SC(=S)SCCC(=O)OCC(COCC(COC(=O)CCSC(=S)SC(C)C(=O)OC)(COC(=O)CCSC(=S)SC(C)C(=O)OC)COC(=O)CCSC(=S)SC(C)C(=O)OC)(COC(=O)CCSC(=S)SC(C)C(=O)OC)COC(=O)CCSC(=S)SC(C)C(=O)OC</smiles>
Abbildung 4-77 Weitere in diesem Projekt erprobte RAFT-Agenzien:

a) 1,4-Butandiolbis(2-(hexyltrithiocarbonat)propanoat).

b) Dipentaerythriol-hexakis(3-(S-methoxopropionat-trithiocarbonat))propanoat

Paare, die von links nach rechts einen steigenden Massenanteil des RAFT-Agens EPHT enthalten. Die linke Formulierung der Paare ist jeweils ein Norrish-I-System, die rechte ein Norrish-IISystem.

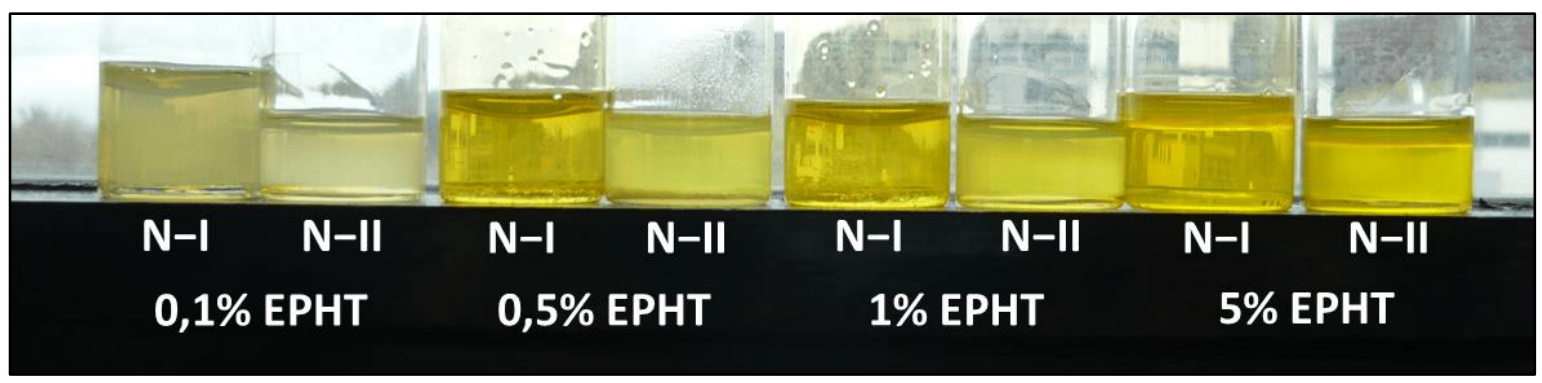

Abbildung 4-78 Foto von Norrish-I- und Norrish-II-Systemen mit von links nach rechts steigenden Massenanteilen des RAFT-Agens EPHT nach Härtung im Sonnenlicht. 
Abbildung 4-78 macht deutlich, dass es Unterschiede zwischen den Norrish-I- und Norrish-IISystemen gibt. Während die Norrish-I-Systeme mit steigendem EPHT-Anteil immer klarer werden, blieben die Norrish-II-Systeme trüb. Die Trübung geht darauf zurück, dass das einfallende Licht an Nanogel-Domänen in den Netzwerken gestreut wird. Es kann vermutet werden, dass der RAFT-Mechanismus in den Norrish-I-Systemen dazu geführt hat, dass diese Nanogel-Domänen reduziert wurden, weshalb weniger Licht in diesen gestreut wird. Allerdings war ein Massenanteil von 0,1 Gew.-\% EPHT noch nicht ausreichend, wie die entsprechende Probe zeigt. Diese ist ebenfalls noch sehr trüb. Bei den Norrish-II-Systemen scheint der RAFTMechanismus keinen oder nur sehr wenig Einfluss auf die Mikrostruktur des Netzwerks zu haben. Die Proben sind alle deutlich trüber als die entsprechenden Norrish-I-Systeme. Eine mögliche Erklärung hierfür wurde durch ESR-Spektroskopie erhalten.

Bei diesen Untersuchungen wurde, im Gegensatz zu den Norrish-I-Systemen, bei den Norrish-IISystemen kein Hinweis darauf gefunden, dass das Intermediat-Radikal des RAFT-Gleichgewichts gebildet wird. ${ }^{[228]}$ Dieses ist normalerweise sehr gut mittels ESR-Spektroskopie nachweisbar. ${ }^{[230]}$ Das bedeutet, dass im Norrish-II-System keine RAFT-Polymerisation stattgefunden haben kann und somit immer noch eine hohe Heterogenität in den ausgehärteten Netzwerken vorliegt. Allerdings muss dazu erwähnt werden, dass für die Untersuchungen auf ein anderes Präpolymer zurückgegriffen werden musste, da das Laromer $9013^{\circledR}$ schon ohne die Zugabe anderer Substanzen wie z. B. Initiatoren oder EPHT ein sehr starkes Radikal-Signal zeigte, welches anhand einer nachträglichen Simulation mit dem Programm Simfonia auf das ESR-Signal von 2,2,6,6-Tetramethylpiperidinyloxyl (TEMPO) zurückgeführt werden konnte (s. Abbildung 4-80). Dieses machte eine sichere Interpretation der ESR-Spektren der Laromer $9013^{\circledR}$ Polymerisationen nahezu unmöglich. Es kann nur vermutet werden, dass dieses stabile Radikal (s. Abbildung 4-79) bei der Herstellung von Laromer $9013^{\circledR}$ hinzugesetzt wird, um die Reaktion zum Abbruch zu bringen. Bei dem anderen Präpolymer handelte es sich um Rocracure VP 5468, das im reinen Zustand ohne stattfindende Polymerisation kein solches Signal im ESR-Spektrometer zeigte. ${ }^{[228]}$

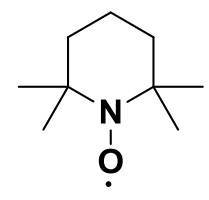

Abbildung 4-79 Strukturformel von 2,2,6,6Tetramethylpiperidinyloxyl (TEMPO). 


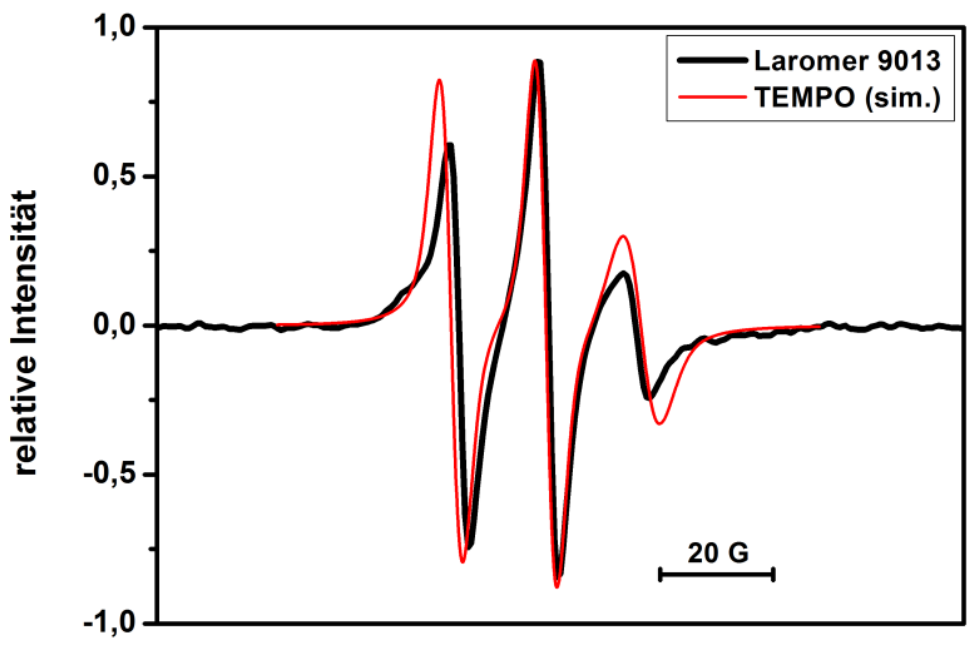

Abbildung 4-80 ESR-Spektrum von reinem Laromer $9013^{\circledR}$ ohne UV-Bestrahlung bei $21{ }^{\circ} \mathrm{C}$. In Rot: Simuliertes ESRSpektrum von 2,2,6,6-Tetramethylpiperidinyloxyl (TEMPO). Die Durchlaufzeit einer Messung betrug 5,24 s, als Modulationsamplitude wurden $1 \mathrm{G}$ gewählt, die Modulationsfrequenz betrug $100 \mathrm{kHz}$. Die Verstärkung des Empfängers betrug $60 \mathrm{~dB}$, der G-Faktor betrug 2. Die Mikrowellenbrücke wurde mit einer Leistung von 0,63 $\mathrm{mW}$ bei einer Dämpfung von $25 \mathrm{~dB}$ betrieben. 


\subsubsection{Mechanische Eigenschaften der ausgehärteten Formulierungen}

Um zu überprüfen, ob der RAFT-Mechanismus wie bei den Elastomeren einen Einfluss auf die mechanischen Eigenschaften der Netzwerke hatte, wurden Zugversuch-Probekörper aus dem Norrish-I-System mit steigendem Massenanteil des RAFT-Agens EPHT (bezogen auf die Masse des Monomers) hergestellt. Diese entsprachen in ihren Abmessungen allerdings nicht den Elastomer-Probekörpern. Sie hatten nur eine Höhe von $0,2 \mathrm{~mm}$, da die Probekörper so flexibel genug waren, um sie unbeschadet aus den Aushärtungsformen zu entnehmen.

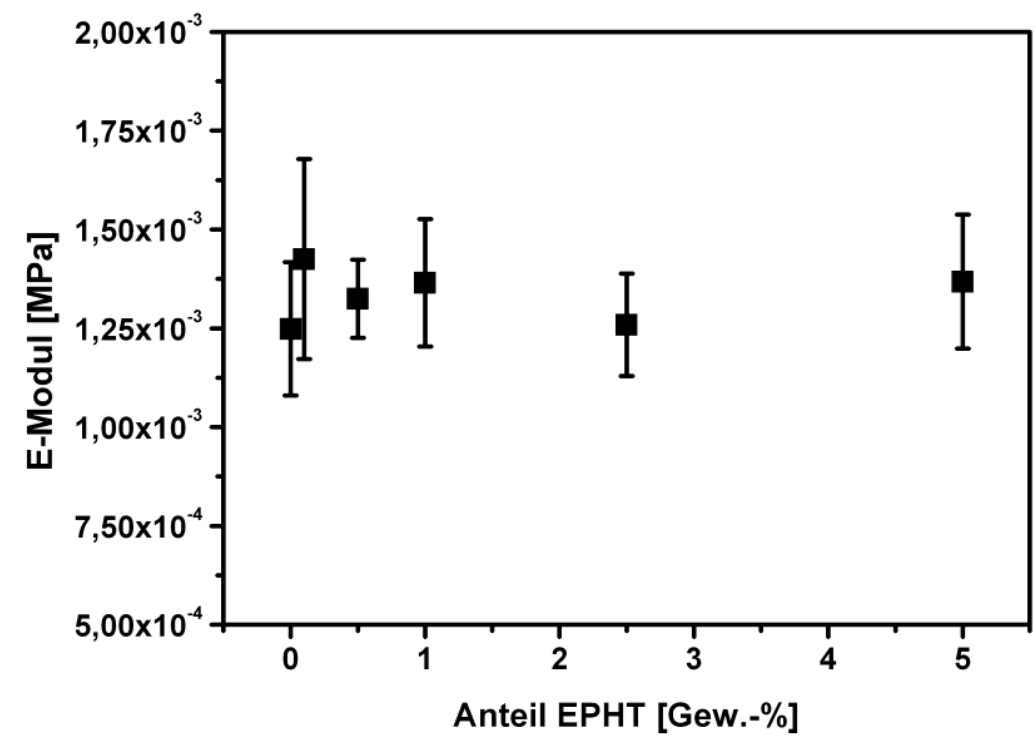

Abbildung 4-81 Elastizitätsmoduln von Probekörpern aus Norrish-I-Systemen mit unterschiedlichen Massenanteilen des RAFT-Agens EPHT.

Wie zu erkennen ist, hat die Zugabe von bis zu 5 Gew.-\% keinen Einfluss auf den E-Modul der Druckfarben. Alle Werte streuen in einem Bereich zwischen 1,25 und $1,5 \cdot 10^{-3} \mathrm{MPa}$. Da dies aber genauso eine Folge der sehr dünnen Probekörper sein könnte, sind diese Messungen somit ungeeignet, um mögliche Auswirkungen des RAFT-Mechanismus auf die Mikrostrukturen der ausgehärteten Formulierungen zu erkennen.

\subsubsection{Oberflächenstrukturen der ausgehärteten Formulierungen}

Eine weitere Möglichkeit, den Einfluss des RAFT-Mechanismus auf die Mikrostrukturen der ausgehärteten Formulierungen zu untersuchen, bietet, wie schon vorgestellt (s. Abschnitt 4.2.1.3), die Rasterkraftmikroskopie. Sie ist dafür optimal geeignet, da sie neben den Höhenprofilen der Oberflächen ebenfalls die Möglichkeit bietet, Informationen über die mechanischen Eigenschaften der Oberflächen zu erhalten. 
Die im Folgenden untersuchten Formulierungen wurden in den Laboren der FOGRA mittels eines Rakels auf Glasplättchen aufgetragen und gehärtet. Zunächst erfolgte die Untersuchung von Proben eines Norrish-I-Systems, wobei es sich bei der einen Probe um ein konventionelles und bei der anderen um ein RAFT-Netzwerk mit 1 Gew.-\% EPHT handelte.

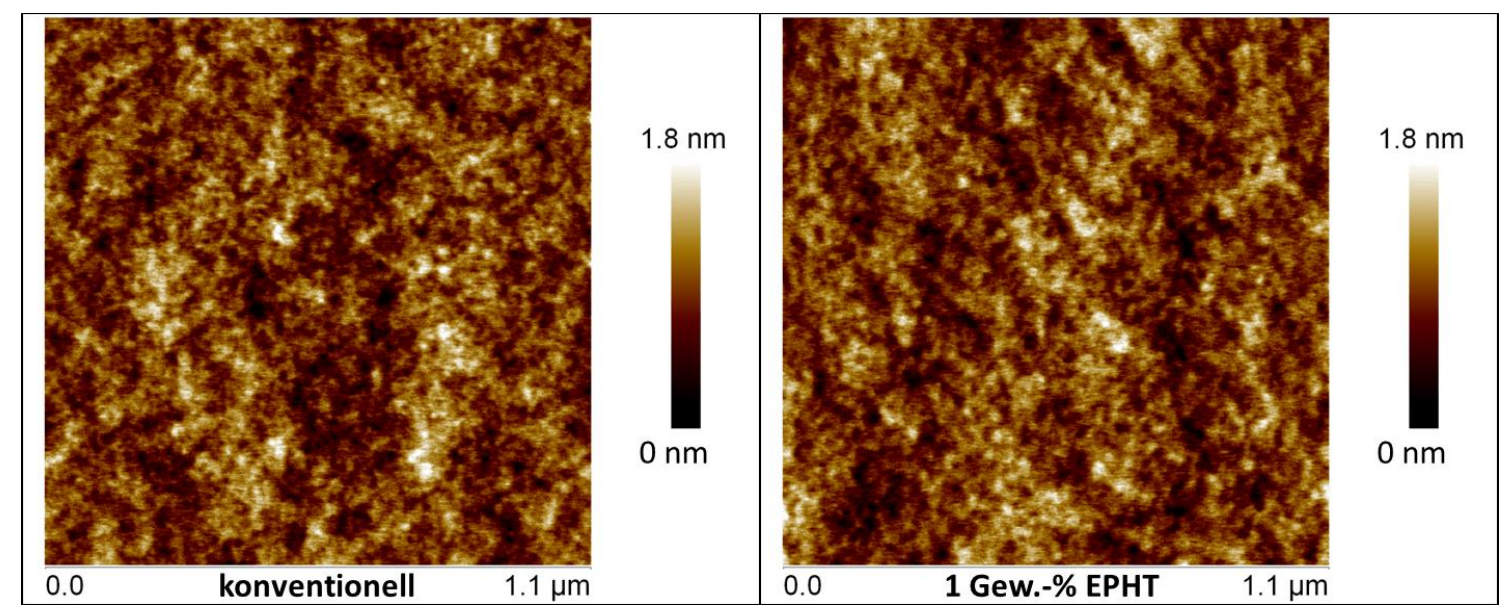

Abbildung 4-82 Rasterkraftmikroskop-Aufnahmen der Höhenprofile der Oberflächen von ausgehärteten Norrish-IMusterformulierungen. Links: aus konventioneller radikalischer Polymerisation. Rechts: aus RAFT-Polymerisation mit 1 Gew.-\% EPHT. Die Aufnahmen wurden im ScanAsyst-Modus durchgeführt. Die Kantenlänge beträgt 1,1×1,1 $\mu$ m. Die Scan-Frequenz betrug 0,9 Hz. Es wurden 1024 Linien pro Bild aufgenommen.

An den Höhenprofilen der beiden Proben lassen sich keine signifikanten Unterschiede zwischen der konventionellen und der Probe aus RAFT-Polymerisation feststellen (s. Abbildung 4-82). Bei beiden Proben hat sich eine sehr ähnliche Oberflächenstruktur ausgebildet. Beide sind insgesamt sehr eben (die höchste Erhebung beträgt 1,8 nm) und lassen eine ähnlich einheitliche Verteilung von höheren und tieferen Bereichen erkennen. Es scheint als ob die Zugabe von EPHT keinen Einfluss auf die ausgebildeten Oberflächen der Druckfarben hatte. Weitere Informationen liefert der Messkanal der Adhäsionen der Oberflächen (s. Abbildung 4-83). 


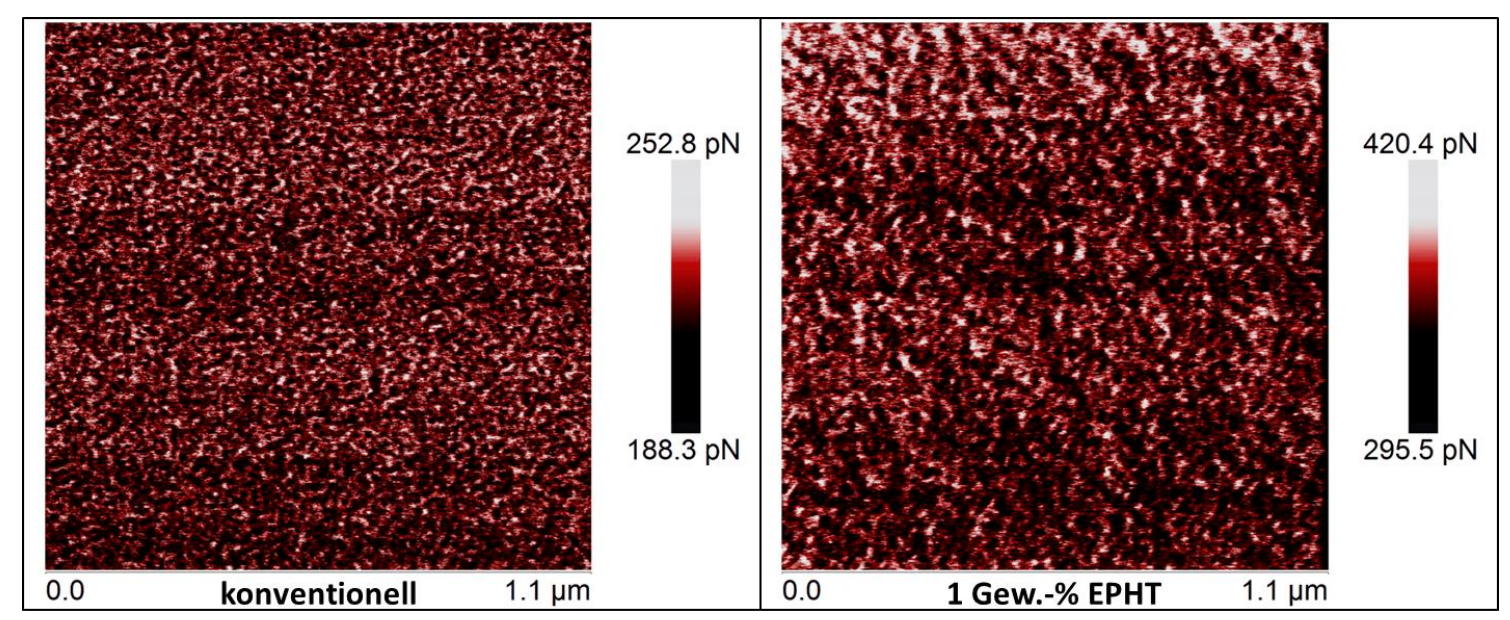

Abbildung 4-83 Rasterkraftmikroskop-Aufnahmen der Adhäsionen von ausgehärteten Norrish-IMusterformulierungen. Links: aus konventioneller radikalischer Polymerisation. Rechts: aus RAFT-Polymerisation mit 1 Gew.-\% EPHT. Die Aufnahmen wurden im ScanAsyst-Modus durchgeführt. Die Kantenlänge beträgt 1,1×1,1 $\mu$ m. Die Scan-Frequenz betrug 0,9 Hz. Es wurden 1024 Linien pro Bild aufgenommen.

Bei diesen Messungen lässt sich eine Veränderung durch die Zugabe von EPHT feststellen. Im Vergleich zu der konventionellen Probe sind die Bereiche unterschiedlicher Adhäsion bei dem Lack aus RAFT-Polymerisation größer. Aus einer Analyse ergibt sich, dass der mittlere Durchmesser dieser Bereiche von 76,0 $\mathrm{nm}^{2}$ auf 143,0 $\mathrm{nm}^{2}$ anwächst. Wie schon bei den PEGAcrylaten geht dies wahrscheinlich auf eine nicht so weit fortgeschrittene Reaktion bei den RAFT-Netzwerken zurück, sodass die nicht so stark vernetzten Bereiche (in Abbildung 4-83 als rote bzw. weiße Bereiche zu erkennen) insgesamt größer werden. Dies kann möglicherweise wieder auf die Absorption des RAFT-Agens zurückgeführt werden, die trotz der sehr hohen Lichtintensität beim Härten der Formulierungen und der sehr großen Photoinitiatormenge immer noch zu einer deutlichen Verlangsamung der Reaktion geführt hat. Dabei sollte vor allem beachtet werden, dass die Aushärtungszeit in der Regel zwischen einer und fünf Minuten beträgt. Genau wie für die PEG-Acrylate (s. Abschnitt 4.2.1.2) gilt hier, dass die Bereiche mit hoher Adhäsion über einen niedrigen DMT-Modul verfügen und umgekehrt.

Analog zu den Norrish-I-Systemen wurden ebenfalls Proben von Norrish-II-Systemen hergestellt und mittels AFM untersucht. Abbildung 4-84 zeigt die Höhenprofile der Oberflächen von einer konventionellen und einer Probe aus RAFT-Polymerisation. Der Massenanteil des RAFT-Agens betrug wieder 1 Gew.-\%. 


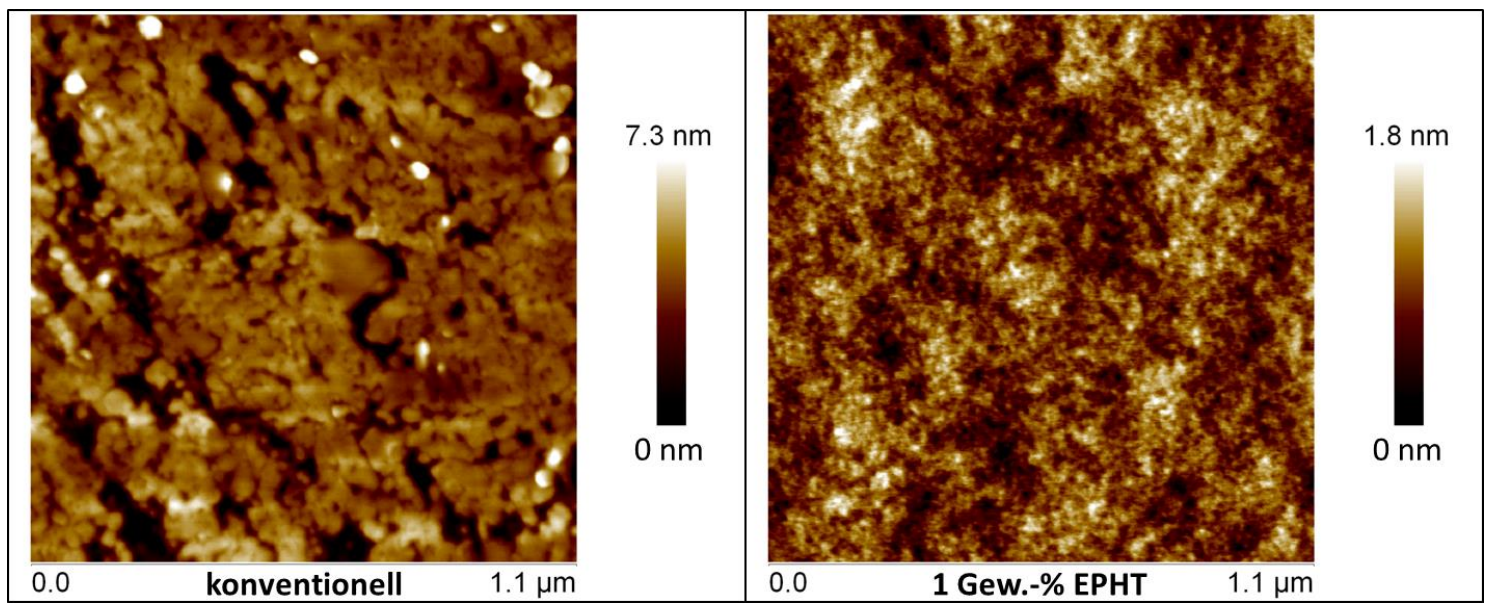

Abbildung 4-84 Rasterkraftmikroskop-Aufnahmen der Höhenprofile der Oberflächen von ausgehärteten Norrish-IIMusterformulierungen. Links: aus konventioneller radikalischer Polymerisation. Rechts: aus RAFT-Polymerisation mit 1 Gew.-\% EPHT. Die Aufnahmen wurden im ScanAsyst-Modus durchgeführt. Die Kantenlänge beträgt 1,1×1,1 $\mu$ m. Die Scan-Frequenz betrug 0,9 Hz. Es wurden 1024 Linien pro Bild aufgenommen.

Die Höhenprofile der beiden ausgehärteten Formulierungen zeigen deutliche Unterschiede in ihrer Struktur. Während die konventionelle Probe eine hohe Diversität in der Oberflächenstruktur besitzt, ist die Oberfläche der RAFT-Probe ähnlich homogen wie die der Norrish-I-Systeme. Die in Abbildung 4-85 gezeigten Adhäsionen der Proben sind den Höhenprofilen dieser Systeme sehr ähnlich.
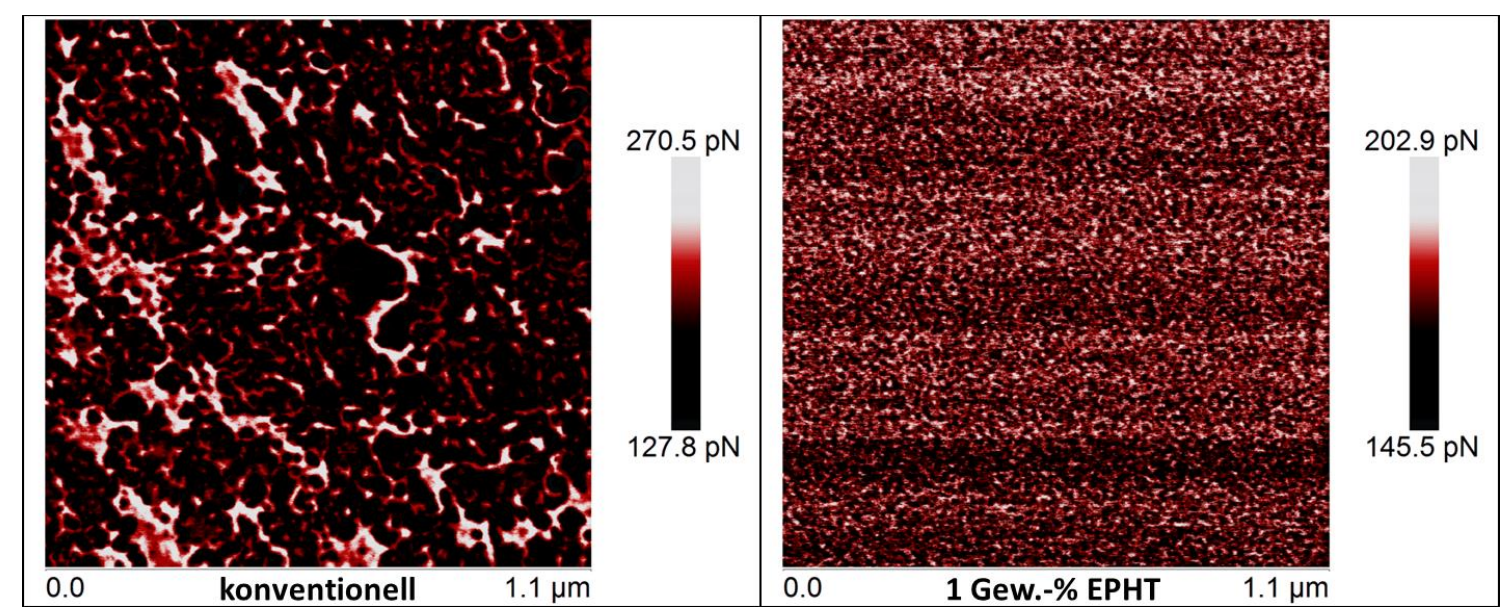

Abbildung 4-85 Rasterkraftmikroskop-Aufnahmen der Adhäsionen von ausgehärteten Norrish-IMusterformulierungen. Links: aus konventioneller radikalischer Polymerisation. Rechts: aus RAFT-Polymerisation mit 1 Gew.-\% EPHT. Die Aufnahmen wurden im ScanAsyst-Modus durchgeführt. Die Kantenlänge beträgt 1,1×1,1 $\mu \mathrm{m}$. Die Scan-Frequenz betrug 0,9 Hz. Es wurden 1024 Linien pro Bild aufgenommen.

Es scheint, dass das RAFT-Agens bei diesen Systemen einen deutlich stärkeren Einfluss auf die Mikrostrukturen der ausgehärteten Formulierungen besitzt. Dies ist so nicht zu erwarten gewesen, da in ESR-Experimenten bei diesen Systemen keine Intermediat-Radikale nachgewiesen 
werden konnten. Allerdings ist es möglich, dass der Einfluss des RAFT-Agens vor allem in der veränderten Viskosität der Formulierungen vor dem Härten zu finden ist. ${ }^{[228]}$ Durch die Zugabe des flüssigen RAFT-Agens wurde die Viskosität der sehr zähen Formulierung des konventionellen Norrish-II-Systems deutlich reduziert. Dass die Viskosität einer Reaktionsmischung einen Einfluss auf die Kinetik der Reaktion hat, ist bekannt. Somit ist es nachvollziehbar, dass die Zugabe des RAFT-Agens EPHT, trotz des sehr wahrscheinlich nicht stattfindenden RAFT-Mechanismus, bei den Norrish-II-Systemen $\mathrm{zu}$ veränderten Mikrostrukturen der ausgehärteten Lacke führt. Dazu sei erwähnt, dass die Viskosität des konventionellen Norrish-I-Systems ebenfalls deutlich geringer war als die des konventionellen Norrish-II-Systems.

\subsubsection{Ergebnisse der Recyclierbarkeitsversuche}

Um den Einfluss des RAFT-Mechanismus auf die Recyclierbarkeit der ausgehärteten Druckfarben zu untersuchen, wurden in den Laboren der FOGRA Musterformulierungen mit einem Norrish-IInitiatorsystem, sowohl ohne als auch mit dem RAFT-Agens EPHT, zusammengestellt. Mit diesen wurden mittels einer Bogenoffsetdruckmaschine bei der Firma IST METZ GmbH in Nürtingen spezielle Testdrucke erstellt, die anschließend in den Laboren der FOGRA auf ihr DeinkingVerhalten hin untersucht wurden. Dabei zeigte sich, dass das Deinking-Verhalten von gut deinkbaren UV-härtenden Druckfarben durch die Zugabe von RAFT-Agenzien nicht signifikant verbessert werden konnte. ${ }^{[228]}$

\subsubsection{Abschließende Bemerkungen}

Es konnte gezeigt werden, dass die Zugabe des RAFT-Agens EPHT zu einem typischen, als UVhärtenden Lack genutzten Systems durchaus zu Veränderungen der daraus resultierenden Netzwerkstrukturen führen kann. Die RAFT-Netzwerke aus Norrish-I-Reaktion sind weniger trüb, als konventionelle oder entsprechende Norrish-II-Systeme, was für das geringere Auftreten von Nanogel-Domänen in diesen Netzwerken spricht. Dies konnte darauf zurückgeführt werden, dass im Falle der Norrish-II-Reaktionen wahrscheinlich keine RAFT-Polymerisation stattgefunden hat, wie ESR-Experimente gezeigt haben. Trotzdem zeigte sich bei den aus Norrish-II-Reaktion resultierten Netzwerken eine deutliche Veränderung der Oberflächenstrukturen. Dies wurde aber auf die geringere Viskosität der Systeme zurückgeführt. Darum führten die beobachteten Veränderungen auch nicht dazu, dass die ausgehärteten Farben besser recycliert werden konnten. Weiterhin konnte nachgewiesen werden, dass es aufgrund der unsicheren chemischen Zusammensetzung der UV-härtenden Druckfarben sehr herausfordernd ist, mit den zur Verfügung stehenden Messmethoden belastbare Ergebnisse zu erhalten. 



\section{Fazit und Ausblick}

Das Ziel der vorliegenden Arbeit war es, den Einfluss der RAFT-Polymerisation auf die Mikrostruktur und den Aufbau von Polymernetzwerken aus UV-initiierter radikalischer Polymerisation zu untersuchen, sowie die Herkunft dieser Unterschiede aufzuklären und mit Bezug auf die theoretischen Grundlagen einzuordnen. Dazu wurden statistische Poly(BA-coBDDA)- und Poly(EA-co-BDDA)-Netzwerke sowie duroplastische PEGDA-Netzwerke in Gegenwart des RAFT-Agens EPHT synthetisiert, hinsichtlich ihrer mechanischen Eigenschaften untersucht und mit analog hergestellten konventionellen Netzwerken verglichen. Dies ermöglichte es, einen direkten Vergleich zwischen den konventionellen und den RAFTNetzwerken herzustellen, sodass die speziell durch den RAFT-Mechanismus hervorgerufenen Veränderungen in den Mikrostrukturen der Netzwerke spezifiziert werden konnten.

Es sind umfangreiche Untersuchungen an statistischen Poly(BA-co-BDDA)- und Poly(EA-coBDDA)-Netzwerken durchgeführt worden. Die mechanischen Eigenschaften der Netzwerke wurden mit Hilfe von Zugversuchen untersucht. Dabei erwiesen sich die RAFT-Netzwerke als weicher als ihre konventionellen Gegenstücke. Durch Einordnen der Ergebnisse in die Theorie der Entropieelastizität konnte eine geringere Netzkettendichte der RAFT-Netzwerke als Grund für diesen Effekt ermittelt werden. Eine Möglichkeit, die mechanischen Eigenschaften der RAFTNetzwerke noch detaillierter zu untersuchen, würde vor allem die Dynamisch-Mechanische Analyse (DMA) bieten. Diese ermöglicht es, die mechanischen Eigenschaften der Netzwerke über einen sehr weiten Temperaturbereich zu beobachten. Die DMA könnte es in Kombination mit kinetischen Messungen ermöglichen, die Gelpunkte der Netzwerke während der Synthese genau zu bestimmen. Außerdem würde die DMA auch die Möglichkeit bieten, die mechanischen Eigenschaften von duroplastischen Netzwerken wie z. B. PEGDA-Netzwerken zu ermitteln. Zusätzlich ist sie in der Lage, direkte Informationen über die Homogenität der Polymernetzwerke zu liefern.

Weiterhin wurden das Quellungsverhalten der Netzwerke und ihre Klebrigkeit beobachtet. Dabei erwiesen sich die RAFT-Netzwerke als stärker quellend und klebriger als ihre konventionellen Gegenstücke. Dies geht zum einen auf den größeren Anteil von Extrahierbaren, aber auch auf eine Reduzierung der Nanogel-Domänen in den RAFT-Netzwerken zurück. Dieser Zusammenhang zwischen der RAFT-Polymerisation und der daraus zunehmenden Klebrigkeit der statistischen Polymernetzwerke auf Acrylat-Basis wurde im Rahmen der vorliegenden Arbeit erstmals gefunden und untersucht. Die RAFT-Polymerisation bietet somit das Potential, mit Polymernetzwerken eine neue PSA-Klasse zu etablieren, da nur durch die Zugabe des RAFT- 
Agens zu den Polymerisationsmischungen der Netzwerke die dafür nötige Klebrigkeit erreicht wird. Dies könnte zum Durchbruch in der Verwendung der RAFT-Polymerisation in industriellen Anwendungen werden, da gerade PSAs weite Verbreitung in Alltag, Wissenschaft und Technik finden. Dazu sollte in Zukunft speziell der Einfluss der RAFT-Polymerisation auf diese besondere Eigenschaft der Netzwerke tiefergehend untersucht werden. So könnten z. B. verschiedene RAFT-Agenzien wie multifunktionelle oder mit speziellen Funktionen ausgestattete Z- und RGruppen dazu beitragen, die Klebrigkeit der Netzwerke noch weiter zu optimieren und auf die entsprechenden Verwendungen einzustellen. Dazu könnten als Z- oder R-Gruppe des RAFTAgens z. B. Silanderivate gewählt werden, die als Ankergruppen für Glasoberflächen fungieren. Weiterhin bietet die RAFT-Polymerisation die Möglichkeit, relativ einfach durch Copolymerisation andere Monomere in die Netzwerke einzubringen, welche die Mikrostrukturen der Netzwerke und somit ihre kohäsiven und adhäsiven Eigenschaften noch weiter verändern könnten. Gerade in Kombination mit der UV-Härtung bieten sich somit vielfältige Möglichkeiten, aus einer Flüssigkeit nach der Härtung hochkohäsive PSAs herzustellen, die durch die RAFTPolymerisation auf ihren speziellen Verwendungszweck ausgerichtet sind.

Weiterhin sollte die Rolle der Extrahierbaren bei der Klebrigkeit der Netzwerke genauer betrachtet werden. So könnte z. B. die Fibrillenbildung mittels apparativ optimierten Probe-TackTests untersucht werden, wozu z. B. ein genauer, auflösender und feinerer Kraftaufnehmer zählen würde. Außerdem könnten die Extrahierbaren vor den Rasterkraftmikroskop-Aufnahmen und den Rollkugel- bzw. Probe-Tack-Tests aus den Netzwerken entfernt werden, um deren Auswirkung auf die Oberflächenstrukturen und die Klebrigkeiten der Netzwerke zu untersuchen.

Im Rahmen der Quellungsexperimente erwiesen sich die RAFT-Netzwerke, aufgrund ihres homogenen Aufbaus und der daraus resultierenden gleichmäßigeren Aufspaltung der NMRSignale, als sehr gut geeignete Netzwerke für SSE-NMR-Experimente. Sie bieten daher das Potential, die Messgenauigkeit dieser Methode deutlich zu verbessern. Weiterhin ermöglichen es speziell die Poly(BA-co-BDDA)-Netzwerke Tieftemperaturexperimente durchzuführen, wodurch es möglich wird, temperaturempfindliche Moleküle, die sich in den Kavitäten des Netzwerks befinden, mit der SSE-NMR-Technik zu untersuchen.

Neben der Veränderung der mechanischen Eigenschaften der Poly(BA-co-BDDA)- und Poly(EAco-BDDA)-Netzwerke ist die Retardierung der Polymerisationen, die auch bei den PEGDANetzwerken deutlich wurde, hervorgerufen durch das RAFT-Agens EPHT der deutlichste Einfluss auf die Polymerisation der Netzwerke. Als Grund für diese Retardierung konnte die Absorption von Strahlung bei der eingestrahlten Wellenlänge von $366 \mathrm{~nm}$ identifiziert werden. Für eine potentielle, industrielle Nutzung der RAFT-Polymerisation in UV-härtenden Systemen könnte diese Retardierung allerdings ein Problem sein, da es dort häufig auf eine sehr schnelle Polymerisation ankommt. Darum ist es sinnvoll, die Konzentrationsverhältnisse aller an der 
Polymerisation beteiligten Spezies so zu optimieren, dass die maximal gewünschte Wirkung des RAFT-Mechanismus auf die Mikrostrukturen der Netzwerke bei einem minimalen Einsatz des verwendeten RAFT-Agens erreicht werden. Dazu wären intensive Studien mit verschiedensten Kombinationen der an der Polymerisation beteiligten Spezies notwendig. Weiterhin könnte durch eine aufeinander optimierte Wahl von Initiator, RAFT-Agens und Initiierungswellenlänge ein System gefunden werden, bei dem die Absorptionen von Photoinitiator und RAFT-Agens nicht mehr in Konkurrenz zueinander stehen. Eine Möglichkeit hierfür würde ein für sichtbares Licht optimierter Photoinitiator bieten. Außerdem sollte der Einfluss der thermisch-initiierten RAFT-Polymerisation auf die Eigenschaften der Netzwerke untersucht werden, da erste im Rahmen dieser Arbeit durchgeführte thermische Polymerisationen nahelegen, dass die bei den UV-initiierten Systemen gefundene Retardierung in den thermischen Systemen nicht stattfindet.

Am deutlichsten wurden die Unterschiede zwischen den RAFT- und den konventionellen Poly(BA-co-BDDA)- und Poly(EA-co-BDDA)-Netzwerken bei sehr geringen BDDA-Anteilen von unter 1 mol-\%. Dies legt nahe, dass der Einfluss des RAFT-Mechanismus auf Polymernetzwerke mit steigender Netzkettendichte der Netzwerke geringer wird. Dies zeigte sich sehr deutlich bei den ebenfalls untersuchten PEGDA-Netzwerken. Während bei PEGDA-575- und PEGDA-700Netzwerken durchaus kleinere Unterschiede zwischen den Oberflächenstrukturen von RAFTund konventionellen Netzwerken gefunden werden konnten, waren diese bei PEGDA-250Netzwerken nicht mehr eindeutig nachweisbar. Trotzdem können diese oder andere multifunktionellen Monomere helfen, den Einfluss der RAFT-Polymerisation auch auf Systeme, deren genaue Zusammensetzung unbekannt ist, wie z. B. UV-härtende Druckfarben besser zu verstehen.

Insgesamt ist es im Rahmen dieser Arbeit gelungen, die Informationen aus verschiedenen Messmethoden zu einem Bild über Polymernetzwerke aus UV-initiierter RAFT-Polymerisation zu vereinen und somit die generellen Unterschiede in Mikrostruktur und Aufbau von konventionellen und RAFT-Netzwerken zu benennen. Weiterhin wurden die Gründe für diese Unterschiede aufgeklärt und die Ergebnisse in existierende theoretische Modelle zur Beschreibung von Polymernetzwerken eingeordnet. In Zukunft sollte vor allem der Einfluss der RAFT-Polymerisation auf die Klebrigkeit von Polymeren und Polymernetzwerken näher untersucht werden, da die RAFT-Polymerisation ein großes Potential bietet, diese entsprechend der gewünschten Eigenschaften zu beeinflussen. 



\section{Experimentelles}

\subsection{Chemikalien}

\subsubsection{Verwendete Chemikalien}

2-Methyl-4'-(methylthio)-2-morpholinopropiophenon (MMMP)<smiles>CSc1ccc(C(=O)C(C)(C)N2CCOCC2)cc1</smiles>

Der Photoinitiator 2-Methyl-4'-(methylthio)-2-morpholinopropiophenon $\quad(M=279,13 \mathrm{~g} / \mathrm{mol}$, SIGMA ALDRICH, > 98\%) wurde ohne weitere Aufarbeitung verwendet.

$n$-Butylacrylat (BA)<smiles>C=CC(=O)OCCCC</smiles>

Das Monomer $n$-Butylacrylat ( $M=128,17 \mathrm{~g} / \mathrm{mol}$, SIGMA ALDRICH, 99\%) wurde mittels einer mit Inhibitor Remover (SIGMA ALDRICH) gepackten Chromatographie-Säule vom enthaltenen Inhibitor befreit.

Ethylacrylat (EA)<smiles>C=CC(=O)OCC</smiles>

Das Monomer Ethylacrylat $(M=100,12 \mathrm{~g} / \mathrm{mol}$, SIGMA ALDRICH, 99\%) wurde mittels einer mit Inhibitor Remover (SIGMA ALDRICH) gepackten Chromatographie-Säule vom enthaltenen Inhibitor befreit. 


\section{1,4-Butandioldiacrylat (BDDA)}<smiles>C=CC(=O)OCCCCOC(=O)C=C</smiles>

Das als Vernetzungsreagenz verwendete, bifunktionelle Monomer 1,4-Butandioldiacrylat ( $M=100,12 \mathrm{~g} / \mathrm{mol}$, ABCR, 99\%) wurde mittels Rühren mit Inhibitor Remover (SIGMA ALDRICH) und anschließender Filtration vom enthaltenen Inhibitor befreit.

\section{Polyethylenglycoldiacrylat (PEGDA)}<smiles>C=CC(=O)OCC(C)(C)OC(=O)C=C</smiles>

1<smiles>C=CC(=O)OCC(C)(C)OC(=O)C=C</smiles>

2<smiles>C=CC(=O)OCC(C)(C)OC(=O)C=C</smiles>

3

Das Präpolymer Polyethylenglycoldiacrylat $\quad\left(1: \bar{M}_{\mathrm{n}}=250 \mathrm{~g} / \mathrm{mol}, \quad 2: \bar{M}_{\mathrm{n}}=575 \mathrm{~g} / \mathrm{mol}\right.$, 3: $\bar{M}_{\mathrm{n}}=700 \mathrm{~g} / \mathrm{mol}$, alle SIGMA ALDRICH) wurde mittels Rühren mit Inhibitor Remover (SIGMA ALDRICH) und anschließender Filtration vom enthaltenen Inhibitor befreit.

\section{Für Musterformulierungen verwendete Chemikalien}<smiles>CCOP(=O)(C(=O)c1c(C)cc(C)cc1C)c1ccccc1</smiles>

Luricin TPO-L

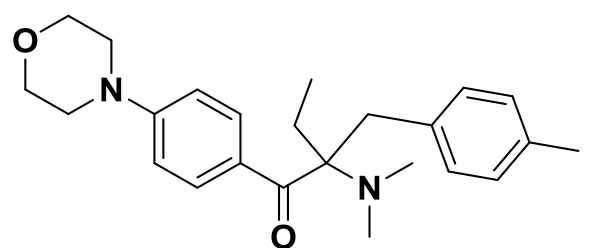

Irgacure 379

Laromer $9013^{\circledR}$, Luricin TPO-L und Irgacure 379 wurden von der BASF zur Verfügung gestellt. MDEA wurde von der RAHN AG zur Verfügung gestellt. Wenn nicht anders im Text erwähnt, wurden die Musterformulierungen für die Druckfarbenexperimente in den Laboren der FOGRA zusammengestellt.

\section{Weitere Chemikalien}

Alle weiteren Chemikalien, wie z. B. Lösungsmittel oder Edukte zur Synthese von RAFTAgenzien wurden, sofern im Text nicht anders erwähnt, kommerziell erworben und so verwendet wie erhalten. 


\subsubsection{Synthesen von RAFT-Agenzien}

\subsubsection{S-Ethylpropan-2-ylonat-S'-hexyltrithiocarbonat (EPHT)}

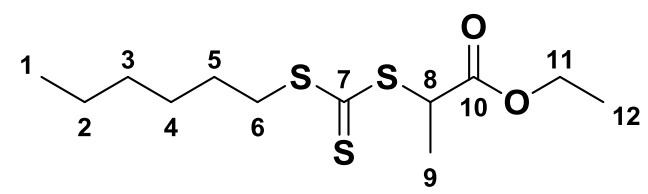

$S$-Ethylpropan-2-ylonat-S'-hexyltrithiocarbonat (EPHT, $M=294,50 \mathrm{~g} / \mathrm{mol}$ ) wurde nach folgender Vorschrift synthetisiert. In einem $500 \mathrm{~mL}$ Rundkolben wurde Hexanthiol (4,0 g, 0,034 mol) in Chloroform $(220 \mathrm{~mL})$ vorgelegt. Es wurde Triethylamin $(4,1 \mathrm{~g}, 0,040 \mathrm{~mol})$ hinzugetropft. Die Mischung wurde eine Stunde bei Raumtemperatur gerührt. Anschließend wurde Kohlenstoffdisulfid $(20 \mathrm{~mL})$ langsam hinzugegeben und weitere 30 Minuten gerührt. Nach Zugabe von Ethyl-2-Bromopropionat (7,4 g, 0,040 mol) wurde drei Tage bei Raumtemperatur gerührt. Die Reaktion wurde durch Zugabe von 10\%iger Salzsäure $(250 \mathrm{~mL})$ abgebrochen. Die organische Phase wurde abgetrennt, dreimal mit demineralisiertem Wasser gewaschen und über Natriumsulfat getrocknet. Das Lösungsmittel wurde am Rotationsverdampfer entfernt. Anschließend wurde das Rohprodukt mittels Säulenchromatographie über Kieselgel (Porengröße: $60 \AA, 70-230$ mesh, 63-200 $\mu \mathrm{m}$ ) gereinigt. Als Eluent wurde eine Mischung von Ethylacetat / Hexan (1/10) verwendet. Das Produkt EPHT (8,3 g, 0,028 mol, 83\%) wurde als gelbes Öl erhalten. Über NMR-Spektroskopie wurde eine Reinheit von $98 \%$ ermittelt.

${ }^{1} \mathbf{H}$ NMR (300 MHz, $\mathrm{CDCl}_{3}, \delta$, ppm): 0,84 (t, $\left.\mathcal{F}=6,8 \mathrm{~Hz}, 3 \mathrm{H}, 3-\mathrm{H}\right), 1,23(\mathrm{~m}, 9 \mathrm{H}, 12-\mathrm{H}, 2-\mathrm{H}, 3-\mathrm{H}$, 4-H), 1,54 (d, $\mathcal{f}=7,4 \mathrm{~Hz}, 3 \mathrm{H}, 9-\mathrm{H}), 1,65$ (q, $\mathcal{f}=7,2 \mathrm{~Hz}, 2 \mathrm{H}, 5-\mathrm{H}) 3,31$ (t, $\mathcal{f}=7,5 \mathrm{~Hz}, 2 \mathrm{H}, 6-\mathrm{H}), 4,14$ (q, $\mathcal{F}=7,2 \mathrm{~Hz}, 2 \mathrm{H}, 11-\mathrm{H}), 4,75$ (q, $\mathcal{F}=7,4 \mathrm{~Hz}, 1 \mathrm{H}, 8-\mathrm{H}$ ).

${ }^{13}$ C NMR (75 MHz, $\mathrm{CDCl}_{3}, \delta$, ppm): 13,9 (C-1), 14,0 (C-12), 16,8 (C-9), 22,4 (C-2), 27,8 (C-4), 28,5 (C-5), 31,2 (C-3), 37,1 (C-6), 47,9 (C-8), 61,7 (C-11), 170,9 (C-10), 221,9 (C-7).

\subsubsection{1,4-Butandiolbis(2-bromopropanoat)}

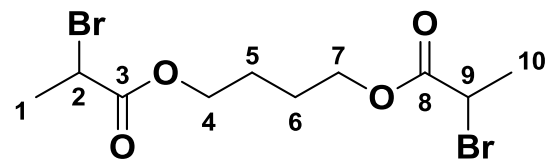

Das RAFT-Agens-Edukt 1,4-Butandiolbis(2-bromopropanoat) $\left(M=360,04 \mathrm{~g} \cdot \mathrm{mol}^{-1}\right)$ wurde durch eine Veresterung nach Steglich ${ }^{[231]}$ hergestellt. In einem $250 \mathrm{~mL}$ Rundkolben wurden ( \pm )-2- 
Brompropionsäure (7,14 g, 0,047 mol) in trockenem Dichlormethan (DCM, $100 \mathrm{~mL}$ ) vorgelegt. Unter Rühren wurden 4-Dimethylaminopyridin (DMAP, 321,4 mg) und 1,4-Butandiol (1,27 g, 0,014 mol) hinzugegeben. Die Reaktionsmischung wurde im Eisbad gekühlt und Dicyclocarbodiimid (DCC, 6,230 g) zugegeben. Anschließend wurde fünf Minuten bei $0{ }^{\circ} \mathrm{C}$ und weitere drei Stunden bei Raumtemperatur gerührt. Der ausgefallene Harnstoff wurde abfiltriert und das Lösungsmittel am Rotationsverdampfer abgezogen. Nachdem das Rohprodukt mit DCM $(100 \mathrm{~mL})$ aufgefüllt wurde, wurde erneut filtriert. Das Filtrat wurde über Nacht bei $-21^{\circ} \mathrm{C}$ im Gefrierschrank gelagert, erneut filtriert und jeweils zweimal mit 0,5 N Salzsäure (100 mL) und gesättigter Natriumhydrogencarbonat-Lösung $(100 \mathrm{~mL})$ gewaschen. Anschließend wurde über Natriumsulfat $\left(\mathrm{Na}_{2} \mathrm{SO}_{4}\right)$ getrocknet, das Lösungsmittel am Rotationsverdampfer abgezogen und am Hochvakuum getrocknet. Das Produkt (2,245 g, 6,2 mmol, 44,1 \%) wurde als leicht bräunliches Ỏl erhalten. Über NMR-Spektroskopie wurde eine Reinheit von 99 \% ermittelt.

${ }^{1} \mathbf{H}$ NMR $\left(200 \mathrm{MHz}, \mathrm{CDCL}_{3}, \delta\right.$, ppm): 1,79 (t, $\left.\mathcal{f}=2,8 \mathrm{~Hz}, 4 \mathrm{H}, 5-\mathrm{H}, 6-\mathrm{H}\right), 1,81(\mathrm{~d}, \mathcal{J}=7,0 \mathrm{~Hz}, 6 \mathrm{H}$, 1-H, 10-H), 4,20 (m, 4 H, 4-H, 7-H), 4,36 (q, J = 7,0 Hz, 2 H, 3-H, 9-H)

${ }^{13}$ C NMR (75 MHz, $\mathrm{CDCL}_{3}, \delta$, ppm): 21,6 (C-1,C-10), 25,0 (C-5, C-6), 40,0 (C-2, C-9), 65,2 (C-4, C-7), 170,2 (C-3, C-8)

\subsubsection{1,4-Butandiolbis(2-(hexyltrithiocarbonat)propanoat) (BDHTP)}

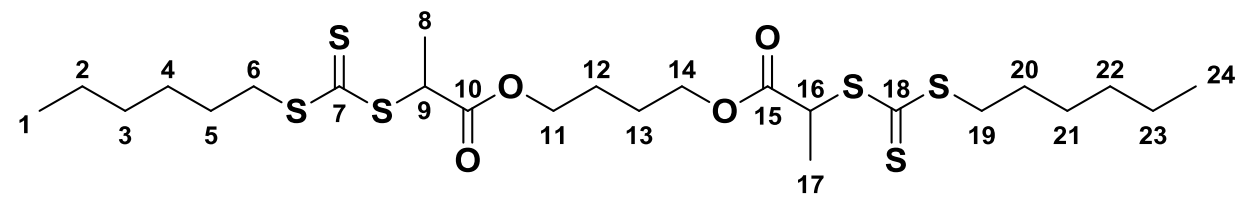

1,4-Butandiolbis(2-(hexyltrithiocarbonat)propanoat) (BDHTP, $M=586,98 \mathrm{~g} \cdot \mathrm{mol}^{-1}$ ) wurde nach folgender Vorschrift synthetisiert. In einem $250 \mathrm{~mL}$ Rundkolben wurde Hexanthiol $(2,0 \mathrm{~g}$, $0,017 \mathrm{~mol})$ in Dichlormethan $(100 \mathrm{~mL})$ vorgelegt. Es wurde Triethylamin $(2,0 \mathrm{~g}, 0,020 \mathrm{~mol})$ hinzugetropft. Diese Mischung wurde eine Stunde bei Raumtemperatur gerührt. Anschließend wurde Kohlenstoffdisulfid (10 mL) langsam hinzugegeben und weitere 30 Minuten gerührt. Nach Zugabe von 1,4-Butandiolbis(2-bromopropylester) (2,7 g, 7,5 mmol) wurde drei Tage bei Raumtemperatur gerührt. Die Reaktion wurde durch Zugabe von 2\%iger Salzsäure (100 mL) abgebrochen. Die organische Phase wurde abgetrennt, dreimal mit demineralisiertem Wasser gewaschen und über $\mathrm{Na}_{2} \mathrm{SO}_{4}$ getrocknet. Das Lösungsmittel wurde am Rotationsverdampfer entfernt. Anschließend wurde das Rohprodukt mittels Säulenchromatographie über Kieselgel (Porengröße: $60 \AA, 70-230$ mesh, 63-200 $\mu \mathrm{m}$ ) gereinigt. Als Eluent wurde eine Mischung von DCM/Hexan (1/4) verwendet. Die Produktfraktion wurde anschließend mit reinem DCM von der 
Säule gespült. Das Produkt BDHTP (3,75 g, 6,4 mmol, 85,2\%) wurde als gelbes Öl erhalten. Über NMR-Spektroskopie wurde eine Reinheit von $99 \%$ ermittelt.

${ }^{1} \mathbf{H}$ NMR $\left(200 \mathrm{MHz}, \mathrm{CDCL}_{3}, \delta\right.$, ppm): 0,82 (t, $\left.\mathcal{J}=6,7 \mathrm{~Hz}, 6 \mathrm{H}, 1-\mathrm{H}, 24-\mathrm{H}\right), 1,23(\mathrm{~m}, 8 \mathrm{H}, 2-\mathrm{H}, 3-\mathrm{H}$, 22-H, 23-H), 1,33 (m, 4 H, 4-H, 21-H), 1,52 (d, J = 7,4 Hz, 6 H, 8-H, 17-H) 1,65 (m, 8 H, 12-H, 13-H, 5-H, 20-H), 3,28 (t, J = 7,2 Hz, 4 H, 6-H, 19-H), 4,09 (m, 4 H, 11-H, 14-H), 4,7 (q, J = 7,4 Hz, $2 \mathrm{H}$, 9-H, 16-H).

${ }^{13} \mathrm{C}$ NMR $\left(75 \mathrm{MHz}, \mathrm{CDCL}_{3}, \delta\right.$, ppm): 14,0 (C-1, C-24), 16,8 (C-8, C-17), 22,5 (C-2, C-23), 25,1 (C-12, C-13), 27,9 (C-4, C-21), 28,6 (C-5, C-20), 31,3 (C-3, C-22), 37,3 (C-6, C-19), 47,9 (C-9, C-16), 65,1 (C-11, C-14), 171,1 (C-10, C-15), 222,0 (C-7, C-18).

\subsubsection{Bis(S-Ethylpropan-2-ylonat)-S'-1,4-butylbistrithiocarbonat}

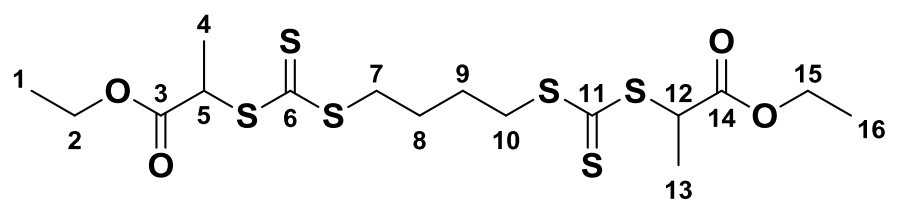

Bis(S-Ethylpropan-2-ylonat)-S-1,4-butylbistrithiocarbonat (BEPBT, $\quad M=474,77 \mathrm{~g} / \mathrm{mol}$ ) wurde nach folgender Vorschrift synthetisiert. In einem $250 \mathrm{~mL}$ Rundkolben wurde 1,4-Hexandithiol $(1,4 \mathrm{~g}, 9,0 \mathrm{mmol})$ in Dichlormethan $(100 \mathrm{~mL})$ vorgelegt. Es wurde Triethylamin $(2,7 \mathrm{~g}, 0,027 \mathrm{~mol})$ hinzugetropft. Diese Mischung wurde eine Stunde bei Raumtemperatur gerührt. Anschließend wurde im Überschuss Kohlenstoffdisulfid $(10 \mathrm{~mL})$ langsam hinzugegeben und weitere 30 Minuten gerührt. Nach Zugabe von Ethyl-2-Bromopropionat (5,0 g, 0,028 mol) wurde 15 Stunden bei Raumtemperatur gerührt. Die Reaktion wurde durch Zugabe von 10\%iger Salzsäure (100 mL) abgebrochen. Die organische Phase wurde abgetrennt, dreimal mit demineralisiertem Wasser gewaschen und über Natriumsulfat getrocknet. Das Lösungsmittel wurde am Rotationsverdampfer entfernt. Anschließend wurde das Rohprodukt mittels Säulenchromatographie über Kieselgel (Porengröße: $60 \AA$, 70 - 230 mesh, $63-200 \mu \mathrm{m}$ ) gereinigt. Als Eluent wurde eine Mischung von Ethylacetat / Hexan (1/20) verwendet. Das Produkt wurde anschließend mit einem modifizierten Eluentenverhältnis von Ethylacetat / Hexan (1/4) von der Säule gespült. Das Produkt BEPBT (4,19 g, 8,8 mmol, 98\%) wurde als gelbes Öl erhalten. Über NMR-Spektroskopie wurde eine Reinheit von $99 \%$ ermittelt.

${ }^{1} \mathbf{H}$ NMR (300 MHz, $\left.\mathrm{CDCl}_{3}, \delta, \mathrm{ppm}\right): 1,21$ (t, $\left.\mathcal{f}=7,1 \mathrm{~Hz}, 6 \mathrm{H}, 1-\mathrm{H}, 16-\mathrm{H}\right), 1,53(\mathrm{~d}, \mathcal{F}=7,4 \mathrm{~Hz}, 6 \mathrm{H}$, 4-H, 13-H), 1,75 (m, 4 H, 8-H, 9-H), 3,32 (m, 4 H, 7-H, 10-H) 4,12 (q, f = 7,1 Hz, 4 H, 2-H, 15-H), $4,72$ (q, $\mathcal{f}=7,4 \mathrm{~Hz}, 2 \mathrm{H}, 5-\mathrm{H}, 12-\mathrm{H})$. 
${ }^{13}$ C NMR (75 MHz, $\mathrm{CDCl}_{3}, \delta$, ppm): 14,1 (C-1, C-16), 16,9 (C-4, C-13), 27,3 (C-8, C-9), 36,2 (C-7, C-10), 48,1 (C-5, C-12), 61,9 (C-2, C-15), 171,0 (C-3, C-14), 221,6 (C-6, C-11).

\subsubsection{S-S'-Diethyltrithiocarbonat}

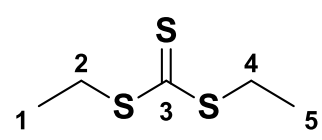

$S$-S -Diethyltrithiocarbonat (DET, $M=166,33 \mathrm{~g} / \mathrm{mol}$ ) wurde analog zu einer Vorschrift von Aoyagi et al. ${ }^{[42]}$ synthetisiert. In einem $25 \mathrm{~mL}$ Rundkolben wurden Cäsiumcarbonat $(3,29 \mathrm{~g}$, $10 \mathrm{mmol}$ ) und Kohlenstoffdisulfid $(0,76 \mathrm{~g}, 10 \mathrm{mmol})$ in Dimethylacetamid (DMAc, $8 \mathrm{~mL}$ ) vorgelegt. Es wurde 30 Minuten bei Raumtemperatur gerührt. Anschließend wurden Ethyliodid (1,64 g, $10 \mathrm{mmol})$ in DMAc (2 mL) hinzugegeben. Es wurde 20 Stunden bei Raumtemperatur gerührt. Um die Reaktion zu stoppen, wurde die Reaktionslösung in Eiswasser gegeben, welches anschließend dreimal mit Ethylacetat ausgeschüttelt wurde, um das Produkt zu extrahieren. Die zusammengeführten Extrakte wurden über Magnesiumsulfat getrocknet und filtriert. Das Lösungsmittel wurde am Rotationsverdampfer abgezogen und das Produkt unter Hochvakuum getrocknet. Das Produkt DET (0,33 g, 2,0 mmol, 38\%) wurde als orangefarbenes Öl erhalten. Über NMR-Spektroskopie wurde eine Reinheit von $98 \%$ ermittelt.

${ }^{1} \mathbf{H}$ NMR (300 MHz, $\left.\mathrm{CDCl}_{3}, \delta, \mathrm{ppm}\right): 1,28(\mathrm{t}, \mathcal{J}=7,5 \mathrm{~Hz}, 6 \mathrm{H}, 1-\mathrm{H}, 5-\mathrm{H}), 3,29$ (q, $\mathcal{f}=7,4 \mathrm{~Hz}, 4 \mathrm{H}$, $2-\mathrm{H}, 4-\mathrm{H})$.

${ }^{13}$ C NMR (75 MHz, $\mathrm{CDCL}_{3}, \delta$, ppm): 13,1 (C-1, C-5), 31,1 (C-2, C-4), 224,3 (C-3).

\subsubsection{Dipentaerythriol-hexakis(3-(S-methoxopropionat-trithiocarbonat))-propanoat}

Dipentaerythriol-hexakis(3-(S-methoxopropionat-trithiocarbonat))-propanoat wurde von Nadja Förster synthetisiert und analysiert. ${ }^{[232]}$ 


\subsection{Polymerisationen}

\subsubsection{Synthese von Probekörpern für Zugversuche}

Die Synthese, der für die Zugversuche verwendeten Probekörper nach ISO 37-Norm, Typ $1^{\text {[233] }}$ (s. Abbildung 6-1), erfolgte in einer entsprechenden Teflonform, die von der Mechanik-Werkstatt des Instituts für Physikalische Chemie der Universität Göttingen speziell hergestellt worden ist. Die Form wurde in einem Exsikkator platziert, der anschließend zunächst unter Vakuum gesetzt und dann mit Argon geflutet wurde. Unter konstantem Argonzufluss wurde der Exsikkator geöffnet und 3,5 mL der Polymerisationsmischung mittels einer mit Argon gespülten Spritze in die Teflonform gegeben. Jede Polymerisationsmischung wurde zuvor 15 Minuten mit Argon gespült.

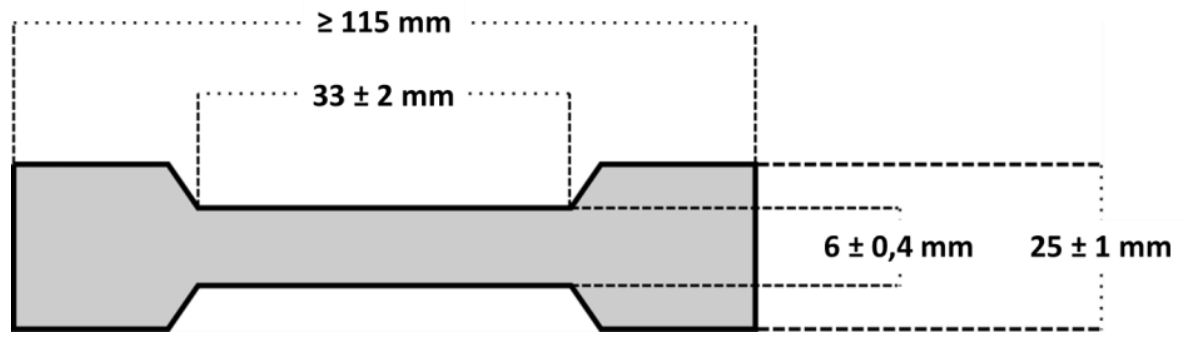

Abbildung 6-1 Abmessungen eines ISO 37-Norm, Typ 1-Zugversuchprobekörpers. Die Höhe des Probekörpers beträgt: $2 \pm 0,2 \mathrm{~mm}$.

Als Lichtquelle wurde eine N-8 UV-Handlampe der Firma BENDA KONRAD bei einer Emissionswellenlänge von $366 \mathrm{~nm}$ verwendet. Diese wurde so auf dem Exsikkator platziert, dass der Abstand zur Teflonform $5 \mathrm{~cm}$ betrug, was eine Lichtintensität von $9 \mathrm{~mW} / \mathrm{cm}^{2}$ ergab. Die Polymerisation der Probekörper erfolgte unter konstantem leichtem Argonstrom. Zusätzlich wurde der Exsikkator mit Alufolie abgedeckt. Die Polymerisationszeit betrug, je nach eingesetzter Polymerisationsmischung, zwischen 30 Minuten für die konventionellen Netzwerke und die Netzwerke mit 1 mol-\%o EPHT, 45 Minuten für die Netzwerke mit 2 mol-\%o EPHT und 80 Minuten für die Netzwerke mit 4 mol-\%o EPHT. Die Zusammensetzungen der verwendeten Polymerisationsmischungen sind in Kapitel 6.2.4 zu finden. Alle Synthesen wurden bei Raumtemperatur durchgeführt. Die fertigen Probekörper wurden vor der Messung mindestens 24 Stunden in einem dunklen abgesaugten Chemikalienschrank gelagert. 


\subsubsection{Synthese von Probekörpern für Rasterkraftmikroskop-Messungen}

Die Proben der Poly(BA-co-BDDA)- und Poly(EA-co-BDDA)-Netzwerke wurden für die Messungen mit dem Rasterkraftmikroskop direkt nach der Synthese aus ZugversuchProbekörpern mittels einer Lochstanze $(d=1 \mathrm{~cm})$ ausgestanzt und auf ein AFMProbeträgerplättchen aus Stahl geklebt.

Die Probekörper für die PEG-ArcrylatNetzwerke wurden nach folgender Methode hergestellt: Eine $2 \mathrm{~mm}$ Dicke Teflonscheibe mit einem Loch in der Mitte $(d=0,8 \mathrm{~cm})$ wurde einseitig mit einem glatten Stück Aluminiumfolie umwickelt. Diese Form (s. Abbildung 6-2) wurde in einem mit Argon durchspülten Kunststoffbecher (Höhe: $5 \mathrm{~cm}$ ) platziert. Anschließend wurden $0,1 \mathrm{~mL}$ der jeweiligen Polymerisationsmischung in die Form gegeben. Die Polymerisationsmischungen wurden zuvor jeweils 20 Minuten mit Argon gespült, um Sauerstoff zu entfernen. Die

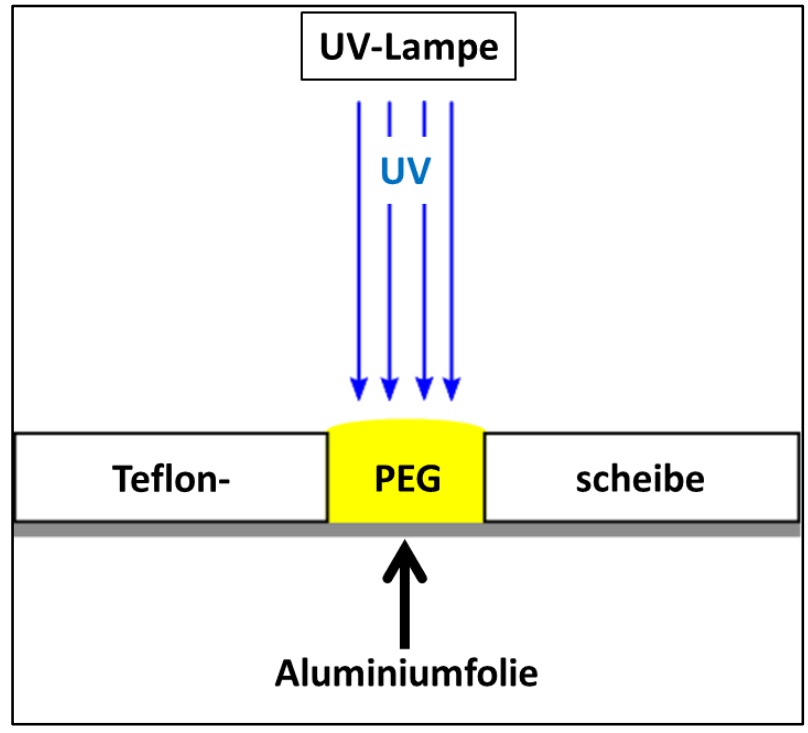

Abbildung 6-2 Schematischer Querschnitt durch den Versuchsaufbau zur Synthese von PEG-Probekörpern für die Rasterkraftmikroskopie. UV-Lampe (s. Kapitel 6.2.1) wurde so über dem Kunststoffbecher platziert, dass sie $5 \mathrm{~cm}$ von der Form entfernt war, sodass die Lichtintensität $9 \mathrm{~mW} / \mathrm{cm}^{2}$ betrug. Die Polymerisationszeit betrug 15 Minuten. Alle Synthesen wurden bei Raumtemperatur durchgeführt. Nach erfolgter Polymerisation wurde die Aluminiumfolie entfernt und der Probekörper von unten aus der Teflonscheibe vorsichtig herausgedrückt und auf ein AFM-Probenträgerplättchen aus Stahl geklebt. Anschließend wurden sie in einer Polystyrol-Petrischale mit Deckel, die zusätzlich mit Parafilm ${ }^{\circledR}$ umwickelt wurde, im Dunkeln aufbewahrt. Für alle Rasterkraftmikroskop-Proben galt, dass sie erst einen Tag nach dem Aufkleben auf die Probenträger mit dem Rasterkraftmikroskop untersucht wurden. Die Zusammensetzungen der verwendeten Polymerisationsmischungen sind in Kapitel 6.2.4 zu finden.

\subsubsection{Synthese von Probekörpern für die NMR-Untersuchungen}

Die Synthese der Probekörper für die NMR-Untersuchungen erfolgte in $12 \mathrm{~cm}$ langen Borosilikatglas-Röhrchen (innerer Durchmesser: $3,8 \mathrm{~mm}$ ), die extra für diese Synthesen 
hergestellt wurden. Diese wurden in einem $100 \mathrm{~mL}$ Schlenk-Rohr platziert, welches mit einem Butyl-Kautschuk-Septum verschlossen wurde. Anschließend wurde das Schlenk-Rohr evakuiert und mit Argon gefüllt.

Vor der Polymerisation wurde jede Polymerisationsmischung 15 Minuten mit Argon gespült. $2 \mathrm{~mL}$ der gespülten Mischung wurde mittels einer mit Argon gespülten Spritze durch das Septum in das Glasröhrchen gegeben. Die UV-Lampe (s. Kapitel 6.2.1) wurde in einem Abstand von $5 \mathrm{~cm}$ von dem Glasröhrchen entfernt platziert, was eine Lichtintensität von $9 \mathrm{~mW} / \mathrm{cm}^{2}$ ergab. Um die Polymerisation zu starten, wurde eine Blende, die sich zwischen der UV-Lampe und dem Schlenk-Rohr befand, entfernt. Die Polymerisationszeit betrug 30 Minuten für konventionelle Netzwerke und 60 Minuten für RAFT-Netzwerke. Alle Synthesen wurden bei Raumtemperatur durchgeführt.

Nach der Polymerisation wurde das Glasröhrchen aus dem Schlenk-Rohr entnommen und in flüssigem Stickstoff eingefroren. Anschließend wurde das Glas zerbrochen und die noch gefrorene Probe von den Bruchstücken befreit. Nachdem die Probe wieder aufgetaut war, wurde sie in $1 \mathrm{~cm}$ lange Stücke geschnitten. Diese wurden bis zu ihrer Verwendung in abgedunkelten Schnappdeckelgläsern gelagert. Die Zusammensetzungen der verwendeten Polymerisationsmischungen sind in Kapitel 6.2.4 zu finden.

\subsubsection{Zusammensetzungen der Polymerisationsmischungen}

\subsubsection{Poly(BA-Co-BDDA)-Netzwerke}

Die molaren Anteile und die entsprechenden Konzentrationen sowie die Massen der verwendeten Chemikalien sind in den folgenden Tabellen aufgelistet. Entsprechend dieser Tabellen wurden die Proben für jede durchgeführte Polymersynthese und Messmethode hergestellt, die Massen wurden entsprechend an den Bedarf angepasst. Bei allen Polymerisationen wurde ohne Lösungsmittel gearbeitet.

Tabelle 6-1 Molare Anteile und entsprechende Konzentrationen sowie Massen des Vernetzers BDDA und des RAFTAgens EPHT bei Poly(BA-co-BDDA)-Netzwerken aus RAFT-Polymerisation. Das Volumen des Monomers BA beträgt für die Berechnungen $1 \mathrm{~mL}\left(1,12 \mathrm{~g}, 8,7 \cdot 10^{-3} \mathrm{~mol} / \mathrm{mL}\right)$. Für konventionelle Netzwerke gelten die gleichen Werte, allerdings ohne EPHT. Bei jeder Polymerisation waren 2 Gew.- $\%\left(0,0178 \mathrm{~g}, 7,95 \cdot 10^{-5} \mathrm{~mol} / \mathrm{mL}\right)$ MMMP als Photoinitiator vorhanden.

\begin{tabular}{cccccc}
\hline $\begin{array}{c}\text { BDDA } \\
{[\mathbf{m o l}-\%]}\end{array}$ & $\boldsymbol{c}_{\text {BDDA }}[\mathbf{m o l} / \mathbf{m L}]$ & $\begin{array}{c}\boldsymbol{m}_{\text {BDDA }} \\
{[\mathbf{g}]}\end{array}$ & EPHT [mol-\%o $]$ & $\boldsymbol{c}_{\text {EPHT }}[\mathbf{m o l} / \mathbf{m L}]$ & $\begin{array}{c}\boldsymbol{m}_{\text {EPHT }} \\
{[\mathbf{g}]}\end{array}$ \\
\hline 0,125 & $1,08 \cdot 10^{-5}$ & 0,002 & 2 & $1,74 \cdot 10^{-5}$ & 0,005 \\
0,25 & $2,17 \cdot 10^{-5}$ & 0,004 & 2 & $1,74 \cdot 10^{-5}$ & 0,005 \\
0,375 & $3,24 \cdot 10^{-5}$ & 0,006 & 2 & $1,74 \cdot 10^{-5}$ & 0,005 \\
\hline
\end{tabular}




\begin{tabular}{cccccc}
\hline 0,5 & $4,33 \cdot 10^{-5}$ & 0,009 & 2 & $1,74 \cdot 10^{-5}$ & 0,005 \\
0,625 & $5,40 \cdot 10^{-5}$ & 0,011 & 2 & $1,74 \cdot 10^{-5}$ & 0,005 \\
0,75 & $6,48 \cdot 10^{-5}$ & 0,013 & 2 & $1,74 \cdot 10^{-5}$ & 0,005 \\
0,875 & $7,56 \cdot 10^{-5}$ & 0,015 & 2 & $1,74 \cdot 10^{-5}$ & 0,005 \\
1 & $8,66 \cdot 10^{-5}$ & 0,017 & 2 & $1,74 \cdot 10^{-5}$ & 0,005 \\
1,125 & $9,72 \cdot 10^{-5}$ & 0,019 & 2 & $1,74 \cdot 10^{-5}$ & 0,005 \\
1,25 & $1,08 \cdot 10^{-4}$ & 0,021 & 2 & $1,74 \cdot 10^{-5}$ & 0,005 \\
1,375 & $1,19 \cdot 10^{-4}$ & 0,024 & 2 & $1,74 \cdot 10^{-5}$ & 0,005 \\
1,5 & $1,30 \cdot 10^{-4}$ & 0,026 & 2 & $1,74 \cdot 10^{-5}$ & 0,005 \\
1,625 & $1,40 \cdot 10^{-4}$ & 0,028 & 2 & $1,74 \cdot 10^{-5}$ & 0,005 \\
1,75 & $1,51 \cdot 10^{-4}$ & 0,030 & 2 & $1,74 \cdot 10^{-5}$ & 0,005 \\
1,875 & $1,62 \cdot 10^{-4}$ & 0,032 & 2 & $1,74 \cdot 10^{-5}$ & 0,005 \\
2 & $1,73 \cdot 10^{-4}$ & 0,034 & 2 & $1,74 \cdot 10^{-5}$ & 0,005 \\
5 & $4,33 \cdot 10^{-4}$ & 0,086 & 2 & $1,74 \cdot 10^{-5}$ & 0,005 \\
\hline
\end{tabular}

Die Zusammensetzungen für die Synthesen der konventionellen Netzwerke entsprechen Tabelle 6-1. Das RAFT-Agens EPHT wurde dabei weggelassen.

Tabelle 6-2 Molare Anteile und entsprechende Konzentrationen sowie die Massen des RAFT-Agens EPHT und des Vernetzers BDDA bei Poly(BA-co-BDDA)-Netzwerken. Das Volumen des Monomers BA beträgt für die Berechnungen $1 \mathrm{~mL}\left(1,12 \mathrm{~g}, 8,7 \cdot 10^{-3} \mathrm{~mol} / \mathrm{mL}\right)$. Bei jeder Polymerisation waren 2 Gew.-\% (0,0178 g, 7, $\left.95 \cdot 10^{-5} \mathrm{~mol} / \mathrm{mL}\right) \mathrm{MMMP}$ als Photoinitiator vorhanden.

\begin{tabular}{cccccc}
\hline $\begin{array}{c}\text { EPHT } \\
{\left[\mathbf{m o l}-\%_{0}\right]}\end{array}$ & $\begin{array}{c}\boldsymbol{c}_{\text {EPHT }} \\
{[\mathbf{m o l} / \mathbf{m L}]}\end{array}$ & $\begin{array}{c}\boldsymbol{m}_{\text {EPHT }} \\
{[\mathbf{g}]}\end{array}$ & BDDA [mol-\%] & $\boldsymbol{c}_{\text {BDDA }}[\mathbf{m o l} / \mathbf{m L}]$ & $\begin{array}{c}\boldsymbol{m}_{\text {BDDA }} \\
{[\mathbf{g}]}\end{array}$ \\
\hline 0 & 0 & 0 & 0,5 & $4,33 \cdot 10^{-5}$ & 0,009 \\
1 & $0,87 \cdot 10^{-5}$ & 0,003 & 0,5 & $4,33 \cdot 10^{-5}$ & 0,009 \\
2 & $1,74 \cdot 10^{-5}$ & 0,005 & 0,5 & $4,33 \cdot 10^{-5}$ & 0,009 \\
4 & $3,47 \cdot 10^{-5}$ & 0,011 & 0,5 & $4,33 \cdot 10^{-5}$ & 0,009 \\
\hline
\end{tabular}

Tabelle 6-3 Molare Anteile und entsprechende Konzentrationen sowie die Massen des Trithiocarbonats DET und desVernetzers BDDA bei Poly(BA-co-BDDA)-Netzwerken. Das Volumen des Monomers BA beträgt für die Berechnungen $1 \mathrm{~mL} \quad\left(1,12 \mathrm{~g}, \quad 8,7 \cdot 10^{-3} \mathrm{~mol} / \mathrm{mL}\right)$. Bei jeder Polymerisation waren 2 Gew.-\% $(0,0178 \mathrm{~g}$, $\left.7,95 \cdot 10^{-5} \mathrm{~mol} / \mathrm{mL}\right) \mathrm{MMMP}$ als Photoinitiator vorhanden.

\begin{tabular}{cccccc}
\hline $\begin{array}{c}\text { EPHT } \\
{[\mathbf{m o l}-\boldsymbol{\%} \mathbf{c}]}\end{array}$ & $\begin{array}{c}\boldsymbol{c}_{\text {DET }} \\
{[\mathbf{m o l} / \mathbf{m L}]}\end{array}$ & $\begin{array}{c}\boldsymbol{m}_{\text {DET }} \\
{[\mathbf{g}]}\end{array}$ & BDDA [mol-\%] & $\boldsymbol{c}_{\text {BDDA }}[\mathbf{m o l} / \mathbf{m L}]$ & $\begin{array}{c}\boldsymbol{m}_{\text {BDDA }} \\
{[\mathbf{g}]}\end{array}$ \\
\hline 0 & 0 & 0 & 0,5 & $4,33 \cdot 10^{-5}$ & 0,009 \\
1 & $0,87 \cdot 10^{-5}$ & 0,001 & 0,5 & $4,33 \cdot 10^{-5}$ & 0,009 \\
2 & $1,74 \cdot 10^{-5}$ & 0,003 & 0,5 & $4,33 \cdot 10^{-5}$ & 0,009 \\
4 & $3,47 \cdot 10^{-5}$ & 0,006 & 0,5 & $4,33 \cdot 10^{-5}$ & 0,009 \\
\hline
\end{tabular}




\subsubsection{Poly(EA-co-BDDA)-Netzwerke}

Die molaren Anteile und die entsprechenden Konzentrationen sowie die Massen der verwendeten Chemikalien sind in den folgenden Tabellen aufgelistet. Entsprechend dieser Tabellen wurden die Proben für jede durchgeführte Polymersynthese und Messmethode hergestellt, die Massen wurden entsprechend an den Bedarf angepasst. Bei allen Polymerisationen wurde ohne Lösungsmittel gearbeitet.

Tabelle 6-4 Molare Anteile und entsprechende Konzentrationen sowie die Massen des Vernetzers BDDA und des RAFT-Agens EPHT bei Poly(EA-co-BDDA)-Netzwerken aus RAFT-Polymerisation. Das Volumen des Monomers EA beträgt für die Berechnungen $1 \mathrm{~mL}\left(1,09 \mathrm{~g}, 1,1 \cdot 10^{-2} \mathrm{~mol} / \mathrm{mL}\right)$. Für konventionelle Netzwerke gelten die gleichen Werte, allerdings ohne EPHT. Bei jeder Polymerisation waren 2 Gew.- $\%\left(0,0184 \mathrm{~g}, 7,79 \cdot 10^{-5} \mathrm{~mol} / \mathrm{mL}\right) \mathrm{MMMP}$ als Photoinitiator vorhanden.

\begin{tabular}{cccccc}
\hline $\begin{array}{c}\text { BDDA } \\
{[\mathbf{m o l - \%}]}\end{array}$ & $\boldsymbol{c}_{\text {BDDA }}[\mathbf{m o l} / \mathbf{m L}]$ & $\begin{array}{c}\boldsymbol{m}_{\text {BDDA }} \\
{[\mathbf{g}]}\end{array}$ & EPHT [mol-\%o $]$ & $\boldsymbol{c}_{\text {EPHT }}[\mathbf{m o l} / \mathbf{m L}]$ & $\begin{array}{c}\boldsymbol{m}_{\text {EPHT }} \\
{[\mathbf{g}]}\end{array}$ \\
\hline 0,25 & $2,71 \cdot 10^{-5}$ & 0,005 & 2 & $2,17 \cdot 10^{-5}$ & 0,006 \\
0,5 & $5,42 \cdot 10^{-5}$ & 0,011 & 2 & $2,17 \cdot 10^{-5}$ & 0,006 \\
1 & $1,08 \cdot 10^{-4}$ & 0,021 & 2 & $2,17 \cdot 10^{-5}$ & 0,006 \\
2 & $2,17 \cdot 10^{-4}$ & 0,043 & 2 & $2,17 \cdot 10^{-5}$ & 0,006 \\
3 & $3,24 \cdot 10^{-4}$ & 0,064 & 2 & $2,17 \cdot 10^{-5}$ & 0,006 \\
\hline
\end{tabular}

Die Zusammensetzungen für die Synthesen der konventionellen Netzwerke entsprechen Tabelle 6-4. Das RAFT-Agens EPHT wurde dabei weggelassen.

Tabelle 6-5 Molare Anteile und entsprechende Konzentrationen sowie die Massen des RAFT-Agens EPHT und des Vernetzers BDDA bei Poly(EA-co-BDDA)-Netzwerken. Das Volumen des Monomers EA beträgt für die Berechnungen $1 \mathrm{~mL}\left(1,09 \mathrm{~g}, 1,1 \cdot 10^{-2} \mathrm{~mol} / \mathrm{mL}\right)$. Bei jeder Polymerisation waren 2 Gew.-\% (0,0184 g, 7, $\left.79 \cdot 10^{-5} \mathrm{~mol} / \mathrm{mL}\right) \mathrm{MMMP}$ als Photoinitiator vorhanden.

\begin{tabular}{cccccc}
\hline $\begin{array}{c}\text { EPHT } \\
{[\mathbf{m o l - \%}]}\end{array}$ & $\begin{array}{c}\boldsymbol{c}_{\text {EPHT }} \\
{[\mathbf{m o l} / \mathbf{m L}]}\end{array}$ & $\begin{array}{c}\boldsymbol{m}_{\text {EPHT }} \\
{[\mathbf{g}]}\end{array}$ & BDDA [mol-\%] & $\boldsymbol{c}_{\text {BDDA }}[\mathbf{m o l} / \mathbf{m L}]$ & $\begin{array}{c}\boldsymbol{m}_{\text {BDDA }} \\
{[\mathbf{g}]}\end{array}$ \\
\hline 0 & 0 & 0 & 0,5 & $5,42 \cdot 10^{-5}$ & 0,011 \\
1 & $1,09 \cdot 10^{-5}$ & 0,003 & 0,5 & $5,42 \cdot 10^{-5}$ & 0,011 \\
2 & $2,17 \cdot 10^{-5}$ & 0,005 & 0,5 & $5,42 \cdot 10^{-5}$ & 0,011 \\
4 & $4,43 \cdot 10^{-5}$ & 0,013 & 0,5 & $5,42 \cdot 10^{-5}$ & 0,011 \\
\hline
\end{tabular}

\subsubsection{Polyethylenglycoldiacrylat-Netzwerke}

Die Massenanteile und die entsprechenden Konzentrationen sowie die Massen der verwendeten Chemikalien sind in der folgenden Tabelle aufgelistet. Entsprechend dieser Tabellen wurden die Proben für jede durchgeführte Polymersynthese und Messmethode hergestellt, die Massen 
wurden entsprechend an den Bedarf angepasst. Bei allen Polymerisationen wurde ohne Lösungsmittel gearbeitet.

Tabelle 6-6 Konzentrationen der PEG-Diacrylate bezogen auf eine Probenmasse von $1 \mathrm{~g}(0,9 \mathrm{~mL})$ sowie Massenanteile und entsprechende Konzentrationen und Massen vom RAFT-Agens EPHT. Bei jeder Polymerisation waren 2 Gew.- $\%$ $\left(0,02 \mathrm{~g}, 7,96 \cdot 10^{-5} \mathrm{~mol} / \mathrm{mL}\right) \mathrm{MMMP}$ als Photoinitiator vorhanden.

\begin{tabular}{ccccc}
\hline PEGDA & $\boldsymbol{c}_{\text {PEGDA }}[\mathbf{m o l} / \mathbf{m L}]$ & EPHT [Gew.- $\%]$ & $\boldsymbol{c}_{\text {EPHT }}[\mathbf{m o l} / \mathbf{m L}]$ & $\boldsymbol{m}_{\text {BDDA }}[\mathbf{g} / \mathbf{m o l}]$ \\
\hline PEGDA-250 & $4,44 \cdot 10^{-3}$ & 0 & 0 & 0 \\
& $4,44 \cdot 10^{-3}$ & 1 & $3,78 \cdot 10^{-5}$ & 0,01 \\
& $4,44 \cdot 10^{-3}$ & 2 & $7,55 \cdot 10^{-5}$ & 0,02 \\
& $4,44 \cdot 10^{-3}$ & 3 & $1,13 \cdot 10^{-4}$ & 0,03 \\
& $4,44 \cdot 10^{-3}$ & 4 & $1,51 \cdot 10^{-4}$ & 0,04 \\
& $4,44 \cdot 10^{-3}$ & 5 & $1,89 \cdot 10^{-4}$ & 0,05 \\
\hline PEGDA-575 & $1,93 \cdot 10^{-3}$ & 0 & 0 & 0 \\
& $1,93 \cdot 10^{-3}$ & 1 & $3,78 \cdot 10^{-5}$ & 0,01 \\
& $1,93 \cdot 10^{-3}$ & 2 & $7,55 \cdot 10^{-5}$ & 0,02 \\
& $1,93 \cdot 10^{-3}$ & 3 & $1,13 \cdot 10^{-4}$ & 0,03 \\
& $1,93 \cdot 10^{-3}$ & 4 & $1,51 \cdot 10^{-4}$ & 0,04 \\
& $1,93 \cdot 10^{-3}$ & 5 & $1,89 \cdot 10^{-4}$ & 0,05 \\
\hline PEGDA-700 & $1,59 \cdot 10^{-3}$ & 0 & 0 & 0 \\
& $1,59 \cdot 10^{-3}$ & 1 & $3,78 \cdot 10^{-5}$ & 0,01 \\
& $1,59 \cdot 10^{-3}$ & 2 & $7,55 \cdot 10^{-5}$ & 0,02 \\
& $1,59 \cdot 10^{-3}$ & 3 & $1,13 \cdot 10^{-4}$ & 0,03 \\
& $1,59 \cdot 10^{-3}$ & 4 & $1,51 \cdot 10^{-4}$ & 0,04 \\
& $1,59 \cdot 10^{-3}$ & 5 & $1,89 \cdot 10^{-4}$ & 0,05 \\
\hline
\end{tabular}




\subsection{Messmethoden}

\subsubsection{Zugversuche}

\subsubsection{Apparatives}

Die Zugversuche dienten der Bestimmung der Elastizitätsmoduln und der Bruchdehnungen der verschiedenen Netzwerke. Sie wurden an einer Zwicki-Line Z 2.5 Materialprüfmaschine der Firma ZWICK/RoELl durchgeführt. Alle Messungen fanden bei einer Temperatur von $21^{\circ} \mathrm{C}$ statt. Die Zuggeschwindigkeit betrug $25 \mathrm{~mm} / \mathrm{min}$.

\subsubsection{Auswertung der Messungen}

Für jede Probenzusammensetzung wurden fünf Probekörper vermessen. Die Auswertung der aufgenommenen Spannungs-Dehnungs-Diagramme erfolgte nachträglich mit dem Programm ORIGIN (Version 8.5). Dazu wurde die Steigung des linearen Teils der Spannungs-DehnungsKurven bestimmt, indem Geraden an die linearen Bereiche der Messkurven angepasst wurden. Dieser Bereich umfasste immer 0,05 bis maximal $5 \%$ Dehnung (s. Abbildung 6-3 und Abbildung 6-4). Somit war sichergestellt, dass der ermittelte Elastizitätsmodul dem Gleichgewichtsmodul entsprach. Außerdem ist bei den daraus resultierenden, geringen Dehnverhältnissen $l$ die technische Zugspannung $\sigma$, die bei Zugversuchen in der Regel erhalten wird, nahezu gleich der wahren Zugspannung $\sigma_{\mathrm{w}}$. Für inkompressible Werkstoffe wie z. B. Polymernetzwerke gilt: ${ }^{[158]}$

$$
\sigma_{\mathrm{w}}=l \cdot \sigma
$$

Aus allen erhaltenen Moduln einer Messreihe wurde der Mittelwert gebildet. Die Bruchdehnungen wurden direkt vom zur Materialprüfmaschine gehörenden Messprogramm testXpert II (Version 3.0) bestimmt. Die Deformationsarbeiten wurden durch Integration der Spannungs-Dehnungs-Kurven mit der Software ORIGIN (Version 8.5) bestimmt. 


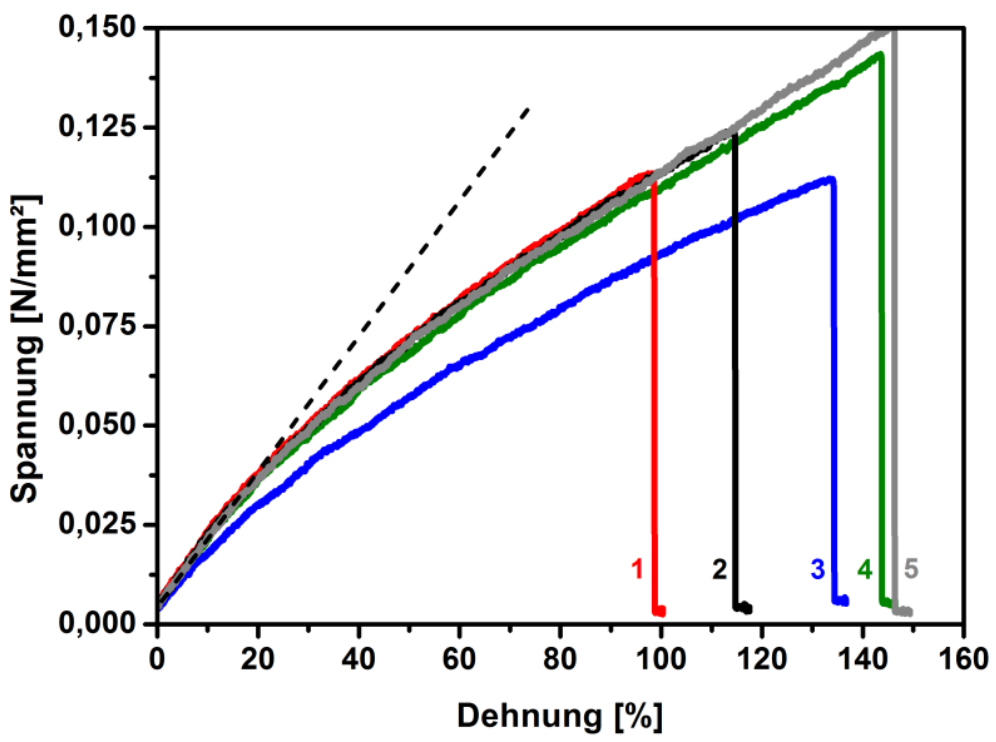

Abbildung 6-3 Mittels Zugversuch gemessene Spannungs-Dehnungs-Kurven aller Probekörper eines Poly(BA-coBDDA)-Netzwerks gleicher Zusammensetzung mit einem molaren Anteil von 0,5 mol-\% BDDA und 2 mol-\%o EPHT. Zusätzlich: Eine lineare Anpassung zur Ermittlung der Anfangssteigungen. Die Zugversuche wurden mit einer Zuggeschwindigkeit von $25 \mathrm{~mm} / \mathrm{min}$ bei einer Temperatur von $21^{\circ} \mathrm{C}$ durchgeführt. Die gezeigten Kurven stehen beispielhaft für alle durchgeführten Messungen.

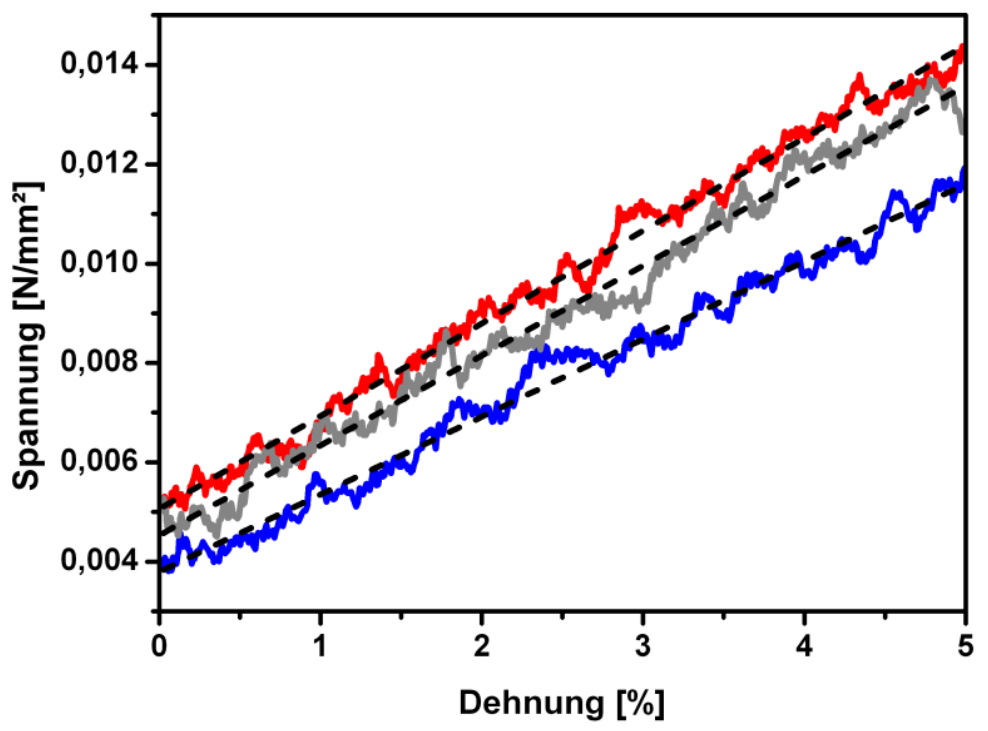

Abbildung 6-4 Verläufe der Spannungs-Dehnungs-Kurven von drei ausgewählten Probeköpern bei einer Dehnung von 0-5 \% mit den jeweils dazugehörigen linearen Anpassung (--). Die Messkurven entsprechen den SDKs 1, 3 und 5 aus Abbildung 6-3. Auf die Darstellung aller Messkurven wurde aus Gründen der Übersichtlichkeit verzichtet. 


\subsubsection{Quellungsexperimente}

Alle Quellungsexperimente wurden mit HPLC-Aceton als Lösungsmittel durchgeführt. Dazu wurden aus den Probenkörpern, die für die Zugversuche hergestellt wurden, jeweils zwei kleine Stücke herausgeschnitten (ca. 0,1 g). Die Proben wurden jeweils in ein Schnappdeckelglas gegeben, das anschließend mit $10 \mathrm{~mL}$ Aceton aufgefüllt wurde. Alle Quellungsexperimente wurden bei Raumtemperatur durchgeführt.

Die Proben wurden täglich äußerlich abgetrocknet und ausgewogen. Anschließend wurde das Lösungsmittel gewechselt, um den Anteil der Extrahierbaren im Polymer zu ermitteln. Dazu wurde das Lösungsmittel in einem separaten Schnappdeckelglas aufgefangen. Dieses wurde bei Raumtemperatur offen gelagert, sodass das Lösungsmittel abdampfen konnte. Dieser Vorgang wurde solange wiederholt, bis sich das Quellungsgleichgewicht (s. Kapitel 2.3.2) eingestellt hatte und die Massen der Proben mindestens drei Tage konstant waren. Anschließend wurden sowohl die Netzwerk-Proben als auch die Schnappdeckelgläser mit den Extrahierbaren zwei Tage bei Raumtemperatur und einige Stunden bei $40{ }^{\circ} \mathrm{C}$ in einem VT 6025-Vakuumtrockenschrank der Firma THERMO SCIENTIFIC getrocknet und ausgewogen.

\subsubsection{Messung der Klebrigkeit}

\subsubsection{Rollkugel-Test}

Die Rolling Ball Tack- oder Rollkugel-Tests wurden mittels eines Rollkugeltesters, der entsprechend der PSTC-6-Norm ${ }^{[190]}$ von der Werkstatt am Institut für Physikalische Chemie der Universität Göttingen gefertigt wurde, durchgeführt. Dazu wurden $1,2 \mathrm{~mL}$ der jeweiligen Reaktionsmischung in eine speziell angefertigte Aluminiumschale gegeben. Die Synthese der Netzwerke erfolgte ansonsten entsprechend der Synthesen der Probekörper für die Zugversuche (s. Kapitel 6.2.1). Es wurden $\mathrm{zu}$ drei verschiedenen Zeitpunkten jeweils fünf Messungen durchgeführt; direkt nach der Synthese, 30 Minuten nach der Synthese und 24 Stunden nach der Synthese.

Als Rollkugel wurden für die Poly(BA-co-BDDA)-Netzwerke eine Hartmetallkugel und für die Poly(EA-co-BDDA)-Netzwerke eine Edelstahlkugel verwendet. Der Durchmesser betrug für beide Kugeln $1 \mathrm{~cm}$. Die Laufstrecke der Kugel wurde mit einem Gliedermaßstab, der sich neben der Probenform befand, bestimmt. Abgelesen wurde dieser mittels einer Spritzenkanüle, die auf die Mitte der Kugel gerichtet wurde. Es wurde dabei sehr genau darauf geachtet, dass die Oberfläche der Probe nicht berührt wurde. Nach jeder Messung wurde die Kugel in Aceton gereinigt und anschließend getrocknet. Dabei wurde sie nur mit einer sauberen Pinzette 
gehandhabt um Verunreinigen zu vermeiden. Alle Messungen wurden bei Raumtemperatur durchgeführt.

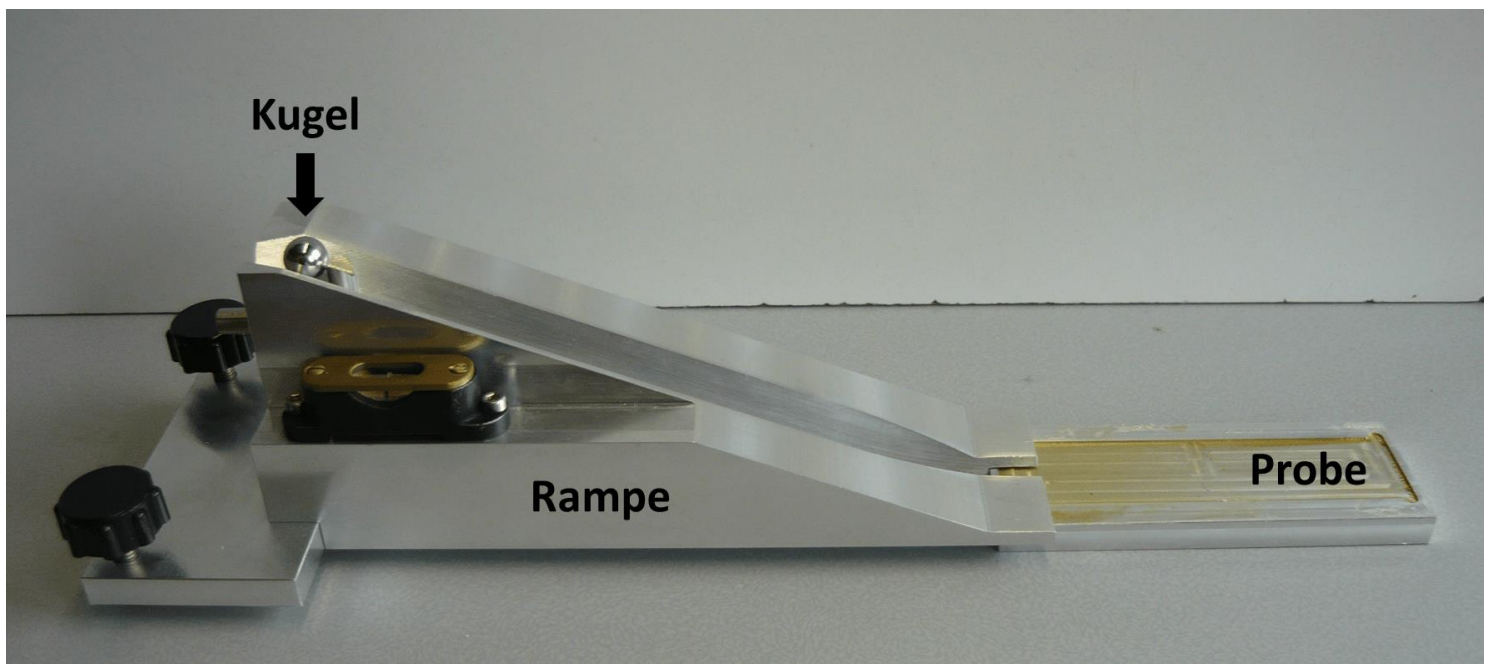

Abbildung 6-5 Rollkugeltester zur Durchführung der Rollkugel-Tests.

\subsubsection{Probe Tack-Test}

Die Probe Tack-Tests wurden mittels einer von der Werkstatt am Institut für Physikalische Chemie der Universität Göttingen gefertigten Apparatur durchgeführt.

Als Stempel wurde ein Edelstahlzylinder (Gewicht: $12,7 \mathrm{~g} ; \quad$ Durchmesser: $1 \mathrm{~cm}$ ) verwendet. Die Messwerte des Kraftaufnehmers wurden nach dem Anhängen des Stempels tariert. Die Proben wurden direkt nach der Synthese aus Zugversuchsprobekörpern ausgeschnitten. Ihre Abmessungen betrugen jeweils $1 \times 2 \mathrm{~cm}$. Für die Versuche wurden die Proben auf einer Glasplatte positioniert. Diese zeigen auf Glas eine sehr starke Haftung. So war sichergestellt, dass ein möglicher Bruch zwischen dem Edelstahlstempel und der Probe stattfand.

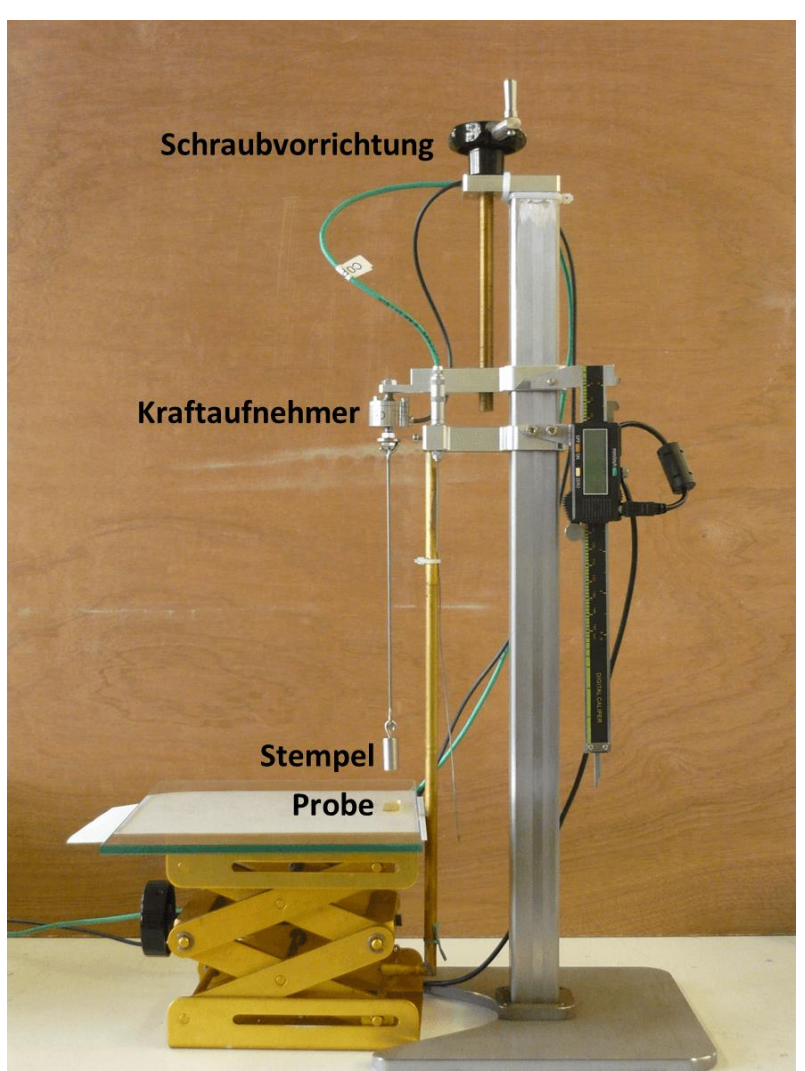

Abbildung 6-6 Messapparatur zur Durchführung der Probe Tack-Tests. 
Der Stempel wurde über die Schraubvorrichtung sehr langsam auf der Probe abgestellt und 10 Sekunden gewartet. Anschließend wurde der Stempel über die Schraubvorrichtung von der Probe hochgezogen. Für jede Probe wurden drei Wiederholungen durchgeführt. Die auftretende Kraft wurde mittels eines von Hans-Peter Vögele in der Programmiersprache AGILENTVEE programmierten Messprogramms aufgezeichnet. Die vom Kraftaufnehmer in der Einheit Gramm gemessenen Werte wurden über die Erdbeschleunigung ${ }^{\mathrm{a}}$ in Newton umgerechnet. Alle Messungen wurden bei Raumtemperatur durchgeführt.

\footnotetext{
${ }^{a}$ Da es sich bei den Messungen nicht um absolute Messungen handelte, wurde hier mit dem vereinfachten Wert für $g$ von $10 \mathrm{~m} / \mathrm{s}$ gearbeitet. Es sollten nur die relativen Unterschiede zwischen konventionellen und RAFT-Netzwerken aufgezeigt werden.
} 


\subsubsection{Fourier-Transformations-Infrarot-(FTIR)-Spektroskopie}

\subsubsection{Apparatives}

Die kinetischen Fourier-Transformations-Infrarot-(FTIR)-Messungen wurden an einem IFS 88 FTIR-Spektrometer der Firma BRUKER durchgeführt. Bei der Strahlungsquelle handelte es sich um eine Wolfram-Halogen-Lampe, der Strahlteiler (Modell T8401) bestand aus $\mathrm{CaF}_{2}$. Es wurde ein stickstoffgekühlter InSb-Detektor (Modell D413, Durchmesser $1 \mathrm{~mm}$ ) der Firma INFRARED ASSOCIATES verwendet. Die Apertur des Spektrometers wurde für die Messungen auf 1,5 mm eingestellt.

\subsubsection{Vorbereitung und Durchführung der FTIR-Messungen}

Als Küvetten wurden NMR-Röhrchen (VWR, $d=5 \mathrm{~mm}, 100 \mathrm{MHz}$ ) verwendet. Diese wurden in einem $100 \mathrm{~mL}$ Schlenk-Rohr, das mit einem weiteren Schlenk-Hahn verschlossen wurde, platziert. Anschließend wurde das Schlenk-Rohr evakuiert und mit Argon befüllt. Unter konstantem Argonstrom wurde der obere Schlenk-Hahn geöffnet und über diesen mittels einer, mit Argon gespülten Spritze, 0,3 mL der zuvor mit Argon gespülten Polymerisationsmischung (20 min: PEG-Diacrylate, 10 min: EA) eingefüllt. Anschließend wurde weiterhin unter konstantem Argonstrom der obere SchlenkHahn abgenommen und das NMR-Röhrchen mit einem Deckel verschlossen. Zusätzlich wurde es mit PARAFILM@ verschlossen und mit Alu-Folie umwickelt. Die beim Befüllen der Röhrchen mit den PEG-Diacrylaten entstandenen Blasen wurden entfernt, indem das NMR-Röhrchen mit der Probe kurz in ein Ultraschallbad gehalten

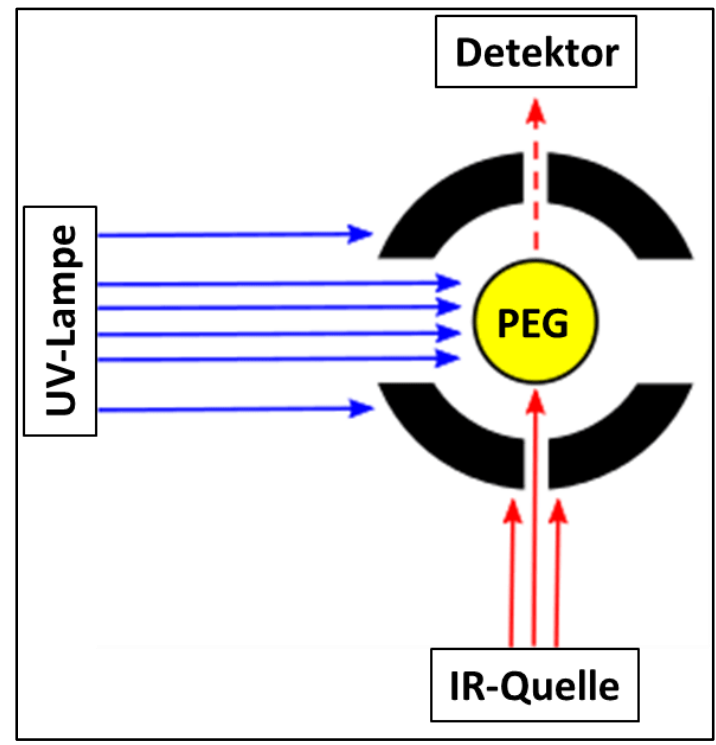

Abbildung 6-7 Schematischer Versuchsaufbau der durchgeführten IR-Messungen. wurde. Die NMR-Röhrchen mit den Polymerisationsmischungen wurden in einem speziell für diese Messungen hergestellten Probenhalter im Spektrometer platziert (s. Abbildung 6-7). Dieser sollte sicherstellen, dass kein Streulicht von der Röhrchenoberfläche den Detektor erreichte, die Proben aber trotzdem vollständig mit UV-Strahlung bestrahlt wurden.

Um eine Basislinie zu ermitteln, wurden zunächst fünf Spektren ohne Bestrahlung mit der UVLampe gemessen. Anschließend wurde eine Blende, die sich zwischen Lampe und Probe befand, 
entfernt, um die Polymerisation zu starten. Für die PEG-Diacrylate wurden jeweils 120 Spektren aufgenommen. Für die Poly(EA-co-BDDA)-Proben wurden jeweils 400 Spektren aufgenommen. Alle Messungen wurden bei Raumtemperatur $\left(21^{\circ} \mathrm{C}\right)$ durchgeführt.

\subsubsection{Auswertung der Spektren}

Darstellung und Auswertung der gemessenen Spektren erfolgte mit der Software OPUS (Version 6.0) der Firma BRUKER. Die Absorptionsbande bei einer Wellenzahl $\tilde{v}$ von $6170 \mathrm{~cm}^{-1}$ entspricht dem zweiten Oberton der asymmetrischen Streckschwingung einer C-H-Bindung an einer $\mathrm{C}=\mathrm{C}$-Doppelbindung. Mit zunehmendem Umsatz der Acrylat-Doppelbindung nimmt die Absorption bei dieser Wellenzahl ab. Abbildung 6-8 zeigt Ausschnitte von ausgewählten Spektren einer Poly(EA-co-BDDA)-Polymerisationsmischung bei verschiedenen Monomerumsätzen.

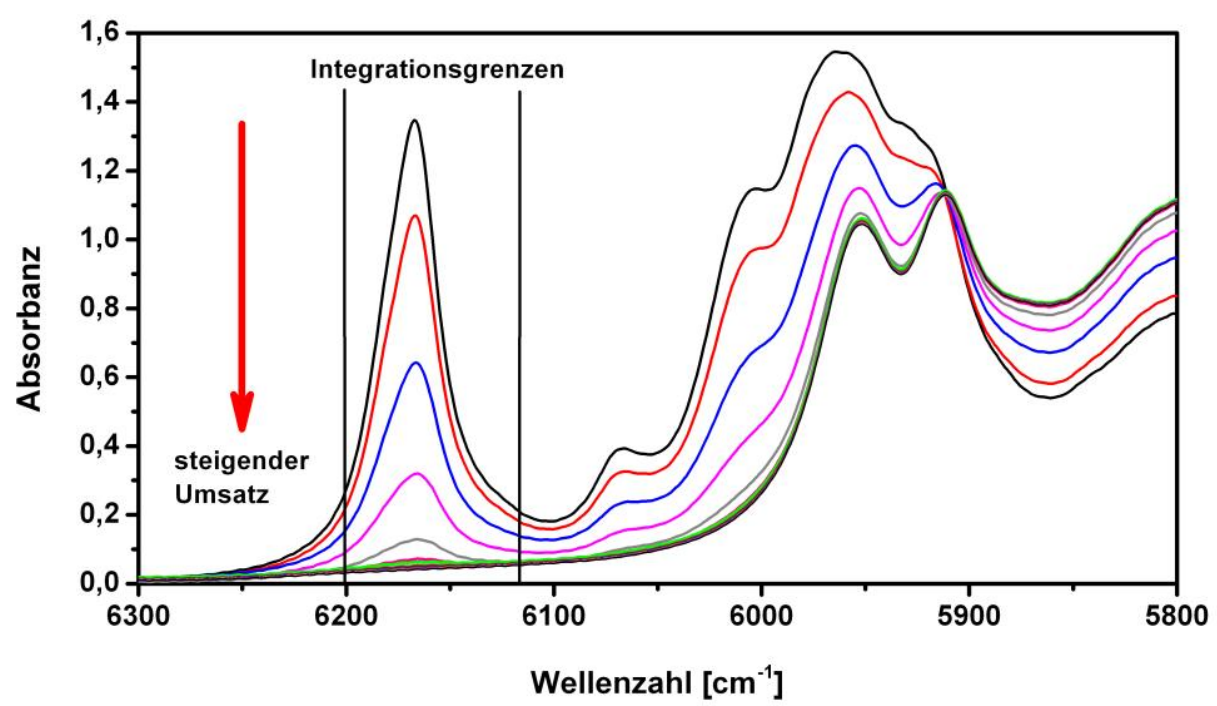

Abbildung 6-8 Auswahl von FTIR-Spektren aufgenommen zu verschiedenen Zeitpunkten (zu Beginn der Polymerisation und im Folgenden alle 10 Minuten) während der Polymerisation eines Poly(EA-co-BDDA)-Netzwerks mit einem molaren von 0,5 mol-\% BDDA als Vernetzer und 2 mol-\%o EPHT als RAFT-Agens. Die Messtemperatur betrug $21^{\circ} \mathrm{C}$. Als Photoinitiator wurden 2 Gew.-\% MMMP verwendet.

Zur Bestimmung des Monomerumsatzes wurde die markierte Absorptionsbande in den Grenzen zwischen 6200 und $6110 \mathrm{~cm}^{-1}$ integriert. Dazu wurde die OPUS-Methode F verwendet. Die dafür notwendigen weiteren Grundlinienpunkte wurden bei 6350, 6250, 6100 und $6060 \mathrm{~cm}^{-1}$ gesetzt. Über das Lambert-Beer'sche Gesetz ist die dekadische Absorbanz bei der entsprechenden Wellenzahl $A_{\widetilde{v}}$ mit der Konzentration des Monomers verknüpft. Es gilt: 


$$
A_{\widetilde{v}}=\log \left(\frac{I_{0}(\tilde{v})}{I_{1}(\tilde{v})}\right)=\varepsilon(\tilde{v}) \cdot c_{\mathrm{M}} \cdot d
$$

mit $I_{0}(\widetilde{v})$ der Intensität der einfallenden Strahlung und $I_{t}(\tilde{v})$ der Strahlung nach der Probe. Unter Verwendung der integrierten dekadischen Absorbanz $A_{\text {int }}$, die gegeben ist durch:

$$
A_{\text {int }}=\int A_{\widetilde{v}} \mathrm{~d} \tilde{v}=\varepsilon_{\text {int }} \cdot c_{\mathrm{M}} \cdot d
$$

kann somit mittels Gleichung (57) der Umsatz des Monomers zum Zeitpunkt $t$ berechnet werden.

$$
C_{\mathrm{M}}(t)=1-\frac{c_{\mathrm{M}}(t)}{c_{\mathrm{M}}(t=0)}=1-\frac{A_{\mathrm{int}}(t)}{A_{\mathrm{int}}(t=0)}
$$

\subsubsection{Rasterkraftmikroskopie}

\subsubsection{Apparatives}

Rasterkraftmikroskopie-Messungen (engl.: Atomic Force Microscopy, AFM) wurden an einem Multimode 8 Rasterkraftmikroskop der Firma BRUKER durchgeführt. Alle Messungen wurden im PeakForce $^{\mathrm{TM}}$-Quantitative-Nanomechanical-Mapping (QNM) ${ }^{\mathrm{TM}}$-Modus durchgeführt. Dieser Modus bietet neben der Information über die Höhenprofile der Oberflächen die Möglichkeit, ebenfalls deren mechanische Eigenschaften wie Adhäsion, Dejarguin-Muller-Toporov (DMT)Modul $^{[219,220]}$ oder die Deformationen der Oberfläche zu ermitteln. Bei den verwendeten Cantilevern handelte es sich um ScanAsyst-Air-Cantilever $\left(k=0,4 \mathrm{~N} / \mathrm{m}, f_{0}=50-90 \mathrm{kHz}\right)$ der Firma BRUKER.

\subsubsection{Kalibrierung, Messungen und Auswertung}

Zur Ermittlung der mechanischen Eigenschaften wurde das Rasterkraftmikroskop vor dem Messen kalibriert. Dabei wurden die Ablenkungsempfindlichkeit (engl.: deflection sensitivity), und die genaue Federkonstante des Cantilevers sowie der genaue Durchmesser der Messspitze ermittelt. Mit Hilfe dieser Werte ist es dem Messprogramm Research NanoScope (Version 8.15) möglich, aus den Kraft-Abstandskurven des Cantilevers die entsprechenden mechanischen Informationen zu berechnen. ${ }^{[234]}$ Eine detaillierte Beschreibung aller für die Kalibrierung des 
Multimode 8 Rasterkraftmikroskops notwendigen Schritte kann in der Arbeit von fan-Hendrik Schütz nachgelesen werden. ${ }^{[235]}$ Sofern nicht anders kenntlich gemacht, erfolgten alle Messungen bei einer Auflösung von 512 Linien pro Bild und einer Messfrequenz von 0,5 Hz. Die Wahl der Außenmaße des Messbereichs erfolgte nach Bedarf.

Alle Auswertungen und Bildbearbeitungen erfolgten mit der Software NanoScope Analysis (Version 1.4). Die Höhenprofil-Bilder wurden in 3. Ordnung geglättet, und von Bildstörungen gereinigt (Remove Spikes: off, Remove Streaks: on, Spike Cutoff: 3.00, Streak Cutoff: 3.00). Anschließend wurde durch einen Lowpass-Filter das Rauschen geglättet. Die Auswertung der Bilder erfolgte mittels der Particle-Analysis-Funktion von NanoScope Analysis 1.4. Die mittleren Höhen, Adhäsionen und DMT-Moduln wurden mittels der Funktion Depth ermittelt. Dabei wurde der in der Verteilung häufigste Wert ausgewählt.

Weiterhin wurde bei allen Bildern die Höhenskala relativ zum Minimum eingestellt. Für alle dreidimensionalen Abbildungen wurden eine perspektivische Darstellung, ein gemischter PlotTypus, ein Zoom von 1.2 und ein z-Achsen-Aspekt-Verhältnis von 0.1 gewählt. Weitere Kanäle auf der Oberfläche wurden nach Bedarf gewählt. Jegliche Informationen aus den Messkanälen der mechanischen Eigenschaften wurden nicht weiter bearbeitet.

\subsubsection{Dynamische Differenzkalorimetrie}

\subsubsection{Apparatives und durchgeführte Messungen}

Kinetische DSC-Messungen wurden an einem DSC 820 der Firma METTLER TOLEDO durchgeführt. Die Wärme der Polymerisationsreaktion wurde dabei gegen ein leeres Aluminiumpfännchen als Referenz gemessen. Die Messkammer wurde konstant bei $21^{\circ} \mathrm{C}$ gehalten und mit Stickstoff gespült, außerdem wurde sie, zum Schutz vor Sauerstoff, mit einer DURAN ${ }^{\circledR}$-Glasscheibe abgedeckt, die bei der verwendeten Wellenlänge von $366 \mathrm{~nm}$ noch vollständig lichtdurchlässig ist. Die N-8 UV-Handlampe wurde $30 \mathrm{~s}$ nach Beginn der Messung auf dem Kalorimeter platziert. Der Abstand zu den Pfännchen betrug $5 \mathrm{~cm}$, so dass die Lichtintensität $9 \mathrm{~mW} / \mathrm{cm}^{2}$ betrug. Die Messdauer betrug jeweils 60 Minuten. Jeder Tiegel wurde leer, sowie nach erfolgter Polymerisation mit dem Probenvolumen gefüllt, ausgewogen und die Differenz der beiden Werte bestimmt. Auf diese Weise konnten die Massen der einzelnen Proben ermittelt werden.

Die Messungen der Glastemperaturen der Poly(BA-co-BDDA)- und Poly(EA-co-BDDA)Netzwerke wurden an einem DSC-60 der Firma SHIMATZU durchgeführt. Dabei wurde jeweils eine kleine Probemenge (max. $10 \mathrm{mg}$ ) aus den Probekörpern für die Zugversuche entnommen und abgewogen. Zur Ermittlung der Glastemperatur wurden anschließend zwei Zyklen mit einer 
Heiz-/Kühlrate von $10 \mathrm{~K} / \mathrm{min}$ durchgeführt. Der durchlaufene Temperaturbereich lag dabei immer zwischen $-100{ }^{\circ} \mathrm{C}$ und $+100{ }^{\circ} \mathrm{C}$.

\subsubsection{Berechnung der theoretischen Wärmen für die kinetischen Messungen}

Um die theoretisch möglichen Reaktionswärmen der einzelnen Proben zu bestimmen, wurde die Anzahl der vor der Polymerisation vorhandenen Doppelbindungen über das Probengewicht bestimmt. Dazu wurde dieses zunächst korrigiert, indem die Gewichte des Initiators $m_{\mathrm{I}}$, des verwendeten RAFT-Agens $m_{\text {RAFT }}$ und des Vernetzers $m_{\text {Vern }}$ vom Probengewicht $m_{\text {Probe }}$ subtrahiert wurden:

$$
m_{\mathrm{korr}}=m_{\text {Probe }}-m_{\mathrm{I}}-m_{\mathrm{RAFT}}-m_{\mathrm{Vern}}
$$

Anschließend wurde anhand der korrigierten Probenmasse $m_{\mathrm{korr}}$ die Stoffmenge der Monomere bestimmt:

$$
n_{\mathrm{M}}=\frac{m_{\mathrm{korr}}}{M_{\mathrm{M}}}
$$

Zusätzlich wurde über $m_{\text {Vern }}$ mittels Gleichung (59) die Stoffmenge des Vernetzers berechnet. Mit Hilfe der Avogadro-Konstanten $N_{\mathrm{A}}$ wurde für beide Stoffmengen die Anzahl der vorhandenen Moleküle $N$ bestimmt:

$$
N=n \cdot N_{\mathrm{A}}=n \cdot 6,022 \cdot 10^{23} \mathrm{~mol}^{-1}
$$

Da pro Monomer jeweils eine Doppelbindung und pro Vernetzer zwei Doppelbindungen vorhanden sind, konnte durch Addition der Anzahl der Monomere $N_{\mathrm{M}}$ und der Anzahl des Vernetzers $N_{\text {Vern }}$ die gesamte Anzahl der in der Probe vorhandenen Doppelbindungen $N_{\text {DB }}$ berechnet werden:

$$
N_{\text {DB }}=N_{\mathrm{M}}+2 N_{\text {Vern }}
$$


Die theoretische Reaktionswärme eines Mols Acrylat-Doppelbindungen beträgt $86250 \mathrm{~J} / \mathrm{mol}$. Durch Division durch $N_{\mathrm{A}}$ kann die Reaktionswärme einer Doppelbindung $\Delta H_{\mathrm{DB}}$ bestimmt werden:

$$
\Delta H_{\mathrm{DB}}=\frac{\Delta H_{0}^{\text {th }}}{N_{\mathrm{A}}}=1,4322 \cdot 10^{-19} \mathrm{~J}
$$

Durch Multiplikation von $N_{\mathrm{DB}}$ und $\Delta H_{\mathrm{DB}}$ konnte anschließend die theoretische Reaktionswärme für jede Probe einzeln $\Delta H_{\text {Probe }}^{\text {th }}$ bestimmt werden.

$$
\Delta H_{\text {Probe }}^{\text {th }}=N_{\mathrm{DB}} \cdot \Delta H_{\mathrm{DB}}
$$

\subsubsection{Elektronen-Spin-Resonanz-(ESR)-Spektroskopie}

\subsubsection{Apparatives und durchgeführte Messungen}

Die ESR-Spektroskopie wurde an einem Exelsys-II 500T-Spektrometer der Firma BRUKER durchgeführt. Als Lichtquelle wurde bei diesen Messungen die Mitteldruck UV-Lampe (Standard Hg, 2 kW) eines UVACube Inert der Firma HOENLE verwendet.

Für die Untersuchung der PEG-Diacrylate wurde die Strahlung des mittels eines Lichtleiters (Liquid Lightguide series 250) der Firma LUMATEC aus dem UVACube ausgekoppelt und direkt in die Messzelle des Spektrometers geleitet. Am Beginn des Lichtleiters befand sich ein Bandpassfilter (Transmissionswellenlänge $365 \pm 5 \mathrm{~nm}$ ) der Firma EDMUND OPTICS. Es wurden ESRRöhrchen (712-SQ-250M, $d=2 \mathrm{~mm}$ ) von WILMAD verwendet. Die Polymerisationsmischungen wurden vor dem Einfüllen in die Röhrchen 10 Minuten mit Argon gespült. Die ESR-Röhrchen wurden in einer Handschuhbox (Lab Master 130) der Firma BRAUN befüllt und mit Parafilm ${ }^{\circledR}$ versiegelt. Um eine vorzeitige Polymerisation zu verhindern, wurden alle Proben mit Aluminiumfolie umwickelt. Die Durchlaufzeit einer Messung betrug 5,24 s, als Modulationsamplitude wurden $3 \mathrm{G}$ gewählt, die Modulationsfrequenz betrug $100 \mathrm{kHz}$. Die Verstärkung des Empfängers betrug $60 \mathrm{~dB}$, der G-Faktor betrug 2. Die Mikrowellenbrücke wurde mit einer Leistung von $0,63 \mathrm{~mW}$ bei einer Dämpfung von $25 \mathrm{~dB}$ betrieben. Es wurden pro Messung 60 Spektren aufgenommen. Alle Messungen wurden bei Raumtemperatur $\left(21^{\circ} \mathrm{C}\right)$ durchgeführt. Die Spektren wurden mit dem Programm Origin (Version 8.5) normiert, indem sie 
durch die jeweils höchste gemessene Signalintensität der jeweiligen Spektrenserie geteilt wurden.

Für die Untersuchungen, die in Kooperation mit der FOGRA durchgeführt wurden, wurde das Licht aus dem UVACube mittels eines Lichtleiters (A10014) der Firma HAMAMATSU ausgekoppelt und direkt in die Messzelle des Spektrometers geleitet. Es wurden ESR-Röhrchen (707-SQ-250M, $d=4 \mathrm{~mm}$ ) der Firma WILMAD verwendet. Diese wurden in den Laboren der FOGRA mittels einer speziell dafür entwickelten Spritze befüllt. Alle Messungen wurden bei Raumtemperatur $\left(21^{\circ} \mathrm{C}\right)$ durchgeführt. Die Durchlaufzeit einer Messung betrug 5,24 s, als Modulationsamplitude wurde $1 \mathrm{G}$ gewählt, die Modulationsfrequenz betrug $100 \mathrm{kHz}$. Die Verstärkung des Empfängers betrug $60 \mathrm{~dB}$, der G-Faktor betrug 2. Die Mikrowellenbrücke wurde mit einer Leistung von 0,63 $\mathrm{mW}$ bei einer Dämpfung von $25 \mathrm{~dB}$ betrieben. Es wurden pro Messung 60 Spektren aufgenommen. Alle Messungen wurden bei Raumtemperatur $\left(21^{\circ} \mathrm{C}\right)$ durchgeführt. Die Spektren wurden mit dem Programm Origin (Version 8.5) normiert, indem sie durch die jeweils höchste gemessene Signalintensität der jeweiligen Spektrenserie geteilt wurden.

\subsubsection{Ultraviolett/sichtbares Licht (UV/Vis)-Spektroskopie}

UV-Vis-Spektren wurden mit einem Cary-300-Spektrometer der Firma VARIAN über einen Wellenlängenbereich von 190 bis $800 \mathrm{~nm}$ gemessen. Für die Messungen wurden QuarzSuprasilKüvetten $(110-Q S, d=1 \mathrm{~cm})$ der Firma Hellma verwendet. Die Proben wurden gegen eine Referenz, bei der es sich um das reine Lösungsmittel handelte, gemessen.

\subsubsection{Weitere verwendete Apparaturen}

Die Einwaagen aller Substanzen sowie die gravimetrischen Messungen für die Quellungsuntersuchungen wurden an einer Analysenwaage (CPA 324S) der Firma SARTORIUS durchgeführt. Die Lichtmikroskop-Bilder wurden an einem Lichtmikroskop (S6D) der Firma LEICA aufgenommen. Das Entfernen der Argon-Bläschen aus den PEG-Diacrylaten wurde mit einem Ultraschallbad (Elmasonic S30 H) der Firma ELMA durchgeführt. Die Strahlungsintensität des UVACube wurde mit einem Intensitätsmessgerät (Handheld HI 1, der Firma MEYER GMBH ermittelt), welches auf Mitteldruck UV-Strahler kalibriert war. 


\section{Literaturverzeichnis}

[1] Plastics - the Facts 2013 An Analysis of European Latest Plastics Production, Demand and Waste Data, 2013.

[2] "Gesamtverband Kunststoffverarbeitende Industrie e. V., http://www.gkv.de/statistik.html, 03.02.2014

[3] K. Matyjaszewski, J. Xia, Chem. Rev. 2001, 101, 2921-2990.

[4] M. Swarc, Nature 1956, 178, 1168-1169.

[5] C. N. Bowman, C. J. Kloxin, AIChE F. 2008, 54, 2775-2795.

[6] H. Staudinger, Berichte der Dtsch. Chem. Gesellschaft 1920, 59, 1073-1085.

[7] H. Staudinger, Berichte der Dtsch. Chem. Gesellschaft 1926, 59, 3019-3043.

[8] H. Staudinger, M. Brunner, K. Frey, P. Garbsch, R. Signer, S. Wehrli, Berichte der Dtsch. Chem. Gesellschaft 1929, 62, 241-263.

[9] D. Braun, Int. F. Polym. Sci. 2009, 2009, 1-10.

[10] J. Flory, J. Am. Chem. Soc. 1937, 59, 241-253.

[11] A. H. E. Müller, K. Matyjaszewski, in Control. Living Polym., Wiley-VCH, Weinheim, 2009, S. 103.

[12] M. Destarac, Macromol. React. Eng. 2010, 4, 165-179.

[13] P. Nesvadba, Encycl. Radicals Chem. Biol. Mater. 2012, DOI 10.1002/9780470971253.rad080.

[14] Y. Yagci, S. Jockusch, N. J. Turro, Macromolecules 2010, 43, 6245-6260.

[15] U. Schubert, Chemie unserer Zeit 2005, 39, 3.

[16] R. Dersch, A. Greiner, M. Steinhardt, J. Wendorff, Chemie unserer Zeit 2005, 39, 26-35.

[17] S. Steuer, Bioabbaubare Polymernetzwerke Mit Formgedächtniseffekt Als Matrix Für Wirkstofffreisetzungssysteme, Rheinisch-Westfälische Technische Hochschule Aachen, 2003.

[18] J. Nie, B. Du, W. Oppermann, Macromolecules 2005, 38, 5729-5736. 
[19] C. Boyer, M. H. Stenzel, T. P. Davis, J. Polym. Sci. Part A Polym. Chem. 2011, 49, 551-595.

[20] D. F. Grishin, I. D. Grishin, Russ. F. Appl. Chem. 2012, 84, 2021-2028.

[21] B. Springer, Polybutadien Und Butadien Enthaltende Copolymere Mit Kontrollierter Kettenstruktur Durch RAFTPolymerisation, Georg-AugustUniversität Göttingen, 2011.

[22] M. D. Lechner, K. Gehrke, E. Lehrbuch, Makromolekulare Chemie, Birkhäuser, Basel, 2010.

[23] B. Tieke, Makromolekulare Chemie - Eine Einführung, Wiley-VCH, Weinheim, 2005.

[24] V. E. Trommsdorff, H. Kohle, Makromol. Chemie 1947, 1, 169-198.

[25] A. D. Jenkins, R. G. Jones, G. Moad, Pure Appl. Chem. 2010, 82, 483-491.

[26] C. J. Hawker, a W. Bosman, E. Harth, Chem. Rev. 2001, 101, 3661-88.

[27] M. Kato, M. Kamigaito, M. Sawamoto, T. Higashimuras, Macromolecules 1996, 28, 1721-1723.

[28] J. Wang, K. Matyjaszewski, f. Am. Chem. Soc. 1995, 117, 5614-5615.

[29] J. Chiefari, Y. K. B. Chong, F. Ercole, J. Krstina, J. Jeffery, T. P. T. Le, R. T. A. Mayadunne, G. F. Meijs, C. L. Moad, G. Moad, et al., Macromolecules 1998, 31, 5559-5562.

[30] H. Fischer, f. Polym. Sci. Part A Polym. Chem. 1999, 37, 1885-1901.

[31] H. Gao, K. Matyjaszewski, Prog. Polym. Sci. 2009, 34, 317-350.

[32] J.-F. Lutz, Polym. Int. 2006, 55, 979-993.

[33] K. Matyjaszewski, A. H. E. Müller, in Control. Living Polym., Wiley-VCH, Weinheim, 2009, S. 125-130.

[34] B. Y. K. Chong, J. Krstina, T. P. T. Le, G. Moad, A. Postma, E. Rizzardo, S. H. Thang, Macromolecules 2003, 60, 2256-2272.

[35] A. Favier, M.-T. Charreyre, Macromol. Rapid Commun. 2006, 27, 653-692.

[36] M. L. Coote, D. J. Henry, Macromolecules 2005, 38, 1415-1433.

[37] E. Rizzardo, M. Chen, B. Chong, G. Moad, M. Skidmore, S. H. Thang, Macromol. Symp. 2007, 248, 104-116. 
[38] T. Arita, M. Buback, P. Vana, Macromolecules 2005, 38, 7935-7943.

[39] D. B. Thomas, A. J. Convertine, L. J. Myrick, C. W. Scales, A. E. Smith, A. B. Lowe, Y. A. Vasilieva, N. Ayres, C. L. Mccormick, Macromolecules 2004, 37, 8941-8950.

[40] C. Li, B. C. Benicewicz, f. Polym. Sci. Part A Polym. Chem. 2005, 43, 1535-1543.

[41] A. Goto, K. Sato, Y. Tsujii, T. Fukuda, G. Moad, E. Rizzardo, S. H. Thang, Macromolecules 2001, 34, 402-408.

[42] N. Aoyagi, B. Ochiai, H. Mori, T. Endo, Synlett 2006, 4, 636-638.

[43] N. Aoygi, T. Endo, 2009, 47, 1535-1543.

[44] M. Buback, P. Hesse, T. Junkers, P. Vana, Macromol. Rapid Commun. 2006, 27, 182-187.

[45] S. Perrier, P. Takolpuckdee, J. Polym. Sci. Part A Polym. Chem. 2005, 43, 5347-5393.

[46] M. Ham, J. HoYouk, Y.-K. Kwon, Y.-J. Kwark, F. Polym. Sci. Part A Polym. Chem. 2012, 50, 2389-2397.

[47] J. Skey, R. K. O’Reilly, Chem. Commun. (Camb). 2008, 4183-5.

[48] J. Chiefari, R. T. A. Mayadunne, C. L. Moad, G. Moad, E. Rizzardo, A. Postma, M. A. Skidmore, S. H. Thang, Macromolecules 2003, 36, 2273-2283.

[49] P. Vana, T. P. Davis, C. Barner-Kowollik, Macromol. Theory Simulations 2002, 11, 823-835.

[50] B. Ebeling, P. Vana, Polymers (Basel). 2011, 3, 719-739.

[51] W. Zhang, F. D. Agosto, O. Boyron, J. Rieger, B. Charleux, Macromolecules 2011, 44, 7584-7593.

[52] C. K. Ullal, S. Primpke, R. Schmidt, U. Böhm, A. Egner, P. Vana, S. W. Hell, Macromolecules 2011, 44, 7508-7510.

[53] T. Arita, M. Buback, O. Janssen, P. Vana, Macromol. Rapid Commun. 2004, 25, 1376-1381.

[54] P. Yang, L. P. D. Ratcli, S. P. Armes, Macromolecules 2013, 46, 8545-8556.

[55] A. Chakrabarty, N. K. Singha, f. Colloid Interface Sci. 2013, 408, 66-74.

[56] T. Arita, S. Beuermann, M. Buback, P. Vana, Macromol. Mater. Eng. 2005, 290, 283293. 
[57] X. Wang, S. Li, Y. Su, F. Huo, W. Zhang, f. Polym. Sci. Part A Polym. Chem. 2013, $51,2188-2198$.

[58] J.-P. Fouassier, Photoinitiation, Photopolymerization, and Photocuring: Fundamentals and Applications, Rapra Technology Ltd, Shawbury, 1998.

[59] S. Yamago, Y. Nakamura, Polymer 2013, 54, 981-994.

[60] K. Ikemura, T. Endo, Dent. Mater. F. 2010, 29, 481-501.

[61] A. Ibrahim, L. Di Stefano, O. Tarzi, H. Tar, C. Ley, X. Allonas, F. Photochem. Photobiol. Photobiol. 2013, 89, 1283-1290.

[62] "Grundlagen zur UV-Strahlung, http://www.bfs.de/de/uv/uv2/uv_strahlung.html, 27.01.2014

[63] W. A. Green, in Ind. Photoinitiators A Tech. Guid., CRC Press, Boca Raton, 2010, S. $18-20$.

[64] W. A. Green, in Ind. Photoinitiators A Tech. Guid., CRC Press, Boca Raton, 2010, S. 139-147.

[65] N. S. Allen, F. Photochem. Photobiol. 1996, 100, 101-107.

[66] C. Decker, K. Moussa, Macromolecules 1989, 22, 4455-4462.

[67] L. Lu, N. Yang, Y. Cai, Chem. Commun. (Camb). 2005, 5287-5288.

[68] J. F. Quinn, L. Barner, C. Barner-Kowollik, E. Rizzardo, T. P. Davis, Macromolecules 2002, 35, 7620-7627.

[69] L. Lu, H. Zhang, N. Yang, Y. Cai, C. Chemistry, X. U. V, R. V January, V. Re, M. Recei, V. April, Macromolecules 2006, 39, 3770-3776.

[70] H. Yin, H. Zheng, L. Lu, P. Liu, Y. Cai, f. Polym. Sci. Part A Polym. Chem. 2007, 45, 5091-5102.

[71] H. Zhang, J. Deng, L. Lu, Y. Cai, Macromolecules 2007, 40, 2978-2980.

[72] W. Jiang, L. Lu, Y. Cai, Macromol. Rapid Commun. 2007, 28, 725-728.

[73] R. Ran, Y. Yu, T. Wan, f. Appl. Polym. Sci. 2007, 105, 398-404.

[74] Y. Shi, G. Liu, H. Gao, L. Lu, Y. Cai, Macromolecules 2009, 42, 3917-3926.

[75] S. Muthukrishnan, E. H. Pan, M. H. Stenzel, C. Barner-Kowollik, T. P. Davis, D. Lewis, L. Barner, Macromolecules 2007, 40, 2978-2980. 
[76] B. Ki, Y. C. Yu, H. J. Jeon, W.-R. Yu, H. W. Ryu, J. H. Youk, Fibers Polym. 2012, 13, 135-138.

[77] D. Leung, C. N. Bowman, Macromol. Chem. Phys. 2012, 213, 198-204.

[78] D. Zhuo, Y. Ruan, X. Zhao, R. Ran, J. Appl. Polym. Sci. 2011, 121, 660-665.

[79] Q. Yu, Y. Zhu, Y. Ding, S. Zhu, Macromol. Chem. Phys. 2008, 209, 551-556.

[80] H.-G. Elias, in Makromoleküle Band 1 Chem. Strukt. Und Synth., Wiley-VCH, Weinheim, 1999, S. 57-60.

[81] M. Rubinstein, R. H. Colby, in Polym. Phys., Oxford University Press, New York, 2003, S. 199-204.

[82] S. Sepeur, Nanotechnologie: Grundlagen Und Anwendungen, Vincentz Network $\mathrm{GmBH}$, Hannover, 2008.

[83] R. Henkel, Synthese Und Charakterisierung von Linearen Und Vernetzten Polymeren Über ATRP, Diplomarbeit, Technische Universität Clausthal, 2010.

[84] W. Funke, W. Beer, U. Seitz, Prog. Colloid Polym. Sci. 1975, 57, 48-53.

[85] S. Frahn, Einfluß Der Stoffzustände Des Quellmittels Auf Das Quellverhalten von Chemisch Vernetzten Polymeren in Lösemitteln, Universität Duisburg-Essen, 1997.

[86] C. GOODYEAR, Improvement in India-Rubber Fabrics, 1844, 3633.

[87] L. Wang, X. Yang, H. Chen, T. Gong, W. Li, G. Yang, S. Zhou, ACS Appl. Mater. Interfaces 2013, 5, 10520-8.

[88] T. C. Krasia, C. S. Patrickios, Macromolecules 2006, 39, 2467-2473.

[89] M. Achilleos, T. Krasia-Christoforou, C. S. Patrickios, Macromolecules 2007, 40, $5575-5581$.

[90] K. S. Pafiti, E. Loizou, C. S. Patrickios, L. Porcar, Macromolecules 2010, 43, 51955204.

[91] K. S. Anseth, C. M. Wang, C. N. Bowman, Polymer 1994, 35, 3243-3250.

[92] K. S. Anseth, N. A. Peppas, C. N. Bowman, f. Polym. Sci. Part A Polym. Chem. 1994, 32, 139-147.

[93] K.-M. Jeong, K. Koseki, J. Photopolym. Sci. Technol. 2005, 18, 165-170.

[94] A. Hancock, L. Lin, Pigment Resin Technol. 2004, 32, 280-286. 
[95] B. B. Yang, Investigation of UV Curable Coatings and Adhesives by Real Time FTIR, Exton, 2005.

[96] L. G. Lovell, K. a. Berchtold, J. E. Elliott, H. Lu, C. N. Bowman, Polym. Adv. Technol. 2001, 12, 335-345.

[97] K. S. Anseth, K. J. Anderson, C. N. Bowman, Macromol. Chem. Phys. 1996, 848, 833-848.

[98] Q. Yu, S. Nauman, J. P. Santerre, S. Zhu, F. Appl. Polym. Sci. 2001, 82, 1107-1117.

[99] K. A. Berchtold, T. W. Randolph, C. N. Bowman, Macromol. Chem. Phys. 2005, 38, 6954-6964.

[100] J. E. Elliott, C. N. Bowman, Macromolecules 2001, 34, 4642-4649.

[101] N. Hayki, L. Lecamp, N. Désilles, P. Lebaudy, Macromolecules 2010, 43, 177-184.

[102] B. Youssef, U. De Rouen, P. E. Blondel, M. Saint, Polymer 1997, 38, 6089-6096.

[103] E. Andrzejewska, M. B. Bogacki, Macromol. Chem. Phys. 1997, 198, 1649-1664.

[104] L. Lecamp, B. Youssef, C. Bunel, P. Lebaudy, Polymer 1999, 40, 6313-6320.

[105] X. Ramis, J. M. Morancho, A. Cadenato, J. M. Salla, X. Fernández-Francos, Thermochim. Acta 2007, 463, 81-86.

[106] A. K. O. Brien, C. N. Bowman, Macromolecules 2006, 39, 2501-2506.

[107] M. Pilkenton, J. Lewman, R. Chartoff, J. Appl. Polym. Sci. 2010, 119, 2359-2370.

[108] H. L. Bos, J. J. H. Nusselder, Polymer 1994, 35, 2793-2799.

[109] M. Podgorski, f. Appl. Polym. Sci. 2009, 112, 2942-2952.

[110] L. F. Francis, A. V. M. C. Cormick, D. M. Vaessen, E. K. Company, f. Mater. Sci. 2002, 7, 4717-4731.

[111] M. Podgórski, f. Therm. Anal. Calorim. 2012, 111, 1235-1242.

[112] S. G. Croll, f. Coatings Technol. Res. 2009, 7, 49-55.

[113] M. Shibayama, Macromol. Chem. Phys. 1998, 199, 1-30.

[114] F. Asgarzadeh, P. Ourdouillie, E. Beyou, P. Chaumont, Macromolecules 1999, 32, 6996-7002.

[115] R. Liu, W. Oppermann, Macromolecules 2006, 39, 4159-4167. 
[116] K. Dusek, H. Galina, J. Mikes, Polym. Bull. 1980, 25, 19-25.

[117] M. Wen, L. E. Scriven, A. V Mccormick, Macromolecules 2003, 36, 4140-4150.

[118] M. Krzeminski, M. Molinari, M. Troyon, X. Coqueret, Macromolecules 2010, 43, 3757-3763.

[119] N. Ide, T. Fukuda, Macromolecules 1999, 32, 95-99.

[120] Q. Yu, M. Zhou, Y. Ding, B. Jiang, S. Zhu, Polymer 2007, 48, 7058-7064.

[121] T. Norisuye, T. Morinaga, Q. Tran-Cong-Miyata, A. Goto, T. Fukuda, M. Shibayama, Polymer 2005, 46, 1982-1994.

[122] R. Wang, Y. Luo, B.-G. Li, S. Zhu, Macromolecules 2009, 42, 85-94.

[123] Q. Liu, P. Zhang, A. Qing, Y. Lan, M. Lu, Polymer 2006, 47, 2330-2336.

[124] J. Bünsow, M. Mänz, P. Vana, D. Johannsmann, Macromol. Chem. Phys. 2010, 211, 761-767.

[125] J. Huang, W.-J. Wang, B.-G. Li, S. Zhu, Macromol. Mater. Eng. 2013, 298, 391-399.

[126] R. Nicolaÿ, J. Kamada, A. Van Wassen, K. Matyjaszewski, Macromolecules 2010, 43, 4355-4361.

[127] B. Tieke, in Makromol. Chemie, Wiley-VCH, Weinheim, 2005, S. 306-310.

[128] F. C. Auluck, D. S. Kothari, f. Chem. Phys. 1943, 11, 387.

[129] L. R. G. Treloar, in Phys. Rubber Elast., Oxford University Press, New York, 1975, S. $28-30$.

[130] M. Rubinstein, R. H. Colby, in Polym. Phys., Oxford University Press, New York, 2003, S. 253.

[131] B. Tieke, in Makromol. Chemie, Wiley-VCH, Weinheim, 2005, S. 310-320.

[132] H. Meyer, C. Ferri, Helv. Chim. Acta 1934, 18, 570-589.

[133] W. Kuhn, Kolloid-Zeitschrift 1936, 76, 258-271.

[134] W. Kuhn, Kolloid-Zeitschrift 1934, 68, 2-15.

[135] B. M. Brunner, Untersuchungen Zur Inneren Struktur von Hydrogelen Aus N Isopropylacrylamid Mittels Statischer Lichtstreuung, Universität Stuttgart, 2005.

[136] M. Rubinstein, R. H. Colby, in Polym. Phys., Oxford University Press, New York, 2003, S. 255-266. 
[137] M. L. Susoff, Dynamik Polymerer Sonden in Halbverdünnten Polystyrol-Lösungen Und -Gelen, TU Clausthal, 2009.

[138] P. J. Flory, f. Chem. Phys. 1977, 66, 5720-5729.

[139] F. Wall, P. J. Flory, f. Chem. Phys. 1951, 19, 1435-1439.

[140] J. J. Hermans, Trans. Faraday Soc. 1946, 43, 591-600.

[141] P. J. Flory, J. Rehner, f. Chem. Phys. 1943, 11, 521-526.

[142] P. J. Flory, J. Rehner, f. Chem. Phys. 1944, 12, 412-414.

[143] F. T. Wall, f. Chem. Phys. 1942, 10, 132.

[144] F. T. Wall, f. Chem. Phys. 1943, 11, 527.

[145] H. M. James, E. Guth, f. Chem. Phys. 1943, 11, 455.

[146] H. M. James, E. Guth, f. Chem. Phys. 1947, 15, 669.

[147] B. E. Eichinger, Macromolecules 1972, 5, 647-648.

[148] W. W. Graessley, Macromolecules 1975, 8, 186-190.

[149] W. W. Graessley, Macromolecules 1975, 8, 865-868.

[150] P. J. Flory, f. Chem. Phys. 1942, 10, 51.

[151] M. Huggins, f. Phys. Chem. 1942, 46, 151-158.

[152] G. Rehage, Kolloid-Zeitschrift + Zeitschrift für Polym. 1963, 194, 16-34.

[153] G. Rehage, Kolloid-Zeitschrift + Zeitschrift für Polym. 1964, 196, 97-125.

[154] G. Rehage, f. Polym. Sci. 1958, 30, 271-284.

[155] H.-G. Elias, in Makromoleküle Band 2 Phys. Strukt. Und Eig., Wiley-VCH, Weinheim, 2001, S. 304-310.

[156] J. Frenkel, Rubber Chem. Tech. 1940, 13, 264.

[157] M. Rubinstein, R. H. Colby, in Polym. Phys., Oxford University Press, New York, 2003, S. 274-280.

[158] F. R. Schwarzl, in Polymermechanik, Springer-Verlag, Berlin, 1990, S. 123-130.

[159] H.-G. Elias, in Makromoleküle Band 2 Phys. Strukt. Und Eig., Wiley-VCH, Weinheim, 1990, S. 519-532. 
[160] T. Young, A Course of Lectures on Natural Philosophy and the Mechanical Arts, Taylor And Walton, London, 1845.

[161] V. F. Babich, Y. M. Sivergin, A. A. Berlin, A. L. Rabinovich, Polym. Mechnics 1966, 2, 1-3.

[162] M. Gottlieb, W. Macosko, G. S. Benjamin, Macromolecules 1981, 1039-1046.

[163] B. J. R. Scholtens, L. T. Hillegers, f. Polym. Sci. Polym. Phys. Ed. 1985, 23, 23772385.

[164] M. A. Semsarzadeh, S. M. Barikani, Macromol. Symp. 2006, 239, 245-250.

[165] B. Tieke, in Makromol. Chemie, Wiley-VCH, Weinheim, 2005, S. 330-333.

[166] R. Rotzoll, Tailored Silica - Polymer Composites and ABA Type Copolymers: Polymerization Kinetics, Structural Design and Mechanical Properties, GeorgAugust-Universität Göttingen, 2011.

[167] H.-G. Elias, in Makromoleküle Band 2 Phys. Strukt. Und Eig., Wiley-VCH, Weinheim, 1990, S. 627-631.

[168] S. Sun, M. Li, A. Liu, Int. F. Adhes. Adhes. 2013, 41, 98-106.

[169] B. K. Ahn, J. Sung, N. Kim, S. Kraft, X. S. Sun, Polym. Int. 2013, 62, 1293-1301.

[170] A. Kowalski, Z. Czech, Ł. Byczyński, f. Coatings Technol. Res. 2013, 10, 879-885.

[171] Z. Czech, A. Butwin, U. Głuch, J. Kabatc, f. Appl. Polym. Sci. 2012, 123, 118-123.

[172] Z. Czech, A. Kowalczyk, J. Kabatc, J. Świderska, Eur. Polym. f. 2012, 48, 1446-1454.

[173] J. Chauvet, J. M. Asua, J. R. Leiza, Polymer 2005, 46, 9555-9561.

[174] S. Mishra, J. Singh, V. Choudhary, f. Appl. Polym. Sci. 2009, 115, 549-557.

[175] A. Aymonier, E. Papon, G. Castelein, M. Brogly, P. Tordjeman, F. Colloid Interface Sci. 2003, 268, 341-347.

[176] A. Falsafi, M. Tirrell, A. V. Pocius, Langmuir 2000, 16, 1816-1824.

[177] Z. Czech, A. Butwin, J. Kabatc, Eur. Polym. f. 2011, 47, 225-229.

[178] S. D. Tobing, A. Klein, J. Appl. Polym. Sci. 2001, 79, 2230-2244.

[179] I. Benedek, Pressure-Sensitive Adhesives and Applications, Marcel Dekker, Inc., New York, 2004. 
[180] C. Bischof, A. Bauer, W. Possart, R. Kapelle, R. D. Schulze, Acta Polym. 1989, 40, 214-221.

[181] C. Verdier, J. Piau, f. Polym. Sci. Part B Polym. Phys. 2003, 41, 3139-3149.

[182] I. Benedek, in Press. Anhesives Appl., Marcel Dekker, Inc., New York, 2004, S. 89145.

[183] K. W. Allen, Encycl. Phys. Sci. Tech. 2001, 237-250.

[184] C. Y. Hui, Y. Y. Lin, J. M. Baney, f. Polym. Sci. Part B Polym. Phys. 2000, 38, 14851495.

[185] C. Creton, L. Leibler, F. Polym. Sci. Part B Polym. Phys. 1996, 34, 545-554.

[186] H. Murakami, K. Futashima, M. Nanchi, S. Kawahara, Eur. Polym. J. 2011, 47, 378384.

[187] A. Zosel, Int. f. Adhes. Adhes. 1998, 18, 265-271.

[188] P. Tordjeman, E. Papon, J.-J. Villenave, f. Polym. Sci. Part B Polym. Phys. 2000, 38, 1201-1208.

[189] H. Lakrout, P. Sergot, C. Creton, f. Adhes. 1999, 69, 307-359.

[190] Tack Rolling Ball (Metric). Norm PSTC-6M, American Pressure Sensitive Tape Council, 2003.

[191] M. Rubinstein, in Polym. Phys., Oxford University Press, New York, 2003, S. 264268.

[192] J. M. G. Cowie, Chemie Und Physik Der Polymeren, Wiley-VCH, Weinhiem, 1976.

[193] H.-G. Elias, in Makromoleküle Band 2 Phys. Strukt. Und Eig., Wiley-VCH, Weinheim, 1990, S.452-461.

[194] B. Tieke, in Makromol. Chemie, Wiley-VCH, Weinheim, 2005, p. S. 300-306.

[195] H.-G. Elias, in Makromoleküle Band 2 Phys. Strukt. Und Eig., Wiley-VCH, Weinheim, 1990, S.452-461.

[196] W. Li, H. Gao, K. Matyjaszewski, Macromolecules 2009, 42, 927-932.

[197] H. Gao, K. Min, K. Matyjaszewski, Macromolecules 2007, 40, 7763-7770.

[198] B. Luy, K. Kobzar, H. Kessler, Angew. Chem. Int. Ed. Engl. 2004, 43, 1092-1094. 
[199] A.-C. Poeppler, Advanced NMR Methodology for the Investigation of Organometallic Compounds in Solution, Georg-August-Universität Göttingen, 2013.

[200] C. Thiele, Concepts Magn. Reson. Part A 2007, 30A, 65-80.

[201] M. Knörgen, K.-F. Arndt, S. Richter, D. Kuckling, H. Schneider, f. Mol. Struct. 2000, $554,69-79$.

[202] P. Trigo-Mouriño, C. Merle, M. R. M. Kroos, B. Luy, R. Gil, Chem. - A Eur. F. 2013, 19, 7013-7019.

[203] A.-C. Poeppler, S. Frischkorn, D. Stalke, M. John, ChemPhysChem 2013, 14, 31033107.

[204] Q. Yu, J. Zhang, M. Cheng, S. Zhu, Macromol. Chem. Phys. 2006, 207, 287-294.

[205] A. N. Nikitin, R. A. Hutchinson, M. Buback, P. Hesse, Macromolecules 2007, 40, 8631-8641.

[206] J. M. Asua, S. Beuermann, M. Buback, P. Castignolles, B. Charleux, R. G. Gilbert, R. a. Hutchinson, J. R. Leiza, A. N. Nikitin, J.-P. Vairon, et al., Macromol. Chem. Phys. 2004, 205, 2151-2160.

[207] J. Barth, M. Buback, P. Hesse, T. Sergeeva, Macromolecules 2010, 43, 4023-4031.

[208] C. Plessis, G. Arzamendi, J. M. Alberdi, A. M. van Herk, J. R. Leiza, J. M. Asua, Macromol. Rapid Commun. 2003, 24, 173-177.

[209] C. Plessis, G. Arzamendi, J. R. Leiza, H. A. S. Schoonbrood, D. Charmot, J. M. Asua, Macromolecules 2000, 33, 5041-5047.

[210] A. N. F. Peck, R. A. Hutchinson, Macromolecules 2004, 37, 5944-5951.

[211] M. Buback, P. Hesse, I. Lacík, Macromol. Rapid Commun. 2007, 28, 2049-2054.

[212] N. M. Ahmad, B. Charleux, C. Farcet, C. J. Ferguson, S. G. Gaynor, B. S. Hawkett, F. Heatley, B. Klumperman, D. Konkolewicz, P. a Lovell, et al., Macromol. Rapid Commun. 2009, 30, 2002-21.

[213] J. P. Heuts, G. T. Russell, Eur. Polym. f. 2006, 42, 3-20.

[214] Y. Reyes, J. M. Asua, Macromol. Rapid Commun. 2011, 32, 63-7.

[215] C. Barner-Kowollik, M. Buback, B. Charleux, M. L. Coote, M. Drache, T. Fukuda, A. Goto, B. Klumperman, A. B. Lowe, J. B. Mcleary, et al., F. Polym. Sci. Part A Polym. Chem. 2006, 44, 5809-5831.

[216] J. Barth, M. Buback, W. Meiser, P. Vana, Macromolecules 2010, 43, 51-54. 
[217] E. Meyer, Prog. Surf. Sci. 1992, 41, 3-49.

[218] H.-J. Butt, B. Cappella, M. Kappl, Surf. Sci. Rep. 2005, 59, 1-152.

[219] B. V Derjaguin, V. M. Muller, Y. P. Toporov, f. Colloid Interface Sci. 1975, 53, 314326.

[220] V. M. Muller, B. V. Derjaguin, Y. P. Toporov., Colloids and Surfaces 1983, 7, 251259.

[221] T. J. Young, M. a Monclus, T. L. Burnett, W. R. Broughton, S. L. Ogin, P. Smith, Meas. Sci. Technol. 2011, 22, 1-6.

[222] M. Krzeminski, M. Molinari, M. Troyon, X. Coqueret, Macromolecules 2010, 43, 8121-8127.

[223] W. Meiser, J. Barth, M. Buback, H. Kattner, P. Vana, Macromolecules 2011, 44, 2474-2480.

[224] W. Meiser, M. Buback, Macromol. Rapid Commun. 2011, 32, 1490-4.

[225] W. Meiser, Investigation of the Kinetics and Mechanism of RAFT Polymerization via EPR Spectroscopy, Georg-August-Universität Göttingen, 2012.

[226] Y. Mai, A. Eisenberg, Chem. Soc. Rev. 2012, 41, 5969-85.

[227] P. Mischke, Filmbildung in Modernen Lacksystemen, Vincentz Network GmBH, Hannover, 2007.

[228] S. Dietzel, W. Rauh, A. Schiller, P. Stolper, P. Vana, R. Henkel, Beeinflussung Der Polymerisation von UV-Härtenden Druckfarben Durch Zugabe von RAFT-Agenzien Zur Verbesserung Der Recyclierbarkeit von Druckprodukten, München, 2013.

[229] BASF, Laromer 9013 Technisches Datenblatt, 2010.

[230] W. Meiser, Investigation of the Kinetics and Mechanism of RAFT Polymerization via EPR Spectroscopy, Georg-August-Universität Göttingen, 2012.

[231] B. Neises, W. Steglich, Angew. Chem. 1976, 90, 556-557.

[232] N. Förster, Nano-Carrier Synthesis via Z-RAFT Star Polymerisation, GeorgAugust-Universität Göttingen, 2012.

[233] Prüfsysteme Prüfmaschinen Und Prüfsysteme Für Kunststoffe Und Gummi, Zwick Roell AG, Ulm.

[234] B. Pittenger, N. Erina, C. Su, Bruker Corporation, 2010. 
[235] J.-H. Schütz, Bioinspirierte Titin-analoge Polymere, Georg-August-Universität Göttingen, 2014.

[236] M. Buback, C. H. Kurz, C. Schmaltz, Macromol. Chem. Phys. 1998, 199, 1721-1727.

[237] L. Fuente, M. Ferna, J. Appl. Polym. Sci. 2000, 80, 783-789.

[238] S. Beuermann, M. Buback, Prog. Polym. Sci. 2002, 27, 191-254.

[239] L. J. Hughes, G. L. Brown, f. Appl. Polym. Sci. 1961, 5, 580-588.

[240] A. F. M. Barton, in CRC Handb. Polmyer-Liquid Interact. Parameters Solubility Parameters, CRC Press, Boca Raton, 1990, S. 153.

[241] G. Meichsner, T. G. Mezger, J. Schröder, Lackeigenschaften Messen Und Steuern, Vincentz Network GmBH, Hannover, 2003. 



\section{Verzeichnis der Abkürzungen}

AFM

AM

ATRP

BA

BDDA

BEPBT

bzw.

CTA

DET

DMA

DMT

DSC

EA

ESR

EPHT

FOGRA

FTIR

HDPE

i. d. R.

MA

MCR

MMA

MMMP

NMR

NMP

NVP

PEG

PEGDA

PRE

PSA

QNM

RAFT

RDRP
Atomic Force Microscopy

Acrylamid

Atom Transfer Radical Polymerization

n-Butylacrylat

1,4-Butandioldiacrylat

$\operatorname{Bis(S-Ethylpropan-2-ylonat)-S-1,4-butylbistrithiocarbonat~}$

beziehungsweise

Chain Transfer Agent

$S$-S'-Diethyltrithiocarbonat

Dynamisch-Mechanische Analyse

Dejarguin-Muller-Toporov

Differential Scanning Calorimetry

Ethylacrylat

Elektronen-Spin-Resonanz

$S$-Ethylpropan-2-ylonat- $S^{\prime}$-hexyltrithiocarbonat

Forschungsgesellschaft Druck e. V.

Fourier-Transformations-Infrarot-Spetroskopie

High Density Polyethylen

in der Regel

Methylacrylat

midchain radical

Methylmethacrylat

2-Methyl-4'-(methylthio)-2-morpholinopropiophenon

Nuclear Magnetic Resonance

Nitroxide Mediated Polymerisation

$N$-Vinylpyrrolidon

Polyethylenglycol

Polyethylenglycoldiacrylat

Persistent Radical Effect

Pressure Sensitive Adhesiv

Quantitative Nanomechanical Mapping

Reversible Addition Fragmentation chain-Transfer

Reversibel-Desaktivierte Radikalische Polymerisation 
SDK

SPR

TEMPO

u. a.

UV

VAc

Vis

z. B.

\section{Symbolverzeichnis}

A

$\Delta A_{\mathrm{def}}$

$\Delta \bar{A}_{\text {mix }}$

$\Delta A_{\mathrm{Q}}$

$A_{\lambda}$

$B$

$\mathrm{C}-\mathrm{C}$

$C_{\mathrm{M}}$

$c_{\mathrm{I}-\mathrm{I}}$

c

c

$c_{\mathrm{M}}$

$c_{\mathrm{X}}$

$d$

$d$

$\delta$

$\chi$

E

E

$\varepsilon_{\mathrm{E}}$

$\eta$

F

$f$

$f_{\mathrm{I}}$

G

$\gamma$

$\gamma_{\mathrm{B}}$
Spannungs-Dehnungs-Kurve

secondary chain-end radical

2,2,6,6-Tetramethylpiperidinyloxyl

unter anderem

ultraviolett

Vinylacetat

visible: engl. für sichtbar

zum Beispiel

Strukturfaktor

Änderung der freien Energie

Änderung der molaren freien Mischungsenthalpie

Änderung der freien Enthalpie der Quellung

Absorbanz

Volumenfaktor

Kohlenstoff-Kohlenstoff-Bindung

Monomerumsatz

Initiatorkonzentration

Konzentration

Lichtgeschwindigkeit

Konzentration des Monomers

Konzentration des Transferagens

Schichtdicke

Durchmesser

Löslichkeitsparameter

Flory-Huggins-Wechselwirkungsparameter

Elastizitätsmodul

Energie

molarer dekadischer Extinktionskoeffzient

Dilatationsfaktor

Kraft

Funktionalität

Initiatoreffizienz

Schermodul

Dehnung

Bruchdehnung 


\begin{tabular}{|c|c|}
\hline$\Delta \bar{H}_{\text {mix }}$ & Änderung der Mischungsenthalpie \\
\hline$\Delta H_{t}$ & Reaktionswärme \\
\hline$\Delta H_{0}^{\text {th }}$ & theoretische Reaktionswärme \\
\hline$h$ & Planck'sche Wirkungsquantum \\
\hline$I$ & Intensität \\
\hline I $\cdot$ & Primärradikal \\
\hline$k_{\mathrm{d}}$ & Geschwindigkoeffizient der Zerfallsreaktion des Initiators \\
\hline$K_{\mathrm{P}}$ & Proportionalitätsfaktor der radikalischen Polymerisation \\
\hline$k_{\mathrm{B}}$ & Boltzmann-Konstante \\
\hline$k_{\mathrm{p}}$ & Geschwindigkeitskoeffizient der Propagation \\
\hline$k_{\mathrm{t}}$ & Geschwindigkeitskoeffizient der Terminierung \\
\hline$K_{\mathrm{tr}}$ & Transferkonstante \\
\hline$k_{\mathrm{tr}}$ & Geschwindigkeitskoeffizient der Transferreaktion \\
\hline M & Monomer \\
\hline$M$ & molare Masse \\
\hline $\bar{M}_{\mathrm{e}}$ & zahlenmittlere Molmasse zwischen zwei Verhakungen \\
\hline $\bar{M}_{n}$ & zahlenmittlere Molmasse \\
\hline $\bar{M}_{\mathrm{s}}$ & theoretische, zahlenmittlere Molmasse der Netzketten \\
\hline $\bar{M}_{\mathrm{x}}$ & scheinbare, zahlenmittlere Molmasse der Netzketten \\
\hline$m$ & Masse \\
\hline$N_{\mathrm{A}}$ & Avogadro-Konstante \\
\hline$n$ & Stoffmenge \\
\hline$n$ & Anzahl \\
\hline$n_{\mathrm{x}}$ & Stoffmenge des Vernetzers \\
\hline$v$ & Frequenz \\
\hline$\tilde{v}$ & Wellenzahl \\
\hline$v_{\text {eff }}$ & effektive Netzkettendichte \\
\hline$v_{\text {th }}$ & theoretische Netzkettendichte \\
\hline$L$ & Länge \\
\hline$L_{\mathrm{D}}$ & lineare Deformation \\
\hline$l$ & Dehnungsverhältnis \\
\hline$\lambda$ & Wellenlänge \\
\hline$\Omega$ & Effizienz der Vernetzungsreaktion \\
\hline$P$. & Polymerradikal \\
\hline$\phi_{\mathrm{Q}}$ & Quantenausbeute \\
\hline$\Phi$ & Volumenbruch \\
\hline$Q_{m}$ & Massenquellungsgrad \\
\hline$Q_{V}$ & Volumenquellungsgrad \\
\hline
\end{tabular}




$\begin{array}{ll}r_{\mathrm{d}} & \text { Geschwindigkeit der Zerfallsreaktion des Initiators } \\ \mathrm{R} & \text { Molekülrest } \\ R_{0} & \text { End-zu-End-Abstand der Netzketten } \\ R & \text { ideale Gaskonstante } \\ R_{\mathrm{P}} & \text { Gesamtgeschwindigkeit der radikalischen Polymerisation } \\ r_{\mathrm{p}} & \text { Geschwindigkeit der Propagation } \\ r_{\mathrm{t}} & \text { Geschwindigkeit der Terminierung } \\ r_{\mathrm{tr}} & \text { Geschwindigkeit des Radikaltransfers } \\ \rho & \text { Dichte } \\ \Delta S & \text { Änderung der Entropie } \\ \Delta \bar{S}_{\mathrm{mix}} & \text { Änderung der Mischungsentropie } \\ \sigma & \text { Spannung } \\ \sigma_{\mathrm{w}} & \text { wahre Zugspannung } \\ T & \text { Temperatur } \\ t & \text { Zeit } \\ t_{\mathrm{Gel}} & \text { Zeitpunkt, an dem der Gel-Effekt einsetzt } \\ \Delta U & \text { Änderung der inneren Energie } \\ V & \text { Volumen } \\ w & \text { Deformationsarbeit pro Einheitsvolumen } \\ \mathrm{X} & \text { Transferagens }\end{array}$

\section{Indizes}

0

DB

eq

I

int

korr

LM

Pol

M

$\tilde{v}$

mol

Vern

X

$\mathrm{y}$

$\mathrm{Z}$
Anfangswert

Doppelbindung

Gleichgewicht

Initiator

integriert

korrigiert

Lösungsmittel

Polymer

Monomer

Wellenzahl

molar

Vernetzer

$\mathrm{x}$-Richtung

$y$-Richtung

z-Richtung 




\section{Lebenslauf}

\section{Persönliche Daten:}

Name:

Rouven Christoph Henkel

Geburtsdatum:

31. Mai 1983 in Salzgitter

Nationalität:

Deutsch

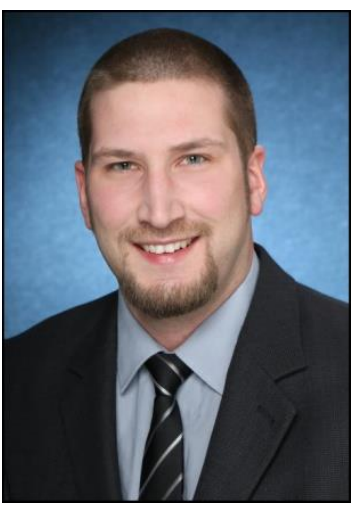

Schulische Ausbildung:

$1995-2002$

Gymnasium am Fredenberg in Salzgitter

Abschluss: Abitur $(2,4)$

\section{Grundwehrdienst:}

07/2002 - 03/2003 Wehrpflichtiger, Panzerlehrbatallion 334/Celle

\section{Studium:}

$04 / 2003-04 / 2010$

Technische Universität Clausthal

Studiengang: Chemie; Abschluss: Diplom $(1,2)$

$11 / 2005$

Diplomvorprüfung $(1,7)$

$08 / 2009-04 / 2010$

Diplomarbeit in der Arbeitsgruppe von Prof. Dr. W. Oppermann zu dem Thema:

„Synthese und Charakterisierung von linearen und vernetzten Polymeren über ATRP“ $(1,1)$

$08 / 2010-06 / 2014$

Promotion an der Georg-August-Universität Göttingen in der Arbeitsgruppe von Prof. Dr. P. Vana zu dem Thema:

„Der Einfluss der UV-initiierten RAFT-Polymerisation auf die Strukturen und Eigenschaften von Polymernetzwerken“ 